Surveillance for Certain Health Behaviors, Chronic Diseases, and Conditions, Access to Health Care, and Use of Preventive Health Services Among States and Selected Local Areas - Behavioral Risk Factor Surveillance System, United States, 2012

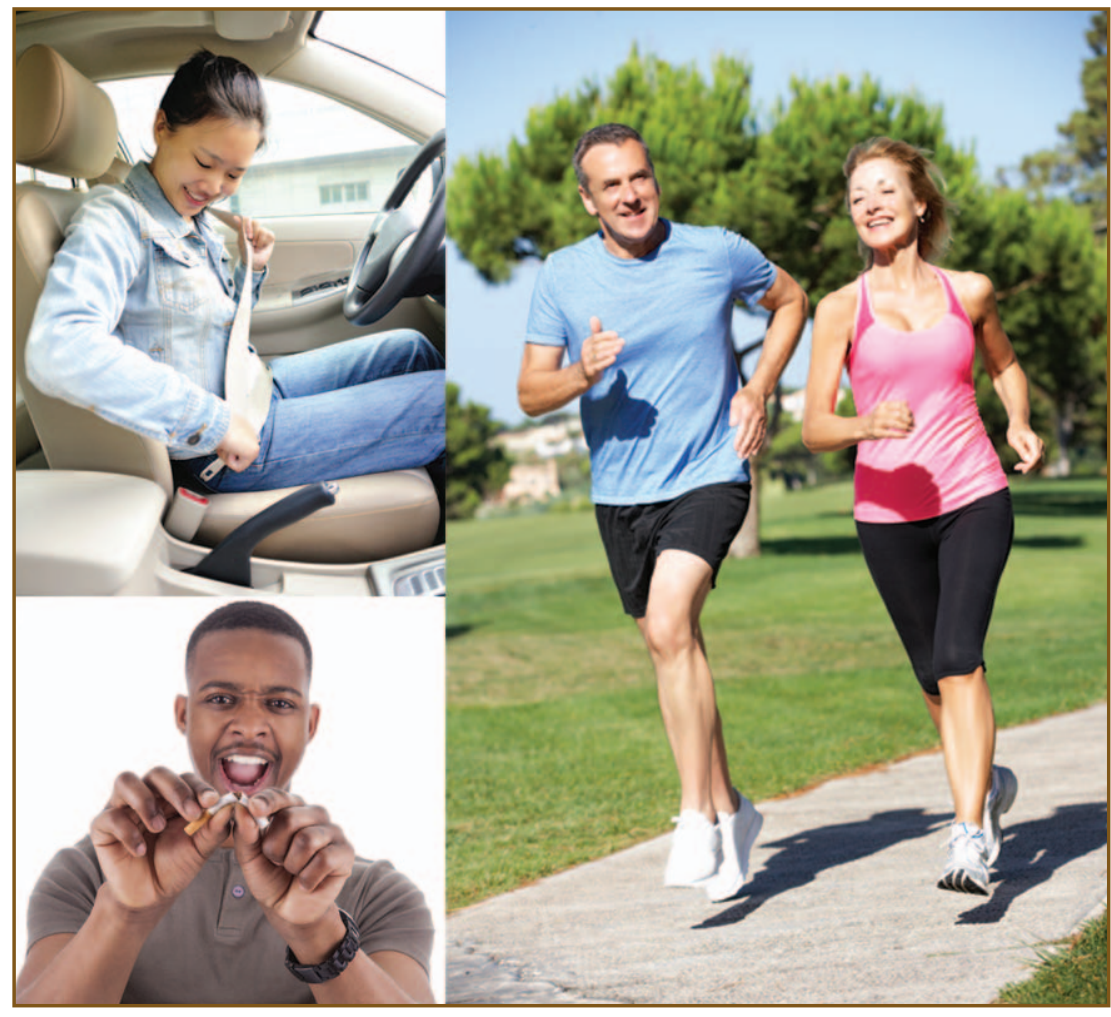




\section{CONTENTS}

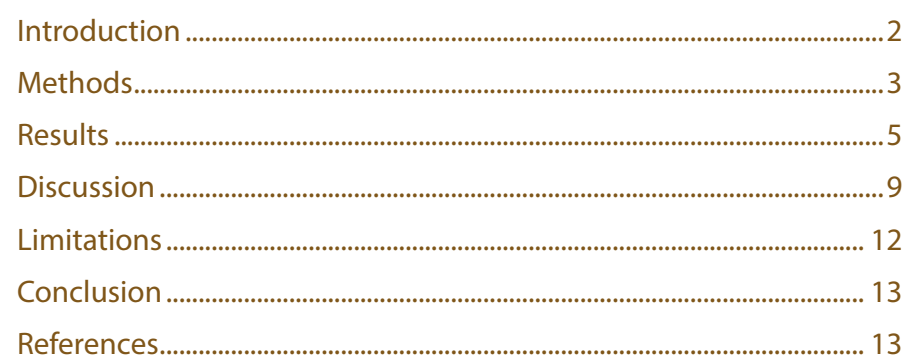

The MMWR series of publications is published by the Center for Surveillance, Epidemiology, and Laboratory Services, Centers for Disease Control and Prevention (CDC), U.S. Department of Health and Human Services, Atlanta, GA 30329-4027.

Suggested citation: [Author names; first three, then et al., if more than six.] [Title]. MMWR Surveill Summ 2016;65(No. SS-\#):[inclusive page numbers].

\section{Centers for Disease Control and Prevention}

Thomas R. Frieden, MD, MPH, Director

Harold W. Jaffe, MD, MA, Associate Director for Science

Joanne Cono, MD, ScM, Director, Office of Science Quality

Chesley L. Richards, MD, MPH, Deputy Director for Public Health Scientific Services

Michael F. Iademarco, MD, MPH, Director, Center for Surveillance, Epidemiology, and Laboratory Services

\section{MMWR Editorial and Production Staff (Serials)}

Sonja A. Rasmussen, MD, MS, Editor-in-Chief

Charlotte K. Kent, $\mathrm{PhD}$, MPH, Executive Editor

Christine G. Casey, MD, Editor

Teresa F. Rutledge, Managing Editor

David C. Johnson, Lead Technical Writer-Editor

Denise Williams, MBA, Project Editor
Martha F. Boyd, Lead Visual Information Specialist

Maureen A. Leahy, Julia C. Martinroe,

Stephen R. Spriggs, Moua Yang, Tong Yang, Visual Information Specialists

Quang M. Doan, MBA, Phyllis H. King, Terraye M. Starr, Information Technology Specialists
MMWR Editorial Board

Timothy F. Jones, MD, Chairman Matthew L. Boulton, MD, MPH Virginia A. Caine, MD

Katherine Lyon Daniel, $\mathrm{PhD}$

Jonathan E. Fielding, MD, MPH, MBA

David W. Fleming, MD
William E. Halperin, MD, DrPH, MPH

King K. Holmes, MD, PhD

Robin Ikeda, MD, MPH

Rima F. Khabbaz, MD

Phyllis Meadows, PhD, MSN, RN

Jewel Mullen, MD, MPH, MPA
Jeff Niederdeppe, PhD

Patricia Quinlisk, MD, MPH

Patrick L. Remington, MD, MPH Carlos Roig, MS, MA

William L. Roper, MD, MPH

William Schaffner, MD 


\title{
Surveillance for Certain Health Behaviors, Chronic Diseases, and Conditions, Access to Health Care, and Use of Preventive Health Services Among States and Selected Local Areas - Behavioral Risk Factor Surveillance System, United States, 2012
}

\author{
Pranesh P. Chowdhury, $\mathrm{MD}^{1}$ \\ Tebitha Mawokomatanda, MSPH ${ }^{1}$ \\ Fang $\mathrm{Xu}, \mathrm{PhD}^{1}$ \\ Sonya Gamble, $\mathrm{MS}^{1}$ \\ David Flegel, MS ${ }^{2}$ \\ Carol Pierannunzi, $\mathrm{PhD}^{1}$ \\ William Garvin, $\mathrm{MS}^{1}$ \\ Machell Town, $\mathrm{PhD}^{1}$ \\ ${ }^{1}$ Division of Population Health, National Center for Chronic Disease Prevention and Health Promotion, CDC \\ ${ }^{2}$ Northrop Grumman Corporation, Atlanta, Georgia
}

\begin{abstract}
Problem: Chronic diseases (e.g., heart diseases, cancer, chronic lower respiratory disease, stroke, diabetes, and arthritis) and unintentional injuries are the leading causes of morbidity and mortality in the United States. Behavioral risk factors (e.g., tobacco use, poor diet, physical inactivity, excessive alcohol consumption, failure to use seat belts, and insufficient sleep) are linked to the leading causes of death. Modifying these behavioral risk factors and using preventive health services (e.g., cancer screenings and influenza and pneumococcal vaccination of adults aged $\geq 65$ years) can substantially reduce morbidity and mortality in the U.S. population. Continuous monitoring of these health-risk behaviors, chronic conditions, and use of preventive services are essential to the development of health promotion strategies, intervention programs, and health policies at the state, city, and county level.
\end{abstract}

Reporting Period: January-December 2012.

Description of the System: The Behavioral Risk Factor Surveillance System (BRFSS) is an ongoing, state-based, randomdigit-dialed landline- and cellular-telephone survey of noninstitutionalized adults aged $\geq 18$ years residing in the United States. BRFSS collects data on health-risk behaviors, chronic diseases and conditions, access to health care, and use of preventive health services related to the leading causes of death and disability. This report presents results for all 50 states, the District of Columbia, participating U.S. territories that include the Commonwealth of Puerto Rico (Puerto Rico) and Guam, 187 Metropolitan/ Micropolitan Statistical Areas (MMSAs), and 210 counties ( $\mathrm{n}=$ 475,687 survey respondents) for the year 2012.

Results: In 2012, the estimated prevalence of health-risk behaviors, chronic diseases or conditions, access to health care, and use of preventive health services substantially varied by state and territory, MMSA, and county. The following portion of the abstract lists a summary of results by selected BRFSS measures. Each set of proportions refers to the range of estimated prevalence for health-risk behaviors, chronic diseases or conditions, and use of preventive health care services among geographical units, as reported by survey respondents. Adults with good or better health: 64.0\%-88.3\% for states and territories, $62.7 \%-90.5 \%$ for MMSAs, and $68.1 \%-$ $92.4 \%$ for counties. Adults aged 18-64 years with health care coverage: $64.2 \%-93.1 \%$ for states and territories, $35.4 \%-93.7 \%$ for MMSAs, and 35.4\%-96.7\% for counties. Adults who received a routine physical checkup during the preceding 12 months: $55.7 \%-80.1 \%$ for states and territories, $50.6 \%-85.0 \%$ for MMSAs, and $52.4 \%-85.0 \%$ for counties. An influenza vaccination received during the preceding 12 months among adults aged $\geq 65$ years: $26.3 \%-70.1 \%$ for states and territories, $20.8 \%-77.8 \%$ for MMSAs, and 24.1\%-77.6\% for counties. Ever received pneumococcal vaccination among adults aged $\geq 65$ years: $22.2 \%-76.2 \%$ for states and territories, $15.3 \%-83.4 \%$ for MMSAs, and $25.8 \%-85.2 \%$ for counties. Adults who had a dental visit in the past year: $53.7 \%-76.2 \%$ for states and territories, and $44.8 \%-81.7 \%$ for MMSAs and counties. Adults aged $\geq 65$ years who have lost all of their natural teeth from tooth decay or gum disease: $7.0 \%-33.7 \%$ for states and territories, $5.8 \%-39.6 \%$ for MMSAs, and $5.8 \%-37.1 \%$ for counties. Adults aged 50-75 years who received a colorectal cancer screening on the basis of the U.S. Preventive

Corresponding author: Machell Town, PhD, Division of Population Health, National Center for Chronic Disease Prevention and Health Promotion, CDC. Telephone: 770-488-4681; E-mail: mpt2@cdc.gov.
Services Task Force recommendation: $40.0 \%-76.4 \%$ for states and territories, $47.1 \%-80.7 \%$ for MMSAs, and $47.0 \%-81.0 \%$ for counties. Women aged 21-65 years who had a Papanicolaou test during the preceding 3 years: $68.5 \%$ to $89.6 \%$ for states 
and territories, $70.3 \%$ to $92.8 \%$ for MMSAs, and $65.7 \%-94.6 \%$ for counties. Women aged $50-74$ years who had a mammogram during the preceding 2 years: $66.5 \%-89.7 \%$ for states and territories, $61.1 \%-91.5 \%$ for MMSAs, and 61.8\%-91.6\% for counties. Current cigarette smoking among adults: $10.6 \%-28.3 \%$ for states and territories, $5.1 \%-30.1 \%$ for MMSAs, and 5.1\%-28.3\% for counties. Binge drinking among adults during the preceding month: 10.2\%-25.2\% for states and territories, $6.2 \%-28.1 \%$ for MMSAs, and 6.2\%-29.5\% for counties. Heavy drinking among adults during the preceding month: 3.5\%-8.5\% for states and territories, $2.0 \%-11.0 \%$ for MMSAs, and 1.9\%-11.0\% for counties. Adults who reported no leisure-time physical activity: $16.3 \%-42.4 \%$ for states and territories, $9.2 \%-47.3 \%$ for MMSAs, and 9.2\%-39.0\% for counties. Self- reported seat belt use: 62.0\%-93.7\% for states and territories, 54.1\%-97.1\% for MMSAs, and 50.1\%-97.4\% for counties. Adults who were obese: 20.5\%-34.7\% for states and territories, $14.8 \%-44.5 \%$ for MMSAs and counties. Adults with diagnosed diabetes: $7.0 \%-16.4 \%$ for states and territories, 3.4\%-17.4\% for MMSAs, and 3.1\%-17.4\% for counties. Adults who ever had any type of cancer: $3.0 \%-13.7 \%$ for states and territories, 3.8\%-19.2\% for MMSAs, and 4.5\%-19.2\% for counties. Adults with current asthma: 5.8\%-11.1\% for states and territories, 3.1\%-15.0\% for MMSAs, and 3.1\%-15.7\% for counties. Adults with some form of arthritis: $15.6 \%-36.4 \%$ for states and territories, $16.8 \%-45.8 \%$ for MMSAs, and $14.8 \%-35.9 \%$ for counties. Adults having had a depressive disorder: $9.0 \%-23.5 \%$ for states and territories, 9.2\%-28.3\% for MMSAs, and $8.5 \%-28.4 \%$ for counties. Adults aged $\geq 45$ years who have had coronary heart disease: $7.4 \%-19.0 \%$ for states and territories, $6.1 \%-23.3 \%$ for MMSAs, and 6.1\%-20.6\% for counties. Adults aged $\geq 45$ years who have had a stroke: $3.1 \%-7.3 \%$ for states and territories, $2.1 \%-9.3 \%$ for MMSAs, and $1.5 \%-9.3 \%$ for counties. Adults with limited activities because of physical, mental, or emotional problems: $15.0 \%-28.6 \%$ for states and territories, $12.0 \%-31.7 \%$ for MMSAs, and $11.3 \%-31.7 \%$ for counties. Adults using special equipment because of any health problem: $4.8 \%-11.6 \%$ for states and territories, $4.0 \%-14.7 \%$ for MMSAs, and $2.8 \%-13.6 \%$ for counties.

Interpretation: This report underscores the need for continuous surveillance of health-risk behaviors, chronic diseases or conditions, health care access, and use of preventive care services at state and local levels. It will help to identify high-risk populations and to evaluate public health intervention programs and policies designed to reduce morbidity and mortality from chronic disease and injury.

Public Health Action: State and local health departments and agencies can continue to use BRFSS data to identify populations at high risk for unhealthy behaviors and chronic diseases or conditions, lack of health care access, and inadequate use of preventive care services. Additionally, states can use the data to design, implement, monitor, and evaluate public health programs and policies at state and local levels.

\section{Introduction}

The goals of national health promotion and disease prevention are to prevent or delay disease, decrease premature mortality, and improve health-related quality of life for all U.S. residents (1). Chronic diseases (e.g., heart disease, cancer, chronic lower-respiratory disease, stroke, diabetes, and arthritis) and unintentional injuries are the leading causes of death and disability in the United States (2). Engaging in healthy behaviors (e.g., quitting smoking, being more physically active, wearing seat belts while riding in vehicles, getting sufficient sleep, reducing alcohol consumption, and eating a nutritious diet) and using preventive services (e.g., routine medical checkup, blood pressure and cholesterol screening, cancer screening, and recommended vaccinations) can reduce morbidity and premature mortality from these chronic diseases and injuries (3). Monitoring health-risk behaviors, chronic diseases or conditions, and the use of preventive services to help identify high-risk groups with the greatest need for intervention is important for preventing morbidity and mortality and unintentional injuries.

The Behavioral Risk Factor Surveillance System (BRFSS) is a state-based landline and cellular telephone survey conducted by state health departments with assistance from CDC (4). Since 1984, BRFSS has been a unique source of data for health-risk behaviors, chronic diseases or conditions, health care access, and the use of preventive health services for states/territories. Since 2002, the large sample size in BRFSS has facilitated calculation of prevalence estimates for selected Metropolitan and Micropolitan Statistical Areas (MMSAs), metropolitan divisions, and selected counties (5). BRFSS data are frequently used to set health goals as well as to monitor progress of public health programs and policy implementation at national, state, and local levels (G). This report provides prevalence estimates for selected health-risk behaviors, health care access, use of preventive care services, and chronic diseases or conditions by states/territories, selected MMSAs, and selected counties for 2012 . 


\section{Methods}

BRFSS is the largest continuously conducted telephone health survey in the world with approximately 400,000 adult interviews completed each year. BRFSS is conducted by states with assistance from CDC. BRFSS uses a multistage sampling design to select a representative sample of the noninstitutionalized civilian population aged $\geq 18$ years residing in states and territories of the Unites States (7). All the responses were self-reported; proxy interviews are not conducted by BRFSS.

Since 2011, BRFSS includes both landline telephone- and cellular telephone-based surveys. In conducting the BRFSS landline telephone survey, interviewers collect data from a randomly selected adult in a household. Cellular telephone interviews are treated as one-person adult household, and survey interviewers collect data from adults answering the cellular telephones (4). Using a dual-frame survey including combined landline and cellular telephones improved validity, data quality, and representativeness of BRFSS data (8).

Details on methodology, random sampling procedures, design, and reliability and validity of measures used in BRFSS have been described previously (9,10). MMSA and metropolitan divisions are defined by the Office of Management and Budget. County names were collected from the respondents during the demographics section of the interviews and were used to determine the corresponding American National Standards Institute county codes. Respondents were assigned to MMSAs on the basis of their county codes. MMSAs were included in this report if there were $\geq 500$ respondents; similarly counties that had $\geq 500$ respondents were included for county level estimates (11).

This report provides prevalence estimates for selected health risk behaviors, use of preventive health care services, and chronic conditions among residents living in the 50 states, the District of Columbia (DC), Guam, Puerto Rico, 187 MMSAs, and 210 counties.

\section{Questionnaire}

All questions included in BRFSS go through technical review, cognitive testing, and field testing before being placed in the questionnaire. The standard BRFSS questionnaire consists of three sections: core questions, optional modules, and stateadded questions. Eligible respondents answer the same core questions. Optional modules were selected by states on the basis of the specific needs and goals of the programs in each state's health department. Although core questions are always collected on both landline telephone and cellular telephones, optional module data might have been collected by landline telephone or cellular telephone. Not all modules are used by every state, and states can opt out of collecting any module data. States could have chosen to split the modules by dividing the samples so only a portion of the respondents answer certain module questions. In 2012, there were as many as three module versions; there were no split versions of a module whose data were collected only by cellular telephone. State-added questions are developed or acquired by participating states and are added to their questionnaires; they are not edited or evaluated by CDC.

The 2012 core questions (12) were used to inquire about participants' health status, number of healthy days in the past 30 days, health care access, exercise, inadequate sleep, chronic health conditions, oral health, demographics, disability, tobacco use, alcohol consumption, immunization, falls, seat belt use, drinking and driving, breast and cervical cancer screening, prostate cancer screening, colorectal cancer screening, and human immunodeficiency virus/acquired immunodeficiency syndrome (HIV/AIDS). The following optional models were used by at least one state in both the landline and cellular telephone survey during 2012 data collection: adult asthma history (two states), adult human papilloma virus (HPV) (six states), adverse childhood experience (four states), cancer survivorship (six states), childhood asthma prevalence (33 states), childhood immunization (12 states), chronic obstructive pulmonary disease (11 states), diabetes (28 states), excess sun exposure (four states), consumption of fruits and vegetables (five states), general preparedness (two states), HIV/ AIDS (two states), inadequate sleep (four states), mental illness and stigma (11 states), prediabetes (26 states), prostate cancer screening decision making module (three states), random child selection (36 states), reaction to race (two states), shingles (Zostavax or ZOS) (four states), social context (five states), sugar-sweetened beverages and menu labeling (11 states), tetanus-diphtheria vaccination in adults (four states), and veteran's health (two states).

\section{Data Collection and Processing}

BRFSS data are collected according to standard BRFSS protocol across all states, DC, and participating territories (4). States can conduct interviews internally or opt to contract with a private company or university to conduct interviews but maintain standard procedures to ensure respondents' confidentiality, document the quality of the interviewing process, and supervise and monitor the interviewers. Since 2007, BRFSS surveys have been collected monthly in all 50 states, DC, and participating U.S. territories. State-collected data are submitted to CDC for processing, checking, and weighting. 


\section{Sampling}

A BRFSS sample record is one telephone number in the list of all telephone numbers selected for dialing. States obtain two types of samples of telephone numbers from CDC: one for landline telephone respondents and one for cellular telephone respondents. For the landline survey, all 50 states and DC used a disproportionate stratified sample (DSS) design; but Guam and Puerto Rico used a simple random sample design (4). In a DSS design, landline telephone numbers are divided into two groups, or strata: high-density and medium-density strata containing telephone numbers that are expected to belong mostly to households. The two strata are sampled to obtain a probability sample of all households with telephones. For the cellular telephone survey, phone samples are randomly drawn from confirmed cellular telephone sampling frames for each state (4). The target population for cellular telephone samples consist of adults aged $\geq 18$ years, living in households or college housing who have a working cellular telephone, and receive $\geq 90 \%$ of their calls on cellular telephones (13). Because of the portability of the cellular telephone, some of the numbers in the cellular telephone sample will reach respondents who have moved into other states. In those cases, the contacting state completes the core BRFSS interview with respondents from other states; data from out-of-state interviews are transferred to the appropriate states at the end of each data-collection period.

\section{Data Weighting}

In 2011, a new weighting methodology called iterative proportional fitting (or "raking") replaced post stratification. The latter had been used to weight the data every year before 2011. Raking allows incorporation of cellular telephone survey data and permits the introduction of additional demographic characteristics (e.g., education level, marital status, and home renter/owner) in addition to age-race/ethnicity-sex that improves the degree and extent to which the BRFSS sample properly reflects the sociodemographic make-up of both individual states and, when aggregated, the entire United States (14). After combining landline and cellular telephone data, BRFSS performs raking by adjusting one or a combination of demographic categories at a time in an iterative process until a convergence of a set value is reached. During 2012, state-level BRFSS raking included the following demographic categories: sex by age, detailed race and ethnicity groups, education levels, marital status, home renter/owner, sex by race and ethnicity, age groups by race and ethnicity, and phone-source. Those states that used regional weighting also included four additional categories: region, region by age, region by sex, and region by race and ethnicity. The state-level weights were raked to five margins including age group, sex, race and ethnicity group, sex by age group, and sex by race and ethnicity group at the MMSA or county level to produce MMSA or county weights. Information about weighting MMSA and county BRFSS data can be found on the BRFSS SMART webpage (11).

\section{Statistical Analyses}

To account for the complex sampling design of BRFSS, all the prevalence estimates were computed on the basis of a statistical analysis (SAS version 9.3, SAS Institute Inc., Cary, NC, USA) using weights and strata. The prevalence estimates in this report are direct estimates. This report provides unweighted sample size, weighted prevalence estimates with standard errors, and $95 \%$ confidence intervals for prevalence of health-risk behaviors, chronic diseases or conditions, and use of preventive health care services by states and territories, MMSAs, and counties on the basis of 2012 BRFSS data. If the unweighted sample size was represented by $<50$ survey participants or the relative standard error (RSE) was $>30 \%$, the results were suppressed to avoid unstable estimates. RSE was calculated by dividing the standard error by the estimated prevalence and multiplying by 100 (for percent). Responses coded as "do not know" or "refused" were excluded from the analyses.

\section{About This Report}

This report presents the results and a discussion of the following topics: 1) health status indicators (self-rated health status and health care coverage for persons aged 18-64 years), 2) preventive practices (recent routine physical checkup, influenza vaccination, and pneumococcal vaccination for persons aged $\geq 65$ years), 3 ) oral health (dental visit in the past year and loss of all natural teeth among adults aged $\geq 65$ years), 4) cancer screening (e.g., colorectal cancer screening, cervical cancer screening, and breast cancer screening), 5) healthrisk behaviors (current cigarette smoking, binge drinking, heavy drinking, no leisure-time physical activity, and selfreported seat belt use), 6) chronic health conditions (obesity, diagnosed diabetes, cancer survivors, current asthma, arthritis, depression, coronary heart disease, and stroke for persons aged $\geq 45$ years), and 7) disability (activity limitation and use of special equipment because of physical, mental, or emotional problems). The 2012 questionnaire and all related support documents are available on the BRFSS webpage (http://www. cdc.gov/brfss/annual_data/annual_2012.html). 


\section{Results}

In 2012, a total of 475,687 adults completed interviews by landline and cellular telephones. For data collected by landline telephone, 377,013 respondents completed the interview and the numbers of participants ranged from 1,728 in Guam to 18,325 in Massachusetts (median: 6,085). For data collected by cellular telephone, 98,674 respondents completed the interview, with participant numbers ranging from 303 in Guam to 3,990 in Nebraska (median: 1,580). Response rates for BRFSS were calculated using the standard set by the American Association of Public Opinion Research (AAPOR) response rate formula 4 (RR4), which is the number of respondents who completed the survey as a proportion of all eligible and likely eligible persons (15). For landline telephone data, the RR4 response rate ranged from 28.2\% in California to $62.9 \%$ in Puerto Rico (median: $49.1 \%$ ) and the RR4 response rate for cellular telephone data ranged from $16.4 \%$ in Washington to $55.7 \%$ in Iowa (median: $35.3 \%$ ). For combined landline telephone and cellular telephone data, the weighted response rate (based on a combination of the landline telephone response rate with the cellular telephone response rate proportional to the total sample used to collect the data for a state) ranged from $27.7 \%$ in California to $60.4 \%$ in South Dakota (median: 45.2\%). Detailed information on response, cooperation, and refusal rates for landline and cellular telephone data can be found in the BRFSS 2012 Summary Data Quality Report (16).

\section{Health Status Indicators}

\section{Health Status}

Respondents were asked to rate their general health as excellent, very good, good, fair, or poor. Those who reported their general health as excellent, very good, or good were categorized in one group, and the other group included those who reported their general health as fair or poor. In 2012, the estimated prevalence of self-reported good or better health among adults aged $\geq 18$ years ranged from $64.0 \%$ in Puerto Rico to $88.3 \%$ in Minnesota (median: $82.9 \%$ ) (Table 1). Among the selected 187 MMSAs, the estimated prevalence of self-reported good or better health among respondents ranged from $62.7 \%$ in Aguadilla-Isabela, Puerto Rico, to $90.5 \%$ in Fargo, North Dakota-Minnesota, Boulder, Colorado, and Hilton Head Island-Beaufort, South Carolina (median: 83.6\%) (Table 2). Among the selected 210 counties, the estimated prevalence of self-reported good or better health among respondents ranged from $68.1 \%$ in San Juan Municipio, Puerto Rico to $92.4 \%$ in Douglas County, Colorado (median: $84.4 \%$ ) (Table 3).

\section{Health Care Coverage}

Health care coverage was defined as respondents having reported that they had private health insurance, prepaid plans (e.g., health maintenance organizations) or government health plans (e.g., Medicare or Medicaid) among adults aged 18-64 years. In 2012, the estimated prevalence of health care coverage among persons aged 18-64 years ranged from 64.2\% in Texas to $93.1 \%$ in Massachusetts (median: $79.6 \%$ ) (Table 4). Among selected MMSAs, the estimated prevalence ranged from $35.4 \%$ in McAllen-Edinburg-Mission, Texas, to 93.7\% in Cambridge-Newton-Framingham, Massachusetts (median: 80.7\%) (Table 5). Among selected counties, the estimated prevalence ranged from $35.4 \%$ in Hidalgo County, Texas, to $96.7 \%$ in Norfolk County, Massachusetts (median: $81.4 \%$ ) (Table 6).

\section{Preventive Practices}

\section{Recent Routine Physical Checkup}

A recent routine physical checkup was defined as a visit to a doctor for a general physical examination rather than for a specific injury, illness, or condition during the preceding 12 months. In 2012, the estimated prevalence of having a recent routine physical checkup among adults aged $\geq 18$ years ranged from $55.7 \%$ in Idaho to $80.1 \%$ in Delaware (median: 67.7\%) (Table 7). Among selected MMSAs, the estimated prevalence ranged from $50.6 \%$ in Norfolk, Nebraska, to $85.0 \%$ in Barnstable Town, Massachusetts (median: 68.3\%) (Table 8). Among selected counties, the estimated prevalence ranged from $52.4 \%$ in Utah County, Utah, to $85.0 \%$ in Barnstable County, Massachusetts (median: 68.0\%) (Table 9).

\section{Annual Influenza Vaccination for Adults Aged $\geq 65$ Years}

Respondents were asked whether they had the annual influenza vaccination. In 2012, the estimated prevalence of influenza vaccination among adults aged $\geq 65$ years during the preceding 12 months ranged from $26.3 \%$ in Puerto Rico to $70.1 \%$ in Iowa (median: $60.1 \%$ ) (Table 10). Among selected MMSAs, the estimated prevalence of annual influenza vaccination ranged from $20.8 \%$ in Aguadilla-Isabela, Puerto Rico, to $77.8 \%$ in Greensboro-High Point, North Carolina (median: 60.5\%) (Table 11); among selected counties, the estimated prevalence ranged from $24.1 \%$ in San Juan Municipio, Puerto Rico, to $77.6 \%$ in Kanawha County, West Virginia (median: 60.8\%) (Table 12). 


\section{Pneumococcal Vaccination for Adults Aged $\geq 65$ Years}

In 2012, the estimated prevalence of ever having received a pneumococcal vaccination among adults aged $\geq 65$ years ranged from $22.2 \%$ in Puerto Rico to $76.2 \%$ in Oregon (median: 68.5\%) (Table 13). Among selected MMSAs, the estimated prevalence ranged from $15.3 \%$ in Aguadilla-Isabela, Puerto Rico, to $83.4 \%$ in Eugene, Oregon (median: 70.0\%) (Table 14). Among selected counties, the estimated prevalence ranged from $25.8 \%$ in San Juan Municipio, Puerto Rico, to $85.2 \%$ in Denver County, Colorado (median: 70.1\%) (Table 15).

\section{Oral Health}

\section{Dental Visit in the Past Year}

BRFSS assessed use of dental care services by asking when an adult last visited a dentist or a dental clinic for any reason. In 2012, the estimated prevalence of having had a dental visit in the past year among adults aged $\geq 18$ years ranged from $53.7 \%$ in Guam to $76.2 \%$ in Massachusetts (median: $67.2 \%$ ) (Table 16). Among selected MMSAs, the estimated prevalence ranged from $44.8 \%$ in McAllen-Edinburg-Mission, Texas, to 81.7\% in Barnstable Town, Massachusetts (median: 67.5\%) (Table 17). Among selected counties, the estimated prevalence ranged from $44.8 \%$ in Hidalgo County, Texas to $81.7 \%$ in Barnstable County, Massachusetts and Norfolk County, Massachusetts (median 68.9\%) (Table 18).

\section{Loss of All Natural Teeth for Adults Aged $\geq 65$ Years}

BRFSS assessed oral health status by asking adults the number of their permanent teeth were removed because of tooth decay or gum diseases. In 2012, the estimated prevalence of adults aged $\geq 65$ years who had lost all of their natural teeth ranged from $7.0 \%$ in Hawaii to $33.7 \%$ in West Virginia (median: 16.2\%) (Table 19). Among selected MMSAs, the estimated prevalence ranged from $5.8 \%$ in San Diego-Carlsbad, California, to $39.6 \%$ in Lafayette, Louisiana, (median: 15.8\%) (Table 20). Among selected counties, the estimated prevalence ranged from 5.8\% in San Diego County, California, to $37.1 \%$ in Aroostook County, Maine (median: $14.5 \%$ ) (Table 21).

\section{Cancer Screening}

\section{Colorectal Cancer Screening for Adults Aged 50-75 Years}

The 2008 U.S. Preventive Services Task Force (USPSTF) recommendation for colorectal cancer screening in adults aged 50-75 years is a blood stool test (either a guaiac fecal occult blood testing [FOBT] or fecal immunochemical test [FIT]) every year, a colonoscopy every 10 years, or a flexible sigmoidoscopy every 5 years with FOBT every 3 years. In 2012, the estimated prevalence of adults aged 50-75 years who received a colorectal cancer screening on the basis of the USPSTF recommendation ranged from $40.0 \%$ in Guam to 76.4\% in Massachusetts (median: 64.9\%) (Table 22). Among selected MMSAs, the estimated prevalence ranged from $47.1 \%$ in El Paso, Texas, to $80.7 \%$ in Barnstable Town, Massachusetts (median: 67.0\%) (Table 23). Among selected counties, the estimated prevalence ranged from $47.0 \%$ in El Paso County, Texas to $81.0 \%$ in Washington County, Rhode Island (median: 67.3\%) (Table 24).

\section{Cervical Cancer Screening for Women Aged 21-65 Years}

In 2012, the estimated prevalence of women aged 21-65 years who have not had a hysterectomy and had a Papanicolaou (Pap) test during the preceding 3 years ranged from $68.5 \%$ in Guam to $89.6 \%$ in Massachusetts (median: 84.0\%) (Table 25). Among selected MMSAs, the estimated prevalence ranged from $70.3 \%$ in Aguadilla-Isabela, Puerto Rico, to $92.8 \%$ in Lewiston-Auburn, Maine (median: $85.2 \%$ ) (Table 26). Among selected counties, the estimated prevalence ranged from $65.7 \%$ in Canyon County, Idaho to $94.6 \%$ in Lorain County, Ohio (median: 85.9\%) (Table 27).

\section{Breast Cancer Screening for Women Aged 50-74 Years}

A mammogram is a radiograph of each breast used to test for breast cancer. The state-specific estimated prevalence of having a mammogram during the preceding 2 years among women aged $50-74$ years ranged from $66.5 \%$ in Wyoming to $89.7 \%$ in Massachusetts (median: 78.4\%) (Table 28). Among selected MMSAs, the estimated prevalence ranged from $61.1 \%$ in Fort Wayne, Indiana to $91.5 \%$ in Barnstable Town, Massachusetts (median: 79.6\%) (Table 29). Among selected counties, the estimated prevalence ranged from $61.8 \%$ in Natrona County, Wyoming to $91.6 \%$ in Suffolk County, Massachusetts (median: 79.7\%) (Table 30). 


\section{Health-Risk Behaviors}

\section{Current Cigarette Smoking}

Respondents were classified as current smokers if they reported having smoked at least 100 cigarettes during their lifetime and indicated that they smoked every day or some days at the time of survey participation. The estimated prevalence of current cigarette smoking among adults aged $\geq 18$ years ranged from $10.6 \%$ in Utah to $28.3 \%$ in Kentucky (median: 19.6\%) (Table 31). Among selected MMSAs, the estimated prevalence ranged from 5.1\% in Provo-Orem, Utah to 30.1\% in Huntington-Ashland, West Virginia-Kentucky-Ohio (median: 19.5\%) (Table 32). Among selected counties, the estimated prevalence ranged from $5.1 \%$ in Utah County, Utah, to $28.3 \%$ in Lorain County, Ohio (median: $18.3 \%$ ) (Table 33).

\section{Binge Drinking}

Binge drinking was defined for men aged $\geq 18$ years as having on average five or more drinks during one occasion and for women aged $\geq 18$ years as having on average four or more drinks on one occasion during the preceding month. In 2012, the estimated prevalence of binge drinking among adults aged $\geq 18$ years ranged from $10.2 \%$ in West Virginia to $25.2 \%$ in Wisconsin (median: $16.9 \%$ ) (Table 34). Among selected MMSAs, the estimated prevalence ranged from 6.2\% in Provo-Orem, Utah to $28.1 \%$ in Fargo, North DakotaMinnesota (median: 17.2\%) (Table 35). Among selected counties, the estimated prevalence ranged from $6.2 \%$ in Utah County, Utah, to $29.5 \%$ in Lincoln County, South Dakota (median: 17.5\%) (Table 36).

\section{Heavy Drinking}

For men aged $\geq 18$ years, heavy drinking was defined as having, on average, more than two drinks per day during the preceding month; women aged $\geq 18$ years were heavy drinkers if they had, on average, more than one drink per day during the preceding month. In 2012, the estimated prevalence of heavy drinking among adults aged $\geq 18$ years ranged from $3.5 \%$ in West Virginia to $8.5 \%$ in Wisconsin and Montana (median: 6.1\%) (Table 37). Among selected MMSAs, the estimated prevalence ranged from $2.0 \%$ in Provo-Orem, Utah to $11.0 \%$ in Hilo, Hawaii (median: 6.1\%) (Table 38). Among selected counties, the estimated prevalence ranged from $1.9 \%$ in Utah County, Utah to $11.0 \%$ in Hawaii County, Hawaii (median: 6.1\%) (Table 39).

\section{No Leisure-Time Physical Activity}

No leisure-time physical activity was defined from the respondent's indication of no participation in any physical activities or exercise (e.g., running, calisthenics, golf, gardening, or walking for exercise) other than their regular job during the preceding month. In 2012, the estimated prevalence of no leisure-time physical activity among adults aged $\geq 18$ years ranged from $16.3 \%$ in Oregon to $42.4 \%$ in Puerto Rico (median: 23.1\%) (Table 40). Among selected MMSAs, the estimated prevalence ranged from $9.2 \%$ in Boulder, Colorado, to $47.3 \%$ in Aguadilla-Isabela, Puerto Rico (median: 22.2\%) (Table 41). Among selected counties, the estimated prevalence ranged from $9.2 \%$ in Boulder County, Colorado, to $39.0 \%$ in Robeson County, North Carolina (median: 21.5\%) (Table 42).

\section{Seat Belt Use}

Respondents were asked how often (always, nearly always, sometimes, seldom, and never) they use a seat belt when they drive or ride in a car. In 2012, the estimate of always wearing a seat belt among adults aged $\geq 18$ years ranged from $62.0 \%$ in South Dakota to $93.7 \%$ in California (median: $84.7 \%$ ) (Table 43). Among selected MMSAs, the estimated prevalence ranged from 54.1\% in Berlin, New Hampshire-Vermont, to $97.1 \%$ in San Jose-Sunnyvale-Santa Clara, California (median: 85.7\%) (Table 44). Among selected counties, the estimated prevalence ranged from $50.1 \%$ in Coos County, New Hampshire, to $97.4 \%$ in Clackamas County, Oregon (median: 86.0\%) (Table 45).

\section{Chronic Health Conditions}

\section{Obesity}

Self-reported weight and height were used to calculate body mass index (BMI) (weight $[\mathrm{kg}] /$ height $[\mathrm{m} 2]$ ). Respondents were obese if their BMI was $\geq 30.0$. In 2012, the estimated prevalence of adults aged $\geq 18$ years with obesity ranged from $20.5 \%$ in Colorado to $34.7 \%$ in Louisiana (median: 28.1\%) (Table 46). Among selected MMSAs, the estimated prevalence ranged from $14.8 \%$ in Boulder, Colorado, to 44.5\% in McAllen-Edinburg-Mission, Texas (median: 28.3\%) (Table 47). Among selected counties, the estimated prevalence ranged from $14.8 \%$ in Boulder County, Colorado, to $44.5 \%$ in Hidalgo County, Texas (median: 26.7\%) (Table 48).

\section{Diabetes}

Respondents were identified as having diabetes if they reported ever being told by a doctor that they have diabetes. For this report, gestational diabetes, prediabetes, or borderline diabetes were not included in the estimates. In 2012, the estimated prevalence of diagnosed diabetes among adults aged $\geq 18$ years ranged from $7.0 \%$ in Alaska to $16.4 \%$ in Puerto Rico (median: 9.7\%) (Table 49). Among selected MMSAs, the estimated prevalence ranged from $3.4 \%$ in Boulder, Colorado, 
to $17.4 \%$ in Lumberton, North Carolina (median: $9.6 \%$ ) (Table 50). Among selected counties, the estimated prevalence ranged from 3.1\% in Douglas County, Colorado, to $17.4 \%$ in Robeson County, North Carolina (median: 9.3\%) (Table 51).

\section{Cancer Survivors}

Respondents were identified as being a cancer survivor if they had ever been told by a doctor, nurse, or other health professional that they had any type of cancer, including skin cancer. In 2012, the estimated prevalence of cancer survivors among adults aged $\geq 18$ years ranged from $3.0 \%$ in Guam to $13.7 \%$ in Florida (median: $11.0 \%$ ) (Table 52). Among selected MMSAs, the estimated prevalence ranged from 3.8\% in Aguadilla-Isabela, Puerto Rico, to $19.2 \%$ in Ocean City, New Jersey (median: 10.8\%) (Table 53). Among selected counties, the estimated prevalence ranged from $4.5 \%$ in Hudson County, New Jersey, to $19.2 \%$ in Cape May County, New Jersey (median: 10.8\%) (Table 54).

\section{Currently Have Asthma}

Respondents were identified as currently having asthma if they reported having ever been told by a doctor, nurse, or other health care professional that they had asthma and still had it during the time of the survey. In 2012, the estimated prevalence of current asthma among adults aged $\geq 18$ years ranged from $5.8 \%$ in Guam to $11.1 \%$ in Kentucky and Maine (median: 8.9\%) (Table 55). Among selected MMSAs, the estimated prevalence ranged from $3.1 \%$ in McAllenEdinburg-Mission, Texas, to $15.0 \%$ in Vineland-Bridgeton, New Jersey (median: 8.9\%) (Table 56). Among selected counties, the estimated prevalence ranged from $3.1 \%$ in Hidalgo County, Texas, to $15.7 \%$ in Hampden County, Massachusetts (median: 9.2\%) (Table 57).

\section{Arthritis}

Respondents were identified as having arthritis if they had ever been told by a health professional that they had some form of arthritis, rheumatoid arthritis, gout, lupus, or fibromyalgia. In 2012, the estimated prevalence of arthritis among adults aged $\geq 18$ years ranged from $15.6 \%$ in Guam to $36.4 \%$ in West Virginia (median: 25.5\%) (Table 58). Among selected MMSAs, the estimated prevalence ranged from $16.8 \%$ in Heber, Utah, to $45.8 \%$ in Kingsport-Bristol-Bristol, TennesseeVirginia (median: 25.3\%) (Table 59). Among selected counties, the estimated prevalence ranged from $14.8 \%$ in Travis County, Texas, to $35.9 \%$ in Aroostook County, Maine and Mobile County, Alabama (median: 24.5\%) (Table 60).

\section{Depression}

Depression was defined as having ever been told by a health professional that the participants had a depressive disorder, which includes depression, major depression, dysthymia, or minor depression. In 2012, the estimated prevalence of depression among adults aged $\geq 18$ years ranged from 9.0\% in Guam to $23.5 \%$ in Kentucky (median: $17.6 \%$ ) (Table 61). Among selected MMSAs, the estimated prevalence ranged from 9.2\% in Anaheim-Santa Ana-Irvine, metropolitan division, California, to $28.3 \%$ in Lewiston-Auburn, Maine (median: 18.2\%) (Table 62). Among selected counties, the estimated prevalence ranged from $8.5 \%$ in Union County, New Jersey, to $28.4 \%$ in Androscoggin County, Maine (median: $17.8 \%$ ) (Table 63).

\section{Coronary Heart Disease}

Respondents were identified as having coronary heart disease if they reported that they had ever been told by a doctor, nurse, or other health care professional that they had a heart attack (also known as myocardial infarction, or MI) or angina (i.e., coronary heart disease). In 2012, the estimated prevalence of coronary heart disease among adults aged $\geq 45$ years ranged from $7.4 \%$ in Hawaii to $19.0 \%$ in West Virginia (median: 11.4\%) (Table 64). Among selected MMSAs, the estimated prevalence ranged from $6.1 \%$ in Boulder, Colorado, to 23.3\% in Charleston, West Virginia (median: 11.0\%) (Table 65). Among selected counties, the estimated prevalence ranged from $6.1 \%$ in Boulder County, Colorado, to $20.6 \%$ in Kanawha County, West Virginia (median: 10.0\%) (Table 66).

\section{Stroke}

Respondents were identified as having had a stroke if they had ever been told by a doctor, nurse, or other health care professional that they had a stroke. In 2012, the estimated prevalence of stroke among adults aged $\geq 45$ years ranged from $3.1 \%$ in Colorado to $7.3 \%$ in Mississippi (median: $4.7 \%$ ) (Table 67). Among selected MMSAs, the estimated prevalence ranged from $2.1 \%$ in Silver Spring-Frederick-Rockville, Maryland, to $9.3 \%$ in Mobile, Alabama (median: 4.4\%) (Table 68). Among selected counties, the estimated prevalence ranged from $1.5 \%$ in Montgomery County, Maryland, to 9.3\% in Mobile County, Alabama (median: 4.4\%) (Table 69).

\section{Disability}

\section{Activity Limitation}

Respondents were asked if they were limited in any way from performing any activities because of physical, mental, or emotional problems. In 2012, the estimated prevalence of 
activity limitation among adults aged $\geq 18$ years ranged from $15.0 \%$ in Guam to $28.6 \%$ in West Virginia (median: $20.0 \%$ ) (Table 70). Among selected MMSAs, the estimated prevalence ranged from $12.0 \%$ in Heber, Utah, to $31.7 \%$ in Eugene, Oregon (median: 20.3\%) (Table 71). Among selected counties, the estimated prevalence ranged from $11.3 \%$ in Wake County, North Carolina, to $31.7 \%$ in Lane County, Oregon (median: 19.5\%) (Table 72).

\section{Use of Special Equipment}

Respondents were asked if they currently had any health problem that required them to use special equipment (e.g., a cane, a wheelchair, a special bed, or a special telephone), which included occasional use or use in certain circumstances. In 2012, the estimated prevalence of using special equipment because of any health problem among adults aged $\geq 18$ years ranged from $4.8 \%$ in Guam to $11.6 \%$ in Kentucky (median: 8.0\%) (Table 73). Among selected MMSAs, the estimated prevalence ranged from $4.0 \%$ in Boulder, Colorado, to $14.7 \%$ in Kingsport-Bristol-Bristol, Tennessee-Virginia (median: 7.8\%) (Table 74). Among selected counties, the estimated prevalence ranged from 2.8\% in Douglas County, Colorado, to $13.6 \%$ in Thurston County, Washington (median: $7.5 \%$ ) (Table 75).

\section{Discussion}

The findings in this report indicate substantial geographic variations in the estimated prevalence of health status indicators, preventive practices, oral health, cancer screening, health-risk behaviors, chronic health conditions, and disability status among adults in the United States at the state and territory, MMSA, and county level. These variations in crude (unadjusted) estimates might reflect differences in demographic characteristics (e.g., age, race, and sex distribution), socioeconomic conditions (e.g., education attainment, income, and employment status), cultural contexts, health care access and cost, state laws and local ordinances, or combinations of these factors. The results provided in this report were estimated on the basis of survey results (i.e., direct estimates) and might differ from those derived by other methods.

\section{Health Status Indicators}

Although measured by a single question, self-reported health status encompasses physical health, mental health, and functional capacity of a person (17). It has been validated as an independent predictor of mortality (18) and is a useful proxy indicator for perceived prevalence of acute and chronic health conditions (19). For this report, self-assessed health status was reported for good or better health. Large variations in selfrated health at the state and local levels suggest differences in the underlying etiology and severity of specific diseases and illnesses, health care access, and residents' health related behaviors among states and territories, MMSAs, and counties. Health services research has identified that having no health insurance is a risk indicator for lower overall health status, exacerbation of chronic disease indicators (e.g., uncontrolled and undiagnosed hypertension, and hypercholesterolemia), and less access to and use of preventive health care services (e.g., blood pressure monitoring, cholesterol screening, and age appropriate cancer screenings) $(20,21)$. In 2012, the median prevalence of health care coverage among adults aged $\leq 65$ years at the state and local levels was approximately $81 \%$.

\section{Preventive Practices}

A routine physical checkup can help persons stay healthy and prevent or delay disease and disability. A visit to a doctor for a routine checkup can help to identify a disease (e.g., diabetes, heart disease, and cancer) and other health problems in the early stages of development when they are most successfully treated (22); these visits also can provide opportunities for disease prevention and patient education. In 2012, the median prevalence of having received a routine physical checkup among adults, at the state or local level, was approximately $66 \%$. In addition to geographic variation, access to health care, socioeconomic factors (e.g., education and income), and marital status are associated with routine medical checkups (23).

Influenza and pneumonia together are the seventh leading cause of death among adults aged $\geq 65$ years (2). The vaccinations against influenza and pneumonia are safe, effective, and cost efficient ways to reduce the morbidity and mortality from these diseases in older adults (24). In 2012, the median prevalence of having received an influenza vaccination during the preceding 12 months or ever having a pneumococcal vaccination among adults aged $\geq 65$ was approximately $61 \%$ and $71 \%$, respectively. Estimates of influenza vaccination prevalence measured in this report are calculated from interview data collected during the calendar year and measures the proportion of persons reporting influenza vaccination during the 12 months preceding the survey. Therefore, annual estimates represent a weighted average of incomplete estimates for up to three influenza seasons. The variation in influenza and pneumococcal vaccination coverage levels observed among states and local areas suggests that coverage levels for both vaccines can be improved. Strategies such as clinician reminders, team change, and patient outreach along with patient financial incentives, audit, and feedback (for influenza 
vaccination only) and clinician education, case management (for pneumococcal vaccination only) can be employed at the state and local levels to increase the rates of influenza and pneumococcal vaccinations among older adults (25).

\section{Oral Health}

The Surgeon General's report on oral health in America described the mouth as a mirror of health (26). A thorough oral examination can detect signs of numerous general health problems (e.g., nutritional deficiencies), systemic diseases (e.g., microbial infections and immune disorders), injuries, and some cancers. Routine dental visits can help prevent and control the most common oral diseases including dental caries (tooth decay) and periodontal disease (26). The loss of one's natural teeth can reduce quality of life in many ways (e.g., bringing pain and distress, lowering self-esteem, and making it more difficult to eat, relax, or socialize) (27). Periodontal disease and dental caries are the leading causes of tooth loss (28); tooth loss is associated with poor nutrition, coronary artery disease, diabetes, smoking, and rheumatoid arthritis (29). In 2012, at the state and local levels, the median prevalence of having had a dental visit in the past year was $69 \%$; $14 \%$ of adults aged $\geq 65$ years had lost all their natural teeth. These findings suggest an unmet need for oral health care and needs for oral disease prevention and health promotion programs at state and local levels.

\section{Cancer Screening}

Cancer is the second-leading cause of death in the United States following heart disease (2) and it is the leading cause of death among both men and women aged 40-79 years (30). Colorectal cancer is the third most-commonly diagnosed of new cancer cases and the third leading cause of cancer-related death in both men and women (30). Colorectal cancer usually begins from polyps present in the rectum or colon. The FOBT/FIT, colonoscopy, and a flexible-sigmoidoscopy, are the recommended screening tests for early detection of precancerous polyps and prevention of colorectal cancer (31). In 2012, the median prevalence of colorectal cancer screening based on the 2008 USPSTF recommendation was $67 \%$ at the state and local levels-a finding that suggests the need for population-based strategies to improve colorectal cancer screening efforts at the state and local levels.

The primary cause of cervical cancer is HPV. Early-stages of cervical cancer often can be detected easily with two tests: 1) a Pap test and 2) an HPV test (32). A Pap test that detects precancerous or abnormal cells in the cervix and a HPV test looks for the HPV that caused the precancerous or abnormal cervical cells (32). The numbers of cases and deaths from cervical cancer have declined substantially during the past decades because of early detection of cervical cancer by the Pap test and early treatment (33). USPSTF recommends that women aged 21-29 years should receive the Pap test to screen for cervical cancer every 3 years (34). Women aged 30-65 years can continue receiving the Pap test every 3 years or the Pap test in combination with the HPV test every 5 years (34). Among women aged 21-65 years, the median prevalence of having received a Pap test varied in 2012 from $66 \%$ to $93 \%$ at the state and local levels. The variation in Pap test screening rates among women in this age group at state and local levels might be related to health care access and other barriers to screening. Breast cancer is the most commonly diagnosed cancer and second leading cause of cancer mortality in women (30). Mammograms can detect breast cancer at an early stage, when it is most treatable. This combination of early detection and advances in treatment has helped to reduce the rate of death from breast cancer in the United States (35). USPSTF recommends biennial screening mammography for women aged $50-74$ years (36). In 2012, the median prevalence of having had a mammogram in the preceding 2 years for women aged 50-74 varied from 78\% to $80 \%$ for the state, MMSAs, and counties.

\section{Health-risk Behaviors}

Health-risk behaviors are unhealthy behaviors that persons can change. Tobacco use, diet and activity patterns, and alcohol use are the major contributors to deaths in the United States (37). Cigarette smoking is the single-most preventable cause of morbidity and mortality in the United States (38). The prevalence of disease and death from tobacco use is overwhelmingly caused by cigarettes and other combusted tobacco products. Cigarette smoking has been causally linked to diseases of nearly all organs of the body and can harm the fetus during pregnancy (38). Moreover, secondhand tobacco smoke has been causally linked to lung cancer as well as respiratory and cardiovascular diseases, and it can damage the health of infants and children (38). The prevalence of current cigarette smoking among U.S. adults aged $\geq 18$ years declined during 2005-2010 (39); however, current cigarette smoking remains widespread. The median prevalence of current cigarette smoking (19.6\%) indicates the need for sustained, adequately funded, comprehensive tobacco control programs at the state and local levels (40).

Excessive alcohol consumption, including binge and heavy drinking, is one of the leading preventable causes of death in the United States (41). Binge and heavy drinking can lead to risky sexual activity, unintentional injuries (e.g., motor-vehicle crashes), falls, violence, and suicide (42); excessive alcohol consumption can lead to development of high blood pressure, 
liver disease, some cancers, dementia, and alcohol dependence (42). This report highlighted the variation in the prevalence of binge and heavy drinking among states/territories, MMSAs, and counties that might be attributable to cultural factors as well as the state/local laws governing the price, availability, and marketing of alcoholic beverages. Evidence-based populationlevel strategies recommended by the Community Preventive Services Task Force can be implemented to reduce and prevent excessive alcohol consumption (43).

Being physically active is an important step persons can take to improve their health; physical activity improves cardiorespiratory and muscular fitness and bone health. It helps to control weight, reduces risk for early death, cardiovascular diseases (e.g., heart disease, stroke, and high blood pressure), diabetes, and cancer (e.g., colon and breast), and prevents falls (44). The 2012 BRFSS survey measured the prevalence of participation in any physical activities or exercises (e.g., running, calisthenics, golf, gardening, or walking during the preceding month). This report indicates the need for continued effort to increase the physical activity in the population at the state and local levels.

Accidents or unintentional injuries are the fifth-leading cause of death in the United States (2). Unintentional motor-vehicle traffic injury is the second-leading cause of injury deaths among all U.S. residents (45). Seat belt use can reduce the risk for fatal injury to front-seat car occupants by $45 \%$ and the risk for moderate-to-critical injury by 50\% (46). Laws and law enforcement levels related to seat belt use vary by state (47). The findings in this report indicate a great variation in seat belt use across states (62\%-92\%), MMSAs (54\%-97\%), and counties $(50 \%-97 \%)$, despite the fact that all states have seat belt laws. Populations with lower use of seat belts are at risk for injury and death from motor vehicle crashes.

\section{Chronic Conditions}

Chronic diseases represented seven of the top 10 leading causes of death in the United States in 2013. Heart disease, malignant neoplasms (cancers), cerebrovascular diseases, and diabetes mellitus are the leading, second-, fourth-, and fifthleading causes of death, respectively (2). BRFSS helps monitor these chronic diseases, along with chronic conditions like obesity, asthma, arthritis, and depression, to help states and local areas plan, implement, and track health interventions.

\section{Obesity}

Obesity continues to be a critical public health problem (48); it increases the risk for various chronic conditions like type 2 diabetes, hypertension, dyslipidemia, coronary heart disease, and certain types of cancer (49).The National
Health and Nutrition Examination Survey (NHANES) data indicated that the obesity prevalence among U.S. adults had not changed significantly from 2003-2004 to 2011-2012; however, approximately one third of adults are obese (50). The high median prevalence of obesity at the state and local levels (range: $26.7 \%-28.1 \%$ ) underscores the need for close monitoring of the obesity trend at the state and local levels and for implementing interventions that address obesity.

\section{Diabetes}

Diabetes is associated with extensive and serious complications that might involve many organs including loss of vision, lower-extremity amputation, heart attack, stroke, high blood pressure, kidney disease, skin infection (e.g., bacterial and fungal infection), periodontitis, erectile dysfunction, depression, and complications of pregnancy (51). In 2012, the median prevalence of diagnosed diabetes ranged from $9.3 \%$ to $9.7 \%$ at state and local levels. The disease and its complications often can be prevented, delayed, or controlled by closely monitoring and controlling blood glucose through healthy eating, increasing physical activity, taking prescribed medications, and receiving proper diabetes-related preventive care services that frequently focus on areas like foot health and diabetic patient education (52).

\section{Cancer Survivors}

A cancer survivor is any person living with a history of cancer (53). The population of cancer survivors in this country has been steadily increasing since 1970; the prevalence is projected to approach 18 million by 2022 (54). The increases in the number of cancer survivors are largely because of aging and growth of the population as well as advances in cancer detection and treatments (55). At the state and local levels, the median prevalence of cancer survivorship (11\%) among BRFSS participants indicates a growing need to monitor cancer survivors to address their special health issues and needs (55) at state and local levels, including quitting tobacco use, being active and maintaining a healthy weight, and discussing follow-up care with a health care provider.

\section{Current Asthma}

Asthma is a common chronic disorder of the airways involved with breathing and respiration, characterized by periods of reversible airflow obstruction known as asthma exacerbations or attacks (56). Many persons with asthma have only occasional, mild symptoms, but others have severe asthma that can interfere with daily activity or even be life-threatening. The report on national surveillance of asthma indicated that current asthma prevalence increased from 2001 to 2010 and that there were no significant changes in rates for hospital 
outpatient department visits, emergency department visits, and hospitalizations for asthma (57). In 2012, the overall median prevalence of current asthma was 9\%; BRFSS data indicated variability in estimated current asthma prevalence at the state, MMSA, and county level indicates the need for continued asthma surveillance in the United States.

\section{Arthritis}

Arthritis is the most common cause of disability among U.S. adults (58). Data from the 2010-2012 National Health Interview Survey indicated that $22.7 \%$ of adults reported having doctor-diagnosed arthritis and $43.2 \%$ of those with arthritis reported arthritis-attributable activity limitations. Moreover, approximately half of the adults with heart disease and diabetes and about one third of the adults who were obese had arthritis (59). In the 2012 BRFSS, the median prevalence of reported doctor-diagnosed arthritis was $25 \%$ at state and local levels, which indicates a substantial personal and societal prevalence of arthritis in the United States.

\section{Depression}

Depression is the leading cause of disability in the Global Burden of Disease during 2010 as measured by Years Lived with Disability and Disability Adjusted Life Years (60). Depressive disorders are more common among persons with chronic conditions (e.g., obesity, cardiovascular disease, diabetes, asthma, arthritis, and cancer) and among those with unhealthy behaviors (e.g., smoking, physical inactivity, and binge drinking) $(61,62)$. It is also associated with decreased productivity in the workplace and an increased risk for absenteeism from work (63). In 2012, the median prevalence of depression was $18 \%$ at the state and local levels; the variations of prevalence among states, MMSAs, and counties indicate the need for targeted prevention and intervention efforts and the allocation of mental health treatment resources at the state and local levels.

\section{Heart Disease and Stroke}

Heart disease and stroke are the leading causes of death in the United States. During 2011-2012, heart disease accounted for 170.5 deaths and stroke accounted for 36.9 deaths per 100,000 population (2). Stroke is the leading cause of serious long-term disability in the United States and costs $\$ 34$ billion annually (64). In 2012, the prevalence of self-reported coronary heart disease and stroke among adults aged $\geq 45$ years ranged from $6 \%-21 \%$ and $2 \%-9 \%$, respectively, at the state and local levels. This report indicates wide variation in prevalence of coronary heart disease and stroke. It is essential for states and local areas to take initiatives to improve access and quality of health care systems and to continue to build environments that support healthy behaviors through community and clinical prevention strategies (65).

\section{Disability}

Disability or health impairment caused by limitation of activities because of physical, mental, or emotional problems can affect health, functioning, and quality of life (66). During 2010 in the United States, approximately 56.7 million persons were living with some kind of disability and $12.6 \%$ of them had severe disability (67). Disability is associated with low socioeconomic status; persons with disabilities are more likely to be poor, experience barriers to education, employment, and health care (68). Persons with disabilities often require the use of special equipment and access to assistive technology to improve functioning, independence, and participation in community life (e.g., work, school, and social functions) (69).

In the 2012 BRFSS, the median prevalence of disability or health impairment was $20 \%$ at the state and local levels and the prevalence of special equipment usage (e.g., a cane, a wheelchair, a special bed, or special telephone) because of any health problem ranged from $5 \%$ to $14 \%$ at state, MMSA, and county levels. Because of the aging of the population, the number of adults reporting a disability is likely to increase, along with the need for appropriate medical and public health services; therefore, it is essential to continue surveillance of disability rates and the need for special equipment in the United States, to address quality of life issues for persons living with disabilities.

\section{Limitations}

The findings in this report are subject to at least four limitations. First, BRFSS results might not be generalizable to the entire U.S. population because the BRFSS survey design excludes persons living in institutions, nursing homes, longterm-care facilities, military installations, and correctional institutions. In addition, because participants answer the survey on a cellular or landline telephone, persons without access to either type of telephone are excluded. Second, BRFSS data are self-reported, so the information is subject to recall (e.g., diagnosis of diabetes and duration of physical activity) and social desirability bias (e.g., underreporting of actual weight). Third, although BRFSS surveys are conducted in several languages other than English (i.e., Spanish, Mandarin, and Portuguese), the survey does not apply to persons who speak other languages exclusively. Finally, because of the small sample size producing unreliable estimates, the prevalence of certain variables (e.g., influenza and pneumococcal vaccination among 
adults aged $\geq 65$ years) could not be obtained among residents living within certain MMSAs or counties.

Overall, BRFSS is a cost-effective, timely, and flexible survey that makes data available to state health departments and local communities so they can assess and monitor the health risk behaviors, chronic conditions, use of preventive health care services, health impairments, and disabilities of their residents. The response over time within BRFSS and the prevalence rates from the BRFSS survey might differ from other national and state surveys because of differences in wording of questions, the number of questions focusing on a measure or topic, survey modes (telephone versus in person), length of questionnaire, format of the questionnaire, and sampling frame.

Researchers have found BRFSS data to be reliable and to have an overall high level of validity when compared with data from other surveys $(9,10)$; prevalence estimates from BRFSS were consistent with other national survey databases (70). Despite concerns about declines in telephone survey response rates, BRFSS response rates compare favorably to those of telephone surveys (16). The raking weighting methods used by the BRFSS survey reduces the nonresponse and noncoverage bias and helps to match more accurately the sample distribution of BRFSS to known demographic characteristics of state populations.

BRFSS data have been used in a variety of ways to improve health. Federal, state, and local health officials continue to use BRFSS as a tool for monitoring and responding to public health emergencies $(71)$, such as the seasonal influenza vaccine shortage (2004-2005) and the effects of hurricanes Katrina, Rita, and Wilma in 2005 as well as to monitor prevalence rates of influenza-like illness and the use of $\mathrm{H} 1 \mathrm{~N} 1$ vaccination that are a part of pandemic planning (since 2009). The asthma callback survey, funded by the National Asthma Control Program and conducted with BRFSS respondents (who reported an asthma diagnosis), helps in asthma surveillance in the United States (72). Since 2002, the Safety and Health Assessment and Research for Prevention program at the Washington State Department of Labor and Industries has been adding industry and occupation questions to the Washington BRFSS to identify worker populations with a high prevalence of chronic diseases or conditions and injury to target disease prevention efforts in work places (73).

\section{Conclusion}

In the United States, major risk factors contributing to the prevalence of chronic disease and injury include tobacco use, poor diet (e.g., low in fruits and vegetables and high in sodium and saturated fat), physical inactivity, low seat belt use, excessive alcohol consumption, and high blood pressure. All of these risk factors can be effectively addressed at both the individual and population levels. CDC works to prevent chronic diseases and injuries through four domains: epidemiology and surveillance, environmental approaches, health care system interventions, and community-clinical links (74).

State and local health departments and agencies can use data from BRFSS to monitor risk factors, chronic conditions, and use of preventive health care practices related to chronic disease and injury. Specifically, BRFSS data can be used to evaluate public health policies and programs, to identify the needs for additional support or resources for programs, to identify emerging health problems, to educate leaders and decision makers about health-related issues, and to monitor progress toward achieving health objectives at state and local levels.

\section{References}

1. US Department of Health and Human Services, Office of the Surgeon General. National Prevention Council, National Prevention Strategy, Washington, DC, 2011.

2. Kochanek KD, Murphy SL, Xu JQ, Arias E. Mortality in the United States, 2013. NCHS data brief, no 178. Hyattsville, MD: National Center for Health Statistics. 2014.

3. CDC. Chronic diseases and health promotion. Atlanta, GA: US Department of Health and Human Services, CDC; 2012. http://www. cdc.gov/chronicdisease/overview/index.htm

4. CDC. The BRFSS Data User Guide August 15, 2013. http://www.cdc. gov/brfss/data_documentation/PDF/UserguideJune2013.pdf

5. CDC. Notice to readers: SMART BRFSS provides data comparisons by metropolitan and micropolitan statistical area (MMSA). MMWR Morb Mortal Wkly Rep 2003a;51:453-6.

6. Mokdad AH, Stroup DF, Giles WH; Behavioral Risk Factor Surveillance Team. Public health surveillance for behavioral risk factors in a changing environment. Recommendations from the Behavioral Risk Factor Surveillance Team. MMWR Recomm Rep 2003;52(RR-9).

7. CDC. At a glance: conducting the 2011 Behavioral Risk Factor Surveillance System (BRFSS). US Department of Health and Human Services, 2011. http://www.cdc.gov/brfss/factsheets/pdf/238974_BRFSS-AAG.pdf

8. Hu SS, Pierannunzi C, Balluz L. Integrating a multimode design into a national random-digit-dialed telephone survey. Prev Chronic Dis 2011;8:A145.

9. Pierannunzi C, Hu SS, Balluz L. A systematic review of publications assessing reliability and validity of the Behavioral Risk Factor Surveillance System (BRFSS), 2004-2011. BMC Med Res Methodol 2013;13:49.

10. Nelson DE, Holtzman D, Bolen J, Stanwyck CA, Mack KA. Reliability and validity of measures from the Behavioral Risk Factor Surveillance System (BRFSS). Soz Praventivmed 2001;46(Suppl 1):S3-42.

11. CDC. 2012 SMART BRFSS MMSA methodology, 2012. Atlanta, GA: US Department of Health and Human Services, CDC. http://www.cdc. gov/brfss/smart/2012/2012_SMART_BRFSS_MMSA_Methodology.pdf

12. CDC. 2012 Behavioral Risk Factor Surveillance System questionnaire. Atlanta, GA: US Department of Health and Human Services. http:// www.cdc.gov/brfss/questionnaires/pdf-ques/2012_BRFSS.pdf

13. CDC. Behavioral Risk Factor Surveillance System, overview: BRFSS 2012. Atlanta, GA: US Department of Health and Human Services, CDC; 2015. http://www.cdc.gov/brfss/annual_data/2012/pdf/ Overview_2012.pdf

14. Pierannunzi C, Town M, Garvin W, Shaw FE, Balluz L; Centers for Disease Control and Prevention (CDC). Methodologic changes in the Behavioral Risk Factor Surveillance System in 2011 and potential effects on prevalence estimates. MMWR Morb Mortal Wkly Rep 2012;61:410-3. 
15. The American Association of Public Opinion Research. 2011. Standard definitions: final dispositions of case codes and outcome rates for surveys. 7th edition. AAPOR. Standard definitions. Deerfield, IL: AAPOR; 2011.

16. CDC. Behavioral Risk Factor Surveillance System, 2012 summary data quality report, Atlanta, GA: US Department of Health and Human Services. http:// www.cdc.gov/brfss/pdf/2011_Summary_Data_Quality_ Report.pdf

17. Simon JG, De Boer JB, Joung IM, Bosma H, Mackenbach JP. How is your health in general? A qualitative study on self-assessed health. Eur J Public Health 2005; 15:200-8.

18. DeSalvo KB, Bloser N, Reynolds K, He J, Muntner P. Mortality prediction with a single general self-rated health question. A metaanalysis. J Gen Intern Med 2006;21:267-75.

19. CDC. Measuring healthy days. Atlanta, Georgia: CDC. November 2000. http://www.cdc.gov/hrqol/pdfs/mhd.pdf

20. CDC. Health insurance coverage and receipt of preventive health services-United States, 1993. MMWR Morb Mortal Wkly Rep 1995;44:219-25.

21. Schober SE, Makuc DM, Zhang C, Kennedy-Stephenson J, Burt V. Health insurance affects diagnosis and control of hypercholesterolemia and hypertension among adults aged 20-64: United States, 2005-2008. NCHS data brief, no 57. Hyattsville, MD: National Center for Health Statistics. 2011.

22. CDC. Regular check-ups are important. Atlanta, GA: US Department of Health and Human Services, CDC; 2013. http://www.cdc.gov/ family/checkup

23. Culica D, Rohrer J, Ward M, Hilsenrath P, Pomrehn P. Medical checkups: who does not get them? Am J Public Health 2002;92:88-91.

24. American Lung Association. Missed opportunities: influenza and pneumonia vaccination in older adults, disparities in lung health series. American Lung Association, Washington DC, 2013. http://www.lung. org/lung-disease/influenza/reports/vaccination-disparities

25. Lau D, Hu J, Majumdar SR, et al. Interventions to improve influenza and pneumococcal vaccination rates among community-dwelling adults: a systematic review and meta-analysis. Ann Fam Med 2012;10:538-46 10.1370/afm.1405.

26. US Department of Health and Human Services. Oral health in America: a report of the Surgeon General-Executive Summary. Rockville, MD: US Department of Health and Human Services, National Institute of Dental and Craniofacial Research, National Institutes of Health, 2000.

27. Brennan DS, Spencer AJ, Roberts-Thomson KF. Tooth loss, chewing ability and quality of life. Qual Life Res 2008;17:227-35.

28. Beltran-Aguilar ED, Beltran-Neira RJ. Oral diseases and conditions throughout the lifespan. I. Diseases and conditions directly associated with tooth loss. Gen Dent 2004;52:21-7.

29. Felton DA. Edentulism and comorbid factors. Tex Dent J 2010;127:389-401.

30. US Cancer Statistics Working Group. United States Cancer Statistics: 1999-2012 incidence and mortality web-based report. Atlanta: US Department of Health and Human Services, Centers for Disease Control and Prevention and National Cancer Institute; 2015. https://nccd.cdc.gov/uscs/

31. U.S. Preventive Services Task Force. Screening for colorectal cancer: U.S. Preventive Services Task Force recommendation statement. Ann Intern Med 2008;149:627-37.

32. CDC. Cervical cancer. Atlanta, GA: US Department of Health and Human Services, CDC; 2012. http://www.cdc.gov/cancer/cervical/pdf/ cervical_facts.pdf

33. Benard VB, Thomas CC, King J, et al.; Centers for Disease Control and Prevention (CDC). Vital signs: cervical cancer incidence, mortality, and screening_United States, 2007-2012. MMWR Morb Mortal Wkly Rep 2014;63:1004-9.

34. Moyer VA; US Preventive Services Task Force. Screening for cervical cancer: US Preventive Services Task Force recommendation statement. Ann Intern Med 2012;156:880-91, W312.
35. Berry DA, Cronin KA, Plevritis SK, et al.; Cancer Intervention and Surveillance Modeling Network (CISNET) Collaborators. Effect of screening and adjuvant therapy on mortality from breast cancer. N Engl J Med 2005;353:1784-92.

36. US Preventive Services Task Force. Screening for breast cancer: US Preventive Services Task Force recommendation statement. Ann Intern Med 2009;151:716-26, W-236.

37. McGinnis JM, Foege WH. Actual causes of death in the United States. JAMA 1993;270:2207-12.

38. US Department of Health and Human Services. The health consequences of smoking - 50 years of progress: a report of the Surgeon General.Atlanta, GA.: US Department of Health and Human Services, Centers for Disease Control and Prevention, National Center for Chronic Disease Prevention and Health Promotion, Office on Smoking and Health, 2014. CDC.

39. Jamal A, Agaku IT, O'Connor E, et al. CDC. Current cigarette smoking among adults-United States, 2005-2013. MMWR Morb Mortal Wkly Rep 2014;63:1108-12.

40. CDC. Best practices for comprehensive tobacco control programs-2014. Atlanta, GA: US Department of Health and Human Services, CDC; 2014. http://www.cdc.gov/tobacco/stateandcommunity/best_practices/ pdfs/2014/comprehensive.pdf

41. Mokdad AH, Marks JS, Stroup DF, Gerberding JL. Actual causes of death in the United States, 2000. JAMA 2004;291:1238-45.

42. US Department of Health and Human Services, National Institute of Alcohol Abuse and Alcoholism. Tenth special report to the US Congress on alcohol and health. Bethesda, MD: National Institute of Health; 2000. http://pubs.niaaa.nih.gov/publications/10Report/10thSpecialReport.pdf

43. Community Preventive Services Task Force. Preventing excessive alcohol consumption. Atlanta, GA: Community Preventive Services Task Force; 2013. http://www.thecommunityguide.org/alcohol/index.html

44. US Department of Health and Human Services. Physical activity and health: a report of the Surgeon General. Atlanta, GA: US Department of Health and Human Services, CDC; 1996.

45. CDC. WISQARS (Web-based Injury Statistics Query and Reporting System), National Center for Health Statistics (NCHS), National Vital Statistics System. Atlanta, GA: U.S. Department of Health and Human Services, CDC. http://www.cdc.gov/injury/images/lc-charts/leading_ causes_of_injury_deaths_highlighting_unintentional_injury_2012-a.gif

46. CDC. Vital signs: nonfatal, motor vehicle-occupant injuries (2009) and seat belt use (2008) among adults-United States. MMWR Morb Mortal Wkly Rep 2011;59:1681-6.

47. National Highway Traffic Safety Administration (NHTSA). Seat belt use in 2011 - overall results. Traffic safety facts research note, 2011(DOT HS1 1 544). US Department of Transportation, National Highway Traffic Safety Administration, Washington, DC.

48. US Department of Health and Human Services. The Surgeon General's vision for a healthy and fit nation. Rockville, MD: US Department of Health and Human Services, Office of the Surgeon General; 2010.

49. National Institutes of Health; National Heart, Lung, and Blood Institute. Obesity education initiative, clinical guidelines on the identification, evaluation, and treatment of overweight and obesity in adults, the evidence report. HIH-NHLBI 1998. http://www.nhlbi.nih.gov/ guidelines/obesity/ob gdlns.pdf

50. Ogden CL, Carroll MD, Kit BK, Flegal KM. Prevalence of childhood and adult obesity in the United States, 2011-2012. JAMA 2014;311:806-14.

51. CDC. National diabetes statistics report: estimates of diabetes and its burden in the United States, 2014. Atlanta, GA: US Department of Health and Human Services. http://www.cdc.gov/diabetes/pubs/ statsreport14/national-diabetes-report-web.pdf

52. American Diabetes Association. Standards of medical care in diabetes-2012. Diabetes Care 2012;35(Suppl 1):S11-63.

53. CDC. Basic information about cancer survivorship. Atlanta, GA: CDC; 2011. http://www.cdc.gov/cancer/survivorship/basic_info 
54. De Moor JS, Mariotto AB, Parry C, et al. Cancer survivors in the United States: prevalence across the survivorship trajectory and implications for care. Cancer epidemiology, biomarkers \& prevention : a publication of the American Association for Cancer Research, cosponsored by the American Society of Preventive Oncology. 2013;22:561-70.

55. CDC. Surveillance or demographic characteristics and health behaviors among adult cancer survivors - behavioral risk factor surveillance system, United States. MMWR Morb Mortal Wkly Rep 2009;61:1.

56. US Department of Health and Human Services. Expert panel report 3: guidelines for the diagnosis and management of asthma, full report 2007. Bethesda, MD: US Department of Health and Human Services, NIH, National Heart, Lung and Blood Institute; 2007. NIH Publication No. 07-4051. http://www.nhlbi.nih.gov/files/docs/guidelines/asthgdln.pdf

57. Moorman JE, Akinbami LJ, Bailey CM, et al. National surveillance of asthma: United States, 2001-2010. National Center for Health Statistics. Vital Health Stat 3;2012:1-58.

58. CDC. Prevalence and most common causes of disability among adultsUnited States, 2005. MMWR Morb Mortal Wkly Rep 2009;58:421-6.

59. CDC. Prevalence of doctor-diagnosed arthritis and arthritis-attributable activity limitation-United States, 2010-2012. MMWR Morb Mortal Wkly Rep 2013;62:869-73.

60. Ferrari AJ, Charlson FJ, Norman RE, et al. Burden of depressive disorders by country, sex, age, and year: findings from the global burden of disease study 2010. PLoS Med 2013;10:e1001547.

61. Chapman DP, Perry GS, Strine TW. The vital link between chronic disease and depressive disorders. Prev Chronic Dis 2005;2:A14.

62. Strine TW, Mokdad AH, Balluz LS, et al. Depression and anxiety in the United States: findings from the 2006 Behavioral Risk Factor Surveillance System. Psychiatr Serv 2008;59:1383-90.

63. Lépine J-P, Briley M. The increasing burden of depression. Neuropsychiatr Dis Treat 2011;7(Suppl 1):3-7 10.2147/NDT.S19617.

64. Mozaffarian D, Benjamin EJ, Go AS, et al.; American Heart Association Statistics Committee and Stroke Statistics Subcommittee. Heart disease and stroke statistics-2015 update: a report from the American Heart Association. Circulation 2015;131:e29-322.
65. CDC. Vital signs: avoidable deaths from heart disease, stroke, and hypertensive disease- United States, 2001-2010. MMWR Morb Mortal Wkly Rep 2013;62:721-7.

66. Okoro CA, Strine TW, Balluz LS, et al. Serious psychological distress among adults with and without disabilities. Int J Public Health 2009;54(Suppl 1):52-60.

67. Brault MW. Americans with disabilities: 2010, current population reports, US Census Bureau, Washington, DC, 2012:70-131.

68. American Psychological Association. Disability and socioeconomic status. Washington, DC: American Psychological Association; 2013. http:// www.apa.org/pi/ses/resources/publications/factsheet-disability.aspx

69. Carlson D, Ehrlich N. US Department of Education, National Institute on Disability and Rehabilitation Research. Assistive technology and information technology use and need by persons with disabilities in the United States. Washington, DC; 2005.

70. Li C, Balluz LS, Ford ES, Okoro CA, Zhao G, Pierannunzi C. A comparison of prevalence estimates for selected health indicators and chronic diseases or conditions from the Behavioral Risk Factor Surveillance System, the National Health Interview Survey, and the National Health and Nutrition Examination Survey, 2007-2008. Prev Med 2012;54:381-7.

71. CDC. Behavioral Risk Factor Surveillance System history. Atlanta, GA: US Department of Health and Human Services, CDC; 2011. http:// www.cdc.gov/brfss/factsheets/pdf/brfss-history.pdf

72. CDC. 2011-2012 Behavioral Risk Factor Surveillance System asthma call-back survey history and analysis guidance (version 1.0.0), National Asthma Control Program. 2014. http://www.cdc.gov/brfss/acbs/2012/ pdf/acbs_2012.pdf

73. CDC. BRFSS facts \& news data making a difference. Atlanta, GA: US Department of Health and Human Services, CDC; 2014. http://www. cdc.gov/brfss/factsheets/pdf/2014newsletter_summer.pdf

74. Bauer UE, Briss PA, Goodman RA, Bowman BA. Prevention of chronic disease in the 21 st century: elimination of the leading preventable causes of premature death and disability in the USA. Lancet 2014;384:45-52. 
TABLE 1. Estimated prevalence of adults aged $\geq 18$ years who reported good or better health, ${ }^{*}$ by state/territory - Behavioral Risk Factor Surveillance System, United States, 2012

\begin{tabular}{|c|c|c|c|c|}
\hline State/Territory & $\begin{array}{l}\text { Sample } \\
\text { size }\end{array}$ & $\%$ & SE & $95 \% \mathrm{Cl}$ \\
\hline Alabama & 9,002 & 75.0 & 0.7 & $(73.7-76.3)$ \\
\hline Alaska & 4,328 & 85.9 & 0.7 & $(84.5-87.3)$ \\
\hline Arizona & 7,289 & 81.9 & 0.8 & $(80.4-83.4)$ \\
\hline Arkansas & 5,167 & 76.3 & 0.8 & (74.8-77.9) \\
\hline California & 14,570 & 82.3 & 0.5 & $(81.4-83.2)$ \\
\hline Colorado & 12,210 & 85.5 & 0.4 & $(84.6-86.3)$ \\
\hline Connecticut & 8,751 & 85.8 & 0.5 & $(84.8-86.9)$ \\
\hline Delaware & 5,168 & 83.6 & 0.7 & $(82.2-85.0)$ \\
\hline District of Columbia & 3,809 & 87.9 & 0.8 & (86.4-89.4) \\
\hline Florida & 7,572 & 80.1 & 0.7 & (78.6-81.5) \\
\hline Georgia & 6,089 & 82.5 & 0.7 & $(81.2-83.8)$ \\
\hline Hawaii & 7,571 & 85.2 & 0.6 & $(84.0-86.5)$ \\
\hline Idaho & 5,872 & 84.4 & 0.8 & $(82.8-85.9)$ \\
\hline Illinois & 5,576 & 82.6 & 0.8 & $(81.1-84.2)$ \\
\hline Indiana & 8,578 & 80.0 & 0.6 & (78.9-81.1) \\
\hline lowa & 7,146 & 86.0 & 0.5 & $(85.1-87.0)$ \\
\hline Kansas & 11,775 & 84.0 & 0.5 & $(83.1-85.0)$ \\
\hline Kentucky & 11,188 & 76.1 & 0.6 & (74.9-77.3) \\
\hline Louisiana & 9,046 & 77.5 & 0.7 & (76.2-78.8) \\
\hline Maine & 9,861 & 83.9 & 0.5 & $(83.0-84.9)$ \\
\hline Maryland & 12,788 & 84.2 & 0.6 & (83.1-85.4) \\
\hline Massachusetts & 21,671 & 86.6 & 0.4 & (85.9-87.3) \\
\hline Michigan & 10,485 & 82.9 & 0.5 & (81.9-83.9) \\
\hline Minnesota & 12,225 & 88.3 & 0.4 & $(87.5-89.0)$ \\
\hline Mississippi & 7,765 & 76.6 & 0.6 & (75.4-77.9) \\
\hline Missouri & 6,731 & 81.3 & 0.7 & $(80.0-82.6)$ \\
\hline Montana & 8,655 & 84.2 & 0.5 & $(83.3-85.2)$ \\
\hline Nebraska & 19,132 & 85.6 & 0.4 & $(84.9-86.3)$ \\
\hline Nevada & 4,832 & 81.1 & 0.8 & $(79.4-82.7)$ \\
\hline New Hampshire & 7,510 & 86.5 & 0.6 & (85.4-87.6) \\
\hline New Jersey & 15,702 & 83.9 & 0.4 & $(83.1-84.7)$ \\
\hline New Mexico & 8,757 & 78.9 & 0.6 & (77.8-80.0) \\
\hline New York & 6,004 & 82.5 & 0.7 & $(81.1-84.0)$ \\
\hline North Carolina & 11,844 & 80.7 & 0.5 & $(79.8-81.6)$ \\
\hline North Dakota & 4,845 & 86.6 & 0.6 & (85.4-87.8) \\
\hline Ohio & 13,001 & 81.7 & 0.5 & (80.8-82.6) \\
\hline Oklahoma & 7,990 & 81.0 & 0.6 & $(79.9-82.1)$ \\
\hline Oregon & 5,286 & 81.8 & 0.7 & $(80.4-83.2)$ \\
\hline Pennsylvania & 19,798 & 83.1 & 0.4 & $(82.3-83.9)$ \\
\hline Rhode Island & 5,468 & 83.7 & 0.7 & $(82.3-85.0)$ \\
\hline South Carolina & 12,738 & 81.1 & 0.5 & $(80.1-82.0)$ \\
\hline South Dakota & 7,862 & 86.9 & 0.6 & $(85.8-88.1)$ \\
\hline Tennessee & 7,036 & 78.9 & 0.6 & $(77.7-80.2)$ \\
\hline Texas & 9,051 & 80.8 & 0.6 & (79.7-81.9) \\
\hline Utah & 12,385 & 86.9 & 0.4 & $(86.1-87.7)$ \\
\hline Vermont & 6,041 & 88.0 & 0.5 & (86.9-89.0) \\
\hline Virginia & 7,374 & 82.5 & 0.6 & $(81.4-83.7)$ \\
\hline Washington & 15,288 & 83.8 & 0.4 & $(83.0-84.7)$ \\
\hline West Virginia & 5,400 & 74.8 & 0.7 & (73.5-76.1) \\
\hline Wisconsin & 5,292 & 86.0 & 0.7 & (84.6-87.4) \\
\hline Wyoming & 6,259 & 85.1 & 0.7 & (83.7-86.5) \\
\hline Guam & 2,025 & 78.2 & 1.2 & $(75.7-80.6)$ \\
\hline Puerto Rico & 6,316 & 64.0 & 0.7 & $(62.6-65.3)$ \\
\hline Median & & 82.9 & & \\
\hline Range & & $64.0-88.3$ & & \\
\hline
\end{tabular}

Abbreviations: $\mathrm{Cl}=$ confidence interval; $\mathrm{SE}=$ standard error.

* Respondents were asked to rate general health as poor, fair, good, very good, or excellent. Respondents were classified into two groups: those who reported fair or poor health and those with good, very good, or excellent health.

${ }^{\dagger}$ Metropolitan division.
TABLE 2. Estimated prevalence of adults aged $\geq 18$ years who reported good or better health, ${ }^{*}$ by metropolitan and micropolitan statistical area - Behavioral Risk Factor Surveillance System, United States, 2012

\begin{tabular}{|c|c|c|c|c|}
\hline MMSA(s) & $\begin{array}{l}\text { Sample } \\
\text { size }\end{array}$ & $\%$ & SE & $95 \% \mathrm{Cl}$ \\
\hline Aguadilla-Isabela, Puerto Rico & 549 & 62.7 & 2.3 & $(58.1-67.3)$ \\
\hline Akron, Ohio & 744 & 79.5 & 2.1 & $(75.4-83.5)$ \\
\hline Albuquerque, New Mexico & 3,266 & 82.3 & 0.8 & $(80.6-83.9)$ \\
\hline $\begin{array}{l}\text { Allentown-Bethlehem-Easton, } \\
\text { Pennsylvania-New Jersey }\end{array}$ & 1,344 & 83.2 & 1.6 & $(80.1-86.2)$ \\
\hline Anaheim-Santa Ana-Irvine, California ${ }^{\dagger}$ & 1,037 & 87.0 & 1.4 & $(84.3-89.7)$ \\
\hline Anchorage, Alaska & 1,501 & 86.3 & 1.1 & $(84.1-88.4)$ \\
\hline Asheville, North Carolina & 592 & 83.6 & 1.8 & $(80.1-87.1)$ \\
\hline $\begin{array}{l}\text { Atlanta-Sandy Springs-Roswell, } \\
\text { Georgia }\end{array}$ & 2,535 & 86.5 & 0.9 & $(84.8-88.3)$ \\
\hline Atlantic City-Hammonton, New Jersey & 1,021 & 83.5 & 1.5 & $(80.6-86.5)$ \\
\hline $\begin{array}{l}\text { Augusta-Richmond County, Georgia- } \\
\text { South Carolina }\end{array}$ & 1,034 & 82.4 & 2.1 & $(78.3-86.5)$ \\
\hline Augusta-Waterville, Maine & 830 & 82.2 & 1.6 & $(79.1-85.4)$ \\
\hline Austin-Round Rock, Texas & 1,380 & 84.2 & 1.6 & $(81.0-87.4)$ \\
\hline $\begin{array}{l}\text { Baltimore-Columbia-Towson, } \\
\text { Maryland }\end{array}$ & 4,716 & 83.5 & 0.8 & $(81.8-85.1)$ \\
\hline Bangor, Maine & 920 & 82.4 & 1.6 & $(79.2-85.6)$ \\
\hline Barnstable Town, Massachusetts & 557 & 88.4 & 1.7 & $(85.0-91.7)$ \\
\hline Barre, Vermont & 515 & 89.5 & 1.7 & $(86.2-92.8)$ \\
\hline Baton Rouge, Louisiana & 1,390 & 80.0 & 1.6 & $(76.8-83.1)$ \\
\hline Bellingham, Washington & 846 & 88.0 & 1.4 & $(85.3-90.7)$ \\
\hline Berlin, New Hampshire-Vermont & 709 & 79.1 & 2.4 & $(74.3-83.8)$ \\
\hline Billings, Montana & 846 & 84.6 & 1.4 & $(81.9-87.3)$ \\
\hline Birmingham-Hoover, Alabama & 1,820 & 78.3 & 1.3 & $(75.8-80.7)$ \\
\hline Bismarck, North Dakota & 817 & 86.1 & 1.5 & $(83.2-89.1)$ \\
\hline Boise City, Idaho & 1,479 & 85.9 & 1.4 & $(83.2-88.5)$ \\
\hline Boston, Massachusetts $^{\dagger}$ & 5,902 & 87.1 & 0.6 & $(85.8-88.3)$ \\
\hline Boulder, Colorado & 515 & 90.5 & 1.5 & $(87.6-93.4)$ \\
\hline Bremerton-Silverdale, Washington & 566 & 81.5 & 2.2 & $(77.2-85.8)$ \\
\hline $\begin{array}{l}\text { Bridgeport-Stamford-Norwalk, } \\
\text { Connecticut }\end{array}$ & 2,180 & 86.0 & 1.1 & $(83.8-88.2)$ \\
\hline Burlington-South Burlington, Vermont & 1,520 & 90.1 & 0.9 & $(88.4-91.9)$ \\
\hline $\begin{array}{l}\text { Cambridge-Newton-Framingham, } \\
\text { Massachusetts }{ }^{\dagger}\end{array}$ & 6,924 & 88.0 & 0.6 & $(86.9-89.2)$ \\
\hline Camden, New Jersey ${ }^{\dagger}$ & 1,978 & 82.5 & 1.2 & $(80.2-84.8)$ \\
\hline Canton-Massillon, Ohio & 681 & 79.9 & 2.1 & $(75.8-84.0)$ \\
\hline Casper, Wyoming & 827 & 83.4 & 1.9 & $(79.6-87.1)$ \\
\hline Cedar Rapids, lowa & 585 & 87.3 & 1.6 & $(84.2-90.4)$ \\
\hline Charleston, West Virginia & 771 & 74.4 & 1.8 & $(70.9-77.9)$ \\
\hline $\begin{array}{l}\text { Charleston-North Charleston, } \\
\text { South Carolina }\end{array}$ & 1,700 & 84.5 & 1.2 & $(82.2-86.8)$ \\
\hline $\begin{array}{l}\text { Charlotte-Concord-Gastonia, } \\
\text { North Carolina-South Carolina }\end{array}$ & 2,515 & 82.6 & 1.0 & $(80.7-84.5)$ \\
\hline Chattanooga, Tennessee-Georgia & 631 & 75.7 & 2.7 & $(70.5-81.0)$ \\
\hline Cheyenne, Wyoming & 958 & 82.2 & 1.9 & $(78.4-86.0)$ \\
\hline $\begin{array}{l}\text { Chicago-Naperville-Elgin, } \\
\text { Illinois-Indiana-Wisconsin }\end{array}$ & 3,725 & 83.6 & 1.0 & $(81.7-85.6)$ \\
\hline Cincinnati, Ohio-Kentucky-Indiana & 2,360 & 82.3 & 1.0 & $(80.3-84.3)$ \\
\hline $\begin{array}{l}\text { Claremont-Lebanon, New } \\
\text { Hampshire-Vermont }\end{array}$ & 1,948 & 87.7 & 0.9 & $(85.9-89.6)$ \\
\hline Cleveland-Elyria, Ohio & 1,779 & 82.5 & 1.2 & $(80.2-84.9)$ \\
\hline Colorado Springs, Colorado & 1,157 & 87.3 & 1.1 & $(85.0-89.5)$ \\
\hline Columbia, South Carolina & 1,798 & 83.1 & 1.3 & $(80.5-85.6)$ \\
\hline Columbus, Ohio & 1,602 & 84.6 & 1.3 & $(82.0-87.1)$ \\
\hline Concord, New Hampshire & 704 & 86.8 & 1.6 & $(83.7-90.0)$ \\
\hline Dallas-Plano-Irving, Texas ${ }^{\dagger}$ & 899 & 83.3 & 1.5 & $(80.4-86.1)$ \\
\hline Dayton, Ohio & 855 & 81.5 & 1.7 & $(78.3-84.8)$ \\
\hline Denver-Aurora-Lakewood, Colorado & 4,836 & 85.0 & 0.7 & $(83.6-86.3)$ \\
\hline Des Moines-West Des Moines, lowa & 1,156 & 87.0 & 1.2 & $(84.7-89.3)$ \\
\hline
\end{tabular}
See table footnotes on page 18. 
TABLE 2. (Continued) Estimated prevalence of adults aged $\geq 18$ years who reported good or better health,* by metropolitan and micropolitan statistical area - Behavioral Risk Factor Surveillance System, United States, 2012

\begin{tabular}{|c|c|c|c|c|}
\hline MMSA(s) & $\begin{array}{l}\text { Sample } \\
\text { size }\end{array}$ & $\%$ & SE & $95 \% \mathrm{Cl}$ \\
\hline Detroit-Dearborn-Livonia, Michigan $^{\dagger}$ & 2,218 & 78.1 & 1.5 & $(75.3-81.0)$ \\
\hline Dover, Delaware & 1,439 & 82.1 & 1.4 & $(79.3-84.8)$ \\
\hline Duluth, Minnesota-Wisconsin & 514 & 84.9 & 1.9 & $(81.1-88.7)$ \\
\hline Durham-Chapel Hill, North Carolina & 790 & 84.6 & 1.6 & $(81.4-87.8)$ \\
\hline El Paso, Texas & 622 & 76.6 & 2.3 & $(72.0-81.1)$ \\
\hline Eugene, Oregon & 525 & 84.5 & 1.9 & $(80.8-88.1)$ \\
\hline Fairbanks, Alaska & 597 & 86.2 & 1.6 & $(83.0-89.4)$ \\
\hline Fargo, North Dakota-Minnesota & 937 & 90.5 & 1.2 & $(88.2-92.8)$ \\
\hline Farmington, New Mexico & 650 & 79.1 & 2.0 & $(75.2-82.9)$ \\
\hline Fayetteville, North Carolina & 503 & 83.6 & 2.0 & $(79.6-87.6)$ \\
\hline $\begin{array}{l}\text { Fayetteville-Springdale-Rogers, } \\
\text { Arkansas-Missouri }\end{array}$ & 811 & 79.7 & 2.2 & $(75.4-84.0)$ \\
\hline Fort Collins, Colorado & 596 & 88.8 & 1.6 & $(85.6-91.9)$ \\
\hline Fort Wayne, Indiana & 534 & 82.3 & 2.1 & $(78.1-86.5)$ \\
\hline Fort Worth-Arlington, Texas ${ }^{\dagger}$ & 722 & 84.3 & 1.8 & $(80.8-87.7)$ \\
\hline Grand Island, Nebraska & 853 & 85.4 & 1.6 & $(82.3-88.4)$ \\
\hline Grand Rapids-Wyoming, Michigan & 892 & 88.1 & 1.4 & $(85.4-90.8)$ \\
\hline Great Falls, Montana & 707 & 83.0 & 1.6 & $(79.8-86.2)$ \\
\hline Greeley, Colorado & 534 & 84.5 & 1.9 & $(80.8-88.2)$ \\
\hline $\begin{array}{l}\text { Greensboro-High Point, } \\
\text { North Carolina }\end{array}$ & 805 & 81.5 & 1.6 & $(78.3-84.7)$ \\
\hline $\begin{array}{l}\text { Greenville-Anderson-Mauldin, South } \\
\text { Carolina }\end{array}$ & 1,687 & 81.0 & 1.2 & $(78.6-83.4)$ \\
\hline Gulfport-Biloxi-Pascagoula, Mississippi & 742 & 76.4 & 1.9 & $(72.6-80.2)$ \\
\hline $\begin{array}{l}\text { Hagerstown-Martinsburg, Maryland- } \\
\text { West Virginia }\end{array}$ & 760 & 84.0 & 2.0 & $(80.2-87.9)$ \\
\hline Harrisburg-Carlisle, Pennsylvania & 659 & 85.9 & 1.7 & $(82.6-89.3)$ \\
\hline $\begin{array}{l}\text { Hartford-West Hartford-East Hartford, } \\
\text { Connecticut }\end{array}$ & 2,661 & 86.3 & 0.9 & $(84.6-88.0)$ \\
\hline Heber, Utah & 510 & 88.4 & 2.6 & $(83.2-93.5)$ \\
\hline Hilo, Hawaii & 1,348 & 84.7 & 1.4 & $(81.9-87.4)$ \\
\hline $\begin{array}{l}\text { Hilton Head Island-Bluffton-Beaufort, } \\
\text { South Carolina }\end{array}$ & 932 & 90.5 & 1.3 & $(88.0-93.0)$ \\
\hline $\begin{array}{l}\text { Houston-The Woodlands-Sugar Land, } \\
\text { Texas }\end{array}$ & 1,139 & 80.9 & 1.5 & $(78.1-83.8)$ \\
\hline $\begin{array}{l}\text { Huntington-Ashland, West } \\
\text { Virginia-Kentucky-Ohio }\end{array}$ & 1,114 & 73.3 & 1.6 & $(70.2-76.4)$ \\
\hline Huntsville, Alabama & 614 & 78.8 & 2.3 & $(74.4-83.3)$ \\
\hline Idaho Falls, Idaho & 540 & 85.1 & 2.4 & $(80.3-89.9)$ \\
\hline $\begin{array}{l}\text { Indianapolis-Carmel-Anderson, } \\
\text { Indiana }\end{array}$ & 2,182 & 81.0 & 1.1 & $(78.8-83.1)$ \\
\hline Jackson, Mississippi & 920 & 80.0 & 1.5 & $(77.0-83.0)$ \\
\hline Jacksonville, Florida & 518 & 81.4 & 2.5 & $(76.5-86.3)$ \\
\hline Kahului-Wailuku-Lahaina, Hawaii & 1,216 & 86.3 & 1.4 & $(83.6-89.1)$ \\
\hline Kalispell, Montana & 560 & 82.6 & 1.9 & $(78.8-86.3)$ \\
\hline Kansas City, Missouri-Kansas & 4,733 & 84.0 & 1.0 & $(82.1-85.9)$ \\
\hline Kapaa, Hawaii & 669 & 83.4 & 2.2 & $(79.2-87.7)$ \\
\hline Keene, New Hampshire & 546 & 84.8 & 2.4 & $(80.0-89.5)$ \\
\hline Kennewick-Richland, Washington & 535 & 80.8 & 2.2 & $(76.6-85.1)$ \\
\hline $\begin{array}{l}\text { Kingsport-Bristol-Bristol, } \\
\text { Tennessee-Virginia }\end{array}$ & 568 & 73.0 & 2.7 & $(67.8-78.2)$ \\
\hline Knoxville, Tennessee & 833 & 79.3 & 1.8 & $(75.8-82.9)$ \\
\hline Laconia, New Hampshire & 566 & 81.1 & 3.1 & $(75.0-87.3)$ \\
\hline Lafayette, Louisiana & 555 & 83.9 & 1.9 & $(80.2-87.5)$ \\
\hline Las Cruces, New Mexico & 706 & 71.2 & 2.3 & $(66.7-75.8)$ \\
\hline $\begin{array}{l}\text { Las Vegas-Henderson-Paradise, } \\
\text { Nevada }\end{array}$ & 007 & 80.3 & 1.1 & $(78.1-82.5)$ \\
\hline Lewiston-Auburn, Maine & 695 & 81.6 & 1.9 & $(78.0-85.3)$ \\
\hline Lexington-Fayette, Kentucky & 531 & 83.5 & 1.9 & $(79.8-87.2)$ \\
\hline Lincoln, Nebraska & 1,667 & 87.9 & 1.0 & $(86.0-89.8)$ \\
\hline
\end{tabular}

TABLE 2. (Continued) Estimated prevalence of adults aged $\geq 18$ years who reported good or better health,* by metropolitan and micropolitan statistical area - Behavioral Risk Factor Surveillance System, United States, 2012

\begin{tabular}{|c|c|c|c|c|}
\hline MMSA(s) & $\begin{array}{l}\text { Sample } \\
\text { size }\end{array}$ & $\%$ & SE & $95 \% \mathrm{Cl}$ \\
\hline $\begin{array}{l}\text { Little Rock-North Little Rock-Conway, } \\
\text { Arkansas }\end{array}$ & 1,172 & 80.7 & 1.5 & $(77.8-83.5)$ \\
\hline Logan, Utah-Idaho & 504 & 90.1 & 1.7 & $(86.8-93.4)$ \\
\hline $\begin{array}{l}\text { Los Angeles-Long Beach-Glendale, } \\
\text { California }^{\dagger}\end{array}$ & 3,502 & 78.4 & 1.0 & $(76.5-80.4)$ \\
\hline $\begin{array}{l}\text { Louisville/Jefferson County, } \\
\text { Kentucky-Indiana }\end{array}$ & 2,178 & 78.2 & 1.4 & $(75.5-80.8)$ \\
\hline Lumberton, North Carolina & 545 & 72.7 & 3.6 & $(65.7-79.8)$ \\
\hline Manchester-Nashua, New Hampshire & 1,903 & 87.2 & 0.9 & $(85.3-89.0)$ \\
\hline McAllen-Edinburg-Mission, Texas & 614 & 71.9 & 2.8 & $(66.4-77.4)$ \\
\hline $\begin{array}{l}\text { Memphis, } \\
\text { Tennessee-Mississippi-Arkansas }\end{array}$ & 1,309 & 79.8 & 1.5 & $(76.9-82.7)$ \\
\hline $\begin{array}{l}\text { Miami-Fort Lauderdale-West Palm } \\
\text { Beach, Florida }\end{array}$ & 1,659 & 81.0 & 1.4 & $(78.2-83.8)$ \\
\hline $\begin{array}{l}\text { Milwaukee-Waukesha-West Allis, } \\
\text { Wisconsin }\end{array}$ & 1,256 & 85.3 & 1.5 & $(82.3-88.2)$ \\
\hline $\begin{array}{l}\text { Minneapolis-St. Paul-Bloomington, } \\
\text { Minnesota-Wisconsin }\end{array}$ & 7,887 & 89.2 & 0.5 & $(88.2-90.3)$ \\
\hline Missoula, Montana & 778 & 87.2 & 1.3 & $(84.6-89.8)$ \\
\hline Mobile, Alabama & 815 & 74.3 & 2.3 & $(69.7-78.9)$ \\
\hline Montgomery, Alabama & 534 & 78.5 & 2.3 & $(74.0-82.9)$ \\
\hline $\begin{array}{l}\text { Montgomery County-Bucks County- } \\
\text { Chester County, Pennsylvania }{ }^{\dagger}\end{array}$ & 1,311 & 87.5 & 1.2 & $(85.2-89.8)$ \\
\hline $\begin{array}{l}\text { Myrtle Beach-Conway-North Myrtle } \\
\text { Beach, South Carolina-North Carolina }\end{array}$ & 945 & 83.3 & 1.5 & $(80.3-86.2)$ \\
\hline $\begin{array}{l}\text { Nashville-Davidson-Murfreesboro- } \\
\text { Franklin, Tennessee }\end{array}$ & 1,339 & 84.7 & 1.2 & $(82.3-87.0)$ \\
\hline $\begin{array}{l}\text { Nassau County-Suffolk County, } \\
\text { New York }^{\dagger}\end{array}$ & 894 & 88.8 & 1.3 & $(86.3-91.3)$ \\
\hline Newark, New Jersey-Pennsylvania ${ }^{\dagger}$ & 6,530 & 85.9 & 0.7 & $(84.6-87.2)$ \\
\hline New Haven-Milford, Connecticut & 2,006 & 84.2 & 1.2 & $(81.9-86.6)$ \\
\hline New Orleans-Metairie, Louisiana & 1,274 & 80.7 & 1.5 & $(77.8-83.5)$ \\
\hline $\begin{array}{l}\text { New York-Jersey City-White Plains, } \\
\text { New York-New Jersey }{ }^{\dagger}\end{array}$ & 7,403 & 81.4 & 0.9 & $(79.6-83.1)$ \\
\hline Norfolk, Nebraska & 569 & 85.6 & 1.9 & $(81.9-89.2)$ \\
\hline North Platte, Nebraska & 613 & 85.6 & 1.9 & $(81.9-89.2)$ \\
\hline Norwich-New London, Connecticut & 997 & 87.1 & 1.5 & $(84.2-89.9)$ \\
\hline $\begin{array}{l}\text { Oakland-Hayward-Berkeley, } \\
\text { California }{ }^{\dagger}\end{array}$ & 976 & 87.0 & 1.8 & $(83.6-90.5)$ \\
\hline Ocean City, New Jersey & 552 & 80.4 & 2.8 & $(74.9-85.9)$ \\
\hline Ogden-Clearfield, Utah & 2,423 & 87.1 & 0.8 & $(85.5-88.7)$ \\
\hline Oklahoma City, Oklahoma & 2,434 & 82.0 & 0.9 & $(80.1-83.8)$ \\
\hline Olympia-Tumwater, Washington & 515 & 81.1 & 2.4 & $(76.5-85.8)$ \\
\hline Omaha-Council Bluffs, Nebraska-lowa & 5,580 & 86.0 & 0.6 & $(84.7-87.2)$ \\
\hline Orlando-Kissimmee-Sanford, Florida & 566 & 76.8 & 2.8 & $(71.3-82.4)$ \\
\hline Philadelphia, Pennsylvania $^{\dagger}$ & 2,431 & 81.6 & 1.0 & $(79.6-83.7)$ \\
\hline Phoenix-Mesa-Scottsdale, Arizona & 2,600 & 83.2 & 1.0 & $(81.2-85.2)$ \\
\hline Pittsburgh, Pennsylvania & 3,351 & 83.4 & 0.8 & $(81.9-84.9)$ \\
\hline Ponce, Puerto Rico & 548 & 64.5 & 2.4 & $(59.8-69.2)$ \\
\hline Portland-South Portland, Maine & 3,314 & 86.6 & 0.8 & $(85.1-88.1)$ \\
\hline $\begin{array}{l}\text { Portland-Vancouver-Hillsboro, } \\
\text { Oregon-Washington }\end{array}$ & 3,126 & 84.5 & 0.9 & $(82.8-86.3)$ \\
\hline $\begin{array}{l}\text { Providence-Warwick, Rhode } \\
\text { Island-Massachusetts }\end{array}$ & 8,116 & 83.5 & 0.6 & $(82.3-84.7)$ \\
\hline Provo-Orem, Utah & 1,750 & 88.6 & 0.9 & $(86.8-90.5)$ \\
\hline Raleigh, North Carolina & 944 & 85.5 & 1.3 & $(82.9-88.1)$ \\
\hline Rapid City, South Dakota & 1,051 & 85.8 & 1.4 & $(83.0-88.6)$ \\
\hline Reno, Nevada & 1,508 & 83.6 & 1.4 & $(80.8-86.3)$ \\
\hline Richmond, Virginia & 1,011 & 84.6 & 1.5 & $(81.7-87.6)$ \\
\hline
\end{tabular}

See table footnotes on page 18. 
TABLE 2. (Continued) Estimated prevalence of adults aged $\geq 18$ years who reported good or better health,* by metropolitan and micropolitan statistical area - Behavioral Risk Factor Surveillance System, United States, 2012

\begin{tabular}{|c|c|c|c|c|}
\hline MMSA(s) & $\begin{array}{l}\text { Sample } \\
\text { size }\end{array}$ & $\%$ & SE & $95 \% \mathrm{Cl}$ \\
\hline $\begin{array}{l}\text { Riverside-San Bernardino-Ontario, } \\
\text { California }\end{array}$ & 1,529 & 81.8 & 1.3 & $(79.2-84.4)$ \\
\hline $\begin{array}{l}\text { Rockingham County-Strafford County, } \\
\text { New Hampshire }\end{array}$ & 1,671 & 86.9 & 1.1 & $(84.8-89.1)$ \\
\hline Rutland, Vermont & 596 & 84.9 & 1.9 & $(81.2-88.7)$ \\
\hline $\begin{array}{l}\text { Sacramento-Roseville-Arden-Arcade, } \\
\text { California }\end{array}$ & 998 & 85.8 & 1.8 & $(82.3-89.4)$ \\
\hline St. Louis, Missouri-Illinois & 2,065 & 83.6 & 1.1 & $(81.4-85.8)$ \\
\hline Salisbury, Maryland-Delaware & 2,123 & 78.9 & 1.7 & $(75.6-82.2)$ \\
\hline Salt Lake City, Utah & 4,598 & 85.8 & 0.7 & $(84.4-87.2)$ \\
\hline San Antonio-New Braunfels, Texas & 775 & 81.0 & 1.8 & $(77.5-84.6)$ \\
\hline San Diego-Carlsbad, California & 1,128 & 84.2 & 1.7 & $(81.0-87.5)$ \\
\hline $\begin{array}{l}\text { San Francisco-Redwood City-South } \\
\text { San Francisco, California }{ }^{\dagger}\end{array}$ & 595 & 86.6 & 2.0 & $(82.7-90.4)$ \\
\hline $\begin{array}{l}\text { San Jose-Sunnyvale-Santa Clara, } \\
\text { California }\end{array}$ & 684 & 88.2 & 1.8 & $(84.7-91.7)$ \\
\hline San Juan-Carolina-Caguas, Puerto Rico & 3,955 & 65.8 & 0.9 & $(64.1-67.5)$ \\
\hline Santa Fe, New Mexico & 691 & 83.6 & 1.7 & $(80.3-86.8)$ \\
\hline Sayre, Pennsylvania & 1,845 & 84.5 & 1.2 & $(82.2-86.8)$ \\
\hline Scottsbluff, Nebraska & 629 & 80.3 & 2.6 & $(75.2-85.5)$ \\
\hline $\begin{array}{l}\text { Scranton-Wilkes-Barre-Hazleton, } \\
\text { Pennsylvania }\end{array}$ & 738 & 82.9 & 1.8 & $(79.4-86.3)$ \\
\hline Seattle-Bellevue-Everett, Washington ${ }^{\dagger}$ & 5,095 & 86.2 & 0.7 & $(84.9-87.5)$ \\
\hline Shreveport-Bossier City, Louisiana & 579 & 76.2 & 2.3 & $(71.7-80.7)$ \\
\hline $\begin{array}{l}\text { Silver Spring-Frederick-Rockville, } \\
\text { Maryland }{ }^{\dagger}\end{array}$ & 2,295 & 86.4 & 1.3 & $(83.9-88.9)$ \\
\hline $\begin{array}{l}\text { Sioux City, lowa-Nebraska-South } \\
\text { Dakota }\end{array}$ & 1,203 & 82.0 & 2.3 & $(77.5-86.4)$ \\
\hline Sioux Falls, South Dakota & 1,465 & 88.7 & 1.1 & $(86.6-90.8)$ \\
\hline Spartanburg, South Carolina & 706 & 77.2 & 2.3 & $(72.8-81.7)$ \\
\hline Spokane-Spokane Valley, Washington & 1,096 & 85.1 & 1.4 & $(82.4-87.8)$ \\
\hline Springfield, Massachusetts & 2,343 & 82.4 & 1.2 & $(80.0-84.7)$ \\
\hline Tacoma-Lakewood, Washington ${ }^{\dagger}$ & 1,181 & 83.2 & 1.5 & $(80.4-86.1)$ \\
\hline $\begin{array}{l}\text { Tampa-St. Petersburg-Clearwater, } \\
\text { Florida }\end{array}$ & 823 & 81.0 & 2.1 & $(77.0-85.1)$ \\
\hline
\end{tabular}

TABLE 2. (Continued) Estimated prevalence of adults aged $\geq 18$ years who reported good or better health,* by metropolitan and micropolitan statistical area - Behavioral Risk Factor Surveillance System, United States, 2012

\begin{tabular}{|c|c|c|c|c|}
\hline MMSA(s) & $\begin{array}{l}\text { Sample } \\
\text { size }\end{array}$ & $\%$ & SE & $95 \% \mathrm{Cl}$ \\
\hline Toledo, Ohio & 972 & 81.2 & 1.8 & $(77.7-84.8)$ \\
\hline Topeka, Kansas & 1,086 & 82.9 & 1.5 & $(79.9-85.9)$ \\
\hline Torrington, Connecticut & 662 & 88.3 & 1.6 & $(85.1-91.5)$ \\
\hline Trenton, New Jersey & 576 & 86.1 & 1.8 & (82.7-89.6) \\
\hline Tucson, Arizona & 999 & 81.2 & 1.9 & $(77.5-84.9)$ \\
\hline Tulsa, Oklahoma & 1,737 & 83.6 & 1.0 & $(81.6-85.6)$ \\
\hline Tuscaloosa, Alabama & 580 & 73.4 & 3.0 & $(67.5-79.3)$ \\
\hline Urban Honolulu, Hawaii & 4,338 & 85.3 & 0.8 & $(83.7-86.9)$ \\
\hline Vineland-Bridgeton, New Jersey & 558 & 76.5 & 2.9 & $(70.9-82.2)$ \\
\hline $\begin{array}{l}\text { Virginia Beach-Norfolk-Newport News, } \\
\text { Virginia-North Carolina }\end{array}$ & 1,501 & 82.8 & 1.3 & $(80.2-85.4)$ \\
\hline $\begin{array}{l}\text { Warren-Troy-Farmington Hills, } \\
\text { Michigan }\end{array}$ & 2,183 & 86.5 & 0.9 & $(84.7-88.3)$ \\
\hline $\begin{array}{l}\text { Washington-Arlington-Alexandria, } \\
\text { District of Columbia-Virginia- } \\
\text { Maryland-West Virginia }{ }^{\dagger}\end{array}$ & 8,178 & 86.5 & 0.7 & $(85.2-87.8)$ \\
\hline Wichita, Kansas & 2,355 & 84.8 & 1.0 & $(82.8-86.7)$ \\
\hline $\begin{array}{l}\text { Wilmington, Delaware-Maryland- } \\
\text { New Jersey }{ }^{\dagger}\end{array}$ & 3,230 & 85.1 & 0.9 & $(83.4-86.8)$ \\
\hline Winston-Salem, North Carolina & 793 & 76.9 & 1.8 & (73.3-80.5) \\
\hline Worcester, Massachusetts-Connecticut & t 2,841 & 87.3 & 0.9 & $(85.6-89.0)$ \\
\hline Yakima, Washington & 531 & 73.5 & 2.7 & $(68.3-78.8)$ \\
\hline $\begin{array}{l}\text { Youngstown-Warren-Boardman, } \\
\text { Ohio-Pennsylvania }\end{array}$ & 926 & 79.5 & 2.1 & $(75.3-83.7)$ \\
\hline Median & & 83.6 & & \\
\hline Range & & $62.7-90.5$ & & \\
\hline
\end{tabular}

Abbreviations: $\mathrm{Cl}=$ confidence interval; $\mathrm{MMSA}=$ metropolitan and micropolitan statistical area; $\mathrm{SE}=$ standard error.

* Respondents were asked to rate general health as poor, fair, good, very good, or excellent. Respondents were classified into two groups: those who reported fair or poor health and those with good, very good, or excellent health.

+ Metropolitan division. 
TABLE 3. Estimated prevalence of adults aged $\geq 18$ years who reported good or better health, ${ }^{*}$ by county - Behavioral Risk Factor Surveillance System, United States, 2012

\begin{tabular}{|c|c|c|c|c|}
\hline County & $\begin{array}{l}\text { Sample } \\
\text { size }\end{array}$ & $\%$ & SE & $95 \% \mathrm{Cl}$ \\
\hline Jefferson County, Alabama & 960 & 79.5 & 1.5 & $(76.6-82.5)$ \\
\hline Madison County, Alabama & 511 & 80.9 & 2.4 & $(76.2-85.7)$ \\
\hline Mobile County, Alabama & 815 & 74.3 & 2.3 & $(69.7-78.9)$ \\
\hline Anchorage Municipality, Alaska & 902 & 87.5 & 1.3 & $(85.0-90.0)$ \\
\hline Fairbanks North Star Borough, Alaska & 597 & 86.2 & 1.6 & $(83.0-89.4)$ \\
\hline Matanuska-Susitna Borough, Alaska & 599 & 82.3 & 1.9 & $(78.6-86.0)$ \\
\hline Maricopa County, Arizona & 2,119 & 83.2 & 1.1 & $(81.1-85.3)$ \\
\hline Pima County, Arizona & 999 & 81.2 & 1.9 & $(77.6-84.9)$ \\
\hline Pulaski County, Arkansas & 749 & 82.9 & 1.8 & $(79.4-86.3)$ \\
\hline Alameda County, California & 619 & 86.2 & 2.5 & $(81.3-91.0)$ \\
\hline Los Angeles County, California & 3,502 & 78.4 & 1.0 & $(76.5-80.4)$ \\
\hline Orange County, California & 1,037 & 87.0 & 1.4 & $(84.3-89.7)$ \\
\hline Riverside County, California & 824 & 79.7 & 1.9 & $(75.9-83.4)$ \\
\hline Sacramento County, California & 609 & 82.4 & 2.5 & $(77.5-87.3)$ \\
\hline San Bernardino County, California & 705 & 84.8 & 1.8 & $(81.3-88.3)$ \\
\hline San Diego County, California & 1,128 & 84.2 & 1.7 & $(81.0-87.5)$ \\
\hline Santa Clara County, California & 665 & 88.3 & 1.8 & $(84.7-91.9)$ \\
\hline Adams County, Colorado & 804 & 79.1 & 1.9 & $(75.4-82.7)$ \\
\hline Arapahoe County, Colorado & 853 & 85.7 & 1.4 & $(83.0-88.5)$ \\
\hline Boulder County, Colorado & 515 & 90.5 & 1.5 & $(87.6-93.4)$ \\
\hline Denver County, Colorado & 998 & 82.1 & 1.4 & $(79.2-84.9)$ \\
\hline Douglas County, Colorado & 545 & 92.4 & 1.6 & $(89.3-95.5)$ \\
\hline El Paso County, Colorado & 1,017 & 87.3 & 1.2 & $(85.0-89.6)$ \\
\hline Jefferson County, Colorado & 1,114 & 88.7 & 1.2 & $(86.5-91.0)$ \\
\hline Larimer County, Colorado & 596 & 88.8 & 1.6 & $(85.6-91.9)$ \\
\hline Weld County, Colorado & 534 & 84.5 & 1.9 & $(80.8-88.2)$ \\
\hline Fairfield County, Connecticut & 2,180 & 86.0 & 1.1 & $(83.8-88.2)$ \\
\hline Hartford County, Connecticut & 1,979 & 85.3 & 1.0 & $(83.2-87.3)$ \\
\hline Litchfield County, Connecticut & 662 & 88.3 & 1.6 & $(85.1-91.5)$ \\
\hline New Haven County, Connecticut & 2,006 & 84.2 & 1.2 & $(81.9-86.6)$ \\
\hline New London County, Connecticut & 997 & 87.1 & 1.5 & $(84.2-89.9)$ \\
\hline Kent County, Delaware & 1,439 & 82.1 & 1.4 & (79.3-84.8) \\
\hline New Castle County, Delaware & 2,331 & 85.6 & 1.0 & $(83.7-87.5)$ \\
\hline Sussex County, Delaware & 1,398 & 79.9 & 1.5 & $(77.0-82.8)$ \\
\hline $\begin{array}{l}\text { District of Columbia, } \\
\text { District of Columbia }\end{array}$ & 3,809 & 87.9 & 0.8 & $(86.4-89.4)$ \\
\hline Broward County, Florida & 523 & 83.4 & 2.2 & $(79.0-87.8)$ \\
\hline Miami-Dade County, Florida & 812 & 78.4 & 2.2 & $(74.0-82.8)$ \\
\hline Hawaii County, Hawaii & 1,348 & 84.7 & 1.4 & $(81.9-87.4)$ \\
\hline Honolulu County, Hawaii & 4,338 & 85.3 & 0.8 & $(83.7-86.9)$ \\
\hline Kauai County, Hawaii & 669 & 83.4 & 2.2 & $(79.2-87.7)$ \\
\hline Maui County, Hawaii & 1,216 & 86.3 & 1.4 & $(83.6-89.1)$ \\
\hline Ada County, Idaho & 808 & 87.5 & 1.7 & $(84.2-90.7)$ \\
\hline Canyon County, Idaho & 502 & 81.3 & 2.8 & $(75.9-86.7)$ \\
\hline Cook County, Illinois & 1,503 & 82.5 & 1.4 & $(79.8-85.3)$ \\
\hline Lake County, Indiana & 883 & 76.6 & 2.6 & $(71.5-81.7)$ \\
\hline Marion County, Indiana & 1,269 & 80.4 & 1.4 & $(77.6-83.2)$ \\
\hline Polk County, lowa & 805 & 87.0 & 1.4 & $(84.2-89.7)$ \\
\hline Johnson County, Kansas & 2,175 & 90.2 & 0.9 & $(88.4-91.9)$ \\
\hline Sedgwick County, Kansas & 1,794 & 84.1 & 1.1 & $(81.9-86.3)$ \\
\hline Shawnee County, Kansas & 768 & 82.5 & 1.8 & $(79.0-86.1)$ \\
\hline Wyandotte County, Kansas & 871 & 71.5 & 2.8 & $(66.0-77.0)$ \\
\hline Jefferson County, Kentucky & 1,693 & 76.9 & 1.8 & $(73.4-80.3)$ \\
\hline East Baton Rouge Parish, Louisiana & 573 & 80.9 & 2.2 & $(76.5-85.3)$ \\
\hline Androscoggin County, Maine & 695 & 81.6 & 1.9 & $(78.0-85.3)$ \\
\hline Aroostook County, Maine & 537 & 77.6 & 2.1 & $(73.5-81.6)$ \\
\hline Cumberland County, Maine & 1,753 & 87.1 & 1.0 & $(85.0-89.1)$ \\
\hline Kennebec County, Maine & 830 & 82.2 & 1.6 & $(79.1-85.4)$ \\
\hline Penobscot County, Maine & 920 & 82.4 & 1.6 & (79.2-85.6) \\
\hline York County, Maine & 1,187 & 86.6 & 1.2 & $(84.2-89.0)$ \\
\hline
\end{tabular}

See table footnotes on page 20.
TABLE 3. (Continued) Estimated prevalence of adults aged $\geq 18$ years who reported good or better health, ${ }^{*}$ by county - Behavioral Risk Factor Surveillance System, United States, 2012

\begin{tabular}{|c|c|c|c|c|}
\hline County & $\begin{array}{c}\text { Sample } \\
\text { size }\end{array}$ & $\%$ & SE & $95 \% \mathrm{Cl}$ \\
\hline Anne Arundel County, Maryland & 894 & 85.5 & 1.7 & $(82.2-88.8)$ \\
\hline Baltimore County, Maryland & 1,521 & 83.3 & 1.4 & $(80.6-86.0)$ \\
\hline Charles County, Maryland & 512 & 83.2 & 2.8 & $(77.6-88.8)$ \\
\hline Frederick County, Maryland & 755 & 85.8 & 2.5 & $(80.9-90.7)$ \\
\hline Montgomery County, Maryland & 1,540 & 86.4 & 1.5 & $(83.5-89.3)$ \\
\hline Prince George's County, Maryland & 1,142 & 85.2 & 1.8 & $(81.7-88.7)$ \\
\hline Washington County, Maryland & 538 & 85.5 & 2.5 & $(80.5-90.5)$ \\
\hline Baltimore city, Maryland & 743 & 76.9 & 2.4 & $(72.2-81.6)$ \\
\hline Barnstable County, Massachusetts & 557 & 88.4 & 1.7 & $(85.0-91.7)$ \\
\hline Bristol County, Massachusetts & 2,648 & 82.2 & 1.2 & $(79.8-84.6)$ \\
\hline Essex County, Massachusetts & 2,565 & 85.8 & 1.1 & $(83.6-87.9)$ \\
\hline Hampden County, Massachusetts & 1,961 & 81.3 & 1.3 & $(78.8-83.8)$ \\
\hline Middlesex County, Massachusetts & 4,359 & 89.1 & 0.7 & $(87.8-90.4)$ \\
\hline Norfolk County, Massachusetts & 1,758 & 90.7 & 1.0 & $(88.8-92.7)$ \\
\hline Plymouth County, Massachusetts & 1,833 & 85.9 & 1.3 & $(83.4-88.5)$ \\
\hline Suffolk County, Massachusetts & 2,311 & 84.4 & 1.1 & $(82.2-86.6)$ \\
\hline Worcester County, Massachusetts & 2,596 & 87.6 & 0.9 & $(85.8-89.3)$ \\
\hline Kent County, Michigan & 525 & 89.8 & 1.6 & $(86.7-93.0)$ \\
\hline Macomb County, Michigan & 612 & 85.5 & 1.6 & $(82.3-88.6)$ \\
\hline Oakland County, Michigan & 1,168 & 89.0 & 1.1 & $(86.8-91.2)$ \\
\hline Wayne County, Michigan & 2,218 & 78.1 & 1.4 & $(75.3-81.0)$ \\
\hline Anoka County, Minnesota & 545 & 90.1 & 1.4 & $(87.4-92.8)$ \\
\hline Dakota County, Minnesota & 672 & 90.5 & 1.5 & $(87.6-93.4)$ \\
\hline Hennepin County, Minnesota & 3,297 & 90.0 & 0.8 & $(88.3-91.6)$ \\
\hline Ramsey County, Minnesota & 1,941 & 86.5 & 1.4 & $(83.7-89.3)$ \\
\hline Jackson County, Missouri & 881 & 81.1 & 2.1 & $(76.9-85.3)$ \\
\hline St. Louis County, Missouri & 950 & 84.8 & 1.5 & $(81.8-87.8)$ \\
\hline Cascade County, Montana & 707 & 83.0 & 1.6 & $(79.8-86.2)$ \\
\hline Flathead County, Montana & 560 & 82.6 & 1.9 & $(78.8-86.3)$ \\
\hline Hill County, Montana & 584 & 79.7 & 2.6 & $(74.5-84.9)$ \\
\hline Lake County, Montana & 899 & 79.7 & 2.1 & $(75.6-83.9)$ \\
\hline Missoula County, Montana & 778 & 87.2 & 1.3 & $(84.6-89.8)$ \\
\hline Yellowstone County, Montana & 749 & 85.1 & 1.4 & $(82.3-87.8)$ \\
\hline Dakota County, Nebraska & 732 & 75.6 & 4.5 & $(66.8-84.5)$ \\
\hline Douglas County, Nebraska & 3,567 & 83.8 & 0.8 & $(82.3-85.4)$ \\
\hline Hall County, Nebraska & 533 & 84.8 & 2.0 & $(80.8-88.7)$ \\
\hline Lancaster County, Nebraska & 1,445 & 88.0 & 1.0 & $(86.0-90.0)$ \\
\hline Lincoln County, Nebraska & 589 & 85.5 & 1.9 & $(81.8-89.2)$ \\
\hline Sarpy County, Nebraska & 1,175 & 91.6 & 0.9 & $(89.7-93.4)$ \\
\hline Scotts Bluff County, Nebraska & 576 & 79.5 & 2.7 & $(74.2-84.8)$ \\
\hline Clark County, Nevada & 2,007 & 80.3 & 1.1 & $(78.1-82.5)$ \\
\hline Washoe County, Nevada & 1,489 & 83.6 & 1.4 & $(80.8-86.4)$ \\
\hline Belknap County, New Hampshire & 566 & 81.1 & 3.1 & $(75.0-87.3)$ \\
\hline Carroll County, New Hampshire & 528 & 86.1 & 2.5 & $(81.2-91.1)$ \\
\hline Cheshire County, New Hampshire & 546 & 84.8 & 2.4 & $(80.1-89.5)$ \\
\hline Coos County, New Hampshire & 539 & 79.5 & 2.9 & $(73.8-85.2)$ \\
\hline Grafton County, New Hampshire & 566 & 89.0 & 1.5 & (86.1-91.9) \\
\hline Hillsborough County, New Hampshire & 1,903 & 87.2 & 0.9 & $(85.3-89.0)$ \\
\hline Merrimack County, New Hampshire & 704 & 86.8 & 1.6 & $(83.7-90.0)$ \\
\hline Rockingham County, New Hampshire & 1,050 & 88.3 & 1.3 & $(85.6-90.9)$ \\
\hline Strafford County, New Hampshire & 621 & 83.9 & 1.9 & $(80.1-87.7)$ \\
\hline Atlantic County, New Jersey & 1,021 & 83.5 & 1.5 & $(80.6-86.5)$ \\
\hline Bergen County, New Jersey & 1,009 & 86.7 & 1.3 & $(84.1-89.3)$ \\
\hline Burlington County, New Jersey & 684 & 83.4 & 2.0 & $(79.6-87.3)$ \\
\hline Camden County, New Jersey & 744 & 78.7 & 2.1 & $(74.5-82.8)$ \\
\hline Cape May County, New Jersey & 552 & 80.4 & 2.8 & $(74.9-85.9)$ \\
\hline Cumberland County, New Jersey & 558 & 76.5 & 2.9 & $(70.9-82.2)$ \\
\hline Essex County, New Jersey & 1,336 & 81.3 & 1.3 & $(78.7-84.0)$ \\
\hline Gloucester County, New Jersey & 550 & 86.5 & 1.8 & $(83.0-90.0)$ \\
\hline Hudson County, New Jersey & 1,303 & 78.3 & 1.5 & $(75.4-81.2)$ \\
\hline
\end{tabular}
See table footnotes on page 20 . 
TABLE 3. (Continued) Estimated prevalence of adults aged $\geq 18$ years who reported good or better health,* by county - Behavioral Risk Factor Surveillance System, United States, 2012

\begin{tabular}{|c|c|c|c|c|}
\hline County & $\begin{array}{l}\text { Sample } \\
\text { size }\end{array}$ & $\%$ & SE & $95 \% \mathrm{Cl}$ \\
\hline Hunterdon County, New Jersey & 571 & 91.5 & 1.4 & $(88.8-94.3)$ \\
\hline Mercer County, New Jersey & 576 & 86.1 & 1.8 & $(82.7-89.6)$ \\
\hline Middlesex County, New Jersey & 880 & 86.5 & 1.4 & $(83.7-89.3)$ \\
\hline Monmouth County, New Jersey & 710 & 86.4 & 1.7 & $(83.1-89.8)$ \\
\hline Morris County, New Jersey & 848 & 89.9 & 1.3 & $(87.4-92.4)$ \\
\hline Ocean County, New Jersey & 662 & 83.2 & 1.8 & $(79.7-86.6)$ \\
\hline Passaic County, New Jersey & 688 & 75.6 & 2.3 & $(71.1-80.1)$ \\
\hline Salem County, New Jersey & 583 & 77.1 & 3.3 & $(70.7-83.5)$ \\
\hline Somerset County, New Jersey & 638 & 91.3 & 1.5 & $(88.3-94.3)$ \\
\hline Sussex County, New Jersey & 546 & 85.3 & 2.2 & $(81.1-89.6)$ \\
\hline Union County, New Jersey & 711 & 84.3 & 1.6 & $(81.1-87.5)$ \\
\hline Warren County, New Jersey & 532 & 83.5 & 2.8 & $(78.0-89.0)$ \\
\hline Bernalillo County, New Mexico & 2,062 & 83.4 & 1.0 & $(81.4-85.3)$ \\
\hline Dona Ana County, New Mexico & 706 & 71.2 & 2.3 & $(66.7-75.8)$ \\
\hline Sandoval County, New Mexico & 656 & 84.5 & 1.8 & $(81.0-88.0)$ \\
\hline San Juan County, New Mexico & 650 & 79.1 & 2.0 & $(75.2-82.9)$ \\
\hline Santa Fe County, New Mexico & 691 & 83.6 & 1.7 & $(80.3-86.8)$ \\
\hline Kings County, New York & 490 & 83.0 & 2.3 & $(78.5-87.6)$ \\
\hline Guilford County, North Carolina & 511 & 87.8 & 1.6 & $(84.6-91.0)$ \\
\hline Mecklenburg County, North Carolina & 726 & 83.9 & 1.6 & $(80.9-87.0)$ \\
\hline Robeson County, North Carolina & 545 & 72.7 & 3.6 & $(65.7-79.8)$ \\
\hline Wake County, North Carolina & 700 & 88.1 & 1.4 & $(85.2-90.9)$ \\
\hline Burleigh County, North Dakota & 563 & 86.0 & 1.9 & $(82.3-89.7)$ \\
\hline Cass County, North Dakota & 841 & 90.3 & 1.3 & $(87.7-92.8)$ \\
\hline Cuyahoga County, Ohio & 868 & 80.0 & 1.6 & $(76.8-83.2)$ \\
\hline Franklin County, Ohio & 837 & 84.6 & 1.8 & $(81.1-88.0)$ \\
\hline Hamilton County, Ohio & 772 & 82.1 & 1.7 & $(78.8-85.5)$ \\
\hline Lorain County, Ohio & 612 & 85.7 & 1.9 & $(81.9-89.6)$ \\
\hline Lucas County, Ohio & 623 & 78.6 & 2.4 & $(73.9-83.3)$ \\
\hline Mahoning County, Ohio & 590 & 81.5 & 2.5 & $(76.7-86.3)$ \\
\hline Montgomery County, Ohio & 663 & 80.8 & 1.9 & $(77.1-84.6)$ \\
\hline Stark County, Ohio & 623 & 80.1 & 2.2 & $(75.9-84.4)$ \\
\hline Summit County, Ohio & 631 & 78.0 & 2.4 & $(73.3-82.6)$ \\
\hline Oklahoma County, Oklahoma & 1,188 & 80.9 & 1.3 & (78.4-83.4) \\
\hline Tulsa County, Oklahoma & 1,200 & 86.8 & 1.1 & $(84.6-88.9)$ \\
\hline Clackamas County, Oregon & 511 & 83.5 & 2.6 & (78.5-88.5) \\
\hline Lane County, Oregon & 525 & 84.5 & 1.9 & $(80.8-88.1)$ \\
\hline Multnomah County, Oregon & 895 & 83.6 & 1.7 & $(80.3-87.0)$ \\
\hline Washington County, Oregon & 619 & 85.6 & 2.0 & $(81.8-89.4)$ \\
\hline Allegheny County, Pennsylvania & 1,882 & 85.1 & 1.0 & $(83.1-87.0)$ \\
\hline Bradford County, Pennsylvania & 1,845 & 84.5 & 1.2 & $(82.2-86.8)$ \\
\hline Montgomery County, Pennsylvania & 546 & 90.6 & 1.3 & $(88.0-93.2)$ \\
\hline Philadelphia County, Pennsylvania & 2,041 & 79.0 & 1.2 & $(76.5-81.4)$ \\
\hline Pike County, Pennsylvania & 1,880 & 86.0 & 1.3 & $(83.5-88.6)$ \\
\hline Kent County, Rhode Island & 812 & 85.2 & 1.7 & $(81.9-88.5)$ \\
\hline Providence County, Rhode Island & 3,338 & 81.1 & 0.9 & $(79.3-82.9)$ \\
\hline Washington County, Rhode Island & 657 & 89.9 & 1.7 & $(86.4-93.3)$ \\
\hline Aiken County, South Carolina & 554 & 80.3 & 2.1 & $(76.1-84.5)$ \\
\hline Beaufort County, South Carolina & 800 & 91.8 & 1.2 & $(89.4-94.2)$ \\
\hline
\end{tabular}

TABLE 3. (Continued) Estimated prevalence of adults aged $\geq 18$ years who reported good or better health, ${ }^{*}$ by county - Behavioral Risk Factor Surveillance System, United States, 2012

\begin{tabular}{|c|c|c|c|c|}
\hline County & $\begin{array}{l}\text { Sample } \\
\text { size }\end{array}$ & $\%$ & SE & $95 \% \mathrm{Cl}$ \\
\hline Charleston County, South Carolina & 1,010 & 85.3 & 1.6 & $(82.2-88.3)$ \\
\hline Greenville County, South Carolina & 904 & 82.7 & 1.6 & $(79.5-85.9)$ \\
\hline Horry County, South Carolina & 776 & 84.6 & 1.5 & $(81.6-87.6)$ \\
\hline Richland County, South Carolina & 963 & 84.6 & 1.7 & $(81.3-88.0)$ \\
\hline Spartanburg County, South Carolina & 652 & 79.4 & 2.3 & $(75.0-83.8)$ \\
\hline Lincoln County, South Dakota & 500 & 90.2 & 2.1 & $(86.0-94.4)$ \\
\hline Minnehaha County, South Dakota & 878 & 87.6 & 1.3 & $(85.0-90.2)$ \\
\hline Pennington County, South Dakota & 604 & 86.4 & 1.6 & $(83.2-89.5)$ \\
\hline Davidson County, Tennessee & 555 & 85.6 & 1.8 & $(82.0-89.2)$ \\
\hline Shelby County, Tennessee & 537 & 83.2 & 1.9 & $(79.6-86.8)$ \\
\hline Bexar County, Texas & 582 & 79.9 & 2.2 & $(75.6-84.1)$ \\
\hline Dallas County, Texas & 508 & 79.6 & 2.2 & $(75.4-83.8)$ \\
\hline El Paso County, Texas & 621 & 76.5 & 2.3 & $(71.9-81.1)$ \\
\hline Harris County, Texas & 771 & 78.6 & 1.9 & $(75.0-82.3)$ \\
\hline Hidalgo County, Texas & 614 & 71.9 & 2.8 & $(66.4-77.4)$ \\
\hline Tarrant County, Texas & 567 & 84.1 & 2.0 & $(80.1-88.1)$ \\
\hline Travis County, Texas & 1,042 & 84.3 & 2.0 & $(80.5-88.1)$ \\
\hline Davis County, Utah & 1,144 & 89.6 & 1.0 & $(87.6-91.6)$ \\
\hline Salt Lake County, Utah & 4,033 & 85.7 & 0.7 & $(84.3-87.2)$ \\
\hline Tooele County, Utah & 565 & 87.3 & 2.0 & $(83.3-91.2)$ \\
\hline Utah County, Utah & 1,682 & 88.6 & 1.0 & $(86.7-90.5)$ \\
\hline Wasatch County, Utah & 510 & 88.4 & 2.6 & $(83.2-93.5)$ \\
\hline Weber County, Utah & 1,045 & 83.9 & 1.4 & $(81.1-86.7)$ \\
\hline Chittenden County, Vermont & 918 & 92.0 & 1.0 & $(90.0-94.0)$ \\
\hline Rutland County, Vermont & 596 & 84.9 & 1.9 & $(81.2-88.7)$ \\
\hline Washington County, Vermont & 515 & 89.5 & 1.7 & $(86.2-92.8)$ \\
\hline Windsor County, Vermont & 545 & 89.0 & 1.6 & $(85.9-92.1)$ \\
\hline Fairfax County, Virginia & 742 & 88.7 & 1.7 & $(85.4-92.1)$ \\
\hline Clark County, Washington & 789 & 85.7 & 1.4 & $(82.9-88.5)$ \\
\hline King County, Washington & 3,923 & 87.0 & 0.8 & $(85.5-88.6)$ \\
\hline Kitsap County, Washington & 566 & 81.5 & 2.2 & $(77.2-85.8)$ \\
\hline Pierce County, Washington & 1,181 & 83.2 & 1.4 & $(80.4-86.1)$ \\
\hline Snohomish County, Washington & 1,172 & 83.7 & 1.5 & $(80.8-86.6)$ \\
\hline Spokane County, Washington & 950 & 85.4 & 1.5 & $(82.5-88.3)$ \\
\hline Thurston County, Washington & 515 & 81.1 & 2.4 & $(76.5-85.8)$ \\
\hline Whatcom County, Washington & 846 & 88.0 & 1.4 & $(85.3-90.7)$ \\
\hline Yakima County, Washington & 531 & 73.5 & 2.7 & $(68.3-78.8)$ \\
\hline Kanawha County, West Virginia & 640 & 76.1 & 1.9 & $(72.4-79.9)$ \\
\hline Milwaukee County, Wisconsin & 963 & 83.2 & 1.8 & $(79.7-86.7)$ \\
\hline Laramie County, Wyoming & 958 & 82.2 & 1.9 & $(78.4-86.0)$ \\
\hline Natrona County, Wyoming & 827 & 83.4 & 1.9 & $(79.6-87.1)$ \\
\hline San Juan Municipio, Puerto Rico & 667 & 68.1 & 2.1 & $(63.9-72.3)$ \\
\hline Median & & 84.4 & & \\
\hline Range & & $68.1-92$ & & \\
\hline
\end{tabular}

Abbreviations: $\mathrm{Cl}=$ confidence interval; $\mathrm{SE}=$ standard error.

* Respondents were asked to rate general health as poor, fair, good, very good, or excellent. Respondents were classified into two groups: those who reported fair or poor health and those with good, very good, or excellent health. 
TABLE 4. Estimated prevalence of adults aged 18-64 years who have health care coverage, ${ }^{*}$ by state/territory - Behavioral Risk Factor Surveillance System, United States, 2012

\begin{tabular}{|c|c|c|c|c|}
\hline State/Territory & $\begin{array}{l}\text { Sample } \\
\text { size }\end{array}$ & $\%$ & SE & $95 \% \mathrm{Cl}$ \\
\hline Alabama & 5,768 & 76.5 & 0.9 & $(74.8-78.2)$ \\
\hline Alaska & 3,487 & 80.1 & 1.0 & (78.3-82.0) \\
\hline Arizona Alaska & 4,297 & 75.3 & 1.1 & (73.2-77.4) \\
\hline Arkansas & 3,167 & 68.9 & 1.1 & $(66.7-71.1)$ \\
\hline California & 10,219 & 74.9 & 0.6 & (73.7-76.1) \\
\hline Colorado & 8,532 & 78.6 & 0.6 & (77.3-79.8) \\
\hline Connecticut & 5,900 & 87.2 & 0.6 & $(86.0-88.4)$ \\
\hline Delaware & 3,412 & 86.1 & 0.9 & (84.3-87.9) \\
\hline District of Columbia & 2,479 & 90.2 & 1.0 & (88.4-92.1) \\
\hline Florida & 4,436 & 72.4 & 1.0 & $(70.4-74.4)$ \\
\hline Georgia & 4,099 & 72.7 & 1.0 & (70.7-74.6) \\
\hline Hawaii & 5,505 & 87.3 & 0.7 & $(85.9-88.7)$ \\
\hline Idaho & 3,630 & 76.6 & 1.2 & (74.2-78.9) \\
\hline Illinois & 3,586 & 80.0 & 1.0 & (78.0-82.1) \\
\hline Indiana & 5,795 & 78.2 & 0.7 & (76.8-79.7) \\
\hline lowa & 4,578 & 86.6 & 0.7 & (85.3-87.9) \\
\hline Kansas & 7,753 & 79.5 & 0.7 & (78.2-80.8) \\
\hline Kentucky & 7,680 & 79.1 & 0.7 & (77.6-80.6) \\
\hline Louisiana & 5,769 & 74.3 & 0.9 & (72.5-76.2) \\
\hline Maine & 6,656 & 84.3 & 0.6 & (83.1-85.4) \\
\hline Maryland & 8,404 & 84.3 & 0.8 & (82.8-85.8) \\
\hline Massachusetts & 14,920 & 93.1 & 0.3 & (92.4-93.7) \\
\hline Michigan & 6,820 & 83.4 & 0.6 & $(82.2-84.7)$ \\
\hline Minnesota & 8,646 & 86.8 & 0.5 & $(85.8-87.8)$ \\
\hline Mississippi & 4,869 & 72.7 & 0.9 & (70.9-74.5) \\
\hline Missouri & 4,245 & 79.7 & 0.9 & (77.9-81.4) \\
\hline Montana & 5,776 & 76.8 & 0.7 & (75.4-78.2) \\
\hline Nebraska & 12,310 & 82.0 & 0.5 & $(81.0-83.0)$ \\
\hline Nevada & 3,191 & 69.8 & 1.2 & $(67.5-72.1)$ \\
\hline New Hampshire & 4,780 & 84.2 & 0.8 & (82.6-85.7) \\
\hline New Jersey & 11,036 & 81.4 & 0.5 & (80.4-82.5) \\
\hline New Mexico & 6,060 & 73.2 & 0.7 & (71.8-74.7) \\
\hline New York & 4,231 & 81.4 & 0.9 & (79.6-83.2) \\
\hline North Carolina & 8,064 & 74.6 & 0.6 & (73.4-75.8) \\
\hline North Dakota & 3,240 & 84.0 & 0.9 & (82.1-85.8) \\
\hline Ohio & 8,810 & 82.2 & 0.6 & $(81.1-83.4)$ \\
\hline Oklahoma & 5,364 & 77.9 & 0.8 & $(76.5-79.4)$ \\
\hline Oregon & 3,387 & 76.7 & 1.0 & $(74.8-78.7)$ \\
\hline Pennsylvania & 13,020 & 83.8 & 0.5 & (82.8-84.8) \\
\hline Rhode Island & 3,711 & 81.6 & 0.9 & $(79.8-83.4)$ \\
\hline South Carolina & 8,211 & 73.5 & 0.7 & (72.1-74.9) \\
\hline South Dakota & 5,432 & 86.2 & 0.7 & (84.8-87.6) \\
\hline Tennessee & 4,616 & 78.1 & 0.9 & (76.3-79.8) \\
\hline Texas & 6,311 & 64.2 & 0.8 & $(62.6-65.9)$ \\
\hline Utah & 8,924 & 79.6 & 0.6 & (78.4-80.8) \\
\hline Vermont & 4,011 & 88.1 & 0.7 & $(86.7-89.5)$ \\
\hline Virginia & 5,033 & 81.8 & 0.8 & (80.3-83.3) \\
\hline Washington & 10,135 & 78.8 & 0.6 & (77.6-79.9) \\
\hline West Virginia & 3,665 & 75.8 & 0.9 & (74.0-77.5) \\
\hline Wisconsin & 3,608 & 85.3 & 0.9 & $(83.5-87.1)$ \\
\hline Wyoming & 3,762 & 75.2 & 1.2 & (72.9-77.5) \\
\hline Guam & 1,778 & 73.4 & 1.4 & $(70.6-76.2)$ \\
\hline Puerto Rico & 4,417 & 90.2 & 0.6 & (89.1-91.3) \\
\hline Median & & 79.6 & & \\
\hline Range & & $64.2-93.1$ & & \\
\hline
\end{tabular}

Abbreviations: $\mathrm{Cl}=$ confidence interval; $\mathrm{SE}=$ standard error.

* Including health insurance, prepaid plans (e.g., health maintenance organizations), or government plans (e.g., Medicare).
TABLE 5. Estimated prevalence of adults aged 18-64 years who have health care coverage,* by metropolitan and micropolitan statistical area - Behavioral Risk Factor Surveillance System, United States, 2012

\begin{tabular}{|c|c|c|c|c|}
\hline MMSA(s) & $\begin{array}{l}\text { Sample } \\
\text { size }\end{array}$ & $\%$ & SE & $95 \% \mathrm{Cl}$ \\
\hline Aguadilla-Isabela, Puerto Rico & 390 & 92.3 & 1.6 & $(89.2-95.4)$ \\
\hline Akron, Ohio & 503 & 79.1 & 2.7 & $(73.8-84.5)$ \\
\hline Albuquerque, New Mexico & 2,318 & 76.4 & 1.1 & $(74.2-78.7)$ \\
\hline $\begin{array}{l}\text { Allentown-Bethlehem-Easton, } \\
\text { Pennsylvania-New Jersey }\end{array}$ & 902 & 89.1 & 1.5 & $(86.2-92.0)$ \\
\hline Anaheim-Santa Ana-Irvine, California ${ }^{\dagger}$ & 705 & 82.4 & 1.8 & (78.9-86.0) \\
\hline Anchorage, Alaska & 1,239 & 80.9 & 1.4 & $(78.2-83.6)$ \\
\hline Asheville, North Carolina & 364 & 72.1 & 3.0 & $(66.2-78.0)$ \\
\hline $\begin{array}{l}\text { Atlanta-Sandy Springs-Roswell, } \\
\text { Georgia }\end{array}$ & 1,809 & 74.2 & 1.5 & $(71.4-77.1)$ \\
\hline Atlantic City-Hammonton, New Jersey & 655 & 78.6 & 2.3 & $(74.1-83.2)$ \\
\hline $\begin{array}{l}\text { Augusta-Richmond County, Georgia- } \\
\text { South Carolina }\end{array}$ & 657 & 74.3 & 3.2 & $(68.1-80.5)$ \\
\hline Augusta-Waterville, Maine & 599 & 86.4 & 1.8 & $(82.9-89.9)$ \\
\hline Austin-Round Rock, Texas & 964 & 71.9 & 2.4 & $(67.2-76.6)$ \\
\hline $\begin{array}{l}\text { Baltimore-Columbia-Towson, } \\
\text { Maryland }\end{array}$ & 3,212 & 86.2 & 1.1 & $(84.1-88.3)$ \\
\hline Bangor, Maine & 663 & 80.9 & 2.1 & $(76.7-85.0)$ \\
\hline Barnstable Town, Massachusetts & 280 & 91.8 & 2.1 & $(87.7-96.0)$ \\
\hline Barre, Vermont & 377 & 87.9 & 2.3 & $(83.3-92.5)$ \\
\hline Baton Rouge, Louisiana & 887 & 79.7 & 2.1 & $(75.5-83.9)$ \\
\hline Bellingham, Washington & 550 & 77.2 & 3.2 & (70.9-83.5) \\
\hline Berlin, New Hampshire-Vermont & 422 & 72.4 & 3.5 & $(65.5-79.2)$ \\
\hline Billings, Montana & 580 & 80.0 & 1.8 & $(76.4-83.6)$ \\
\hline Birmingham-Hoover, Alabama & 1,216 & 77.4 & 1.7 & $(74.0-80.8)$ \\
\hline Bismarck, North Dakota & 557 & 88.1 & 2.1 & $(83.9-92.3)$ \\
\hline Boise City, Idaho & 957 & 76.3 & 2.1 & $(72.2-80.4)$ \\
\hline Boston, Massachusetts $^{\dagger}$ & 4,103 & 93.4 & 0.6 & $(92.3-94.6)$ \\
\hline Boulder, Colorado & 381 & 79.3 & 2.8 & $(73.7-84.9)$ \\
\hline Bremerton-Silverdale, Washington & 357 & 81.8 & 3.0 & $(75.8-87.7)$ \\
\hline $\begin{array}{l}\text { Bridgeport-Stamford-Norwalk, } \\
\text { Connecticut }\end{array}$ & 1,525 & 83.5 & 1.5 & $(80.7-86.4)$ \\
\hline Burlington-South Burlington, Vermont & 1,089 & 91.3 & 1.0 & $(89.2-93.3)$ \\
\hline $\begin{array}{l}\text { Cambridge-Newton-Framingham, } \\
\text { Massachusetts }{ }^{\dagger}\end{array}$ & 4,881 & 93.7 & 0.6 & $(92.6-94.8)$ \\
\hline Camden, New Jersey ${ }^{\dagger}$ & 1,385 & 86.6 & 1.4 & $(83.9-89.3)$ \\
\hline Canton-Massillon, Ohio & 445 & 83.9 & 2.6 & $(78.8-89.1)$ \\
\hline Casper, Wyoming & 485 & 74.7 & 3.0 & $(68.8-80.7)$ \\
\hline Cedar Rapids, lowa & 375 & 89.5 & 2.1 & $(85.4-93.6)$ \\
\hline Charleston, West Virginia & 527 & 76.7 & 2.3 & (72.1-81.3) \\
\hline $\begin{array}{l}\text { Charleston-North Charleston, } \\
\text { South Carolina }\end{array}$ & 1,145 & 77.7 & 1.7 & $(74.4-80.9)$ \\
\hline $\begin{array}{l}\text { Charlotte-Concord-Gastonia, } \\
\text { North Carolina-South Carolina }\end{array}$ & 1,789 & 76.3 & 1.2 & $(73.9-78.8)$ \\
\hline Chattanooga, Tennessee-Georgia & 397 & 77.9 & 3.3 & $(71.4-84.4)$ \\
\hline Cheyenne, Wyoming & 546 & 81.2 & 2.6 & $(76.0-86.4)$ \\
\hline $\begin{array}{l}\text { Chicago-Naperville-Elgin, } \\
\text { Illinois-Indiana-Wisconsin }\end{array}$ & 2,547 & 78.8 & 1.3 & $(76.3-81.3)$ \\
\hline Cincinnati, Ohio-Kentucky-Indiana & 1,680 & 84.5 & 1.2 & $(82.2-86.9)$ \\
\hline $\begin{array}{l}\text { Claremont-Lebanon, } \\
\text { New Hampshire-Vermont }\end{array}$ & 1,217 & 84.6 & 1.6 & $(81.5-87.8)$ \\
\hline Cleveland-Elyria, Ohio & 1,197 & 83.4 & 1.5 & $(80.4-86.3)$ \\
\hline Colorado Springs, Colorado & 849 & 80.9 & 1.7 & $(77.5-84.4)$ \\
\hline Columbia, South Carolina & 1,241 & 75.4 & 1.8 & $(71.9-79.0)$ \\
\hline Columbus, Ohio & 1,176 & 82.9 & 1.4 & $(80.2-85.7)$ \\
\hline Concord, New Hampshire & 457 & 85.2 & 2.1 & $(81.0-89.4)$ \\
\hline Dallas-Plano-Irving, Texas ${ }^{\dagger}$ & 681 & 63.9 & 2.3 & $(59.3-68.4)$ \\
\hline Dayton, Ohio & 562 & 80.2 & 2.3 & $(75.7-84.8)$ \\
\hline Denver-Aurora-Lakewood, Colorado & 3,575 & 79.0 & 0.9 & (77.3-80.8) \\
\hline Des Moines-West Des Moines, lowa & 784 & 89.5 & 1.4 & $(86.8-92.2)$ \\
\hline
\end{tabular}

See table footnotes on page 23. 
TABLE 5. (Continued) Estimated prevalence of adults aged 18-64 years who have health care coverage, ${ }^{*}$ by metropolitan and micropolitan statistical area - Behavioral Risk Factor Surveillance System, United States, 2012

\begin{tabular}{|c|c|c|c|c|}
\hline MMSA(s) & $\begin{array}{l}\text { Sample } \\
\text { size }\end{array}$ & $\%$ & SE & $95 \% \mathrm{Cl}$ \\
\hline 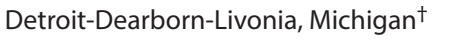 & 1,416 & 77.9 & 1.7 & $(74.6-81.2)$ \\
\hline Dover, Delaware & 939 & 87.3 & 1.7 & $(84.0-90.7)$ \\
\hline Duluth, Minnesota-Wisconsin & 351 & 86.1 & 2.6 & $(81.0-91.3)$ \\
\hline Durham-Chapel Hill, North Carolina & 580 & 72.8 & 2.3 & $(68.2-77.3)$ \\
\hline El Paso, Texas & 421 & 52.1 & 3.8 & $(44.7-59.4)$ \\
\hline Eugene, Oregon & 338 & 74.8 & 3.2 & $(68.5-81.0)$ \\
\hline Fairbanks, Alaska & 486 & 83.7 & 2.3 & $(79.2-88.2)$ \\
\hline Fargo, North Dakota-Minnesota & 662 & 82.2 & 2.2 & $(78.0-86.5)$ \\
\hline Farmington, New Mexico & 458 & 76.9 & 2.4 & $(72.1-81.7)$ \\
\hline Fayetteville, North Carolina & 369 & 78.6 & 3.0 & $(72.7-84.5)$ \\
\hline $\begin{array}{l}\text { Fayetteville-Springdale-Rogers, } \\
\text { Arkansas-Missouri }\end{array}$ & 506 & 67.0 & 3.3 & $(60.5-73.6)$ \\
\hline Fort Collins, Colorado & 407 & 80.9 & 2.5 & $(76.0-85.7)$ \\
\hline Fort Wayne, Indiana & 377 & 73.6 & 3.0 & $(67.7-79.5)$ \\
\hline Fort Worth-Arlington, Texas ${ }^{\dagger}$ & 495 & 70.7 & 2.7 & $(65.5-76.0)$ \\
\hline Grand Island, Nebraska & 533 & 81.2 & 2.7 & $(76.0-86.4)$ \\
\hline Grand Rapids-Wyoming, Michigan & 616 & 87.7 & 1.7 & $(84.4-91.0)$ \\
\hline Great Falls, Montana & 441 & 77.0 & 2.6 & $(72.0-82.0)$ \\
\hline Greeley, Colorado & 399 & 75.9 & 2.7 & $(70.5-81.2)$ \\
\hline $\begin{array}{l}\text { Greensboro-High Point, } \\
\text { North Carolina }\end{array}$ & 544 & 74.3 & 2.4 & $(69.6-79.0)$ \\
\hline $\begin{array}{l}\text { Greenville-Anderson-Mauldin, } \\
\text { South Carolina }\end{array}$ & 1,116 & 72.6 & 1.8 & $(69.1-76.1)$ \\
\hline Gulfport-Biloxi-Pascagoula, Mississippi & 475 & 75.1 & 2.6 & $(70.1-80.1)$ \\
\hline $\begin{array}{l}\text { Hagerstown-Martinsburg, Maryland- } \\
\text { West Virginia }\end{array}$ & 488 & 81.3 & 3.0 & $(75.5-87.1)$ \\
\hline Harrisburg-Carlisle, Pennsylvania & 465 & 83.2 & 2.3 & $(78.7-87.8)$ \\
\hline $\begin{array}{l}\text { Hartford-West Hartford-East Hartford, } \\
\text { Connecticut }\end{array}$ & 1,792 & 89.4 & 1.0 & $(87.6-91.3)$ \\
\hline Heber, Utah & 347 & 72.3 & 5.5 & $(61.6-82.9)$ \\
\hline Hilo, Hawaii & 951 & 83.9 & 1.8 & $(80.5-87.4)$ \\
\hline $\begin{array}{l}\text { Hilton Head Island-Bluffton-Beaufort, } \\
\text { South Carolina }\end{array}$ & 430 & 64.1 & 4.6 & $(55.1-73.2)$ \\
\hline $\begin{array}{l}\text { Houston-The Woodlands-Sugar Land, } \\
\text { Texas }\end{array}$ & 903 & 64.1 & 2.0 & $(60.3-68.0)$ \\
\hline $\begin{array}{l}\text { Huntington-Ashland, West } \\
\text { Virginia-Kentucky-Ohio }\end{array}$ & 756 & 78.9 & 2.1 & $(74.9-83.0)$ \\
\hline Huntsville, Alabama & 451 & 81.3 & 2.4 & $(76.6-86.1)$ \\
\hline Idaho Falls, Idaho & 353 & 83.0 & 3.3 & $(76.5-89.5)$ \\
\hline $\begin{array}{l}\text { Indianapolis-Carmel-Anderson, } \\
\text { Indiana }\end{array}$ & 1,523 & 82.6 & 1.2 & $(80.2-85.0)$ \\
\hline Jackson, Mississippi & 630 & 73.5 & 2.2 & $(69.2-77.7)$ \\
\hline Jacksonville, Florida & 340 & 80.8 & 2.9 & $(75.1-86.6)$ \\
\hline Kahului-Wailuku-Lahaina, Hawaii & 842 & 87.0 & 1.6 & $(83.9-90.2)$ \\
\hline Kalispell, Montana & 374 & 74.4 & 2.5 & $(69.5-79.3)$ \\
\hline Kansas City, Missouri-Kansas & 3,144 & 83.7 & 1.2 & $(81.4-86.0)$ \\
\hline Kapaa, Hawaii & 434 & 88.9 & 2.1 & $(84.8-93.1)$ \\
\hline Keene, New Hampshire & 319 & 80.1 & 3.4 & $(73.4-86.8)$ \\
\hline Kennewick-Richland, Washington & 372 & 73.0 & 3.1 & $(67.0-79.1)$ \\
\hline $\begin{array}{l}\text { Kingsport-Bristol-Bristol, } \\
\text { Tennessee-Virginia }\end{array}$ & 358 & 79.3 & 3.4 & $(72.7-85.9)$ \\
\hline Knoxville, Tennessee & 552 & 80.7 & 2.2 & $(76.4-85.0)$ \\
\hline Laconia, New Hampshire & 320 & 81.9 & 3.6 & $(74.8-89.0)$ \\
\hline Lafayette, Louisiana & 386 & 72.6 & 3.0 & $(66.7-78.5)$ \\
\hline Las Cruces, New Mexico & 489 & 65.4 & 2.8 & $(60.0-70.8)$ \\
\hline $\begin{array}{l}\text { Las Vegas-Henderson-Paradise, } \\
\text { Nevada }\end{array}$ & 1,458 & 69.4 & 1.5 & $(66.5-72.3)$ \\
\hline Lewiston-Auburn, Maine & 505 & 86.6 & 1.8 & $(83.0-90.2)$ \\
\hline Lexington-Fayette, Kentucky & 399 & 84.7 & 2.3 & $(80.2-89.2)$ \\
\hline Lincoln, Nebraska & 1,287 & 82.7 & 1.3 & $(80.2-85.2)$ \\
\hline
\end{tabular}

See table footnotes on page 23.
TABLE 5. (Continued) Estimated prevalence of adults aged 18-64 years who have health care coverage, ${ }^{*}$ by metropolitan and micropolitan statistical area - Behavioral Risk Factor Surveillance System, United States, 2012

\begin{tabular}{|c|c|c|c|c|}
\hline MMSA(s) & $\begin{array}{l}\text { Sample } \\
\text { size }\end{array}$ & $\%$ & SE & $95 \% \mathrm{Cl}$ \\
\hline $\begin{array}{l}\text { Little Rock-North Little Rock-Conway, } \\
\text { Arkansas }\end{array}$ & 779 & 75.9 & 2.1 & $(71.8-80.1)$ \\
\hline Logan, Utah-Idaho & 370 & 82.5 & 2.7 & (77.2-87.8) \\
\hline $\begin{array}{l}\text { Los Angeles-Long Beach-Glendale, } \\
\text { California }^{\dagger}\end{array}$ & 2,609 & 68.3 & 1.3 & $(65.9-70.8)$ \\
\hline $\begin{array}{l}\text { Louisville/Jefferson County, } \\
\text { Kentucky-Indiana }\end{array}$ & 1,466 & 79.9 & 1.6 & $(76.7-83.0)$ \\
\hline Lumberton, North Carolina & 376 & 65.9 & 5.0 & $(56.2-75.7)$ \\
\hline Manchester-Nashua, New Hampshire & 1,332 & 84.8 & 1.5 & $(82.0-87.7)$ \\
\hline McAllen-Edinburg-Mission, Texas & 420 & 35.4 & 3.4 & $(28.8-42.1)$ \\
\hline $\begin{array}{l}\text { Memphis, } \\
\text { Tennessee-Mississippi-Arkansas }\end{array}$ & 865 & 76.9 & 2.0 & $(73.0-80.9)$ \\
\hline $\begin{array}{l}\text { Miami-Fort Lauderdale-West Palm } \\
\text { Beach, Florida }\end{array}$ & 1,028 & 67.9 & 2.1 & $(63.8-72.0)$ \\
\hline $\begin{array}{l}\text { Milwaukee-Waukesha-West Allis, } \\
\text { Wisconsin }\end{array}$ & 912 & 85.6 & 1.8 & $(82.1-89.0)$ \\
\hline $\begin{array}{l}\text { Minneapolis-St. Paul-Bloomington, } \\
\text { Minnesota-Wisconsin }\end{array}$ & 5,704 & 87.3 & 0.7 & $(86.0-88.6)$ \\
\hline Missoula, Montana & 547 & 73.4 & 2.4 & $(68.7-78.1)$ \\
\hline Mobile, Alabama & 501 & 73.5 & 3.1 & $(67.3-79.6)$ \\
\hline Montgomery, Alabama & 350 & 78.8 & 3.1 & $(72.8-84.9)$ \\
\hline $\begin{array}{l}\text { Montgomery County-Bucks County- } \\
\text { Chester County, Pennsylvania }{ }^{\dagger}\end{array}$ & 940 & 88.2 & 1.7 & $(84.9-91.4)$ \\
\hline $\begin{array}{l}\text { Myrtle Beach-Conway-North Myrtle } \\
\text { Beach, South Carolina-North Carolina }\end{array}$ & 583 & 68.4 & 2.6 & $(63.3-73.6)$ \\
\hline $\begin{array}{l}\text { Nashville-Davidson-Murfreesboro- } \\
\text { Franklin, Tennessee }\end{array}$ & 981 & 82.0 & 1.7 & $(78.7-85.4)$ \\
\hline $\begin{array}{l}\text { Nassau County-Suffolk County, } \\
\text { New York }^{\dagger}\end{array}$ & 612 & 83.1 & 2.2 & $(78.8-87.4)$ \\
\hline Newark, New Jersey-Pennsylvania ${ }^{\dagger}$ & 4,414 & 80.5 & 1.0 & $(78.6-82.4)$ \\
\hline New Haven-Milford, Connecticut & 1,341 & 86.6 & 1.3 & $(84.1-89.1)$ \\
\hline New Orleans-Metairie, Louisiana & 862 & 76.1 & 2.1 & $(72.1-80.1)$ \\
\hline $\begin{array}{l}\text { New York-Jersey City-White Plains, } \\
\text { New York-New Jersey }{ }^{\dagger}\end{array}$ & 5,525 & 78.3 & 1.1 & $(76.2-80.5)$ \\
\hline Norfolk, Nebraska & 367 & 76.3 & 3.5 & $(69.5-83.1)$ \\
\hline North Platte, Nebraska & 349 & 82.4 & 3.0 & $(76.6-88.2)$ \\
\hline Norwich-New London, Connecticut & 629 & 90.2 & 1.6 & $(87.1-93.4)$ \\
\hline $\begin{array}{l}\text { Oakland-Hayward-Berkeley, } \\
\text { California }{ }^{\dagger}\end{array}$ & 672 & 86.3 & 2.1 & $(82.2-90.5)$ \\
\hline Ocean City, New Jersey & 298 & 81.9 & 4.5 & $(73.0-90.8)$ \\
\hline Ogden-Clearfield, Utah & 1,750 & 85.3 & 1.1 & $(83.1-87.5)$ \\
\hline Oklahoma City, Oklahoma & 1,791 & 78.7 & 1.2 & $(76.3-81.1)$ \\
\hline Olympia-Tumwater, Washington & 349 & 85.6 & 2.5 & $(80.7-90.5)$ \\
\hline Omaha-Council Bluffs, Nebraska-lowa & 3,991 & 82.9 & 0.8 & $(81.3-84.5)$ \\
\hline Orlando-Kissimmee-Sanford, Florida & 348 & 73.0 & 3.4 & $(66.5-79.6)$ \\
\hline Philadelphia, Pennsylvania $^{\dagger}$ & 1,715 & 79.4 & 1.4 & $(76.7-82.1)$ \\
\hline Phoenix-Mesa-Scottsdale, Arizona & 1,697 & 75.3 & 1.4 & $(72.6-78.1)$ \\
\hline Pittsburgh, Pennsylvania & 2,167 & 85.9 & 1.0 & $(84.0-87.8)$ \\
\hline Ponce, Puerto Rico & 389 & 91.0 & 1.7 & $(87.6-94.4)$ \\
\hline Portland-South Portland, Maine & 2,235 & 86.8 & 0.9 & $(85.0-88.6)$ \\
\hline $\begin{array}{l}\text { Portland-Vancouver-Hillsboro, } \\
\text { Oregon-Washington }\end{array}$ & 2,141 & 81.5 & 1.2 & $(79.1-83.8)$ \\
\hline $\begin{array}{l}\text { Providence-Warwick, Rhode } \\
\text { Island-Massachusetts }\end{array}$ & 5,446 & 85.1 & 0.8 & $(83.6-86.6)$ \\
\hline Provo-Orem, Utah & 1,339 & 79.5 & 1.4 & $(76.7-82.2)$ \\
\hline Raleigh, North Carolina & 753 & 78.3 & 1.8 & $(74.8-81.9)$ \\
\hline Rapid City, South Dakota & 741 & 87.1 & 1.5 & $(84.1-90.1)$ \\
\hline Reno, Nevada & 968 & 71.1 & 2.2 & $(66.8-75.4)$ \\
\hline Richmond, Virginia & 666 & 84.3 & 2.0 & $(80.5-88.1)$ \\
\hline
\end{tabular}

See table footnotes on page 23. 
TABLE 5. (Continued) Estimated prevalence of adults aged 18-64 years who have health care coverage, ${ }^{*}$ by metropolitan and micropolitan statistical area - Behavioral Risk Factor Surveillance System, United States, 2012

\begin{tabular}{|c|c|c|c|c|}
\hline MMSA(s) & $\begin{array}{l}\text { Sample } \\
\text { size }\end{array}$ & $\%$ & SE & $95 \% \mathrm{Cl}$ \\
\hline $\begin{array}{l}\text { Riverside-San Bernardino-Ontario, } \\
\text { California }\end{array}$ & 1,078 & 72.6 & 1.8 & $(69.0-76.2)$ \\
\hline $\begin{array}{l}\text { Rockingham County-Strafford County, } \\
\text { New Hampshire }{ }^{\dagger}\end{array}$ & 1,098 & 86.2 & 1.5 & $(83.3-89.1)$ \\
\hline Rutland, Vermont & 390 & 84.8 & 2.8 & $(79.2-90.3)$ \\
\hline $\begin{array}{l}\text { Sacramento-Roseville-Arden-Arcade, } \\
\text { California }\end{array}$ & 707 & 81.2 & 2.2 & $(76.9-85.5)$ \\
\hline St. Louis, Missouri-Illinois & 1,374 & 83.4 & 1.4 & $(80.6-86.3)$ \\
\hline Salisbury, Maryland-Delaware & 1,209 & 86.0 & 1.5 & $(83.0-89.0)$ \\
\hline Salt Lake City, Utah & 3,361 & 77.6 & 1.0 & $(75.6-79.7)$ \\
\hline San Antonio-New Braunfels, Texas & 525 & 68.3 & 2.7 & $(63.0-73.6)$ \\
\hline San Diego-Carlsbad, California & 787 & 77.1 & 2.1 & $(72.9-81.3)$ \\
\hline $\begin{array}{l}\text { San Francisco-Redwood City-South } \\
\text { San Francisco, California }{ }^{\dagger}\end{array}$ & 416 & 84.5 & 2.4 & $(79.8-89.2)$ \\
\hline $\begin{array}{l}\text { San Jose-Sunnyvale-Santa Clara, } \\
\text { California }\end{array}$ & 496 & 82.6 & 2.3 & $(78.0-87.1)$ \\
\hline San Juan-Carolina-Caguas, Puerto Rico & 2,783 & 89.5 & 0.7 & $(88.1-91.0)$ \\
\hline Santa Fe, New Mexico & 482 & 65.9 & 2.7 & $(60.6-71.2)$ \\
\hline Sayre, Pennsylvania & 1,127 & 75.9 & 4.1 & $(67.8-84.0)$ \\
\hline Scottsbluff, Nebraska & 356 & 76.4 & 3.8 & $(68.9-83.9)$ \\
\hline $\begin{array}{l}\text { Scranton-Wilkes-Barre-Hazleton, } \\
\text { Pennsylvania }\end{array}$ & 459 & 82.1 & 3.1 & $(76.0-88.3)$ \\
\hline Seattle-Bellevue-Everett, Washington ${ }^{\dagger}$ & 3,603 & 82.4 & 0.9 & $(80.7-84.1)$ \\
\hline Shreveport-Bossier City, Louisiana & 373 & 74.5 & 3.2 & $(68.3-80.7)$ \\
\hline $\begin{array}{l}\text { Silver Spring-Frederick-Rockville, } \\
\text { Maryland }^{\dagger}\end{array}$ & 1,553 & 83.0 & 1.8 & $(79.4-86.6)$ \\
\hline $\begin{array}{l}\text { Sioux City, lowa-Nebraska-South } \\
\text { Dakota }\end{array}$ & 734 & 82.4 & 2.8 & $(77.0-87.8)$ \\
\hline Sioux Falls, South Dakota & 1,149 & 85.9 & 1.4 & $(83.1-88.7)$ \\
\hline Spartanburg, South Carolina & 460 & 74.9 & 2.9 & $(69.1-80.6)$ \\
\hline Spokane-Spokane Valley, Washington & 710 & 77.5 & 2.2 & $(73.3-81.7)$ \\
\hline Springfield, Massachusetts & 1,627 & 92.3 & 1.0 & $(90.3-94.2)$ \\
\hline Tacoma-Lakewood, Washington $^{\dagger}$ & 844 & 77.8 & 1.9 & $(74.1-81.6)$ \\
\hline
\end{tabular}

TABLE 5. (Continued) Estimated prevalence of adults aged 18-64 years who have health care coverage, ${ }^{*}$ by metropolitan and micropolitan statistical area - Behavioral Risk Factor Surveillance System, United States, 2012

\begin{tabular}{|c|c|c|c|c|}
\hline MMSA(s) & $\begin{array}{l}\text { Sample } \\
\text { size }\end{array}$ & $\%$ & SE & $95 \% \mathrm{Cl}$ \\
\hline $\begin{array}{l}\text { Tampa-St. Petersburg-Clearwater, } \\
\text { Florida }\end{array}$ & 498 & 76.7 & 2.6 & $(71.6-81.8)$ \\
\hline Toledo, Ohio & 645 & 82.1 & 2.9 & $(76.5-87.8)$ \\
\hline Topeka, Kansas & 745 & 78.8 & 2.2 & $(74.4-83.1)$ \\
\hline Torrington, Connecticut & 443 & 88.7 & 2.1 & $(84.6-92.9)$ \\
\hline Trenton, New Jersey & 414 & 85.1 & 2.3 & $(80.7-89.6)$ \\
\hline Tucson, Arizona & 596 & 79.7 & 2.3 & $(75.2-84.2)$ \\
\hline Tulsa, Oklahoma & 1,191 & 79.3 & 1.5 & $(76.4-82.2)$ \\
\hline Tuscaloosa, Alabama & 378 & 70.9 & 4.3 & $(62.5-79.3)$ \\
\hline Urban Honolulu, Hawaii & 3,278 & 87.8 & 0.9 & $(86.0-89.7)$ \\
\hline Vineland-Bridgeton, New Jersey & 384 & 74.2 & 4.1 & $(66.2-82.1)$ \\
\hline $\begin{array}{l}\text { Virginia Beach-Norfolk-Newport News, } \\
\text { Virginia-North Carolina }\end{array}$ & 1,023 & 80.4 & 1.8 & $(76.9-83.9)$ \\
\hline $\begin{array}{l}\text { Warren-Troy-Farmington Hills, } \\
\text { Michigan }^{\dagger}\end{array}$ & 1,477 & 85.7 & 1.2 & $(83.3-88.1)$ \\
\hline $\begin{array}{l}\text { Washington-Arlington-Alexandria, } \\
\text { District of Columbia-Virginia- } \\
\text { Maryland-West Virginia }{ }^{\dagger}\end{array}$ & 5,695 & 84.5 & 0.9 & $(82.7-86.3)$ \\
\hline Wichita, Kansas & 1,586 & 76.4 & 1.5 & $(73.5-79.4)$ \\
\hline $\begin{array}{l}\text { Wilmington, Delaware- } \\
\text { Maryland-New Jersey }{ }^{\dagger}\end{array}$ & 2,287 & 85.6 & 1.3 & $(83.0-88.1)$ \\
\hline Winston-Salem, North Carolina & 522 & 73.4 & 2.5 & $(68.6-78.3)$ \\
\hline Worcester, Massachusetts-Connecticut & 1,992 & 91.8 & 1.0 & $(89.9-93.8)$ \\
\hline Yakima, Washington & 357 & 63.5 & 3.5 & $(56.5-70.4)$ \\
\hline $\begin{array}{l}\text { Youngstown-Warren-Boardman, } \\
\text { Ohio-Pennsylvania }\end{array}$ & 573 & 81.3 & 2.8 & $(75.9-86.8)$ \\
\hline Median & & 80.7 & & \\
\hline Range & & $35.4-93.7$ & & \\
\hline
\end{tabular}

Abbreviations: $\mathrm{Cl}=$ confidence interval; $\mathrm{MMSA}=$ metropolitan and micropolitan statistical area; $\mathrm{SE}=$ standard error.

* Including health insurance, prepaid plans (e.g., health maintenance organizations), or government plans (e.g., Medicare).

† Metropolitan division. 
TABLE 6. Estimated prevalence of adults aged 18-64 years who have health care coverage, ${ }^{*}$ by county - Behavioral Risk Factor Surveillance System, United States, 2012

\begin{tabular}{|c|c|c|c|c|}
\hline County & $\begin{array}{l}\text { Sample } \\
\text { size }\end{array}$ & $\%$ & SE & $95 \% \mathrm{Cl}$ \\
\hline Jefferson County, Alabama & 668 & 77.3 & 2.2 & $(73.0-81.6)$ \\
\hline Madison County, Alabama & 376 & 81.8 & 2.5 & $(76.9-86.7)$ \\
\hline Mobile County, Alabama & 501 & 73.5 & 3.1 & $(67.3-79.6)$ \\
\hline Anchorage Municipality, Alaska & 754 & 81.6 & 1.7 & $(78.3-84.9)$ \\
\hline Fairbanks North Star Borough, Alaska & 486 & 83.7 & 2.3 & $(79.2-88.2)$ \\
\hline Matanuska-Susitna Borough, Alaska & 485 & 78.4 & 2.3 & $(73.8-82.9)$ \\
\hline Maricopa County, Arizona & 1,446 & 75.6 & 1.5 & $(72.7-78.6)$ \\
\hline Pima County, Arizona & 596 & 79.7 & 2.3 & $(75.2-84.2)$ \\
\hline Pulaski County, Arkansas & 489 & 75.4 & 3.0 & $(69.5-81.3)$ \\
\hline Alameda County, California & 434 & 86.4 & 2.5 & $(81.4-91.4)$ \\
\hline Los Angeles County, California & 2,609 & 68.3 & 1.3 & $(65.9-70.8)$ \\
\hline Orange County, California & 705 & 82.4 & 1.8 & $(78.9-86.0)$ \\
\hline Riverside County, California & 550 & 72.7 & 2.5 & $(67.7-77.7)$ \\
\hline Sacramento County, California & 440 & 80.1 & 2.9 & $(74.5-85.8)$ \\
\hline San Bernardino County, California & 528 & 72.6 & 2.6 & $(67.4-77.8)$ \\
\hline San Diego County, California & 787 & 77.1 & 2.1 & $(72.9-81.3)$ \\
\hline Santa Clara County, California & 487 & 82.7 & 2.4 & $(78.1-87.3)$ \\
\hline Adams County, Colorado & 589 & 69.6 & 2.6 & $(64.6-74.6)$ \\
\hline Arapahoe County, Colorado & 635 & 79.7 & 2.0 & $(75.7-83.7)$ \\
\hline Boulder County, Colorado & 381 & 79.3 & 2.8 & $(73.7-84.9)$ \\
\hline Denver County, Colorado & 750 & 74.2 & 1.9 & $(70.5-78.0)$ \\
\hline Douglas County, Colorado & 429 & 92.2 & 1.8 & $(88.7-95.8)$ \\
\hline El Paso County, Colorado & 763 & 80.9 & 1.8 & $(77.4-84.5)$ \\
\hline Jefferson County, Colorado & 789 & 84.5 & 1.6 & $(81.3-87.7)$ \\
\hline Larimer County, Colorado & 407 & 80.9 & 2.5 & $(76.0-85.7)$ \\
\hline Weld County, Colorado & 399 & 75.9 & 2.7 & $(70.5-81.2)$ \\
\hline Fairfield County, Connecticut & 1,525 & 83.5 & 1.5 & $(80.7-86.4)$ \\
\hline Hartford County, Connecticut & 1,341 & 88.8 & 1.2 & $(86.5-91.1)$ \\
\hline Litchfield County, Connecticut & 443 & 88.7 & 2.1 & $(84.6-92.9)$ \\
\hline New Haven County, Connecticut & 1,341 & 86.6 & 1.3 & $(84.1-89.1)$ \\
\hline New London County, Connecticut & 629 & 90.2 & 1.6 & $(87.1-93.4)$ \\
\hline Kent County, Delaware & 939 & 87.3 & 1.7 & $(84.0-90.7)$ \\
\hline New Castle County, Delaware & 1,677 & 86.8 & 1.2 & $(84.4-89.2)$ \\
\hline Sussex County, Delaware & 796 & 82.6 & 2.0 & $(78.6-86.6)$ \\
\hline $\begin{array}{l}\text { District of Columbia, } \\
\text { District of Columbia }\end{array}$ & 2,479 & 90.2 & 1.0 & $(88.3-92.1)$ \\
\hline Broward County, Florida & 335 & 73.7 & 3.4 & $(67.0-80.5)$ \\
\hline Miami-Dade County, Florida & 528 & 60.7 & 3.2 & $(54.4-67.0)$ \\
\hline Hawaii County, Hawaii & 951 & 83.9 & 1.8 & $(80.5-87.4)$ \\
\hline Honolulu County, Hawaii & 3,278 & 87.8 & 0.9 & $(86.0-89.7)$ \\
\hline Kauai County, Hawaii & 434 & 88.9 & 2.1 & $(84.8-93.1)$ \\
\hline Maui County, Hawaii & 842 & 87.0 & 1.6 & $(83.9-90.2)$ \\
\hline Ada County, Idaho & 542 & 81.6 & 2.4 & $(76.8-86.3)$ \\
\hline Canyon County, Idaho & 306 & 66.8 & 4.2 & $(58.5-75.0)$ \\
\hline Cook County, Illinois & 1,037 & 76.3 & 1.8 & $(72.7-79.9)$ \\
\hline Lake County, Indiana & 564 & 74.2 & 3.3 & $(67.7-80.7)$ \\
\hline Marion County, Indiana & 874 & 79.7 & 1.8 & $(76.2-83.2)$ \\
\hline Polk County, lowa & 556 & 88.2 & 1.7 & $(84.9-91.6)$ \\
\hline Johnson County, Kansas & 1,502 & 88.4 & 1.2 & $(86.0-90.8)$ \\
\hline Sedgwick County, Kansas & 1,241 & 74.8 & 1.7 & $(71.5-78.2)$ \\
\hline Shawnee County, Kansas & 524 & 80.7 & 2.4 & $(76.0-85.4)$ \\
\hline Wyandotte County, Kansas & 542 & 59.8 & 3.7 & $(52.4-67.1)$ \\
\hline Jefferson County, Kentucky & 1,099 & 79.3 & 2.0 & $(75.3-83.3)$ \\
\hline East Baton Rouge Parish, Louisiana & 388 & 82.6 & 2.6 & $(77.5-87.7)$ \\
\hline Androscoggin County, Maine & 505 & 86.6 & 1.8 & $(83.0-90.2)$ \\
\hline Aroostook County, Maine & 360 & 83.8 & 2.4 & $(79.1-88.6)$ \\
\hline Cumberland County, Maine & 1,217 & 87.9 & 1.2 & $(85.6-90.3)$ \\
\hline Kennebec County, Maine & 599 & 86.4 & 1.8 & $(82.9-89.9)$ \\
\hline Penobscot County, Maine & 663 & 80.9 & 2.1 & $(76.7-85.0)$ \\
\hline York County, Maine & 774 & 85.8 & 1.6 & $(82.7-88.9)$ \\
\hline
\end{tabular}

See table footnotes on page 25 .
TABLE 6. (Continued) Estimated prevalence of adults aged 18-64 years who have health care coverage, ${ }^{*}$ by county - Behavioral Risk Factor Surveillance System, United States, 2012

\begin{tabular}{|c|c|c|c|c|}
\hline County & $\begin{array}{l}\text { Sample } \\
\text { size }\end{array}$ & $\%$ & SE & $95 \% \mathrm{Cl}$ \\
\hline Anne Arundel County, Maryland & 621 & 85.2 & 2.5 & $(80.3-90.1)$ \\
\hline Baltimore County, Maryland & 1,033 & 86.2 & 1.7 & $(82.9-89.6)$ \\
\hline Charles County, Maryland & 378 & 84.4 & 3.7 & $(77.1-91.7)$ \\
\hline Frederick County, Maryland & 497 & 84.9 & 3.9 & $(77.3-92.6)$ \\
\hline Montgomery County, Maryland & 1,056 & 82.4 & 2.1 & $(78.4-86.5)$ \\
\hline Prince George's County, Maryland & 801 & 79.1 & 2.7 & $(73.8-84.3)$ \\
\hline Washington County, Maryland & 330 & 83.4 & 4.2 & $(75.2-91.7)$ \\
\hline Baltimore city, Maryland & 497 & 80.5 & 2.9 & $(74.8-86.1)$ \\
\hline Barnstable County, Massachusetts & 280 & 91.8 & 2.1 & $(87.7-96.0)$ \\
\hline Bristol County, Massachusetts & 1,735 & 92.2 & 1.2 & $(89.8-94.7)$ \\
\hline Essex County, Massachusetts & 1,678 & 94.2 & 1.0 & $(92.3-96.1)$ \\
\hline Hampden County, Massachusetts & 1,350 & 91.0 & 1.3 & $(88.6-93.5)$ \\
\hline Middlesex County, Massachusetts & 3,203 & 93.7 & 0.7 & $(92.4-95.0)$ \\
\hline Norfolk County, Massachusetts & 1,206 & 96.7 & 0.8 & $(95.1-98.2)$ \\
\hline Plymouth County, Massachusetts & 1,217 & 91.8 & 1.2 & $(89.4-94.2)$ \\
\hline Suffolk County, Massachusetts & 1,680 & 92.3 & 0.9 & $(90$ \\
\hline Worcester County, Massachusetts & 1,822 & 92.2 & 1.1 & $(90.1-94.3)$ \\
\hline Kent County, Michigan & 368 & 88.0 & 2.3 & $(83.4-92.6)$ \\
\hline Macomb County, Michigan & 417 & 88.6 & 1.8 & $(85.1-92.0)$ \\
\hline Oakland County, Michigan & 768 & 84.5 & 1.8 & $(80.9-88.1)$ \\
\hline Wayne County, Michigan & 1,416 & 77.9 & 1.7 & $(74.6-81.2)$ \\
\hline Anoka County, Minnesota & 421 & 89.5 & 1.9 & $(85.8-93.3)$ \\
\hline Dakota County, Minnesota & 512 & 91.0 & 1.5 & $(88.1-93.8)$ \\
\hline Hennepin County, Minnesota & 2,386 & 86.6 & 1.0 & $(84.6-88.6)$ \\
\hline Ramsey County, Minnesota & 1,309 & 84.3 & 2.3 & $(79.7-88.8)$ \\
\hline Jackson County, Missouri & 551 & 81.9 & 2.6 & $(76.7-87.0)$ \\
\hline St. Louis County, Missouri & 636 & 80.2 & 2.2 & $(75.8-84.7)$ \\
\hline ntana & 441 & 77.0 & 2.6 & $(72$ \\
\hline Flathead County, Montana & 374 & 74.4 & 2.5 & $(69.5-79.3)$ \\
\hline Hill County, Montana & 394 & 73.8 & 4.0 & $(65.9$ \\
\hline Lake County, Montana & 544 & 69.2 & 3.4 & $(62.5-75.8)$ \\
\hline Missoula County, Montana & 547 & 73.4 & 2.4 & $(68.7-78.1)$ \\
\hline Yellowstone County, Montana & 520 & 80.0 & 1.9 & $(76.3-83.7)$ \\
\hline Dakota County, Nebraska & 436 & 66.9 & 5.8 & $(55.5-78.2)$ \\
\hline Douglas County, Nebraska & 2,543 & 81.4 & 1.0 & $(79.5-83.3)$ \\
\hline Hall County, Nebraska & 340 & 79.2 & 3.3 & $(72.7-85.8)$ \\
\hline Lancaster County, Nebraska & 1,143 & 82.5 & 1.3 & $(79.9-85.1)$ \\
\hline Lincoln County, Nebraska & 335 & 82.1 & 3.1 & $(76.1-88.0)$ \\
\hline Sarpy County, Nebraska & 860 & 88.9 & 1.5 & $(86.0-91.8)$ \\
\hline Scotts Bluff County, Nebraska & 325 & 77.3 & 3.8 & $(69.9-84.7)$ \\
\hline Clark County, Nevada & 1,458 & 69.4 & 1.5 & $(66.5-72.3)$ \\
\hline Washoe County, Nevada & 957 & 71.2 & 2.2 & $(66.9-75.5)$ \\
\hline Belknap County, New Hampshire & 320 & 81.9 & 3.6 & $(74.8-89.0)$ \\
\hline Carroll County, New Hampshire & 279 & 80.2 & 3.5 & $(73.2-87.2)$ \\
\hline Cheshire County, New Hampshire & 319 & 80.1 & 3.4 & $(73.4-86.8)$ \\
\hline Coos County, New Hampshire & 317 & 68.7 & 4.2 & $(60.5-76.9)$ \\
\hline Grafton County, New Hampshire & 341 & 80.8 & 3.4 & $(74.2-87.4)$ \\
\hline Hillsborough County, New Hampshire & 1,332 & 84.8 & 1.5 & $(82.0-87.7)$ \\
\hline Merrimack County, New Hampshire & 457 & 85.2 & 2.1 & $(81.0-89.4)$ \\
\hline Rockingham County, New Hampshire & 696 & 86.2 & 1.8 & $(82.6-89.8)$ \\
\hline Strafford County, New Hampshire & 402 & 86.2 & 2.6 & $(81.2-91.3)$ \\
\hline Atlantic County, New Jersey & 655 & 78.6 & 2.3 & $(74.1-83.2)$ \\
\hline Bergen County, New Jersey & 754 & 80.4 & 1.9 & $(76.7-84.2)$ \\
\hline Burlington County, New Jersey & 467 & 87.5 & 2.5 & $(82.5-92.5)$ \\
\hline Camden County, New Jersey & 515 & 85.1 & 2.2 & $(80.7-89.5)$ \\
\hline Cape May County, New Jersey & 298 & 81.9 & 4.5 & $(73.0-90.8)$ \\
\hline Cumberland County, New Jersey & 384 & 74.2 & 4.1 & $(66.2-82.1)$ \\
\hline Essex County, New Jersey & 1,017 & 71.4 & 2.0 & $(67.5-75.2)$ \\
\hline Gloucester County, New Jersey & 403 & 87.4 & 2.0 & $(83.5-91.4)$ \\
\hline Hudson County, New Jersey & 1,009 & 70.9 & 2.0 & $(67.0-74.7)$ \\
\hline
\end{tabular}
See table footnotes on page 25 . 
TABLE 6. (Continued) Estimated prevalence of adults aged 18-64 years who have health care coverage, ${ }^{*}$ by county - Behavioral Risk Factor Surveillance System, United States, 2012

\begin{tabular}{|c|c|c|c|c|}
\hline County & $\begin{array}{l}\text { Sample } \\
\text { size }\end{array}$ & $\%$ & SE & $95 \% \mathrm{Cl}$ \\
\hline Hunterdon County, New Jersey & 397 & 91.2 & 1.9 & $(87.5-95.0)$ \\
\hline Mercer County, New Jersey & 414 & 85.1 & 2.3 & $(80.7-89.6)$ \\
\hline Middlesex County, New Jersey & 660 & 86.0 & 1.7 & $(82.7-89.3)$ \\
\hline Monmouth County, New Jersey & 479 & 89.1 & 1.8 & $(85.5-92.7)$ \\
\hline Morris County, New Jersey & 580 & 86.5 & 2.3 & $(82.1-91.0)$ \\
\hline Ocean County, New Jersey & 403 & 84.8 & 2.3 & $(80.2-89.3)$ \\
\hline Passaic County, New Jersey & 527 & 69.2 & 2.8 & $(63.7-74.7)$ \\
\hline Salem County, New Jersey & 393 & 74.7 & 5.2 & $(64.5-84.9)$ \\
\hline Somerset County, New Jersey & 425 & 91.1 & 1.9 & $(87.4-94.8)$ \\
\hline Sussex County, New Jersey & 366 & 84.8 & 2.6 & $(79.7-89.8)$ \\
\hline Union County, New Jersey & 534 & 79.0 & 2.2 & $(74.7-83.4)$ \\
\hline Warren County, New Jersey & 356 & 86.4 & 3.3 & (79.9-92.9) \\
\hline Bernalillo County, New Mexico & 1,508 & 75.8 & 1.4 & (73.1-78.4) \\
\hline Dona Ana County, New Mexico & 489 & 65.4 & 2.8 & $(60.0-70.8)$ \\
\hline Sandoval County, New Mexico & 444 & 80.0 & 2.6 & $(75.0-85.0)$ \\
\hline San Juan County, New Mexico & 458 & 76.9 & 2.4 & $(72.1-81.7)$ \\
\hline Santa Fe County, New Mexico & 482 & 65.9 & 2.7 & $(60.6-71.2)$ \\
\hline Kings County, New York & 421 & 76.4 & 2.8 & $(70.9-81.9)$ \\
\hline Guilford County, North Carolina & 353 & 77.8 & 2.7 & $(72.6-83.1)$ \\
\hline Mecklenburg County, North Carolina & 573 & 75.8 & 2.0 & $(71.8-79.7)$ \\
\hline Robeson County, North Carolina & 376 & 65.9 & 5.0 & $(56.1-75.7)$ \\
\hline Wake County, North Carolina & 576 & 78.5 & 2.2 & $(74.2-82.7)$ \\
\hline Burleigh County, North Dakota & 392 & 88.5 & 2.6 & $(83.5-93.6)$ \\
\hline Cass County, North Dakota & 593 & 81.1 & 2.3 & (76.6-85.6) \\
\hline Cuyahoga County, Ohio & 622 & 80.2 & 2.1 & $(76.1-84.4)$ \\
\hline Franklin County, Ohio & 640 & 82.7 & 1.9 & $(79.0-86.4)$ \\
\hline Hamilton County, Ohio & 556 & 87.1 & 1.8 & $(83.5-90.6)$ \\
\hline Lorain County, Ohio & 375 & 89.9 & 2.4 & $(85.3-94.6)$ \\
\hline Lucas County, Ohio & 406 & 79.7 & 3.7 & $(72.4-87.0)$ \\
\hline Mahoning County, Ohio & 349 & 84.9 & 3.3 & (78.4-91.3) \\
\hline Montgomery County, Ohio & 423 & 78.6 & 2.8 & $(73.0-84.1)$ \\
\hline Stark County, Ohio & 405 & 84.2 & 2.7 & $(78.9-89.5)$ \\
\hline Summit County, Ohio & 418 & 78.7 & 3.1 & $(72.7-84.8)$ \\
\hline Oklahoma County, Oklahoma & 893 & 76.0 & 1.7 & (72.7-79.3) \\
\hline Tulsa County, Oklahoma & 830 & 77.1 & 1.8 & $(73.5-80.7)$ \\
\hline Clackamas County, Oregon & 331 & 79.8 & 3.3 & (73.4-86.2) \\
\hline Lane County, Oregon & 338 & 74.8 & 3.2 & $(68.5-81.0)$ \\
\hline Multnomah County, Oregon & 637 & 80.8 & 2.2 & $(76.5-85.0)$ \\
\hline Washington County, Oregon & 434 & 83.4 & 2.4 & $(78.7-88.1)$ \\
\hline Allegheny County, Pennsylvania & 1,191 & 86.3 & 1.3 & $(83.8-88.9)$ \\
\hline Bradford County, Pennsylvania & 1,127 & 75.9 & 4.1 & $(67.8-84.0)$ \\
\hline Montgomery County, Pennsylvania & 399 & 88.2 & 2.3 & $(83.8-92.7)$ \\
\hline Philadelphia County, Pennsylvania & 1,445 & 76.6 & 1.6 & (73.4-79.8) \\
\hline Pike County, Pennsylvania & 1,095 & 83.8 & 1.9 & $(80.1-87.4)$ \\
\hline Kent County, Rhode Island & 568 & 86.7 & 2.0 & $(82.7-90.6)$ \\
\hline Providence County, Rhode Island & 2,324 & 78.5 & 1.3 & $(76.0-80.9)$ \\
\hline Washington County, Rhode Island & 394 & 83.1 & 3.0 & $(77.3-89.0)$ \\
\hline Aiken County, South Carolina & 341 & 77.8 & 3.2 & $(71.5-84.1)$ \\
\hline Beaufort County, South Carolina & 355 & 65.1 & 4.8 & $(55.6-74.6)$ \\
\hline
\end{tabular}

TABLE 6. (Continued) Estimated prevalence of adults aged 18-64 years who have health care coverage, ${ }^{*}$ by county - Behavioral Risk Factor Surveillance System, United States, 2012

\begin{tabular}{|c|c|c|c|c|}
\hline County & $\begin{array}{c}\text { Sample } \\
\text { size }\end{array}$ & $\%$ & SE & $95 \% \mathrm{Cl}$ \\
\hline Charleston County, South Carolina & 663 & 76.0 & 2.5 & $(71.1-80.9)$ \\
\hline Greenville County, South Carolina & 627 & 72.2 & 2.5 & $(67.3-77.1)$ \\
\hline Horry County, South Carolina & 492 & 70.9 & 2.7 & $(65.6-76.2)$ \\
\hline Richland County, South Carolina & 659 & 75.6 & 2.5 & $(70.6-80.5)$ \\
\hline Spartanburg County, South Carolina & 424 & 76.7 & 3.0 & $(70.9-82.5)$ \\
\hline Lincoln County, South Dakota & 366 & 85.8 & 3.8 & $(78.4-93.2)$ \\
\hline Minnehaha County, South Dakota & 715 & 85.8 & 1.6 & $(82.8-88.9$ \\
\hline Pennington County, South Dakota & 451 & 86.4 & 1.8 & $(82.7-90.0)$ \\
\hline Davidson County, Tennessee & 393 & 82.3 & 2.4 & $(77.5-87.0$ \\
\hline Shelby County, Tennessee & 393 & 78.1 & 2.5 & $(73.2-83.0$ \\
\hline Bexar County, Texas & 405 & 67.8 & 3.1 & $(61.7-73.9)$ \\
\hline Dallas County, Texas & 379 & 53.6 & 3.1 & $(47.5-59.7)$ \\
\hline El Paso County, Texas & 420 & 52.1 & 3.8 & $(44.8-59.5)$ \\
\hline Harris County, Texas & 626 & 61.2 & 2.3 & $(56.6-65.8$ \\
\hline Hidalgo County, Texas & 420 & 35.4 & 3.4 & $(28.8-42.1)$ \\
\hline Tarrant County, Texas & 410 & 69.9 & 3.0 & $(63.9-75.8$ \\
\hline Travis County, Texas & 735 & 70.1 & 2.9 & $(64.5-75.8$ \\
\hline Davis County, Utah & 859 & 86.6 & 1.6 & $(83.4-89.7)$ \\
\hline Salt Lake County, Utah & 2,954 & 77.3 & 1.1 & $(75.2-79.4$ \\
\hline Tooele County, Utah & 407 & 84.3 & 3.1 & $(78.3-90.3$ \\
\hline Utah County, Utah & 1,298 & 79.3 & 1.4 & $(76.5-82.1)$ \\
\hline Wasatch County, Utah & 347 & 72.3 & 5.5 & $(61.6-82.9$ \\
\hline Weber County, Utah & 741 & 82.6 & 1.8 & $(79.1-86.2$ \\
\hline Chittenden County, Vermont & 673 & 92.2 & 1.2 & $(89.8-94.7)$ \\
\hline Rutland County, Vermont & 390 & 84.8 & 2.8 & $(79.2-90.3)$ \\
\hline Washington County, Vermont & 377 & 87.9 & 2.3 & $(83.3-92.5)$ \\
\hline Windsor County, Vermont & 332 & 89.5 & 2.0 & $(85.7-93.3)$ \\
\hline Fairfax County, Virginia & 559 & 85.6 & 2.0 & $(81.6-89.6)$ \\
\hline Clark County, Washington & 537 & 83.0 & 2.1 & $(78.8-87.2$ \\
\hline King County, Washington & 2,743 & 82.6 & 1.0 & $(80.6-84.6)$ \\
\hline Kitsap County, Washington & 357 & 81.8 & 3.0 & $(75.8-87.7)$ \\
\hline Pierce County, Washington & 844 & 77.8 & 1.9 & $(74.1-81.6)$ \\
\hline Snohomish County, Washington & 860 & 81.4 & 1.8 & $(78.0-84.9$ \\
\hline Spokane County, Washington & 611 & 79.4 & 2.3 & $(75.0-83.8$ \\
\hline Thurston County, Washington & 349 & 85.6 & 2.5 & $(80.7-90.5)$ \\
\hline Whatcom County, Washington & 550 & 77.2 & 3.2 & $(70.9-83.5)$ \\
\hline Yakima County, Washington & 357 & 63.5 & 3.5 & $(56.5-70.4$ \\
\hline Kanawha County, West Virginia & 435 & 79.0 & 2.3 & $(74.5-83.5)$ \\
\hline Milwaukee County, Wisconsin & 704 & 81.9 & 2.3 & $(77.5-86.3)$ \\
\hline Laramie County, Wyoming & 546 & 81.2 & 2.6 & $(76.0-86.4$ \\
\hline Natrona County, Wyoming & 485 & 74.7 & 3.0 & $(68.8-80.7)$ \\
\hline San Juan Municipio, Puerto Rico & 450 & 84.4 & 2.1 & $(80.3-88.6$ \\
\hline Median & & 81.4 & & \\
\hline Range & & $35.4-96$ & & \\
\hline
\end{tabular}

Abbreviations: $\mathrm{Cl}=$ confidence interval; $\mathrm{SE}=$ standard error.

* Including health insurance, prepaid plans (e.g., health maintenance organizations), or government plans (e.g., Medicare). 
TABLE 7. Estimated prevalence of adults aged $\geq 18$ years who visited a doctor for a routine checkup during the preceding 12 months, by state/territory - Behavioral Risk Factor Surveillance System, United States, 2012

\begin{tabular}{|c|c|c|c|c|}
\hline State/Territory & $\begin{array}{c}\text { Sample } \\
\text { size }\end{array}$ & $\%$ & SE & $95 \% \mathrm{Cl}$ \\
\hline Alabama & 8,874 & 71.6 & 0.8 & $(70.1-73.1)$ \\
\hline Alaska & 4,209 & 64.3 & 1.0 & $(62.2-66.3)$ \\
\hline Arizona & 7,211 & 63.6 & 1.0 & $(61.7-65.5)$ \\
\hline Arkansas & 5,054 & 62.7 & 1.0 & $(60.8-64.6)$ \\
\hline California & 14,502 & 62.9 & 0.6 & $(61.7-64.0)$ \\
\hline Colorado & 12,034 & 60.1 & 0.6 & $(59.0-61.3)$ \\
\hline Connecticut & 8,686 & 71.7 & 0.7 & $(70.3-73.0)$ \\
\hline Delaware & 5,140 & 80.1 & 0.8 & $(78.6-81.7)$ \\
\hline District of Columbia & 3,796 & 74.1 & 1.2 & $(71.7-76.5)$ \\
\hline Florida & 7,553 & 70.3 & 0.9 & $(68.6-72.0)$ \\
\hline Georgia & 6,018 & 70.3 & 0.9 & $(68.6-72.0)$ \\
\hline Hawaii & 7,530 & 64.0 & 0.8 & $(62.4-65.7)$ \\
\hline Idaho & 5,841 & 55.7 & 1.2 & $(53.4-58.0)$ \\
\hline Illinois & 5,564 & 67.7 & 0.9 & $(65.9-69.5)$ \\
\hline Indiana & 8,559 & 63.8 & 0.7 & $(62.4-65.2)$ \\
\hline lowa & 7,084 & 67.7 & 0.7 & $(66.3-69.1)$ \\
\hline Kansas & 11,612 & 68.1 & 0.6 & $(66.9-69.3)$ \\
\hline Kentucky & 10,981 & 67.2 & 0.7 & $(65.8-68.7)$ \\
\hline Louisiana & 8,891 & 72.4 & 0.8 & $(70.8-74.0)$ \\
\hline Maine & 9,879 & 70.9 & 0.6 & $(69.7-72.0)$ \\
\hline Maryland & 12,689 & 76.1 & 0.7 & $(74.8-77.5)$ \\
\hline Massachusetts & 21,562 & 78.7 & 0.4 & $(77.8-79.5)$ \\
\hline Michigan & 10,400 & 66.5 & 0.7 & $(65.2-67.8)$ \\
\hline Minnesota & 12,098 & 68.9 & 0.6 & $(67.8-70.1)$ \\
\hline Mississippi & 7,666 & 69.1 & 0.8 & $(67.6-70.7)$ \\
\hline Missouri & 6,651 & 65.8 & 0.9 & $(64.1-67.5)$ \\
\hline Montana & 8,560 & 57.0 & 0.7 & $(55.6-58.4)$ \\
\hline Nebraska & 18,929 & 60.4 & 0.5 & (59.4-61.4) \\
\hline Nevada & 4,780 & 63.9 & 1.0 & $(61.9-65.9)$ \\
\hline New Hampshire & 7,458 & 70.5 & 0.8 & $(68.9-72.1)$ \\
\hline New Jersey & 15,561 & 75.0 & 0.5 & (74.0-76.0) \\
\hline New Mexico & 8,644 & 57.7 & 0.7 & $(56.4-59.1)$ \\
\hline New York & 6,010 & 71.0 & 0.9 & $(69.3-72.7)$ \\
\hline North Carolina & 11,749 & 73.5 & 0.6 & (72.4-74.6) \\
\hline North Dakota & 4,849 & 62.5 & 1.0 & $(60.6-64.3)$ \\
\hline Ohio & 12,869 & 71.0 & 0.6 & $(69.9-72.1)$ \\
\hline Oklahoma & 7,887 & 60.1 & 0.7 & (58.7-61.6) \\
\hline Oregon & 5,145 & 57.2 & 0.9 & $(55.4-59.1)$ \\
\hline Pennsylvania & 19,807 & 70.3 & 0.5 & $(69.3-71.3)$ \\
\hline Rhode Island & 5,450 & 78.7 & 0.8 & (77.2-80.3) \\
\hline South Carolina & 12,578 & 64.3 & 0.7 & $(63.0-65.6)$ \\
\hline South Dakota & 7,780 & 66.6 & 0.9 & $(64.9-68.3)$ \\
\hline Tennessee & 6,955 & 75.4 & 0.8 & (73.9-76.9) \\
\hline Texas & 9,003 & 63.4 & 0.7 & $(61.9-64.8)$ \\
\hline Utah & 12,095 & 56.7 & 0.6 & $(55.5-58.0)$ \\
\hline Vermont & 6,002 & 66.6 & 0.8 & $(65.0-68.2)$ \\
\hline Virginia & 7,304 & 72.5 & 0.7 & (71.1-74.0) \\
\hline Washington & 15,095 & 59.0 & 0.6 & $(57.9-60.1)$ \\
\hline West Virginia & 5,340 & 75.3 & 0.8 & (73.8-76.7) \\
\hline Wisconsin & 5,271 & 69.8 & 1.0 & $(67.9-71.7)$ \\
\hline Wyoming & 6,137 & 58.6 & 1.1 & $(56.5-60.7)$ \\
\hline Guam & 2,007 & 62.8 & 1.5 & (59.9-65.6) \\
\hline Puerto Rico & 6,238 & 78.9 & 0.6 & $(77.6-80.1)$ \\
\hline Median & & 67.7 & & \\
\hline Range & & $55.7-80.1$ & & \\
\hline
\end{tabular}

Abbreviations: $\mathrm{Cl}=$ confidence interval; $\mathrm{SE}=$ standard error.
TABLE 8. Estimated prevalence of adults aged $\geq 18$ years who visited a doctor for a routine checkup during the preceding 12 months, by metropolitan and micropolitan statistical area - Behavioral Risk Factor Surveillance System, United States, 2012

\begin{tabular}{|c|c|c|c|c|}
\hline MMSA(s) & $\begin{array}{l}\text { Sample } \\
\text { size }\end{array}$ & $\%$ & SE & $95 \% \mathrm{Cl}$ \\
\hline Aguadilla-Isabela, Puerto Rico & 540 & 75.6 & 2.3 & $(71.0-80.1)$ \\
\hline Akron, Ohio & 741 & 69.9 & 2.4 & $(65.2-74.6)$ \\
\hline Albuquerque, New Mexico & 3,229 & 56.7 & 1.1 & $(54.5-58.9)$ \\
\hline $\begin{array}{l}\text { Allentown-Bethlehem-Easton, } \\
\text { Pennsylvania-New Jersey }\end{array}$ & 1,340 & 71.6 & 1.8 & $(68.0-75.2)$ \\
\hline Anaheim-Santa Ana-Irvine, California* & 1,035 & 66.6 & 2.3 & $(62.1-71.1)$ \\
\hline Anchorage, Alaska & 1,470 & 66.6 & 1.5 & $(63.6-69.6)$ \\
\hline Asheville, North Carolina & 586 & 70.4 & 2.6 & $(65.4-75.4)$ \\
\hline $\begin{array}{l}\text { Atlanta-Sandy Springs-Roswell, } \\
\text { Georgia }\end{array}$ & 2,505 & 70.1 & 1.3 & $(67.6-72.6)$ \\
\hline Atlantic City-Hammonton, New Jersey & 1,012 & 77.4 & 1.9 & $(73.7-81.1)$ \\
\hline $\begin{array}{l}\text { Augusta-Richmond County, Georgia- } \\
\text { South Carolina }\end{array}$ & 1,024 & 76.1 & 2.6 & $(71.1-81.2)$ \\
\hline Augusta-Waterville, Maine & 831 & 72.9 & 2.0 & $(69.0-76.8)$ \\
\hline Austin-Round Rock, Texas & 1,370 & 62.1 & 2.2 & $(57.9-66.4)$ \\
\hline $\begin{array}{l}\text { Baltimore-Columbia-Towson, } \\
\text { Maryland }\end{array}$ & 4,682 & 78.3 & 1.0 & $(76.4-80.2)$ \\
\hline Bangor, Maine & 924 & 71.6 & 2.0 & $(67.8-75.5)$ \\
\hline Barnstable Town, Massachusetts & 554 & 85.0 & 1.9 & $(81.3-88.6)$ \\
\hline Barre, Vermont & 512 & 66.6 & 2.8 & $(61.2-72.1)$ \\
\hline Baton Rouge, Louisiana & 1,367 & 76.3 & 1.8 & $(72.7-79.8)$ \\
\hline Bellingham, Washington & 835 & 60.0 & 2.8 & $(54.5-65.6)$ \\
\hline Berlin, New Hampshire-Vermont & 703 & 68.3 & 2.9 & $(62.6-73.9)$ \\
\hline Billings, Montana & 837 & 58.7 & 2.0 & $(54.9-62.5)$ \\
\hline Birmingham-Hoover, Alabama & 1,792 & 75.5 & 1.5 & $(72.6-78.4)$ \\
\hline Bismarck, North Dakota & 815 & 62.7 & 2.3 & $(58.1-67.2)$ \\
\hline Boise City, Idaho & 1,473 & 55.2 & 2.0 & $(51.3-59.1)$ \\
\hline Boston, Massachusetts* & 5,870 & 79.3 & 0.8 & $(77.7-80.9)$ \\
\hline Boulder, Colorado & 509 & 53.4 & 2.8 & $(47.9-58.8)$ \\
\hline Bremerton-Silverdale, Washington & 561 & 65.1 & 2.9 & $(59.5-70.7)$ \\
\hline $\begin{array}{l}\text { Bridgeport-Stamford-Norwalk, } \\
\text { Connecticut }\end{array}$ & 2,165 & 67.9 & 1.5 & $(65.0-70.8)$ \\
\hline Burlington-South Burlington, Vermont & 1,510 & 66.5 & 1.5 & $(63.5-69.5)$ \\
\hline $\begin{array}{l}\text { Cambridge-Newton-Framingham, } \\
\text { Massachusetts* }\end{array}$ & 6,884 & 78.6 & 0.8 & $(77.1-80.2)$ \\
\hline Camden, New Jersey* & 1,958 & 75.6 & 1.4 & $(73.0-78.2)$ \\
\hline Canton-Massillon, Ohio & 676 & 77.8 & 2.4 & $(73.1-82.4)$ \\
\hline Casper, Wyoming & 807 & 58.6 & 2.9 & $(53.0-64.2)$ \\
\hline Cedar Rapids, lowa & 581 & 69.2 & 2.6 & $(64.2-74.2)$ \\
\hline Charleston, West Virginia & 765 & 77.8 & 1.8 & $(74.2-81.3)$ \\
\hline $\begin{array}{l}\text { Charleston-North Charleston, } \\
\text { South Carolina }\end{array}$ & 1,684 & 63.2 & 1.8 & $(59.7-66.7)$ \\
\hline $\begin{array}{l}\text { Charlotte-Concord-Gastonia, } \\
\text { North Carolina-South Carolina }\end{array}$ & 2,486 & 71.4 & 1.2 & $(69.1-73.8)$ \\
\hline Chattanooga, Tennessee-Georgia & 627 & 71.1 & 3.1 & $(65.0-77.2)$ \\
\hline Cheyenne, Wyoming & 938 & 64.9 & 2.6 & $(59.8-69.9)$ \\
\hline $\begin{array}{l}\text { Chicago-Naperville-Elgin, } \\
\text { Illinois-Indiana-Wisconsin }\end{array}$ & 3,714 & 67.4 & 1.2 & $(65.2-69.7)$ \\
\hline Cincinnati, Ohio-Kentucky-Indiana & 2,332 & 70.8 & 1.3 & $(68.3-73.3)$ \\
\hline $\begin{array}{l}\text { Claremont-Lebanon, New } \\
\text { Hampshire-Vermont }\end{array}$ & 1,935 & 68.9 & 1.6 & $(65.8-72.0)$ \\
\hline Cleveland-Elyria, Ohio & 1,758 & 72.1 & 1.5 & $(69.1-75.0)$ \\
\hline Colorado Springs, Colorado & 1,149 & 64.2 & 1.8 & $(60.7-67.7)$ \\
\hline Columbia, South Carolina & 1,781 & 64.8 & 1.7 & $(61.5-68.1)$ \\
\hline Columbus, Ohio & 1,583 & 69.1 & 1.4 & $(66.3-72.0)$ \\
\hline Concord, New Hampshire & 700 & 72.4 & 2.3 & $(67.9-76.9)$ \\
\hline Dallas-Plano-Irving, Texas* & 898 & 64.8 & 2.0 & $(60.9-68.8)$ \\
\hline Dayton, Ohio & 845 & 69.7 & 2.2 & $(65.4-74.0)$ \\
\hline Denver-Aurora-Lakewood, Colorado & 4,785 & 61.3 & 0.9 & $(59.6-63.1)$ \\
\hline
\end{tabular}

See table footnotes on page 28. 
TABLE 8. (Continued) Estimated prevalence of adults aged $\geq 18$ years who visited a doctor for a routine checkup during the preceding 12 months, by metropolitan and micropolitan statistical area Behavioral Risk Factor Surveillance System, United States, 2012

\begin{tabular}{|c|c|c|c|c|}
\hline MMSA(s) & $\begin{array}{l}\text { Sample } \\
\text { size }\end{array}$ & $\%$ & SE & $95 \% \mathrm{Cl}$ \\
\hline Des Moines-West Des Moines, lowa & 1,147 & 67.0 & 1.8 & $(63.6-70.5)$ \\
\hline Detroit-Dearborn-Livonia, Michigan* & 2,203 & 69.9 & 1.6 & $(66.8-73.0)$ \\
\hline Dover, Delaware & 1,428 & 82.4 & 1.5 & $(79.4-85.4)$ \\
\hline Duluth, Minnesota-Wisconsin & 508 & 74.8 & 2.5 & $(69.9-79.7)$ \\
\hline Durham-Chapel Hill, North Carolina & 790 & 69.8 & 2.1 & $(65.7-73.9)$ \\
\hline El Paso, Texas & 621 & 59.2 & 3.2 & $(52.9-65.6)$ \\
\hline Eugene, Oregon & 505 & 54.4 & 3.0 & $(48.6-60.3)$ \\
\hline Fairbanks, Alaska & 582 & 62.6 & 2.7 & $(57.3-67.8)$ \\
\hline Fargo, North Dakota-Minnesota & 938 & 65.0 & 2.4 & $(60.4-69.7)$ \\
\hline Farmington, New Mexico & 639 & 57.1 & 2.5 & $(52.2-62.0)$ \\
\hline Fayetteville, North Carolina & 498 & 78.8 & 2.5 & $(74.0-83.6)$ \\
\hline $\begin{array}{l}\text { Fayetteville-Springdale-Rogers, } \\
\text { Arkansas-Missouri }\end{array}$ & 799 & 57.4 & 2.8 & $(51.9-62.8)$ \\
\hline Fort Collins, Colorado & 585 & 61.1 & 2.5 & $(56.2-66.0)$ \\
\hline Fort Wayne, Indiana & 533 & 61.4 & 2.7 & $(56.2-66.6)$ \\
\hline Fort Worth-Arlington, Texas* & 720 & 69.9 & 2.3 & $(65.3-74.5)$ \\
\hline Grand Island, Nebraska & 841 & 55.7 & 2.4 & $(50.9-60.4)$ \\
\hline Grand Rapids-Wyoming, Michigan & 885 & 65.6 & 2.3 & $(61.1-70.1)$ \\
\hline Great Falls, Montana & 697 & 61.4 & 2.4 & $(56.6-66.2)$ \\
\hline Greeley, Colorado & 521 & 58.6 & 2.7 & $(53.2-63.9)$ \\
\hline $\begin{array}{l}\text { Greensboro-High Point, North } \\
\text { Carolina }\end{array}$ & 799 & 72.1 & 2.1 & $(68.1-76.2)$ \\
\hline $\begin{array}{l}\text { Greenville-Anderson-Mauldin, } \\
\text { South Carolina }\end{array}$ & 1,664 & 60.7 & 1.7 & $(57.4-63.9)$ \\
\hline Gulfport-Biloxi-Pascagoula, Mississippi & 734 & 68.7 & 2.3 & $(64.2-73.2)$ \\
\hline $\begin{array}{l}\text { Hagerstown-Martinsburg, Maryland- } \\
\text { West Virginia }\end{array}$ & 756 & 78.2 & 2.4 & $(73.5-82.9)$ \\
\hline Harrisburg-Carlisle, Pennsylvania & 659 & 69.4 & 2.3 & $(65.0-73.8)$ \\
\hline $\begin{array}{l}\text { Hartford-West Hartford-East Hartford, } \\
\text { Connecticut }\end{array}$ & 2,634 & 74.1 & 1.2 & $(71.8-76.3)$ \\
\hline Heber, Utah & 503 & 53.0 & 5.0 & $(43.2-62.8)$ \\
\hline Hilo, Hawaii & 1,342 & 58.8 & 2.0 & $(54.9-62.6)$ \\
\hline $\begin{array}{l}\text { Hilton Head Island-Bluffton-Beaufort, } \\
\text { South Carolina }\end{array}$ & 923 & 65.8 & 3.5 & $(59.0-72.6)$ \\
\hline $\begin{array}{l}\text { Houston-The Woodlands-Sugar Land, } \\
\text { Texas }\end{array}$ & 1,125 & 60.6 & 1.8 & $(57.0-64.1)$ \\
\hline $\begin{array}{l}\text { Huntington-Ashland, West } \\
\text { Virginia-Kentucky-Ohio }\end{array}$ & 1,090 & 73.4 & 1.9 & $(69.6-77.2)$ \\
\hline Huntsville, Alabama & 610 & 69.0 & 2.6 & $(63.8-74.2)$ \\
\hline Idaho Falls, Idaho & 536 & 54.9 & 3.7 & $(47.7-62.2)$ \\
\hline $\begin{array}{l}\text { Indianapolis-Carmel-Anderson, } \\
\text { Indiana }\end{array}$ & 2,182 & 65.9 & 1.3 & $(63.4-68.5)$ \\
\hline Jackson, Mississippi & 907 & 72.9 & 1.9 & $(69.1-76.7)$ \\
\hline Jacksonville, Florida & 514 & 72.6 & 3.0 & $(66.7-78.5)$ \\
\hline Kahului-Wailuku-Lahaina, Hawaii & 1,210 & 61.3 & 2.1 & $(57.2-65.3)$ \\
\hline Kalispell, Montana & 554 & 55.2 & 2.5 & $(50.3-60.1)$ \\
\hline Kansas City, Missouri-Kansas & 4,686 & 66.1 & 1.3 & $(63.4-68.7)$ \\
\hline Kapaa, Hawaii & 664 & 62.9 & 2.9 & $(57.3-68.6)$ \\
\hline Keene, New Hampshire & 541 & 65.1 & 3.4 & $(58.5-71.7)$ \\
\hline Kennewick-Richland, Washington & 528 & 58.2 & 2.8 & $(52.7-63.8)$ \\
\hline $\begin{array}{l}\text { Kingsport-Bristol-Bristol, } \\
\text { Tennessee-Virginia }\end{array}$ & 559 & 78.8 & 2.9 & $(73.0-84.5)$ \\
\hline Knoxville, Tennessee & 823 & 76.3 & 2.2 & $(72.0-80.6)$ \\
\hline Laconia, New Hampshire & 564 & 67.3 & 3.4 & $(60.5-74.0)$ \\
\hline Lafayette, Louisiana & 543 & 71.6 & 2.7 & $(66.3-76.9)$ \\
\hline Las Cruces, New Mexico & 701 & 58.9 & 2.5 & $(54.0-63.7)$ \\
\hline $\begin{array}{l}\text { Las Vegas-Henderson-Paradise, } \\
\text { Nevada }\end{array}$ & 1,977 & 65.2 & 1.3 & $(62.7-67.8)$ \\
\hline Lewiston-Auburn, Maine & 696 & 69.5 & 2.2 & $(65.2-73.9)$ \\
\hline
\end{tabular}

See table footnotes on page 28.
TABLE 8. (Continued) Estimated prevalence of adults aged $\geq 18$ years who visited a doctor for a routine checkup during the preceding 12 months, by metropolitan and micropolitan statistical area Behavioral Risk Factor Surveillance System, United States, 2012

\begin{tabular}{|c|c|c|c|c|}
\hline MMSA(s) & $\begin{array}{l}\text { Sample } \\
\text { size }\end{array}$ & $\%$ & SE & $95 \% \mathrm{Cl}$ \\
\hline Lexington-Fayette, Kentucky & 522 & 69.4 & 2.5 & $(64.5-74.3)$ \\
\hline Lincoln, Nebraska & 1,640 & 58.5 & 1.5 & $(55.7-61.4)$ \\
\hline $\begin{array}{l}\text { Little Rock-North Little Rock-Conway, } \\
\text { Arkansas }\end{array}$ & 1,151 & 64.0 & 2.0 & $(60.1-67.8)$ \\
\hline Logan, Utah-Idaho & 491 & 54.6 & 3.1 & $(48.6-60.6)$ \\
\hline $\begin{array}{l}\text { Los Angeles-Long Beach-Glendale, } \\
\text { California* }\end{array}$ & 3,488 & 64.7 & 1.1 & $(62.5-67.0)$ \\
\hline $\begin{array}{l}\text { Louisville/Jefferson County, } \\
\text { Kentucky-Indiana }\end{array}$ & 2,155 & 65.2 & 1.6 & $(62.0-68.3)$ \\
\hline Lumberton, North Carolina & 543 & 71.5 & 4.4 & $(62.8-80.2)$ \\
\hline Manchester-Nashua, New Hampshire & 1,891 & 71.1 & 1.4 & $(68.3-73.9)$ \\
\hline McAllen-Edinburg-Mission, Texas & 606 & 56.3 & 3.2 & $(50.0-62.6)$ \\
\hline $\begin{array}{l}\text { Memphis, } \\
\text { Tennessee-Mississippi-Arkansas }\end{array}$ & 1,297 & 75.6 & 1.8 & $(72.1-79.1)$ \\
\hline $\begin{array}{l}\text { Miami-Fort Lauderdale-West Palm } \\
\text { Beach, Florida }\end{array}$ & 1,661 & 70.8 & 1.7 & $(67.4-74.2)$ \\
\hline $\begin{array}{l}\text { Milwaukee-Waukesha-West Allis, } \\
\text { Wisconsin }\end{array}$ & 1,250 & 72.4 & 1.9 & $(68.6-76.1)$ \\
\hline $\begin{array}{l}\text { Minneapolis-St. Paul-Bloomington, } \\
\text { Minnesota-Wisconsin }\end{array}$ & 7,813 & 69.2 & 0.8 & $(67.7-70.7)$ \\
\hline Missoula, Montana & 775 & 53.1 & 2.3 & $(48.7-57.6)$ \\
\hline Mobile, Alabama & 799 & 75.2 & 2.7 & $(69.9-80.5)$ \\
\hline Montgomery, Alabama & 530 & 74.8 & 2.9 & $(69.2-80.5)$ \\
\hline $\begin{array}{l}\text { Montgomery County-Bucks County- } \\
\text { Chester County, Pennsylvania* }\end{array}$ & 1,303 & 68.2 & 1.6 & $(65.1-71.4)$ \\
\hline $\begin{array}{l}\text { Myrtle Beach-Conway-North Myrtle } \\
\text { Beach, South Carolina-North Carolina }\end{array}$ & 933 & 64.8 & 2.2 & $(60.6-69.1)$ \\
\hline $\begin{array}{l}\text { Nashville-Davidson-Murfreesboro- } \\
\text { Franklin, Tennessee }\end{array}$ & 1,324 & 74.6 & 1.7 & $(71.3-78.0)$ \\
\hline $\begin{array}{l}\text { Nassau County-Suffolk County, } \\
\text { New York* }\end{array}$ & 889 & 70.9 & 2.2 & $(66.6-75.2)$ \\
\hline Newark, New Jersey-Pennsylvania* & 6,499 & 74.6 & 0.9 & $(72.9-76.4)$ \\
\hline New Haven-Milford, Connecticut & 1,995 & 70.1 & 1.5 & $(67.1-73.0)$ \\
\hline New Orleans-Metairie, Louisiana & 1,261 & 70.4 & 1.9 & $(66.6-74.1)$ \\
\hline $\begin{array}{l}\text { New York-Jersey City-White Plains, } \\
\text { New York-New Jersey* }\end{array}$ & 7,357 & 71.9 & 1.0 & $(70.0-73.9)$ \\
\hline Norfolk, Nebraska & 557 & 50.6 & 3.0 & $(44.8-56.4)$ \\
\hline North Platte, Nebraska & 606 & 54.4 & 3.1 & $(48.3-60.4)$ \\
\hline Norwich-New London, Connecticut & 993 & 79.3 & 2.0 & $(75.4-83.1)$ \\
\hline $\begin{array}{l}\text { Oakland-Hayward-Berkeley, } \\
\text { California* }\end{array}$ & 973 & 66.1 & 2.3 & $(61.6-70.6)$ \\
\hline Ocean City, New Jersey & 547 & 74.8 & 4.0 & $(67.0-82.6)$ \\
\hline Ogden-Clearfield, Utah & 2,371 & 59.9 & 1.3 & $(57.4-62.4)$ \\
\hline Oklahoma City, Oklahoma & 2,400 & 60.7 & 1.3 & $(58.1-63.2)$ \\
\hline Olympia-Tumwater, Washington & 512 & 66.0 & 2.8 & $(60.6-71.5)$ \\
\hline Omaha-Council Bluffs, Nebraska-lowa & 5,540 & 64.0 & 0.9 & $(62.2-65.7)$ \\
\hline Orlando-Kissimmee-Sanford, Florida & 560 & 73.8 & 2.8 & $(68.2-79.3)$ \\
\hline Philadelphia, Pennsylvania** & 2,442 & 72.0 & 1.3 & $(69.5-74.6)$ \\
\hline Phoenix-Mesa-Scottsdale, Arizona & 2,571 & 62.9 & 1.3 & $(60.3-65.5)$ \\
\hline Pittsburgh, Pennsylvania & 3,352 & 69.7 & 1.1 & $(67.6-71.8)$ \\
\hline Ponce, Puerto Rico & 543 & 79.9 & 2.0 & (75.9-83.9) \\
\hline Portland-South Portland, Maine & 3,321 & 71.9 & 1.0 & (69.9-73.8) \\
\hline $\begin{array}{l}\text { Portland-Vancouver-Hillsboro, } \\
\text { Oregon-Washington }\end{array}$ & 3,064 & 58.8 & 1.2 & $(56.4-61.2)$ \\
\hline $\begin{array}{l}\text { Providence-Warwick, Rhode } \\
\text { Island-Massachusetts }\end{array}$ & 8,089 & 79.1 & 0.8 & $(77.6-80.6)$ \\
\hline Provo-Orem, Utah & 1,692 & 52.3 & 1.5 & $(49.3-55.4)$ \\
\hline Raleigh, North Carolina & 930 & 72.9 & 1.8 & $(69.3-76.4)$ \\
\hline Rapid City, South Dakota & 1,046 & 64.3 & 2.1 & $(60.2-68.4)$ \\
\hline
\end{tabular}


TABLE 8. (Continued) Estimated prevalence of adults aged $\geq 18$ years who visited a doctor for a routine checkup during the preceding 12 months, by metropolitan and micropolitan statistical area Behavioral Risk Factor Surveillance System, United States, 2012

\begin{tabular}{|c|c|c|c|c|}
\hline MMSA(s) & $\begin{array}{l}\text { Sample } \\
\text { size }\end{array}$ & $\%$ & SE & $95 \% \mathrm{Cl}$ \\
\hline Reno, Nevada & 1,497 & 60.5 & 1.9 & $(56.8-64.2)$ \\
\hline Richmond, Virginia & 1,003 & 75.0 & 1.9 & (71.3-78.8) \\
\hline $\begin{array}{l}\text { Riverside-San Bernardino-Ontario, } \\
\text { California }\end{array}$ & 1,522 & 63.2 & 1.8 & $(59.8-66.7)$ \\
\hline $\begin{array}{l}\text { Rockingham County-Strafford County, } \\
\text { New Hampshire* }\end{array}$ & 1,662 & 71.9 & 1.6 & $(68.8-75.0)$ \\
\hline Rutland, Vermont & 589 & 63.6 & 2.8 & $(58.1-69.1)$ \\
\hline $\begin{array}{l}\text { Sacramento-Roseville-Arden-Arcade, } \\
\text { California }\end{array}$ & 992 & 63.4 & 2.2 & $(59.1-67.7)$ \\
\hline St. Louis, Missouri-Illinois & 2,056 & 70.9 & 1.5 & $(68.1-73.8)$ \\
\hline Salisbury, Maryland-Delaware & 2,105 & 79.5 & 1.8 & $(75.9-83.1)$ \\
\hline Salt Lake City, Utah & 4,500 & 55.8 & 1.0 & $(53.9-57.8)$ \\
\hline San Antonio-New Braunfels, Texas & 770 & 67.0 & 2.4 & $(62.3-71.6)$ \\
\hline San Diego-Carlsbad, California & 1,125 & 63.5 & 2.1 & $(59.4-67.6)$ \\
\hline $\begin{array}{l}\text { San Francisco-Redwood City-South } \\
\text { San Francisco, California* }\end{array}$ & 593 & 62.2 & 2.8 & $(56.7-67.8)$ \\
\hline $\begin{array}{l}\text { San Jose-Sunnyvale-Santa Clara, } \\
\text { California }\end{array}$ & 684 & 58.7 & 2.6 & $(53.6-63.8)$ \\
\hline San Juan-Carolina-Caguas, Puerto Rico & 3,913 & 79.2 & 0.8 & $(77.7-80.8)$ \\
\hline Santa Fe, New Mexico & 687 & 60.2 & 2.3 & $(55.7-64.6)$ \\
\hline Sayre, Pennsylvania & 1,852 & 68.4 & 2.4 & $(63.6-73.1)$ \\
\hline Scottsbluff, Nebraska & 623 & 55.9 & 3.2 & $(49.5-62.3)$ \\
\hline $\begin{array}{l}\text { Scranton-Wilkes-Barre-Hazleton, } \\
\text { Pennsylvania }\end{array}$ & 736 & 75.3 & 2.3 & $(70.8-79.8)$ \\
\hline Seattle-Bellevue-Everett, Washington* & 5,043 & 59.7 & 0.9 & $(57.9-61.6)$ \\
\hline Shreveport-Bossier City, Louisiana & 569 & 69.5 & 2.8 & $(64.1-75.0)$ \\
\hline $\begin{array}{l}\text { Silver Spring-Frederick-Rockville, } \\
\text { Maryland* }\end{array}$ & 2,282 & 73.1 & 1.5 & $(70.0-76.1)$ \\
\hline $\begin{array}{l}\text { Sioux City, lowa-Nebraska- } \\
\text { South Dakota }\end{array}$ & 1,194 & 67.2 & 3.0 & $(61.4-73.0)$ \\
\hline Sioux Falls, South Dakota & 1,447 & 67.6 & 1.6 & $(64.4-70.8)$ \\
\hline Spartanburg, South Carolina & 700 & 65.8 & 2.7 & $(60.5-71.2)$ \\
\hline Spokane-Spokane Valley, Washington & 1,082 & 58.2 & 2.1 & $(54.1-62.3)$ \\
\hline
\end{tabular}

TABLE 8. (Continued) Estimated prevalence of adults aged $\geq 18$ years who visited a doctor for a routine checkup during the preceding 12 months, by metropolitan and micropolitan statistical area Behavioral Risk Factor Surveillance System, United States, 2012

\begin{tabular}{|c|c|c|c|c|}
\hline MMSA(s) & $\begin{array}{l}\text { Sample } \\
\text { size }\end{array}$ & $\%$ & SE & $95 \% \mathrm{Cl}$ \\
\hline Springfield, Massachusetts & 2,327 & 76.9 & 1.5 & $(74.0-79.8)$ \\
\hline Tacoma-Lakewood, Washington* & 1,157 & 56.0 & 2.0 & $(52.1-59.8)$ \\
\hline $\begin{array}{l}\text { Tampa-St. Petersburg-Clearwater, } \\
\text { Florida }\end{array}$ & 824 & 69.9 & 2.3 & $(65.5-74.4)$ \\
\hline Toledo, Ohio & 963 & 68.7 & 2.6 & $(63.7-73.7)$ \\
\hline Topeka, Kansas & 1,071 & 70.4 & 1.9 & $(66.7-74.1)$ \\
\hline Torrington, Connecticut & 655 & 71.1 & 2.3 & $(66.5-75.7)$ \\
\hline Trenton, New Jersey & 574 & 78.7 & 2.2 & $(74.5-83.0)$ \\
\hline Tucson, Arizona & 987 & 66.3 & 2.1 & $(62.1-70.5)$ \\
\hline Tulsa, Oklahoma & 1,723 & 61.6 & 1.5 & $(58.7-64.6)$ \\
\hline Tuscaloosa, Alabama & 573 & 76.0 & 3.1 & $(70.1-82.0)$ \\
\hline Urban Honolulu, Hawaii & 4,314 & 65.6 & 1.1 & $(63.5-67.7)$ \\
\hline Vineland-Bridgeton, New Jersey & 556 & 77.8 & 3.1 & $(71.7-83.9)$ \\
\hline $\begin{array}{l}\text { Virginia Beach-Norfolk-Newport News, } \\
\text { Virginia-North Carolina }\end{array}$ & 1,492 & 76.5 & 1.6 & $(73.4-79.6)$ \\
\hline $\begin{array}{l}\text { Warren-Troy-Farmington Hills, } \\
\text { Michigan* }\end{array}$ & 2,167 & 67.3 & 1.3 & $(64.7-69.9)$ \\
\hline $\begin{array}{l}\text { Washington-Arlington-Alexandria, } \\
\text { District of Columbia-Virginia- } \\
\text { Maryland-West Virginia* }\end{array}$ & 8,137 & 71.4 & 0.9 & $(69.6-73.2)$ \\
\hline Wichita, Kansas & 2,324 & 67.9 & 1.3 & $(65.3-70.5)$ \\
\hline $\begin{array}{l}\text { Wilmington, Delaware-Maryland- } \\
\text { New Jersey* }\end{array}$ & 3,208 & 77.4 & 1.2 & $(75.1-79.8)$ \\
\hline Winston-Salem, North Carolina & 787 & 77.8 & 1.9 & (74.2-81.4) \\
\hline Worcester, Massachusetts-Connecticut & 2,832 & 77.0 & 1.2 & $(74.6-79.4)$ \\
\hline Yakima, Washington & 521 & 55.6 & 3.1 & $(49.6-61.7)$ \\
\hline $\begin{array}{l}\text { Youngstown-Warren-Boardman, } \\
\text { Ohio-Pennsylvania }\end{array}$ & 923 & 73.6 & 2.6 & $(68.4-78.7)$ \\
\hline Median & & 68.3 & & \\
\hline Range & & $50.6-85.0$ & & \\
\hline
\end{tabular}

Abbreviations: $\mathrm{Cl}=$ confidence interval; $\mathrm{MMSA}=$ metropolitan and micropolitan statistical area; $\mathrm{SE}=$ standard error.

* Metropolitan division. 
TABLE 9. Estimated prevalence of adults aged $\geq 18$ years who visited a doctor for a routine checkup during the preceding 12 months, by county - Behavioral Risk Factor Surveillance System, United States, 2012

\begin{tabular}{|c|c|c|c|c|}
\hline County & $\begin{array}{l}\text { Sample } \\
\text { size }\end{array}$ & $\%$ & SE & $95 \% \mathrm{Cl}$ \\
\hline Jefferson County, Alabama & 943 & 75.5 & 1.9 & $(71.8-79.2)$ \\
\hline Madison County, Alabama & 509 & 70.2 & 2.8 & $(64.7-75.7)$ \\
\hline Mobile County, Alabama & 799 & 75.2 & 2.7 & $(69.9-80.5)$ \\
\hline Anchorage Municipality, Alaska & 886 & 68.1 & 1.8 & $(64.5-71.7)$ \\
\hline Fairbanks North Star Borough, Alaska & 582 & 62.6 & 2.7 & $(57.3-67.8)$ \\
\hline Matanuska-Susitna Borough, Alaska & 584 & 61.4 & 2.7 & $(56.2-66.7)$ \\
\hline Maricopa County, Arizona & 2,093 & 62.2 & 1.4 & $(59.5-65.0)$ \\
\hline Pima County, Arizona & 987 & 66.3 & 2.1 & $(62.1-70.5)$ \\
\hline Pulaski County, Arkansas & 733 & 68.0 & 2.6 & $(62.9-73.2)$ \\
\hline Alameda County, California & 618 & 64.4 & 2.9 & $(58.7-70.1)$ \\
\hline Los Angeles County, California & 3,488 & 64.7 & 1.1 & $(62.5-67.0)$ \\
\hline Orange County, California & 1,035 & 66.6 & 2.3 & $(62.1-71.1)$ \\
\hline Riverside County, California & 822 & 64.4 & 2.4 & $(59.7-69.2)$ \\
\hline Sacramento County, California & 607 & 63.5 & 2.9 & $(57.9-69.1)$ \\
\hline San Bernardino County, California & 700 & 61.6 & 2.5 & $(56.7-66.6)$ \\
\hline San Diego County, California & 1,125 & 63.5 & 2.1 & $(59.4-67.6)$ \\
\hline Santa Clara County, California & 665 & 59.1 & 2.6 & $(53.9-64.2)$ \\
\hline Adams County, Colorado & 797 & 54.9 & 2.3 & $(50.3-59.4)$ \\
\hline Arapahoe County, Colorado & 848 & 64.0 & 2.0 & $(60.1-67.9)$ \\
\hline Boulder County, Colorado & 509 & 53.4 & 2.8 & $(47.9-58.8)$ \\
\hline Denver County, Colorado & 981 & 60.2 & 1.9 & $(56.5-63.9)$ \\
\hline Douglas County, Colorado & 540 & 64.5 & 2.5 & $(59.6-69.4)$ \\
\hline El Paso County, Colorado & 1,009 & 64.5 & 1.8 & $(60.8-68.1)$ \\
\hline Jefferson County, Colorado & 1,104 & 63.8 & 1.8 & $(60.3-67.2)$ \\
\hline Larimer County, Colorado & 585 & 61.1 & 2.5 & $(56.2-66.0)$ \\
\hline Weld County, Colorado & 521 & 58.6 & 2.7 & $(53.2-63.9)$ \\
\hline Fairfield County, Connecticut & 2,165 & 67.9 & 1.5 & $(65.0-70.8)$ \\
\hline Hartford County, Connecticut & 1,963 & 75.2 & 1.3 & $(72.7-77.7)$ \\
\hline Litchfield County, Connecticut & 655 & 71.1 & 2.3 & $(66.5-75.7)$ \\
\hline New Haven County, Connecticut & 1,995 & 70.1 & 1.5 & $(67.1-73.0)$ \\
\hline New London County, Connecticut & 993 & 79.3 & 2.0 & $(75.4-83.1)$ \\
\hline Kent County, Delaware & 1,428 & 82.4 & 1.5 & $(79.4-85.4)$ \\
\hline New Castle County, Delaware & 2,319 & 78.7 & 1.2 & $(76.4-81.0)$ \\
\hline Sussex County, Delaware & 1,393 & 81.5 & 1.6 & $(78.4-84.5)$ \\
\hline $\begin{array}{l}\text { District of Columbia, } \\
\text { District of Columbia }\end{array}$ & 3,796 & 74.1 & 1.2 & $(71.7-76.5)$ \\
\hline Broward County, Florida & 525 & 70.3 & 3.1 & $(64.2-76.4)$ \\
\hline Miami-Dade County, Florida & 812 & 69.2 & 2.8 & $(63.8-74.6)$ \\
\hline Hawaii County, Hawaii & 1,342 & 58.8 & 2.0 & $(54.9-62.6)$ \\
\hline Honolulu County, Hawaii & 4,314 & 65.6 & 1.1 & $(63.5-67.7)$ \\
\hline Kauai County, Hawaii & 664 & 62.9 & 2.9 & $(57.3-68.6)$ \\
\hline Maui County, Hawaii & 1,210 & 61.3 & 2.1 & $(57.2-65.3)$ \\
\hline Ada County, Idaho & 806 & 54.7 & 2.6 & $(49.7-59.8)$ \\
\hline Canyon County, Idaho & 500 & 54.4 & 3.7 & $(47.2-61.6)$ \\
\hline Cook County, Illinois & 1,503 & 68.4 & 1.6 & $(65.2-71.6)$ \\
\hline Lake County, Indiana & 877 & 64.3 & 2.8 & $(58.8-69.9)$ \\
\hline Marion County, Indiana & 1,270 & 64.9 & 1.8 & $(61.4-68.5)$ \\
\hline Polk County, lowa & 801 & 66.1 & 2.1 & $(62.1-70.1)$ \\
\hline Johnson County, Kansas & 2,164 & 73.0 & 1.3 & $(70.4-75.5)$ \\
\hline Sedgwick County, Kansas & 1,773 & 67.3 & 1.5 & $(64.3-70.2)$ \\
\hline Shawnee County, Kansas & 763 & 71.3 & 2.3 & $(66.8-75.9)$ \\
\hline Wyandotte County, Kansas & 855 & 66.5 & 3.1 & $(60.5-72.6)$ \\
\hline Jefferson County, Kentucky & 1,673 & 68.0 & 1.9 & $(64.3-71.7)$ \\
\hline East Baton Rouge Parish, Louisiana & 566 & 77.2 & 2.4 & $(72.5-81.9)$ \\
\hline Androscoggin County, Maine & 696 & 69.5 & 2.2 & $(65.2-73.9)$ \\
\hline Aroostook County, Maine & 535 & 70.3 & 2.6 & $(65.3-75.3)$ \\
\hline Cumberland County, Maine & 1,749 & 72.5 & 1.3 & $(69.9-75.1)$ \\
\hline Kennebec County, Maine & 831 & 72.9 & 2.0 & $(69.0-76.8)$ \\
\hline Penobscot County, Maine & 924 & 71.6 & 2.0 & $(67.8-75.5)$ \\
\hline
\end{tabular}

See table footnotes on page 30 .
TABLE 9. (Continued) Estimated prevalence of adults aged $\geq 18$ years who visited a doctor for a routine checkup during the preceding 12 months, by county - Behavioral Risk Factor Surveillance System, United States, 2012

\begin{tabular}{|c|c|c|c|c|}
\hline County & $\begin{array}{l}\text { Sample } \\
\text { size }\end{array}$ & $\%$ & SE & $95 \% \mathrm{Cl}$ \\
\hline York County, Maine & 1,192 & 72.4 & 1.7 & $(69.1-75.6)$ \\
\hline Anne Arundel County, Maryland & 887 & 76.7 & 2.1 & $(72.5-80.8)$ \\
\hline Baltimore County, Maryland & 1,505 & 80.6 & 1.5 & $(77.6-83.6)$ \\
\hline Charles County, Maryland & 510 & 69.9 & 3.8 & $(62.4-77.4)$ \\
\hline Frederick County, Maryland & 748 & 69.3 & 3.4 & $(62.7-75.9)$ \\
\hline Montgomery County, Maryland & 1,534 & 74.1 & 1.7 & $(70.7-77.4)$ \\
\hline Prince George's County, Maryland & 1,135 & 75.3 & 2.3 & $(70.8-79.8)$ \\
\hline Washington County, Maryland & 536 & 79.4 & 3.1 & $(73.3-85.5)$ \\
\hline Baltimore city, Maryland & 742 & 78.0 & 2.5 & $(73.0-83.0)$ \\
\hline Barnstable County, Massachusetts & 554 & 85.0 & 1.9 & $(81.3-88.6)$ \\
\hline Bristol County, Massachusetts & 2,639 & 80.3 & 1.6 & $(77.2-83.4)$ \\
\hline Essex County, Massachusetts & 2,544 & 82.4 & 1.3 & $(79.9-85.0)$ \\
\hline Hampden County, Massachusetts & 1,949 & 79.1 & 1.5 & $(76.0-82.1)$ \\
\hline Middlesex County, Massachusetts & 4,340 & 76.9 & 1.0 & $(75.1-78.8)$ \\
\hline Norfolk County, Massachusetts & 1,753 & 79.3 & 1.4 & $(76.5-82.1)$ \\
\hline Plymouth County, Massachusetts & 1,823 & 80.6 & 1.5 & $(77.6-83.5)$ \\
\hline Suffolk County, Massachusetts & 2,294 & 78.1 & 1.3 & $(75.5-80.7)$ \\
\hline Worcester County, Massachusetts & 2,588 & 77.7 & 1.3 & $(75.2-80.2)$ \\
\hline Kent County, Michigan & 522 & 64.5 & 3.1 & $(58.5-70.5)$ \\
\hline Macomb County, Michigan & 608 & 68.0 & 2.3 & $(63.6-72.5)$ \\
\hline Oakland County, Michigan & 1,163 & 68.9 & 1.9 & $(65.3-72.6)$ \\
\hline Wayne County, Michigan & 2,203 & 69.9 & 1.6 & $(66.8-73.0)$ \\
\hline Anoka County, Minnesota & 536 & 62.7 & 2.7 & $(57.3-68.0)$ \\
\hline Dakota County, Minnesota & 665 & 74.5 & 2.0 & $(70.5-78.4)$ \\
\hline Hennepin County, Minnesota & 3,271 & 69.3 & 1.2 & $(67.0-71.6)$ \\
\hline Ramsey County, Minnesota & 1,925 & 68.0 & 2.2 & $(63.7-72.4)$ \\
\hline Jackson County, Missouri & 878 & 63.7 & 2.8 & $(58.3-69.1)$ \\
\hline St. Louis County, Missouri & 948 & 72.3 & 2.1 & $(68.2-76.4)$ \\
\hline Cascade County, Montana & 697 & 61.4 & 2.4 & $(56.6-66.2)$ \\
\hline Flathead County, Montana & 554 & 55.2 & 2.5 & $(50.3-60.1)$ \\
\hline Hill County, Montana & 575 & 58.8 & 3.5 & $(52.0-65.6)$ \\
\hline Lake County, Montana & 886 & 54.0 & 2.8 & $(48.6-59.5)$ \\
\hline Missoula County, Montana & 775 & 53.1 & 2.3 & $(48.7-57.6)$ \\
\hline Yellowstone County, Montana & 742 & 58.7 & 2.0 & $(54.7-62.7)$ \\
\hline Dakota County, Nebraska & 726 & 69.8 & 3.8 & $(62.2-77.3)$ \\
\hline Douglas County, Nebraska & 3,539 & 62.7 & 1.1 & $(60.6-64.7)$ \\
\hline Hall County, Nebraska & 526 & 55.9 & 3.0 & $(50.0-61.9)$ \\
\hline Lancaster County, Nebraska & 1,421 & 58.5 & 1.5 & $(55.5-61.5)$ \\
\hline Lincoln County, Nebraska & 583 & 54.1 & 3.2 & $(47.9-60.3)$ \\
\hline Sarpy County, Nebraska & 1,167 & 66.5 & 1.8 & $(62.9-70.1)$ \\
\hline Scotts Bluff County, Nebraska & 571 & 55.7 & 3.3 & $(49.2-62.2)$ \\
\hline Clark County, Nevada & 1,977 & 65.2 & 1.3 & $(62.7-67.8)$ \\
\hline Washoe County, Nevada & 1,478 & 60.6 & 1.9 & $(56.9-64.4)$ \\
\hline Belknap County, New Hampshire & 564 & 67.3 & 3.4 & $(60.5-74.0)$ \\
\hline Carroll County, New Hampshire & 525 & 67.3 & 3.4 & $(60.7-73.9)$ \\
\hline Cheshire County, New Hampshire & 541 & 65.1 & 3.4 & $(58.5-71.7)$ \\
\hline Coos County, New Hampshire & 532 & 68.4 & 3.3 & $(61.9-75.0)$ \\
\hline Grafton County, New Hampshire & 561 & 68.8 & 3.1 & $(62.8-74.9)$ \\
\hline Hillsborough County, New Hampshire & 1,891 & 71.1 & 1.4 & $(68.3-73.9)$ \\
\hline Merrimack County, New Hampshire & 700 & 72.4 & 2.3 & $(67.9-76.9)$ \\
\hline Rockingham County, New Hampshire & 1,043 & 74.1 & 1.9 & $(70.4-77.7)$ \\
\hline Strafford County, New Hampshire & 619 & 67.1 & 3.0 & $(61.2-73.0)$ \\
\hline Atlantic County, New Jersey & 1,012 & 77.4 & 1.9 & $(73.7-81.1)$ \\
\hline Bergen County, New Jersey & 1,000 & 75.2 & 1.9 & $(71.5-78.9)$ \\
\hline Burlington County, New Jersey & 679 & 76.2 & 2.2 & $(71.8-80.6)$ \\
\hline Camden County, New Jersey & 734 & 75.7 & 2.1 & $(71.5-79.9)$ \\
\hline Cape May County, New Jersey & 547 & 74.8 & 4.0 & $(67.0-82.6)$ \\
\hline Cumberland County, New Jersey & 556 & 77.8 & 3.1 & $(71.7-83.9)$ \\
\hline Essex County, New Jersey & 1,324 & 75.0 & 1.7 & $(71.7-78.2)$ \\
\hline
\end{tabular}

See table footnotes on page 30 . 
TABLE 9. (Continued) Estimated prevalence of adults aged $\geq 18$ years who visited a doctor for a routine checkup during the preceding 12 months, by county - Behavioral Risk Factor Surveillance System, United States, 2012

\begin{tabular}{|c|c|c|c|c|}
\hline County & $\begin{array}{l}\text { Sample } \\
\text { size }\end{array}$ & $\%$ & SE & $95 \% \mathrm{Cl}$ \\
\hline Gloucester County, New Jersey & 545 & 75.2 & 2.6 & $(70.0-80.4)$ \\
\hline Hudson County, New Jersey & 1,287 & 74.2 & 1.7 & $(70.8-77.5)$ \\
\hline Hunterdon County, New Jersey & 564 & 72.1 & 2.7 & $(66.8-77.4)$ \\
\hline Mercer County, New Jersey & 574 & 78.7 & 2.2 & $(74.5-83.0)$ \\
\hline Middlesex County, New Jersey & 872 & 75.3 & 2.1 & $(71.2-79.4)$ \\
\hline Monmouth County, New Jersey & 702 & 72.7 & 2.2 & $(68.3-77.0)$ \\
\hline Morris County, New Jersey & 845 & 74.4 & 2.1 & $(70.2-78.6)$ \\
\hline Ocean County, New Jersey & 652 & 76.4 & 2.2 & $(72.1-80.8)$ \\
\hline Passaic County, New Jersey & 684 & 71.0 & 2.4 & $(66.3-75.7)$ \\
\hline Salem County, New Jersey & 574 & 71.4 & 4.1 & $(63.3-79.5)$ \\
\hline Somerset County, New Jersey & 635 & 74.3 & 2.4 & $(69.5-79.0)$ \\
\hline Sussex County, New Jersey & 539 & 72.0 & 2.9 & $(66.3-77.6)$ \\
\hline Union County, New Jersey & 709 & 75.8 & 2.0 & $(71.9-79.7)$ \\
\hline Warren County, New Jersey & 527 & 76.7 & 2.7 & (71. \\
\hline Bernalillo County, New Mexico & 2,041 & 55.5 & 1.3 & $(52$. \\
\hline Dona Ana County, New Mexico & 701 & 58.9 & 2.5 & 3.7) \\
\hline Sandoval County, New Mexico & 650 & 58.4 & 2.5 & 3.4) \\
\hline San Juan County, New Mexico & 639 & 57.1 & .5 & 2.0) \\
\hline Santa Fe County, New Mexico & 687 & 60.2 & 2.3 & $(55.7-64.6)$ \\
\hline Kings County, New York & 496 & 66.9 & 2.9 & $(61.3-72.5)$ \\
\hline Guilford County, North Carolina & 506 & 72.1 & 2.7 & $(66.9-77.3)$ \\
\hline Mecklenburg County, North Carolina & 723 & 72.1 & 2.0 & $(68.2-76.0)$ \\
\hline Robeson County, North Carolina & 543 & 71.5 & 4.4 & $(62.8-80.2)$ \\
\hline Wake County, North Carolina & 689 & 72.3 & 2.1 & $(68.2-76.3)$ \\
\hline Burleigh County, North Dakota & 563 & 63.6 & 2.8 & $(58.0-69.1)$ \\
\hline Cass County, North Dakota & 842 & 62.1 & 2.4 & $(57.3-66.8)$ \\
\hline Cuyahoga County, Ohio & 854 & 73.1 & 1.9 & $(69.4-76.8)$ \\
\hline Franklin County, Ohio & 831 & 68.4 & 1.9 & $(64.6-72.2)$ \\
\hline Har & 764 & 77.3 & 1.9 & $0.9)$ \\
\hline Lorain County, Ohio & 604 & 77.1 & 2.7 & $(71.8-82.5)$ \\
\hline Lucas County, Ohio & 618 & 70.4 & 3.1 & $(64.4-76.4)$ \\
\hline Mahoning County, Ohio & 590 & 76.3 & 2.9 & $(70.7-81.9)$ \\
\hline Montgomery County, Ohio & 654 & 69.0 & 2.6 & $(63.9-74.1)$ \\
\hline Stark County, Ohio & 618 & 78.5 & 2.4 & $(73.7-83.3)$ \\
\hline Summit County, Ohio & 628 & 71.9 & 2.6 & $(66.8-77.1)$ \\
\hline Oklahoma County, Oklahoma & 1,175 & 61.3 & 1.7 & $(57.9-64.7)$ \\
\hline Tulsa County, Oklahoma & 1,192 & 60.3 & 1.8 & $(56.8-63.9)$ \\
\hline Clackamas County, Oregon & 501 & 52.9 & 3.1 & $(46.9-59.0)$ \\
\hline Lane County, Oregon & 505 & 54.4 & 3.0 & $(48.6-60.3)$ \\
\hline Multnomah County, Oregon & 871 & 58.4 & 2.3 & $(54.0-62.8)$ \\
\hline Washington County, Oregon & 605 & 63.7 & 2.6 & $(58.6-68.8)$ \\
\hline Allegheny County, Pennsylvania & 1,881 & 69.2 & 1.4 & $(66.5-71.9)$ \\
\hline Bradford County, Pennsylvania & 1,852 & 68.4 & 2.4 & $(63.6-73.1)$ \\
\hline Montgomery County, Pennsylvania & 541 & 66.4 & 2.5 & $(61.5-71.2)$ \\
\hline Philadelphia County, Pennsylvania & 2,049 & 71.7 & 1.5 & $(68.8-74.6)$ \\
\hline Pike County, Pennsylvania & 1,883 & 72.9 & 2.2 & $(68.6-77.1)$ \\
\hline Kent County, Rhode Island & 812 & 82.6 & 1.8 & $(79.1-86.1)$ \\
\hline Providence County, Rhode Island & 3,328 & 78.6 & 1.0 & $(76.5-80.6)$ \\
\hline
\end{tabular}

TABLE 9. (Continued) Estimated prevalence of adults aged $\geq 18$ years who visited a doctor for a routine checkup during the preceding 12 months, by county - Behavioral Risk Factor Surveillance System, United States, 2012

\begin{tabular}{|c|c|c|c|c|}
\hline County & $\begin{array}{l}\text { Sample } \\
\text { size }\end{array}$ & $\%$ & SE & $95 \% \mathrm{Cl}$ \\
\hline Washington County, Rhode Island & 650 & 75.3 & 2.6 & $(70.2-80.5)$ \\
\hline Aiken County, South Carolina & 550 & 70.0 & 2.8 & $(64.5-75.5)$ \\
\hline Beaufort County, South Carolina & 793 & 67.1 & 3.5 & $(60.2-74.0)$ \\
\hline Charleston County, South Carolina & 1,004 & 62.8 & 2.4 & $(58.2-67.5)$ \\
\hline Greenville County, South Carolina & 895 & 61.6 & 2.3 & $(57.2-66.1)$ \\
\hline Horry County, South Carolina & 768 & 61.0 & 2.3 & $(56.4-65.6)$ \\
\hline Richland County, South Carolina & 956 & 67.5 & 2.3 & $(62.9-72.1)$ \\
\hline Spartanburg County, South Carolina & 649 & 66.9 & 2.8 & $(61.4-72.4)$ \\
\hline Lincoln County, South Dakota & 493 & 68.1 & 3.4 & $(61.3-74.8)$ \\
\hline Minnehaha County, South Dakota & 869 & 67.2 & 1.9 & $(63.4-70.9)$ \\
\hline Pennington County, South Dakota & 602 & 64.3 & 2.4 & $(59.6-69.1)$ \\
\hline Davidson County, Tennessee & 551 & 74.1 & 2.7 & $(68.8-79.4)$ \\
\hline Shelby County, Tennessee & 535 & 78.2 & 2.3 & $(73.7-82.6)$ \\
\hline Bexar County, Texas & 579 & 64.5 & 2.8 & $(59.0-69.9)$ \\
\hline Dallas County, Texas & 509 & 63.0 & 2.7 & $(57.7-68.2)$ \\
\hline El Paso County, Texas & 620 & 59.3 & 3.2 & $(53.0-65.7)$ \\
\hline Harris County, Texas & 755 & 58.4 & 2.2 & $(54.1-62.7)$ \\
\hline Hidalgo County, Texas & 606 & 56.3 & 3.2 & $(50.0-62.6)$ \\
\hline Tarrant County, Texas & 566 & 71.1 & 2.6 & $(66.1-76.2)$ \\
\hline Travis County, Texas & 1,038 & 59.5 & 2.6 & $(54.4-64.6)$ \\
\hline Davis County, Utah & 1,116 & 59.0 & 1.8 & $(55.4-62.6)$ \\
\hline Salt Lake County, Utah & 3,957 & 55.6 & 1.0 & $(53.6-57.6)$ \\
\hline Tooele County, Utah & 543 & 60.4 & 3.5 & $(53.5-67.3)$ \\
\hline Utah County, Utah & 1,625 & 52.4 & 1.6 & $(49.3-55.5)$ \\
\hline Wasatch County, Utah & 503 & 53.0 & 5.0 & $(43.2-62.8)$ \\
\hline Weber County, Utah & 1,025 & 60.7 & 2.0 & $(56.8-64.7)$ \\
\hline Chittenden County, Vermont & 910 & 65.6 & 1.9 & $(62.0-69.3)$ \\
\hline Rutland County, Vermont & 589 & 63.6 & 2.8 & $(58.1-69.1)$ \\
\hline Washington County, Vermont & 512 & 66.6 & 2.8 & $(61.2-72.1)$ \\
\hline Windsor County, Vermont & 543 & 70.0 & 2.5 & $(65.0-75.0)$ \\
\hline Fairfax County, Virginia & 735 & 71.2 & 2.2 & $(67.0-75.4)$ \\
\hline Clark County, Washington & 780 & 57.6 & 2.3 & $(53.1-62.2)$ \\
\hline King County, Washington & 3,881 & 60.6 & 1.1 & $(58.5-62.7)$ \\
\hline Kitsap County, Washington & 561 & 65.1 & 2.9 & $(59.5-70.7)$ \\
\hline Pierce County, Washington & 1,157 & 56.0 & 2.0 & $(52.1-59.8)$ \\
\hline Snohomish County, Washington & 1,162 & 56.7 & 1.9 & $(53.0-60.5)$ \\
\hline Spokane County, Washington & 939 & 59.4 & 2.3 & $(55.0-63.9)$ \\
\hline Thurston County, Washington & 512 & 66.0 & 2.8 & $(60.6-71.5)$ \\
\hline Whatcom County, Washington & 835 & 60.0 & 2.8 & $(54.5-65.6)$ \\
\hline Yakima County, Washington & 521 & 55.6 & 3.1 & $(49.6-61.7)$ \\
\hline Kanawha County, West Virginia & 636 & 78.3 & 2.0 & $(74.4-82.1)$ \\
\hline Milwaukee County, Wisconsin & 958 & 71.3 & 2.4 & $(66.7-75.9)$ \\
\hline Laramie County, Wyoming & 938 & 64.9 & 2.6 & $(59.8-69.9)$ \\
\hline Natrona County, Wyoming & 807 & 58.6 & 2.9 & $(53.0-64.2)$ \\
\hline San Juan Municipio, Puerto Rico & 662 & 78.3 & 2.0 & $(74.5-82.2)$ \\
\hline Median & \multicolumn{4}{|c|}{68.0} \\
\hline Range & \multicolumn{4}{|c|}{$52.4-85.0$} \\
\hline
\end{tabular}

Abbreviations: $\mathrm{Cl}=$ confidence interval; $\mathrm{SE}=$ standard error. 
TABLE 10. Estimated prevalence of adults aged $\geq 65$ years who received an influenza vaccination during the preceding 12 months, by state/territory - Behavioral Risk Factor Surveillance System, United States, 2012

\begin{tabular}{|c|c|c|c|c|}
\hline State/Territory & $\begin{array}{l}\text { Sample } \\
\text { size }\end{array}$ & $\%$ & SE & $95 \% \mathrm{Cl}$ \\
\hline Alabama & 3,123 & 61.2 & 1.2 & $(58.9-63.5)$ \\
\hline Alaska & 765 & 50.8 & 2.5 & $(45.9-55.6)$ \\
\hline Arizona & 2,808 & 52.3 & 1.5 & $(49.5-55.2)$ \\
\hline Arkansas & 1,909 & 57.2 & 1.4 & $(54.4-60.0)$ \\
\hline California & 3,764 & 57.9 & 1.2 & $(55.5-60.3)$ \\
\hline Colorado & 3,374 & 66.2 & 1.0 & $(64.2-68.3)$ \\
\hline Connecticut & 2,682 & 59.5 & 1.3 & $(57.0-62.0)$ \\
\hline Delaware & 1,682 & 63.1 & 1.5 & $(60.1-66.1)$ \\
\hline District of Columbia & 1,202 & 56.7 & 2.0 & $(52.8-60.6)$ \\
\hline Florida & 2,977 & 54.7 & 1.4 & $(52.0-57.4)$ \\
\hline Georgia & 1,853 & 60.1 & 1.5 & $(57.2-63.0)$ \\
\hline Hawaii & 1,965 & 62.7 & 1.6 & $(59.5-65.9)$ \\
\hline Idaho & 2,154 & 52.0 & 1.7 & $(48.7-55.2)$ \\
\hline Illinois & 1,939 & 52.5 & 1.5 & $(49.6-55.4)$ \\
\hline Indiana & 2,698 & 57.1 & 1.2 & $(54.8-59.5)$ \\
\hline lowa & 2,417 & 70.1 & 1.0 & $(68.0-72.1)$ \\
\hline Kansas & 3,929 & 66.7 & 0.9 & $(65.0-68.5)$ \\
\hline Kentucky & 3,274 & 61.8 & 1.3 & $(59.3-64.4)$ \\
\hline Louisiana & 3,184 & 63.8 & 1.3 & $(61.2-66.3)$ \\
\hline Maine & 3,120 & 61.3 & 1.1 & $(59.2-63.4)$ \\
\hline Maryland & 4,056 & 63.2 & 1.2 & $(60.9-65.5)$ \\
\hline Massachusetts & 6,045 & 63.6 & 1.0 & $(61.7-65.5)$ \\
\hline Michigan & 3,481 & 55.4 & 1.1 & $(53.3-57.5)$ \\
\hline Minnesota & 3,320 & 65.5 & 1.1 & (63.4-67.6) \\
\hline Mississippi & 2,805 & 62.4 & 1.1 & $(60.1-64.6)$ \\
\hline Missouri & 2,381 & 67.3 & 1.4 & $(64.6-70.1)$ \\
\hline Montana & 2,779 & 57.5 & 1.2 & $(55.2-59.9)$ \\
\hline Nebraska & 6,625 & 62.9 & 0.9 & $(61.2-64.6)$ \\
\hline Nevada & 1,535 & 50.0 & 2.0 & $(46.1-54.0)$ \\
\hline New Hampshire & 2,584 & 58.9 & 1.2 & $(56.5-61.3)$ \\
\hline New Jersey & 4,259 & 61.2 & 1.1 & (59.0-63.4) \\
\hline New Mexico & 2,494 & 57.8 & 1.2 & $(55.4-60.1)$ \\
\hline New York & 1,670 & 55.1 & 1.9 & $(51.4-58.7)$ \\
\hline North Carolina & 3,601 & 68.4 & 1.0 & $(66.5-70.3)$ \\
\hline North Dakota & 1,567 & 59.7 & 1.5 & (56.7-62.6) \\
\hline Ohio & 3,916 & 61.0 & 1.1 & $(58.8-63.1)$ \\
\hline Oklahoma & 2,576 & 67.8 & 1.1 & $(65.6-70.0)$ \\
\hline Oregon & 1,770 & 53.9 & 1.4 & $(51.1-56.7)$ \\
\hline Pennsylvania & 6,463 & 60.2 & 0.9 & $(58.4-62.0)$ \\
\hline Rhode Island & 1,632 & 57.6 & 1.5 & $(54.7-60.6)$ \\
\hline South Carolina & 4,337 & 60.1 & 1.1 & (57.9-62.3) \\
\hline South Dakota & 2,353 & 66.4 & 1.8 & $(62.8-70.0)$ \\
\hline Tennessee & 2,240 & 69.9 & 1.3 & $(67.4-72.5)$ \\
\hline Texas & 2,653 & 59.4 & 1.4 & $(56.8-62.1)$ \\
\hline Utah & 3,263 & 56.0 & 1.1 & $(53.9-58.1)$ \\
\hline Vermont & 1,955 & 64.2 & 1.3 & (61.6-66.8) \\
\hline Virginia & 2,164 & 60.1 & 1.3 & $(57.5-62.6)$ \\
\hline Washington & 4,905 & 60.1 & 0.9 & (58.4-61.9) \\
\hline West Virginia & 1,698 & 68.9 & 1.3 & $(66.4-71.4)$ \\
\hline Wisconsin & 1,574 & 50.5 & 1.9 & $(46.8-54.3)$ \\
\hline Wyoming & 2,351 & 53.3 & 1.5 & $(50.3-56.3)$ \\
\hline Guam & 218 & 39.2 & 3.9 & (31.6-46.7) \\
\hline Puerto Rico & 1,838 & 26.3 & 1.2 & $(24.0-28.6)$ \\
\hline Median & & 60.1 & & \\
\hline Range & & $26.3-70.1$ & & \\
\hline
\end{tabular}

Abbreviations: $\mathrm{Cl}=$ confidence interval; $\mathrm{SE}=$ standard error.
TABLE 11. Estimated prevalence of adults aged $\geq 65$ years who received an influenza vaccination during the preceding 12 months, by metropolitan and micropolitan statistical area - Behavioral Risk Factor Surveillance System, United States, 2012

\begin{tabular}{|c|c|c|c|c|}
\hline MMSA(s) & $\begin{array}{l}\text { Sample } \\
\text { size }\end{array}$ & $\%$ & SE & $95 \% \mathrm{Cl}$ \\
\hline Aguadilla-Isabela, Puerto Rico & 158 & 20.8 & 3.8 & $(13.4-28.2)$ \\
\hline Akron, Ohio & 224 & 62.6 & 3.9 & $(54.9-70.3)$ \\
\hline Albuquerque, New Mexico & 864 & 60.1 & 2.0 & $(56.1-64.1)$ \\
\hline $\begin{array}{l}\text { Allentown-Bethlehem-Easton, } \\
\text { Pennsylvania-New Jersey }\end{array}$ & 421 & 72.1 & 2.7 & $(66.8-77.4)$ \\
\hline Anaheim-Santa Ana-Irvine, California* & 274 & 65.0 & 4.6 & $(56.0-74.0)$ \\
\hline Anchorage, Alaska & 239 & 54.5 & 4.0 & $(46.8-62.3)$ \\
\hline Asheville, North Carolina & 223 & 73.9 & 3.4 & $(67.2-80.5)$ \\
\hline $\begin{array}{l}\text { Atlanta-Sandy Springs-Roswell, } \\
\text { Georgia }\end{array}$ & 668 & 57.2 & 2.4 & $(52.4-62.0)$ \\
\hline Atlantic City-Hammonton, New Jersey & 341 & 58.0 & 3.5 & $(51.1-64.9)$ \\
\hline $\begin{array}{l}\text { Augusta-Richmond County, Georgia- } \\
\text { South Carolina }\end{array}$ & 354 & 59.6 & 4.4 & $(50.9-68.2)$ \\
\hline Augusta-Waterville, Maine & 221 & 68.8 & 3.8 & $(61.4-76.2)$ \\
\hline Austin-Round Rock, Texas & 399 & 64.0 & 3.4 & $(57.3-70.7)$ \\
\hline $\begin{array}{l}\text { Baltimore-Columbia-Towson, } \\
\text { Maryland }\end{array}$ & 1,388 & 66.1 & 1.7 & $(62.8-69.4)$ \\
\hline Bangor, Maine & 253 & 59.0 & 3.6 & $(51.9-66.2)$ \\
\hline Barnstable Town, Massachusetts & 252 & 58.9 & 4.0 & (51.0-66.8) \\
\hline Barre, Vermont & 135 & 68.8 & 4.6 & $(59.8-77.7)$ \\
\hline Baton Rouge, Louisiana & 489 & 64.6 & 3.2 & $(58.4-70.8)$ \\
\hline Bellingham, Washington & 278 & 60.5 & 3.5 & $(53.6-67.4)$ \\
\hline Berlin, New Hampshire-Vermont & 276 & 58.8 & 3.6 & $(51.7-65.8)$ \\
\hline Billings, Montana & 250 & 66.6 & 3.3 & $(60.1-73.1)$ \\
\hline Birmingham-Hoover, Alabama & 575 & 63.7 & 2.6 & $(58.7-68.8)$ \\
\hline Bismarck, North Dakota & 252 & 59.4 & 3.9 & $(51.8-67.0)$ \\
\hline Boise City, Idaho & 495 & 51.1 & 3.1 & $(45.0-57.3)$ \\
\hline Boston, Massachusetts* & 1,589 & 61.9 & 1.9 & $(58.1-65.7)$ \\
\hline Boulder, Colorado & 126 & 72.2 & 4.4 & $(63.5-80.8)$ \\
\hline Bremerton-Silverdale, Washington & 201 & 65.1 & 4.0 & $(57.4-72.9)$ \\
\hline $\begin{array}{l}\text { Bridgeport-Stamford-Norwalk, } \\
\text { Connecticut }\end{array}$ & 601 & 57.1 & 2.7 & $(51.8-62.4)$ \\
\hline Burlington-South Burlington, Vermont & 418 & 65.7 & 2.8 & $(60.1-71.2)$ \\
\hline $\begin{array}{l}\text { Cambridge-Newton-Framingham, } \\
\text { Massachusetts* }\end{array}$ & 1,814 & 67.0 & 1.6 & $(63.8-70.1)$ \\
\hline Camden, New Jersey* & 550 & 65.5 & 2.6 & $(60.5-70.5)$ \\
\hline Canton-Massillon, Ohio & 216 & 58.8 & 4.3 & $(50.5-67.2)$ \\
\hline Casper, Wyoming & 318 & 50.7 & 4.2 & $(42.5-58.9)$ \\
\hline Cedar Rapids, lowa & 198 & 71.2 & 3.5 & $(64.3-78.1)$ \\
\hline Charleston, West Virginia & 237 & 74.8 & 3.3 & $(68.4-81.2)$ \\
\hline $\begin{array}{l}\text { Charleston-North Charleston, South } \\
\text { Carolina }\end{array}$ & 540 & 68.1 & 3.0 & $(62.2-73.9)$ \\
\hline $\begin{array}{l}\text { Charlotte-Concord-Gastonia, North } \\
\text { Carolina-South Carolina }\end{array}$ & 675 & 63.2 & 2.5 & $(58.3-68.0)$ \\
\hline Chattanooga, Tennessee-Georgia & 221 & 61.8 & 4.4 & $(53.2-70.4)$ \\
\hline Cheyenne, Wyoming & 390 & 62.1 & 3.5 & $(55.2-69.0)$ \\
\hline $\begin{array}{l}\text { Chicago-Naperville-Elgin, } \\
\text { Illinois-Indiana-Wisconsin }\end{array}$ & 1,141 & 52.2 & 2.1 & $(48.0-56.3)$ \\
\hline Cincinnati, Ohio-Kentucky-Indiana & 632 & 63.3 & 2.6 & $(58.1-68.4)$ \\
\hline $\begin{array}{l}\text { Claremont-Lebanon, New } \\
\text { Hampshire-Vermont }\end{array}$ & 700 & 60.1 & 2.1 & $(55.9-64.3)$ \\
\hline Cleveland-Elyria, Ohio & 535 & 58.3 & 3.1 & $(52.3-64.3)$ \\
\hline Colorado Springs, Colorado & 283 & 62.0 & 3.4 & $(55.3-68.7)$ \\
\hline Columbia, South Carolina & 524 & 62.3 & 3.1 & $(56.2-68.5)$ \\
\hline Columbus, Ohio & 396 & 67.4 & 3.0 & $(61.5-73.4)$ \\
\hline Concord, New Hampshire & 231 & 51.3 & 3.9 & $(43.6-59.0)$ \\
\hline Dallas-Plano-Irving, Texas* & 216 & 56.2 & 4.1 & $(48.1-64.3)$ \\
\hline Dayton, Ohio & 276 & 57.5 & 3.6 & $(50.3-64.6)$ \\
\hline Denver-Aurora-Lakewood, Colorado & 1,155 & 68.3 & 1.6 & $(65.1-71.5)$ \\
\hline Des Moines-West Des Moines, lowa & 346 & 72.2 & 2.7 & $(66.8-77.5)$ \\
\hline
\end{tabular}

See table footnotes on page 33 . 
TABLE 11. (Continued) Estimated prevalence of adults aged $\geq 65$ years who received an influenza vaccination during the preceding 12 months, by metropolitan and micropolitan statistical area Behavioral Risk Factor Surveillance System, United States, 2012

\begin{tabular}{|c|c|c|c|c|}
\hline MMSA(s) & $\begin{array}{l}\text { Sample } \\
\text { size }\end{array}$ & $\%$ & SE & $95 \% \mathrm{Cl}$ \\
\hline Detroit-Dearborn-Livonia, Michigan* & 750 & 46.5 & 3.0 & $(40.7-52.3)$ \\
\hline Dover, Delaware & 484 & 59.3 & 2.8 & $(53.7-64.8)$ \\
\hline Duluth, Minnesota-Wisconsin & 152 & 59.9 & 4.9 & $(50.2-69.6)$ \\
\hline Durham-Chapel Hill, North Carolina & 206 & 72.4 & 3.6 & $(65.3-79.5)$ \\
\hline El Paso, Texas & 195 & 50.9 & 4.8 & $(41.4-60.4)$ \\
\hline Eugene, Oregon & 176 & 49.9 & 4.5 & $(41.2-58.7)$ \\
\hline Fairbanks, Alaska & 100 & 44.0 & 6.0 & $(32.2-55.7)$ \\
\hline Fargo, North Dakota-Minnesota & 263 & 65.3 & 4.0 & $(57.4-73.1)$ \\
\hline Farmington, New Mexico & 172 & 50.7 & 4.6 & $(41.7-59.6)$ \\
\hline Fayetteville, North Carolina & 123 & 61.5 & 5.3 & $(51.1-71.9)$ \\
\hline $\begin{array}{l}\text { Fayetteville-Springdale-Rogers, } \\
\text { Arkansas-Missouri }\end{array}$ & 289 & 56.6 & 3.8 & $(49.1-64.1)$ \\
\hline Fort Collins, Colorado & 170 & 70.3 & 3.8 & $(62.8-77.7)$ \\
\hline Fort Wayne, Indiana & 147 & 51.8 & 4.7 & $(42.6-61.0)$ \\
\hline Fort Worth-Arlington, Texas* & 221 & 60.0 & 4.2 & $(51.8-68.2)$ \\
\hline Grand Island, Nebraska & 312 & 62.4 & 3.8 & $(54.9-69.8)$ \\
\hline Grand Rapids-Wyoming, Michigan & 268 & 57.9 & 3.6 & $(50.8-65.0)$ \\
\hline Great Falls, Montana & 256 & 55.8 & 3.5 & $(48.8-62.7)$ \\
\hline Greeley, Colorado & 123 & 59.6 & 5.3 & $(49.3-69.9)$ \\
\hline $\begin{array}{l}\text { Greensboro-High Point, } \\
\text { North Carolina }\end{array}$ & 246 & 77.8 & 3.0 & $(71.9-83.7)$ \\
\hline $\begin{array}{l}\text { Greenville-Anderson-Mauldin, } \\
\text { South Carolina }\end{array}$ & 541 & 62.6 & 2.9 & $(57.0-68.2)$ \\
\hline Gulfport-Biloxi-Pascagoula, Mississippi & 257 & 62.9 & 3.6 & $(55.9-69.9)$ \\
\hline $\begin{array}{l}\text { Hagerstown-Martinsburg, Maryland- } \\
\text { West Virginia }\end{array}$ & 262 & 63.6 & 4.1 & $(55.6-71.7)$ \\
\hline Harrisburg-Carlisle, Pennsylvania & 186 & 59.8 & 4.2 & $(51.5-68.0)$ \\
\hline $\begin{array}{l}\text { Hartford-West Hartford-East Hartford, } \\
\text { Connecticut }\end{array}$ & 817 & 60.9 & 2.2 & $(56.5-65.3)$ \\
\hline Heber, Utah & 154 & 58.6 & 4.4 & $(50.0-67.2)$ \\
\hline Hilo, Hawaii & 381 & 53.1 & 3.6 & $(46.1-60.1)$ \\
\hline $\begin{array}{l}\text { Hilton Head Island-Bluffton-Beaufort, } \\
\text { South Carolina }\end{array}$ & 485 & 60.4 & 2.6 & $(55.3-65.5)$ \\
\hline $\begin{array}{l}\text { Houston-The Woodlands-Sugar Land, } \\
\text { Texas }\end{array}$ & 219 & 61.7 & 3.9 & $(54.0-69.3)$ \\
\hline $\begin{array}{l}\text { Huntington-Ashland, West } \\
\text { Virginia-Kentucky-Ohio }\end{array}$ & 343 & 66.9 & 3.0 & $(61.0-72.8)$ \\
\hline Huntsville, Alabama & 153 & 54.8 & 5.2 & $(44.7-64.9)$ \\
\hline Idaho Falls, Idaho & 180 & 54.0 & 5.8 & $(42.6-65.3)$ \\
\hline $\begin{array}{l}\text { Indianapolis-Carmel-Anderson, } \\
\text { Indiana }\end{array}$ & 634 & 61.5 & 2.4 & $(56.7-66.3)$ \\
\hline Jackson, Mississippi & 277 & 62.7 & 3.4 & $(56.1-69.3)$ \\
\hline Jacksonville, Florida & 168 & 55.5 & 4.7 & $(46.2-64.8)$ \\
\hline Kahului-Wailuku-Lahaina, Hawaii & 363 & 57.7 & 3.9 & $(50.2-65.3)$ \\
\hline Kalispell, Montana & 177 & 58.1 & 4.3 & $(49.8-66.5)$ \\
\hline Kansas City, Missouri-Kansas & 1,532 & 66.5 & 2.3 & $(62.0-71.0)$ \\
\hline Kapaa, Hawaii & 225 & 62.8 & 4.0 & $(55.1-70.6)$ \\
\hline Keene, New Hampshire & 212 & 59.7 & 4.1 & $(51.6-67.8)$ \\
\hline Kennewick-Richland, Washington & 155 & 51.8 & 4.7 & $(42.6-61.0)$ \\
\hline $\begin{array}{l}\text { Kingsport-Bristol-Bristol, } \\
\text { Tennessee-Virginia }\end{array}$ & 194 & 68.4 & 4.7 & $(59.2-77.7)$ \\
\hline Knoxville, Tennessee & 266 & 75.7 & 3.4 & $(69.1-82.3)$ \\
\hline Laconia, New Hampshire & 236 & 55.5 & 4.0 & $(47.6-63.4)$ \\
\hline Lafayette, Louisiana & 167 & 60.5 & 5.2 & $(50.4-70.6)$ \\
\hline Las Cruces, New Mexico & 203 & 61.1 & 3.9 & $(53.5-68.7)$ \\
\hline $\begin{array}{l}\text { Las Vegas-Henderson-Paradise, } \\
\text { Nevada }\end{array}$ & 511 & 50.6 & 2.8 & $(45.0-56.1)$ \\
\hline Lewiston-Auburn, Maine & 187 & 68.7 & 4.0 & $(60.8-76.6)$ \\
\hline Lexington-Fayette, Kentucky & 117 & 61.9 & 5.7 & $(50.7-73.1)$ \\
\hline Lincoln, Nebraska & 367 & 69.4 & 3.1 & $(63.4-75.4)$ \\
\hline
\end{tabular}

See table footnotes on page 33 .
TABLE 11. (Continued) Estimated prevalence of adults aged $\geq 65$ years who received an influenza vaccination during the preceding 12 months, by metropolitan and micropolitan statistical area Behavioral Risk Factor Surveillance System, United States, 2012

\begin{tabular}{|c|c|c|c|c|}
\hline MMSA(s) & $\begin{array}{l}\text { Sample } \\
\text { size }\end{array}$ & $\%$ & SE & $95 \% \mathrm{Cl}$ \\
\hline $\begin{array}{l}\text { Little Rock-North Little Rock-Conway, } \\
\text { Arkansas }\end{array}$ & 371 & 59.2 & 3.2 & $(53.0-65.5)$ \\
\hline Logan, Utah-Idaho & 128 & 52.8 & 5.2 & $(42.6-63.0)$ \\
\hline $\begin{array}{l}\text { Los Angeles-Long Beach-Glendale, } \\
\text { California* }\end{array}$ & 744 & 55.6 & 2.8 & $(50.1-61.2)$ \\
\hline $\begin{array}{l}\text { Louisville/Jefferson County, } \\
\text { Kentucky-Indiana }\end{array}$ & 644 & 62.2 & 3.1 & $(56.2-68.2)$ \\
\hline Lumberton, North Carolina & 155 & 47.4 & 5.9 & $(35.8-59.0)$ \\
\hline Manchester-Nashua, New Hampshire & 534 & 56.8 & 2.6 & $(51.8-61.8)$ \\
\hline McAllen-Edinburg-Mission, Texas & 189 & 63.8 & 4.3 & $(55.4-72.2)$ \\
\hline $\begin{array}{l}\text { Memphis, } \\
\text { Tennessee-Mississippi-Arkansas }\end{array}$ & 414 & 70.5 & 3.4 & $(63.9-77.1)$ \\
\hline $\begin{array}{l}\text { Miami-Fort Lauderdale-West Palm } \\
\text { Beach, Florida }\end{array}$ & 589 & 53.0 & 3.2 & $(46.7-59.4)$ \\
\hline $\begin{array}{l}\text { Milwaukee-Waukesha-West Allis, } \\
\text { Wisconsin }\end{array}$ & 315 & 53.4 & 4.1 & $(45.4-61.3)$ \\
\hline $\begin{array}{l}\text { Minneapolis-St. Paul-Bloomington, } \\
\text { Minnesota-Wisconsin }\end{array}$ & 2,004 & 67.6 & 1.5 & $(64.7-70.5)$ \\
\hline Missoula, Montana & 221 & 56.5 & 3.9 & $(48.8-64.2)$ \\
\hline Mobile, Alabama & 304 & 65.8 & 3.7 & $(58.6-73.0)$ \\
\hline Montgomery, Alabama & 177 & 62.4 & 4.5 & $(53.6-71.2)$ \\
\hline $\begin{array}{l}\text { Montgomery County-Bucks County- } \\
\text { Chester County, Pennsylvania* }\end{array}$ & 346 & 63.7 & 3.1 & $(57.6-69.7)$ \\
\hline $\begin{array}{l}\text { Myrtle Beach-Conway-North Myrtle } \\
\text { Beach, South Carolina-North Carolina }\end{array}$ & 353 & 62.1 & 3.1 & $(56.1-68.1)$ \\
\hline $\begin{array}{l}\text { Nashville-Davidson-Murfreesboro- } \\
\text { Franklin, Tennessee }\end{array}$ & 320 & 71.9 & 3.1 & $(65.7-78.0)$ \\
\hline $\begin{array}{l}\text { Nassau County-Suffolk County, } \\
\text { New York* }\end{array}$ & 258 & 59.2 & 3.9 & $(51.5-66.9)$ \\
\hline Newark, New Jersey-Pennsylvania* & 1,950 & 59.4 & 2.0 & $(55.4-63.4)$ \\
\hline New Haven-Milford, Connecticut & 626 & 57.3 & 2.7 & $(52.0-62.6)$ \\
\hline New Orleans-Metairie, Louisiana & 394 & 66.4 & 3.0 & $(60.6-72.2)$ \\
\hline $\begin{array}{l}\text { New York-Jersey City-White Plains, } \\
\text { New York-New Jersey* }\end{array}$ & 1,704 & 56.0 & 2.4 & $(51.2-60.7)$ \\
\hline Norfolk, Nebraska & 196 & 53.9 & 4.5 & $(45.1-62.7)$ \\
\hline North Platte, Nebraska & 262 & 50.0 & 4.3 & $(41.6-58.3)$ \\
\hline Norwich-New London, Connecticut & 355 & 62.3 & 3.8 & $(54.8-69.7)$ \\
\hline $\begin{array}{l}\text { Oakland-Hayward-Berkeley, } \\
\text { California* }\end{array}$ & 260 & 59.2 & 5.1 & $(49.2-69.2)$ \\
\hline Ocean City, New Jersey & 236 & 63.2 & 3.8 & $(55.8-70.7)$ \\
\hline Ogden-Clearfield, Utah & 632 & 55.4 & 2.2 & $(51.0-59.8)$ \\
\hline Oklahoma City, Oklahoma & 625 & 69.5 & 2.2 & $(65.2-73.9)$ \\
\hline Olympia-Tumwater, Washington & 158 & 53.1 & 5.0 & $(43.3-62.9)$ \\
\hline Omaha-Council Bluffs, Nebraska-lowa & 1,515 & 68.2 & 1.6 & $(65.1-71.4)$ \\
\hline Orlando-Kissimmee-Sanford, Florida & 210 & 54.6 & 5.1 & $(44.5-64.6)$ \\
\hline Philadelphia, Pennsylvania* & 680 & 54.0 & 2.5 & $(49.1-59.0)$ \\
\hline Phoenix-Mesa-Scottsdale, Arizona & 836 & 54.1 & 2.2 & $(49.7-58.4)$ \\
\hline Pittsburgh, Pennsylvania & 1,136 & 60.8 & 1.8 & $(57.3-64.3)$ \\
\hline Ponce, Puerto Rico & 155 & 21.2 & 3.9 & $(13.7-28.8)$ \\
\hline Portland-South Portland, Maine & 1,052 & 60.3 & 1.8 & $(56.8-63.9)$ \\
\hline $\begin{array}{l}\text { Portland-Vancouver-Hillsboro, } \\
\text { Oregon-Washington }\end{array}$ & 914 & 59.7 & 2.0 & $(55.8-63.6)$ \\
\hline $\begin{array}{l}\text { Providence-Warwick, Rhode } \\
\text { Island-Massachusetts }\end{array}$ & 2,468 & 58.7 & 1.5 & $(55.8-61.5)$ \\
\hline Provo-Orem, Utah & 387 & 51.1 & 2.9 & $(45.5-56.8)$ \\
\hline Raleigh, North Carolina & 183 & 74.3 & 4.1 & $(66.3-82.2)$ \\
\hline Rapid City, South Dakota & 296 & 64.4 & 3.9 & $(56.7-72.1)$ \\
\hline Reno, Nevada & 498 & 52.6 & 2.8 & $(47.2-58.0)$ \\
\hline Richmond, Virginia & 311 & 61.1 & 3.1 & $(55.0-67.2)$ \\
\hline
\end{tabular}

See table footnotes on page 33. 
TABLE 11. (Continued) Estimated prevalence of adults aged $\geq 65$ years who received an influenza vaccination during the preceding 12 months, by metropolitan and micropolitan statistical area Behavioral Risk Factor Surveillance System, United States, 2012

\begin{tabular}{|c|c|c|c|c|}
\hline MMSA(s) & $\begin{array}{l}\text { Sample } \\
\text { size }\end{array}$ & $\%$ & SE & $95 \% \mathrm{Cl}$ \\
\hline $\begin{array}{l}\text { Riverside-San Bernardino-Ontario, } \\
\text { California }\end{array}$ & 395 & 56.7 & 3.6 & $(49.7-63.7)$ \\
\hline $\begin{array}{l}\text { Rockingham County-Strafford County, } \\
\text { New Hampshire* }\end{array}$ & 536 & 63.8 & 2.5 & $(58.9-68.6)$ \\
\hline Rutland, Vermont & 194 & 59.7 & 4.2 & $(51.4-68.0)$ \\
\hline $\begin{array}{l}\text { Sacramento-Roseville-Arden-Arcade, } \\
\text { California }\end{array}$ & 260 & 61.6 & 4.1 & $(53.5-69.6)$ \\
\hline St. Louis, Missouri-Illinois & 659 & 63.6 & 2.8 & $(58.1-69.1)$ \\
\hline Salisbury, Maryland-Delaware & 871 & 63.1 & 2.0 & $(59.3-67.0)$ \\
\hline Salt Lake City, Utah & 1,168 & 58.8 & 1.8 & $(55.2-62.3)$ \\
\hline San Antonio-New Braunfels, Texas & 237 & 63.6 & 4.2 & $(55.5-71.8)$ \\
\hline San Diego-Carlsbad, California & 300 & 55.1 & 3.9 & $(47.5-62.7)$ \\
\hline $\begin{array}{l}\text { San Francisco-Redwood City-South } \\
\text { San Francisco, California* }\end{array}$ & 156 & 64.6 & 6.1 & $(52.6-76.7)$ \\
\hline $\begin{array}{l}\text { San Jose-Sunnyvale-Santa Clara, } \\
\text { California }\end{array}$ & 162 & 54.1 & 6.0 & $(42.3-65.9)$ \\
\hline San Juan-Carolina-Caguas, Puerto Rico & 1,141 & 27.9 & 1.6 & $(24.8-30.9)$ \\
\hline Santa Fe, New Mexico & 195 & 62.4 & 4.0 & $(54.6-70.2)$ \\
\hline Sayre, Pennsylvania & 690 & 52.2 & 2.2 & $(47.9-56.4)$ \\
\hline Scottsbluff, Nebraska & 269 & 52.4 & 4.1 & $(44.3-60.4)$ \\
\hline $\begin{array}{l}\text { Scranton-Wilkes-Barre-Hazleton, } \\
\text { Pennsylvania }\end{array}$ & 264 & 59.7 & 3.7 & $(52.4-67.0)$ \\
\hline Seattle-Bellevue-Everett, Washington* & 1,399 & 60.7 & 1.7 & $(57.4-64.1)$ \\
\hline Shreveport-Bossier City, Louisiana & 192 & 60.2 & 4.4 & $(51.5-68.9)$ \\
\hline $\begin{array}{l}\text { Silver Spring-Frederick-Rockville, } \\
\text { Maryland* }\end{array}$ & 688 & 62.8 & 2.7 & $(57.5-68.2)$ \\
\hline $\begin{array}{l}\text { Sioux City, lowa-Nebraska- } \\
\text { South Dakota }\end{array}$ & 448 & 56.2 & 4.7 & $(47.0-65.4)$ \\
\hline Sioux Falls, South Dakota & 301 & 68.3 & 3.8 & $(60.8-75.8)$ \\
\hline Spartanburg, South Carolina & 237 & 56.0 & 4.7 & $(46.8-65.3)$ \\
\hline Spokane-Spokane Valley, Washington & 374 & 59.1 & 3.0 & $(53.3-65.0)$ \\
\hline Springfield, Massachusetts & 633 & 64.1 & 3.1 & $(58.1-70.1)$ \\
\hline
\end{tabular}

TABLE 11. (Continued) Estimated prevalence of adults aged $\geq 65$ years who received an influenza vaccination during the preceding 12 months, by metropolitan and micropolitan statistical area Behavioral Risk Factor Surveillance System, United States, 2012

\begin{tabular}{|c|c|c|c|c|}
\hline MMSA(s) & $\begin{array}{l}\text { Sample } \\
\text { size }\end{array}$ & $\%$ & SE & $95 \% \mathrm{Cl}$ \\
\hline Tacoma-Lakewood, Washington* & 314 & 60.6 & 3.4 & $(54.0-67.2)$ \\
\hline $\begin{array}{l}\text { Tampa-St. Petersburg-Clearwater, } \\
\text { Florida }\end{array}$ & 304 & 56.0 & 3.7 & $(48.8-63.3)$ \\
\hline Toledo, Ohio & 298 & 61.9 & 3.5 & $(55.0-68.8)$ \\
\hline Topeka, Kansas & 336 & 65.9 & 3.0 & $(60.1-71.8)$ \\
\hline Torrington, Connecticut & 210 & 62.5 & 4.1 & $(54.5-70.4)$ \\
\hline Trenton, New Jersey & 152 & 62.7 & 5.1 & $(52.8-72.7)$ \\
\hline Tucson, Arizona & 368 & 50.6 & 3.2 & $(44.4-56.8)$ \\
\hline Tulsa, Oklahoma & 537 & 66.4 & 2.4 & $(61.7-71.0)$ \\
\hline Tuscaloosa, Alabama & 198 & 63.4 & 4.2 & $(55.1-71.7)$ \\
\hline Urban Honolulu, Hawaii & 996 & 65.4 & 2.1 & $(61.3-69.5)$ \\
\hline Vineland-Bridgeton, New Jersey & 159 & 61.7 & 5.0 & $(52.0-71.5)$ \\
\hline $\begin{array}{l}\text { Virginia Beach-Norfolk-Newport News, } \\
\text { Virginia-North Carolina }\end{array}$ & 445 & 59.0 & 3.0 & $(53.1-64.8)$ \\
\hline $\begin{array}{l}\text { Warren-Troy-Farmington Hills, } \\
\text { Michigan* }\end{array}$ & 670 & 57.4 & 2.3 & $(52.9-61.9)$ \\
\hline $\begin{array}{l}\text { Washington-Arlington-Alexandria, } \\
\text { District of Columbia-Virginia- } \\
\text { Maryland-West Virginia* }\end{array}$ & 2,255 & 58.7 & 1.8 & $(55.3-62.2)$ \\
\hline Wichita, Kansas & 743 & 68.2 & 2.0 & $(64.3-72.0)$ \\
\hline $\begin{array}{l}\text { Wilmington, Delaware-Maryland- } \\
\text { New Jersey* }\end{array}$ & 892 & 62.6 & 2.3 & $(58.1-67.1)$ \\
\hline Winston-Salem, North Carolina & 260 & 64.8 & 3.5 & $(57.9-71.7)$ \\
\hline Worcester, Massachusetts-Connecticut & 783 & 63.8 & 2.5 & $(58.8-68.7)$ \\
\hline Yakima, Washington & 169 & 60.4 & 4.5 & $(51.5-69.3)$ \\
\hline $\begin{array}{l}\text { Youngstown-Warren-Boardman, } \\
\text { Ohio-Pennsylvania }\end{array}$ & 337 & 55.2 & 4.2 & $(46.9-63.5)$ \\
\hline Median & & 60.5 & & \\
\hline Range & & $20.8-77.8$ & & \\
\hline
\end{tabular}

Abbreviations: $\mathrm{Cl}=$ confidence interval; $\mathrm{MMSA}=$ metropolitan and micropolitan statistical area; $\mathrm{SE}=$ standard error.

* Metropolitan division. 
TABLE 12. Estimated prevalence of adults aged $\geq 65$ years who received an influenza vaccination during the preceding 12 months, by county - Behavioral Risk Factor Surveillance System, United States, 2012

\begin{tabular}{|c|c|c|c|c|}
\hline County & $\begin{array}{l}\text { Sample } \\
\text { size }\end{array}$ & $\%$ & SE & $95 \% \mathrm{Cl}$ \\
\hline Jefferson County, Alabama & 271 & 66.6 & 3.6 & $(59.5-73.6)$ \\
\hline Madison County, Alabama & 130 & 55.5 & 5.8 & $(44.2-66.8)$ \\
\hline Mobile County, Alabama & 304 & 65.8 & 3.7 & $(58.6-73.0)$ \\
\hline Anchorage Municipality, Alaska & 132 & 56.1 & 4.9 & $(46.5-65.8)$ \\
\hline Fairbanks North Star Borough, Alaska & 100 & 44.0 & 6.0 & $(32.2-55.7)$ \\
\hline Matanuska-Susitna Borough, Alaska & 107 & 48.5 & 5.6 & $(37.5-59.6)$ \\
\hline Maricopa County, Arizona & 614 & 53.6 & 2.4 & $(48.9-58.3)$ \\
\hline Pima County, Arizona & 368 & 50.6 & 3.2 & $(44.4-56.9)$ \\
\hline Pulaski County, Arkansas & 245 & 60.8 & 4.0 & $(52.9-68.7)$ \\
\hline Alameda County, California & 159 & 54.3 & 7.0 & $(40.6-68.0)$ \\
\hline Los Angeles County, California & 744 & 55.6 & 2.8 & $(50.1-61.2)$ \\
\hline Orange County, California & 274 & 65.0 & 4.6 & $(56.0-74.0)$ \\
\hline Riverside County, California & 239 & 56.9 & 4.9 & $(47.3-66.6)$ \\
\hline Sacramento County, California & 150 & 63.8 & 5.3 & $(53.4-74.1)$ \\
\hline San Bernardino County, California & 156 & 56.5 & 5.2 & $(46.2-66.7)$ \\
\hline San Diego County, California & 300 & 55.1 & 3.9 & $(47.5-62.7)$ \\
\hline Santa Clara County, California & 152 & 53.2 & 6.2 & $(41.1-65.3)$ \\
\hline Adams County, Colorado & 204 & 69.2 & 4.0 & $(61.4-77.0)$ \\
\hline Arapahoe County, Colorado & 204 & 69.4 & 3.6 & $(62.3-76.5)$ \\
\hline Boulder County, Colorado & 126 & 72.2 & 4.4 & $(63.5-80.9)$ \\
\hline Denver County, Colorado & 213 & 67.9 & 3.7 & $(60.7-75.1)$ \\
\hline Douglas County, Colorado & 101 & 67.9 & 5.2 & $(57.8-78.1)$ \\
\hline El Paso County, Colorado & 230 & 62.3 & 3.6 & $(55.2-69.4)$ \\
\hline Jefferson County, Colorado & 309 & 67.0 & 3.3 & $(60.6-73.4)$ \\
\hline Larimer County, Colorado & 170 & 70.3 & 3.8 & $(62.8-77.7)$ \\
\hline Weld County, Colorado & 123 & 59.6 & 5.3 & $(49.3-69.9)$ \\
\hline Fairfield County, Connecticut & 601 & 57.1 & 2.7 & $(51.8-62.4)$ \\
\hline Hartford County, Connecticut & 597 & 60.8 & 2.7 & $(55.6-66.1)$ \\
\hline Litchfield County, Connecticut & 210 & 62.5 & 4.1 & $(54.5-70.4)$ \\
\hline New Haven County, Connecticut & 626 & 57.3 & 2.7 & $(52.0-62.6)$ \\
\hline New London County, Connecticut & 355 & 62.3 & 3.8 & $(54.8-69.7)$ \\
\hline Kent County, Delaware & 484 & 59.3 & 2.8 & $(53.7-64.8)$ \\
\hline New Castle County, Delaware & 617 & 63.6 & 2.5 & $(58.7-68.5)$ \\
\hline Sussex County, Delaware & 581 & 64.1 & 2.3 & $(59.6-68.6)$ \\
\hline $\begin{array}{l}\text { District of Columbia, } \\
\text { District of Columbia }\end{array}$ & 1,202 & 57.1 & 1.9 & $(53.3-60.9)$ \\
\hline Broward County, Florida & 176 & 51.6 & 5.7 & $(40.5-62.7)$ \\
\hline Miami-Dade County, Florida & 257 & 49.8 & 5.9 & $(38.2-61.3)$ \\
\hline Hawaii County, Hawaii & 381 & 53.1 & 3.6 & $(46.1-60.1)$ \\
\hline Honolulu County, Hawaii & 996 & 65.4 & 2.1 & $(61.3-69.5)$ \\
\hline Kauai County, Hawaii & 225 & 62.8 & 3.9 & $(55.1-70.6)$ \\
\hline Maui County, Hawaii & 363 & 57.7 & 3.8 & $(50.2-65.3)$ \\
\hline Ada County, Idaho & 248 & 55.5 & 4.2 & $(47.2-63.9)$ \\
\hline Canyon County, Idaho & 189 & 45.8 & 5.4 & $(35.3-56.3)$ \\
\hline Cook County, Illinois & 453 & 48.6 & 2.9 & $(42.9-54.3)$ \\
\hline Lake County, Indiana & 312 & 45.5 & 4.6 & $(36.5-54.5)$ \\
\hline Marion County, Indiana & 378 & 63.4 & 3.2 & $(57.1-69.7)$ \\
\hline Polk County, lowa & 232 & 73.5 & 3.3 & $(67.0-80.0)$ \\
\hline Johnson County, Kansas & 660 & 70.1 & 2.0 & $(66.1-74.1)$ \\
\hline Sedgwick County, Kansas & 537 & 67.9 & 2.4 & $(63.2-72.5)$ \\
\hline Shawnee County, Kansas & 240 & 68.2 & 3.6 & $(61.1-75.2)$ \\
\hline Wyandotte County, Kansas & 321 & 59.2 & 3.7 & $(52.0-66.3)$ \\
\hline Jefferson County, Kentucky & 530 & 65.4 & 3.7 & $(58.1-72.7)$ \\
\hline East Baton Rouge Parish, Louisiana & 181 & 59.7 & 4.5 & $(50.9-68.4)$ \\
\hline Androscoggin County, Maine & 187 & 68.7 & 4.0 & $(60.8-76.6)$ \\
\hline Aroostook County, Maine & 172 & 45.4 & 4.4 & $(36.7-54.1)$ \\
\hline Cumberland County, Maine & 516 & 60.5 & 2.6 & $(55.3-65.6)$ \\
\hline Kennebec County, Maine & 221 & 68.8 & 3.8 & $(61.4-76.2)$ \\
\hline Penobscot County, Maine & 253 & 59.0 & 3.6 & $(51.9-66.2)$ \\
\hline York County, Maine & 403 & 61.2 & 2.8 & $(55.7-66.7)$ \\
\hline
\end{tabular}

See table footnotes on page 35.
TABLE 12. (Continued) Estimated prevalence of adults aged $\geq 65$ years who received an influenza vaccination during the preceding 12 months, by county - Behavioral Risk Factor Surveillance System, United States, 2012

\begin{tabular}{|c|c|c|c|c|}
\hline County & $\begin{array}{l}\text { Sample } \\
\text { size }\end{array}$ & $\%$ & SE & $95 \% \mathrm{Cl}$ \\
\hline Anne Arundel County, Maryland & 253 & 65.0 & 3.8 & $(57.5-72.5)$ \\
\hline Baltimore County, Maryland & 454 & 63.9 & 2.9 & $(58.3-69.6)$ \\
\hline Charles County, Maryland & 118 & 60.5 & 6.2 & $(48.4-72.6)$ \\
\hline Frederick County, Maryland & 242 & 55.9 & 5.8 & $(44.5-67.3)$ \\
\hline Montgomery County, Maryland & 446 & 64.6 & 3.1 & $(58.5-70.7)$ \\
\hline Prince George's County, Maryland & 305 & 54.7 & 4.2 & $(46.5-63.0)$ \\
\hline Washington County, Maryland & 199 & 66.4 & 4.9 & $(56.8-75.9)$ \\
\hline Baltimore city, Maryland & 223 & 66.1 & 4.4 & $(57.5-74.6)$ \\
\hline Barnstable County, Massachusetts & 252 & 58.9 & 4.0 & $(51.0-66.8)$ \\
\hline Bristol County, Massachusetts & 836 & 60.6 & 3.1 & $(54.6-66.6)$ \\
\hline Essex County, Massachusetts & 799 & 64.3 & 2.6 & $(59.1-69.4)$ \\
\hline Hampden County, Massachusetts & 539 & 62.2 & 3.5 & -69.2) \\
\hline Middlesex County, Massachusetts & 1,015 & 68.3 & 2.0 & $(64.4-72.3)$ \\
\hline Norfolk County, Massachusetts & 490 & 66.8 & 3.2 & $(60.7-73.0)$ \\
\hline Plymouth County, Massachusetts & 551 & 57.0 & 3.5 & $(50.2-63.7)$ \\
\hline Suffolk County, Massachusetts & 548 & 60.5 & 3.4 & $(53.9-67.1)$ \\
\hline Worcester County, Massachusetts & 710 & 63.1 & 2.7 & $(57.8-68.5)$ \\
\hline Kent County, Michigan & 153 & 61.7 & 5.0 & $(51.9-71.5)$ \\
\hline Macomb County, Michigan & 191 & 60.6 & 4.0 & $(52.7-68.4)$ \\
\hline Oakland County, Michigan & 371 & 59.5 & 3.0 & $(53.6-65.5)$ \\
\hline Wayne County, Michigan & 750 & 46.5 & 3.0 & $(40.7-52.3)$ \\
\hline Anoka County, Minnesota & 116 & 62.7 & 5.8 & $(51.4-74.0)$ \\
\hline Dakota County, Minnesota & 145 & 74.7 & 4.0 & $(66.8-82.6)$ \\
\hline Hennepin County, Minnesota & 829 & 66.3 & 2.4 & $(61.6-70.9)$ \\
\hline Ramsey County, Minnesota & 583 & 71.2 & 2.6 & $(66.0-76.4)$ \\
\hline Jackson County, Missouri & 303 & 62.3 & 4.7 & $(53.1-71.5)$ \\
\hline St. Louis County, Missouri & 298 & 72.5 & 4.2 & $(64.3-80.7)$ \\
\hline Cascade County, Montana & 256 & 55.8 & 3.5 & $(48.8-62.7)$ \\
\hline Flathead County, Montana & 177 & 58.2 & 4.3 & $(49.8-66.5)$ \\
\hline Hill County, Montana & 179 & 58.7 & 5.5 & $(48.0-69.5)$ \\
\hline Lake County, Montana & 343 & 55.5 & 3.5 & $(48.6-62.4)$ \\
\hline Missoula County, Montana & 221 & 56.5 & 3.9 & $(48.8-64.2)$ \\
\hline Yellowstone County, Montana & 214 & 67.3 & 3.5 & $(60.4-74.3)$ \\
\hline Dakota County, Nebraska & 283 & 51.4 & 3.5 & $(44.6-58.3)$ \\
\hline Douglas County, Nebraska & 972 & 68.1 & 2.0 & $(64.3-72.0)$ \\
\hline Hall County, Nebraska & 190 & 60.1 & 5.3 & $(49.8-70.5)$ \\
\hline Lancaster County, Nebraska & 289 & 69.8 & 3.3 & $(63.4-76.3)$ \\
\hline Lincoln County, Nebraska & 252 & 51.6 & 4.4 & $(42.9-60.3)$ \\
\hline Sarpy County, Nebraska & 304 & 62.1 & 3.5 & $(55.3-68.9)$ \\
\hline Scotts Bluff County, Nebraska & 248 & 54.8 & 4.3 & $(46.3-63.3)$ \\
\hline Clark County, Nevada & 511 & 50.6 & 2.8 & $(45.0-56.1)$ \\
\hline Washoe County, Nevada & 490 & 52.3 & 2.8 & $(46.8-57.8)$ \\
\hline Belknap County, New Hampshire & 236 & 55.5 & 4.0 & $(47.6-63.4)$ \\
\hline Carroll County, New Hampshire & 246 & 65.3 & 3.5 & $(58.3-72.2)$ \\
\hline Cheshire County, New Hampshire & 212 & 59.7 & 4.1 & $(51.6-67.8)$ \\
\hline Coos County, New Hampshire & 212 & 55.6 & 4.0 & $(47.8-63.5)$ \\
\hline Grafton County, New Hampshire & 213 & 60.1 & 3.8 & $(52.6-67.6)$ \\
\hline Hillsborough County, New Hampshire & 534 & 56.8 & 2.6 & $(51.8-61.8)$ \\
\hline Merrimack County, New Hampshire & 231 & 51.3 & 3.9 & $(43.6-59.0)$ \\
\hline Rockingham County, New Hampshire & 326 & 62.8 & 3.1 & $(56.8-68.8)$ \\
\hline Strafford County, New Hampshire & 210 & 66.5 & 3.9 & $(58.8-74.1)$ \\
\hline Atlantic County, New Jersey & 341 & 58.0 & 3.5 & $(51.1-64.9)$ \\
\hline Bergen County, New Jersey & 220 & 58.3 & 4.5 & $(49.5-67.1)$ \\
\hline Burlington County, New Jersey & 201 & 66.9 & 4.2 & $(58.8-75.1)$ \\
\hline Camden County, New Jersey & 212 & 61.6 & 4.1 & $(53.4-69.7)$ \\
\hline Cape May County, New Jersey & 236 & 63.2 & 3.8 & $(55.8-70.7)$ \\
\hline Cumberland County, New Jersey & 159 & 61.7 & 5.0 & $(52.0-71.5)$ \\
\hline Essex County, New Jersey & 291 & 56.3 & 3.8 & $(48.7-63.8)$ \\
\hline Gloucester County, New Jersey & 137 & 70.5 & 5.1 & $(60.5-80.5)$ \\
\hline Hudson County, New Jersey & 259 & 49.4 & 4.2 & $(41.1-57.7)$ \\
\hline
\end{tabular}

See table footnotes on page 35 . 
TABLE 12. (Continued) Estimated prevalence of adults aged $\geq 65$ years who received an influenza vaccination during the preceding 12 months, by county - Behavioral Risk Factor Surveillance System, United States, 2012

\begin{tabular}{|c|c|c|c|c|}
\hline County & $\begin{array}{l}\text { Sample } \\
\text { size }\end{array}$ & $\%$ & SE & $95 \% \mathrm{Cl}$ \\
\hline Hunterdon County, New Jersey & 155 & 58.3 & 5.7 & $(47.2-69.4)$ \\
\hline Mercer County, New Jersey & 152 & 62.7 & 5.1 & $(52.8-72.7)$ \\
\hline Middlesex County, New Jersey & 199 & 67.7 & 4.2 & $(59.4-76.0)$ \\
\hline Monmouth County, New Jersey & 212 & 63.4 & 4.1 & $(55.3-71.5)$ \\
\hline Morris County, New Jersey & 240 & 74.9 & 3.4 & $(68.1-81.6)$ \\
\hline Ocean County, New Jersey & 244 & 62.6 & 3.6 & $(55.5-69.7)$ \\
\hline Passaic County, New Jersey & 140 & 56.8 & 5.0 & $(46.9-66.7)$ \\
\hline Salem County, New Jersey & 184 & 56.2 & 4.3 & $(47.8-64.5)$ \\
\hline Somerset County, New Jersey & 189 & 64.5 & 5.2 & $(54.4-74.6)$ \\
\hline Sussex County, New Jersey & 163 & 63.4 & 4.9 & $(53.9-72.9)$ \\
\hline Union County, New Jersey & 158 & 48.6 & 5.6 & $(37.7-59.5)$ \\
\hline Warren County, New Jersey & 167 & 66.1 & 4.1 & $(58.0-74.2)$ \\
\hline Bernalillo County, New Mexico & 505 & 59.6 & 2.6 & $(54.6-64.6)$ \\
\hline Dona Ana County, New Mexico & 203 & 61.1 & 3.9 & $(53.5-68.7)$ \\
\hline Sandoval County, New Mexico & 189 & 62.4 & 4.2 & $(54.2-70.7)$ \\
\hline San Juan County, New Mexico & 172 & 50.7 & 4.6 & $(41.7-59.6)$ \\
\hline Santa Fe County, New Mexico & 195 & 62.4 & 4.0 & $(54.6-70.2)$ \\
\hline Kings County, New York & 60 & 37.3 & 7.9 & $(21.9-52.7)$ \\
\hline Guilford County, North Carolina & 146 & 72.4 & 4.5 & $(63.5-81.2)$ \\
\hline Mecklenburg County, North Carolina & 131 & 70.0 & 4.8 & $(60.5-79.5)$ \\
\hline Robeson County, North Carolina & 155 & 47.5 & 5.9 & $(35.9-59.1)$ \\
\hline Wake County, North Carolina & 118 & 73.5 & 5.0 & $(63.6-83.3)$ \\
\hline Burleigh County, North Dakota & 168 & 57.0 & 4.9 & $(47.4-66.6)$ \\
\hline Cass County, North Dakota & 236 & 63.2 & 4.0 & $(55.4-70.9)$ \\
\hline Cuyahoga County, Ohio & 221 & 59.7 & 4.2 & $(51.5-67.8)$ \\
\hline Franklin County, Ohio & 177 & 63.9 & 4.6 & $(55.0-72.9)$ \\
\hline Hamilton County, Ohio & 202 & 67.1 & 4.1 & $(59.0-75.2)$ \\
\hline Lorain County, Ohio & 222 & 66.6 & 4.4 & $(58.1-75.1)$ \\
\hline Lucas County, Ohio & 198 & 64.3 & 4.3 & $(56.0-72.7)$ \\
\hline Mahoning County, Ohio & 227 & 45.8 & 4.6 & $(36.7-54.8)$ \\
\hline Montgomery County, Ohio & 228 & 54.5 & 4.1 & $(46.5-62.5)$ \\
\hline Stark County, Ohio & 199 & 60.0 & 4.4 & $(51.4-68.7)$ \\
\hline Summit County, Ohio & 197 & 62.8 & 4.1 & $(54.8-70.7)$ \\
\hline Oklahoma County, Oklahoma & 285 & 68.9 & 3.2 & $(62.7-75.2)$ \\
\hline Tulsa County, Oklahoma & 365 & 66.9 & 2.9 & $(61.3-72.5)$ \\
\hline Clackamas County, Oregon & 164 & 53.5 & 4.7 & $(44.3-62.6)$ \\
\hline Lane County, Oregon & 176 & 49.9 & 4.5 & $(41.2-58.7)$ \\
\hline Multnomah County, Oregon & 234 & 62.2 & 3.9 & $(54.6-69.8)$ \\
\hline Washington County, Oregon & 177 & 54.8 & 4.8 & $(45.4-64.2)$ \\
\hline Allegheny County, Pennsylvania & 662 & 60.8 & 2.3 & $(56.4-65.2)$ \\
\hline Bradford County, Pennsylvania & 690 & 52.2 & 2.1 & $(48.0-56.4)$ \\
\hline Montgomery County, Pennsylvania & 134 & 67.9 & 4.7 & $(58.6-77.2)$ \\
\hline Philadelphia County, Pennsylvania & 566 & 54.3 & 2.8 & $(48.9-59.7)$ \\
\hline Pike County, Pennsylvania & 754 & 54.9 & 1.9 & $(51.1-58.6)$ \\
\hline Kent County, Rhode Island & 227 & 56.2 & 3.9 & $(48.5-63.8)$ \\
\hline Providence County, Rhode Island & 940 & 57.1 & 2.0 & $(53.1-61.1)$ \\
\hline Washington County, Rhode Island & 241 & 62.6 & 3.5 & $(55.8-69.5)$ \\
\hline
\end{tabular}

TABLE 12. (Continued) Estimated prevalence of adults aged $\geq 65$ years who received an influenza vaccination during the preceding 12 months, by county - Behavioral Risk Factor Surveillance System, United States, 2012

\begin{tabular}{|c|c|c|c|c|}
\hline County & $\begin{array}{l}\text { Sample } \\
\text { size }\end{array}$ & $\%$ & SE & $95 \% \mathrm{Cl}$ \\
\hline Aiken County, South Carolina & 201 & 58.8 & 4.3 & $(50.4-67.3)$ \\
\hline Beaufort County, South Carolina & 429 & 60.4 & 2.8 & $(55.0-65.8)$ \\
\hline Charleston County, South Carolina & 337 & 66.9 & 3.6 & $(59.8-73.9)$ \\
\hline Greenville County, South Carolina & 261 & 63.0 & 4.1 & $(55.0-71.0)$ \\
\hline Horry County, South Carolina & 274 & 58.1 & 3.4 & $(51.4-64.8)$ \\
\hline Richland County, South Carolina & 280 & 62.4 & 4.4 & $(53.9-71.0)$ \\
\hline Spartanburg County, South Carolina & 219 & 53.8 & 5.0 & $(44.0-63.6)$ \\
\hline Lincoln County, South Dakota & 129 & 75.1 & 5.3 & $(64.8-85.5)$ \\
\hline Minnehaha County, South Dakota & 153 & 65.7 & 4.5 & $(56.8-74.6)$ \\
\hline Pennington County, South Dakota & 147 & 66.5 & 4.5 & $(57.7-75.4)$ \\
\hline Davidson County, Tennessee & 147 & 71.0 & 4.5 & $(62.1-79.9)$ \\
\hline Shelby County, Tennessee & 128 & 69.3 & 4.8 & $(60.0-78.7)$ \\
\hline Bexar County, Texas & 167 & 61.2 & 5.1 & $(51.2-71.2)$ \\
\hline Dallas County, Texas & 126 & 52.3 & 5.5 & $(41.4-63.1)$ \\
\hline El Paso County, Texas & 195 & 50.9 & 4.9 & $(41.4-60.4)$ \\
\hline Harris County, Texas & 128 & 59.3 & 5.2 & $(49.1-69.5)$ \\
\hline Hidalgo County, Texas & 189 & 63.8 & 4.3 & $(55.4-72.2)$ \\
\hline Tarrant County, Texas & 152 & 64.9 & 4.9 & $(55.3-74.5)$ \\
\hline Travis County, Texas & 296 & 59.6 & 3.7 & $(52.5-66.8)$ \\
\hline Davis County, Utah & 266 & 51.4 & 3.4 & $(44.8-58.1)$ \\
\hline Salt Lake County, Utah & 1,018 & 59.1 & 1.9 & $(55.4-62.7)$ \\
\hline Tooele County, Utah & 150 & 52.8 & 6.5 & $(40.0-65.6)$ \\
\hline Utah County, Utah & 360 & 50.6 & 3.0 & $(44.8-56.4)$ \\
\hline Wasatch County, Utah & 154 & 58.6 & 4.4 & $(50.0-67.2)$ \\
\hline Weber County, Utah & 285 & 61.6 & 3.4 & $(54.9-68.3)$ \\
\hline Chittenden County, Vermont & 238 & 66.7 & 3.6 & $(59.7-73.7)$ \\
\hline Rutland County, Vermont & 194 & 59.7 & 4.2 & $(51.4-68.0)$ \\
\hline Washington County, Vermont & 135 & 68.8 & 4.6 & $(59.8-77.7)$ \\
\hline Windsor County, Vermont & 206 & 65.2 & 3.7 & $(57.9-72.5)$ \\
\hline Fairfax County, Virginia & 163 & 61.9 & 5.0 & $(52.2-71.6)$ \\
\hline Clark County, Washington & 238 & 66.7 & 3.6 & $(59.6-73.9)$ \\
\hline King County, Washington & 1,106 & 60.8 & 1.9 & $(57.0-64.6)$ \\
\hline Kitsap County, Washington & 201 & 65.2 & 3.9 & $(57.4-72.9)$ \\
\hline Pierce County, Washington & 314 & 60.6 & 3.4 & $(54.0-67.2)$ \\
\hline Snohomish County, Washington & 293 & 60.3 & 3.7 & $(53.0-67.5)$ \\
\hline Spokane County, Washington & 327 & 60.1 & 3.2 & $(53.9-66.4)$ \\
\hline Thurston County, Washington & 158 & 53.1 & 5.0 & $(43.3-62.9)$ \\
\hline Whatcom County, Washington & 278 & 60.5 & 3.5 & $(53.6-67.4)$ \\
\hline Yakima County, Washington & 169 & 60.4 & 4.5 & $(51.5-69.3)$ \\
\hline Kanawha County, West Virginia & 198 & 77.6 & 3.4 & $(70.8-84.3)$ \\
\hline Milwaukee County, Wisconsin & 235 & 61.8 & 4.7 & $(52.6-71.0)$ \\
\hline Laramie County, Wyoming & 390 & 62.1 & 3.5 & $(55.2-69.0)$ \\
\hline Natrona County, Wyoming & 318 & 50.7 & 4.2 & $(42.5-58.9)$ \\
\hline San Juan Municipio, Puerto Rico & 210 & 24.1 & 3.3 & $(17.6-30.6)$ \\
\hline Median & \multicolumn{4}{|c|}{60.8} \\
\hline Range & \multicolumn{4}{|c|}{$24.1-77.6$} \\
\hline
\end{tabular}

Abbreviations: $\mathrm{Cl}=$ confidence interval; $\mathrm{SE}=$ standard error. 
TABLE 13. Estimated prevalence of adults aged $\geq 65$ years who had ever received a pneumococcal vaccination, by state/territory Behavioral Risk Factor Surveillance System, United States, 2012

\begin{tabular}{|c|c|c|c|c|}
\hline State/Territory & $\begin{array}{l}\text { Sample } \\
\text { size }\end{array}$ & $\%$ & SE & $95 \% \mathrm{Cl}$ \\
\hline Alabama & 2,998 & 67.1 & 1.1 & $(64.8-69.3)$ \\
\hline Alaska & 714 & 62.5 & 2.5 & $(57.6-67.5)$ \\
\hline Arizona & 2,713 & 66.2 & 1.5 & $(63.3-69.0)$ \\
\hline Arkansas & 1,846 & 63.5 & 1.4 & $(60.7-66.3)$ \\
\hline California & 3,658 & 67.5 & 1.2 & $(65.1-69.9)$ \\
\hline Colorado & 3,193 & 73.8 & 1.0 & (71.9-75.7) \\
\hline Connecticut & 2,546 & 67.6 & 1.3 & $(65.1-70.1)$ \\
\hline Delaware & 1,624 & 70.7 & 1.5 & $(67.7-73.6)$ \\
\hline District of Columbia & 1,109 & 64.0 & 2.1 & $(59.9-68.1)$ \\
\hline Florida & 2,852 & 65.8 & 1.4 & $(63.0-68.5)$ \\
\hline Georgia & 1,774 & 66.2 & 1.5 & $(63.3-69.1)$ \\
\hline Hawaii & 1,830 & 65.1 & 1.7 & $(61.8-68.5)$ \\
\hline Idaho & 2,071 & 68.5 & 1.6 & $(65.4-71.6)$ \\
\hline Illinois & 1,907 & 63.7 & 1.5 & $(60.8-66.6)$ \\
\hline Indiana & 2,589 & 68.0 & 1.1 & $(65.7-70.2)$ \\
\hline lowa & 2,332 & 70.8 & 1.0 & $(68.7-72.8)$ \\
\hline Kansas & 3,787 & 70.3 & 0.9 & $(68.6-72.0)$ \\
\hline Kentucky & 3,184 & 65.6 & 1.3 & $(63.0-68.1)$ \\
\hline Louisiana & 3,111 & 67.7 & 1.3 & $(65.2-70.2)$ \\
\hline Maine & 2,985 & 70.7 & 1.0 & $(68.7-72.7)$ \\
\hline Maryland & 3,893 & 67.4 & 1.2 & $(65.1-69.7)$ \\
\hline Massachusetts & 5,710 & 70.2 & 0.9 & $(68.4-72.0)$ \\
\hline Michigan & 3,383 & 66.8 & 1.0 & $(64.7-68.8)$ \\
\hline Minnesota & 3,146 & 73.6 & 1.0 & $(71.5-75.6)$ \\
\hline Mississippi & 2,721 & 65.8 & 1.2 & $(63.5-68.1)$ \\
\hline Missouri & 2,305 & 71.1 & 1.4 & (68.4-73.8) \\
\hline Montana & 2,674 & 69.5 & 1.1 & $(67.2-71.7)$ \\
\hline Nebraska & 6,413 & 70.0 & 0.8 & $(68.4-71.6)$ \\
\hline Nevada & 1,464 & 64.1 & 2.1 & $(59.9-68.3)$ \\
\hline New Hampshire & 2,464 & 75.0 & 1.1 & (72.9-77.2) \\
\hline New Jersey & 4,036 & 61.6 & 1.1 & (59.4-63.9) \\
\hline New Mexico & 2,392 & 70.8 & 1.1 & $(68.7-73.0)$ \\
\hline New York & 1,585 & 67.1 & 1.8 & $(63.5-70.6)$ \\
\hline North Carolina & 3,482 & 70.2 & 1.0 & $(68.2-72.1)$ \\
\hline North Dakota & 1,496 & 68.8 & 1.5 & $(65.9-71.7)$ \\
\hline Ohio & 3,800 & 69.5 & 1.0 & $(67.4-71.5)$ \\
\hline Oklahoma & 2,509 & 74.9 & 1.0 & (72.9-77.0) \\
\hline Oregon & 1,688 & 76.2 & 1.2 & (73.8-78.6) \\
\hline Pennsylvania & 6,171 & 71.1 & 0.9 & $(69.4-72.8)$ \\
\hline Rhode Island & 1,564 & 71.4 & 1.5 & $(68.5-74.3)$ \\
\hline South Carolina & 4,166 & 69.4 & 1.1 & $(67.3-71.5)$ \\
\hline South Dakota & 2,262 & 64.1 & 1.9 & $(60.3-67.9)$ \\
\hline Tennessee & 2,197 & 69.6 & 1.4 & $(66.9-72.3)$ \\
\hline Texas & 2,529 & 70.3 & 1.2 & (67.9-72.8) \\
\hline Utah & 3,172 & 70.1 & 1.0 & $(68.1-72.1)$ \\
\hline Vermont & 1,864 & 70.8 & 1.3 & $(68.2-73.3)$ \\
\hline Virginia & 2,088 & 65.7 & 1.3 & $(63.2-68.2)$ \\
\hline Washington & 4,651 & 72.8 & 0.9 & (71.1-74.5) \\
\hline West Virginia & 1,648 & 68.0 & 1.3 & $(65.4-70.5)$ \\
\hline Wisconsin & 1,527 & 70.4 & 1.8 & $(67.0-73.9)$ \\
\hline Wyoming & 2,280 & 68.4 & 1.5 & (65.4-71.3) \\
\hline Guam & 202 & 39.5 & 4.8 & $(30.2-48.9)$ \\
\hline Puerto Rico & 1,739 & 22.2 & 1.1 & (19.9-24.4) \\
\hline Median & & 68.5 & & \\
\hline Range & & $22.2-76.2$ & & \\
\hline
\end{tabular}

Abbreviations: $\mathrm{Cl}=$ confidence interval; $\mathrm{SE}=$ standard error.
TABLE 14. Estimated prevalence of adults aged $\geq 65$ years who had ever received a pneumococcal vaccination, by metropolitan and micropolitan statistical area - Behavioral Risk Factor Surveillance System, United States, 2012

\begin{tabular}{|c|c|c|c|c|}
\hline MMSA(s) & $\begin{array}{l}\text { Sample } \\
\text { size }\end{array}$ & $\%$ & SE & $95 \% \mathrm{Cl}$ \\
\hline Aguadilla-Isabela, Puerto Rico & 156 & 15.3 & 3.4 & $(8.6-21.9)$ \\
\hline Akron, Ohio & 220 & 70.6 & 3.9 & $(62.9-78.3)$ \\
\hline Albuquerque, New Mexico & 829 & 77.0 & 1.8 & $(73.5-80.4)$ \\
\hline $\begin{array}{l}\text { Allentown-Bethlehem-Easton, } \\
\text { Pennsylvania-New Jersey }\end{array}$ & 393 & 72.6 & 3.0 & $(66.7-78.5)$ \\
\hline Anaheim-Santa Ana-Irvine, California* & 264 & 67.4 & 4.9 & $(57.8-76.9)$ \\
\hline Anchorage, Alaska & 224 & 67.1 & 3.9 & $(59.4-74.8)$ \\
\hline Asheville, North Carolina & 211 & 73.7 & 3.4 & $(67.0-80.4)$ \\
\hline $\begin{array}{l}\text { Atlanta-Sandy Springs-Roswell, } \\
\text { Georgia }\end{array}$ & 641 & 64.5 & 2.5 & $(59.5-69.4)$ \\
\hline Atlantic City-Hammonton, New Jersey & 331 & 64.1 & 3.6 & $(56.9-71.2)$ \\
\hline $\begin{array}{l}\text { Augusta-Richmond County, Georgia- } \\
\text { South Carolina }\end{array}$ & 342 & 71.5 & 4.1 & $(63.5-79.4)$ \\
\hline Augusta-Waterville, Maine & 212 & 75.9 & 3.6 & $(68.9-82.9)$ \\
\hline Austin-Round Rock, Texas & 375 & 79.4 & 2.9 & $(73.6-85.1)$ \\
\hline $\begin{array}{l}\text { Baltimore-Columbia-Towson, } \\
\text { Maryland }\end{array}$ & 1,327 & 69.0 & 1.7 & $(65.6-72.4)$ \\
\hline Bangor, Maine & 243 & 67.7 & 3.6 & $(60.6-74.8)$ \\
\hline Barnstable Town, Massachusetts & 247 & 62.7 & 3.9 & $(55.0-70.4)$ \\
\hline Barre, Vermont & 126 & 65.7 & 5.2 & $(55.5-75.9)$ \\
\hline Baton Rouge, Louisiana & 481 & 70.4 & 3.0 & $(64.5-76.2)$ \\
\hline Bellingham, Washington & 271 & 76.6 & 3.2 & $(70.4-82.9)$ \\
\hline Berlin, New Hampshire-Vermont & 261 & 73.0 & 3.1 & $(66.9-79.0)$ \\
\hline Billings, Montana & 241 & 78.3 & 3.0 & $(72.4-84.2)$ \\
\hline Birmingham-Hoover, Alabama & 551 & 69.5 & 2.5 & $(64.5-74.4)$ \\
\hline Bismarck, North Dakota & 242 & 63.1 & 4.0 & $(55.2-71.0)$ \\
\hline Boise City, Idaho & 480 & 74.2 & 2.8 & $(68.7-79.7)$ \\
\hline Boston, Massachusetts* & 1,476 & 66.3 & 1.9 & $(62.5-70.0)$ \\
\hline Boulder, Colorado & 120 & 67.3 & 4.6 & $(58.2-76.3)$ \\
\hline Bremerton-Silverdale, Washington & 191 & 77.9 & 3.3 & $(71.5-84.3)$ \\
\hline $\begin{array}{l}\text { Bridgeport-Stamford-Norwalk, } \\
\text { Connecticut }\end{array}$ & 567 & 61.7 & 2.8 & $(56.3-67.2)$ \\
\hline Burlington-South Burlington, Vermont & 403 & 69.4 & 2.8 & $(63.9-74.9)$ \\
\hline $\begin{array}{l}\text { Cambridge-Newton-Framingham, } \\
\text { Massachusetts* }\end{array}$ & 1,709 & 74.8 & 1.5 & (71.9-77.8) \\
\hline Camden, New Jersey* & 518 & 67.0 & 2.7 & $(61.7-72.4)$ \\
\hline Canton-Massillon, Ohio & 211 & 63.3 & 4.4 & $(54.8-71.9)$ \\
\hline Casper, Wyoming & 313 & 76.8 & 3.6 & $(69.8-83.8)$ \\
\hline Cedar Rapids, lowa & 190 & 75.5 & 3.3 & $(69.0-82.0)$ \\
\hline Charleston, West Virginia & 231 & 76.9 & 3.0 & $(70.9-82.8)$ \\
\hline $\begin{array}{l}\text { Charleston-North Charleston, } \\
\text { South Carolina }\end{array}$ & 513 & 73.7 & 2.8 & $(68.2-79.2)$ \\
\hline $\begin{array}{l}\text { Charlotte-Concord-Gastonia, } \\
\text { North Carolina-South Carolina }\end{array}$ & 651 & 68.3 & 2.4 & $(63.5-73.0)$ \\
\hline Chattanooga, Tennessee-Georgia & 218 & 70.0 & 4.2 & $(61.8-78.1)$ \\
\hline Cheyenne, Wyoming & 374 & 67.5 & 3.8 & $(60.0-74.9)$ \\
\hline $\begin{array}{l}\text { Chicago-Naperville-Elgin, } \\
\text { Illinois-Indiana-Wisconsin }\end{array}$ & 1,113 & 59.4 & 2.1 & $(55.2-63.6)$ \\
\hline Cincinnati, Ohio-Kentucky-Indiana & 613 & 73.6 & 2.5 & $(68.7-78.4)$ \\
\hline $\begin{array}{l}\text { Claremont-Lebanon, New } \\
\text { Hampshire-Vermont }\end{array}$ & 664 & 71.0 & 2.1 & $(66.9-75.0)$ \\
\hline Cleveland-Elyria, Ohio & 515 & 72.1 & 2.8 & $(66.7-77.5)$ \\
\hline Colorado Springs, Colorado & 268 & 69.3 & 3.4 & $(62.7-75.9)$ \\
\hline Columbia, South Carolina & 506 & 74.4 & 2.8 & $(68.8-79.9)$ \\
\hline Columbus, Ohio & 383 & 70.8 & 2.9 & $(65.0-76.5)$ \\
\hline Concord, New Hampshire & 222 & 74.3 & 3.5 & $(67.5-81.1)$ \\
\hline Dallas-Plano-Irving, Texas* & 203 & 76.5 & 3.5 & $(69.7-83.3)$ \\
\hline Dayton, Ohio & 268 & 68.9 & 3.5 & $(62.0-75.9)$ \\
\hline Denver-Aurora-Lakewood, Colorado & 1,091 & 78.0 & 1.5 & $(75.1-81.0)$ \\
\hline Des Moines-West Des Moines, lowa & 333 & 75.5 & 2.5 & $(70.6-80.5)$ \\
\hline
\end{tabular}

See table footnotes on page 38 . 
TABLE 14. (Continued) Estimated prevalence of adults aged $\geq 65$ years who had ever received a pneumococcal vaccination, by metropolitan and micropolitan statistical area - Behavioral Risk Factor Surveillance System, United States, 2012

\begin{tabular}{|c|c|c|c|c|}
\hline MMSA(s) & $\begin{array}{l}\text { Sample } \\
\text { size }\end{array}$ & $\%$ & SE & $95 \% \mathrm{Cl}$ \\
\hline Detroit-Dearborn-Livonia, Michigan* & 726 & 55.7 & 3.1 & $(49.5-61.8)$ \\
\hline Dover, Delaware & 465 & 72.7 & 2.7 & $(67.5-77.9)$ \\
\hline Duluth, Minnesota-Wisconsin & 147 & 72.5 & 4.6 & $(63.4-81.5)$ \\
\hline Durham-Chapel Hill, North Carolina & 198 & 76.9 & 3.4 & $(70.3-83.5)$ \\
\hline El Paso, Texas & 186 & 57.0 & 5.1 & $(47.1-66.9)$ \\
\hline Eugene, Oregon & 169 & 83.4 & 3.4 & $(76.7-90.1)$ \\
\hline Fairbanks, Alaska & 89 & 67.5 & 6.2 & $(55.3-79.7)$ \\
\hline Fargo, North Dakota-Minnesota & 252 & 70.1 & 4.2 & $(61.9-78.3)$ \\
\hline Farmington, New Mexico & 166 & 71.7 & 4.1 & $(63.6-79.8)$ \\
\hline Fayetteville, North Carolina & 120 & 69.6 & 4.9 & $(60.0-79.2)$ \\
\hline $\begin{array}{l}\text { Fayetteville-Springdale-Rogers, } \\
\text { Arkansas-Missouri }\end{array}$ & 281 & 68.6 & 3.7 & $(61.3-75.8)$ \\
\hline Fort Collins, Colorado & 158 & 77.4 & 3.6 & $(70.3-84.6)$ \\
\hline Fort Wayne, Indiana & 141 & 61.7 & 4.6 & $(52.7-70.8)$ \\
\hline Fort Worth-Arlington, Texas* & 213 & 70.7 & 3.9 & $(63.0-78.4)$ \\
\hline Grand Island, Nebraska & 300 & 63.3 & 3.9 & $(55.6-71.0)$ \\
\hline Grand Rapids-Wyoming, Michigan & 262 & 71.8 & 3.4 & $(65.2-78.4)$ \\
\hline Great Falls, Montana & 249 & 65.3 & 3.5 & $(58.5-72.2)$ \\
\hline Greeley, Colorado & 119 & 65.1 & 5.5 & $(54.4-75.8)$ \\
\hline $\begin{array}{l}\text { Greensboro-High Point, } \\
\text { North Carolina }\end{array}$ & 242 & 73.6 & 3.2 & $(67.3-79.8)$ \\
\hline $\begin{array}{l}\text { Greenville-Anderson-Mauldin, } \\
\text { South Carolina }\end{array}$ & 526 & 65.9 & 2.9 & $(60.3-71.6)$ \\
\hline Gulfport-Biloxi-Pascagoula, Mississippi & 250 & 68.4 & 3.4 & $(61.6-75.1)$ \\
\hline $\begin{array}{l}\text { Hagerstown-Martinsburg, Maryland- } \\
\text { West Virginia }\end{array}$ & 248 & 67.9 & 4.3 & $(59.5-76.3)$ \\
\hline Harrisburg-Carlisle, Pennsylvania & 175 & 65.0 & 4.1 & $(57.0-73.1)$ \\
\hline $\begin{array}{l}\text { Hartford-West Hartford-East Hartford, } \\
\text { Connecticut }\end{array}$ & 765 & 71.1 & 2.2 & $(66.7-75.5)$ \\
\hline Heber, Utah & 153 & 75.2 & 3.6 & $(68.2-82.3)$ \\
\hline Hilo, Hawaii & 358 & 61.1 & 3.6 & $(53.9-68.2)$ \\
\hline $\begin{array}{l}\text { Hilton Head Island-Bluffton-Beaufort, } \\
\text { South Carolina }\end{array}$ & 467 & 65.5 & 2.6 & $(60.3-70.6)$ \\
\hline $\begin{array}{l}\text { Houston-The Woodlands-Sugar Land, } \\
\text { Texas }\end{array}$ & 210 & 69.3 & 3.8 & $(61.9-76.7)$ \\
\hline $\begin{array}{l}\text { Huntington-Ashland, West } \\
\text { Virginia-Kentucky-Ohio }\end{array}$ & 340 & 71.5 & 2.8 & $(66.1-76.9)$ \\
\hline Huntsville, Alabama & 147 & 61.6 & 5.3 & $(51.2-72.0)$ \\
\hline Idaho Falls, Idaho & 166 & 59.2 & 5.2 & $(49.0-69.3)$ \\
\hline $\begin{array}{l}\text { Indianapolis-Carmel-Anderson, } \\
\text { Indiana }\end{array}$ & 611 & 74.3 & 2.2 & $(70.0-78.6)$ \\
\hline Jackson, Mississippi & 267 & 68.6 & 3.4 & $(62.0-75.3)$ \\
\hline Jacksonville, Florida & 160 & 69.5 & 4.5 & $(60.7-78.3)$ \\
\hline Kahului-Wailuku-Lahaina, Hawaii & 341 & 59.2 & 4.6 & $(50.2-68.2)$ \\
\hline Kalispell, Montana & 168 & 66.9 & 4.4 & $(58.3-75.4)$ \\
\hline Kansas City, Missouri-Kansas & 1,472 & 74.5 & 2.1 & $(70.4-78.5)$ \\
\hline Kapaa, Hawaii & 209 & 67.8 & 4.0 & $(59.9-75.6)$ \\
\hline Keene, New Hampshire & 204 & 73.5 & 3.5 & $(66.6-80.3)$ \\
\hline Kennewick-Richland, Washington & 150 & 72.4 & 4.4 & $(63.7-81.0)$ \\
\hline $\begin{array}{l}\text { Kingsport-Bristol-Bristol, } \\
\text { Tennessee-Virginia }\end{array}$ & 187 & 77.2 & 3.9 & $(69.6-84.8)$ \\
\hline Knoxville, Tennessee & 262 & 70.9 & 3.8 & $(63.4-78.4)$ \\
\hline Laconia, New Hampshire & 228 & 70.3 & 3.9 & $(62.6-77.8)$ \\
\hline Lafayette, Louisiana & 160 & 62.4 & 5.2 & $(52.1-72.6)$ \\
\hline Las Cruces, New Mexico & 197 & 70.3 & 3.6 & $(63.1-77.4)$ \\
\hline $\begin{array}{l}\text { Las Vegas-Henderson-Paradise, } \\
\text { Nevada }\end{array}$ & 487 & 60.4 & 3.0 & $(54.5-66.3)$ \\
\hline Lewiston-Auburn, Maine & 183 & 77.9 & 3.6 & $(70.7-85.0)$ \\
\hline Lexington-Fayette, Kentucky & 113 & 62.8 & 6.3 & $(50.5-75.1)$ \\
\hline Lincoln, Nebraska & 351 & 74.3 & 2.7 & $(69.0-79.7)$ \\
\hline
\end{tabular}

See table footnotes on page 38 .
TABLE 14. (Continued) Estimated prevalence of adults aged $\geq 65$ years who had ever received a pneumococcal vaccination, by metropolitan and micropolitan statistical area - Behavioral Risk Factor Surveillance System, United States, 2012

\begin{tabular}{|c|c|c|c|c|}
\hline MMSA(s) & $\begin{array}{l}\text { Sample } \\
\text { size }\end{array}$ & $\%$ & SE & $95 \% \mathrm{Cl}$ \\
\hline $\begin{array}{l}\text { Little Rock-North Little Rock-Conway, } \\
\text { Arkansas }\end{array}$ & 361 & 63.9 & 3.2 & $(57.6-70.2)$ \\
\hline Logan, Utah-Idaho & 127 & 59.8 & 5.2 & $(49.7-70.0)$ \\
\hline $\begin{array}{l}\text { Los Angeles-Long Beach-Glendale, } \\
\text { California* }\end{array}$ & 723 & 65.4 & 2.8 & $(60.0-70.8)$ \\
\hline $\begin{array}{l}\text { Louisville/Jefferson County, } \\
\text { Kentucky-Indiana }\end{array}$ & 625 & 69.7 & 2.9 & $(64.1-75.4)$ \\
\hline Lumberton, North Carolina & 151 & 52.5 & 6.3 & $(40.3-64.8)$ \\
\hline Manchester-Nashua, New Hampshire & 510 & 75.3 & 2.1 & $(71.1-79.5)$ \\
\hline McAllen-Edinburg-Mission, Texas & 175 & 56.1 & 5.1 & $(46.2-66.0)$ \\
\hline $\begin{array}{l}\text { Memphis, } \\
\text { Tennessee-Mississippi-Arkansas }\end{array}$ & 397 & 66.7 & 3.5 & $(59.8-73.7)$ \\
\hline $\begin{array}{l}\text { Miami-Fort Lauderdale-West Palm } \\
\text { Beach, Florida }\end{array}$ & 557 & 55.9 & 3.4 & $(49.3-62.5)$ \\
\hline $\begin{array}{l}\text { Milwaukee-Waukesha-West Allis, } \\
\text { Wisconsin }\end{array}$ & 306 & 70.9 & 3.9 & $(63.2-78.5)$ \\
\hline $\begin{array}{l}\text { Minneapolis-St. Paul-Bloomington, } \\
\text { Minnesota-Wisconsin }\end{array}$ & 1,882 & 74.3 & 1.5 & $(71.4-77.2)$ \\
\hline Missoula, Montana & 214 & 70.7 & 3.8 & $(63.3-78.0)$ \\
\hline Mobile, Alabama & 295 & 80.0 & 2.8 & (74.4-85.5) \\
\hline Montgomery, Alabama & 168 & 71.5 & 4.2 & $(63.2-79.8)$ \\
\hline $\begin{array}{l}\text { Montgomery County-Bucks County- } \\
\text { Chester County, Pennsylvania* }\end{array}$ & 335 & 74.7 & 2.8 & $(69.2-80.1)$ \\
\hline $\begin{array}{l}\text { Myrtle Beach-Conway-North Myrtle } \\
\text { Beach, South Carolina-North Carolina }\end{array}$ & 336 & 73.3 & 2.8 & $(67.9-78.7)$ \\
\hline $\begin{array}{l}\text { Nashville-Davidson-Murfreesboro- } \\
\text { Franklin, Tennessee }\end{array}$ & 317 & 71.9 & 3.2 & $(65.5-78.2)$ \\
\hline $\begin{array}{l}\text { Nassau County-Suffolk County, } \\
\text { New York* }\end{array}$ & 248 & 64.4 & 4.0 & $(56.6-72.3)$ \\
\hline Newark, New Jersey-Pennsylvania* & 1,843 & 62.1 & 2.1 & $(58.0-66.3)$ \\
\hline New Haven-Milford, Connecticut & 600 & 68.3 & 2.4 & $(63.5-73.1)$ \\
\hline New Orleans-Metairie, Louisiana & 386 & 67.7 & 2.9 & $(62.0-73.5)$ \\
\hline $\begin{array}{l}\text { New York-Jersey City-White Plains, } \\
\text { New York-New Jersey* }\end{array}$ & 1,612 & 61.5 & 2.4 & $(56.7-66.3)$ \\
\hline Norfolk, Nebraska & 193 & 68.7 & 4.0 & $(60.8-76.6)$ \\
\hline North Platte, Nebraska & 256 & 60.0 & 4.5 & $(51.1-68.8)$ \\
\hline Norwich-New London, Connecticut & 342 & 66.1 & 3.8 & $(58.6-73.6)$ \\
\hline $\begin{array}{l}\text { Oakland-Hayward-Berkeley, } \\
\text { California* }\end{array}$ & 253 & 72.5 & 4.7 & $(63.3-81.7)$ \\
\hline Ocean City, New Jersey & 225 & 65.7 & 4.0 & $(57.9-73.6)$ \\
\hline Ogden-Clearfield, Utah & 608 & 69.0 & 2.2 & $(64.8-73.3)$ \\
\hline Oklahoma City, Oklahoma & 613 & 78.3 & 2.0 & $(74.3-82.3)$ \\
\hline Olympia-Tumwater, Washington & 148 & 79.0 & 3.7 & $(71.8-86.2)$ \\
\hline Omaha-Council Bluffs, Nebraska-lowa & 1,466 & 73.9 & 1.5 & $(70.9-76.9)$ \\
\hline Orlando-Kissimmee-Sanford, Florida & 196 & 70.5 & 4.8 & $(61.1-80.0)$ \\
\hline Philadelphia, Pennsylvania** & 633 & 66.3 & 2.5 & $(61.3-71.2)$ \\
\hline Phoenix-Mesa-Scottsdale, Arizona & 813 & 65.3 & 2.2 & $(60.9-69.6)$ \\
\hline Pittsburgh, Pennsylvania & 1,098 & 75.3 & 1.6 & $(72.3-78.4)$ \\
\hline Ponce, Puerto Rico & 147 & 19.0 & 3.6 & $(11.9-26.1)$ \\
\hline Portland-South Portland, Maine & 1,001 & 71.2 & 1.7 & $(67.9-74.6)$ \\
\hline $\begin{array}{l}\text { Portland-Vancouver-Hillsboro, } \\
\text { Oregon-Washington }\end{array}$ & 877 & 75.0 & 1.8 & $(71.5-78.6)$ \\
\hline $\begin{array}{l}\text { Providence-Warwick, Rhode } \\
\text { Island-Massachusetts }\end{array}$ & 2,364 & 70.5 & 1.4 & $(67.8-73.1)$ \\
\hline Provo-Orem, Utah & 378 & 68.9 & 2.8 & $(63.5-74.4)$ \\
\hline Raleigh, North Carolina & 176 & 70.1 & 4.4 & $(61.5-78.6)$ \\
\hline Rapid City, South Dakota & 291 & 64.7 & 4.0 & $(56.8-72.6)$ \\
\hline Reno, Nevada & 473 & 78.3 & 2.3 & $(73.8-82.9)$ \\
\hline Richmond, Virginia & 301 & 68.2 & 3.1 & $(62.2-74.2)$ \\
\hline
\end{tabular}

See table footnotes on page 38 . 
TABLE 14. (Continued) Estimated prevalence of adults aged $\geq 65$ years who had ever received a pneumococcal vaccination, by metropolitan and micropolitan statistical area - Behavioral Risk Factor Surveillance System, United States, 2012

\begin{tabular}{|c|c|c|c|c|}
\hline MMSA(s) & $\begin{array}{l}\text { Sample } \\
\text { size }\end{array}$ & $\%$ & SE & $95 \% \mathrm{Cl}$ \\
\hline $\begin{array}{l}\text { Riverside-San Bernardino-Ontario, } \\
\text { California }\end{array}$ & 382 & 66.9 & 3.8 & $(59.5-74.3)$ \\
\hline $\begin{array}{l}\text { Rockingham County-Strafford County, } \\
\text { New Hampshire* }\end{array}$ & 504 & 78.5 & 2.2 & $(74.2-82.9)$ \\
\hline Rutland, Vermont & 190 & 74.5 & 3.9 & $(66.8-82.2)$ \\
\hline $\begin{array}{l}\text { Sacramento-Roseville-Arden-Arcade, } \\
\text { California }\end{array}$ & 254 & 73.3 & 4.0 & $(65.5-81.1)$ \\
\hline St. Louis, Missouri-Illinois & 638 & 70.8 & 2.6 & $(65.7-76.0)$ \\
\hline Salisbury, Maryland-Delaware & 841 & 74.3 & 1.8 & $(70.8-77.9)$ \\
\hline Salt Lake City, Utah & 1,131 & 74.1 & 1.7 & $(70.8-77.3)$ \\
\hline San Antonio-New Braunfels, Texas & 220 & 68.2 & 4.2 & $(59.9-76.5)$ \\
\hline San Diego-Carlsbad, California & 292 & 70.5 & 3.9 & $(62.9-78.0)$ \\
\hline $\begin{array}{l}\text { San Francisco-Redwood City-South } \\
\text { San Francisco, California* }\end{array}$ & 149 & 60.8 & 6.5 & $(48.1-73.5)$ \\
\hline $\begin{array}{l}\text { San Jose-Sunnyvale-Santa Clara, } \\
\text { California }\end{array}$ & 160 & 65.0 & 5.8 & $(53.7-76.3)$ \\
\hline San Juan-Carolina-Caguas, Puerto Rico & 1,079 & 23.6 & 1.5 & $(20.7-26.6)$ \\
\hline Santa Fe, New Mexico & 184 & 72.0 & 3.8 & $(64.6-79.5)$ \\
\hline Sayre, Pennsylvania & 656 & 64.6 & 2.0 & $(60.7-68.6)$ \\
\hline Scottsbluff, Nebraska & 261 & 66.0 & 3.9 & $(58.4-73.5)$ \\
\hline $\begin{array}{l}\text { Scranton-Wilkes-Barre-Hazleton, } \\
\text { Pennsylvania }\end{array}$ & 252 & 65.9 & 3.8 & $(58.4-73.4)$ \\
\hline Seattle-Bellevue-Everett, Washington* & 1,304 & 69.6 & 1.8 & $(66.0-73.2)$ \\
\hline Shreveport-Bossier City, Louisiana & 187 & 71.0 & 4.0 & $(63.2-78.8)$ \\
\hline $\begin{array}{l}\text { Silver Spring-Frederick-Rockville, } \\
\text { Maryland* }\end{array}$ & 660 & 70.3 & 2.5 & $(65.5-75.2)$ \\
\hline $\begin{array}{l}\text { Sioux City, lowa-Nebraska- } \\
\text { South Dakota }\end{array}$ & 432 & 65.7 & 4.7 & $(56.5-75.0)$ \\
\hline Sioux Falls, South Dakota & 289 & 67.3 & 4.1 & $(59.2-75.4)$ \\
\hline Spartanburg, South Carolina & 232 & 75.8 & 3.9 & $(68.1-83.5)$ \\
\hline Spokane-Spokane Valley, Washington & 357 & 74.8 & 2.7 & $(69.6-80.0)$ \\
\hline Springfield, Massachusetts & 608 & 68.0 & 3.1 & $(61.9-74.2)$ \\
\hline
\end{tabular}

TABLE 14. (Continued) Estimated prevalence of adults aged $\geq 65$ years who had ever received a pneumococcal vaccination, by metropolitan and micropolitan statistical area - Behavioral Risk Factor Surveillance System, United States, 2012

\begin{tabular}{|c|c|c|c|c|}
\hline MMSA(s) & $\begin{array}{l}\text { Sample } \\
\text { size }\end{array}$ & $\%$ & SE & $95 \% \mathrm{Cl}$ \\
\hline Tacoma-Lakewood, Washington* & 301 & 75.0 & 3.1 & $(68.9-81.1)$ \\
\hline $\begin{array}{l}\text { Tampa-St. Petersburg-Clearwater, } \\
\text { Florida }\end{array}$ & 292 & 71.0 & 3.6 & $(63.9-78.1)$ \\
\hline Toledo, Ohio & 291 & 71.8 & 3.4 & $(65.1-78.4)$ \\
\hline Topeka, Kansas & 325 & 69.8 & 3.0 & $(63.9-75.8)$ \\
\hline Torrington, Connecticut & 201 & 74.1 & 3.6 & $(66.9-81.2)$ \\
\hline Trenton, New Jersey & 144 & 63.7 & 5.2 & $(53.6-73.8)$ \\
\hline Tucson, Arizona & 360 & 70.0 & 3.1 & $(63.9-76.2)$ \\
\hline Tulsa, Oklahoma & 518 & 76.0 & 2.2 & $(71.8-80.3)$ \\
\hline Tuscaloosa, Alabama & 191 & 72.3 & 3.7 & $(65.1-79.5)$ \\
\hline Urban Honolulu, Hawaii & 922 & 67.0 & 2.1 & $(62.8-71.2)$ \\
\hline Vineland-Bridgeton, New Jersey & 150 & 65.4 & 5.1 & $(55.3-75.4)$ \\
\hline $\begin{array}{l}\text { Virginia Beach-Norfolk-Newport News, } \\
\text { Virginia-North Carolina }\end{array}$ & 428 & 61.5 & 3.0 & $(55.6-67.3)$ \\
\hline $\begin{array}{l}\text { Warren-Troy-Farmington Hills, } \\
\text { Michigan* }\end{array}$ & 651 & 66.0 & 2.2 & $(61.6-70.4)$ \\
\hline $\begin{array}{l}\text { Washington-Arlington-Alexandria, } \\
\text { District of Columbia-Virginia- } \\
\text { Maryland-West Virginia* }\end{array}$ & 2,111 & 65.1 & 1.8 & $(61.7-68.6)$ \\
\hline Wichita, Kansas & 718 & 75.2 & 1.8 & (71.6-78.8) \\
\hline $\begin{array}{l}\text { Wilmington, Delaware-Maryland- } \\
\text { New Jersey* }\end{array}$ & 868 & 65.8 & 2.3 & $(61.3-70.2)$ \\
\hline Winston-Salem, North Carolina & 249 & 75.1 & 3.2 & $(68.8-81.5)$ \\
\hline Worcester, Massachusetts-Connecticut & 741 & 73.6 & 2.3 & $(69.1-78.2)$ \\
\hline Yakima, Washington & 156 & 73.0 & 4.2 & $(64.8-81.2)$ \\
\hline $\begin{array}{l}\text { Youngstown-Warren-Boardman, } \\
\text { Ohio-Pennsylvania }\end{array}$ & 327 & 62.2 & 4.1 & $(54.3-70.2)$ \\
\hline Median & & 70.0 & & \\
\hline Range & & $15.3-83$. & & \\
\hline
\end{tabular}

Abbreviations: $\mathrm{Cl}=$ confidence interval; $\mathrm{MMSA}=$ metropolitan and micropolitan statistical area; $\mathrm{SE}=$ standard error.

* Metropolitan division. 
TABLE 15. Estimated prevalence of adults aged $\geq 65$ years who had ever received a pneumococcal vaccination, by county - Behavioral Risk Factor Surveillance System, United States, 2012

\begin{tabular}{|c|c|c|c|c|}
\hline County & $\begin{array}{l}\text { Sample } \\
\text { size }\end{array}$ & $\%$ & SE & $95 \% \mathrm{Cl}$ \\
\hline Jefferson County, Alabama & 259 & 71.6 & 3.3 & $(65.1-78.2)$ \\
\hline Madison County, Alabama & 125 & 62.7 & 6.0 & $(51.0-74.5)$ \\
\hline Mobile County, Alabama & 295 & 80.0 & 2.8 & $(74.4-85.5)$ \\
\hline Anchorage Municipality, Alaska & 123 & 66.5 & 5.0 & $(56.8-76.3)$ \\
\hline Fairbanks North Star Borough, Alaska & 89 & 67.5 & 6.2 & $(55.3-79.7)$ \\
\hline Matanuska-Susitna Borough, Alaska & 101 & 67.1 & 5.7 & $(55.8-78.3)$ \\
\hline Maricopa County, Arizona & 592 & 65.1 & 2.4 & $(60.3-69.8)$ \\
\hline Pima County, Arizona & 360 & 70.0 & 3.1 & $(63.9-76.2)$ \\
\hline Pulaski County, Arkansas & 240 & 67.1 & 4.0 & $(59.3-74.9)$ \\
\hline Alameda County, California & 154 & 75.4 & 5.6 & $(64.5-86.4)$ \\
\hline Los Angeles County, California & 723 & 65.4 & 2.8 & $(60.0-70.8)$ \\
\hline Orange County, California & 264 & 67.4 & 4.9 & $(57.8-76.9)$ \\
\hline Riverside County, California & 234 & 66.6 & 5.2 & $(56.3-76.9)$ \\
\hline Sacramento County, California & 147 & 76.7 & 5.5 & $(66.0-87.5)$ \\
\hline San Bernardino County, California & 148 & 67.3 & 5.3 & $(57.0-77.7)$ \\
\hline San Diego County, California & 292 & 70.5 & 3.9 & $(62.9-78.0)$ \\
\hline Santa Clara County, California & 150 & 64.2 & 6.0 & $(52.5-75.9)$ \\
\hline Adams County, Colorado & 197 & 74.0 & 3.9 & $(66.4-81.6)$ \\
\hline Arapahoe County, Colorado & 194 & 76.9 & 3.5 & $(70.1-83.8)$ \\
\hline Boulder County, Colorado & 120 & 67.3 & 4.6 & $(58.2-76.3)$ \\
\hline Denver County, Colorado & 198 & 85.2 & 2.8 & $(79.7-90.7)$ \\
\hline Douglas County, Colorado & 92 & 78.9 & 4.6 & $(69.8-88.0)$ \\
\hline El Paso County, Colorado & 215 & 69.3 & 3.6 & $(62.3-76.2)$ \\
\hline Jefferson County, Colorado & 292 & 75.3 & 3.0 & $(69.4-81.2)$ \\
\hline Larimer County, Colorado & 158 & 77.4 & 3.6 & $(70.3-84.6)$ \\
\hline Weld County, Colorado & 119 & 65.1 & 5.5 & $(54.3-75.8)$ \\
\hline Fairfield County, Connecticut & 567 & 61.7 & 2.8 & $(56.3-67.2)$ \\
\hline Hartford County, Connecticut & 554 & 71.0 & 2.7 & $(65.6-76.3)$ \\
\hline Litchfield County, Connecticut & 201 & 74.1 & 3.6 & $(66.9-81.2)$ \\
\hline New Haven County, Connecticut & 600 & 68.3 & 2.5 & $(63.5-73.1)$ \\
\hline New London County, Connecticut & 342 & 66.1 & 3.8 & $(58.6-73.6)$ \\
\hline Kent County, Delaware & 465 & 72.7 & 2.7 & $(67.5-77.9)$ \\
\hline New Castle County, Delaware & 596 & 66.5 & 2.5 & $(61.6-71.4)$ \\
\hline Sussex County, Delaware & 563 & 75.9 & 2.1 & $(71.8-80.1)$ \\
\hline $\begin{array}{l}\text { District of Columbia, } \\
\text { District of Columbia }\end{array}$ & 1,109 & 64.0 & 2.1 & $(59.9-68.1)$ \\
\hline Broward County, Florida & 167 & 52.4 & 5.7 & $(41.1-63.6)$ \\
\hline Miami-Dade County, Florida & 243 & 52.0 & 6.0 & $(40.2-63.8)$ \\
\hline Hawaii County, Hawaii & 358 & 61.1 & 3.6 & $(53.9-68.2)$ \\
\hline Honolulu County, Hawaii & 922 & 67.0 & 2.1 & $(62.8-71.2)$ \\
\hline Kauai County, Hawaii & 209 & 67.8 & 4.0 & $(59.9-75.6)$ \\
\hline Maui County, Hawaii & 341 & 59.2 & 4.6 & $(50.2-68.2)$ \\
\hline Ada County, Idaho & 242 & 74.1 & 4.0 & $(66.3-81.8)$ \\
\hline Canyon County, Idaho & 183 & 77.0 & 3.6 & $(69.9-84.0)$ \\
\hline Cook County, Illinois & 441 & 59.4 & 3.0 & $(53.6-65.2)$ \\
\hline Lake County, Indiana & 304 & 53.7 & 4.5 & $(44.9-62.5)$ \\
\hline Marion County, Indiana & 365 & 75.4 & 3.1 & $(69.4-81.3)$ \\
\hline Polk County, lowa & 224 & 78.1 & 2.9 & $(72.4-83.9)$ \\
\hline Johnson County, Kansas & 633 & 76.1 & 2.0 & $(72.2-79.9)$ \\
\hline Sedgwick County, Kansas & 517 & 76.4 & 2.2 & $(72.1-80.7)$ \\
\hline Shawnee County, Kansas & 232 & 73.7 & 3.6 & $(66.7-80.8)$ \\
\hline Wyandotte County, Kansas & 311 & 71.8 & 3.3 & $(65.4-78.3)$ \\
\hline Jefferson County, Kentucky & 513 & 71.1 & 3.6 & $(63.9-78.2)$ \\
\hline East Baton Rouge Parish, Louisiana & 176 & 68.9 & 4.2 & $(60.7-77.1)$ \\
\hline Androscoggin County, Maine & 183 & 77.9 & 3.6 & $(70.7-85.0)$ \\
\hline Aroostook County, Maine & 168 & 63.9 & 4.4 & $(55.2-72.6)$ \\
\hline Cumberland County, Maine & 497 & 71.9 & 2.4 & $(67.2-76.6)$ \\
\hline Kennebec County, Maine & 212 & 75.9 & 3.6 & $(68.9-82.9)$ \\
\hline Penobscot County, Maine & 243 & 67.7 & 3.6 & $(60.6-74.8)$ \\
\hline York County, Maine & 379 & 69.3 & 2.8 & $(63.9-74.8)$ \\
\hline Anne Arundel County, Maryland & 246 & 67.8 & 3.7 & $(60.5-75.2)$ \\
\hline
\end{tabular}

See table footnotes on page 40.
TABLE 15. (Continued) Estimated prevalence of adults aged $\geq 65$ years who had ever received a pneumococcal vaccination, by county Behavioral Risk Factor Surveillance System, United States, 2012

\begin{tabular}{|c|c|c|c|c|}
\hline County & $\begin{array}{l}\text { Sample } \\
\text { size }\end{array}$ & $\%$ & SE & $95 \% \mathrm{Cl}$ \\
\hline Baltimore County, Maryland & 434 & 68.3 & 2.9 & $(62.5-74.1)$ \\
\hline Charles County, Maryland & 109 & 72.0 & 5.3 & $(61.7-82.3)$ \\
\hline Frederick County, Maryland & 237 & 65.7 & 5.9 & $(54.1-77.3)$ \\
\hline Montgomery County, Maryland & 423 & 71.3 & 2.7 & $(66.1-76.6)$ \\
\hline Prince George's County, Maryland & 293 & 56.0 & 4.4 & $(47.4-64.5)$ \\
\hline Washington County, Maryland & 187 & 77.0 & 5.1 & $(66.9-87.1)$ \\
\hline Baltimore city, Maryland & 213 & 76.0 & 3.5 & $(69.1-82.9)$ \\
\hline Barnstable County, Massachusetts & 247 & 62.7 & 3.9 & $(55.0-70.4)$ \\
\hline Bristol County, Massachusetts & 800 & 69.1 & 2.8 & $(63.7-74.6)$ \\
\hline Essex County, Massachusetts & 764 & 69.4 & 2.6 & $(64.2-74.6)$ \\
\hline Hampden County, Massachusetts & 519 & 65.6 & 3.6 & $(58.5-72.7)$ \\
\hline Middlesex County, Massachusetts & 945 & 77.9 & 1.8 & $(74.4-81.4)$ \\
\hline Norfolk County, Massachusetts & 466 & 71.7 & 3.0 & $(65.8-77.7)$ \\
\hline Plymouth County, Massachusetts & 509 & 67.6 & 3.3 & $(61.1-74.1)$ \\
\hline Suffolk County, Massachusetts & 501 & 55.5 & 3.5 & $(48.6-62.4)$ \\
\hline Worcester County, Massachusetts & 670 & 74.3 & 2.5 & $(69.4-79.1)$ \\
\hline Kent County, Michigan & 148 & 73.9 & 4.7 & $(64.8-83.1)$ \\
\hline Macomb County, Michigan & 185 & 71.3 & 3.8 & $(63.8-78.7)$ \\
\hline Oakland County, Michigan & 359 & 66.0 & 3.0 & $(60.2-71.8)$ \\
\hline Wayne County, Michigan & 726 & 55.7 & 3.1 & $(49.6-61.8)$ \\
\hline Anoka County, Minnesota & 114 & 72.2 & 5.5 & $(61.5-83.0)$ \\
\hline Dakota County, Minnesota & 137 & 76.5 & 5.3 & $(66.1-86.8)$ \\
\hline Hennepin County, Minnesota & 761 & 73.4 & 2.4 & $(68.8-78.1)$ \\
\hline Ramsey County, Minnesota & 550 & 74.9 & 2.8 & $(69.3-80.4)$ \\
\hline Jackson County, Missouri & 288 & 71.2 & 4.3 & $(62.7-79.6)$ \\
\hline St. Louis County, Missouri & 283 & 68.6 & 4.4 & $(59.9-77.2)$ \\
\hline Cascade County, Montana & 249 & 65.3 & 3.5 & $(58.5-72.2)$ \\
\hline Flathead County, Montana & 168 & 66.9 & 4.4 & $(58.3-75.4)$ \\
\hline Hill County, Montana & 168 & 57.4 & 5.6 & $(46.4-68.5)$ \\
\hline Lake County, Montana & 333 & 68.2 & 3.6 & $(61.2-75.2)$ \\
\hline Missoula County, Montana & 214 & 70.7 & 3.8 & $(63.3-78.1)$ \\
\hline Yellowstone County, Montana & 206 & 77.7 & 3.3 & $(71.3-84.1)$ \\
\hline Dakota County, Nebraska & 274 & 62.3 & 3.5 & $(55.4-69.2)$ \\
\hline Douglas County, Nebraska & 945 & 72.6 & 1.9 & $(68.8-76.4)$ \\
\hline Hall County, Nebraska & 183 & 61.1 & 5.5 & $(50.4-71.8)$ \\
\hline Lancaster County, Nebraska & 276 & 74.5 & 2.9 & $(68.8-80.2)$ \\
\hline Lincoln County, Nebraska & 247 & 60.7 & 4.6 & $(51.7-69.7)$ \\
\hline Sarpy County, Nebraska & 291 & 72.1 & 3.3 & $(65.6-78.6)$ \\
\hline Scotts Bluff County, Nebraska & 242 & 65.5 & 4.0 & $(57.7-73.3)$ \\
\hline Clark County, Nevada & 487 & 60.4 & 3.0 & $(54.5-66.3)$ \\
\hline Washoe County, Nevada & 466 & 78.4 & 2.4 & $(73.8-83.1)$ \\
\hline Belknap County, New Hampshire & 228 & 70.3 & 3.9 & $(62.6-77.9)$ \\
\hline Carroll County, New Hampshire & 236 & 73.5 & 3.3 & $(67.1-79.9)$ \\
\hline Cheshire County, New Hampshire & 204 & 73.5 & 3.5 & $(66.6-80.3)$ \\
\hline Coos County, New Hampshire & 202 & 71.8 & 3.5 & $(64.9-78.7)$ \\
\hline Grafton County, New Hampshire & 200 & 73.7 & 3.7 & $(66.6-80.9)$ \\
\hline Hillsborough County, New Hampshire & 510 & 75.3 & 2.1 & $(71.1-79.5)$ \\
\hline Merrimack County, New Hampshire & 222 & 74.3 & 3.4 & $(67.6-81.1)$ \\
\hline Rockingham County, New Hampshire & 306 & 77.5 & 2.8 & $(72.0-83.0)$ \\
\hline Strafford County, New Hampshire & 198 & 81.1 & 3.4 & $(74.5-87.7)$ \\
\hline Atlantic County, New Jersey & 331 & 64.1 & 3.7 & $(56.9-71.2)$ \\
\hline Bergen County, New Jersey & 207 & 53.6 & 4.5 & $(44.8-62.4)$ \\
\hline Burlington County, New Jersey & 187 & 67.1 & 4.8 & $(57.8-76.5)$ \\
\hline Camden County, New Jersey & 200 & 64.8 & 4.2 & $(56.6-72.9)$ \\
\hline Cape May County, New Jersey & 225 & 65.7 & 4.0 & $(57.9-73.6)$ \\
\hline Cumberland County, New Jersey & 150 & 65.4 & 5.1 & $(55.3-75.4)$ \\
\hline Essex County, New Jersey & 273 & 63.6 & 3.9 & $(56.1-71.2)$ \\
\hline Gloucester County, New Jersey & 131 & 72.0 & 4.9 & $(62.4-81.6)$ \\
\hline Hudson County, New Jersey & 244 & 58.4 & 4.2 & $(50.2-66.6)$ \\
\hline Hunterdon County, New Jersey & 149 & 61.7 & 5.7 & $(50.6-72.8)$ \\
\hline Mercer County, New Jersey & 144 & 63.7 & 5.2 & $(53.6-73.9)$ \\
\hline
\end{tabular}

See table footnotes on page 40 . 
TABLE 15. (Continued) Estimated prevalence of adults aged $\geq 65$ years who had ever received a pneumococcal vaccination, by county Behavioral Risk Factor Surveillance System, United States, 2012

\begin{tabular}{|c|c|c|c|c|}
\hline County & $\begin{array}{l}\text { Sample } \\
\text { size }\end{array}$ & $\%$ & SE & $95 \% \mathrm{Cl}$ \\
\hline Middlesex County, New Jersey & 192 & 65.0 & 4.8 & $(55.6-74.4)$ \\
\hline Monmouth County, New Jersey & 201 & 62.7 & 4.3 & $(54.3-71.0)$ \\
\hline Morris County, New Jersey & 215 & 68.0 & 4.5 & $(59.2-76.8)$ \\
\hline Ocean County, New Jersey & 231 & 65.0 & 3.7 & $(57.7-72.2)$ \\
\hline Passaic County, New Jersey & 135 & 47.8 & 5.1 & $(37.9-57.8)$ \\
\hline Salem County, New Jersey & 183 & 67.0 & 4.1 & $(59.0-75.0)$ \\
\hline Somerset County, New Jersey & 181 & 66.2 & 4.9 & $(56.7-75.7)$ \\
\hline Sussex County, New Jersey & 154 & 69.5 & 4.8 & $(60.1-79.0)$ \\
\hline Union County, New Jersey & 148 & 50.7 & 5.8 & $(39.2-62.1)$ \\
\hline Warren County, New Jersey & 155 & 69.8 & 4.3 & $(61.4-78.3)$ \\
\hline Bernalillo County, New Mexico & 492 & 78.2 & 2.1 & $(74.0-82.4)$ \\
\hline Dona Ana County, New Mexico & 197 & 70.3 & 3.6 & $(63.1-77.4)$ \\
\hline Sandoval County, New Mexico & 176 & 79.2 & 3.4 & $(72.6-85.8)$ \\
\hline San Juan County, New Mexico & 166 & 71.7 & 4.1 & $(63.6-79.8)$ \\
\hline Santa Fe County, New Mexico & 184 & 72.0 & 3.8 & $(64.6-79.5)$ \\
\hline Kings County, New York & 57 & 60.8 & 9.5 & $(42.1-79.4)$ \\
\hline Guilford County, North Carolina & 143 & 67.9 & 4.8 & $(58.5-77.3)$ \\
\hline Mecklenburg County, North Carolina & 125 & 73.1 & 4.6 & $(64.1-82.1)$ \\
\hline Robeson County, North Carolina & 151 & 52.5 & 6.3 & $(40.2-64.9)$ \\
\hline Wake County, North Carolina & 113 & 68.9 & 5.3 & $(58.5-79.4)$ \\
\hline Burleigh County, North Dakota & 161 & 66.4 & 5.0 & $(56.6-76.2)$ \\
\hline Cass County, North Dakota & 225 & 70.9 & 4.1 & $(62.8-79.0)$ \\
\hline Cuyahoga County, Ohio & 214 & 72.9 & 3.6 & $(65.8-79.9)$ \\
\hline Franklin & 171 & 73.1 & 4.2 & $(64.8$ \\
\hline Hamilton County, Ohio & 194 & 77.1 & 3.9 & $(69.5-84.7)$ \\
\hline Lorain County, Ohio & 213 & 74.3 & .8 & $(66.9-81.7)$ \\
\hline Lucas County, Ohio & 191 & 69.5 & 4.4 & $(60.8-78.2)$ \\
\hline Mahoning County, Ohio & 219 & 59.4 & 4.8 & $(50.1-68.7)$ \\
\hline Montgomery County, Ohio & 221 & 67.5 & 4.0 & $(59.7-75.4)$ \\
\hline Stark County, Ohio & 195 & 64.5 & 4.5 & $(55.6-73.3)$ \\
\hline Summit County, Ohio & 193 & 72.3 & 3.8 & $(64.8-79.8)$ \\
\hline Oklahoma County, Oklahoma & 279 & 81.4 & 2.7 & $(76.1-86.6)$ \\
\hline Tulsa County, Oklahoma & 353 & 74.7 & 2.7 & $(69.4-80.0)$ \\
\hline Clackamas County, Oregon & 160 & 67.3 & 4.8 & $(57.9-76.7)$ \\
\hline Lane County, Oregon & 169 & 83.4 & 3.4 & $(76.7-90.1)$ \\
\hline Multnomah County, Oregon & 221 & 80.1 & 3.1 & $(74.0-86.3)$ \\
\hline Washington County, Oregon & 167 & 78.7 & 3.7 & $(71.4-86.0)$ \\
\hline Allegheny County, Pennsylvania & 645 & 78.6 & 1.9 & $(74.9-82.3)$ \\
\hline Bradford County, Pennsylvania & 656 & 64.7 & 2.0 & $(60.7-68.6)$ \\
\hline Montgomery County, Pennsylvania & 130 & 84.8 & 3.2 & $(78.5-91.2)$ \\
\hline Philadelphia County, Pennsylvania & 533 & 64.8 & 2.8 & $(59.4-70.1)$ \\
\hline Pike County, Pennsylvania & 723 & 65.7 & 1.9 & $(62.1-69.4)$ \\
\hline Kent County, Rhode Island & 217 & 67.0 & 4.0 & $(59.1-74.9)$ \\
\hline Providence County, Rhode Island & 902 & 72.4 & 1.9 & $(68.6-76.2)$ \\
\hline Washington County, Rhode Island & 231 & 73.5 & 3.4 & $(66.9-80.1)$ \\
\hline Aiken County, South Carolina & 193 & 68.9 & 4.3 & $(60.5-77.2)$ \\
\hline
\end{tabular}

TABLE 15. (Continued) Estimated prevalence of adults aged $\geq 65$ years who had ever received a pneumococcal vaccination, by county Behavioral Risk Factor Surveillance System, United States, 2012

\begin{tabular}{|c|c|c|c|c|}
\hline County & $\begin{array}{l}\text { Sample } \\
\text { size }\end{array}$ & $\%$ & SE & $95 \% \mathrm{Cl}$ \\
\hline Beaufort County, South Carolina & 412 & 65.2 & 2.8 & $(59.7-70.6)$ \\
\hline Charleston County, South Carolina & 318 & 70.4 & 3.6 & $(63.3-77.5)$ \\
\hline Greenville County, South Carolina & 252 & 67.6 & 4.0 & $(59.8-75.5)$ \\
\hline Horry County, South Carolina & 261 & 70.1 & 3.2 & $(63.8-76.4)$ \\
\hline Richland County, South Carolina & 271 & 73.4 & 3.9 & $(65.7-81.1)$ \\
\hline Spartanburg County, South Carolina & 214 & 73.6 & 4.4 & $(65.0-82.2)$ \\
\hline Lincoln County, South Dakota & 128 & 55.1 & 7.9 & (39.7-70.5) \\
\hline Minnehaha County, South Dakota & 143 & 70.5 & 4.6 & $(61.6-79.4)$ \\
\hline Pennington County, South Dakota & 144 & 63.7 & 4.7 & $(54.5-72.8)$ \\
\hline Davidson County, Tennessee & 145 & 73.8 & 5.0 & $(64.1-83.6)$ \\
\hline Shelby County, Tennessee & 124 & 63.7 & 5.0 & $(53.8-73.5)$ \\
\hline Bexar County, Texas & 153 & 71.8 & 4.9 & $(62.2-81.5)$ \\
\hline Dallas County, Texas & 118 & 71.2 & 4.9 & $(61.6-80.7)$ \\
\hline El Paso County, Texas & 186 & 57.0 & 5.0 & $(47.1-66.9)$ \\
\hline Harris County, Texas & 122 & 71.2 & 4.7 & $(61.9-80.4)$ \\
\hline Hidalgo County, Texas & 175 & 56.1 & 5.0 & $(46.2-66.0)$ \\
\hline Tarrant County, Texas & 146 & 69.5 & 5.1 & $(59.5-79.5)$ \\
\hline Travis County, Texas & 274 & 81.2 & 2.8 & $(75.7-86.8)$ \\
\hline Davis County, Utah & 252 & 67.9 & 3.2 & $(61.6-74.2)$ \\
\hline Salt Lake County, Utah & 983 & 74.1 & 1.7 & (70.7-77.6) \\
\hline Tooele County, Utah & 148 & 73.3 & 4.5 & $(64.4-82.1)$ \\
\hline Utah County, Utah & 351 & 68.8 & 2.9 & $(63.2-74.5)$ \\
\hline Wasatch County, Utah & 153 & 75.2 & 3.6 & $(68.2-82.3)$ \\
\hline Weber County, Utah & 277 & 72.7 & 3.2 & $(66.5-79.0)$ \\
\hline Chittenden County, Vermont & 225 & 66.8 & 3.6 & $(59.7-73.9)$ \\
\hline Rutland County, Vermont & 190 & 74.5 & 3.9 & $(66.8-82.2)$ \\
\hline Washington County, Vermont & 126 & 65.7 & 5.2 & $(55.5-75.9)$ \\
\hline Windsor County, Vermont & 196 & 69.1 & 3.8 & $(61.6-76.6)$ \\
\hline Fairfax County, Virginia & 154 & 71.2 & 4.2 & $(62.9-79.5)$ \\
\hline Clark County, Washington & 230 & 75.4 & 3.2 & $(69.2-81.7)$ \\
\hline King County, Washington & 1,033 & 68.7 & 2.2 & $(64.5-73.0)$ \\
\hline Kitsap County, Washington & 191 & 77.9 & 3.3 & (71.5-84.3) \\
\hline Pierce County, Washington & 301 & 75.0 & 3.1 & $(68.9-81.1)$ \\
\hline Snohomish County, Washington & 271 & 72.8 & 3.4 & $(66.2-79.4)$ \\
\hline Spokane County, Washington & 311 & 75.9 & 2.8 & $(70.3-81.4)$ \\
\hline Thurston County, Washington & 148 & 79.0 & 3.7 & $(71.8-86.2)$ \\
\hline Whatcom County, Washington & 271 & 76.6 & 3.2 & $(70.4-82.9)$ \\
\hline Yakima County, Washington & 156 & 73.0 & 4.2 & $(64.8-81.2)$ \\
\hline Kanawha County, West Virginia & 193 & 78.2 & 3.3 & $(71.8-84.6)$ \\
\hline Milwaukee County, Wisconsin & 228 & 75.9 & 4.0 & $(68.1-83.7)$ \\
\hline Laramie County, Wyoming & 374 & 67.5 & 3.8 & $(60.0-74.9)$ \\
\hline Natrona County, Wyoming & 313 & 76.8 & 3.6 & $(69.8-83.8)$ \\
\hline San Juan Municipio, Puerto Rico & 204 & 25.8 & 3.6 & $(18.7-32.8)$ \\
\hline Median & & 70.1 & & \\
\hline Range & & $25.8-85$. & & \\
\hline
\end{tabular}

Abbreviations: $\mathrm{Cl}=$ confidence interval; $\mathrm{SE}=$ standard error. 
TABLE 16. Estimated prevalence of adults aged $\geq 18$ years who have visited a dentist, dental hygienist, or dental clinic within the past year, by state/territory - Behavioral Risk Factor Surveillance System, United States, 2012

\begin{tabular}{|c|c|c|c|c|}
\hline State/Territory & $\begin{array}{c}\text { Sample } \\
\text { size }\end{array}$ & $\%$ & SE & $95 \% \mathrm{Cl}$ \\
\hline Alabama & 8,893 & 58.6 & 0.8 & $(57.1-60.2)$ \\
\hline Alaska & 4,295 & 67.4 & 1.0 & $(65.5-69.4)$ \\
\hline Arizona & 7,262 & 61.6 & 1.0 & (59.7-63.5) \\
\hline Arkansas & 5,080 & 54.9 & 1.0 & $(53.0-56.8)$ \\
\hline California & 14,556 & 67.0 & 0.6 & $(65.8-68.1)$ \\
\hline Colorado & 12,154 & 65.3 & 0.6 & $(64.1-66.4)$ \\
\hline Connecticut & 8,699 & 76.1 & 0.7 & $(74.8-77.4)$ \\
\hline Delaware & 5,152 & 70.0 & 0.9 & $(68.3-71.8)$ \\
\hline District of Columbia & 3,801 & 71.1 & 1.2 & (68.7-73.6) \\
\hline Florida & 7,571 & 59.8 & 0.9 & (58.0-61.5) \\
\hline Georgia & 6,016 & 64.1 & 0.9 & $(62.4-65.8)$ \\
\hline Hawaii & 7,558 & 70.4 & 0.8 & $(68.8-72.0)$ \\
\hline Idaho & 5,862 & 67.6 & 1.1 & $(65.4-69.7)$ \\
\hline Illinois & 5,568 & 66.9 & 0.9 & (65.1-68.8) \\
\hline Indiana & 8,576 & 62.6 & 0.7 & $(61.3-64.0)$ \\
\hline lowa & 7,121 & 71.1 & 0.7 & $(69.8-72.5)$ \\
\hline Kansas & 11,680 & 67.3 & 0.6 & $(66.1-68.5)$ \\
\hline Kentucky & 11,030 & 60.3 & 0.7 & (58.9-61.7) \\
\hline Louisiana & 8,872 & 56.1 & 0.9 & (54.4-57.8) \\
\hline Maine & 9,873 & 65.3 & 0.6 & (64.1-66.5) \\
\hline Maryland & 12,689 & 72.7 & 0.7 & $(71.3-74.1)$ \\
\hline Massachusetts & 21,523 & 76.2 & 0.5 & $(75.3-77.1)$ \\
\hline Michigan & 10,401 & 68.0 & 0.7 & $(66.8-69.3)$ \\
\hline Minnesota & 12,167 & 74.8 & 0.6 & (73.7-75.9) \\
\hline Mississippi & 7,684 & 55.4 & 0.8 & $(53.8-57.1)$ \\
\hline Missouri & 6,698 & 61.8 & 0.9 & $(60.1-63.5)$ \\
\hline Montana & 8,610 & 61.0 & 0.7 & (59.6-62.3) \\
\hline Nebraska & 19,052 & 67.6 & 0.5 & (66.6-68.6) \\
\hline Nevada & 4,798 & 60.8 & 1.0 & (58.8-62.9) \\
\hline New Hampshire & 7,450 & 73.1 & 0.8 & (71.5-74.6) \\
\hline New Jersey & 15,598 & 71.2 & 0.5 & (70.1-72.3) \\
\hline New Mexico & 8,709 & 60.9 & 0.7 & $(59.6-62.3)$ \\
\hline New York & 6,020 & 67.5 & 0.9 & $(65.8-69.3)$ \\
\hline North Carolina & 11,712 & 64.9 & 0.6 & $(63.8-66.0)$ \\
\hline North Dakota & 4,845 & 67.2 & 0.9 & $(65.3-69.0)$ \\
\hline Ohio & 12,899 & 67.6 & 0.6 & $(66.4-68.7)$ \\
\hline Oklahoma & 7,973 & 58.9 & 0.7 & $(57.5-60.3)$ \\
\hline Oregon & 5,225 & 65.3 & 0.9 & $(63.5-67.0)$ \\
\hline Pennsylvania & 19,812 & 68.5 & 0.5 & (67.5-69.5) \\
\hline Rhode Island & 5,427 & 73.6 & 0.9 & (71.9-75.3) \\
\hline South Carolina & 12,687 & 59.6 & 0.7 & (58.3-60.9) \\
\hline South Dakota & 7,836 & 70.9 & 0.8 & $(69.3-72.5)$ \\
\hline Tennessee & 6,921 & 61.4 & 0.8 & $(59.8-63.1)$ \\
\hline Texas & 9,040 & 58.8 & 0.7 & $(57.3-60.2)$ \\
\hline Utah & 12,272 & 68.4 & 0.6 & $(67.2-69.5)$ \\
\hline Vermont & 6,012 & 70.8 & 0.8 & $(69.3-72.3)$ \\
\hline Virginia & 7,320 & 70.4 & 0.7 & (69.0-71.8) \\
\hline Washington & 15,235 & 67.6 & 0.5 & $(66.5-68.6)$ \\
\hline West Virginia & 5,359 & 56.4 & 0.8 & $(54.8-58.0)$ \\
\hline Wisconsin & 5,264 & 72.0 & 0.9 & (70.1-73.8) \\
\hline Wyoming & 6,201 & 66.0 & 1.0 & $(64.0-68.0)$ \\
\hline Guam & 1,999 & 53.7 & 1.5 & $(50.8-56.6)$ \\
\hline Puerto Rico & 6,258 & 67.9 & 0.7 & $(66.6-69.3)$ \\
\hline Median & & 67.2 & & \\
\hline Range & & $53.7-76.2$ & & \\
\hline
\end{tabular}

Abbreviations: $\mathrm{Cl}=$ confidence interval; $\mathrm{SE}=$ standard error.
TABLE 17. Estimated prevalence of adults aged $\geq 18$ years who have visited a dentist, dental hygienist, or dental clinic within the past year, by metropolitan and micropolitan statistical area - Behavioral Risk Factor Surveillance System, United States, 2012

\begin{tabular}{|c|c|c|c|c|}
\hline $\operatorname{MMSA}(\mathbf{s})$ & $\begin{array}{l}\text { Sample } \\
\text { size }\end{array}$ & $\%$ & SE & $95 \% \mathrm{Cl}$ \\
\hline Aguadilla-Isabela, Puerto Rico & 544 & 69.5 & 2.2 & $(65.1-73.9)$ \\
\hline Akron, Ohio & 740 & 68.3 & 2.4 & $(63.6-73.0)$ \\
\hline Albuquerque, New Mexico & 3,249 & 63.7 & 1.1 & $(61.6-65.8)$ \\
\hline $\begin{array}{l}\text { Allentown-Bethlehem-Easton, } \\
\text { Pennsylvania-New Jersey }\end{array}$ & 1,337 & 72.3 & 1.8 & $(68.7-75.9)$ \\
\hline Anaheim-Santa Ana-Irvine, California* & 1,037 & 73.0 & 2.0 & $(69.1-77.0)$ \\
\hline Anchorage, Alaska & 1,495 & 69.6 & 1.5 & $(66.6-72.5)$ \\
\hline Asheville, North Carolina & 584 & 67.4 & 2.5 & $(62.5-72.2)$ \\
\hline $\begin{array}{l}\text { Atlanta-Sandy Springs-Roswell, } \\
\text { Georgia }\end{array}$ & 2,512 & 68.3 & 1.3 & $(65.8-70.8)$ \\
\hline Atlantic City-Hammonton, New Jersey & 1,014 & 70.6 & 2.1 & $(66.4-74.7)$ \\
\hline $\begin{array}{l}\text { Augusta-Richmond County, Georgia- } \\
\text { South Carolina }\end{array}$ & 1,025 & 62.2 & 2.8 & (56.7-67.8) \\
\hline Augusta-Waterville, Maine & 828 & 66.8 & 2.0 & $(62.9-70.8)$ \\
\hline Austin-Round Rock, Texas & 1,383 & 65.5 & 2.1 & $(61.3-69.6)$ \\
\hline $\begin{array}{l}\text { Baltimore-Columbia-Towson, } \\
\text { Maryland }\end{array}$ & 4,680 & 72.2 & 1.1 & $(70.1-74.2)$ \\
\hline Bangor, Maine & 924 & 63.2 & 2.0 & $(59.3-67.2)$ \\
\hline Barnstable Town, Massachusetts & 558 & 81.7 & 2.1 & $(77.5-85.8)$ \\
\hline Barre, Vermont & 514 & 73.9 & 2.5 & $(69.0-78.9)$ \\
\hline Baton Rouge, Louisiana & 1,364 & 59.9 & 2.1 & $(55.8-64.0)$ \\
\hline Bellingham, Washington & 844 & 67.5 & 2.6 & $(62.3-72.6)$ \\
\hline Berlin, New Hampshire-Vermont & 703 & 57.7 & 2.9 & $(52.1-63.4)$ \\
\hline Billings, Montana & 845 & 62.2 & 1.9 & $(58.4-66.0)$ \\
\hline Birmingham-Hoover, Alabama & 1,799 & 59.8 & 1.6 & $(56.7-62.9)$ \\
\hline Bismarck, North Dakota & 818 & 71.7 & 2.1 & $(67.5-76.0)$ \\
\hline Boise City, Idaho & 1,474 & 69.9 & 1.9 & $(66.3-73.5)$ \\
\hline Boston, Massachusetts* & 5,857 & 76.2 & 0.9 & $(74.5-77.8)$ \\
\hline Boulder, Colorado & 514 & 71.4 & 2.6 & $(66.3-76.5)$ \\
\hline Bremerton-Silverdale, Washington & 564 & 67.0 & 2.8 & $(61.6-72.5)$ \\
\hline $\begin{array}{l}\text { Bridgeport-Stamford-Norwalk, } \\
\text { Connecticut }\end{array}$ & 2,165 & 73.1 & 1.5 & $(70.2-76.1)$ \\
\hline Burlington-South Burlington, Vermont & 1,517 & 74.8 & 1.4 & $(72.0-77.6)$ \\
\hline $\begin{array}{l}\text { Cambridge-Newton-Framingham, } \\
\text { Massachusetts* }\end{array}$ & 6,875 & 79.1 & 0.8 & $(77.6-80.6)$ \\
\hline Camden, New Jersey* & 1,968 & 72.3 & 1.4 & $(69.5-75.1)$ \\
\hline Canton-Massillon, Ohio & 677 & 65.1 & 2.6 & $(60.1-70.2)$ \\
\hline Casper, Wyoming & 816 & 67.2 & 2.7 & $(61.9-72.5)$ \\
\hline Cedar Rapids, lowa & 581 & 72.4 & 2.5 & $(67.5-77.3)$ \\
\hline Charleston, West Virginia & 769 & 62.3 & 2.1 & $(58.3-66.3)$ \\
\hline $\begin{array}{l}\text { Charleston-North Charleston, } \\
\text { South Carolina }\end{array}$ & 1,696 & 59.1 & 1.7 & $(55.7-62.5)$ \\
\hline $\begin{array}{l}\text { Charlotte-Concord-Gastonia, } \\
\text { North Carolina-South Carolina }\end{array}$ & 2,494 & 66.1 & 1.2 & $(63.7-68.5)$ \\
\hline Chattanooga, Tennessee-Georgia & 620 & 59.2 & 3.2 & $(53.0-65.4)$ \\
\hline Cheyenne, Wyoming & 954 & 71.5 & 2.4 & $(66.9-76.1)$ \\
\hline $\begin{array}{l}\text { Chicago-Naperville-Elgin, } \\
\text { Illinois-Indiana-Wisconsin }\end{array}$ & 3,714 & 67.8 & 1.2 & $(65.4-70.1)$ \\
\hline Cincinnati, Ohio-Kentucky-Indiana & 2,344 & 68.7 & 1.3 & $(66.2-71.2)$ \\
\hline $\begin{array}{l}\text { Claremont-Lebanon, New } \\
\text { Hampshire-Vermont }\end{array}$ & 1,939 & 69.9 & 1.5 & $(66.9-72.9)$ \\
\hline Cleveland-Elyria, Ohio & 1,767 & 70.1 & 1.5 & $(67.1-73.2)$ \\
\hline Colorado Springs, Colorado & 1,157 & 69.5 & 1.7 & $(66.1-72.9)$ \\
\hline Columbia, South Carolina & 1,787 & 63.5 & 1.7 & $(60.2-66.8)$ \\
\hline Columbus, Ohio & 1,593 & 71.1 & 1.5 & $(68.2-73.9)$ \\
\hline Concord, New Hampshire & 700 & 73.2 & 2.3 & $(68.6-77.7)$ \\
\hline Dallas-Plano-Irving, Texas & 899 & 60.5 & 2.1 & $(56.4-64.5)$ \\
\hline Dayton, Ohio & 848 & 66.4 & 2.2 & $(62.1-70.8)$ \\
\hline Denver-Aurora-Lakewood, Colorado & 4,821 & 66.3 & 0.9 & $(64.6-68.0)$ \\
\hline Des Moines-West Des Moines, lowa & 1,151 & 74.1 & 1.6 & $(70.8-77.3)$ \\
\hline
\end{tabular}

See table footnotes on page 43. 
TABLE 17. (Continued) Estimated prevalence of adults aged $\geq 18$ years who have visited a dentist, dental hygienist, or dental clinic within the past year, by metropolitan and micropolitan statistical area Behavioral Risk Factor Surveillance System, United States, 2012

\begin{tabular}{|c|c|c|c|c|}
\hline MMSA(s) & $\begin{array}{l}\text { Sample } \\
\text { size }\end{array}$ & $\%$ & SE & $95 \% \mathrm{Cl}$ \\
\hline Detroit-Dearborn-Livonia, Michigan? & 2,194 & 62.4 & 1.7 & $(59.1-65.7)$ \\
\hline Dover, Delaware & 1,436 & 65.7 & 1.9 & $(62.0-69.3)$ \\
\hline Duluth, Minnesota-Wisconsin & 512 & 73.9 & 2.7 & $(68.7-79.1)$ \\
\hline Durham-Chapel Hill, North Carolina & 785 & 69.3 & 2.1 & $(65.1-73.5)$ \\
\hline El Paso, Texas & 618 & 48.8 & 3.2 & $(42.4-55.1)$ \\
\hline Eugene, Oregon & 521 & 63.9 & 2.9 & $(58.3-69.5)$ \\
\hline Fairbanks, Alaska & 595 & 62.4 & 2.6 & $(57.3-67.6)$ \\
\hline Fargo, North Dakota-Minnesota & 934 & 74.3 & 2.1 & (70.3-78.4) \\
\hline Farmington, New Mexico & 649 & 57.6 & 2.5 & $(52.7-62.4)$ \\
\hline Fayetteville, North Carolina & 500 & 69.4 & 2.6 & $(64.4-74.5)$ \\
\hline $\begin{array}{l}\text { Fayetteville-Springdale-Rogers, } \\
\text { Arkansas-Missouri }\end{array}$ & 802 & 56.1 & 2.7 & $(50.7-61.5)$ \\
\hline Fort Collins, Colorado & 595 & 72.9 & 2.3 & $(68.4-77.3)$ \\
\hline Fort Wayne, Indiana & 531 & 67.7 & 2.7 & $(62.5-72.9)$ \\
\hline Fort Worth-Arlington, Texas* & 723 & 59.9 & 2.4 & $(55.2-64.6)$ \\
\hline Grand Island, Nebraska & 849 & 63.4 & 2.4 & $(58.7-68.0)$ \\
\hline Grand Rapids-Wyoming, Michigan & 882 & 72.4 & 2.2 & $(68.1-76.6)$ \\
\hline Great Falls, Montana & 704 & 61.4 & 2.4 & $(56.7-66.1)$ \\
\hline Greeley, Colorado & 534 & 60.6 & 2.7 & $(55.3-65.8)$ \\
\hline $\begin{array}{l}\text { Greensboro-High Point, } \\
\text { North Carolina }\end{array}$ & 794 & 60.8 & 2.2 & $(56.6-65.1)$ \\
\hline $\begin{array}{l}\text { Greenville-Anderson-Mauldin, } \\
\text { South Carolina }\end{array}$ & 1,683 & 59.6 & 1.6 & $(56.4-62.7)$ \\
\hline Gulfport-Biloxi-Pascagoula, Mississippi & 735 & 58.7 & 2.4 & $(54.1-63.4)$ \\
\hline $\begin{array}{l}\text { Hagerstown-Martinsburg, Maryland- } \\
\text { West Virginia }\end{array}$ & 752 & 72.1 & 2.4 & $(67.3-76.9)$ \\
\hline Harrisburg-Carlisle, Pennsylvania & 660 & 74.8 & 2.0 & $(70.8-78.8)$ \\
\hline $\begin{array}{l}\text { Hartford-West Hartford-East Hartford, } \\
\text { Connecticut }\end{array}$ & 2,644 & 80.7 & 1.1 & $(78.6-82.7)$ \\
\hline Heber, Utah & 504 & 71.0 & 4.8 & $(61.5-80.5)$ \\
\hline Hilo, Hawaii & 1,343 & 66.7 & 1.8 & $(63.2-70.3)$ \\
\hline $\begin{array}{l}\text { Hilton Head Island-Bluffton-Beaufort, } \\
\text { South Carolina }\end{array}$ & 934 & 68.4 & 3.1 & $(62.3-74.5)$ \\
\hline $\begin{array}{l}\text { Houston-The Woodlands-Sugar Land, } \\
\text { Texas }\end{array}$ & 1,128 & 60.3 & 1.8 & $(56.8-63.9)$ \\
\hline $\begin{array}{l}\text { Huntington-Ashland, West } \\
\text { Virginia-Kentucky-Ohio }\end{array}$ & 1,101 & 57.6 & 2.0 & $(53.7-61.5)$ \\
\hline Huntsville, Alabama & 610 & 66.4 & 2.6 & $(61.4-71.5)$ \\
\hline Idaho Falls, Idaho & 538 & 68.8 & 3.4 & $(62.2-75.5)$ \\
\hline $\begin{array}{l}\text { Indianapolis-Carmel-Anderson, } \\
\text { Indiana }\end{array}$ & 2,185 & 68.3 & 1.3 & $(65.8-70.8)$ \\
\hline Jackson, Mississippi & 912 & 60.5 & 2.0 & $(56.6-64.5)$ \\
\hline Jacksonville, Florida & 516 & 62.8 & 3.2 & $(56.5-69.0)$ \\
\hline Kahului-Wailuku-Lahaina, Hawaii & 1,215 & 70.2 & 2.0 & $(66.3-74.1)$ \\
\hline Kalispell, Montana & 555 & 60.7 & 2.4 & $(55.9-65.5)$ \\
\hline Kansas City, Missouri-Kansas & 4,712 & 68.7 & 1.3 & $(66.2-71.2)$ \\
\hline Kapaa, Hawaii & 668 & 64.6 & 2.9 & $(58.9-70.3)$ \\
\hline Keene, New Hampshire & 537 & 74.1 & 2.9 & $(68.4-79.7)$ \\
\hline Kennewick-Richland, Washington & 533 & 66.4 & 2.7 & $(61.1-71.8)$ \\
\hline $\begin{array}{l}\text { Kingsport-Bristol-Bristol, } \\
\text { Tennessee-Virginia }\end{array}$ & 556 & 56.9 & 3.3 & $(50.4-63.4)$ \\
\hline Knoxville, Tennessee & 825 & 61.7 & 2.3 & $(57.2-66.2)$ \\
\hline Laconia, New Hampshire & 560 & 62.0 & 3.6 & $(54.8-69.1)$ \\
\hline Lafayette, Louisiana & 544 & 59.5 & 2.8 & $(53.9-65.0)$ \\
\hline Las Cruces, New Mexico & 701 & 61.8 & 2.4 & $(57.1-66.5)$ \\
\hline $\begin{array}{l}\text { Las Vegas-Henderson-Paradise, } \\
\text { Nevada }\end{array}$ & 1,991 & 60.2 & 1.4 & $(57.6-62.9)$ \\
\hline Lewiston-Auburn, Maine & 697 & 61.1 & 2.2 & $(56.8-65.5)$ \\
\hline Lexington-Fayette, Kentucky & 525 & 67.5 & 2.6 & $(62.4-72.6)$ \\
\hline Lincoln, Nebraska & 1,660 & 73.1 & 1.3 & $(70.5-75.6)$ \\
\hline
\end{tabular}

See table footnotes on page 43.
TABLE 17. (Continued) Estimated prevalence of adults aged $\geq 18$ years who have visited a dentist, dental hygienist, or dental clinic within the past year, by metropolitan and micropolitan statistical area Behavioral Risk Factor Surveillance System, United States, 2012

\begin{tabular}{|c|c|c|c|c|}
\hline MMSA(s) & $\begin{array}{l}\text { Sample } \\
\text { size }\end{array}$ & $\%$ & SE & $95 \% \mathrm{Cl}$ \\
\hline $\begin{array}{l}\text { Little Rock-North Little Rock-Conway, } \\
\text { Arkansas }\end{array}$ & 1,163 & 61.0 & 1.9 & $(57.2-64.8)$ \\
\hline Logan, Utah-Idaho & 499 & 72.2 & 2.7 & $(66.9-77.5)$ \\
\hline $\begin{array}{l}\text { Los Angeles-Long Beach-Glendale, } \\
\text { California* }\end{array}$ & 3,497 & 63.8 & 1.2 & $(61.5-66.1)$ \\
\hline $\begin{array}{l}\text { Louisville/Jefferson County, } \\
\text { Kentucky-Indiana }\end{array}$ & 2,155 & 64.3 & 1.6 & $(61.2-67.5)$ \\
\hline Lumberton, North Carolina & 541 & 48.4 & 4.3 & $(39.9-56.9)$ \\
\hline Manchester-Nashua, New Hampshire & 1,891 & 74.6 & 1.4 & (71.9-77.3) \\
\hline McAllen-Edinburg-Mission, Texas & 610 & 44.8 & 3.2 & $(38.6-51.0)$ \\
\hline $\begin{array}{l}\text { Memphis, } \\
\text { Tennessee-Mississippi-Arkansas }\end{array}$ & 1,288 & 62.8 & 2.0 & $(58.9-66.6)$ \\
\hline $\begin{array}{l}\text { Miami-Fort Lauderdale-West Palm } \\
\text { Beach, Florida }\end{array}$ & 1,662 & 64.0 & 1.8 & $(60.4-67.6)$ \\
\hline $\begin{array}{l}\text { Milwaukee-Waukesha-West Allis, } \\
\text { Wisconsin }\end{array}$ & 1,245 & 73.6 & 1.9 & $(69.9-77.3)$ \\
\hline $\begin{array}{l}\text { Minneapolis-St. Paul-Bloomington, } \\
\text { Minnesota-Wisconsin }\end{array}$ & 7,860 & 75.7 & 0.8 & $(74.2-77.2)$ \\
\hline Missoula, Montana & 778 & 61.2 & 2.2 & $(56.8-65.6)$ \\
\hline Mobile, Alabama & 805 & 53.4 & 2.8 & $(47.9-58.8)$ \\
\hline Montgomery, Alabama & 531 & 61.2 & 3.0 & $(55.4-67.1)$ \\
\hline $\begin{array}{l}\text { Montgomery County-Bucks County- } \\
\text { Chester County, Pennsylvania** }\end{array}$ & 1,309 & 76.1 & 1.5 & $(73.1-79.1)$ \\
\hline $\begin{array}{l}\text { Myrtle Beach-Conway-North Myrtle } \\
\text { Beach, South Carolina-North Carolina }\end{array}$ & 941 & 61.7 & 2.1 & $(57.5-65.8)$ \\
\hline $\begin{array}{l}\text { Nashville-Davidson-Murfreesboro- } \\
\text { Franklin, Tennessee }\end{array}$ & 1,325 & 67.9 & 1.6 & $(64.8-71.1)$ \\
\hline $\begin{array}{l}\text { Nassau County-Suffolk County, } \\
\text { New York* }\end{array}$ & 897 & 69.2 & 2.1 & $(65.1-73.3)$ \\
\hline Newark, New Jersey-Pennsylvania* & 6,515 & 72.4 & 0.9 & $(70.6-74.2)$ \\
\hline New Haven-Milford, Connecticut & 1,993 & 72.6 & 1.4 & $(69.8-75.5)$ \\
\hline New Orleans-Metairie, Louisiana & 1,263 & 58.9 & 1.9 & $(55.1-62.7)$ \\
\hline $\begin{array}{l}\text { New York-Jersey City-White Plains, } \\
\text { New York-New Jersey* }\end{array}$ & 7,381 & 66.6 & 1.1 & $(64.5-68.7)$ \\
\hline Norfolk, Nebraska & 565 & 60.7 & 3.0 & $(54.9-66.4)$ \\
\hline North Platte, Nebraska & 612 & 60.3 & 3.0 & $(54.4-66.3)$ \\
\hline Norwich-New London, Connecticut & 995 & 77.2 & 2.1 & $(73.1-81.3)$ \\
\hline $\begin{array}{l}\text { Oakland-Hayward-Berkeley, } \\
\text { California* }\end{array}$ & 976 & 69.9 & 2.4 & $(65.2-74.6)$ \\
\hline Ocean City, New Jersey & 546 & 74.5 & 3.2 & $(68.1-80.8)$ \\
\hline Ogden-Clearfield, Utah & 2,408 & 71.4 & 1.2 & $(69.1-73.8)$ \\
\hline Oklahoma City, Oklahoma & 2,434 & 63.1 & 1.3 & $(60.6-65.5)$ \\
\hline Olympia-Tumwater, Washington & 515 & 71.4 & 2.7 & $(66.2-76.6)$ \\
\hline Omaha-Council Bluffs, Nebraska-lowa & 5,560 & 69.1 & 0.8 & $(67.4-70.7)$ \\
\hline Orlando-Kissimmee-Sanford, Florida & 567 & 57.5 & 3.1 & $(51.3-63.6)$ \\
\hline Philadelphia, Pennsylvania* & 2,435 & 63.5 & 1.4 & $(60.9-66.2)$ \\
\hline Phoenix-Mesa-Scottsdale, Arizona & 2,589 & 62.7 & 1.3 & $(60.2-65.3)$ \\
\hline Pittsburgh, Pennsylvania & 3,349 & 66.4 & 1.0 & $(64.3-68.4)$ \\
\hline Ponce, Puerto Rico & 547 & 65.6 & 2.4 & $(61.0-70.3)$ \\
\hline Portland-South Portland, Maine & 3,326 & 70.3 & 1.0 & $(68.4-72.3)$ \\
\hline $\begin{array}{l}\text { Portland-Vancouver-Hillsboro, } \\
\text { Oregon-Washington }\end{array}$ & 3,103 & 69.4 & 1.2 & $(67.2-71.7)$ \\
\hline $\begin{array}{l}\text { Providence-Warwick, Rhode } \\
\text { Island-Massachusetts }\end{array}$ & 8,051 & 73.4 & 0.8 & $(71.9-74.9)$ \\
\hline Provo-Orem, Utah & 1,728 & 68.9 & 1.5 & $(66.1-71.8)$ \\
\hline Raleigh, North Carolina & 932 & 71.7 & 1.8 & $(68.2-75.1)$ \\
\hline Rapid City, South Dakota & 1,049 & 69.0 & 1.9 & $(65.1-72.8)$ \\
\hline Reno, Nevada & 1,498 & 65.2 & 1.9 & $(61.5-68.8)$ \\
\hline Richmond, Virginia & 1,008 & 73.9 & 1.9 & (70.2-77.6) \\
\hline
\end{tabular}

See table footnotes on page 43. 
TABLE 17. (Continued) Estimated prevalence of adults aged $\geq 18$ years who have visited a dentist, dental hygienist, or dental clinic within the past year, by metropolitan and micropolitan statistical area Behavioral Risk Factor Surveillance System, United States, 2012

\begin{tabular}{|c|c|c|c|c|}
\hline MMSA(s) & $\begin{array}{l}\text { Sample } \\
\text { size }\end{array}$ & $\%$ & SE & $95 \% \mathrm{Cl}$ \\
\hline $\begin{array}{l}\text { Riverside-San Bernardino-Ontario, } \\
\text { California }\end{array}$ & 1,529 & 63.0 & 1.7 & $(59.6-66.4)$ \\
\hline $\begin{array}{l}\text { Rockingham County-Strafford County, } \\
\text { New Hampshire* }\end{array}$ & 1,654 & 75.5 & 1.5 & $(72.6-78.4)$ \\
\hline Rutland, Vermont & 591 & 64.9 & 2.7 & $(59.6-70.3)$ \\
\hline $\begin{array}{l}\text { Sacramento-Roseville-Arden-Arcade, } \\
\text { California }\end{array}$ & 998 & 72.2 & 2.1 & $(68.2-76.3)$ \\
\hline St. Louis, Missouri-Illinois & 2,052 & 69.1 & 1.5 & $(66.1-72.0)$ \\
\hline Salisbury, Maryland-Delaware & 2,110 & 65.1 & 1.9 & $(61.3-68.9)$ \\
\hline Salt Lake City, Utah & 4,565 & 67.3 & 1.0 & $(65.5-69.2)$ \\
\hline San Antonio-New Braunfels, Texas & 775 & 62.1 & 2.4 & $(57.4-66.8)$ \\
\hline San Diego-Carlsbad, California & 1,127 & 71.5 & 1.9 & $(67.7-75.3)$ \\
\hline $\begin{array}{l}\text { San Francisco-Redwood City-South } \\
\text { San Francisco, California** }\end{array}$ & 596 & 72.7 & 2.7 & $(67.4-78.0)$ \\
\hline $\begin{array}{l}\text { San Jose-Sunnyvale-Santa Clara, } \\
\text { California }\end{array}$ & 684 & 76.4 & 2.2 & $(72.1-80.7)$ \\
\hline San Juan-Carolina-Caguas, Puerto Rico & 3,918 & 69.0 & 0.9 & $(67.3-70.7)$ \\
\hline Santa Fe, New Mexico & 691 & 66.1 & 2.3 & $(61.7-70.5)$ \\
\hline Sayre, Pennsylvania & 1,848 & 66.3 & 2.1 & $(62.1-70.5)$ \\
\hline Scottsbluff, Nebraska & 625 & 59.6 & 3.2 & $(53.3-65.9)$ \\
\hline $\begin{array}{l}\text { Scranton-Wilkes-Barre-Hazleton, } \\
\text { Pennsylvania }\end{array}$ & 739 & 66.9 & 2.4 & $(62.2-71.6)$ \\
\hline Seattle-Bellevue-Everett, Washington* & 5,079 & 71.8 & 0.9 & $(70.1-73.5)$ \\
\hline Shreveport-Bossier City, Louisiana & 567 & 54.3 & 2.8 & $(48.7-59.8)$ \\
\hline $\begin{array}{l}\text { Silver Spring-Frederick-Rockville, } \\
\text { Maryland* }\end{array}$ & 2,289 & 77.9 & 1.5 & $(75.1-80.8)$ \\
\hline $\begin{array}{l}\text { Sioux City, lowa-Nebraska- } \\
\text { South Dakota }\end{array}$ & 1,200 & 68.0 & 2.9 & $(62.3-73.7)$ \\
\hline Sioux Falls, South Dakota & 1,458 & 74.0 & 1.6 & $(70.9-77.0)$ \\
\hline Spartanburg, South Carolina & 702 & 60.0 & 2.7 & $(54.7-65.3)$ \\
\hline Spokane-Spokane Valley, Washington & 1,095 & 64.7 & 2.0 & $(60.7-68.6)$ \\
\hline Springfield, Massachusetts & 2,326 & 72.7 & 1.5 & $(69.7-75.6)$ \\
\hline
\end{tabular}

TABLE 17. (Continued) Estimated prevalence of adults aged $\geq 18$ years who have visited a dentist, dental hygienist, or dental clinic within the past year, by metropolitan and micropolitan statistical area Behavioral Risk Factor Surveillance System, United States, 2012

\begin{tabular}{|c|c|c|c|c|}
\hline MMSA(s) & $\begin{array}{l}\text { Sample } \\
\text { size }\end{array}$ & $\%$ & SE & $95 \% \mathrm{Cl}$ \\
\hline Tacoma-Lakewood, Washington* & 1,172 & 67.0 & 1.8 & $(63.4-70.5)$ \\
\hline $\begin{array}{l}\text { Tampa-St. Petersburg-Clearwater, } \\
\text { Florida }\end{array}$ & 825 & 57.6 & 2.5 & $(52.8-62.4)$ \\
\hline Toledo, Ohio & 966 & 67.8 & 2.6 & $(62.8-72.9)$ \\
\hline Topeka, Kansas & 1,081 & 66.2 & 2.0 & $(62.4-70.1)$ \\
\hline Torrington, Connecticut & 660 & 78.1 & 2.2 & $(73.9-82.4)$ \\
\hline Trenton, New Jersey & 571 & 72.8 & 2.7 & $(67.5-78.1)$ \\
\hline Tucson, Arizona & 990 & 61.9 & 2.2 & $(57.6-66.2)$ \\
\hline Tulsa, Oklahoma & 1,738 & 61.0 & 1.5 & $(58.1-63.9)$ \\
\hline Tuscaloosa, Alabama & 574 & 59.4 & 3.4 & $(52.6-66.1)$ \\
\hline Urban Honolulu, Hawaii & 4,332 & 71.5 & 1.0 & $(69.5-73.5)$ \\
\hline Vineland-Bridgeton, New Jersey & 551 & 67.5 & 3.3 & $(61.0-74.0)$ \\
\hline $\begin{array}{l}\text { Virginia Beach-Norfolk-Newport News, } \\
\text { Virginia-North Carolina }\end{array}$ & 1,486 & 71.8 & 1.6 & $(68.6-75.0)$ \\
\hline $\begin{array}{l}\text { Warren-Troy-Farmington Hills, } \\
\text { Michigan* }\end{array}$ & 2,177 & 73.4 & 1.3 & $(70.9-75.9)$ \\
\hline $\begin{array}{l}\text { Washington-Arlington-Alexandria, } \\
\text { District of Columbia-Virginia- } \\
\text { Maryland-West Virginia* }\end{array}$ & 8,145 & 72.5 & 0.9 & $(70.8-74.3)$ \\
\hline Wichita, Kansas & 2,338 & 69.5 & 1.3 & $(66.9-72.0)$ \\
\hline $\begin{array}{l}\text { Wilmington, Delaware-Maryland- } \\
\text { New Jersey* }\end{array}$ & 3,213 & 71.9 & 1.2 & $(69.5-74.4)$ \\
\hline Winston-Salem, North Carolina & 779 & 67.9 & 2.1 & $(63.8-72.0)$ \\
\hline Worcester, Massachusetts-Connecticut & 2,825 & 73.7 & 1.3 & $(71.2-76.2)$ \\
\hline Yakima, Washington & 528 & 54.1 & 3.0 & $(48.1-60.1)$ \\
\hline $\begin{array}{l}\text { Youngstown-Warren-Boardman, } \\
\text { Ohio-Pennsylvania }\end{array}$ & 924 & 65.9 & 2.7 & $(60.5-71.3)$ \\
\hline Median & & 67.5 & & \\
\hline Range & & $44.8-81.7$ & & \\
\hline
\end{tabular}

Abbreviations: $\mathrm{Cl}=$ confidence interval; $\mathrm{MMSA}=$ metropolitan and micropolitan statistical area; SE = standard error.

* Metropolitan division. 
TABLE 18. Estimated prevalence of adults aged $\geq 18$ years who have visited a dentist, dental hygienist or dental clinic within the past year, by county - Behavioral Risk Factor Surveillance System, United States, 2012

\begin{tabular}{|c|c|c|c|c|}
\hline County & $\begin{array}{l}\text { Sample } \\
\text { size }\end{array}$ & $\%$ & $\mathrm{SE}$ & $95 \% \mathrm{Cl}$ \\
\hline Jefferson County, Alabama & 947 & 59.6 & 2.0 & $(55.7-63.5)$ \\
\hline Madison County, Alabama & 509 & 69.1 & 2.7 & $(63.7-74.5)$ \\
\hline Mobile County, Alabama & 805 & 53.4 & 2.8 & $(47.9-58.8)$ \\
\hline Anchorage Municipality, Alaska & 898 & 71.5 & 1.8 & $(68.0-75.0)$ \\
\hline Fairbanks North Star Borough, Alaska & 595 & 62.4 & 2.6 & $(57.3-67.6)$ \\
\hline Matanuska-Susitna Borough, Alaska & 597 & 62.5 & 2.6 & $(57.4-67.6)$ \\
\hline Maricopa County, Arizona & 2,109 & 63.3 & 1.4 & $(60.5-66.0)$ \\
\hline Pima County, Arizona & 990 & 61.9 & 2.2 & $(57.6-66.2)$ \\
\hline Pulaski County, Arkansas & 741 & 61.8 & 2.6 & $(56.6-66.9)$ \\
\hline Alameda County, California & 619 & 67.1 & 3.1 & $(61.1-73.2)$ \\
\hline Los Angeles County, California & 3,497 & 63.8 & 1.2 & $(61.5-66.1)$ \\
\hline Orange County, California & 1,037 & 73.0 & 2.0 & $(69.1-77.0)$ \\
\hline Riverside County, California & 824 & 63.8 & 2.4 & $(59.2-68.4)$ \\
\hline Sacramento County, California & 609 & 72.6 & 2.6 & $(67.4-77.7)$ \\
\hline San Bernardino County, California & 705 & 62.5 & 2.5 & $(57.6-67.4)$ \\
\hline San Diego County, California & 1,127 & 71.5 & 1.9 & $(67.7-75.3)$ \\
\hline Santa Clara County, California & 665 & 76.3 & 2.3 & (71.9-80.8) \\
\hline Adams County, Colorado & 800 & 56.9 & 2.3 & $(52.4-61.4)$ \\
\hline Arapahoe County, Colorado & 854 & 68.9 & 1.9 & $(65.1-72.7)$ \\
\hline Boulder County, Colorado & 514 & 71.4 & 2.6 & $(66.3-76.5)$ \\
\hline Denver County, Colorado & 988 & 62.2 & 1.9 & $(58.5-65.8)$ \\
\hline Douglas County, Colorado & 543 & 79.0 & 2.2 & (74.8-83.3) \\
\hline El Paso County, Colorado & 1,017 & 70.0 & 1.8 & $(66.5-73.5)$ \\
\hline Jefferson County, Colorado & 1,115 & 70.5 & 1.7 & $(67.2-73.9)$ \\
\hline Larimer County, Colorado & 595 & 72.9 & 2.3 & $(68.4-77.3)$ \\
\hline Weld County, Colorado & 534 & 60.6 & 2.7 & $(55.3-65.8)$ \\
\hline Fairfield County, Connecticut & 2,165 & 73.1 & 1.5 & $(70.2-76.1)$ \\
\hline Hartford County, Connecticut & 1,965 & 80.8 & 1.3 & (78.3-83.3) \\
\hline Litchfield County, Connecticut & 660 & 78.1 & 2.2 & $(73.9-82.4)$ \\
\hline New Haven County, Connecticut & 1,993 & 72.6 & 1.4 & $(69.8-75.5)$ \\
\hline New London County, Connecticut & 995 & 77.2 & 2.1 & (73.1-81.3) \\
\hline Kent County, Delaware & 1,436 & 65.7 & 1.9 & $(62.0-69.3)$ \\
\hline New Castle County, Delaware & 2,323 & 73.7 & 1.2 & $(71.3-76.1)$ \\
\hline Sussex County, Delaware & 1,393 & 63.9 & 1.9 & $(60.3-67.6)$ \\
\hline $\begin{array}{l}\text { District of Columbia, } \\
\text { District of Columbia }\end{array}$ & 3,801 & 71.1 & 1.2 & $(68.6-73.5)$ \\
\hline Broward County, Florida & 528 & 64.2 & 3.3 & $(57.8-70.5)$ \\
\hline Miami-Dade County, Florida & 809 & 61.1 & 2.8 & $(55.6-66.7)$ \\
\hline Hawaii County, Hawaii & 1,343 & 66.7 & 1.8 & $(63.2-70.3)$ \\
\hline Honolulu County, Hawaii & 4,332 & 71.5 & 1.0 & $(69.5-73.5)$ \\
\hline Kauai County, Hawaii & 668 & 64.6 & 2.9 & $(58.9-70.3)$ \\
\hline Maui County, Hawaii & 1,215 & 70.2 & 2.0 & $(66.3-74.1)$ \\
\hline Ada County, Idaho & 805 & 73.3 & 2.3 & $(68.8-77.8)$ \\
\hline Canyon County, Idaho & 501 & 62.1 & 3.6 & $(55.0-69.1)$ \\
\hline Cook County, Illinois & 1,500 & 65.8 & 1.7 & $(62.4-69.2)$ \\
\hline Lake County, Indiana & 878 & 54.6 & 2.8 & $(49.0-60.1)$ \\
\hline Marion County, Indiana & 1,269 & 64.1 & 1.8 & $(60.5-67.7)$ \\
\hline Polk County, lowa & 802 & 74.4 & 2.0 & $(70.6-78.2)$ \\
\hline Johnson County, Kansas & 2,166 & 76.6 & 1.3 & $(74.0-79.2)$ \\
\hline Sedgwick County, Kansas & 1,783 & 69.5 & 1.5 & $(66.6-72.4)$ \\
\hline Shawnee County, Kansas & 767 & 67.7 & 2.3 & $(63.1-72.3)$ \\
\hline Wyandotte County, Kansas & 865 & 53.9 & 3.1 & $(47.8-59.9)$ \\
\hline Jefferson County, Kentucky & 1,670 & 64.9 & 2.0 & $(61.0-68.8)$ \\
\hline East Baton Rouge Parish, Louisiana & 565 & 63.6 & 2.7 & $(58.2-69.0)$ \\
\hline Androscoggin County, Maine & 697 & 61.1 & 2.2 & $(56.8-65.5)$ \\
\hline Aroostook County, Maine & 534 & 51.3 & 2.6 & $(46.1-56.4)$ \\
\hline Cumberland County, Maine & 1,753 & 72.4 & 1.3 & $(69.8-75.1)$ \\
\hline Kennebec County, Maine & 828 & 66.8 & 2.0 & $(62.9-70.8)$ \\
\hline Penobscot County, Maine & 924 & 63.2 & 2.0 & $(59.3-67.2)$ \\
\hline York County, Maine & 1,192 & 68.6 & 1.6 & $(65.4-71.8)$ \\
\hline
\end{tabular}

See table footnotes on page 45.
TABLE 18. (Continued) Estimated prevalence of adults aged $\geq 18$ years who have visited a dentist, dental hygienist or dental clinic within the past year, by county - Behavioral Risk Factor Surveillance System, United States, 2012

\begin{tabular}{|c|c|c|c|c|}
\hline County & $\begin{array}{l}\text { Sample } \\
\text { size }\end{array}$ & $\%$ & SE & $95 \% \mathrm{Cl}$ \\
\hline Anne Arundel County, Maryland & 890 & 70.1 & 2.4 & $(65.4-74.8)$ \\
\hline Baltimore County, Maryland & 1,505 & 73.2 & 1.7 & $(69.8-76.6)$ \\
\hline Charles County, Maryland & 509 & 68.2 & 3.8 & $(60.9-75.6)$ \\
\hline Frederick County, Maryland & 752 & 79.1 & 2.5 & $(74.2-84.1)$ \\
\hline Montgomery County, Maryland & 1,537 & 77.7 & 1.7 & $(74.4-81.0)$ \\
\hline Prince George's County, Maryland & 1,127 & 67.8 & 2.3 & $(63.3-72.4)$ \\
\hline Washington County, Maryland & 532 & 78.9 & 2.7 & $(73.6-84.2)$ \\
\hline Baltimore city, Maryland & 735 & 69.8 & 2.6 & $(64.7-75.0)$ \\
\hline Barnstable County, Massachusetts & 558 & 81.7 & 2.1 & $(77.5-85.8)$ \\
\hline Bristol County, Massachusetts & 2,624 & 73.2 & 1.5 & $(70.1-76.2)$ \\
\hline Essex County, Massachusetts & 2,545 & 79.5 & 1.3 & $(77.0-82.0)$ \\
\hline Hampden County, Massachusetts & 1,944 & 70.5 & 1.8 & $(67.0-73.9)$ \\
\hline Middlesex County, Massachusetts & 4,330 & 79.0 & 0.9 & $(77.2-80.8)$ \\
\hline Norfolk County, Massachusetts & 1,751 & 81.7 & 1.3 & $(79.1-84.3)$ \\
\hline Plymouth County, Massachusetts & 1,823 & 74.6 & 1.7 & $(71.3-77.9)$ \\
\hline Suffolk County, Massachusetts & 2,283 & 72.9 & 1.4 & $(70.1-75.6)$ \\
\hline Worcester County, Massachusetts & 2,583 & 73.8 & 1.4 & $(71.1-76.4)$ \\
\hline Kent County, Michigan & 518 & 74.8 & 2.8 & $(69.4-80.2)$ \\
\hline Macomb County, Michigan & 609 & 72.7 & 2.2 & $(68.4-77.1)$ \\
\hline Oakland County, Michigan & 1,166 & 74.5 & 1.8 & $(70.9-78.1)$ \\
\hline Wayne County, Michigan & 2,194 & 62.4 & 1.7 & $(59.1-65.6)$ \\
\hline Anoka County, Minnesota & 541 & 77.3 & 2.3 & $(72.8-81.9)$ \\
\hline Dakota County, Minnesota & 672 & 78.2 & 2.0 & $(74.2-82.2)$ \\
\hline Hennepin County, Minnesota & 3,284 & 75.7 & 1.2 & $(73.4-77.9)$ \\
\hline Ramsey County, Minnesota & 1,939 & 71.3 & 2.2 & $(67.1-75.6)$ \\
\hline Jackson County, Missouri & 880 & 69.1 & 2.6 & $(64.0-74.2)$ \\
\hline St. Louis County, Missouri & 944 & 71.4 & 2.1 & $(67.3-75.4)$ \\
\hline Cascade County, Montana & 704 & 61.4 & 2.4 & $(56.7-66.1)$ \\
\hline Flathead County, Montana & 555 & 60.7 & 2.4 & $(55.9-65.5)$ \\
\hline Hill County, Montana & 578 & 61.6 & 3.5 & $(54.8-68.4)$ \\
\hline Lake County, Montana & 890 & 57.2 & 2.7 & $(51.8-62.6)$ \\
\hline Missoula County, Montana & 778 & 61.2 & 2.2 & $(56.8-65.6)$ \\
\hline Yellowstone County, Montana & 749 & 62.5 & 2.0 & $(58.5-66.4)$ \\
\hline Dakota County, Nebraska & 731 & 57.9 & 4.7 & $(48.7-67.1)$ \\
\hline Douglas County, Nebraska & 3,552 & 68.4 & 1.0 & $(66.4-70.4)$ \\
\hline Hall County, Nebraska & 531 & 64.6 & 3.0 & $(58.8-70.5)$ \\
\hline Lancaster County, Nebraska & 1,438 & 72.8 & 1.4 & $(70.1-75.5)$ \\
\hline Lincoln County, Nebraska & 588 & 60.3 & 3.1 & $(54.3-66.4)$ \\
\hline Sarpy County, Nebraska & 1,171 & 73.7 & 1.7 & $(70.3-77.0)$ \\
\hline Scotts Bluff County, Nebraska & 573 & 58.2 & 3.3 & $(51.7-64.7)$ \\
\hline Clark County, Nevada & 1,991 & 60.2 & 1.4 & $(57.6-62.9)$ \\
\hline Washoe County, Nevada & 1,479 & 65.0 & 1.9 & $(61.3-68.7)$ \\
\hline Belknap County, New Hampshire & 560 & 62.0 & 3.6 & $(54.8-69.1)$ \\
\hline Carroll County, New Hampshire & 526 & 73.5 & 3.0 & $(67.7-79.3)$ \\
\hline Cheshire County, New Hampshire & 537 & 74.1 & 2.9 & $(68.4-79.7)$ \\
\hline Coos County, New Hampshire & 532 & 56.6 & 3.3 & $(50.1-63.2)$ \\
\hline Grafton County, New Hampshire & 561 & 70.7 & 2.9 & $(65.0-76.4)$ \\
\hline Hillsborough County, New Hampshire & 1,891 & 74.6 & 1.4 & $(71.9-77.3)$ \\
\hline Merrimack County, New Hampshire & 700 & 73.2 & 2.3 & $(68.6-77.7)$ \\
\hline Rockingham County, New Hampshire & 1,039 & 77.6 & 1.7 & $(74.2-81.1)$ \\
\hline Strafford County, New Hampshire & 615 & 70.2 & 2.9 & $(64.6-75.8)$ \\
\hline Atlantic County, New Jersey & 1,014 & 70.6 & 2.1 & $(66.4-74.7)$ \\
\hline Bergen County, New Jersey & 997 & 71.9 & 1.9 & $(68.1-75.7)$ \\
\hline Burlington County, New Jersey & 681 & 74.5 & 2.5 & $(69.7-79.3)$ \\
\hline Camden County, New Jersey & 738 & 68.6 & 2.4 & $(63.9-73.3)$ \\
\hline Cape May County, New Jersey & 546 & 74.5 & 3.2 & $(68.1-80.8)$ \\
\hline Cumberland County, New Jersey & 551 & 67.5 & 3.3 & $(61.0-74.0)$ \\
\hline Essex County, New Jersey & 1,327 & 66.0 & 1.8 & $(62.6-69.5)$ \\
\hline Gloucester County, New Jersey & 549 & 74.7 & 2.6 & $(69.7-79.7)$ \\
\hline Hudson County, New Jersey & 1,296 & 63.7 & 1.8 & $(60.1-67.3)$ \\
\hline
\end{tabular}

See table footnotes on page 45 . 
TABLE 18. (Continued) Estimated prevalence of adults aged $\geq 18$ years who have visited a dentist, dental hygienist or dental clinic within the past year, by county - Behavioral Risk Factor Surveillance System, United States, 2012

\begin{tabular}{|c|c|c|c|c|}
\hline County & $\begin{array}{l}\text { Sample } \\
\text { size }\end{array}$ & $\%$ & SE & $95 \% \mathrm{Cl}$ \\
\hline Hunterdon County, New Jersey & 569 & 79.8 & 2.4 & $(75.0-84.6)$ \\
\hline Mercer County, New Jersey & 571 & 72.8 & 2.7 & $(67.5-78.1)$ \\
\hline Middlesex County, New Jersey & 876 & 69.2 & 2.3 & $(64.8-73.6)$ \\
\hline Monmouth County, New Jersey & 708 & 76.6 & 2.2 & $(72.3-80.8)$ \\
\hline Morris County, New Jersey & 846 & 80.1 & 1.9 & $(76.4-83.9)$ \\
\hline Ocean County, New Jersey & 656 & 74.3 & 2.3 & $(69.8-78.9)$ \\
\hline Passaic County, New Jersey & 681 & 64.8 & 2.5 & $(59.9-69.7)$ \\
\hline Salem County, New Jersey & 579 & 64.9 & 4.0 & $(57.0-72.7)$ \\
\hline Somerset County, New Jersey & 638 & 79.2 & 2.4 & $(74.5-83.9)$ \\
\hline Sussex County, New Jersey & 544 & 67.4 & 2.8 & $(61.9-73.0)$ \\
\hline Union County, New Jersey & 708 & 69.5 & 2.3 & $(65.1-74.0)$ \\
\hline Warren County, New Jersey & 523 & 72.8 & 2.8 & $(67.3-78.4)$ \\
\hline Bernalillo County, New Mexico & 2,048 & 65.0 & 1.3 & $(62.5-67.5)$ \\
\hline Dona Ana County, New Mexico & 701 & 61.8 & 2.4 & $(57.1-66.5)$ \\
\hline Sandoval County, New Mexico & 656 & 63.7 & 2.4 & $(58.9-68.5)$ \\
\hline San Juan County, New Mexico & 649 & 57.6 & 2.5 & $(52.7-62.4)$ \\
\hline Santa Fe County, New Mexico & 691 & 66.1 & 2.3 & $(61.7-70.5)$ \\
\hline Kings County, New York & 499 & 64.1 & 3.0 & $(58.3-70.0)$ \\
\hline Guilford County, North Carolina & 505 & 63.3 & 2.7 & $(58.1-68.6)$ \\
\hline Mecklenburg County, North Carolina & 721 & 69.0 & 2.1 & $(65.0-73.0)$ \\
\hline Robeson County, North Carolina & 541 & 48.4 & 4.3 & $(39.9-56.9)$ \\
\hline Wake County, North Carolina & 693 & 75.1 & 2.0 & $(71.2-79.0)$ \\
\hline Burleigh County, North Dakota & 568 & 75.0 & 2.6 & $(70.0-80.0)$ \\
\hline Cass County, North Dakota & 838 & 72.7 & 2.2 & $(68.4-77.0)$ \\
\hline Cuyahoga County, Ohio & 858 & 68.4 & 2.1 & $(64.3-72.4)$ \\
\hline Franklin County, Ohio & 833 & 71.5 & 1.9 & $(67.7-75.3)$ \\
\hline Hamilton County, Ohio & 768 & 73.0 & 1.9 & $(69.2-76.8)$ \\
\hline Lorain County, Ohio & 609 & 75.6 & 3.0 & $(69.7-81.6)$ \\
\hline Lucas County, Ohio & 619 & 65.5 & 3.2 & $(59.2-71.8)$ \\
\hline Mahoning County, Ohio & 588 & 68.7 & 3.3 & $(62.3-75.1)$ \\
\hline Montgomery County, Ohio & 657 & 65.5 & 2.6 & $(60.5-70.6)$ \\
\hline Stark County, Ohio & 620 & 65.4 & 2.7 & $(60.2-70.7)$ \\
\hline Summit County, Ohio & 627 & 67.1 & 2.7 & $(61.8-72.4)$ \\
\hline Oklahoma County, Oklahoma & 1,189 & 61.9 & 1.7 & $(58.5-65.2)$ \\
\hline Tulsa County, Oklahoma & 1,200 & 61.2 & 1.8 & $(57.7-64.6)$ \\
\hline Clackamas County, Oregon & 506 & 69.9 & 3.0 & $(64.1-75.8)$ \\
\hline Lane County, Oregon & 521 & 63.9 & 2.9 & $(58.3-69.5)$ \\
\hline Multnomah County, Oregon & 885 & 70.4 & 2.1 & $(66.3-74.4)$ \\
\hline Washington County, Oregon & 610 & 71.4 & 2.5 & $(66.6-76.3)$ \\
\hline Allegheny County, Pennsylvania & 1,881 & 68.9 & 1.4 & $(66.2-71.6)$ \\
\hline Bradford County, Pennsylvania & 1,848 & 66.3 & 2.1 & $(62.1-70.5)$ \\
\hline Montgomery County, Pennsylvania & 546 & 76.3 & 2.2 & (71.9-80.6) \\
\hline Philadelphia County, Pennsylvania & 2,043 & 61.7 & 1.5 & $(58.7-64.7)$ \\
\hline Pike County, Pennsylvania & 1,883 & 73.4 & 1.6 & $(70.2-76.6)$ \\
\hline Kent County, Rhode Island & 803 & 74.5 & 2.4 & $(69.9-79.1)$ \\
\hline Providence County, Rhode Island & 3,313 & 70.5 & 1.1 & $(68.2-72.7)$ \\
\hline Washington County, Rhode Island & 653 & 78.3 & 2.5 & $(73.5-83.1)$ \\
\hline
\end{tabular}

TABLE 18. (Continued) Estimated prevalence of adults aged $\geq 18$ years who have visited a dentist, dental hygienist or dental clinic within the past year, by county - Behavioral Risk Factor Surveillance System, United States, 2012

\begin{tabular}{|c|c|c|c|c|}
\hline County & $\begin{array}{l}\text { Sample } \\
\text { size }\end{array}$ & $\%$ & SE & $95 \% \mathrm{Cl}$ \\
\hline Aiken County, South Carolina & 553 & 60.8 & 3.0 & $(55.0-66.6)$ \\
\hline Beaufort County, South Carolina & 802 & 71.5 & 3.1 & $(65.4-77.6)$ \\
\hline Charleston County, South Carolina & 1,009 & 58.5 & 2.3 & $(53.9-63.1)$ \\
\hline Greenville County, South Carolina & 900 & 60.4 & 2.2 & $(56.1-64.7)$ \\
\hline Horry County, South Carolina & 772 & 60.5 & 2.3 & $(56.0-65.0)$ \\
\hline Richland County, South Carolina & 956 & 63.1 & 2.3 & $(58.5-67.7)$ \\
\hline Spartanburg County, South Carolina & 648 & 61.8 & 2.8 & $(56.4-67.3)$ \\
\hline Lincoln County, South Dakota & 496 & 79.2 & 2.5 & $(74.3-84.0)$ \\
\hline Minnehaha County, South Dakota & 876 & 73.1 & 1.8 & $(69.5-76.6)$ \\
\hline Pennington County, South Dakota & 604 & 68.5 & 2.3 & $(64.0-73.0)$ \\
\hline Davidson County, Tennessee & 553 & 65.3 & 2.6 & $(60.1-70.4)$ \\
\hline Shelby County, Tennessee & 524 & 65.7 & 2.6 & $(60.7-70.7)$ \\
\hline Bexar County, Texas & 583 & 60.7 & 2.8 & $(55.3-66.2)$ \\
\hline Dallas County, Texas & 506 & 52.1 & 2.8 & $(46.7-57.6)$ \\
\hline El Paso County, Texas & 617 & 48.9 & 3.2 & $(42.5-55.2)$ \\
\hline Harris County, Texas & 762 & 59.6 & 2.1 & $(55.4-63.8)$ \\
\hline Hidalgo County, Texas & 610 & 44.8 & 3.2 & $(38.6-51.0)$ \\
\hline Tarrant County, Texas & 569 & 59.0 & 2.7 & $(53.6-64.4)$ \\
\hline Travis County, Texas & 1,046 & 65.1 & 2.5 & $(60.3-70.0)$ \\
\hline Davis County, Utah & 1,143 & 73.0 & 1.7 & $(69.7-76.2)$ \\
\hline Salt Lake County, Utah & 4,009 & 67.3 & 1.0 & $(65.3-69.2)$ \\
\hline Tooele County, Utah & 556 & 69.1 & 3.3 & $(62.7-75.5)$ \\
\hline Utah County, Utah & 1,661 & 68.9 & 1.5 & $(66.1-71.8)$ \\
\hline Wasatch County, Utah & 504 & 71.0 & 4.8 & $(61.5-80.5)$ \\
\hline Weber County, Utah & 1,032 & 69.1 & 2.0 & $(65.3-73.0)$ \\
\hline Chittenden County, Vermont & 918 & 76.7 & 1.7 & $(73.4-80.1)$ \\
\hline Rutland County, Vermont & 591 & 64.9 & 2.7 & $(59.6-70.3)$ \\
\hline Washington County, Vermont & 514 & 73.9 & 2.5 & $(69.0-78.9)$ \\
\hline Windsor County, Vermont & 544 & 72.8 & 2.4 & $(68.1-77.4)$ \\
\hline Fairfax County, Virginia & 737 & 77.5 & 2.0 & $(73.6-81.4)$ \\
\hline Clark County, Washington & 789 & 67.1 & 2.2 & $(62.7-71.5)$ \\
\hline King County, Washington & 3,908 & 72.6 & 1.0 & $(70.6-74.6)$ \\
\hline Kitsap County, Washington & 564 & 67.0 & 2.8 & $(61.6-72.5)$ \\
\hline Pierce County, Washington & 1,172 & 67.0 & 1.8 & $(63.4-70.5)$ \\
\hline Snohomish County, Washington & 1,171 & 69.2 & 1.8 & $(65.6-72.7)$ \\
\hline Spokane County, Washington & 950 & 66.3 & 2.2 & $(62.1-70.6)$ \\
\hline Thurston County, Washington & 515 & 71.4 & 2.7 & $(66.2-76.6)$ \\
\hline Whatcom County, Washington & 844 & 67.5 & 2.6 & $(62.3-72.6)$ \\
\hline Yakima County, Washington & 528 & 54.1 & 3.0 & $(48.1-60.1)$ \\
\hline Kanawha County, West Virginia & 638 & 65.6 & 2.2 & $(61.4-69.9)$ \\
\hline Milwaukee County, Wisconsin & 951 & 66.8 & 2.4 & $(62.1-71.6)$ \\
\hline Laramie County, Wyoming & 954 & 71.5 & 2.4 & $(66.9-76.1)$ \\
\hline Natrona County, Wyoming & 816 & 67.2 & 2.7 & $(61.9-72.5)$ \\
\hline San Juan Municipio, Puerto Rico & 665 & 70.5 & 2.1 & $(66.4-74.7)$ \\
\hline Median & & 68.9 & & \\
\hline Range & & $44.8-81.7$ & & \\
\hline
\end{tabular}

Abbreviations: $\mathrm{Cl}=$ confidence interval; $\mathrm{SE}=$ standard error. 
TABLE 19. Estimated prevalence of adults $\geq 65$ years who have lost all of their natural teeth, by state/territory - Behavioral Risk Factor Surveillance System, United States, 2012

\begin{tabular}{|c|c|c|c|c|}
\hline State/Territory & $\begin{array}{l}\text { Sample } \\
\text { size }\end{array}$ & $\%$ & SE & $95 \% \mathrm{Cl}$ \\
\hline Alabama & 3,035 & 23.6 & 1.0 & $(21.5-25.6)$ \\
\hline Alaska & 736 & 15.6 & 1.9 & $(11.9-19.3)$ \\
\hline Arizona & 2,760 & 13.3 & 1.0 & $(11.3-15.2)$ \\
\hline Arkansas & 1,819 & 23.7 & 1.3 & $(21.2-26.2)$ \\
\hline California & 4,243 & 8.7 & 0.7 & $(7.4-10.0)$ \\
\hline Colorado & 3,398 & 12.4 & 0.7 & $(10.9-13.8)$ \\
\hline Connecticut & 2,581 & 13.6 & 1.0 & $(11.6-15.6)$ \\
\hline Delaware & 1,645 & 16.9 & 1.3 & $(14.4-19.4)$ \\
\hline District of Columbia & 1,164 & 13.1 & 1.5 & $(10.3-16.0)$ \\
\hline Florida & 2,955 & 15.8 & 1.1 & (13.7-17.9) \\
\hline Georgia & 1,828 & 18.4 & 1.2 & (16.1-20.6) \\
\hline Hawaii & 1,938 & 7.0 & 0.9 & $(5.3-8.7)$ \\
\hline Idaho & 2,134 & 16.1 & 1.3 & $(13.5-18.6)$ \\
\hline Illinois & 1,944 & 16.1 & 1.1 & $(13.9-18.2)$ \\
\hline Indiana & 2,641 & 19.7 & 1.0 & $(17.7-21.7)$ \\
\hline lowa & 2,421 & 17.3 & 0.9 & $(15.6-19.0)$ \\
\hline Kansas & 3,852 & 18.8 & 0.8 & (17.4-20.3) \\
\hline Kentucky & 3,202 & 24.8 & 1.2 & $(22.5-27.0)$ \\
\hline Louisiana & 3,105 & 28.7 & 1.3 & $(26.2-31.3)$ \\
\hline Maine & 3,075 & 22.1 & 0.9 & $(20.3-23.9)$ \\
\hline Maryland & 3,962 & 14.5 & 0.9 & $(12.8-16.3)$ \\
\hline Massachusetts & 6,058 & 15.5 & 0.7 & $(14.0-16.9)$ \\
\hline Michigan & 3,372 & 13.3 & 0.8 & $(11.8-14.9)$ \\
\hline Minnesota & 3,266 & 12.0 & 0.8 & (10.4-13.5) \\
\hline Mississippi & 2,770 & 25.0 & 1.0 & $(23.0-27.0)$ \\
\hline Missouri & 2,314 & 24.9 & 1.4 & $(22.1-27.6)$ \\
\hline Montana & 2,708 & 18.1 & 1.0 & (16.2-19.9) \\
\hline Nebraska & 6,502 & 13.4 & 0.6 & $(12.2-14.5)$ \\
\hline Nevada & 1,503 & 15.1 & 1.4 & (12.4-17.8) \\
\hline New Hampshire & 2,468 & 13.1 & 0.9 & (11.3-14.8) \\
\hline New Jersey & 4,159 & 14.0 & 0.8 & (12.4-15.5) \\
\hline New Mexico & 2,521 & 16.2 & 0.9 & (14.4-17.9) \\
\hline New York & 1,651 & 15.1 & 1.4 & $(12.3-17.8)$ \\
\hline North Carolina & 3,495 & 21.0 & 0.9 & $(19.2-22.8)$ \\
\hline North Dakota & 1,528 & 17.1 & 1.2 & $(14.8-19.4)$ \\
\hline Ohio & 3,859 & 20.3 & 1.0 & $(18.3-22.2)$ \\
\hline Oklahoma & 2,581 & 21.0 & 1.0 & (19.2-22.9) \\
\hline Oregon & 1,707 & 15.0 & 1.1 & $(12.9-17.1)$ \\
\hline Pennsylvania & 6,341 & 18.2 & 0.7 & (16.8-19.6) \\
\hline Rhode Island & 1,583 & 12.5 & 1.0 & (10.4-14.5) \\
\hline South Carolina & 4,178 & 19.5 & 1.0 & $(17.6-21.4)$ \\
\hline South Dakota & 2,287 & 19.4 & 1.7 & $(16.1-22.8)$ \\
\hline Tennessee & 2,276 & 24.8 & 1.3 & $(22.3-27.3)$ \\
\hline Texas & 2,604 & 13.4 & 1.0 & (11.4-15.4) \\
\hline Utah & 3,181 & 12.9 & 0.8 & (11.4-14.5) \\
\hline Vermont & 1,895 & 17.5 & 1.1 & (15.4-19.6) \\
\hline Virginia & 2,137 & 16.1 & 1.0 & $(14.1-18.0)$ \\
\hline Washington & 4,746 & 10.9 & 0.6 & $(9.7-12.2)$ \\
\hline West Virginia & 1,667 & 33.7 & 1.3 & $(31.1-36.4)$ \\
\hline Wisconsin & 1,579 & 13.5 & 1.5 & $(10.6-16.4)$ \\
\hline Wyoming & 2,277 & 17.7 & 1.2 & (15.4-19.9) \\
\hline Guam & 209 & 19.1 & 4.1 & $(11.2-27.1)$ \\
\hline Puerto Rico & 1,840 & 19.8 & 1.1 & $(17.7-22.0)$ \\
\hline Median & & 16.2 & & \\
\hline Range & & $7.0-33.7$ & & \\
\hline
\end{tabular}

Abbreviations: $\mathrm{Cl}=$ confidence interval; $\mathrm{SE}=$ standard error.
TABLE 20. Estimated prevalence of adults aged $\geq 65$ years who have lost all of their natural teeth, by metropolitan and micropolitan statistical area - Behavioral Risk Factor Surveillance System, United States, 2012

\begin{tabular}{|c|c|c|c|c|}
\hline MMSA(s) & $\begin{array}{l}\text { Sample } \\
\text { size }\end{array}$ & $\%$ & SE & $95 \% \mathrm{Cl}$ \\
\hline Aguadilla-Isabela, Puerto Rico & 158 & 26.4 & 3.9 & $(18.7-34.2)$ \\
\hline Akron, Ohio & 223 & 14.8 & 3.0 & $(8.9-20.6)$ \\
\hline Albuquerque, New Mexico & 880 & 14.8 & 1.5 & $(11.9-17.7)$ \\
\hline $\begin{array}{l}\text { Allentown-Bethlehem-Easton, } \\
\text { Pennsylvania-New Jersey }\end{array}$ & 422 & 14.9 & 2.1 & $(10.7-19.1)$ \\
\hline Anaheim-Santa Ana-Irvine, California* & 323 & 6.4 & 1.9 & $(2.7-10.0)$ \\
\hline Anchorage, Alaska & 230 & 12.7 & 2.9 & $(7.1-18.3)$ \\
\hline Asheville, North Carolina & 213 & 18.6 & 3.4 & $(11.9-25.2)$ \\
\hline $\begin{array}{l}\text { Atlanta-Sandy Springs-Roswell, } \\
\text { Georgia }\end{array}$ & 660 & 14.7 & 1.8 & $(11.2-18.1)$ \\
\hline Atlantic City-Hammonton, New Jersey & 338 & 17.9 & 2.8 & $(12.4-23.4)$ \\
\hline $\begin{array}{l}\text { Augusta-Richmond County, Georgia- } \\
\text { South Carolina }\end{array}$ & 342 & 21.3 & 4.1 & $(13.3-29.4)$ \\
\hline Augusta-Waterville, Maine & 215 & 28.7 & 3.8 & $(21.3-36.1)$ \\
\hline Austin-Round Rock, Texas & 391 & 9.8 & 2.2 & $(5.5-14.1)$ \\
\hline $\begin{array}{l}\text { Baltimore-Columbia-Towson, } \\
\text { Maryland }\end{array}$ & 1,354 & 15.9 & 1.4 & $(13.1-18.7)$ \\
\hline Bangor, Maine & 249 & 28.0 & 3.4 & $(21.4-34.7)$ \\
\hline Barnstable Town, Massachusetts & 260 & 12.2 & 3.0 & $(6.4-18.0)$ \\
\hline Barre, Vermont & 132 & 15.8 & 3.7 & $(8.5-23.2)$ \\
\hline Baton Rouge, Louisiana & 483 & 27.5 & 3.1 & $(21.4-33.5)$ \\
\hline Bellingham, Washington & 268 & 7.6 & 2.1 & $(3.5-11.7)$ \\
\hline Berlin, New Hampshire-Vermont & 260 & 25.3 & 3.1 & $(19.2-31.3)$ \\
\hline Billings, Montana & 249 & 21.7 & 2.9 & $(16.0-27.5)$ \\
\hline Birmingham-Hoover, Alabama & 566 & 23.4 & 2.3 & $(18.9-27.8)$ \\
\hline Bismarck, North Dakota & 245 & 17.0 & 3.4 & $(10.3-23.7)$ \\
\hline Boise City, Idaho & 493 & 13.6 & 2.3 & $(9.1-18.1)$ \\
\hline Boston, Massachusetts* & 1,620 & 13.0 & 1.2 & $(10.7-15.3)$ \\
\hline Boulder, Colorado & 127 & $\mathrm{~N} / \mathrm{A}^{\dagger}$ & $\mathrm{N} / \mathrm{A}$ & $(\mathrm{N} / \mathrm{A}-\mathrm{N} / \mathrm{A})$ \\
\hline Bremerton-Silverdale, Washington & 193 & 10.0 & 2.8 & $(4.6-15.5)$ \\
\hline $\begin{array}{l}\text { Bridgeport-Stamford-Norwalk, } \\
\text { Connecticut }\end{array}$ & 582 & 12.9 & 2.6 & $(7.9-17.9)$ \\
\hline Burlington-South Burlington, Vermont & 405 & 16.4 & 2.3 & $(12.0-20.9)$ \\
\hline $\begin{array}{l}\text { Cambridge-Newton-Framingham, } \\
\text { Massachusetts* }\end{array}$ & 1,800 & 12.7 & 1.2 & $(10.4-15.0)$ \\
\hline Camden, New Jersey* & 533 & 16.0 & 2.4 & $(11.2-20.7)$ \\
\hline Canton-Massillon, Ohio & 220 & 26.9 & 3.8 & $(19.3-34.4)$ \\
\hline Casper, Wyoming & 308 & 18.4 & 3.3 & $(12.0-24.7)$ \\
\hline Cedar Rapids, lowa & 196 & 15.4 & 2.8 & $(9.9-20.8)$ \\
\hline Charleston, West Virginia & 232 & 34.0 & 3.5 & $(27.0-40.9)$ \\
\hline $\begin{array}{l}\text { Charleston-North Charleston, } \\
\text { South Carolina }\end{array}$ & 521 & 15.9 & 2.2 & $(11.6-20.3)$ \\
\hline $\begin{array}{l}\text { Charlotte-Concord-Gastonia, } \\
\text { North Carolina-South Carolina }\end{array}$ & 664 & 21.9 & 2.2 & $(17.6-26.2)$ \\
\hline Chattanooga, Tennessee-Georgia & 227 & 31.4 & 4.1 & $(23.4-39.4)$ \\
\hline Cheyenne, Wyoming & 373 & 14.4 & 2.1 & $(10.2-18.6)$ \\
\hline $\begin{array}{l}\text { Chicago-Naperville-Elgin, } \\
\text { Illinois-Indiana-Wisconsin }\end{array}$ & 1,131 & 14.5 & 1.5 & $(11.7-17.4)$ \\
\hline Cincinnati, Ohio-Kentucky-Indiana & 613 & 20.7 & 2.4 & $(16.0-25.5)$ \\
\hline $\begin{array}{l}\text { Claremont-Lebanon, New } \\
\text { Hampshire-Vermont }\end{array}$ & 680 & 14.7 & 1.6 & $(11.4-17.9)$ \\
\hline Cleveland-Elyria, Ohio & 533 & 19.0 & 2.9 & $(13.3-24.7)$ \\
\hline Colorado Springs, Colorado & 282 & 13.7 & 2.4 & $(9.1-18.4)$ \\
\hline Columbia, South Carolina & 496 & 17.4 & 2.6 & $(12.2-22.5)$ \\
\hline Columbus, Ohio & 401 & 17.6 & 2.5 & $(12.7-22.5)$ \\
\hline Concord, New Hampshire & 221 & 10.6 & 2.6 & $(5.5-15.7)$ \\
\hline Dallas-Plano-Irving, Texas* & 214 & 14.9 & 2.8 & $(9.4-20.3)$ \\
\hline Dayton, Ohio & 260 & 19.3 & 3.1 & $(13.3-25.4)$ \\
\hline Denver-Aurora-Lakewood, Colorado & 1,156 & 11.7 & 1.2 & $(9.2-14.1)$ \\
\hline Des Moines-West Des Moines, lowa & 341 & 12.2 & 2.1 & $(8.2-16.3)$ \\
\hline
\end{tabular}

See table footnotes on page 48. 
TABLE 20. (Continued) Estimated prevalence of adults aged $\geq 65$ years who have lost all of their natural teeth, by metropolitan and micropolitan statistical area - Behavioral Risk Factor Surveillance System, United States, 2012

\begin{tabular}{|c|c|c|c|c|}
\hline MMSA(s) & $\begin{array}{l}\text { Sample } \\
\text { size }\end{array}$ & $\%$ & SE & $95 \% \mathrm{Cl}$ \\
\hline Detroit-Dearborn-Livonia, Michigan* & 720 & 11.9 & 1.7 & $(8.6-15.1)$ \\
\hline Dover, Delaware & 469 & 20.1 & 2.3 & $(15.6-24.5)$ \\
\hline Duluth, Minnesota-Wisconsin & 150 & 12.0 & 3.0 & $(6.2-17.9)$ \\
\hline Durham-Chapel Hill, North Carolina & 202 & 11.1 & 2.7 & $(5.9-16.4)$ \\
\hline El Paso, Texas & 193 & 9.1 & 2.2 & $(4.7-13.5)$ \\
\hline Eugene, Oregon & 171 & 14.8 & 3.3 & $(8.2-21.3)$ \\
\hline Fairbanks, Alaska & 96 & 22.9 & 5.7 & $(11.7-34.2)$ \\
\hline Fargo, North Dakota-Minnesota & 255 & 7.0 & 1.7 & $(3.7-10.3)$ \\
\hline Farmington, New Mexico & 174 & 24.5 & 4.0 & $(16.7-32.3)$ \\
\hline Fayetteville, North Carolina & 112 & 15.9 & 4.0 & $(8.0-23.7)$ \\
\hline $\begin{array}{l}\text { Fayetteville-Springdale-Rogers, } \\
\text { Arkansas-Missouri }\end{array}$ & 283 & 23.7 & 3.4 & $(17.2-30.3)$ \\
\hline Fort Collins, Colorado & 171 & $\mathrm{~N} / \mathrm{A}$ & N/A & $(\mathrm{N} / \mathrm{A}-\mathrm{N} / \mathrm{A})$ \\
\hline Fort Wayne, Indiana & 146 & 18.3 & 4.0 & $(10.6-26.1)$ \\
\hline Fort Worth-Arlington, Texas* & 213 & 14.1 & 3.6 & $(7.1-21.2)$ \\
\hline Grand Island, Nebraska & 296 & 12.7 & 2.4 & $(8.1-17.4)$ \\
\hline Grand Rapids-Wyoming, Michigan & 259 & 12.8 & 2.6 & $(7.6-17.9)$ \\
\hline Great Falls, Montana & 249 & 18.0 & 2.7 & $(12.7-23.2)$ \\
\hline Greeley, Colorado & 124 & 15.4 & 4.1 & $(7.5-23.4)$ \\
\hline $\begin{array}{l}\text { Greensboro-High Point, } \\
\text { North Carolina }\end{array}$ & 240 & 15.8 & 2.6 & $(10.6-20.9)$ \\
\hline $\begin{array}{l}\text { Greenville-Anderson-Mauldin, } \\
\text { South Carolina }\end{array}$ & 521 & 20.9 & 2.6 & $(15.8-26.0)$ \\
\hline Gulfport-Biloxi-Pascagoula, Mississippi & 255 & 21.8 & 3.0 & $(16.0-27.7)$ \\
\hline $\begin{array}{l}\text { Hagerstown-Martinsburg, Maryland- } \\
\text { West Virginia }\end{array}$ & 253 & 20.4 & 3.3 & $(13.9-26.9)$ \\
\hline Harrisburg-Carlisle, Pennsylvania & 185 & 8.0 & 2.0 & $(4.1-11.9)$ \\
\hline $\begin{array}{l}\text { Hartford-West Hartford-East Hartford, } \\
\text { Connecticut }\end{array}$ & 777 & 13.1 & 1.7 & $(9.8-16.4)$ \\
\hline Heber, Utah & 152 & $\mathrm{~N} / \mathrm{A}$ & $\mathrm{N} / \mathrm{A}$ & $(\mathrm{N} / \mathrm{A}-\mathrm{N} / \mathrm{A})$ \\
\hline Hilo, Hawaii & 378 & 11.0 & 2.9 & $(5.3-16.7)$ \\
\hline $\begin{array}{l}\text { Hilton Head Island-Bluffton-Beaufort, } \\
\text { South Carolina }\end{array}$ & 474 & 7.7 & 1.5 & $(4.7-10.7)$ \\
\hline $\begin{array}{l}\text { Houston-The Woodlands-Sugar Land, } \\
\text { Texas }\end{array}$ & 219 & 13.7 & 3.1 & $(7.7-19.7)$ \\
\hline $\begin{array}{l}\text { Huntington-Ashland, West } \\
\text { Virginia-Kentucky-Ohio }\end{array}$ & 334 & 28.0 & 3.0 & $(22.2-33.8)$ \\
\hline Huntsville, Alabama & 152 & 14.4 & 3.5 & $(7.5-21.2)$ \\
\hline Idaho Falls, Idaho & 176 & 25.2 & 6.4 & $(12.6-37.8)$ \\
\hline $\begin{array}{l}\text { Indianapolis-Carmel-Anderson, } \\
\text { Indiana }\end{array}$ & 623 & 18.9 & 2.3 & $(14.4-23.5)$ \\
\hline Jackson, Mississippi & 277 & 22.1 & 2.9 & $(16.4-27.9)$ \\
\hline Jacksonville, Florida & 168 & 18.9 & 3.8 & $(11.4-26.4)$ \\
\hline Kahului-Wailuku-Lahaina, Hawaii & 363 & 9.7 & 2.1 & $(5.6-13.8)$ \\
\hline Kalispell, Montana & 172 & 14.5 & 3.4 & $(7.8-21.2)$ \\
\hline Kansas City, Missouri-Kansas & 1,495 & 19.1 & 2.1 & $(14.9-23.2)$ \\
\hline Kapaa, Hawaii & 227 & $\mathrm{~N} / \mathrm{A}$ & N/A & $(\mathrm{N} / \mathrm{A}-\mathrm{N} / \mathrm{A})$ \\
\hline Keene, New Hampshire & 203 & 19.5 & 4.1 & $(11.4-27.6)$ \\
\hline Kennewick-Richland, Washington & 151 & 16.0 & 3.7 & $(8.6-23.3)$ \\
\hline $\begin{array}{l}\text { Kingsport-Bristol-Bristol, } \\
\text { Tennessee-Virginia }\end{array}$ & 193 & 29.9 & 4.7 & $(20.7-39.2)$ \\
\hline Knoxville, Tennessee & 261 & 25.3 & 3.7 & $(18.1-32.4)$ \\
\hline Laconia, New Hampshire & 225 & 15.6 & 3.1 & $(9.5-21.6)$ \\
\hline Lafayette, Louisiana & 165 & 39.6 & 5.2 & $(29.3-49.9)$ \\
\hline Las Cruces, New Mexico & 209 & 8.9 & 2.6 & $(3.9-14.0)$ \\
\hline $\begin{array}{l}\text { Las Vegas-Henderson-Paradise, } \\
\text { Nevada }\end{array}$ & 493 & 14.2 & 1.9 & $(10.5-18.0)$ \\
\hline Lewiston-Auburn, Maine & 183 & 24.1 & 3.7 & $(16.8-31.5)$ \\
\hline Lexington-Fayette, Kentucky & 116 & 18.9 & 4.3 & $(10.5-27.4)$ \\
\hline Lincoln, Nebraska & 363 & 9.6 & 1.9 & $(5.9-13.3)$ \\
\hline
\end{tabular}

TABLE 20. (Continued) Estimated prevalence of adults aged $\geq 65$ years who have lost all of their natural teeth, by metropolitan and micropolitan statistical area - Behavioral Risk Factor Surveillance System, United States, 2012

\begin{tabular}{|c|c|c|c|c|}
\hline MMSA(s) & $\begin{array}{l}\text { Sample } \\
\text { size }\end{array}$ & $\%$ & SE & $95 \% \mathrm{Cl}$ \\
\hline $\begin{array}{l}\text { Little Rock-North Little Rock-Conway, } \\
\text { Arkansas }\end{array}$ & 354 & 18.3 & 2.6 & $(13.2-23.5)$ \\
\hline Logan, Utah-Idaho & 120 & 12.2 & 3.4 & $(5.5-18.9)$ \\
\hline $\begin{array}{l}\text { Los Angeles-Long Beach-Glendale, } \\
\text { California* }\end{array}$ & 861 & 8.4 & 1.5 & $(5.5-11.4)$ \\
\hline $\begin{array}{l}\text { Louisville/Jefferson County, } \\
\text { Kentucky-Indiana }\end{array}$ & 651 & 22.2 & 2.7 & $(17.0-27.4)$ \\
\hline Lumberton, North Carolina & 158 & 18.9 & 4.1 & $(10.9-26.9)$ \\
\hline Manchester-Nashua, New Hampshire & 506 & 12.7 & 1.9 & $(9.0-16.3)$ \\
\hline McAllen-Edinburg-Mission, Texas & 182 & 12.1 & 3.5 & $(5.3-18.9)$ \\
\hline $\begin{array}{l}\text { Memphis, } \\
\text { Tennessee-Mississippi-Arkansas }\end{array}$ & 405 & 18.6 & 2.6 & $(13.4-23.7)$ \\
\hline $\begin{array}{l}\text { Miami-Fort Lauderdale-West Palm } \\
\text { Beach, Florida }\end{array}$ & 585 & 14.1 & 2.2 & $(9.9-18.4)$ \\
\hline $\begin{array}{l}\text { Milwaukee-Waukesha-West Allis, } \\
\text { Wisconsin }\end{array}$ & 320 & 8.9 & 2.0 & $(4.9-12.9)$ \\
\hline $\begin{array}{l}\text { Minneapolis-St. Paul-Bloomington, } \\
\text { Minnesota-Wisconsin }\end{array}$ & 1,980 & 10.2 & 1.0 & $(8.2-12.2)$ \\
\hline Missoula, Montana & 219 & 17.2 & 3.2 & $(11.0-23.4)$ \\
\hline Mobile, Alabama & 297 & 24.2 & 3.4 & $(17.5-30.9)$ \\
\hline Montgomery, Alabama & 170 & 17.5 & 3.7 & $(10.2-24.7)$ \\
\hline $\begin{array}{l}\text { Montgomery County-Bucks County- } \\
\text { Chester County, Pennsylvania* }\end{array}$ & 340 & 10.7 & 2.0 & $(6.8-14.6)$ \\
\hline $\begin{array}{l}\text { Myrtle Beach-Conway-North Myrtle } \\
\text { Beach, South Carolina-North Carolina }\end{array}$ & 338 & 14.0 & 2.4 & $(9.2-18.8)$ \\
\hline $\begin{array}{l}\text { Nashville-Davidson-Murfreesboro- } \\
\text { Franklin, Tennessee }\end{array}$ & 334 & 21.7 & 3.0 & $(15.8-27.5)$ \\
\hline $\begin{array}{l}\text { Nassau County-Suffolk County, } \\
\text { New York* }\end{array}$ & 262 & 10.5 & 2.2 & $(6.2-14.8)$ \\
\hline Newark, New Jersey-Pennsylvania* & 1,898 & 12.9 & 1.4 & $(10.1-15.7)$ \\
\hline New Haven-Milford, Connecticut & 605 & 13.9 & 1.9 & $(10.3-17.6)$ \\
\hline New Orleans-Metairie, Louisiana & 389 & 20.9 & 3.0 & $(15.1-26.7)$ \\
\hline $\begin{array}{l}\text { New York-Jersey City-White Plains, } \\
\text { New York-New Jersey* }\end{array}$ & 1,680 & 14.3 & 1.8 & $(10.8-17.9)$ \\
\hline Norfolk, Nebraska & 192 & 21.4 & 3.7 & $(14.1-28.7)$ \\
\hline North Platte, Nebraska & 247 & 19.4 & 3.6 & $(12.3-26.4)$ \\
\hline Norwich-New London, Connecticut & 347 & 14.5 & 3.4 & $(7.9-21.1)$ \\
\hline $\begin{array}{l}\text { Oakland-Hayward-Berkeley, } \\
\text { California* }\end{array}$ & 296 & $\mathrm{~N} / \mathrm{A}$ & $\mathrm{N} / \mathrm{A}$ & $(\mathrm{N} / \mathrm{A}-\mathrm{N} / \mathrm{A})$ \\
\hline Ocean City, New Jersey & 225 & 13.7 & 2.6 & $(8.6-18.8)$ \\
\hline Ogden-Clearfield, Utah & 620 & 11.9 & 1.5 & $(8.9-14.8)$ \\
\hline Oklahoma City, Oklahoma & 629 & 16.4 & 1.7 & $(13.2-19.7)$ \\
\hline Olympia-Tumwater, Washington & 156 & 13.6 & 3.7 & $(6.3-21.0)$ \\
\hline Omaha-Council Bluffs, Nebraska-lowa & 1,487 & 12.5 & 1.2 & $(10.2-14.8)$ \\
\hline Orlando-Kissimmee-Sanford, Florida & 208 & 22.1 & 5.2 & $(12.0-32.2)$ \\
\hline Philadelphia, Pennsylvania* & 675 & 16.4 & 1.8 & $(12.8-19.9)$ \\
\hline Phoenix-Mesa-Scottsdale, Arizona & 825 & 11.6 & 1.5 & $(8.6-14.6)$ \\
\hline Pittsburgh, Pennsylvania & 1,115 & 22.5 & 1.7 & $(19.2-25.7)$ \\
\hline Ponce, Puerto Rico & 158 & 24.8 & 4.3 & $(16.5-33.1)$ \\
\hline Portland-South Portland, Maine & 1,042 & 16.0 & 1.3 & $(13.4-18.6)$ \\
\hline $\begin{array}{l}\text { Portland-Vancouver-Hillsboro, } \\
\text { Oregon-Washington }\end{array}$ & 881 & 11.5 & 1.4 & $(8.8-14.2)$ \\
\hline $\begin{array}{l}\text { Providence-Warwick, Rhode } \\
\text { Island-Massachusetts }\end{array}$ & 2,415 & 16.2 & 1.2 & $(13.9-18.6)$ \\
\hline Provo-Orem, Utah & 375 & 10.6 & 1.8 & $(7.0-14.2)$ \\
\hline Raleigh, North Carolina & 180 & 16.9 & 3.3 & $(10.4-23.4)$ \\
\hline Rapid City, South Dakota & 287 & 17.9 & 3.3 & $(11.4-24.4)$ \\
\hline Reno, Nevada & 492 & 14.8 & 2.3 & $(10.3-19.4)$ \\
\hline Richmond, Virginia & 301 & 17.8 & 2.7 & $(12.6-23.0)$ \\
\hline
\end{tabular}

See table footnotes on page 48. 
TABLE 20. (Continued) Estimated prevalence of adults aged $\geq 65$ years who have lost all of their natural teeth, by metropolitan and micropolitan statistical area - Behavioral Risk Factor Surveillance System, United States, 2012

\begin{tabular}{|c|c|c|c|c|}
\hline MMSA(s) & $\begin{array}{l}\text { Sample } \\
\text { size }\end{array}$ & $\%$ & SE & $95 \% \mathrm{Cl}$ \\
\hline $\begin{array}{l}\text { Riverside-San Bernardino-Ontario, } \\
\text { California }\end{array}$ & 445 & 12.5 & 2.1 & $(8.4-16.6)$ \\
\hline $\begin{array}{l}\text { Rockingham County-Strafford County, } \\
\text { New Hampshire* }\end{array}$ & 514 & 11.3 & 1.8 & $(7.7-14.9)$ \\
\hline Rutland, Vermont & 188 & 13.4 & 2.9 & $(7.6-19.1)$ \\
\hline $\begin{array}{l}\text { Sacramento-Roseville-Arden-Arcade, } \\
\text { California }\end{array}$ & 284 & 7.2 & 1.8 & $(3.7-10.7)$ \\
\hline St. Louis, Missouri-Illinois & 651 & 21.2 & 2.5 & $(16.2-26.1)$ \\
\hline Salisbury, Maryland-Delaware & 850 & 17.0 & 1.6 & $(14.0-20.1)$ \\
\hline Salt Lake City, Utah & 1,137 & 12.3 & 1.4 & $(9.6-15.0)$ \\
\hline San Antonio-New Braunfels, Texas & 237 & 9.4 & 2.3 & $(4.9-14.0)$ \\
\hline San Diego-Carlsbad, California & 333 & 5.8 & 1.5 & $(2.8-8.8)$ \\
\hline $\begin{array}{l}\text { San Francisco-Redwood City-South } \\
\text { San Francisco, California* }\end{array}$ & 174 & N/A & $\mathrm{N} / \mathrm{A}$ & $(\mathrm{N} / \mathrm{A}-\mathrm{N} / \mathrm{A})$ \\
\hline $\begin{array}{l}\text { San Jose-Sunnyvale-Santa Clara, } \\
\text { California }\end{array}$ & 187 & N/A & $\mathrm{N} / \mathrm{A}$ & $(\mathrm{N} / \mathrm{A}-\mathrm{N} / \mathrm{A})$ \\
\hline San Juan-Carolina-Caguas, Puerto Rico & 1,137 & 16.3 & 1.3 & $(13.6-18.9)$ \\
\hline Santa Fe, New Mexico & 197 & 15.4 & 3.3 & $(9.0-21.9)$ \\
\hline Sayre, Pennsylvania & 663 & 26.6 & 2.2 & $(22.4-30.9)$ \\
\hline Scottsbluff, Nebraska & 269 & 14.1 & 2.5 & $(9.1-19.1)$ \\
\hline $\begin{array}{l}\text { Scranton-Wilkes-Barre-Hazleton, } \\
\text { Pennsylvania }\end{array}$ & 259 & 21.8 & 3.4 & $(15.2-28.5)$ \\
\hline Seattle-Bellevue-Everett, Washington* & 1,362 & 8.0 & 1.1 & $(5.8-10.1)$ \\
\hline Shreveport-Bossier City, Louisiana & 182 & 22.7 & 3.8 & $(15.3-30.1)$ \\
\hline $\begin{array}{l}\text { Silver Spring-Frederick-Rockville, } \\
\text { Maryland* }\end{array}$ & 664 & 9.4 & 1.5 & $(6.5-12.4)$ \\
\hline $\begin{array}{l}\text { Sioux City, lowa-Nebraska- } \\
\text { South Dakota }\end{array}$ & 450 & 21.7 & 4.2 & $(13.5-29.9)$ \\
\hline Sioux Falls, South Dakota & 287 & 19.2 & 4.0 & $(11.3-27.0)$ \\
\hline Spartanburg, South Carolina & 223 & 29.5 & 4.7 & $(20.4-38.6)$ \\
\hline Spokane-Spokane Valley, Washington & 367 & 13.8 & 2.5 & $(8.9-18.7)$ \\
\hline Springfield, Massachusetts & 630 & 21.5 & 2.9 & $(15.9-27.2)$ \\
\hline Tacoma-Lakewood, Washington* & 298 & 15.3 & 2.8 & $(9.9-20.8)$ \\
\hline
\end{tabular}

TABLE 20. (Continued) Estimated prevalence of adults aged $\geq 65$ years who have lost all of their natural teeth, by metropolitan and micropolitan statistical area - Behavioral Risk Factor Surveillance System, United States, 2012

\begin{tabular}{|c|c|c|c|c|}
\hline MMSA(s) & $\begin{array}{l}\text { Sample } \\
\text { size }\end{array}$ & $\%$ & SE & $95 \% \mathrm{Cl}$ \\
\hline $\begin{array}{l}\text { Tampa-St. Petersburg-Clearwater, } \\
\text { Florida }\end{array}$ & 305 & 15.1 & 2.5 & $(10.1-20.0)$ \\
\hline Toledo, Ohio & 292 & 12.6 & 2.9 & $(6.9-18.3)$ \\
\hline Topeka, Kansas & 332 & 18.7 & 2.5 & $(13.8-23.6)$ \\
\hline Torrington, Connecticut & 202 & 8.1 & 2.3 & $(3.7-12.6)$ \\
\hline Trenton, New Jersey & 147 & 10.8 & 3.2 & $(4.6-17.1)$ \\
\hline Tucson, Arizona & 371 & 12.7 & 2.1 & $(8.6-16.9)$ \\
\hline Tulsa, Oklahoma & 542 & 17.1 & 1.9 & $(13.4-20.7)$ \\
\hline Tuscaloosa, Alabama & 196 & 22.8 & 4.1 & $(14.8-30.8)$ \\
\hline Urban Honolulu, Hawaii & 970 & 6.0 & 1.1 & $(3.9-8.1)$ \\
\hline Vineland-Bridgeton, New Jersey & 159 & 20.0 & 3.9 & $(12.4-27.6)$ \\
\hline $\begin{array}{l}\text { Virginia Beach-Norfolk-Newport News, } \\
\text { Virginia-North Carolina }\end{array}$ & 442 & 12.3 & 1.9 & $(8.6-16.0)$ \\
\hline $\begin{array}{l}\text { Warren-Troy-Farmington Hills, } \\
\text { Michigan* }\end{array}$ & 654 & 11.4 & 1.8 & $(7.9-15.0)$ \\
\hline $\begin{array}{l}\text { Washington-Arlington-Alexandria, } \\
\text { District of Columbia-Virginia- } \\
\text { Maryland-West Virginia* }\end{array}$ & 2,200 & 9.6 & 1.0 & $(7.7-11.5)$ \\
\hline Wichita, Kansas & 739 & 17.0 & 1.6 & $(13.9-20.1)$ \\
\hline $\begin{array}{l}\text { Wilmington, Delaware-Maryland- } \\
\text { New Jersey* }\end{array}$ & 876 & 17.8 & 2.4 & $(13.1-22.5)$ \\
\hline Winston-Salem, North Carolina & 250 & 24.4 & 3.4 & $(17.8-30.9)$ \\
\hline Worcester, Massachusetts-Connecticut & 776 & 19.7 & 2.2 & $(15.3-24.1)$ \\
\hline Yakima, Washington & 155 & 16.8 & 3.6 & $(9.7-23.9)$ \\
\hline $\begin{array}{l}\text { Youngstown-Warren-Boardman, } \\
\text { Ohio-Pennsylvania }\end{array}$ & 333 & 20.8 & 3.4 & $(14.2-27.4)$ \\
\hline Median & & 15.8 & & \\
\hline Range & & $5.8-39.6$ & & \\
\hline
\end{tabular}

Abbreviations: $\mathrm{Cl}=$ confidence interval; $\mathrm{MMSA}=$ metropolitan and micropolitan statistical area; $\mathrm{SE}=$ standard error.

* Metropolitan division.

† Estimate not available (N/A) if the unweighted sample size for the denominator was $<50$ or if the relative standard error is $>0.3$. 
TABLE 21. Estimated prevalence of adults aged $\geq 65$ years who have lost all of their natural teeth, by county - Behavioral Risk Factor Surveillance System, United States, 2012

\begin{tabular}{|c|c|c|c|c|}
\hline County & $\begin{array}{l}\text { Sample } \\
\text { size }\end{array}$ & $\%$ & SE & $95 \% \mathrm{Cl}$ \\
\hline Jefferson County, Alabama & 268 & 24.7 & 3.3 & $(18.3-31.2)$ \\
\hline Madison County, Alabama & 130 & 14.7 & 3.7 & $(7.5-22.0)$ \\
\hline Mobile County, Alabama & 297 & 24.2 & 3.4 & $(17.5-30.9)$ \\
\hline Anchorage Municipality, Alaska & 126 & 13.2 & 3.6 & $(6.2-20.3)$ \\
\hline Fairbanks North Star Borough, Alaska & 96 & 22.9 & 5.7 & $(11.7-34.2)$ \\
\hline Matanuska-Susitna Borough, Alaska & 104 & $N / A^{*}$ & $\mathrm{~N} / \mathrm{A}$ & $(\mathrm{N} / \mathrm{A}-\mathrm{N} / \mathrm{A})$ \\
\hline Maricopa County, Arizona & 611 & 11.8 & 1.6 & $(8.6-15.1)$ \\
\hline Pima County, Arizona & 371 & 12.7 & 2.1 & $(8.6-16.9)$ \\
\hline Pulaski County, Arkansas & 231 & 13.9 & 2.7 & $(8.6-19.2)$ \\
\hline Alameda County, California & 179 & $\mathrm{~N} / \mathrm{A}$ & $\mathrm{N} / \mathrm{A}$ & $(\mathrm{N} / \mathrm{A}-\mathrm{N} / \mathrm{A})$ \\
\hline Los Angeles County, California & 861 & 8.4 & 1.5 & $(5.5-11.4)$ \\
\hline Orange County, California & 323 & 6.4 & 1.9 & $(2.7-10.0)$ \\
\hline Riverside County, California & 269 & 8.9 & 2.1 & $(4.9-13.0)$ \\
\hline Sacramento County, California & 163 & N/A & N/A & $(\mathrm{N} / \mathrm{A}-\mathrm{N} / \mathrm{A})$ \\
\hline San Bernardino County, California & 176 & 19.2 & 4.2 & $(11.0-27.5)$ \\
\hline San Diego County, California & 333 & 5.8 & 1.5 & $(2.8-8.8)$ \\
\hline Santa Clara County, California & 177 & $\mathrm{~N} / \mathrm{A}$ & N/A & $(\mathrm{N} / \mathrm{A}-\mathrm{N} / \mathrm{A})$ \\
\hline Adams County, Colorado & 196 & 17.1 & 3.5 & $(10.3-23.9)$ \\
\hline Arapahoe County, Colorado & 198 & 10.2 & 2.7 & $(4.8-15.5)$ \\
\hline Boulder County, Colorado & 127 & N/A & $\mathrm{N} / \mathrm{A}$ & $(\mathrm{N} / \mathrm{A}-$ \\
\hline Denver County, Colorado & 221 & 11.7 & 2.7 & 7.0) \\
\hline Douglas County, Colorado & 100 & $\mathrm{~N} / \mathrm{A}$ & $\mathrm{N} / \mathrm{A}$ & $(\mathrm{N} / \mathrm{A}-\mathrm{N} / \mathrm{A})$ \\
\hline El Paso County, Colorado & 230 & 13.8 & 2.5 & $(8.9-18.7)$ \\
\hline Jefferson County, Colorado & 313 & 9.2 & 1.9 & $(5.5-12.9)$ \\
\hline Larimer County, Colorado & 171 & $\mathrm{~N} / \mathrm{A}$ & $\mathrm{N} / \mathrm{A}$ & $(\mathrm{N} / \mathrm{A}-\mathrm{N} / \mathrm{A})$ \\
\hline Weld County, Colorado & 124 & 15.4 & 4.1 & $(7.5-23.4)$ \\
\hline Fairfield County, Connecticut & 582 & 12.9 & 2.6 & $(7.9-17.9)$ \\
\hline Hartford County, Connecticut & 566 & 13.1 & 2.0 & $(9.1-17.2)$ \\
\hline Litchfield County, Connecticut & 202 & 8.1 & 2.3 & $(3.7-12.6)$ \\
\hline New Haven County, Connecticut & 605 & 13.9 & 1.9 & $(10.3-17.6)$ \\
\hline New London County, Connecticut & 347 & 14.5 & 3.4 & $(7.9-21.1)$ \\
\hline Kent County, Delaware & 469 & 20.1 & 2.3 & $(15.6-24.5)$ \\
\hline New Castle County, Delaware & 609 & 15.4 & 2.0 & $(11.4-19.4)$ \\
\hline Sussex County, Delaware & 567 & 17.4 & 1.9 & 13.6-21.2) \\
\hline $\begin{array}{l}\text { District of Columbia, } \\
\text { District of Columbia }\end{array}$ & 1,164 & 13.1 & 1.5 & $(10.3-16.0)$ \\
\hline Broward County, Florida & 174 & 12.8 & 3.7 & $(5.6-20.0)$ \\
\hline Miami-Dade County, Florida & 258 & 17.7 & 4.2 & $(9.5-25.9)$ \\
\hline Hawaii County, Hawaii & 378 & 11.0 & 2.9 & $(5.3-16.7)$ \\
\hline Honolulu County, Hawaii & 970 & 6.0 & 1.1 & $(3.9-8.1)$ \\
\hline Kauai County, Hawaii & 227 & $\mathrm{~N} / \mathrm{A}$ & N/A & $(\mathrm{N} / \mathrm{A}-\mathrm{N} / \mathrm{A})$ \\
\hline Maui County, Hawaii & 363 & 9.7 & 2.1 & $(5.6-13.8)$ \\
\hline Ada County, Idaho & 253 & 12.2 & 3.1 & $(6.2-18.2)$ \\
\hline Canyon County, Idaho & 183 & 16.9 & 4.3 & $(8.6-25.2)$ \\
\hline Cook County, Illinois & 452 & 14.9 & 2.0 & $(11.0-18.7)$ \\
\hline Lake County, Indiana & 302 & 21.3 & 3.5 & $(14.5-28.1)$ \\
\hline Marion County, Indiana & 371 & 20.7 & 2.8 & $(15.4-26.1)$ \\
\hline Polk County, lowa & 231 & 9.7 & 2.3 & $(5.1-14.3)$ \\
\hline Johnson County, Kansas & 648 & 11.1 & 1.5 & $(8.1-14.0)$ \\
\hline Sedgwick County, Kansas & 531 & 16.9 & 1.9 & $(13.2-20.6)$ \\
\hline Shawnee County, Kansas & 239 & 14.5 & 2.6 & $(9.4-19.6)$ \\
\hline Wyandotte County, Kansas & 314 & 29.0 & 3.4 & $(22.3-35.7)$ \\
\hline Jefferson County, Kentucky & 536 & 20.8 & 3.2 & $(14.4-27.2)$ \\
\hline East Baton Rouge Parish, Louisiana & 179 & 25.0 & 4.4 & $(16.3-33.7)$ \\
\hline Androscoggin County, Maine & 183 & 24.1 & 3.7 & $(16.8-31.5)$ \\
\hline Aroostook County, Maine & 169 & 37.1 & 4.4 & $(28.4-45.7)$ \\
\hline Cumberland County, Maine & 513 & 14.9 & 1.8 & $(11.4-18.5)$ \\
\hline Kennebec County, Maine & 215 & 28.7 & 3.8 & $(21.3-36.1)$ \\
\hline Penobscot County, Maine & 249 & 28.0 & 3.4 & $(21.4-34.7)$ \\
\hline York County, Maine & 400 & 17.2 & 2.1 & $(13.0-21.3)$ \\
\hline
\end{tabular}

See table footnotes on page 50 .
TABLE 21. (Continued) Estimated prevalence of adults aged $\geq 65$ years who have lost all of their natural teeth, by county - Behavioral Risk Factor Surveillance System, United States, 2012

\begin{tabular}{|c|c|c|c|c|}
\hline County & $\begin{array}{l}\text { Sample } \\
\text { size }\end{array}$ & $\%$ & SE & $95 \% \mathrm{Cl}$ \\
\hline Anne Arundel County, Maryland & 242 & 18.1 & 3.6 & $(11.1-25.2)$ \\
\hline Baltimore County, Maryland & 443 & 14.0 & 2.4 & $(9.3-18.7)$ \\
\hline Charles County, Maryland & 113 & 19.6 & 5.3 & $(9.2-30.0)$ \\
\hline Frederick County, Maryland & 226 & 16.8 & 3.6 & $(9.8-23.8)$ \\
\hline Montgomery County, Maryland & 438 & 7.6 & 1.6 & $(4.4-10.8)$ \\
\hline Prince George's County, Maryland & 302 & 8.2 & 1.7 & $(5.0-11.5)$ \\
\hline Washington County, Maryland & 191 & 16.1 & 3.3 & $(9.7-22.6)$ \\
\hline Baltimore city, Maryland & 220 & 20.4 & 3.9 & $(12.8-27.9)$ \\
\hline Barnstable County, Massachusetts & 260 & 12.2 & 3.0 & $(6.4-18.0)$ \\
\hline Bristol County, Massachusetts & 832 & 23.2 & 2.8 & $(17.8-28.7)$ \\
\hline Essex County, Massachusetts & 787 & 14.3 & 1.9 & $(10.7-18.0)$ \\
\hline Hampden County, Massachusetts & 541 & 21.3 & 3.3 & $(14.9-27.7)$ \\
\hline Middlesex County, Massachusetts & 1,013 & 11.9 & 1.5 & $(8.9-14.8)$ \\
\hline Norfolk County, Massachusetts & 509 & 10.2 & 1.8 & $(6.7-13.8)$ \\
\hline Plymouth County, Massachusetts & 551 & 11.8 & 2.2 & $(7.5-16.0)$ \\
\hline Suffolk County, Massachusetts & 560 & 18.9 & 2.3 & $(14.4-23.5)$ \\
\hline Worcester County, Massachusetts & 708 & 18.1 & 2.3 & $(13.7-22.5)$ \\
\hline Kent County, Michigan & 148 & $\mathrm{~N} / \mathrm{A}$ & N/A & $(\mathrm{N} / \mathrm{A}-\mathrm{N} / \mathrm{A})$ \\
\hline Macomb County, Michigan & 185 & 16.4 & 3.6 & $(9.2-23.5)$ \\
\hline Oakland County, Michigan & 364 & 8.4 & 2.1 & $(4.3-12.4)$ \\
\hline Wayne County, Michigan & 720 & 11.9 & 1.7 & $(8.6-15.1)$ \\
\hline Anoka County, Minnesota & 116 & 10.5 & 3.0 & $(4.6-16.3)$ \\
\hline Dakota County, Minnesota & 143 & $\mathrm{~N} / \mathrm{A}$ & $\mathrm{N} / \mathrm{A}$ & $(\mathrm{N} / \mathrm{A}-\mathrm{N} / \mathrm{A})$ \\
\hline Hennepin County, Minnesota & 813 & 8.9 & 1.5 & $(6.0-11.8)$ \\
\hline Ramsey County, Minnesota & 575 & 10.2 & 2.1 & $(6.1-14.2)$ \\
\hline Jackson County, Missouri & 299 & 22.2 & 4.6 & $(13.2-31.2)$ \\
\hline St. Louis County, Missouri & 288 & 15.8 & 3.9 & $(8.3-23.4)$ \\
\hline Cascade County, Montana & 249 & 18.0 & 2.7 & $(12.7-23.2)$ \\
\hline Flathead County, Montana & 172 & 14.5 & 3.4 & $(7.8-21.2)$ \\
\hline Hill County, Montana & 172 & 15.9 & 3.3 & $(9.5-22.3)$ \\
\hline Lake County, Montana & 336 & 19.1 & 3.0 & $(13.2-25.1)$ \\
\hline Missoula County, Montana & 219 & 17.2 & 3.2 & $(11.0-23.4)$ \\
\hline Yellowstone County, Montana & 216 & 21.6 & 3.1 & $(15.5-27.6)$ \\
\hline Dakota County, Nebraska & 284 & 26.4 & 3.2 & $(20.1-32.8)$ \\
\hline Douglas County, Nebraska & 953 & 12.1 & 1.4 & $(9.4-14.8)$ \\
\hline Hall County, Nebraska & 176 & 13.0 & 3.0 & $(7.1-18.9)$ \\
\hline Lancaster County, Nebraska & 286 & 9.8 & 2.0 & $(5.8-13.7)$ \\
\hline Lincoln County, Nebraska & 238 & 19.3 & 3.7 & $(12.1-26.5)$ \\
\hline Sarpy County, $\mathrm{Ne}$ & 295 & 11.2 & 2.2 & $(7.0-15.4)$ \\
\hline Scotts Bluff County, Nebraska & 247 & 13.9 & 2.7 & $(8.7-19.1)$ \\
\hline Clark County, Nevada & 493 & 14.2 & 1.9 & $(10.5-18.0)$ \\
\hline Washoe County, Nevada & 484 & 15.0 & 2.3 & $(10.5-19.6)$ \\
\hline Belknap County, New Hampshire & 225 & 15.6 & 3.1 & $(9.5-21.6)$ \\
\hline Carroll County, New Hampshire & 233 & 10.8 & 2.6 & $(5.8-15.9)$ \\
\hline Cheshire County, New Hampshire & 203 & 19.5 & 4.1 & $(11.4-27.6)$ \\
\hline Coos County, New Hampshire & 199 & 24.9 & 3.5 & $(18.1-31.8)$ \\
\hline Grafton County, New Hampshire & 209 & 14.9 & 2.9 & $(9.3-20.6)$ \\
\hline Hillsborough County, New Hampshire & 506 & 12.7 & 1.9 & $(9.0-16.3)$ \\
\hline Merrimack County, New Hampshire & 221 & 10.6 & 2.6 & $(5.6-15.7)$ \\
\hline Rockingham County, New Hampshire & 311 & 10.8 & 2.2 & $(6.4-15.2)$ \\
\hline Strafford County, New Hampshire & 203 & 12.2 & 2.9 & $(6.5-17.9)$ \\
\hline Atlantic County, New Jersey & 338 & 17.9 & 2.8 & $(12.4-23.4)$ \\
\hline Bergen County, New Jersey & 220 & 9.5 & 2.2 & $(5.2-13.7)$ \\
\hline Burlington County, New Jersey & 198 & 17.0 & 4.4 & $(8.3-25.7)$ \\
\hline Camden County, New Jersey & 204 & 14.7 & 3.6 & $(7.8-21.7)$ \\
\hline Cape May County, New Jersey & 225 & 13.7 & 2.6 & $(8.6-18.8)$ \\
\hline Cumberland County, New Jersey & 159 & 20.0 & 3.9 & $(12.4-27.6)$ \\
\hline Essex County, New Jersey & 275 & 12.8 & 2.6 & $(7.8-17.8)$ \\
\hline Gloucester County, New Jersey & 131 & 16.5 & 4.0 & $(8.6-24.4)$ \\
\hline Hudson County, New Jersey & 258 & 21.8 & 3.9 & $(14.2-29.4)$ \\
\hline
\end{tabular}
See table footnotes on page 50 . 
TABLE 21. (Continued) Estimated prevalence of adults aged $\geq 65$ years who have lost all of their natural teeth, by county - Behavioral Risk Factor Surveillance System, United States, 2012

\begin{tabular}{|c|c|c|c|c|}
\hline County & $\begin{array}{l}\text { Sample } \\
\text { size }\end{array}$ & $\%$ & SE & $95 \% \mathrm{Cl}$ \\
\hline Hunterdon County, New Jersey & 153 & $\mathrm{~N} / \mathrm{A}$ & $\mathrm{N} / \mathrm{A}$ & $(\mathrm{N} / \mathrm{A}-\mathrm{N} / \mathrm{A})$ \\
\hline Mercer County, New Jersey & 147 & 10.8 & 3.2 & $(4.6-17.1)$ \\
\hline Middlesex County, New Jersey & 181 & 13.5 & 3.5 & $(6.8-20.3)$ \\
\hline Monmouth County, New Jersey & 213 & 9.2 & 2.2 & $(4.9-13.6)$ \\
\hline Morris County, New Jersey & 236 & N/A & N/A & $(\mathrm{N} / \mathrm{A}-\mathrm{N} / \mathrm{A})$ \\
\hline Ocean County, New Jersey & 237 & 11.1 & 2.4 & $(6.3-15.8)$ \\
\hline Passaic County, New Jersey & 142 & 21.8 & 4.3 & $(13.3-30.3)$ \\
\hline Salem County, New Jersey & 180 & 19.7 & 3.3 & $(13.2-26.2)$ \\
\hline Somerset County, New Jersey & 185 & N/A & $\mathrm{N} / \mathrm{A}$ & $(\mathrm{N} / \mathrm{A}-\mathrm{N} / \mathrm{A})$ \\
\hline Sussex County, New Jersey & 158 & 14.4 & 3.5 & $(7.6-21.3)$ \\
\hline Union County, New Jersey & 154 & 20.8 & 4.3 & $(12.4-29.3)$ \\
\hline Warren County, New Jersey & 165 & 20.5 & 3.6 & $(13.5$ \\
\hline Bernalillo County, New Mexico & 518 & 13.7 & 1.8 & $(10.2$ \\
\hline Dona Ana County, New Mexico & 209 & 8.9 & 2.6 & $(3.9-14.0)$ \\
\hline Sandoval County, New Mexico & 189 & 13.6 & 3.2 & $(7.3-19.9)$ \\
\hline San Juan County, New Mexico & 174 & 24.5 & 4.0 & $(16.8-32.3)$ \\
\hline Santa Fe County, New Mexico & 197 & 15.4 & 3.3 & $(9.0-21.9)$ \\
\hline Kings County, New York & 62 & N/A & N/A & $(\mathrm{N} / \mathrm{A}-\mathrm{N} / \mathrm{A})$ \\
\hline Guilford County, North Carolina & 141 & 12.6 & 3.3 & $(6.2-19.0)$ \\
\hline Mecklenburg County, North Carolina & 129 & N/A & N/A & $(\mathrm{N} / \mathrm{A}-\mathrm{N} / \mathrm{A})$ \\
\hline Robeson County, North Carolina & 158 & 18.9 & 4.1 & $(10.8-26.9)$ \\
\hline Wake County, North Carolina & 118 & 14.3 & 3.7 & $(7.0-21.6)$ \\
\hline Burleigh County, North Dakota & 163 & 15.6 & 4.2 & $(7.4-23.9)$ \\
\hline Cass County, North Dakota & 231 & 8.1 & 1.8 & $(4.5-$ \\
\hline Cuyahoga County, Ohio & 226 & 17.4 & 4.1 & $(9.5$ \\
\hline Franklin County, Ohio & 35 & 15.8 & 3.6 & $(8.8$ \\
\hline Hamilton County, Ohio & 196 & 20.9 & 3.7 & $(13.6-28.2)$ \\
\hline Lorain County, Ohio & 218 & 18.1 & 3.7 & $(10.8-25.3)$ \\
\hline Lucas County, Ohio & 192 & 15.8 & 4.2 & $(7.6-23.9)$ \\
\hline Mahoning County, Ohio & 223 & 24.1 & 4.6 & $(15.1-33.2)$ \\
\hline Montgomery County, Ohio & 216 & 22.4 & 3.8 & $(15.0-29.8)$ \\
\hline Stark County, Ohio & 203 & 27.3 & 4.0 & $(19.5-35.1)$ \\
\hline Summit County, Ohio & 197 & 14.9 & 3.1 & $(8.8-21.1)$ \\
\hline Oklahoma County, Oklahoma & 283 & 14.2 & 2.3 & $(9.8-18.7)$ \\
\hline Tulsa County, Oklahoma & 366 & 15.2 & 2.1 & $(11.0-19.4)$ \\
\hline Clackamas County, Oregon & 158 & 13.4 & 3.2 & $(7.1-19.8)$ \\
\hline Lane & 171 & 14.8 & 3.3 & $(8.2-21.3)$ \\
\hline Multnomah County, Oregon & 224 & 14.3 & 3.3 & $(7.8-20.8)$ \\
\hline Washington County, Oregon & 169 & 7.8 & 2.3 & $(3.4-12.2)$ \\
\hline Allegheny County, Pennsylvania & 652 & 19.7 & 2.0 & $(15.7-23.7)$ \\
\hline Bradford County, Pennsylvania & 663 & 26.6 & 2.2 & $(22.4-30.9)$ \\
\hline Montgomery County, Pennsylvania & 133 & 10.4 & 2.9 & $(4.7-16.2)$ \\
\hline Philadelphia County, Pennsylvania & 560 & 19.3 & 2.2 & $(15.0-23.6)$ \\
\hline Pike County, Pennsylvania & 737 & 12.2 & 1.2 & $(9.7-14.6)$ \\
\hline Kent County, Rhode Island & 214 & 13.1 & 2.8 & $(7.6-18.5)$ \\
\hline Providence County, Rhode Isla & 909 & 14.9 & 1.5 & $(12.0-17.8)$ \\
\hline Washington County, Rhode Island & 243 & 8.2 & 2.1 & $(4.1-12.4)$ \\
\hline Aiken County, South Carolina & 188 & 15.0 & 2.9 & $(9.3-20.7)$ \\
\hline
\end{tabular}

TABLE 21. (Continued) Estimated prevalence of adults aged $\geq 65$ years who have lost all of their natural teeth, by county - Behavioral Risk Factor Surveillance System, United States, 2012

\begin{tabular}{|c|c|c|c|c|}
\hline County & $\begin{array}{c}\text { Sample } \\
\text { size }\end{array}$ & $\%$ & SE & $95 \% \mathrm{Cl}$ \\
\hline Beaufort County, South Carolina & 418 & 6.9 & 1.6 & $(3.7-10.0)$ \\
\hline Charleston County, South Carolina & 325 & 14.0 & 2.8 & $(8.5-19.5)$ \\
\hline Greenville County, South Carolina & 249 & 24.3 & 4.0 & $(16.4-32.2)$ \\
\hline Horry County, South Carolina & 264 & 14.2 & 2.5 & $(9.3-19.2)$ \\
\hline Richland County, South Carolina & 262 & 16.4 & 3.4 & $(9.8-23.1)$ \\
\hline Spartanburg County, South Carolina & 207 & 29.0 & 4.9 & $(19.5-38.6)$ \\
\hline Lincoln County, South Dakota & 123 & 9.5 & 2.5 & $(4.5-14.5)$ \\
\hline Minnehaha County, South Dakota & 147 & 19.9 & 4.6 & $(10.9-28.8)$ \\
\hline Pennington County, South Dakota & 142 & 15.9 & 3.5 & $(9.1-22.6)$ \\
\hline Davidson County, Tennessee & 152 & 17.2 & 4.4 & $(8.5-25.9)$ \\
\hline Shelby County, Tennessee & 125 & 17.6 & 3.7 & $(10.3-24.8)$ \\
\hline Bexar County, Texas & 167 & 10.1 & 2.8 & $(4.6-15.7)$ \\
\hline Dallas County, Texas & 124 & 14.5 & 3.8 & $(7.1-21.9)$ \\
\hline El Paso County, Texas & 193 & 9.1 & 2.2 & $(4.7-13.5)$ \\
\hline Harris County, Texas & 130 & 12.8 & 3.9 & $(5.3-20.4)$ \\
\hline Hidalgo County, Texas & 182 & 12.1 & 3.5 & $(5.3-18.9)$ \\
\hline Tarrant County, Texas & 149 & $\mathrm{~N} / \mathrm{A}$ & $\mathrm{N} / \mathrm{A}$ & $(\mathrm{N} / \mathrm{A}-\mathrm{N} / \mathrm{A})$ \\
\hline Travis County, Texas & 290 & 8.3 & 1.9 & $(4.6-12.0)$ \\
\hline Davis County, Utah & 264 & 12.1 & 2.3 & $(7.6-16.6)$ \\
\hline Salt Lake County, Utah & 1,000 & 11.7 & 1.4 & $(9.0-14.4)$ \\
\hline Tooele County, Utah & 137 & $\mathrm{~N} / \mathrm{A}$ & $\mathrm{N} / \mathrm{A}$ & $(\mathrm{N} / \mathrm{A}-\mathrm{N} / \mathrm{A})$ \\
\hline Utah County, Utah & 350 & 10.6 & 1.9 & $(6.9-14.3)$ \\
\hline Wasatch County, Utah & 152 & $N / A^{*}$ & $N / A^{*}$ & $\left(\mathrm{~N} / \mathrm{A}-\mathrm{N} / \mathrm{A}^{*}\right)$ \\
\hline Weber County, Utah & 279 & 9.7 & 2.0 & $(5.7-13.7)$ \\
\hline Chittenden County, Vermont & 228 & 14.9 & 2.9 & $(9.3-20.6)$ \\
\hline Rutland County, Vermont & 188 & 13.4 & 2.9 & $(7.6-19.1)$ \\
\hline Washington County, Vermont & 132 & 15.8 & 3.7 & $(8.5-23.2)$ \\
\hline Windsor County, Vermont & 199 & 13.7 & 2.9 & $(8.0-19.5)$ \\
\hline Fairfax County, Virginia & 156 & $\mathrm{~N} / \mathrm{A}$ & $\mathrm{N} / \mathrm{A}$ & $(\mathrm{N} / \mathrm{A}-\mathrm{N} / \mathrm{A})$ \\
\hline Clark County, Washington & 227 & 8.1 & 1.9 & $(4.3-11.9)$ \\
\hline King County, Washington & 1,084 & 6.1 & 0.9 & $(4.3-8.0)$ \\
\hline Kitsap County, Washington & 193 & 10.0 & 2.8 & $(4.6-15.5)$ \\
\hline Pierce County, Washington & 298 & 15.3 & 2.8 & $(9.9-20.8)$ \\
\hline Snohomish County, Washington & 278 & 13.8 & 3.4 & $(7.1-20.5)$ \\
\hline Spokane County, Washington & 323 & 14.3 & 2.7 & $(8.9-19.7)$ \\
\hline Thurston County, Washington & 156 & 13.6 & 3.7 & $(6.3-21.0)$ \\
\hline Whatcom County, Washington & 268 & 7.6 & 2.1 & $(3.5-11.7)$ \\
\hline Yakima County, Washington & 155 & 16.8 & 3.6 & $(9.7-23.9)$ \\
\hline Kanawha County, West Virginia & 194 & 30.6 & 3.9 & $(23.0-38.2)$ \\
\hline Milwaukee County, Wisconsin & 239 & 12.6 & 3.2 & $(6.3-19.0)$ \\
\hline Laramie County, Wyoming & 373 & 14.4 & 2.1 & $(10.2-18.6)$ \\
\hline Natrona County, Wyoming & 308 & 18.4 & 3.3 & $(12.0-24.7)$ \\
\hline San Juan Municipio, Puerto Rico & 207 & 14.7 & 3.1 & $(8.7-20.7)$ \\
\hline Median & & 14.5 & & \\
\hline Range & & $5.8-37.1$ & & \\
\hline
\end{tabular}

Abbreviations: $\mathrm{Cl}=$ confidence interval; $\mathrm{SE}=$ standard error.

* Estimate not available (N/A) if the unweighted sample size for the denominator was $<50$ or if the relative standard error is $>0.3$. 
TABLE 22. Estimated prevalence of adults aged 50-75 years who received a colorectal cancer screening based on the most recent guidelines,* by state/territory - Behavioral Risk Factor Surveillance System, United States, 2012

\begin{tabular}{|c|c|c|c|c|}
\hline State/Territory & $\begin{array}{l}\text { Sample } \\
\text { size }\end{array}$ & $\%$ & SE & $95 \% \mathrm{Cl}$ \\
\hline Alabama & 4,673 & 65.9 & 1.0 & $(64.0-67.9)$ \\
\hline Alaska & 1,873 & 56.0 & 1.6 & $(52.9-59.2)$ \\
\hline Arizona & 3,525 & 58.5 & 1.4 & $(55.8-61.2)$ \\
\hline Arkansas & 2,485 & 56.8 & 1.3 & $(54.2-59.3)$ \\
\hline California & 5,512 & 67.2 & 0.9 & $(65.3-69.0)$ \\
\hline Colorado & 5,779 & 65.2 & 0.8 & $(63.6-66.8)$ \\
\hline Connecticut & 4,019 & 72.2 & 1.0 & $(70.3-74.1)$ \\
\hline Delaware & 2,490 & 71.5 & 1.3 & $(69.0-74.0)$ \\
\hline District of Columbia & 1,727 & 67.2 & 1.9 & (63.4-70.9) \\
\hline Florida & 3,665 & 66.0 & 1.2 & $(63.6-68.4)$ \\
\hline Georgia & 2,894 & 67.7 & 1.2 & $(65.4-70.0)$ \\
\hline Hawaii & 3,255 & 64.9 & 1.3 & $(62.3-67.5)$ \\
\hline Idaho & 2,973 & 60.4 & 1.5 & $(57.5-63.3)$ \\
\hline Illinois & 2,657 & 60.9 & 1.3 & (58.4-63.5) \\
\hline Indiana & 4,003 & 60.5 & 1.0 & $(58.5-62.4)$ \\
\hline lowa & 3,312 & 66.2 & 0.9 & $(64.3-68.1)$ \\
\hline Kansas & 5,773 & 64.7 & 0.8 & $(63.1-66.3)$ \\
\hline Kentucky & 5,669 & 63.0 & 1.0 & $(61.0-64.9)$ \\
\hline Louisiana & 4,760 & 60.2 & 1.1 & $(58.0-62.3)$ \\
\hline Maine & 5,050 & 73.4 & 0.8 & (72.0-74.9) \\
\hline Maryland & 6,275 & 70.8 & 0.9 & $(68.9-72.6)$ \\
\hline Massachusetts & 9,527 & 76.4 & 0.7 & $(75.1-77.8)$ \\
\hline Michigan & 5,222 & 69.2 & 0.9 & $(67.5-70.9)$ \\
\hline Minnesota & 5,558 & 70.6 & 0.8 & $(69.0-72.2)$ \\
\hline Mississippi & 4,034 & 58.4 & 1.0 & (56.4-60.4) \\
\hline Missouri & 3,261 & 64.1 & 1.2 & $(61.7-66.5)$ \\
\hline Montana & 4,203 & 56.4 & 1.0 & $(54.5-58.4)$ \\
\hline Nebraska & 9,015 & 61.1 & 0.7 & $(59.6-62.5)$ \\
\hline Nevada & 2,302 & 58.6 & 1.7 & (55.3-61.9) \\
\hline New Hampshire & 3,792 & 75.0 & 1.0 & (73.1-76.9) \\
\hline New Jersey & 6,908 & 62.4 & 0.9 & $(60.6-64.1)$ \\
\hline New Mexico & 4,033 & 57.6 & 0.9 & $(55.8-59.5)$ \\
\hline New York & 2,512 & 69.6 & 1.3 & $(67.0-72.2)$ \\
\hline North Carolina & 5,411 & 68.6 & 0.8 & $(67.0-70.2)$ \\
\hline North Dakota & 2,262 & 58.1 & 1.3 & $(55.6-60.6)$ \\
\hline Ohio & 6,310 & 63.4 & 0.8 & $(61.7-65.0)$ \\
\hline Oklahoma & 3,892 & 58.5 & 1.0 & $(56.6-60.4)$ \\
\hline Oregon & 2,562 & 65.5 & 1.2 & $(63.1-67.9)$ \\
\hline Pennsylvania & 9,388 & 66.8 & 0.7 & $(65.3-68.2)$ \\
\hline Rhode Island & 2,580 & 72.9 & 1.1 & $(70.6-75.1)$ \\
\hline South Carolina & 6,229 & 65.1 & 0.9 & $(63.3-66.8)$ \\
\hline South Dakota & 3,576 & 62.5 & 1.4 & $(59.7-65.2)$ \\
\hline Tennessee & 3,458 & 64.9 & 1.1 & $(62.7-67.2)$ \\
\hline Texas & 3,923 & 58.2 & 1.2 & (56.0-60.5) \\
\hline Utah & 5,008 & 67.9 & 0.9 & $(66.2-69.6)$ \\
\hline Vermont & 3,111 & 71.4 & 1.0 & (69.5-73.4) \\
\hline Virginia & 3,470 & 67.9 & 1.0 & $(65.9-69.9)$ \\
\hline Washington & 7,604 & 67.4 & 0.8 & $(65.9-68.9)$ \\
\hline West Virginia & 2,749 & 63.2 & 1.1 & $(61.2-65.3)$ \\
\hline Wisconsin & 2,553 & 71.4 & 1.4 & $(68.7-74.1)$ \\
\hline Wyoming & 3,268 & 55.7 & 1.3 & (53.1-58.3) \\
\hline Guam & 579 & 40.0 & 2.6 & $(34.8-45.1)$ \\
\hline Puerto Rico & 2,688 & 52.3 & 1.1 & $(50.1-54.6)$ \\
\hline Median & & 64.9 & & \\
\hline Range & & $40.0-76.4$ & & \\
\hline
\end{tabular}

Abbreviations: $\mathrm{Cl}=$ confidence interval; $\mathrm{SE}=$ standard error.

* Adults aged 50-75 years who have had a blood stool test in the past year, sigmoidoscopy in the past 5 years, and blood stool test in the past 3 years, or a colonoscopy in the past 10 years.
TABLE 23. Estimated prevalence of adults aged 50-75 years who received a colorectal cancer screening based on the most recent guidelines, ${ }^{*}$ by metropolitan and micropolitan statistical area Behavioral Risk Factor Surveillance System, United States, 2012

\begin{tabular}{|c|c|c|c|c|}
\hline MMSA(s) & $\begin{array}{l}\text { Sample } \\
\text { size }\end{array}$ & $\%$ & SE & $95 \% \mathrm{Cl}$ \\
\hline Aguadilla-Isabela, Puerto Rico & 249 & 50.2 & 3.7 & $(43.1-57.4)$ \\
\hline Akron, Ohio & 365 & 68.4 & 3.1 & $(62.3-74.6)$ \\
\hline Albuquerque, New Mexico & 1,418 & 65.4 & 1.6 & $(62.4-68.5)$ \\
\hline $\begin{array}{l}\text { Allentown-Bethlehem-Easton, } \\
\text { Pennsylvania-New Jersey }\end{array}$ & 641 & 64.0 & 3.0 & $(58.2-69.8)$ \\
\hline Anaheim-Santa Ana-Irvine, California ${ }^{\dagger}$ & 369 & 68.8 & 3.7 & $(61.5-76.2)$ \\
\hline Anchorage, Alaska & 594 & 58.8 & 2.6 & $(53.7-63.9)$ \\
\hline Asheville, North Carolina & 299 & 68.3 & 3.3 & $(61.8-74.8)$ \\
\hline $\begin{array}{l}\text { Atlanta-Sandy Springs-Roswell, } \\
\text { Georgia }\end{array}$ & 1,145 & 69.4 & 1.9 & $(65.7-73.0)$ \\
\hline Atlantic City-Hammonton, New Jersey & 522 & 65.4 & 2.7 & $(60.1-70.7)$ \\
\hline $\begin{array}{l}\text { Augusta-Richmond County, Georgia- } \\
\text { South Carolina }\end{array}$ & 513 & 73.3 & 3.3 & $(66.8-79.9)$ \\
\hline Augusta-Waterville, Maine & 403 & 79.6 & 2.4 & $(74.8-84.4)$ \\
\hline Austin-Round Rock, Texas & 631 & 62.6 & 3.3 & $(56.1-69.0)$ \\
\hline $\begin{array}{l}\text { Baltimore-Columbia-Towson, } \\
\text { Maryland }\end{array}$ & 2,288 & 70.2 & 1.4 & $(67.5-72.9)$ \\
\hline Bangor, Maine & 448 & 73.3 & 2.4 & $(68.5-78.1)$ \\
\hline Barnstable Town, Massachusetts & 295 & 80.7 & 2.8 & $(75.2-86.3)$ \\
\hline Barre, Vermont & 273 & 78.4 & 3.1 & $(72.4-84.5)$ \\
\hline Baton Rouge, Louisiana & 735 & 66.6 & 2.6 & $(61.5-71.8)$ \\
\hline Bellingham, Washington & 447 & 70.0 & 3.1 & $(64.0-76.0)$ \\
\hline Berlin, New Hampshire-Vermont & 379 & 63.4 & 3.3 & $(57.0-69.8)$ \\
\hline Billings, Montana & 345 & 59.5 & 3.0 & $(53.6-65.4)$ \\
\hline Birmingham-Hoover, Alabama & 895 & 69.7 & 2.0 & $(65.7-73.7)$ \\
\hline Bismarck, North Dakota & 381 & 62.0 & 3.1 & $(55.9-68.2)$ \\
\hline Boise City, Idaho & 692 & 63.7 & 2.8 & $(58.2-69.1)$ \\
\hline Boston, Massachusetts ${ }^{\dagger}$ & 2,531 & 74.9 & 1.4 & $(72.2-77.6)$ \\
\hline Boulder, Colorado & 239 & 65.0 & 3.6 & $(57.8-72.1)$ \\
\hline Bremerton-Silverdale, Washington & 309 & 72.3 & 3.1 & $(66.2-78.4)$ \\
\hline $\begin{array}{l}\text { Bridgeport-Stamford-Norwalk, } \\
\text { Connecticut }\end{array}$ & 914 & 70.4 & 2.2 & $(66.1-74.8)$ \\
\hline Burlington-South Burlington, Vermont & 694 & 73.4 & 2.1 & $(69.4-77.4)$ \\
\hline $\begin{array}{l}\text { Cambridge-Newton-Framingham, } \\
\text { Massachusetts }^{\dagger}\end{array}$ & 2,979 & 76.7 & 1.2 & $(74.4-79.1)$ \\
\hline Camden, New Jersey ${ }^{\dagger}$ & 912 & 63.6 & 2.1 & $(59.6-67.7)$ \\
\hline Canton-Massillon, Ohio & 343 & 59.9 & 3.5 & $(53.0-66.9)$ \\
\hline Casper, Wyoming & 400 & 50.1 & 3.6 & $(42.9-57.2)$ \\
\hline Cedar Rapids, lowa & 253 & 73.3 & 3.3 & $(66.8-79.8)$ \\
\hline Charleston, West Virginia & 370 & 70.0 & 2.7 & $(64.6-75.4)$ \\
\hline $\begin{array}{l}\text { Charleston-North Charleston, } \\
\text { South Carolina }\end{array}$ & 807 & 67.2 & 2.3 & $(62.7-71.7)$ \\
\hline $\begin{array}{l}\text { Charlotte-Concord-Gastonia, } \\
\text { North Carolina-South Carolina }\end{array}$ & 1,076 & 68.3 & 2.0 & $(64.5-72.2)$ \\
\hline Chattanooga, Tennessee-Georgia & 311 & 61.4 & 3.9 & $(53.8-69.0)$ \\
\hline Cheyenne, Wyoming & 499 & 58.5 & 3.3 & $(51.9-65.1)$ \\
\hline $\begin{array}{l}\text { Chicago-Naperville-Elgin, } \\
\text { Illinois-Indiana-Wisconsin }\end{array}$ & 1,670 & 58.4 & 1.8 & $(54.9-61.9)$ \\
\hline Cincinnati, Ohio-Kentucky-Indiana & 1,097 & 62.4 & 2.0 & $(58.5-66.2)$ \\
\hline $\begin{array}{l}\text { Claremont-Lebanon, New } \\
\text { Hampshire-Vermont }\end{array}$ & 1,009 & 71.2 & 1.9 & $(67.5-74.8)$ \\
\hline Cleveland-Elyria, Ohio & 859 & 67.6 & 2.2 & $(63.3-71.9)$ \\
\hline Colorado Springs, Colorado & 534 & 68.6 & 2.4 & $(63.9-73.3)$ \\
\hline Columbia, South Carolina & 833 & 70.6 & 2.3 & $(66.0-75.2)$ \\
\hline Columbus, Ohio & 665 & 65.6 & 2.4 & $(60.8-70.3)$ \\
\hline Concord, New Hampshire & 342 & 79.6 & 2.8 & $(74.2-85.1)$ \\
\hline Dallas-Plano-Irving, Texas ${ }^{\dagger}$ & 340 & 59.5 & 3.5 & $(52.6-66.4)$ \\
\hline Dayton, Ohio & 415 & 65.1 & 3.1 & $(59.0-71.2)$ \\
\hline Denver-Aurora-Lakewood, Colorado & 2,084 & 68.4 & 1.3 & $(65.9-70.9)$ \\
\hline Des Moines-West Des Moines, lowa & 540 & 66.6 & 2.4 & $(62.0-71.2)$ \\
\hline
\end{tabular}

See table footnotes on page 53. 
TABLE 23. (Continued) Estimated prevalence of adults aged 50-75 years who received a colorectal cancer screening based on the most recent guidelines, ${ }^{*}$ by metropolitan and micropolitan statistical area — Behavioral Risk Factor Surveillance System, United States, 2012

\begin{tabular}{|c|c|c|c|c|}
\hline MMSA(s) & $\begin{array}{l}\text { Sample } \\
\text { size }\end{array}$ & $\%$ & SE & $95 \% \mathrm{Cl}$ \\
\hline Detroit-Dearborn-Livonia, Michigan $^{\dagger}$ & 1,062 & 64.0 & 2.5 & $(59.1-68.9)$ \\
\hline Dover, Delaware & 688 & 68.8 & 2.5 & $(63.8-73.8)$ \\
\hline Duluth, Minnesota-Wisconsin & 274 & 71.7 & 3.3 & $(65.3-78.1)$ \\
\hline Durham-Chapel Hill, North Carolina & 346 & 75.2 & 2.9 & $(69.5-80.9)$ \\
\hline El Paso, Texas & 297 & 47.1 & 4.3 & $(38.6-55.6)$ \\
\hline Eugene, Oregon & 276 & 65.1 & 3.6 & $(58.1-72.1)$ \\
\hline Fairbanks, Alaska & 234 & 49.5 & 4.0 & $(41.6-57.3)$ \\
\hline Fargo, North Dakota-Minnesota & 417 & 62.1 & 3.3 & $(55.6-68.6)$ \\
\hline Farmington, New Mexico & 286 & 51.5 & 3.7 & $(44.3-58.6)$ \\
\hline Fayetteville, North Carolina & 221 & 72.7 & 3.7 & $(65.4-80.0)$ \\
\hline $\begin{array}{l}\text { Fayetteville-Springdale-Rogers, } \\
\text { Arkansas-Missouri }\end{array}$ & 389 & 52.7 & 3.6 & $(45.6-59.7)$ \\
\hline Fort Collins, Colorado & 264 & 66.1 & 3.4 & $(59.5-72.7)$ \\
\hline Fort Wayne, Indiana & 222 & 56.0 & 4.0 & $(48.2-63.8)$ \\
\hline Fort Worth-Arlington, Texas ${ }^{\dagger}$ & 335 & 61.6 & 3.5 & $(54.8-68.4)$ \\
\hline Grand Island, Nebraska & 420 & 58.6 & 3.1 & $(52.6-64.7)$ \\
\hline Grand Rapids-Wyoming, Michigan & 448 & 71.1 & 2.8 & $(65.6-76.6)$ \\
\hline Great Falls, Montana & 350 & 58.8 & 3.1 & $(52.7-64.9)$ \\
\hline Greeley, Colorado & 229 & 56.2 & 3.8 & $(48.7-63.8)$ \\
\hline $\begin{array}{l}\text { Greensboro-High Point, } \\
\text { North Carolina }\end{array}$ & 365 & 66.0 & 3.2 & $(59.8-72.3)$ \\
\hline $\begin{array}{l}\text { Greenville-Anderson-Mauldin, } \\
\text { South Carolina }\end{array}$ & 736 & 66.8 & 2.3 & $(62.3-71.3)$ \\
\hline Gulfport-Biloxi-Pascagoula, Mississippi & 367 & 65.4 & 3.0 & $(59.5-71.3)$ \\
\hline $\begin{array}{l}\text { Hagerstown-Martinsburg, Maryland- } \\
\text { West Virginia }\end{array}$ & 392 & 66.4 & 3.7 & $(59.2-73.7)$ \\
\hline Harrisburg-Carlisle, Pennsylvania & 301 & 70.4 & 3.3 & $(64.0-76.7)$ \\
\hline $\begin{array}{l}\text { Hartford-West Hartford-East Hartford, } \\
\text { Connecticut }\end{array}$ & 1,216 & 73.1 & 1.7 & $(69.8-76.3)$ \\
\hline Heber, Utah & 223 & 76.3 & 4.1 & $(68.4-84.3)$ \\
\hline Hilo, Hawaii & 670 & 61.5 & 2.7 & $(56.1-66.9)$ \\
\hline $\begin{array}{l}\text { Hilton Head Island-Bluffton-Beaufort, } \\
\text { South Carolina }\end{array}$ & 534 & 63.9 & 3.2 & $(57.6-70.2)$ \\
\hline $\begin{array}{l}\text { Houston-The Woodlands-Sugar Land, } \\
\text { Texas }\end{array}$ & 397 & 62.0 & 3.0 & $(56.2-67.8)$ \\
\hline $\begin{array}{l}\text { Huntington-Ashland, West } \\
\text { Virginia-Kentucky-Ohio }\end{array}$ & 584 & 65.4 & 2.4 & $(60.6-70.2)$ \\
\hline Huntsville, Alabama & 281 & 68.9 & 4.0 & $(61.2-76.7)$ \\
\hline Idaho Falls, Idaho & 272 & 58.5 & 4.7 & $(49.3-67.7)$ \\
\hline $\begin{array}{l}\text { Indianapolis-Carmel-Anderson, } \\
\text { Indiana }\end{array}$ & 979 & 66.9 & 1.9 & $(63.1-70.6)$ \\
\hline Jackson, Mississippi & 438 & 64.2 & 2.8 & $(58.7-69.8)$ \\
\hline Jacksonville, Florida & 253 & 67.6 & 4.1 & $(59.7-75.6)$ \\
\hline Kahului-Wailuku-Lahaina, Hawaii & 577 & 60.5 & 3.2 & $(54.1-66.8)$ \\
\hline Kalispell, Montana & 263 & 59.9 & 3.4 & $(53.2-66.6)$ \\
\hline Kansas City, Missouri-Kansas & 2,312 & 70.5 & 1.7 & $(67.2-73.8)$ \\
\hline Kapaa, Hawaii & 388 & 60.2 & 3.4 & $(53.6-66.8)$ \\
\hline Keene, New Hampshire & 291 & 71.0 & 3.3 & $(64.5-77.6)$ \\
\hline Kennewick-Richland, Washington & 258 & 60.5 & 3.9 & $(52.9-68.2)$ \\
\hline $\begin{array}{l}\text { Kingsport-Bristol-Bristol, } \\
\text { Tennessee-Virginia }\end{array}$ & 300 & 67.2 & 3.7 & $(60.0-74.4)$ \\
\hline Knoxville, Tennessee & 405 & 72.5 & 2.9 & $(66.9-78.1)$ \\
\hline Laconia, New Hampshire & 311 & 70.4 & 3.6 & $(63.4-77.4)$ \\
\hline Lafayette, Louisiana & 279 & 56.7 & 4.0 & $(49.0-64.5)$ \\
\hline Las Cruces, New Mexico & 314 & 57.2 & 3.2 & $(50.9-63.4)$ \\
\hline $\begin{array}{l}\text { Las Vegas-Henderson-Paradise, } \\
\text { Nevada }\end{array}$ & 821 & 57.0 & 2.3 & $(52.5-61.5)$ \\
\hline Lewiston-Auburn, Maine & 324 & 70.0 & 3.1 & $(63.8-76.2)$ \\
\hline Lexington-Fayette, Kentucky & 228 & 69.9 & 4.0 & $(62.1-77.6)$ \\
\hline Lincoln, Nebraska & 641 & 67.1 & 2.3 & $(62.6-71.6)$ \\
\hline
\end{tabular}

See table footnotes on page 53 .
TABLE 23. (Continued) Estimated prevalence of adults aged 50-75 years who received a colorectal cancer screening based on the most recent guidelines, ${ }^{*}$ by metropolitan and micropolitan statistical area — Behavioral Risk Factor Surveillance System, United States, 2012

\begin{tabular}{|c|c|c|c|c|}
\hline $\operatorname{MMSA}(\mathrm{s})$ & $\begin{array}{l}\text { Sample } \\
\text { size }\end{array}$ & $\%$ & SE & $95 \% \mathrm{Cl}$ \\
\hline $\begin{array}{l}\text { Little Rock-North Little Rock-Conway, } \\
\text { Arkansas }\end{array}$ & 557 & 66.8 & 2.6 & $(61.7-71.9)$ \\
\hline Logan, Utah-Idaho & 183 & 71.2 & 3.8 & $(63.8-78.7)$ \\
\hline $\begin{array}{l}\text { Los Angeles-Long Beach-Glendale, } \\
\text { California }^{\dagger}\end{array}$ & 1,132 & 63.4 & 2.2 & $(59.1-67.8)$ \\
\hline $\begin{array}{l}\text { Louisville/Jefferson County, } \\
\text { Kentucky-Indiana }\end{array}$ & 1,046 & 64.2 & 2.3 & $(59.7-68.6)$ \\
\hline Lumberton, North Carolina & 277 & 65.8 & 5.3 & $(55.3-76.2)$ \\
\hline Manchester-Nashua, New Hampshire & 919 & 77.4 & 1.7 & $(74.0-80.8)$ \\
\hline McAllen-Edinburg-Mission, Texas & 246 & 48.0 & 4.9 & $(38.4-57.6)$ \\
\hline $\begin{array}{l}\text { Memphis, } \\
\text { Tennessee-Mississippi-Arkansas }\end{array}$ & 597 & 63.9 & 2.9 & $(58.3-69.5)$ \\
\hline $\begin{array}{l}\text { Miami-Fort Lauderdale-West Palm } \\
\text { Beach, Florida }\end{array}$ & 727 & 57.2 & 2.8 & $(51.6-62.7)$ \\
\hline $\begin{array}{l}\text { Milwaukee-Waukesha-West Allis, } \\
\text { Wisconsin }\end{array}$ & 572 & 71.8 & 3.0 & $(65.9-77.7)$ \\
\hline $\begin{array}{l}\text { Minneapolis-St. Paul-Bloomington, } \\
\text { Minnesota-Wisconsin }\end{array}$ & 3,485 & 70.7 & 1.1 & $(68.5-73.0)$ \\
\hline Missoula, Montana & 370 & 62.1 & 3.0 & $(56.2-68.0)$ \\
\hline Mobile, Alabama & 447 & 61.5 & 3.3 & $(55.1-67.9)$ \\
\hline Montgomery, Alabama & 263 & 72.4 & 3.9 & $(64.8-80.0)$ \\
\hline $\begin{array}{l}\text { Montgomery County-Bucks County- } \\
\text { Chester County, Pennsylvania }{ }^{\dagger}\end{array}$ & 529 & 71.1 & 2.4 & $(66.4-75.8)$ \\
\hline $\begin{array}{l}\text { Myrtle Beach-Conway-North Myrtle } \\
\text { Beach, South Carolina-North Carolina }\end{array}$ & 505 & 68.9 & 2.5 & $(63.9-73.9)$ \\
\hline $\begin{array}{l}\text { Nashville-Davidson-Murfreesboro- } \\
\text { Franklin, Tennessee }\end{array}$ & 588 & 70.6 & 2.4 & $(65.8-75.3)$ \\
\hline $\begin{array}{l}\text { Nassau County-Suffolk County, } \\
\text { New York }\end{array}$ & 385 & 67.7 & 3.1 & $(61.7-73.8)$ \\
\hline Newark, New Jersey-Pennsylvania ${ }^{\dagger}$ & 3,040 & 62.4 & 1.5 & $(59.4-65.3)$ \\
\hline New Haven-Milford, Connecticut & 943 & 71.3 & 2.1 & $(67.2-75.3)$ \\
\hline New Orleans-Metairie, Louisiana & 632 & 62.2 & 2.4 & $(57.5-66.9)$ \\
\hline $\begin{array}{l}\text { New York-Jersey City-White Plains, } \\
\text { New York-New Jersey }{ }^{\dagger}\end{array}$ & 2,748 & 68.2 & 1.7 & $(64.9-71.5)$ \\
\hline Norfolk, Nebraska & 283 & 60.0 & 3.4 & $(53.4-66.6)$ \\
\hline North Platte, Nebraska & 314 & 61.1 & 3.8 & $(53.7-68.6)$ \\
\hline Norwich-New London, Connecticut & 488 & 76.2 & 3.0 & $(70.3-82.2)$ \\
\hline $\begin{array}{l}\text { Oakland-Hayward-Berkeley, } \\
\text { California }^{\dagger}\end{array}$ & 397 & 78.3 & 3.2 & $(72.0-84.6)$ \\
\hline Ocean City, New Jersey & 286 & 68.0 & 3.6 & $(60.8-75.1)$ \\
\hline Ogden-Clearfield, Utah & 988 & 71.2 & 1.8 & $(67.7-74.7)$ \\
\hline Oklahoma City, Oklahoma & 1,080 & 63.1 & 1.8 & $(59.5-66.6)$ \\
\hline Olympia-Tumwater, Washington & 268 & 70.5 & 4.0 & $(62.6-78.4)$ \\
\hline Omaha-Council Bluffs, Nebraska-lowa & 2,345 & 64.9 & 1.3 & $(62.3-67.5)$ \\
\hline Orlando-Kissimmee-Sanford, Florida & 257 & 71.8 & 4.1 & $(63.7-79.8)$ \\
\hline Philadelphia, Pennsylvania ${ }^{\dagger}$ & 1,043 & 69.1 & 2.0 & $(65.2-72.9)$ \\
\hline Phoenix-Mesa-Scottsdale, Arizona & 1,150 & 57.6 & 2.0 & $(53.6-61.5)$ \\
\hline Pittsburgh, Pennsylvania & 1,556 & 63.6 & 1.5 & $(60.7-66.6)$ \\
\hline Ponce, Puerto Rico & 230 & 49.5 & 3.8 & $(42.0-56.9)$ \\
\hline Portland-South Portland, Maine & 1,628 & 73.6 & 1.3 & $(71.0-76.2)$ \\
\hline $\begin{array}{l}\text { Portland-Vancouver-Hillsboro, } \\
\text { Oregon-Washington }\end{array}$ & 1,456 & 71.2 & 1.6 & $(68.0-74.5)$ \\
\hline $\begin{array}{l}\text { Providence-Warwick, Rhode } \\
\text { Island-Massachusetts }\end{array}$ & 3,778 & 74.3 & 1.1 & $(72.2-76.4)$ \\
\hline Provo-Orem, Utah & 550 & 69.0 & 2.4 & $(64.3-73.6)$ \\
\hline Raleigh, North Carolina & 315 & 76.8 & 3.0 & $(70.8-82.8)$ \\
\hline Rapid City, South Dakota & 477 & 60.3 & 3.2 & $(54.0-66.7)$ \\
\hline Reno, Nevada & 747 & 63.7 & 2.3 & $(59.1-68.3)$ \\
\hline Richmond, Virginia & 462 & 72.9 & 2.6 & $(67.7-78.0)$ \\
\hline
\end{tabular}

See table footnotes on page 53. 
TABLE 23. (Continued) Estimated prevalence of adults aged 50-75 years who received a colorectal cancer screening based on the most recent guidelines, ${ }^{*}$ by metropolitan and micropolitan statistical area — Behavioral Risk Factor Surveillance System, United States, 2012

\begin{tabular}{|c|c|c|c|c|}
\hline MMSA(s) & $\begin{array}{l}\text { Sample } \\
\text { size }\end{array}$ & $\%$ & SE & $95 \% \mathrm{Cl}$ \\
\hline $\begin{array}{l}\text { Riverside-San Bernardino-Ontario, } \\
\text { California }\end{array}$ & 595 & 64.4 & 2.7 & $(59.1-69.7)$ \\
\hline $\begin{array}{l}\text { Rockingham County-Strafford County, } \\
\text { New Hampshire }{ }^{\dagger}\end{array}$ & 808 & 76.3 & 2.0 & $(72.4-80.2)$ \\
\hline Rutland, Vermont & 317 & 67.6 & 3.3 & $(61.2-74.0)$ \\
\hline $\begin{array}{l}\text { Sacramento-Roseville-Arden-Arcade, } \\
\text { California }\end{array}$ & 417 & 71.7 & 3.5 & $(64.8-78.6)$ \\
\hline St. Louis, Missouri-Illinois & 970 & 67.3 & 2.2 & $(63.0-71.6)$ \\
\hline Salisbury, Maryland-Delaware & 1,177 & 70.2 & 2.0 & $(66.4-74.1)$ \\
\hline Salt Lake City, Utah & 1,904 & 67.6 & 1.4 & $(64.8-70.4)$ \\
\hline San Antonio-New Braunfels, Texas & 331 & 61.3 & 3.7 & $(54.0-68.5)$ \\
\hline San Diego-Carlsbad, California & 377 & 66.7 & 3.3 & $(60.1-73.2)$ \\
\hline $\begin{array}{l}\text { San Francisco-Redwood City-South } \\
\text { San Francisco, California }{ }^{\dagger}\end{array}$ & 227 & 63.3 & 5.9 & $(51.7-74.9)$ \\
\hline $\begin{array}{l}\text { San Jose-Sunnyvale-Santa Clara, } \\
\text { California }\end{array}$ & 244 & 70.1 & 4.2 & $(61.9-78.2)$ \\
\hline San Juan-Carolina-Caguas, Puerto Rico & 1,665 & 53.4 & 1.4 & $(50.6-56.2)$ \\
\hline Santa Fe, New Mexico & 349 & 60.3 & 3.0 & $(54.3-66.3)$ \\
\hline Sayre, Pennsylvania & 968 & 63.4 & 1.8 & $(59.9-66.9)$ \\
\hline Scottsbluff, Nebraska & 335 & 53.6 & 3.6 & $(46.5-60.7)$ \\
\hline $\begin{array}{l}\text { Scranton-Wilkes-Barre-Hazleton, } \\
\text { Pennsylvania }\end{array}$ & 358 & 69.9 & 3.2 & $(63.8-76.1)$ \\
\hline Seattle-Bellevue-Everett, Washington ${ }^{\dagger}$ & 2,311 & 68.9 & 1.3 & $(66.3-71.5)$ \\
\hline Shreveport-Bossier City, Louisiana & 274 & 65.6 & 3.8 & $(58.2-73.0)$ \\
\hline $\begin{array}{l}\text { Silver Spring-Frederick-Rockville, } \\
\text { Maryland }{ }^{\dagger}\end{array}$ & 1,093 & 70.6 & 2.1 & $(66.5-74.8)$ \\
\hline $\begin{array}{l}\text { Sioux City, lowa-Nebraska- } \\
\text { South Dakota }\end{array}$ & 611 & 71.4 & 3.6 & $(64.3-78.5)$ \\
\hline Sioux Falls, South Dakota & 561 & 64.5 & 2.9 & $(58.8-70.2)$ \\
\hline Spartanburg, South Carolina & 323 & 68.8 & 3.5 & $(61.9-75.7)$ \\
\hline Spokane-Spokane Valley, Washington & 570 & 66.5 & 2.4 & $(61.8-71.2)$ \\
\hline Springfield, Massachusetts & 1,038 & 78.8 & 1.9 & $(75.0-82.5)$ \\
\hline Tacoma-Lakewood, Washington ${ }^{\dagger}$ & 563 & 68.6 & 2.5 & $(63.6-73.5)$ \\
\hline $\begin{array}{l}\text { Tampa-St. Petersburg-Clearwater, } \\
\text { Florida }\end{array}$ & 387 & 67.1 & 3.2 & $(60.8-73.4)$ \\
\hline
\end{tabular}

TABLE 23. (Continued) Estimated prevalence of adults aged 50-75 years who received a colorectal cancer screening based on the most recent guidelines, ${ }^{*}$ by metropolitan and micropolitan statistical area - Behavioral Risk Factor Surveillance System, United States, 2012

\begin{tabular}{|c|c|c|c|c|}
\hline MMSA(s) & $\begin{array}{l}\text { Sample } \\
\text { size }\end{array}$ & $\%$ & SE & $95 \% \mathrm{Cl}$ \\
\hline Toledo, Ohio & 483 & 57.2 & 2.9 & $(51.4-62.9$ \\
\hline Topeka, Kansas & 551 & 69.4 & 2.3 & $(64.8-74.0$ \\
\hline Torrington, Connecticut & 345 & 72.3 & 3.2 & $(66.0-78.7$ \\
\hline Trenton, New Jersey & 256 & 64.0 & 3.8 & $(56.6-71.3$ \\
\hline Tucson, Arizona & 462 & 65.5 & 3.0 & $(59.7-71.3$ \\
\hline Tulsa, Oklahoma & 818 & 63.0 & 2.1 & $(58.9-67.0$ \\
\hline Tuscaloosa, Alabama & 289 & 65.9 & 4.2 & $(57.7-74.2$ \\
\hline Urban Honolulu, Hawaii & 1,620 & 67.0 & 1.7 & $(63.5-70.4$ \\
\hline Vineland-Bridgeton, New Jersey & 268 & 55.5 & 4.6 & $(46.4-64.6$ \\
\hline $\begin{array}{l}\text { Virginia Beach-Norfolk-Newport News, } \\
\text { Virginia-North Carolina }\end{array}$ & 716 & 71.5 & 2.4 & $(66.8-76.2$ \\
\hline $\begin{array}{l}\text { Warren-Troy-Farmington Hills, } \\
\text { Michigan }{ }^{\dagger}\end{array}$ & 1,056 & 69.7 & 1.8 & $(66.1-73.2$ \\
\hline $\begin{array}{l}\text { Washington-Arlington-Alexandria, } \\
\text { District of Columbia-Virginia- } \\
\text { Maryland-West Virginia }{ }^{\dagger}\end{array}$ & 3,640 & 69.9 & 1.3 & $(67.4-72.5$ \\
\hline Wichita, Kansas & 1,124 & 68.6 & 1.8 & $(65.1-72.1$ \\
\hline $\begin{array}{l}\text { Wilmington, Delaware-Maryland- } \\
\text { New Jersey }{ }^{\dagger}\end{array}$ & 1,480 & 71.2 & 1.7 & $(67.8-74.6$ \\
\hline Winston-Salem, North Carolina & 358 & 68.2 & 3.0 & $(62.4-74.1$ \\
\hline Worcester, Massachusetts-Connecticut & 1,242 & 75.8 & 1.8 & $(72.1-79.4$ \\
\hline Yakima, Washington & 232 & 54.5 & 4.9 & $(44.9-64.0$ \\
\hline $\begin{array}{l}\text { Youngstown-Warren-Boardman, } \\
\text { Ohio-Pennsylvania }\end{array}$ & 506 & 61.9 & 3.2 & (55.7-68.1 \\
\hline Median & & 67.0 & & \\
\hline Range & & $47.1-80.7$ & & \\
\hline
\end{tabular}

Abbreviations: $\mathrm{Cl}=$ confidence interval; $\mathrm{MMSA}=$ metropolitan and micropolitan statistical area; $\mathrm{SE}=$ standard error.

* Adults aged 50-75 years who have had a blood stool test in the past year, sigmoidoscopy in the past 5 years, and blood stool test in the past 3 years, or a colonoscopy in the past 10 years.

+ Metropolitan division. 
TABLE 24. Estimated prevalence of adults aged 50-75 years who received a colorectal cancer screening based on the most recent guidelines, * by county - Behavioral Risk Factor Surveillance System, United States, 2012

\begin{tabular}{|c|c|c|c|c|}
\hline County & $\begin{array}{l}\text { Sample } \\
\text { size }\end{array}$ & $\%$ & SE & $95 \% \mathrm{Cl}$ \\
\hline Jefferson County, Alabama & 432 & 69.2 & 2.8 & $(63.8-74.7)$ \\
\hline Madison County, Alabama & 238 & 72.9 & 3.9 & $(65.3-80.5)$ \\
\hline Mobile County, Alabama & 447 & 61.5 & 3.3 & $(55.1-67.9)$ \\
\hline Anchorage Municipality, Alaska & 330 & 60.7 & 3.3 & $(54.3-67.1)$ \\
\hline Fairbanks North Star Borough, Alaska & 234 & 49.5 & 4.0 & $(41.6-57.3)$ \\
\hline Matanuska-Susitna Borough, Alaska & 264 & 53.4 & 3.8 & $(45.9-60.9)$ \\
\hline Maricopa County, Arizona & 886 & 57.2 & 2.1 & $(52.9-61.4)$ \\
\hline Pima County, Arizona & 462 & 65.5 & 3.0 & $(59.7-71.3)$ \\
\hline Pulaski County, Arkansas & 351 & 68.7 & 3.2 & $(62.4-75.0)$ \\
\hline Alameda County, California & 244 & 75.6 & 4.6 & $(66.5-84.6)$ \\
\hline Los Angeles County, California & 1,132 & 63.4 & 2.2 & $(59.1-67.8)$ \\
\hline Orange County, California & 369 & 68.8 & 3.7 & $(61.5-76.2)$ \\
\hline Riverside County, California & 318 & 60.6 & 3.6 & $(53.6-67.6)$ \\
\hline Sacramento County, California & 255 & 66.9 & 4.6 & $(57.8-75.9)$ \\
\hline San Bernardino County, California & 277 & 67.9 & 3.9 & $(60.2-75.6)$ \\
\hline San Diego County, California & 377 & 66.7 & 3.3 & $(60.1-73.2)$ \\
\hline Santa Clara County, California & 237 & 70.0 & 4.2 & $(61.7-78.2)$ \\
\hline Adams County, Colorado & 351 & 64.9 & 3.2 & $(58.6-71.3)$ \\
\hline Arapahoe County, Colorado & 349 & 70.3 & 2.8 & $(64.8-75.8)$ \\
\hline Boulder County, Colorado & 239 & 65.0 & 3.6 & $(57.8-72.1)$ \\
\hline Denver County, Colorado & 369 & 68.4 & 2.9 & $(62.7-74.1)$ \\
\hline Douglas County, Colorado & 219 & 69.0 & 3.6 & $(62.0-76.1)$ \\
\hline El Paso County, Colorado & 439 & 70.1 & 2.5 & $(65.1-75.1)$ \\
\hline Jefferson County, Colorado & 499 & 70.8 & 2.5 & $(65.9-75.7)$ \\
\hline Larimer County, Colorado & 264 & 66.1 & 3.4 & $(59.5-72.7)$ \\
\hline Weld County, Colorado & 229 & 56.2 & 3.8 & $(48.7-63.8)$ \\
\hline Fairfield County, Connecticut & 914 & 70.4 & 2.2 & $(66.1-74.8)$ \\
\hline Hartford County, Connecticut & 911 & 73.8 & 1.9 & $(70.1-77.4)$ \\
\hline Litchfield County, Connecticut & 345 & 72.3 & 3.2 & $(66.0-78.7)$ \\
\hline New Haven County, Connecticut & 943 & 71.3 & 2.1 & $(67.2-75.3)$ \\
\hline New London County, Connecticut & 488 & 76.2 & 3.0 & $(70.3-82.2)$ \\
\hline Kent County, Delaware & 688 & 68.8 & 2.5 & $(63.8-73.8)$ \\
\hline New Castle County, Delaware & 1,004 & 73.1 & 1.9 & $(69.5-76.8)$ \\
\hline Sussex County, Delaware & 798 & 70.1 & 2.2 & $(65.8-74.4)$ \\
\hline $\begin{array}{l}\text { District of Columbia, } \\
\text { District of Columbia }\end{array}$ & 1,727 & 67.3 & 1.9 & $(63.6-71.0)$ \\
\hline Broward County, Florida & 245 & 61.9 & 4.8 & $(52.5-71.2)$ \\
\hline Miami-Dade County, Florida & 333 & 53.3 & 4.8 & $(43.8-62.7)$ \\
\hline Hawaii County, Hawaii & 670 & 61.5 & 2.7 & $(56.1-66.9)$ \\
\hline Honolulu County, Hawaii & 1,620 & 67.0 & 1.7 & $(63.5-70.4)$ \\
\hline Kauai County, Hawaii & 388 & 60.2 & 3.4 & $(53.6-66.8)$ \\
\hline Maui County, Hawaii & 577 & 60.5 & 3.3 & $(54.1-66.8)$ \\
\hline Ada County, Idaho & 369 & 66.5 & 3.6 & $(59.5-73.5)$ \\
\hline Canyon County, Idaho & 242 & 59.5 & 5.1 & $(49.4-69.5)$ \\
\hline Cook County, Illinois & 632 & 55.7 & 2.6 & $(50.6-60.8)$ \\
\hline Lake County, Indiana & 436 & 50.1 & 3.9 & $(42.5-57.8)$ \\
\hline Marion County, Indiana & 561 & 65.4 & 2.7 & $(60.1-70.7)$ \\
\hline Polk County, lowa & 381 & 65.8 & 2.8 & $(60.4-71.2)$ \\
\hline Johnson County, Kansas & 1,067 & 72.7 & 1.7 & $(69.5-76.0)$ \\
\hline Sedgwick County, Kansas & 827 & 70.0 & 2.1 & $(66.0-74.1)$ \\
\hline Shawnee County, Kansas & 365 & 72.1 & 2.8 & $(66.6-77.6)$ \\
\hline Wyandotte County, Kansas & 454 & 61.3 & 4.1 & $(53.3-69.3)$ \\
\hline Jefferson County, Kentucky & 819 & 66.6 & 3.0 & $(60.8-72.4)$ \\
\hline East Baton Rouge Parish, Louisiana & 272 & 70.5 & 3.4 & $(64.0-77.1)$ \\
\hline Androscoggin County, Maine & 324 & 70.0 & 3.1 & $(63.9-76.2)$ \\
\hline Aroostook County, Maine & 289 & 74.5 & 3.0 & $(68.7-80.3)$ \\
\hline Cumberland County, Maine & 812 & 75.8 & 1.8 & $(72.2-79.4)$ \\
\hline Kennebec County, Maine & 403 & 79.6 & 2.4 & $(74.8-84.4)$ \\
\hline Penobscot County, Maine & 448 & 73.3 & 2.4 & $(68.5-78.1)$ \\
\hline
\end{tabular}

See table footnotes on page 55 .
TABLE 24. (Continued) Estimated prevalence of adults aged 50-75 years who received a colorectal cancer screening based on the most recent guidelines,* by county - Behavioral Risk Factor Surveillance System, United States, 2012

\begin{tabular}{|c|c|c|c|c|}
\hline County & $\begin{array}{c}\text { Sample } \\
\text { size }\end{array}$ & $\%$ & SE & $95 \% \mathrm{Cl}$ \\
\hline York County, Maine & 607 & 71.0 & 2.2 & $(66.7-75.4)$ \\
\hline Anne Arundel County, Maryland & 419 & 73.3 & 2.9 & $(67.5-79.1)$ \\
\hline Baltimore County, Maryland & 727 & 71.1 & 2.2 & $(66.9-75.3)$ \\
\hline Charles County, Maryland & 236 & 69.8 & 4.3 & $(61.4-78.1)$ \\
\hline Frederick County, Maryland & 377 & 70.8 & 3.7 & $(63.6-77.9)$ \\
\hline Montgomery County, Maryland & 716 & 70.8 & 2.5 & $(66.0-75.6)$ \\
\hline Prince George's County, Maryland & 537 & 72.6 & 3.1 & $(66.6-78.6)$ \\
\hline Washington County, Maryland & 276 & 70.3 & 4.9 & $(60.6-80.0)$ \\
\hline Baltimore city, Maryland & 358 & 66.4 & 4.5 & $(57.5-75.2)$ \\
\hline Barnstable County, Massachusetts & 295 & 80.7 & 2.8 & $(75.2-86.3)$ \\
\hline Bristol County, Massachusetts & 1,198 & 76.9 & 2.3 & $(72.4-81.5)$ \\
\hline Essex County, Massachusetts & 1,183 & 76.1 & 2.2 & $(71.8-80.5)$ \\
\hline Hampden County, Massachusetts & 852 & 78.5 & 2.2 & $(74.3-82.8)$ \\
\hline Middlesex County, Massachusetts & 1,796 & 77.0 & 1.4 & $(74.2-79.9)$ \\
\hline Norfolk County, Massachusetts & 776 & 76.3 & 2.3 & $(71.9-80.7)$ \\
\hline Plymouth County, Massachusetts & 841 & 75.6 & 2.4 & $(71.0-80.2)$ \\
\hline Suffolk County, Massachusetts & 914 & 71.9 & 2.5 & $(67.0-76.8)$ \\
\hline Worcester County, Massachusetts & 1,129 & 76.1 & 2.0 & $(72.2-80.0)$ \\
\hline Kent County, Michigan & 261 & 72.8 & 3.6 & $(65.7-79.9)$ \\
\hline Macomb County, Michigan & 279 & 67.5 & 3.4 & $(60.8-74.2)$ \\
\hline Oakland County, Michigan & 588 & 71.8 & 2.5 & $(67.0-76.7)$ \\
\hline Wayne County, Michigan & 1,062 & 64.0 & 2.5 & $(59.1-68.9)$ \\
\hline Anoka County, Minnesota & 243 & 71.5 & 3.4 & $(64.8-78.2)$ \\
\hline Dakota County, Minnesota & 268 & 71.7 & 3.3 & $(65.3-78.2)$ \\
\hline Hennepin County, Minnesota & 1,431 & 69.7 & 2.0 & $(65.9-73.6)$ \\
\hline Ramsey County, Minnesota & 965 & 68.6 & 2.8 & $(63.0-74.1)$ \\
\hline Jackson County, Missouri & 413 & 72.8 & 3.3 & $(66.4-79.2)$ \\
\hline St. Louis County, Missouri & 438 & 72.3 & 3.2 & $(66.0-78.6)$ \\
\hline Cascade County, Montana & 350 & 58.8 & 3.1 & $(52.7-64.9)$ \\
\hline Flathead County, Montana & 263 & 59.9 & 3.4 & $(53.2-66.6)$ \\
\hline Hill County, Montana & 300 & 51.2 & 3.9 & $(43.7-58.8)$ \\
\hline Lake County, Montana & 477 & 58.3 & 3.5 & $(51.5-65.0)$ \\
\hline Missoula County, Montana & 370 & 62.1 & 3.0 & $(56.2-68.0)$ \\
\hline Yellowstone County, Montana & 297 & 59.4 & 3.2 & $(53.2-65.7)$ \\
\hline Dakota County, Nebraska & 386 & 59.0 & 5.0 & $(49.1-68.9)$ \\
\hline Douglas County, Nebraska & 1,488 & 64.3 & 1.6 & $(61.2-67.5)$ \\
\hline Hall County, Nebraska & 256 & 59.0 & 4.1 & $(50.9-67.1)$ \\
\hline Lancaster County, Nebraska & 527 & 67.2 & 2.5 & $(62.4-72.0)$ \\
\hline Lincoln County, Nebraska & 304 & 61.9 & 3.8 & $(54.4-69.5)$ \\
\hline Sarpy County, Nebraska & 482 & 69.6 & 2.7 & $(64.4-74.8)$ \\
\hline Scotts Bluff County, Nebraska & 308 & 53.5 & 3.9 & $(46.0-61.1)$ \\
\hline Clark County, Nevada & 821 & 57.0 & 2.3 & $(52.5-61.5)$ \\
\hline Washoe County, Nevada & 737 & 63.7 & 2.3 & $(59.1-68.2)$ \\
\hline Belknap County, New Hampshire & 311 & 70.4 & 3.6 & $(63.4-77.4)$ \\
\hline Carroll County, New Hampshire & 301 & 65.7 & 3.8 & $(58.3-73.0)$ \\
\hline Cheshire County, New Hampshire & 291 & 71.0 & 3.3 & $(64.5-77.6)$ \\
\hline Coos County, New Hampshire & 287 & 65.7 & 3.6 & $(58.6-72.7)$ \\
\hline Grafton County, New Hampshire & 277 & 70.5 & 3.4 & $(63.7-77.2)$ \\
\hline Hillsborough County, New Hampshire & 919 & 77.4 & 1.7 & $(74.0-80.8)$ \\
\hline Merrimack County, New Hampshire & 342 & 79.6 & 2.8 & $(74.2-85.1)$ \\
\hline Rockingham County, New Hampshire & 504 & 77.7 & 2.4 & $(73.0-82.4)$ \\
\hline Strafford County, New Hampshire & 304 & 72.7 & 3.4 & $(66.0-79.4)$ \\
\hline Atlantic County, New Jersey & 522 & 65.4 & 2.7 & $(60.1-70.7)$ \\
\hline Bergen County, New Jersey & 383 & 62.0 & 3.5 & $(55.1-68.9)$ \\
\hline Burlington County, New Jersey & 313 & 66.3 & 3.4 & $(59.7-72.9)$ \\
\hline Camden County, New Jersey & 326 & 61.2 & 3.5 & $(54.4-68.0)$ \\
\hline Cape May County, New Jersey & 286 & 68.0 & 3.6 & $(60.8-75.1)$ \\
\hline Cumberland County, New Jersey & 268 & 55.5 & 4.6 & $(46.4-64.6)$ \\
\hline Essex County, New Jersey & 495 & 61.9 & 2.9 & $(56.1-67.7)$ \\
\hline
\end{tabular}

See table footnotes on page 55 . 
TABLE 24. (Continued) Estimated prevalence of adults aged 50-75 years who received a colorectal cancer screening based on the most recent guidelines, ${ }^{*}$ by county - Behavioral Risk Factor Surveillance System, United States, 2012

\begin{tabular}{|c|c|c|c|c|}
\hline County & $\begin{array}{l}\text { Sample } \\
\text { size }\end{array}$ & $\%$ & SE & $95 \% \mathrm{Cl}$ \\
\hline Gloucester County, New Jersey & 273 & 62.9 & 3.8 & $(55.4-70.3)$ \\
\hline Hudson County, New Jersey & 478 & 51.1 & 3.3 & $(44.7-57.5)$ \\
\hline Hunterdon County, New Jersey & 281 & 67.5 & 3.9 & $(59.9-75.0)$ \\
\hline Mercer County, New Jersey & 256 & 64.0 & 3.8 & $(56.6-71.3)$ \\
\hline Middlesex County, New Jersey & 328 & 62.4 & 3.6 & $(55.4-69.5)$ \\
\hline Monmouth County, New Jersey & 331 & 68.9 & 3.1 & $(62.8-75.1)$ \\
\hline Morris County, New Jersey & 399 & 61.5 & 3.3 & $(54.9-68.0)$ \\
\hline Ocean County, New Jersey & 288 & 62.8 & 3.6 & $(55.7-69.9)$ \\
\hline Passaic County, New Jersey & 260 & 62.3 & 3.6 & $(55.1-69.4)$ \\
\hline Salem County, New Jersey & 315 & 61.0 & 4.3 & $(52.6-69.5)$ \\
\hline Somerset County, New Jersey & 275 & 71.1 & 3.8 & $(63.7-78.5)$ \\
\hline Sussex County, New Jersey & 298 & 55.3 & 3.6 & $(48.2-62.5)$ \\
\hline Union County, New Jersey & 265 & 59.1 & 4.0 & $(51.2-67.1)$ \\
\hline Warren County, New Jersey & 268 & 59.3 & 3.7 & $(52.0-66.6)$ \\
\hline Bernalillo County, New Mexico & 827 & 66.4 & 2.0 & $(62.6-70.3)$ \\
\hline Dona Ana County, New Mexico & 314 & 57.2 & 3.2 & $(50.9$ \\
\hline Sandoval County, New Mexico & 32 & 60.0 & .3 & $(53$. \\
\hline San Juan County, New Mexico & 286 & 51.5 & 3.7 & $(44.3-58.7)$ \\
\hline Santa Fe County, New Mexico & 349 & 60.3 & 3.0 & $(54.3-66.3)$ \\
\hline Kings County, New York & 128 & 71.0 & 5.5 & $(60.2-81.7)$ \\
\hline Guilford County, North Carolina & 212 & 72.5 & 4.2 & $(64.3-80.7)$ \\
\hline Mecklenburg County, North Carolina & 244 & 71.6 & 3.8 & $(64.2-79.1)$ \\
\hline Robeson County, North Carolina & 277 & 65.8 & 5.3 & $(55.3-76.2)$ \\
\hline Wake County, North Carolina & 210 & 77.3 & 3.6 & $(70.3-84.3)$ \\
\hline Burleigh County, North Dakota & 269 & 62.3 & 3.8 & $(54.8-69.8)$ \\
\hline Cass County, North Dakota & 382 & 60.6 & 3.2 & $(54.3-67.0)$ \\
\hline Cuyahoga County, Ohio & 386 & 67.6 & 2.8 & $(62.0-73.2)$ \\
\hline & 304 & 67.3 & 3.3 & $(60$ \\
\hline Hamilton County, Ohio & 321 & 63.6 & 3.5 & $(56.7-70.6)$ \\
\hline Lorain County, Ohio & 337 & 7.1 & 6 & $(60.0-74.1)$ \\
\hline Lucas County, Ohio & 308 & 56.0 & 3.6 & $(48.9-63.2)$ \\
\hline Mahoning County, Ohio & 330 & 55.5 & 3.9 & $(47.9-63.0)$ \\
\hline Montgomery County, Ohio & 325 & 69.6 & 3.2 & $(63.3-76.0)$ \\
\hline Stark County, Ohio & 308 & 60.3 & 3.8 & $(52.9-67.6)$ \\
\hline Summit County, Ohio & 313 & 68.9 & 3.2 & $(62.5-75.2)$ \\
\hline Oklahoma County, Oklahoma & 495 & 64.9 & 2.4 & $(60.2-69.6)$ \\
\hline Tulsa County, Oklahoma & 550 & 64.7 & 2.5 & $(59.8-69.6)$ \\
\hline Clackamas County, Oregon & 264 & 70.7 & 3.8 & $(63.2-78.2)$ \\
\hline Lane County, Oregon & 276 & 65.1 & 3.6 & $(58.1-72.1)$ \\
\hline Multnomah County, Oregon & 390 & 70.4 & 3.2 & $(64.2-76.7)$ \\
\hline Washington County, Oregon & 268 & 74.8 & 3.4 & $(68.1-81.5)$ \\
\hline Allegheny County, Pennsylvania & 830 & 65.6 & 2.1 & $(61.5-69.6)$ \\
\hline Bradford County, Pennsylvania & 968 & 63.4 & 1.8 & $(59.9-66.8)$ \\
\hline Montgomery County, Pennsylvania & 226 & 74.9 & 3.5 & $(68.1-81.8)$ \\
\hline Philadelphia County, Pennsylvania & 891 & 65.4 & 2.2 & $(61.0-69.7)$ \\
\hline Pike County, Pennsylvania & 1,027 & 65.5 & 2.3 & $(60.9-70.1)$ \\
\hline Kent County, Rhode Island & 381 & 79.3 & 2.6 & $(74.3-84.3)$ \\
\hline Providence County, Rhode Islanc & 1,513 & 67.6 & 1.7 & $(64.3-70.8)$ \\
\hline Washington County, Rhode Island & 352 & 81.0 & 2.3 & $(76.4-85.5)$ \\
\hline
\end{tabular}

TABLE 24. (Continued) Estimated prevalence of adults aged 50-75 years who received a colorectal cancer screening based on the most recent guidelines, ${ }^{*}$ by county - Behavioral Risk Factor Surveillance System, United States, 2012

\begin{tabular}{|c|c|c|c|c|}
\hline County & $\begin{array}{l}\text { Sample } \\
\text { size }\end{array}$ & $\%$ & SE & $95 \% \mathrm{Cl}$ \\
\hline Aiken County, South Carolina & 276 & 62.4 & 3.8 & $(55.1-69.8)$ \\
\hline Beaufort County, South Carolina & 460 & 64.0 & 3.2 & $(57.7-70.4)$ \\
\hline Charleston County, South Carolina & 494 & 67.9 & 2.9 & $(62.2-73.7)$ \\
\hline Greenville County, South Carolina & 364 & 70.8 & 3.2 & $(64.5-77.2)$ \\
\hline Horry County, South Carolina & 406 & 63.2 & 2.9 & $(57.5-69.0)$ \\
\hline Richland County, South Carolina & 436 & 75.2 & 2.9 & $(69.4-80.9)$ \\
\hline Spartanburg County, South Carolina & 295 & 66.8 & 3.8 & $(59.4-74.3)$ \\
\hline Lincoln County, South Dakota & 218 & 60.1 & 4.2 & $(51.8-68.3)$ \\
\hline Minnehaha County, South Dakota & 310 & 66.2 & 3.5 & $(59.3-73.1)$ \\
\hline Pennington County, South Dakota & 248 & 62.0 & 3.7 & $(54.8-69.2)$ \\
\hline Davidson County, Tennessee & 233 & 70.6 & 3.7 & $(63.3-77.9)$ \\
\hline Shelby County, Tennessee & 211 & 66.8 & 3.9 & $(59.1-74.5)$ \\
\hline Bexar County, Texas & 232 & 61.4 & 4.5 & $(52.6-70.3)$ \\
\hline Dallas County, Texas & 179 & 60.9 & 4.7 & $(51.6-70.2)$ \\
\hline El Paso County, Texas & 297 & 47.0 & 4.3 & $(38.5-55.5)$ \\
\hline Harris County, Texas & 241 & 60.1 & 3.9 & $(52.4-67.8)$ \\
\hline Hidalgo County, Texas & 246 & 48.0 & 4.9 & $(38.4-57.6)$ \\
\hline Tarrant County, Texas & 260 & 61.6 & 3.9 & $(53.9-69.3)$ \\
\hline Travis County, Texas & 488 & 62.0 & 3.5 & $(55.2-68.9)$ \\
\hline Davis County, Utah & 422 & 72.3 & 2.6 & $(67.2-77.5)$ \\
\hline Salt Lake County, Utah & 1,667 & 67.4 & 1.5 & $(64.4-70.3)$ \\
\hline Tooele County, Utah & 237 & 72.4 & 5.1 & $(62.3-82.5)$ \\
\hline Utah County, Utah & 523 & 68.7 & 2.4 & $(63.9-73.4)$ \\
\hline Wasatch County, Utah & 223 & 76.3 & 4.1 & $(68.4-84.3)$ \\
\hline Weber County, Utah & 454 & 70.9 & 2.7 & $(65.5-76.3)$ \\
\hline Chittenden County, Vermont & 378 & 75.4 & 2.6 & $(70.3-80.6)$ \\
\hline Rutland County, Vermont & 317 & 67.6 & 3.3 & $(61.2-74.0)$ \\
\hline Washington County, Vermont & 273 & 78.4 & 3.1 & $(72.4-84.5)$ \\
\hline Windsor County, Vermont & 297 & 73.1 & 3.3 & $(66.6-79.6)$ \\
\hline Fairfax County, Virginia & 300 & 69.9 & 3.5 & $(63.2-76.7)$ \\
\hline Clark County, Washington & 380 & 73.1 & 3.2 & $(66.8-79.4)$ \\
\hline King County, Washington & 1,761 & 70.5 & 1.5 & $(67.5-73.5)$ \\
\hline Kitsap County, Washington & 309 & 72.3 & 3.1 & $(66.2-78.4)$ \\
\hline Pierce County, Washington & 563 & 68.6 & 2.5 & $(63.6-73.5)$ \\
\hline Snohomish County, Washington & 550 & 64.6 & 2.6 & $(59.5-69.8)$ \\
\hline Spokane County, Washington & 481 & 68.9 & 2.5 & $(64.0-73.9)$ \\
\hline Thurston County, Washington & 268 & 70.5 & 4.0 & $(62.6-78.4)$ \\
\hline Whatcom County, Washington & 447 & 70.0 & 3.1 & $(64.0-76.0)$ \\
\hline Yakima County, Washington & 232 & 54.5 & 4.9 & $(44.9-64.0)$ \\
\hline Kanawha County, West Virginia & 299 & 70.4 & 3.0 & $(64.5-76.4)$ \\
\hline Milwaukee County, Wisconsin & 436 & 66.6 & 3.7 & $(59.3-74.0)$ \\
\hline Laramie County, Wyoming & 499 & 58.5 & 3.3 & $(51.9-65.1)$ \\
\hline Natrona County, Wyoming & 400 & 50.1 & 3.6 & $(42.9-57.2)$ \\
\hline San Juan Municipio, Puerto Rico & 277 & 52.2 & 3.5 & $(45.2-59.1)$ \\
\hline Median & \multicolumn{4}{|c|}{67.3} \\
\hline Range & \multicolumn{4}{|c|}{$47.0-81.0$} \\
\hline
\end{tabular}

Abbreviations: $\mathrm{Cl}=$ confidence interval; $\mathrm{SE}=$ standard error.

* Adults aged 50-75 years who have had a blood stool test in the past year, sigmoidoscopy in the past 5 years, and blood stool test in the past 3 years, or a colonoscopy in the past 10 years. 
TABLE 25. Estimated prevalence of women aged 21-65 years who have not had a hysterectomy and have had a Pap test in the past 3 years, by state/territory - Behavioral Risk Factor Surveillance System, United States, 2012

\begin{tabular}{|c|c|c|c|c|}
\hline State/Territory & $\begin{array}{l}\text { Sample } \\
\text { size }\end{array}$ & $\%$ & SE & $95 \% \mathrm{Cl}$ \\
\hline Alabama & 2,297 & 84.0 & 1.1 & $(81.9-86.1)$ \\
\hline Alaska & 1,456 & 83.5 & 1.5 & $(80.6-86.4)$ \\
\hline Arizona & 1,787 & 78.8 & 1.6 & $(75.6-82.0)$ \\
\hline Arkansas & 1,223 & 79.0 & 1.5 & $(76.1-82.0)$ \\
\hline California & 4,008 & 85.6 & 0.9 & $(83.9-87.3)$ \\
\hline Colorado & 3,433 & 85.5 & 0.8 & $(84.0-87.0)$ \\
\hline Connecticut & 2,775 & 88.0 & 0.9 & $(86.2-89.7)$ \\
\hline Delaware & 1,649 & 88.9 & 1.0 & (86.9-90.9) \\
\hline District of Columbia & 1,182 & 88.1 & 1.7 & (84.7-91.4) \\
\hline Florida & 1,889 & 80.4 & 1.4 & $(77.6-83.1)$ \\
\hline Georgia & 1,724 & 84.4 & 1.2 & $(82.0-86.8)$ \\
\hline Hawaii & 2,357 & 82.2 & 1.2 & $(79.8-84.5)$ \\
\hline Idaho & 1,444 & 74.6 & 1.9 & $(70.8-78.4)$ \\
\hline Illinois & 1,626 & 85.7 & 1.2 & $(83.3-88.0)$ \\
\hline Indiana & 2,408 & 80.4 & 1.1 & (78.3-82.6) \\
\hline lowa & 2,005 & 87.0 & 1.0 & $(85.2-88.9)$ \\
\hline Kansas & 3,286 & 84.8 & 0.8 & $(83.2-86.5)$ \\
\hline Kentucky & 3,327 & 81.6 & 1.0 & (79.7-83.6) \\
\hline Louisiana & 2,422 & 85.0 & 1.1 & $(82.8-87.3)$ \\
\hline Maine & 2,983 & 87.9 & 0.8 & $(86.3-89.4)$ \\
\hline Maryland & 3,942 & 88.2 & 0.9 & $(86.5-90.0)$ \\
\hline Massachusetts & 7,030 & 89.6 & 0.6 & $(88.5-90.8)$ \\
\hline Michigan & 3,010 & 86.2 & 0.8 & $(84.6-87.8)$ \\
\hline Minnesota & 3,801 & 87.8 & 0.8 & $(86.3-89.4)$ \\
\hline Mississippi & 1,995 & 80.9 & 1.2 & $(78.6-83.2)$ \\
\hline Missouri & 1,789 & 82.3 & 1.2 & $(80.0-84.6)$ \\
\hline Montana & 2,351 & 82.2 & 1.0 & $(80.2-84.2)$ \\
\hline Nebraska & 5,055 & 83.9 & 0.7 & $(82.6-85.3)$ \\
\hline Nevada & 1,393 & 77.4 & 1.6 & (74.4-80.5) \\
\hline New Hampshire & 2,218 & 86.9 & 1.1 & $(84.7-89.1)$ \\
\hline New Jersey & 5,184 & 84.9 & 0.8 & $(83.3-86.4)$ \\
\hline New Mexico & 2,524 & 83.0 & 0.9 & $(81.2-84.8)$ \\
\hline New York & 1,942 & 82.6 & 1.4 & (79.9-85.4) \\
\hline North Carolina & 3,483 & 86.3 & 0.7 & (84.9-87.8) \\
\hline North Dakota & 1,226 & 84.3 & 1.4 & $(81.6-87.1)$ \\
\hline Ohio & 3,750 & 84.5 & 0.8 & $(82.9-86.0)$ \\
\hline Oklahoma & 2,153 & 81.0 & 1.0 & (78.9-83.0) \\
\hline Oregon & 1,475 & 80.3 & 1.4 & (77.6-83.0) \\
\hline Pennsylvania & 5,784 & 83.2 & 0.8 & $(81.6-84.9)$ \\
\hline Rhode Island & 1,826 & 88.7 & 1.1 & (86.6-90.9) \\
\hline South Carolina & 3,306 & 82.1 & 1.0 & $(80.2-84.0)$ \\
\hline South Dakota & 2,213 & 86.7 & 1.2 & $(84.2-89.1)$ \\
\hline Tennessee & 1,932 & 85.8 & 1.0 & $(83.8-87.8)$ \\
\hline Texas & 2,642 & 80.6 & 1.1 & $(78.6-82.7)$ \\
\hline Utah & 3,621 & 79.1 & 0.9 & $(77.3-80.8)$ \\
\hline Vermont & 1,923 & 86.8 & 1.0 & $(84.8-88.9)$ \\
\hline Virginia & 2,216 & 87.4 & 0.9 & $(85.6-89.2)$ \\
\hline Washington & 4,389 & 83.0 & 0.8 & $(81.4-84.5)$ \\
\hline West Virginia & 1,472 & 80.9 & 1.3 & $(78.5-83.4)$ \\
\hline Wisconsin & 1,487 & 85.2 & 1.5 & $(82.3-88.0)$ \\
\hline Wyoming & 1,438 & 79.9 & 1.6 & $(76.8-83.0)$ \\
\hline Guam & 807 & 68.5 & 2.2 & $(64.2-72.7)$ \\
\hline Puerto Rico & 2,125 & 75.8 & 1.1 & $(73.6-78.0)$ \\
\hline Median & & 84.0 & & \\
\hline Range & & $68.5-89.6$ & & \\
\hline
\end{tabular}

Abbreviations: $\mathrm{Cl}=$ confidence interval; $\mathrm{SE}=$ standard error.
TABLE 26. Estimated prevalence of women aged 21-65 years who have not had a hysterectomy and have had a Pap test in the past 3 years, by metropolitan and micropolitan statistical area - Behavioral Risk Factor Surveillance System, United States, 2012

\begin{tabular}{|c|c|c|c|c|}
\hline MMSA(s) & $\begin{array}{l}\text { Sample } \\
\text { size }\end{array}$ & $\%$ & SE & $95 \% \mathrm{Cl}$ \\
\hline Aguadilla-Isabela, Puerto Rico & 193 & 70.3 & 4.0 & $(62.5-78.1)$ \\
\hline Akron, Ohio & 216 & 92.0 & 2.1 & $(87.8-96.1)$ \\
\hline Albuquerque, New Mexico & 948 & 84.9 & 1.4 & $(82.1-87.7)$ \\
\hline $\begin{array}{l}\text { Allentown-Bethlehem-Easton, } \\
\text { Pennsylvania-New Jersey }\end{array}$ & 415 & 84.7 & 2.5 & $(79.8-89.6)$ \\
\hline Anaheim-Santa Ana-Irvine, California* & 272 & 88.3 & 2.5 & $(83.3-93.2)$ \\
\hline Anchorage, Alaska & 513 & 85.2 & 2.3 & $(80.7-89.6)$ \\
\hline Asheville, North Carolina & 153 & 88.7 & 3.7 & $(81.5-96.0)$ \\
\hline $\begin{array}{l}\text { Atlanta-Sandy Springs-Roswell, } \\
\text { Georgia }\end{array}$ & 800 & 86.0 & 1.7 & $(82.6-89.3)$ \\
\hline Atlantic City-Hammonton, New Jersey & 284 & 82.7 & 3.0 & $(76.9-88.5)$ \\
\hline $\begin{array}{l}\text { Augusta-Richmond County, Georgia- } \\
\text { South Carolina }\end{array}$ & 249 & 85.4 & 4.4 & $(76.7-94.1)$ \\
\hline Augusta-Waterville, Maine & 270 & 85.1 & 3.2 & (78.9-91.2) \\
\hline Austin-Round Rock, Texas & 420 & 82.3 & 2.7 & $(77.0-87.7)$ \\
\hline $\begin{array}{l}\text { Baltimore-Columbia-Towson, } \\
\text { Maryland }\end{array}$ & 1,549 & 87.6 & 1.4 & $(84.9-90.3)$ \\
\hline Bangor, Maine & 284 & 89.5 & 2.3 & $(85.0-94.0)$ \\
\hline Barnstable Town, Massachusetts & 136 & 90.6 & 4.0 & $(82.7-98.4)$ \\
\hline Barre, Vermont & 186 & 89.7 & 3.6 & $(82.7-96.7)$ \\
\hline Baton Rouge, Louisiana & 381 & 88.0 & 2.5 & $(83.1-92.9)$ \\
\hline Bellingham, Washington & 266 & 88.1 & 3.1 & $(82.1-94.2)$ \\
\hline Berlin, New Hampshire-Vermont & 205 & 77.7 & 5.4 & $(67.1-88.3)$ \\
\hline Billings, Montana & 226 & 84.9 & 2.6 & $(79.8-89.9)$ \\
\hline Birmingham-Hoover, Alabama & 483 & 85.1 & 2.1 & $(80.9-89.3)$ \\
\hline Bismarck, North Dakota & 206 & 87.8 & 3.1 & $(81.7-94.0)$ \\
\hline Boise City, Idaho & 390 & 72.8 & 3.3 & $(66.3-79.4)$ \\
\hline Boston, Massachusetts* & 1,931 & 89.2 & 1.2 & $(86.8-91.6)$ \\
\hline Boulder, Colorado & 177 & 91.1 & 2.2 & $(86.8-95.5)$ \\
\hline Bremerton-Silverdale, Washington & 162 & 85.2 & 3.4 & (78.6-91.8) \\
\hline $\begin{array}{l}\text { Bridgeport-Stamford-Norwalk, } \\
\text { Connecticut }\end{array}$ & 768 & 87.4 & 2.0 & $(83.4-91.3)$ \\
\hline Burlington-South Burlington, Vermont & 500 & 86.5 & 2.1 & $(82.3-90.7)$ \\
\hline $\begin{array}{l}\text { Cambridge-Newton-Framingham, } \\
\text { Massachusetts* }\end{array}$ & 2,285 & 90.4 & 0.9 & $(88.6-92.3)$ \\
\hline Camden, New Jersey* & 673 & 84.1 & 2.2 & $(79.7-88.4)$ \\
\hline Canton-Massillon, Ohio & 185 & 84.6 & 3.8 & $(77.2-92.1)$ \\
\hline Casper, Wyoming & 176 & 85.9 & 3.4 & $(79.3-92.5)$ \\
\hline Cedar Rapids, lowa & 158 & 88.6 & 4.3 & $(80.2-97.0)$ \\
\hline Charleston, West Virginia & 202 & 81.2 & 3.3 & $(74.8-87.6)$ \\
\hline $\begin{array}{l}\text { Charleston-North Charleston, } \\
\text { South Carolina }\end{array}$ & 465 & 86.5 & 1.9 & $(82.7-90.2)$ \\
\hline $\begin{array}{l}\text { Charlotte-Concord-Gastonia, } \\
\text { North Carolina-South Carolina }\end{array}$ & 753 & 86.3 & 1.6 & $(83.1-89.4)$ \\
\hline Chattanooga, Tennessee-Georgia & 160 & 88.8 & 3.1 & $(82.7-94.9)$ \\
\hline Cheyenne, Wyoming & 207 & 83.2 & 4.0 & $(75.3-91.1)$ \\
\hline $\begin{array}{l}\text { Chicago-Naperville-Elgin, } \\
\text { Illinois-Indiana-Wisconsin }\end{array}$ & 1,176 & 86.0 & 1.4 & $(83.2-88.8)$ \\
\hline Cincinnati, Ohio-Kentucky-Indiana & 753 & 82.2 & 1.7 & $(78.8-85.6)$ \\
\hline $\begin{array}{l}\text { Claremont-Lebanon, New } \\
\text { Hampshire-Vermont }\end{array}$ & 541 & 85.3 & 2.1 & $(81.2-89.5)$ \\
\hline Cleveland-Elyria, Ohio & 537 & 88.8 & 1.8 & $(85.3-92.3)$ \\
\hline Colorado Springs, Colorado & 318 & 83.5 & 2.7 & $(78.2-88.7)$ \\
\hline Columbia, South Carolina & 509 & 82.2 & 2.4 & $(77.5-86.9)$ \\
\hline Columbus, Ohio & 512 & 85.4 & 2.0 & $(81.5-89.3)$ \\
\hline Concord, New Hampshire & 192 & 89.0 & 3.4 & $(82.3-95.7)$ \\
\hline Dallas-Plano-Irving, Texas* & 262 & 83.2 & 2.9 & $(77.6-88.8)$ \\
\hline Dayton, Ohio & 222 & 80.3 & 4.0 & $(72.5-88.2)$ \\
\hline Denver-Aurora-Lakewood, Colorado & 1,474 & 87.3 & 1.1 & $(85.1-89.4)$ \\
\hline Des Moines-West Des Moines, lowa & 356 & 88.3 & 2.1 & $(84.2-92.3)$ \\
\hline
\end{tabular}

See table footnotes on page 58. 
TABLE 26. (Continued) Estimated prevalence of women aged 21-65 years who have not had a hysterectomy and have had a Pap test in the past 3 years, by metropolitan and micropolitan statistical area — Behavioral Risk Factor Surveillance System, United States, 2012

\begin{tabular}{|c|c|c|c|c|}
\hline MMSA(s) & $\begin{array}{l}\text { Sample } \\
\text { size }\end{array}$ & $\%$ & SE & $95 \% \mathrm{Cl}$ \\
\hline Detroit-Dearborn-Livonia, Michigan* & 654 & 88.2 & 1.8 & $(84.8-91.7)$ \\
\hline Dover, Delaware & 461 & 90.8 & 1.7 & $(87.5-94.1)$ \\
\hline Duluth, Minnesota-Wisconsin & 144 & 85.5 & 3.9 & $(77.9-93.1)$ \\
\hline Durham-Chapel Hill, North Carolina & 281 & 87.9 & 2.3 & $(83.3-92.4)$ \\
\hline El Paso, Texas & 197 & 71.6 & 5.1 & $(61.6-81.5)$ \\
\hline Eugene, Oregon & 139 & 77.9 & 4.7 & $(68.7-87.2)$ \\
\hline Fairbanks, Alaska & 207 & 79.7 & 4.0 & $(71.8-87.7)$ \\
\hline Fargo, North Dakota-Minnesota & 265 & 88.2 & 2.6 & $(83.1-93.3)$ \\
\hline Farmington, New Mexico & 195 & 83.7 & 3.0 & $(77.8-89.6)$ \\
\hline Fayetteville, North Carolina & 175 & 84.7 & 3.6 & $(77.6-91.8)$ \\
\hline $\begin{array}{l}\text { Fayetteville-Springdale-Rogers, } \\
\text { Arkansas-Missouri }\end{array}$ & 204 & 75.9 & 3.9 & $(68.3-83.5)$ \\
\hline Fort Collins, Colorado & 165 & 91.0 & 2.6 & $(85.8-96.2)$ \\
\hline Fort Wayne, Indiana & 155 & 72.8 & 4.8 & $(63.5-82.2)$ \\
\hline Fort Worth-Arlington, Texas* & 190 & 79.6 & 4.0 & $(71.8-87.4)$ \\
\hline Grand Island, Nebraska & 217 & 81.4 & 3.2 & (75.2-87.6) \\
\hline Grand Rapids-Wyoming, Michigan & 269 & 87.3 & 2.7 & $(82.0-92.7)$ \\
\hline Great Falls, Montana & 176 & 85.9 & 2.9 & $(80.2-91.6)$ \\
\hline Greeley, Colorado & 151 & 83.0 & 3.5 & $(76.2-89.9)$ \\
\hline $\begin{array}{l}\text { Greensboro-High Point, } \\
\text { North Carolina }\end{array}$ & 248 & 86.9 & 2.7 & $(81.7-92.1)$ \\
\hline $\begin{array}{l}\text { Greenville-Anderson-Mauldin, } \\
\text { South Carolina }\end{array}$ & 407 & 77.8 & 2.7 & $(72.5-83.2)$ \\
\hline Gulfport-Biloxi-Pascagoula, Mississippi & i 171 & 77.1 & 3.9 & $(69.5-84.7)$ \\
\hline $\begin{array}{l}\text { Hagerstown-Martinsburg, Maryland- } \\
\text { West Virginia }\end{array}$ & 219 & 80.9 & 4.0 & $(73.1-88.8)$ \\
\hline Harrisburg-Carlisle, Pennsylvania & 198 & 83.8 & 3.5 & $(77.0-90.7)$ \\
\hline $\begin{array}{l}\text { Hartford-West Hartford-East Hartford, } \\
\text { Connecticut }\end{array}$ & 806 & 89.6 & 1.4 & $(86.9-92.3)$ \\
\hline Heber, Utah & 157 & 75.1 & 5.9 & $(63.5-86.7)$ \\
\hline Hilo, Hawaii & 405 & 84.8 & 2.4 & $(80.1-89.6)$ \\
\hline $\begin{array}{l}\text { Hilton Head Island-Bluffton-Beaufort, } \\
\text { South Carolina }\end{array}$ & 206 & 90.1 & 2.7 & $(84.8-95.4)$ \\
\hline $\begin{array}{l}\text { Houston-The Woodlands-Sugar Land, } \\
\text { Texas }\end{array}$ & 364 & 82.5 & 2.3 & $(78.0-86.9)$ \\
\hline $\begin{array}{l}\text { Huntington-Ashland, West } \\
\text { Virginia-Kentucky-Ohio }\end{array}$ & 304 & 78.9 & 3.3 & $(72.5-85.3)$ \\
\hline Huntsville, Alabama & 189 & 87.5 & 3.2 & $(81.3-93.7)$ \\
\hline Idaho Falls, Idaho & 140 & 77.7 & 5.5 & $(67.0-88.5)$ \\
\hline $\begin{array}{l}\text { Indianapolis-Carmel-Anderson, } \\
\text { Indiana }\end{array}$ & 655 & 87.2 & 1.6 & $(84.1-90.4)$ \\
\hline Jackson, Mississippi & 249 & 84.0 & 2.7 & $(78.8-89.3)$ \\
\hline Jacksonville, Florida & 134 & 84.1 & 4.0 & $(76.3-92.0)$ \\
\hline Kahului-Wailuku-Lahaina, Hawaii & 392 & 83.5 & 2.8 & $(77.9-89.1)$ \\
\hline Kalispell, Montana & 134 & 87.3 & 3.2 & $(81.0-93.5)$ \\
\hline Kansas City, Missouri-Kansas & 1,418 & 85.0 & 1.6 & $(81.9-88.2)$ \\
\hline Kapaa, Hawaii & 194 & 79.9 & 5.2 & $(69.6-90.1)$ \\
\hline Keene, New Hampshire & 157 & 87.2 & 4.1 & $(79.1-95.3)$ \\
\hline Kennewick-Richland, Washington & 148 & 86.9 & 3.6 & $(79.9-94.0)$ \\
\hline $\begin{array}{l}\text { Kingsport-Bristol-Bristol, } \\
\text { Tennessee-Virginia }\end{array}$ & 147 & 81.3 & 3.9 & $(73.6-89.0)$ \\
\hline Knoxville, Tennessee & 250 & 89.5 & 2.6 & $(84.4-94.6)$ \\
\hline Laconia, New Hampshire & 142 & 84.4 & 6.6 & (71.5-97.4) \\
\hline Lafayette, Louisiana & 174 & 86.0 & 3.5 & $(79.2-92.8)$ \\
\hline Las Cruces, New Mexico & 202 & 80.2 & 3.6 & $(73.2-87.2)$ \\
\hline $\begin{array}{l}\text { Las Vegas-Henderson-Paradise, } \\
\text { Nevada }\end{array}$ & 659 & 76.6 & 2.0 & $(72.7-80.4)$ \\
\hline Lewiston-Auburn, Maine & 230 & 92.8 & 2.1 & $(88.6-96.9)$ \\
\hline Lexington-Fayette, Kentucky & 161 & 88.0 & 3.3 & $(81.5-94.6)$ \\
\hline Lincoln, Nebraska & 502 & 85.6 & 1.8 & $(82.1-89.1)$ \\
\hline
\end{tabular}

See table footnotes on page 58 .
TABLE 26. (Continued) Estimated prevalence of women aged 21-65 years who have not had a hysterectomy and have had a Pap test in the past 3 years, by metropolitan and micropolitan statistical area — Behavioral Risk Factor Surveillance System, United States, 2012

\begin{tabular}{|c|c|c|c|c|}
\hline MMSA(s) & $\begin{array}{l}\text { Sample } \\
\text { size }\end{array}$ & $\%$ & SE & $95 \% \mathrm{Cl}$ \\
\hline $\begin{array}{l}\text { Little Rock-North Little Rock-Conway, } \\
\text { Arkansas }\end{array}$ & 302 & 83.7 & 2.7 & $(78.4-88.9)$ \\
\hline Logan, Utah-Idaho & 164 & 83.5 & 3.4 & $(76.8-90.3)$ \\
\hline $\begin{array}{l}\text { Los Angeles-Long Beach-Glendale, } \\
\text { California* }\end{array}$ & 948 & 88.0 & 1.8 & $(84.5-91.5)$ \\
\hline $\begin{array}{l}\text { Louisville/Jefferson County, } \\
\text { Kentucky-Indiana }\end{array}$ & 639 & 86.3 & 2.0 & $(82.5-90.2)$ \\
\hline Lumberton, North Carolina & 158 & 80.8 & 5.5 & $(69.9-91.7)$ \\
\hline Manchester-Nashua, New Hampshire & 613 & 86.1 & 2.0 & $(82.2-89.9)$ \\
\hline McAllen-Edinburg-Mission, Texas & 233 & 75.5 & 4.0 & $(67.6-83.4)$ \\
\hline $\begin{array}{l}\text { Memphis, } \\
\text { Tennessee-Mississippi-Arkansas }\end{array}$ & 376 & 84.8 & 2.6 & $(79.7-89.9)$ \\
\hline $\begin{array}{l}\text { Miami-Fort Lauderdale-West Palm } \\
\text { Beach, Florida }\end{array}$ & 464 & 78.4 & 3.1 & $(72.3-84.4)$ \\
\hline $\begin{array}{l}\text { Milwaukee-Waukesha-West Allis, } \\
\text { Wisconsin }\end{array}$ & 378 & 83.0 & 3.2 & $(76.7-89.2)$ \\
\hline $\begin{array}{l}\text { Minneapolis-St. Paul-Bloomington, } \\
\text { Minnesota-Wisconsin }\end{array}$ & 2,565 & 87.6 & 1.0 & $(85.7$ \\
\hline Missoula, Montana & 241 & 86.7 & 2.5 & $(81.9-91.6)$ \\
\hline Mobile, Alabama & 214 & 82.3 & 3.6 & $(75.2-89.4)$ \\
\hline Montgomery, Alabama & 166 & 87.0 & 3.6 & $(80.0-93.9)$ \\
\hline $\begin{array}{l}\text { Montgomery County-Bucks County- } \\
\text { Chester County, Pennsylvania* }\end{array}$ & 421 & 79.1 & 3.4 & $(72.5-85.8)$ \\
\hline $\begin{array}{l}\text { Myrtle Beach-Conway-North Myrtle } \\
\text { Beach, South Carolina-North Carolina }\end{array}$ & 250 & 80.9 & 3.1 & $(74.8-87.1)$ \\
\hline $\begin{array}{l}\text { Nashville-Davidson-Murfreesboro- } \\
\text { Franklin, Tennessee }\end{array}$ & 444 & 86.2 & 2.0 & $(82.3-90.0)$ \\
\hline $\begin{array}{l}\text { Nassau County-Suffolk County, } \\
\text { New York* }\end{array}$ & 298 & 85.1 & 3.2 & $(78.8-91.5)$ \\
\hline Newark, New Jersey-Pennsylvania* & 2,162 & 85.9 & 1.2 & $(83.5-88.2)$ \\
\hline New Haven-Milford, Connecticut & 634 & 86.3 & 1.8 & $(82.8-89.8)$ \\
\hline New Orleans-Metairie, Louisiana & 364 & 86.2 & 2.5 & $(81.3-91.1)$ \\
\hline $\begin{array}{l}\text { New York-Jersey City-White Plains, } \\
\text { New York-New Jersey* }\end{array}$ & 2,497 & 81.1 & 1.7 & $(77.8-84.5)$ \\
\hline Norfolk, Nebraska & 154 & 80.6 & 4.2 & $(72.2-88.9)$ \\
\hline North Platte, Nebraska & 139 & 77.8 & 4.7 & $(68.6-87.0)$ \\
\hline Norwich-New London, Connecticut & 290 & 88.8 & 2.5 & $(83.9-93.8)$ \\
\hline $\begin{array}{l}\text { Oakland-Hayward-Berkeley, } \\
\text { California* }\end{array}$ & 309 & 85.2 & 2.9 & $(79.5-90.8)$ \\
\hline Ocean City, New Jersey & 158 & 81.9 & 4.3 & (73.5-90.3) \\
\hline Ogden-Clearfield, Utah & 685 & 83.7 & 1.8 & $(80.2-87.1)$ \\
\hline Oklahoma City, Oklahoma & 740 & 80.5 & 1.9 & $(76.8-84.1)$ \\
\hline Olympia-Tumwater, Washington & 162 & 82.4 & 3.5 & $(75.5-89.3)$ \\
\hline Omaha-Council Bluffs, Nebraska-lowa & 1,699 & 84.9 & 1.1 & $(82.7-87.2)$ \\
\hline Orlando-Kissimmee-Sanford, Florida & 158 & 81.0 & 4.3 & $(72.6-89.4)$ \\
\hline Philadelphia, Pennsylvania* & 815 & 84.8 & 1.9 & $(81.1-88.6)$ \\
\hline Phoenix-Mesa-Scottsdale, Arizona & 683 & 78.3 & 2.2 & $(73.9-82.7)$ \\
\hline Pittsburgh, Pennsylvania & 963 & 83.8 & 1.5 & $(80.9-86.6)$ \\
\hline Ponce, Puerto Rico & 184 & 72.3 & 4.1 & $(64.4-80.3)$ \\
\hline Portland-South Portland, Maine & 1,027 & 89.0 & 1.3 & $(86.4-91.5)$ \\
\hline $\begin{array}{l}\text { Portland-Vancouver-Hillsboro, } \\
\text { Oregon-Washington }\end{array}$ & 971 & 82.5 & 1.7 & $(79.3-85.8)$ \\
\hline $\begin{array}{l}\text { Providence-Warwick, Rhode } \\
\text { Island-Massachusetts }\end{array}$ & 2,661 & 88.2 & 1.0 & $(86.2-90.2)$ \\
\hline Provo-Orem, Utah & 538 & 73.1 & 2.3 & $(68.6-77.7)$ \\
\hline Raleigh, North Carolina & 326 & 91.1 & 1.8 & $(87.6-94.5)$ \\
\hline Rapid City, South Dakota & 306 & 83.1 & 3.5 & (76.2-90.0) \\
\hline Reno, Nevada & 403 & 79.6 & 3.0 & $(73.6-85.5)$ \\
\hline Richmond, Virginia & 284 & 91.2 & 2.1 & $(87.2-95.3)$ \\
\hline
\end{tabular}


TABLE 26. (Continued) Estimated prevalence of women aged 21-65 years who have not had a hysterectomy and have had a Pap test in the past 3 years, by metropolitan and micropolitan statistical area — Behavioral Risk Factor Surveillance System, United States, 2012

\begin{tabular}{|c|c|c|c|c|}
\hline MMSA(s) & $\begin{array}{l}\text { Sample } \\
\text { size }\end{array}$ & $\%$ & SE & $95 \% \mathrm{Cl}$ \\
\hline $\begin{array}{l}\text { Riverside-San Bernardino-Ontario, } \\
\text { California }\end{array}$ & 432 & 84.6 & 2.3 & $(80.0-89.1)$ \\
\hline $\begin{array}{l}\text { Rockingham County-Strafford County, } \\
\text { New Hampshire* }\end{array}$ & 545 & 89.2 & 2.0 & $(85.3-93.1)$ \\
\hline Rutland, Vermont & 201 & 86.4 & 3.2 & $(80.2-92.7)$ \\
\hline $\begin{array}{l}\text { Sacramento-Roseville-Arden-Arcade, } \\
\text { California }\end{array}$ & 308 & 89.2 & 2.2 & $(84.9-93.6)$ \\
\hline St. Louis, Missouri-Illinois & 609 & 90.6 & 1.5 & $(87.7-93.5)$ \\
\hline Salisbury, Maryland-Delaware & 558 & 85.6 & 2.9 & $(79.8-91.4)$ \\
\hline Salt Lake City, Utah & 1,378 & 79.2 & 1.4 & $(76.3-82.0)$ \\
\hline San Antonio-New Braunfels, Texas & 223 & 84.0 & 3.0 & $(78.2-89.8)$ \\
\hline San Diego-Carlsbad, California & 317 & 82.5 & 3.4 & $(75.9-89.2)$ \\
\hline $\begin{array}{l}\text { San Francisco-Redwood City-South } \\
\text { San Francisco, California* }\end{array}$ & 168 & 89.2 & 3.1 & $(83.1-95.4)$ \\
\hline $\begin{array}{l}\text { San Jose-Sunnyvale-Santa Clara, } \\
\text { California }\end{array}$ & 185 & 87.4 & 3.3 & $(80.9-94.0)$ \\
\hline San Juan-Carolina-Caguas, Puerto Rico & 1,333 & 77.4 & 1.4 & $(74.7-80.1)$ \\
\hline Santa Fe, New Mexico & 228 & 86.1 & 2.8 & $(80.6-91.6)$ \\
\hline Sayre, Pennsylvania & 462 & 70.5 & 6.7 & $(57.4-83.7)$ \\
\hline Scottsbluff, Nebraska & 136 & 75.0 & 5.7 & $(63.9-86.1)$ \\
\hline $\begin{array}{l}\text { Scranton-Wilkes-Barre-Hazleton, } \\
\text { Pennsylvania }\end{array}$ & 213 & 84.0 & 3.0 & $(78.1-89.9)$ \\
\hline Seattle-Bellevue-Everett, Washington* & 1,555 & 85.6 & 1.3 & $(83.0-88.1)$ \\
\hline Shreveport-Bossier City, Louisiana & 160 & 85.2 & 3.8 & $(77.8-92.7)$ \\
\hline $\begin{array}{l}\text { Silver Spring-Frederick-Rockville, } \\
\text { Maryland* }\end{array}$ & 700 & 89.3 & 1.7 & $(85.9-92.7)$ \\
\hline $\begin{array}{l}\text { Sioux City, lowa-Nebraska- } \\
\text { South Dakota }\end{array}$ & 274 & 89.3 & 3.4 & $(82.7-96.0)$ \\
\hline Sioux Falls, South Dakota & 460 & 88.8 & 2.0 & $(84.8-92.7)$ \\
\hline Spartanburg, South Carolina & 178 & 80.3 & 4.1 & $(72.3-88.3)$ \\
\hline Spokane-Spokane Valley, Washington & 298 & 82.6 & 2.9 & (76.9-88.3) \\
\hline Springfield, Massachusetts & 770 & 90.1 & 1.5 & $(87.2-93.0)$ \\
\hline
\end{tabular}

TABLE 26. (Continued) Estimated prevalence of women aged 21-65 years who have not had a hysterectomy and have had a Pap test in the past 3 years, by metropolitan and micropolitan statistical area - Behavioral Risk Factor Surveillance System, United States, 2012

\begin{tabular}{|c|c|c|c|c|}
\hline MMSA(s) & $\begin{array}{l}\text { Sample } \\
\text { size }\end{array}$ & $\%$ & SE & $95 \% \mathrm{Cl}$ \\
\hline Tacoma-Lakewood, Washington* & 335 & 84.1 & 2.5 & $(79.2-89.0)$ \\
\hline $\begin{array}{l}\text { Tampa-St. Petersburg-Clearwater, } \\
\text { Florida }\end{array}$ & 210 & 80.6 & 3.4 & $(74.0-87.1)$ \\
\hline Toledo, Ohio & 285 & 85.2 & 2.9 & $(79.6-90.9)$ \\
\hline Topeka, Kansas & 316 & 84.1 & 2.5 & $(79.1-89.0)$ \\
\hline Torrington, Connecticut & 212 & 89.8 & 2.3 & $(85.3-94.3)$ \\
\hline Trenton, New Jersey & 175 & 88.4 & 3.3 & $(82.0-94.8)$ \\
\hline Tucson, Arizona & 250 & 84.3 & 2.8 & $(78.7-89.8)$ \\
\hline Tulsa, Oklahoma & 483 & 82.5 & 2.2 & $(78.2-86.8)$ \\
\hline Tuscaloosa, Alabama & 158 & 89.6 & 3.1 & $(83.6-95.6)$ \\
\hline Urban Honolulu, Hawaii & 1,366 & 81.8 & 1.5 & $(78.8-84.8)$ \\
\hline Vineland-Bridgeton, New Jersey & 189 & 88.9 & 2.5 & $(84.0-93.8)$ \\
\hline $\begin{array}{l}\text { Virginia Beach-Norfolk-Newport News, } \\
\text { Virginia-North Carolina }\end{array}$ & 424 & 89.4 & 1.8 & $(85.8-93.0)$ \\
\hline $\begin{array}{l}\text { Warren-Troy-Farmington Hills, } \\
\text { Michigan* }\end{array}$ & 691 & 88.1 & 1.6 & $(85.1-91.2)$ \\
\hline $\begin{array}{l}\text { Washington-Arlington-Alexandria, } \\
\text { District of Columbia-Virginia- } \\
\text { Maryland-West Virginia* }\end{array}$ & 2,689 & 88.0 & 1.2 & $(85.7-90.3)$ \\
\hline Wichita, Kansas & 674 & 84.7 & 1.9 & $(80.9-88.5)$ \\
\hline $\begin{array}{l}\text { Wilmington, Delaware-Maryland- } \\
\text { New Jersey* }\end{array}$ & 1,113 & 89.1 & 1.2 & $(86.8-91.4)$ \\
\hline Winston-Salem, North Carolina & 218 & 90.8 & 2.1 & $(86.6-95.0)$ \\
\hline Worcester, Massachusetts-Connecticut & 921 & 88.7 & 1.7 & $(85.4-92.0)$ \\
\hline Yakima, Washington & 160 & 72.7 & 4.7 & $(63.5-81.9)$ \\
\hline $\begin{array}{l}\text { Youngstown-Warren-Boardman, } \\
\text { Ohio-Pennsylvania }\end{array}$ & 271 & 86.6 & 3.1 & $(80.6-92.6)$ \\
\hline Median & & 85.2 & & \\
\hline Range & & $70.3-92.8$ & & \\
\hline
\end{tabular}

Abbreviations: $\mathrm{Cl}=$ confidence interval; $\mathrm{MMSA}=$ metropolitan and micropolitan statistical area; $\mathrm{SE}=$ standard error.

* Metropolitan division. 
TABLE 27. Estimated prevalence of women aged 21-65 years who have not had a hysterectomy and have had a Pap test in the past 3 years, by county - Behavioral Risk Factor Surveillance System, United States, 2012

\begin{tabular}{|c|c|c|c|c|}
\hline County & $\begin{array}{l}\text { Sample } \\
\text { size }\end{array}$ & $\%$ & SE & $95 \% \mathrm{Cl}$ \\
\hline Jefferson County, Alabama & 272 & 85.7 & 2.6 & $(80.6-90.8)$ \\
\hline Madison County, Alabama & 161 & 88.1 & 3.7 & $(80.7-95.4)$ \\
\hline Mobile County, Alabama & 214 & 82.3 & 3.6 & $(75.2-89.4)$ \\
\hline Anchorage Municipality, Alaska & 302 & 85.2 & 2.8 & $(79.7-90.7)$ \\
\hline Fairbanks North Star Borough, Alaska & 207 & 79.7 & 4.0 & $(71.8-87.7)$ \\
\hline Matanuska-Susitna Borough, Alaska & 211 & 84.9 & 3.0 & (79.0-90.8) \\
\hline Maricopa County, Arizona & 579 & 78.1 & 2.3 & $(73.5-82.7)$ \\
\hline Pima County, Arizona & 250 & 84.3 & 2.8 & (78.7-89.8) \\
\hline Pulaski County, Arkansas & 200 & 83.9 & 3.1 & $(77.8-89.9)$ \\
\hline Alameda County, California & 193 & 80.3 & 4.3 & (71.9-88.7) \\
\hline Los Angeles County, California & 948 & 88.0 & 1.8 & $(84.5-91.5)$ \\
\hline Orange County, California & 272 & 88.3 & 2.5 & $(83.3-93.2)$ \\
\hline Riverside County, California & 215 & 82.5 & 3.8 & $(75.0-90.1)$ \\
\hline Sacramento County, California & 189 & 88.6 & 2.6 & $(83.6-93.7)$ \\
\hline San Bernardino County, California & 217 & 86.7 & 2.9 & $(81.1-92.3)$ \\
\hline San Diego County, California & 317 & 82.5 & 3.4 & $(75.9-89.2)$ \\
\hline Santa Clara County, California & 182 & 87.2 & 3.4 & $(80.6-93.9)$ \\
\hline Adams County, Colorado & 252 & 84.4 & 2.9 & $(78.8-90.1)$ \\
\hline Arapahoe County, Colorado & 250 & 85.6 & 2.5 & $(80.8-90.5)$ \\
\hline Boulder County, Colorado & 177 & 91.1 & 2.2 & $(86.8-95.5)$ \\
\hline Denver County, Colorado & 298 & 89.4 & 2.1 & (85.4-93.5) \\
\hline Douglas County, Colorado & 189 & 93.6 & 2.1 & $(89.4-97.7)$ \\
\hline El Paso County, Colorado & 278 & 84.1 & 2.7 & $(78.7-89.4)$ \\
\hline Jefferson County, Colorado & 332 & 87.0 & 2.3 & $(82.5-91.4)$ \\
\hline Larimer County, Colorado & 165 & 91.0 & 2.6 & $(85.8-96.2)$ \\
\hline Weld County, Colorado & 151 & 83.0 & 3.5 & (76.2-89.9) \\
\hline Fairfield County, Connecticut & 768 & 87.4 & 2.0 & (83.4-91.3) \\
\hline Hartford County, Connecticut & 609 & 90.3 & 1.4 & $(87.4-93.1)$ \\
\hline Litchfield County, Connecticut & 212 & 89.8 & 2.3 & $(85.3-94.3)$ \\
\hline New Haven County, Connecticut & 634 & 86.3 & 1.8 & (82.9-89.8) \\
\hline New London County, Connecticut & 290 & 88.8 & 2.5 & (83.8-93.8) \\
\hline Kent County, Delaware & 461 & 90.8 & 1.7 & $(87.5-94.1)$ \\
\hline New Castle County, Delaware & 812 & 88.6 & 1.3 & $(86.0-91.3)$ \\
\hline Sussex County, Delaware & 376 & 88.4 & 2.5 & $(83.5-93.2)$ \\
\hline $\begin{array}{l}\text { District of Columbia, } \\
\text { District of Columbia }\end{array}$ & 1,182 & 88.1 & 1.7 & $(84.7-91.4)$ \\
\hline Broward County, Florida & 153 & 78.6 & 4.8 & $(69.2-87.9)$ \\
\hline Miami-Dade County, Florida & 241 & 80.5 & 3.9 & $(72.8-88.2)$ \\
\hline Hawaii County, Hawaii & 405 & 84.8 & 2.4 & $(80.1-89.6)$ \\
\hline Honolulu County, Hawaii & 1,366 & 81.8 & 1.5 & (78.8-84.8) \\
\hline Kauai County, Hawaii & 194 & 79.9 & 5.2 & $(69.6-90.1)$ \\
\hline Maui County, Hawaii & 392 & 83.5 & 2.9 & $(77.9-89.1)$ \\
\hline Ada County, Idaho & 228 & 75.3 & 4.2 & (67.1-83.5) \\
\hline Canyon County, Idaho & 127 & 65.7 & 6.5 & $(52.9-78.5)$ \\
\hline Cook County, Illinois & 472 & 86.7 & 2.1 & $(82.7-90.8)$ \\
\hline Lake County, Indiana & 258 & 76.2 & 4.3 & $(67.8-84.7)$ \\
\hline Marion County, Indiana & 407 & 86.1 & 2.3 & $(81.6-90.5)$ \\
\hline Polk County, lowa & 256 & 87.1 & 2.5 & $(82.2-92.1)$ \\
\hline Johnson County, Kansas & 696 & 89.1 & 1.5 & $(86.1-92.1)$ \\
\hline Sedgwick County, Kansas & 533 & 85.9 & 2.2 & $(81.6-90.1)$ \\
\hline Shawnee County, Kansas & 234 & 86.2 & 2.9 & $(80.6-91.8)$ \\
\hline Wyandotte County, Kansas & 256 & 86.0 & 2.9 & (80.4-91.6) \\
\hline Jefferson County, Kentucky & 485 & 85.9 & 2.6 & $(80.7-91.1)$ \\
\hline East Baton Rouge Parish, Louisiana & 173 & 87.5 & 3.4 & $(80.7-94.2)$ \\
\hline Androscoggin County, Maine & 230 & 92.8 & 2.1 & $(88.6-96.9)$ \\
\hline Aroostook County, Maine & 152 & 90.3 & 2.7 & $(85.1-95.5)$ \\
\hline Cumberland County, Maine & 553 & 90.2 & 1.8 & (86.7-93.8) \\
\hline Kennebec County, Maine & 270 & 85.1 & 3.2 & (78.9-91.2) \\
\hline Penobscot County, Maine & 284 & 89.5 & 2.3 & $(85.0-94.0)$ \\
\hline
\end{tabular}

See table footnotes on page 60 .
TABLE 27. (Continued) Estimated prevalence of women aged 21-65 years who have not had a hysterectomy and have had a Pap test in the past 3 years, by county - Behavioral Risk Factor Surveillance System, United States, 2012

\begin{tabular}{|c|c|c|c|c|}
\hline County & $\begin{array}{l}\text { Sample } \\
\text { size }\end{array}$ & $\%$ & SE & $95 \% \mathrm{Cl}$ \\
\hline York County, Maine & 354 & 87.0 & 2.1 & $(82.9-91.1)$ \\
\hline Anne Arundel County, Maryland & 294 & 88.2 & 2.7 & $(82.9-93.6)$ \\
\hline Baltimore County, Maryland & 514 & 85.7 & 2.4 & $(81.1-90.3)$ \\
\hline Charles County, Maryland & 187 & 90.7 & 3.4 & $(84.1-97.4)$ \\
\hline Frederick County, Maryland & 229 & 89.4 & 3.1 & $(83.3-95.6)$ \\
\hline Montgomery County, Maryland & 471 & 89.7 & 1.9 & $(85.9-93.5)$ \\
\hline Prince George's County, Maryland & 380 & 89.4 & 2.5 & $(84.5-94.2)$ \\
\hline Washington County, Maryland & 157 & 77.8 & 5.5 & $(67.0-88.6)$ \\
\hline Baltimore city, Maryland & 232 & 90.2 & 2.9 & $(84.5-95.9)$ \\
\hline Barnstable County, Massachusetts & 136 & 90.6 & 4.0 & $(82.7-98.4)$ \\
\hline Bristol County, Massachusetts & 835 & 86.9 & 2.2 & $(82.6-91.3)$ \\
\hline Essex County, Massachusetts & 819 & 91.6 & 1.4 & $(89.0-94.3)$ \\
\hline Hampden County, Massachusetts & 633 & 89.4 & 1.7 & $(86.1-92.7)$ \\
\hline Middlesex County, Massachusetts & 1,466 & 90.0 & 1.2 & $(87.7-92.3)$ \\
\hline Norfolk County, Massachusetts & 568 & 92.2 & 2.2 & $(87.9-96.5)$ \\
\hline Plymouth County, Massachusetts & 567 & 92.0 & 1.5 & $(89.0-95.0)$ \\
\hline Suffolk County, Massachusetts & 796 & 85.8 & 2.0 & $(81.9-89.7)$ \\
\hline Worcester County, Massachusetts & 856 & 89.9 & 1.6 & $(86.7-93.0)$ \\
\hline Kent County, Michigan & 161 & 83.9 & 4.1 & $(75.9-92.0)$ \\
\hline Macomb County, Michigan & 196 & 89.6 & 2.4 & $(85.0-94.3)$ \\
\hline Oakland County, Michigan & 358 & 87.7 & 2.8 & $(82.3-93.2)$ \\
\hline Wayne County, Michigan & 654 & 88.2 & 1.8 & $(84.8-91.7)$ \\
\hline Anoka County, Minnesota & 162 & 87.3 & 3.3 & $(81.0-93.7)$ \\
\hline Dakota County, Minnesota & 232 & 88.7 & 2.5 & $(83.8-93.7)$ \\
\hline Hennepin County, Minnesota & 1,057 & 88.1 & 1.6 & $(85.0-91.1)$ \\
\hline Ramsey County, Minnesota & 649 & 85.7 & 2.9 & $(80.1-91.3)$ \\
\hline Jackson County, Missouri & 241 & 81.7 & 3.7 & $(74.4-89.0)$ \\
\hline St. Louis County, Missouri & 289 & 92.4 & 1.9 & $(88.6-96.1)$ \\
\hline Cascade County, Montana & 176 & 85.9 & 2.9 & $(80.2-91.6)$ \\
\hline Flathead County, Montana & 134 & 87.3 & 3.2 & $(81.0-93.5)$ \\
\hline Hill County, Montana & 161 & 81.2 & 4.3 & $(72.9-89.6)$ \\
\hline Lake County, Montana & 231 & 79.3 & 3.2 & $(73.1-85.5)$ \\
\hline Missoula County, Montana & 241 & 86.7 & 2.5 & $(81.9-91.6)$ \\
\hline Yellowstone County, Montana & 201 & 85.0 & 2.7 & $(79.7-90.3)$ \\
\hline Dakota County, Nebraska & 166 & 90.7 & 2.8 & $(85.1-96.2)$ \\
\hline Douglas County, Nebraska & 1,065 & 85.4 & 1.4 & $(82.8-88.1)$ \\
\hline Hall County, Nebraska & 135 & 82.3 & 4.4 & $(73.7-90.9)$ \\
\hline Lancaster County, Nebraska & 445 & 86.1 & 1.8 & $(82.5-89.6)$ \\
\hline Lincoln County, Nebraska & 133 & 77.5 & 4.8 & $(68.1-87.0)$ \\
\hline Sarpy County, Nebraska & 371 & 86.5 & 2.2 & $(82.1-90.8)$ \\
\hline Scotts Bluff County, Nebraska & 122 & 74.2 & 6.1 & $(62.2-86.3)$ \\
\hline Clark County, Nevada & 659 & 76.6 & 2.0 & $(72.7-80.4)$ \\
\hline Washoe County, Nevada & 399 & 79.5 & 3.1 & $(73.5-85.6)$ \\
\hline Belknap County, New Hampshire & 142 & 84.4 & 6.6 & $(71.5-97.4)$ \\
\hline Carroll County, New Hampshire & 140 & 84.2 & 4.6 & $(75.2-93.1)$ \\
\hline Cheshire County, New Hampshire & 157 & 87.2 & 4.1 & $(79.1-95.3)$ \\
\hline Coos County, New Hampshire & 149 & 79.7 & 6.4 & $(67.1-92.2)$ \\
\hline Grafton County, New Hampshire & 150 & 83.0 & 4.3 & $(74.4-91.5)$ \\
\hline Hillsborough County, New Hampshire & 613 & 86.1 & 2.0 & $(82.2-89.9)$ \\
\hline Merrimack County, New Hampshire & 192 & 89.0 & 3.4 & $(82.3-95.7)$ \\
\hline Rockingham County, New Hampshire & 350 & 88.8 & 2.5 & $(83.8-93.8)$ \\
\hline Strafford County, New Hampshire & 195 & 90.1 & 2.8 & $(84.5-95.6)$ \\
\hline Atlantic County, New Jersey & 284 & 82.7 & 3.0 & $(76.9-88.5)$ \\
\hline Bergen County, New Jersey & 322 & 86.2 & 2.3 & $(81.6-90.7)$ \\
\hline Burlington County, New Jersey & 216 & 87.5 & 2.7 & $(82.1-92.9)$ \\
\hline Camden County, New Jersey & 253 & 78.5 & 4.6 & $(69.5-87.5)$ \\
\hline Cape May County, New Jersey & 158 & 81.9 & 4.3 & $(73.5-90.3)$ \\
\hline Cumberland County, New Jersey & 189 & 88.9 & 2.5 & $(84.0-93.8)$ \\
\hline Essex County, New Jersey & 490 & 83.8 & 2.4 & $(79.1-88.4)$ \\
\hline
\end{tabular}

See table footnotes on page 60. 
TABLE 27. (Continued) Estimated prevalence of women aged 21-65 years who have not had a hysterectomy and have had a Pap test in the past 3 years, by county - Behavioral Risk Factor Surveillance System, United States, 2012

\begin{tabular}{|c|c|c|c|c|}
\hline County & $\begin{array}{l}\text { Sample } \\
\text { size }\end{array}$ & $\%$ & SE & $95 \% \mathrm{Cl}$ \\
\hline Gloucester County, New Jersey & 204 & 87.4 & 2.9 & $(81.7-93.2)$ \\
\hline Hudson County, New Jersey & 465 & 80.9 & 2.7 & $(75.7-86.2)$ \\
\hline Hunterdon County, New Jersey & 189 & 86.7 & 4.4 & (78.0-95.3) \\
\hline Mercer County, New Jersey & 175 & 88.4 & 3.3 & $(82.0-94.8)$ \\
\hline Middlesex County, New Jersey & 287 & 88.3 & 2.6 & $(83.3-93.4)$ \\
\hline Monmouth County, New Jersey & 228 & 84.7 & 3.5 & $(77.8-91.7)$ \\
\hline Morris County, New Jersey & 272 & 90.9 & 2.1 & $(86.9-95.0)$ \\
\hline Ocean County, New Jersey & 177 & 83.9 & 3.7 & $(76.7-91.1)$ \\
\hline Passaic County, New Jersey & 244 & 75.8 & 4.5 & $(67.1-84.6)$ \\
\hline Salem County, New Jersey & 203 & 92.7 & 1.6 & $(89.6-95.9)$ \\
\hline Somerset County, New Jersey & 200 & 85.3 & 4.2 & $(77.1-93.6)$ \\
\hline Sussex County, New Jersey & 184 & 85.1 & 3.6 & $(78.0-92.1)$ \\
\hline Union County, New Jersey & 268 & 84.6 & 2.9 & $(78.9-90.4)$ \\
\hline Warren County, New Jersey & 176 & 90.4 & 2.5 & $(85.5-95.2)$ \\
\hline Bernalillo County, New Mexico & 607 & 84.9 & 1.7 & $(81.6$ \\
\hline Dona Ana County, New Mexico & 202 & 80.2 & 3.6 & $(73.2$ \\
\hline Sandoval County, New Mexico & 175 & 87.1 & 3.0 & $(81.1-93.1)$ \\
\hline San Juan County, New Mexico & 195 & 83.7 & 3.0 & $(77.8-89.6)$ \\
\hline Santa Fe County, New Mexico & 228 & 86.1 & 2.8 & $(80.6-91.6)$ \\
\hline Kings County, New York & 188 & 79.0 & 4.7 & $(69.8-88.2)$ \\
\hline Guilford County, North Carolina & 160 & 92.3 & 2.7 & $(87.1-97.5)$ \\
\hline Mecklenburg County, North Carolina & 248 & 89.4 & 2.3 & $(84.9-93.9)$ \\
\hline Robeson County, North Carolina & 158 & 80.8 & 5.6 & $(69.9-91.7)$ \\
\hline Wake County, North Carolina & 249 & 93.9 & 1.6 & $(90.7-97.2)$ \\
\hline Burleigh County, North Dakota & 151 & 84.7 & 4.2 & $(76.6-92.9)$ \\
\hline s County, North Dakota & 233 & 85.0 & 3.3 & $(78.6-$ \\
\hline Cuyahoga County, Ohio & 285 & 88.3 & 2.2 & $(84.0-92.7)$ \\
\hline Franklin County, Ohio & 277 & 84.6 & 2.8 & $(79.2-90.0)$ \\
\hline Hamilton County, Ohio & 239 & 84.5 & 2.7 & $(79.2-89.7)$ \\
\hline Lorain County, Ohio & 159 & 4.6 & .8 & $(91.1-$ \\
\hline Lucas County, Ohio & 169 & 84.8 & 3.5 & $(77.9-91.6)$ \\
\hline Mahoning County, Ohio & 162 & 85.6 & 4.2 & $(77.3-93.9)$ \\
\hline Montgomery County, Ohio & 174 & 83.0 & 4.5 & $(74.1-91.9)$ \\
\hline Stark County, Ohio & 177 & 86.6 & 3.6 & $(79.6-93.6)$ \\
\hline Summit County, Ohio & 174 & 91.8 & 2.3 & $(87.4-96.2)$ \\
\hline Oklahoma County, Oklahoma & 356 & 80.2 & 2.5 & $(75.2-85.2)$ \\
\hline Tulsa County, Oklahoma & 339 & 82.4 & 2.8 & $(77.0-87.9)$ \\
\hline Clackamas County, Oregon & 144 & 86.0 & 3.4 & $(79.3-92.7)$ \\
\hline Lane County, Oregon & 139 & 77.9 & 4.7 & $(68.6-87.2)$ \\
\hline Multnomah County, Oregon & 288 & 77.8 & 3.3 & (71.4-84.3) \\
\hline Washington County, Oregon & 207 & 85.9 & 3.0 & $(80.0-91.8)$ \\
\hline Allegheny County, Pennsylvania & 559 & 84.6 & 1.8 & $(81.0-88.2)$ \\
\hline Bradford County, Pennsylvania & 462 & 70.5 & 6.7 & $(57.4-83.7)$ \\
\hline Montgomery County, Pennsylvania & 169 & 86.2 & 3.2 & $(80.0-92.4)$ \\
\hline Philadelphia County, Pennsylvania & 695 & 84.3 & 2.0 & $(80.3-88.3)$ \\
\hline Pike County, Pennsylvania & 559 & 81.4 & 2.1 & $(77.3-85.6)$ \\
\hline Kent County, Rhode Island & 283 & 90.4 & 2.7 & $(85.0-95.8)$ \\
\hline Providence County, Rhode Island & 1,124 & 88.8 & 1.3 & $(86.3-91.4)$ \\
\hline
\end{tabular}

TABLE 27. (Continued) Estimated prevalence of women aged 21-65 years who have not had a hysterectomy and have had a Pap test in the past 3 years, by county - Behavioral Risk Factor Surveillance System, United States, 2012

\begin{tabular}{|c|c|c|c|c|}
\hline County & $\begin{array}{l}\text { Sample } \\
\text { size }\end{array}$ & $\%$ & SE & $95 \% \mathrm{Cl}$ \\
\hline Washington County, Rhode Island & 209 & 87.1 & 3.1 & $(81.0-93.1)$ \\
\hline Aiken County, South Carolina & 122 & 89.4 & 3.5 & $(82.5-96.2)$ \\
\hline Beaufort County, South Carolina & 170 & 89.6 & 3.1 & $(83.6-95.7)$ \\
\hline Charleston County, South Carolina & 278 & 86.1 & 2.7 & $(80.8-91.3)$ \\
\hline Greenville County, South Carolina & 238 & 76.6 & 3.9 & $(69.1-84.2)$ \\
\hline Horry County, South Carolina & 209 & 79.2 & 3.4 & $(72.5-85.8)$ \\
\hline Richland County, South Carolina & 261 & 79.8 & 3.6 & $(72.7-86.9)$ \\
\hline Spartanburg County, South Carolina & 168 & 81.2 & 4.2 & $(73.0-89.4)$ \\
\hline Lincoln County, South Dakota & 162 & 92.4 & 2.6 & $(87.2-97.5)$ \\
\hline Minnehaha County, South Dakota & 266 & 88.4 & 2.6 & $(83.4-93.5)$ \\
\hline Pennington County, South Dakota & 178 & 84.6 & 3.6 & $(77.6-91.6)$ \\
\hline Davidson County, Tennessee & 181 & 85.3 & 3.4 & (78.7-91.9) \\
\hline Shelby County, Tennessee & 166 & 88.8 & 3.4 & $(82.2-95.4)$ \\
\hline Bexar County, Texas & 172 & 85.4 & 3.2 & (79.2-91.6) \\
\hline Dallas County, Texas & 144 & 81.8 & 4.2 & $(73.5-90.1)$ \\
\hline El Paso County, Texas & 196 & 72.0 & 5.1 & $(61.9-82.0)$ \\
\hline Harris County, Texas & 253 & 81.4 & 2.8 & $(75.8-87.0)$ \\
\hline Hidalgo County, Texas & 233 & 75.5 & 4.0 & $(67.6-83.4)$ \\
\hline Tarrant County, Texas & 164 & 80.8 & 4.1 & $(72.7-88.9)$ \\
\hline Travis County, Texas & 339 & 80.1 & 3.2 & $(73.9-86.3)$ \\
\hline Davis County, Utah & 344 & 84.9 & 2.2 & $(80.5-89.3)$ \\
\hline Salt Lake County, Utah & 1,218 & 79.0 & 1.5 & $(76.0-81.9)$ \\
\hline Tooele County, Utah & 160 & 83.0 & 3.8 & $(75.4-90.5)$ \\
\hline Utah County, Utah & 525 & 73.2 & 2.3 & $(68.6-77.7)$ \\
\hline Wasatch County, Utah & 157 & 75.1 & 5.9 & $(63.5-86.7)$ \\
\hline Weber County, Utah & 279 & 82.4 & 3.0 & $(76.6-88.2)$ \\
\hline Chittenden County, Vermont & 320 & 86.7 & 2.5 & $(81.8-91.7)$ \\
\hline Rutland County, Vermont & 201 & 86.4 & 3.2 & $(80.2-92.7)$ \\
\hline Washington County, Vermont & 186 & 89.7 & 3.6 & $(82.7-96.7)$ \\
\hline Windsor County, Vermont & 160 & 88.5 & 2.6 & $(83.4-93.6)$ \\
\hline Fairfax County, Virginia & 258 & 88.7 & 2.7 & $(83.4-94.0)$ \\
\hline Clark County, Washington & 236 & 82.2 & 3.4 & $(75.5-88.8)$ \\
\hline King County, Washington & 1,183 & 85.3 & 1.5 & $(82.4-88.3)$ \\
\hline Kitsap County, Washington & 162 & 85.2 & 3.4 & $(78.6-91.8)$ \\
\hline Pierce County, Washington & 335 & 84.1 & 2.5 & $(79.2-89.0)$ \\
\hline Snohomish County, Washington & 372 & 86.9 & 2.1 & $(82.7-91.1)$ \\
\hline Spokane County, Washington & 263 & 86.6 & 2.6 & $(81.4-91.7)$ \\
\hline Thurston County, Washington & 162 & 82.4 & 3.5 & $(75.5-89.3)$ \\
\hline Whatcom County, Washington & 266 & 88.1 & 3.1 & $(82.1-94.2)$ \\
\hline Yakima County, Washington & 160 & 72.7 & 4.7 & $(63.5-81.9)$ \\
\hline Kanawha County, West Virginia & 162 & 81.9 & 3.6 & $(75.0-88.9)$ \\
\hline Milwaukee County, Wisconsin & 293 & 81.8 & 3.6 & $(74.8-88.7)$ \\
\hline Laramie County, Wyoming & 207 & 83.2 & 4.0 & $(75.3-91.1)$ \\
\hline Natrona County, Wyoming & 176 & 85.9 & 3.4 & $(79.3-92.5)$ \\
\hline San Juan Municipio, Puerto Rico & 223 & 83.9 & 3.2 & $(77.7-90.1)$ \\
\hline Median & & 85.9 & & \\
\hline Range & & $65.7-94.6$ & & \\
\hline
\end{tabular}

Abbreviations: $\mathrm{Cl}=$ confidence interval; $\mathrm{SE}=$ standard error. 
TABLE 28. Estimated prevalence of women aged 50-74 years who have had a mammogram in the past 2 years, by state/territory Behavioral Risk Factor Surveillance System, United States, 2012

\begin{tabular}{|c|c|c|c|c|}
\hline State/Territory & $\begin{array}{l}\text { Sample } \\
\text { size }\end{array}$ & $\%$ & SE & $95 \% \mathrm{Cl}$ \\
\hline Alabama & 3,086 & 79.4 & 1.0 & $(77.4-81.3)$ \\
\hline Alaska & 1,013 & 74.3 & 1.9 & $(70.5-78.1)$ \\
\hline Arizona & 2,116 & 74.8 & 1.6 & (71.7-77.9) \\
\hline Arkansas & 1,582 & 69.4 & 1.5 & $(66.5-72.3)$ \\
\hline California & 3,301 & 82.7 & 1.0 & (80.8-84.6) \\
\hline Colorado & 3,317 & 73.4 & 1.0 & (71.4-75.3) \\
\hline Connecticut & 2,414 & 84.1 & 1.0 & $(82.1-86.1)$ \\
\hline Delaware & 1,552 & 84.3 & 1.2 & $(82.0-86.7)$ \\
\hline District of Columbia & 1,058 & 85.4 & 1.5 & (82.4-88.3) \\
\hline Florida & 2,201 & 76.8 & 1.3 & $(74.3-79.4)$ \\
\hline Georgia & 1,835 & 81.8 & 1.2 & $(79.5-84.1)$ \\
\hline Hawaii & 1,800 & 80.8 & 1.4 & (78.1-83.5) \\
\hline Idaho & 1,756 & 69.4 & 1.8 & $(65.8-73.0)$ \\
\hline Illinois & 1,604 & 77.7 & 1.4 & $(75.0-80.4)$ \\
\hline Indiana & 2,444 & 71.4 & 1.2 & $(69.0-73.7)$ \\
\hline lowa & 1,894 & 79.9 & 1.0 & $(77.9-82.0)$ \\
\hline Kansas & 3,459 & 79.2 & 0.9 & (77.5-80.8) \\
\hline Kentucky & 3,671 & 75.6 & 1.1 & (73.5-77.8) \\
\hline Louisiana & 3,238 & 78.4 & 1.1 & (76.2-80.5) \\
\hline Maine & 2,973 & 83.6 & 0.8 & $(82.0-85.2)$ \\
\hline Maryland & 3,862 & 83.8 & 0.9 & $(82.0-85.5)$ \\
\hline Massachusetts & 5,957 & 89.7 & 0.6 & (88.6-90.8) \\
\hline Michigan & 3,177 & 81.0 & 0.9 & (79.2-82.8) \\
\hline Minnesota & 3,280 & 83.7 & 0.9 & $(82.0-85.4)$ \\
\hline Mississippi & 2,563 & 73.7 & 1.1 & (71.5-75.9) \\
\hline Missouri & 2,017 & 77.9 & 1.2 & (75.5-80.3) \\
\hline Montana & 2,434 & 70.6 & 1.2 & $(68.2-72.9)$ \\
\hline Nebraska & 5,200 & 74.9 & 0.8 & (73.2-76.5) \\
\hline Nevada & 1,339 & 73.8 & 2.1 & (69.7-77.9) \\
\hline New Hampshire & 2,243 & 85.1 & 1.0 & $(83.2-86.9)$ \\
\hline New Jersey & 4,147 & 79.4 & 0.9 & (77.6-81.2) \\
\hline New Mexico & 2,422 & 72.7 & 1.1 & (70.4-74.9) \\
\hline New York & 1,483 & 80.9 & 1.5 & (77.9-83.8) \\
\hline North Carolina & 3,340 & 80.3 & 0.9 & $(78.5-82.2)$ \\
\hline North Dakota & 1,273 & 79.2 & 1.4 & (76.4-82.0) \\
\hline Ohio & 3,888 & 78.2 & 0.9 & $(76.5-80.0)$ \\
\hline Oklahoma & 2,300 & 70.3 & 1.2 & $(68.1-72.6)$ \\
\hline Oregon & 1,571 & 75.3 & 1.4 & $(72.6-78.0)$ \\
\hline Pennsylvania & 5,501 & 80.2 & 0.8 & (78.6-81.8) \\
\hline Rhode Island & 1,585 & 85.4 & 1.1 & $(83.1-87.6)$ \\
\hline South Carolina & 3,888 & 75.7 & 1.0 & (73.7-77.7) \\
\hline South Dakota & 2,041 & 79.7 & 1.7 & (76.5-83.0) \\
\hline Tennessee & 2,209 & 77.0 & 1.2 & (74.6-79.3) \\
\hline Texas & 2,385 & 72.7 & 1.4 & (70.0-75.5) \\
\hline Utah & 2,921 & 73.2 & 1.0 & (71.2-75.2) \\
\hline Vermont & 1,842 & 81.8 & 1.1 & (79.7-84.0) \\
\hline Virginia & 2,080 & 81.1 & 1.1 & (78.9-83.2) \\
\hline Washington & 4,474 & 76.4 & 0.9 & (74.7-78.1) \\
\hline West Virginia & 1,536 & 78.1 & 1.2 & (75.7-80.5) \\
\hline Wisconsin & 1,494 & 83.9 & 1.4 & $(81.2-86.6)$ \\
\hline Wyoming & 1,890 & 66.5 & 1.7 & $(63.3-69.8)$ \\
\hline Guam & 323 & 72.5 & 3.1 & $(66.5-78.5)$ \\
\hline Puerto Rico & 1,719 & 81.8 & 1.1 & $(79.6-84.0)$ \\
\hline Median & & 78.4 & & \\
\hline Range & & $66.5-89.7$ & & \\
\hline
\end{tabular}

Abbreviations: $\mathrm{Cl}=$ confidence interval; $\mathrm{SE}=$ standard error.
TABLE 29. Estimated prevalence of women aged 50-74 years who have had a mammogram in the past 2 years, by metropolitan and micropolitan statistical area - Behavioral Risk Factor Surveillance System, United States, 2012

\begin{tabular}{|c|c|c|c|c|}
\hline MMSA(s) & $\begin{array}{l}\text { Sample } \\
\text { size }\end{array}$ & $\%$ & SE & $95 \% \mathrm{Cl}$ \\
\hline Aguadilla-Isabela, Puerto Rico & 159 & 80.4 & 3.7 & $(73.1-87.7)$ \\
\hline Akron, Ohio & 217 & 78.9 & 3.5 & $(72.1-85.7)$ \\
\hline Albuquerque, New Mexico & 831 & 73.0 & 2.0 & $(69.0-76.9)$ \\
\hline $\begin{array}{l}\text { Allentown-Bethlehem-Easton, } \\
\text { Pennsylvania-New Jersey }\end{array}$ & 388 & 76.0 & 3.7 & $(68.8-83.2)$ \\
\hline Anaheim-Santa Ana-Irvine, California* & 209 & 82.9 & 4.7 & $(73.8-92.1)$ \\
\hline Anchorage, Alaska & 354 & 74.0 & 3.0 & $(68.1-79.9)$ \\
\hline Asheville, North Carolina & 180 & 85.0 & 2.9 & $(79.2-90.7)$ \\
\hline $\begin{array}{l}\text { Atlanta-Sandy Springs-Roswell, } \\
\text { Georgia }\end{array}$ & 725 & 80.7 & 1.9 & $(77.0-84.4)$ \\
\hline Atlantic City-Hammonton, New Jersey & 286 & 77.1 & 3.8 & $(69.6-84.6)$ \\
\hline $\begin{array}{l}\text { Augusta-Richmond County, Georgia- } \\
\text { South Carolina }\end{array}$ & 320 & 83.9 & 2.9 & $(78.2-89.5)$ \\
\hline Augusta-Waterville, Maine & 232 & 83.8 & 3.0 & $(77.9-89.6)$ \\
\hline Austin-Round Rock, Texas & 375 & 74.8 & 4.1 & $(66.7-82.8)$ \\
\hline $\begin{array}{l}\text { Baltimore-Columbia-Towson, } \\
\text { Maryland }\end{array}$ & 1,449 & 83.0 & 1.3 & $(80.4-85.6)$ \\
\hline Bangor, Maine & 257 & 86.3 & 2.3 & $(81.9-90.8)$ \\
\hline Barnstable Town, Massachusetts & 188 & 91.5 & 2.0 & $(87.5-95.5)$ \\
\hline Barre, Vermont & 162 & 88.5 & 2.8 & $(83.1-93.9)$ \\
\hline Baton Rouge, Louisiana & 496 & 81.0 & 3.0 & $(75.1-86.9)$ \\
\hline Bellingham, Washington & 277 & 73.2 & 3.8 & $(65.8-80.7)$ \\
\hline Berlin, New Hampshire-Vermont & 207 & 87.0 & 2.4 & $(82.3-91.8)$ \\
\hline Billings, Montana & 206 & 72.0 & 3.6 & $(65.0-78.9)$ \\
\hline Birmingham-Hoover, Alabama & 577 & 84.8 & 2.1 & $(80.8-88.8)$ \\
\hline Bismarck, North Dakota & 217 & 80.9 & 3.2 & $(74.6-87.3)$ \\
\hline Boise City, Idaho & 403 & 67.4 & 3.6 & $(60.4-74.5)$ \\
\hline Boston, Massachusetts* & 1,610 & 90.9 & 1.0 & $(89.0-92.8)$ \\
\hline Boulder, Colorado & 148 & 70.2 & 4.3 & $(61.8-78.6)$ \\
\hline Bremerton-Silverdale, Washington & 174 & 75.6 & 4.2 & $(67.5-83.7)$ \\
\hline $\begin{array}{l}\text { Bridgeport-Stamford-Norwalk, } \\
\text { Connecticut }\end{array}$ & 555 & 79.7 & 2.4 & $(75.1-84.3)$ \\
\hline Burlington-South Burlington, Vermont & 403 & 79.6 & 2.5 & $(74.7-84.5)$ \\
\hline $\begin{array}{l}\text { Cambridge-Newton-Framingham, } \\
\text { Massachusetts* }\end{array}$ & 1,827 & 88.5 & 1.1 & $(86.3-90.7)$ \\
\hline Camden, New Jersey* & 561 & 78.7 & 2.4 & $(74.0-83.4)$ \\
\hline Canton-Massillon, Ohio & 226 & 80.6 & 3.6 & (73.6-87.6) \\
\hline Casper, Wyoming & 245 & 61.8 & 4.6 & $(52.7-70.9)$ \\
\hline Cedar Rapids, lowa & 139 & 89.4 & 2.7 & $(84.1-94.6)$ \\
\hline Charleston, West Virginia & 200 & 82.3 & 3.0 & $(76.4-88.2)$ \\
\hline $\begin{array}{l}\text { Charleston-North Charleston, } \\
\text { South Carolina }\end{array}$ & 483 & 81.1 & 2.4 & $(76.4-85.8)$ \\
\hline $\begin{array}{l}\text { Charlotte-Concord-Gastonia, } \\
\text { North Carolina-South Carolina }\end{array}$ & 650 & 74.4 & 2.6 & $(69.4-79.4)$ \\
\hline Chattanooga, Tennessee-Georgia & 206 & 80.9 & 3.7 & $(73.7-88.2)$ \\
\hline Cheyenne, Wyoming & 297 & 80.4 & 3.7 & $(73.1-87.7)$ \\
\hline $\begin{array}{l}\text { Chicago-Naperville-Elgin, } \\
\text { Illinois-Indiana-Wisconsin }\end{array}$ & 1,046 & 79.2 & 1.8 & $(75.6-82.7)$ \\
\hline Cincinnati, Ohio-Kentucky-Indiana & 683 & 76.0 & 2.0 & $(72.0-80.0)$ \\
\hline $\begin{array}{l}\text { Claremont-Lebanon, New } \\
\text { Hampshire-Vermont }\end{array}$ & 580 & 85.0 & 1.9 & $(81.3-88.7)$ \\
\hline Cleveland-Elyria, Ohio & 517 & 82.9 & 2.3 & $(78.4-87.3)$ \\
\hline Colorado Springs, Colorado & 277 & 74.7 & 3.1 & $(68.5-80.8)$ \\
\hline Columbia, South Carolina & 530 & 80.2 & 2.6 & $(75.1-85.4)$ \\
\hline Columbus, Ohio & 404 & 79.8 & 2.7 & $(74.5-85.2)$ \\
\hline Concord, New Hampshire & 196 & 82.7 & 3.3 & $(76.3-89.1)$ \\
\hline Dallas-Plano-Irving, Texas* & 201 & 72.5 & 4.6 & $(63.5-81.4)$ \\
\hline Dayton, Ohio & 265 & 72.2 & 3.7 & $(64.9-79.5)$ \\
\hline Denver-Aurora-Lakewood, Colorado & 1,201 & 76.3 & 1.5 & $(73.2-79.3)$ \\
\hline Des Moines-West Des Moines, lowa & 303 & 74.5 & 2.9 & $(68.8-80.1)$ \\
\hline
\end{tabular}

See table footnotes on page 63. 
TABLE 29. (Continued) Estimated prevalence of women aged 50-74 years who have had a mammogram in the past 2 years, by metropolitan and micropolitan statistical area - Behavioral Risk Factor Surveillance System, United States, 2012

\begin{tabular}{|c|c|c|c|c|}
\hline MMSA(s) & $\begin{array}{l}\text { Sample } \\
\text { size }\end{array}$ & $\%$ & SE & $95 \% \mathrm{Cl}$ \\
\hline Detroit-Dearborn-Livonia, Michigan* & 700 & 82.7 & 2.3 & $(78.3-87.1)$ \\
\hline Dover, Delaware & 432 & 79.7 & 2.8 & $(74.2-85.2)$ \\
\hline Duluth, Minnesota-Wisconsin & 158 & 80.9 & 4.2 & $(72.7-89.1)$ \\
\hline Durham-Chapel Hill, North Carolina & 207 & 86.9 & 2.9 & $(81.2-92.6)$ \\
\hline El Paso, Texas & 197 & 74.7 & 4.2 & $(66.5-83.0)$ \\
\hline Eugene, Oregon & 172 & 74.1 & 4.0 & $(66.2-82.0)$ \\
\hline Fairbanks, Alaska & 113 & 80.5 & 4.4 & $(71.8-89.2)$ \\
\hline Fargo, North Dakota-Minnesota & 242 & 83.3 & 3.3 & $(76.8-89.7)$ \\
\hline Farmington, New Mexico & 175 & 73.1 & 4.1 & $(65.0-81.1)$ \\
\hline Fayetteville, North Carolina & 130 & 81.9 & 4.0 & $(74.1-89.8)$ \\
\hline $\begin{array}{l}\text { Fayetteville-Springdale-Rogers, } \\
\text { Arkansas-Missouri }\end{array}$ & 227 & 63.6 & 4.4 & $(54.9-72.3)$ \\
\hline Fort Collins, Colorado & 172 & 76.1 & 3.7 & $(69.0-83.3)$ \\
\hline Fort Wayne, Indiana & 130 & 61.1 & 5.5 & $(50.4-71.8)$ \\
\hline Fort Worth-Arlington, Texas* & 194 & 78.0 & 3.7 & $(70.8-85.2)$ \\
\hline Grand Island, Nebraska & 233 & 72.3 & 3.9 & $(64.6-79.9)$ \\
\hline Grand Rapids-Wyoming, Michigan & 272 & 83.3 & 2.8 & $(77.7-88.8)$ \\
\hline Great Falls, Montana & 192 & 75.2 & 3.8 & $(67.7-82.6)$ \\
\hline Greeley, Colorado & 119 & 72.4 & 4.8 & $(63.1-81.8)$ \\
\hline $\begin{array}{l}\text { Greensboro-High Point, } \\
\text { North Carolina }\end{array}$ & 215 & 80.2 & 3.2 & $(74.0-86.4)$ \\
\hline $\begin{array}{l}\text { Greenville-Anderson-Mauldin, } \\
\text { South Carolina }\end{array}$ & 460 & 72.9 & 3.2 & $(66.7-79.1)$ \\
\hline Gulfport-Biloxi-Pascagoula, Mississippi & 228 & 76.1 & 3.3 & $(69.6-82.6)$ \\
\hline $\begin{array}{l}\text { Hagerstown-Martinsburg, Maryland- } \\
\text { West Virginia }\end{array}$ & 244 & 80.3 & 3.6 & $(73.2-87.4)$ \\
\hline Harrisburg-Carlisle, Pennsylvania & 170 & 85.1 & 3.2 & (78.9-91.3) \\
\hline $\begin{array}{l}\text { Hartford-West Hartford-East Hartford, } \\
\text { Connecticut }\end{array}$ & 735 & 87.1 & 1.6 & $(83.9-90.2)$ \\
\hline Heber, Utah & 133 & 68.8 & 6.1 & $(56.9-80.6)$ \\
\hline Hilo, Hawaii & 345 & 79.8 & 2.7 & $(74.5-85.1)$ \\
\hline $\begin{array}{l}\text { Hilton Head Island-Bluffton-Beaufort, } \\
\text { South Carolina }\end{array}$ & 322 & 77.2 & 3.2 & $(70.9-83.5)$ \\
\hline $\begin{array}{l}\text { Houston-The Woodlands-Sugar Land, } \\
\text { Texas }\end{array}$ & 229 & 73.4 & 3.5 & $(66.5-80.3)$ \\
\hline $\begin{array}{l}\text { Huntington-Ashland, West } \\
\text { Virginia-Kentucky-Ohio }\end{array}$ & 362 & 77.4 & 2.6 & $(72.2-82.6)$ \\
\hline Huntsville, Alabama & 187 & 81.9 & 3.7 & $(74.8-89.1)$ \\
\hline Idaho Falls, Idaho & 163 & 69.1 & 5.3 & $(58.7-79.4)$ \\
\hline $\begin{array}{l}\text { Indianapolis-Carmel-Anderson, } \\
\text { Indiana }\end{array}$ & 592 & 73.9 & 2.3 & $(69.3-78.5)$ \\
\hline Jackson, Mississippi & 253 & 81.6 & 2.8 & $(76.1-87.0)$ \\
\hline Jacksonville, Florida & 145 & 69.0 & 5.4 & $(58.3-79.7)$ \\
\hline Kahului-Wailuku-Lahaina, Hawaii & 341 & 79.0 & 2.9 & $(73.4-84.6)$ \\
\hline Kalispell, Montana & 144 & 76.1 & 4.1 & $(68.1-84.1)$ \\
\hline Kansas City, Missouri-Kansas & 1,435 & 81.1 & 1.9 & $(77.5-84.7)$ \\
\hline Kapaa, Hawaii & 218 & 77.3 & 3.8 & $(69.9-84.7)$ \\
\hline Keene, New Hampshire & 173 & 82.5 & 4.2 & (74.3-90.7) \\
\hline Kennewick-Richland, Washington & 149 & 76.5 & 4.2 & $(68.2-84.8)$ \\
\hline $\begin{array}{l}\text { Kingsport-Bristol-Bristol, } \\
\text { Tennessee-Virginia }\end{array}$ & 191 & 76.8 & 4.2 & $(68.7-85.0)$ \\
\hline Knoxville, Tennessee & 259 & 80.9 & 3.0 & $(74.9-86.8)$ \\
\hline Laconia, New Hampshire & 185 & 83.3 & 3.3 & $(76.8-89.9)$ \\
\hline Lafayette, Louisiana & 196 & 79.4 & 4.3 & (71.0-87.8) \\
\hline Las Cruces, New Mexico & 197 & 81.3 & 2.9 & $(75.6-87.1)$ \\
\hline $\begin{array}{l}\text { Las Vegas-Henderson-Paradise, } \\
\text { Nevada }\end{array}$ & 490 & 73.9 & 2.9 & $(68.1-79.6)$ \\
\hline Lewiston-Auburn, Maine & 199 & 82.8 & 3.1 & $(76.7-88.9)$ \\
\hline Lexington-Fayette, Kentucky & 134 & 83.1 & 3.5 & $(76.3-89.9)$ \\
\hline Lincoln, Nebraska & 362 & 77.0 & 2.8 & (71.4-82.5) \\
\hline
\end{tabular}

See table footnotes on page 63.
TABLE 29. (Continued) Estimated prevalence of women aged 50-74 years who have had a mammogram in the past 2 years, by metropolitan and micropolitan statistical area - Behavioral Risk Factor Surveillance System, United States, 2012

\begin{tabular}{|c|c|c|c|c|}
\hline MMSA(s) & $\begin{array}{l}\text { Sample } \\
\text { size }\end{array}$ & $\%$ & SE & $95 \% \mathrm{Cl}$ \\
\hline $\begin{array}{l}\text { Little Rock-North Little Rock-Conway, } \\
\text { Arkansas }\end{array}$ & 373 & 74.8 & 2.9 & $(69.2-80.4)$ \\
\hline Logan, Utah-Idaho & 101 & 78.7 & 4.7 & $(69.6-87.8)$ \\
\hline $\begin{array}{l}\text { Los Angeles-Long Beach-Glendale, } \\
\text { California* }\end{array}$ & 657 & 84.3 & 2.0 & $(80.3-88.3)$ \\
\hline $\begin{array}{l}\text { Louisville/Jefferson County, } \\
\text { Kentucky-Indiana }\end{array}$ & 690 & 79.3 & 2.6 & $(74.3-84.3)$ \\
\hline Lumberton, North Carolina & 194 & 76.1 & 4.8 & $(66.7-85.5)$ \\
\hline Manchester-Nashua, New Hampshire & 543 & 84.5 & 1.8 & $(80.9-88.1)$ \\
\hline McAllen-Edinburg-Mission, Texas & 160 & 69.3 & 5.2 & $(59.1-79.4)$ \\
\hline $\begin{array}{l}\text { Memphis, } \\
\text { Tennessee-Mississippi-Arkansas }\end{array}$ & 381 & 74.5 & 3.5 & $(67.6-81.4)$ \\
\hline $\begin{array}{l}\text { Miami-Fort Lauderdale-West Palm } \\
\text { Beach, Florida }\end{array}$ & 440 & 78.7 & 2.9 & $(73.0-84.5)$ \\
\hline $\begin{array}{l}\text { Milwaukee-Waukesha-West Allis, } \\
\text { Wisconsin }\end{array}$ & 357 & 85.1 & 2.8 & $(79.6-90.7)$ \\
\hline $\begin{array}{l}\text { Minneapolis-St. Paul-Bloomington, } \\
\text { Minnesota-Wisconsin }\end{array}$ & 2,066 & 83.7 & 1.2 & $86.0)$ \\
\hline Missoula, Montana & 210 & 73.7 & 3.4 & $(67.0-80.4)$ \\
\hline Mobile, Alabama & 310 & 76.7 & 3.7 & $(69.4-84.0)$ \\
\hline Montgomery, Alabama & 178 & 82.0 & 3.7 & $(74.8-89.3)$ \\
\hline $\begin{array}{l}\text { Montgomery County-Bucks County- } \\
\text { Chester County, Pennsylvania** }\end{array}$ & 304 & 80.4 & 2.7 & $(75.0-85.7)$ \\
\hline $\begin{array}{l}\text { Myrtle Beach-Conway-North Myrtle } \\
\text { Beach, South Carolina-North Carolina }\end{array}$ & 309 & 79.1 & 2.7 & $(73.8-84.5)$ \\
\hline $\begin{array}{l}\text { Nashville-Davidson-Murfreesboro- } \\
\text { Franklin, Tennessee }\end{array}$ & 363 & 78.3 & 2.7 & $(73.1-83.6)$ \\
\hline $\begin{array}{l}\text { Nassau County-Suffolk County, } \\
\text { New York* }\end{array}$ & 217 & 85.2 & 2.7 & $(79.9-90.5)$ \\
\hline Newark, New Jersey-Pennsylvania* & 1,811 & 79.7 & 1.6 & $(76.6-82.9)$ \\
\hline New Haven-Milford, Connecticut & 564 & 84.1 & 2.2 & $(79.8-88.3)$ \\
\hline New Orleans-Metairie, Louisiana & 416 & 82.2 & 2.2 & $(77.8-86.5)$ \\
\hline $\begin{array}{l}\text { New York-Jersey City-White Plains, } \\
\text { New York-New Jersey* }\end{array}$ & 1,640 & 80.2 & 1.9 & $(76.4-83.9)$ \\
\hline Norfolk, Nebraska & 169 & 76.6 & 3.8 & $(69.2-83.9)$ \\
\hline North Platte, Nebraska & 177 & 73.9 & 4.3 & $(65.4-82.4)$ \\
\hline Norwich-New London, Connecticut & 285 & 84.7 & 3.4 & $(78.1-91.3)$ \\
\hline $\begin{array}{l}\text { Oakland-Hayward-Berkeley, } \\
\text { California* }\end{array}$ & 260 & 86.5 & 3.8 & $(79.1-94.0)$ \\
\hline Ocean City, New Jersey & 178 & 88.4 & 2.7 & $(83.2-93.7)$ \\
\hline Ogden-Clearfield, Utah & 536 & 75.3 & 2.2 & $(71.0-79.5)$ \\
\hline Oklahoma City, Oklahoma & 630 & 74.3 & 2.1 & $(70.1-78.4)$ \\
\hline Olympia-Tumwater, Washington & 151 & 77.2 & 4.3 & $(68.7-85.7)$ \\
\hline Omaha-Council Bluffs, Nebraska-lowa & 1,380 & 77.3 & 1.5 & $(74.5-80.2)$ \\
\hline Orlando-Kissimmee-Sanford, Florida & 159 & 81.6 & 4.2 & $(73.4-89.7)$ \\
\hline Philadelphia, Pennsylvania* & 674 & 84.7 & 1.8 & $(81.2-88.2)$ \\
\hline Phoenix-Mesa-Scottsdale, Arizona & 665 & 74.1 & 2.4 & $(69.5-78.8)$ \\
\hline Pittsburgh, Pennsylvania & 896 & 78.4 & 1.6 & $(75.2-81.6)$ \\
\hline Ponce, Puerto Rico & 149 & 78.5 & 3.9 & $(70.8-86.2)$ \\
\hline Portland-South Portland, Maine & 984 & 84.5 & 1.4 & $(81.7-87.2)$ \\
\hline $\begin{array}{l}\text { Portland-Vancouver-Hillsboro, } \\
\text { Oregon-Washington }\end{array}$ & 919 & 79.5 & 1.9 & $(75.7-83.3)$ \\
\hline $\begin{array}{l}\text { Providence-Warwick, Rhode } \\
\text { Island-Massachusetts }\end{array}$ & 2,357 & 87.1 & 1.0 & $(85.2-89.0)$ \\
\hline Provo-Orem, Utah & 311 & 70.7 & 2.9 & $(65.0-76.5)$ \\
\hline Raleigh, North Carolina & 184 & 81.6 & 3.6 & $(74.6-88.6)$ \\
\hline Rapid City, South Dakota & 271 & 71.8 & 4.7 & $(62.6-80.9)$ \\
\hline Reno, Nevada & 409 & 76.6 & 2.5 & $(71.7-81.6)$ \\
\hline Richmond, Virginia & 277 & 85.4 & 2.6 & $(80.4-90.4)$ \\
\hline
\end{tabular}

See table footnotes on page 63. 
TABLE 29. (Continued) Estimated prevalence of women aged 50-74 years who have had a mammogram in the past 2 years, by metropolitan and micropolitan statistical area - Behavioral Risk Factor Surveillance System, United States, 2012

\begin{tabular}{|c|c|c|c|c|}
\hline MMSA(s) & $\begin{array}{l}\text { Sample } \\
\text { size }\end{array}$ & $\%$ & SE & $95 \% \mathrm{Cl}$ \\
\hline $\begin{array}{l}\text { Riverside-San Bernardino-Ontario, } \\
\text { California }\end{array}$ & 360 & 77.9 & 2.8 & $(72.4-83.4)$ \\
\hline $\begin{array}{l}\text { Rockingham County-Strafford County, } \\
\text { New Hampshire* }\end{array}$ & 515 & 87.8 & 1.7 & $(84.5-91.2)$ \\
\hline Rutland, Vermont & 194 & 75.3 & 3.7 & $(68.0-82.7)$ \\
\hline $\begin{array}{l}\text { Sacramento-Roseville-Arden-Arcade, } \\
\text { California }\end{array}$ & 265 & 86.6 & 2.5 & $(81.7-91.5)$ \\
\hline St. Louis, Missouri-Illinois & 615 & 83.6 & 2.0 & $(79.7-87.5)$ \\
\hline Salisbury, Maryland-Delaware & 730 & 85.6 & 1.7 & $(82.3-89.0)$ \\
\hline Salt Lake City, Utah & 1,140 & 73.1 & 1.7 & $(69.8-76.3)$ \\
\hline San Antonio-New Braunfels, Texas & 196 & 72.2 & 4.7 & $(63.0-81.4)$ \\
\hline San Diego-Carlsbad, California & 225 & 82.3 & 3.1 & $(76.2-88.5)$ \\
\hline $\begin{array}{l}\text { San Francisco-Redwood City-South } \\
\text { San Francisco, California* }\end{array}$ & 119 & 84.2 & 4.4 & $(75.6-92.8)$ \\
\hline $\begin{array}{l}\text { San Jose-Sunnyvale-Santa Clara, } \\
\text { California }\end{array}$ & 148 & 89.4 & 3.5 & $(82.4-96.3)$ \\
\hline San Juan-Carolina-Caguas, Puerto Rico & 1,056 & 82.4 & 1.4 & $(79.6-85.2)$ \\
\hline Santa Fe, New Mexico & 221 & 71.7 & 3.5 & $(64.8-78.6)$ \\
\hline Sayre, Pennsylvania & 550 & 77.3 & 1.9 & $(73.5-81.1)$ \\
\hline Scottsbluff, Nebraska & 191 & 69.1 & 4.4 & $(60.5-77.8)$ \\
\hline $\begin{array}{l}\text { Scranton-Wilkes-Barre-Hazleton, } \\
\text { Pennsylvania }\end{array}$ & 200 & 77.6 & 3.8 & $(70.2-85.0)$ \\
\hline Seattle-Bellevue-Everett, Washington* & 1,353 & 78.0 & 1.5 & $(75.2-80.9)$ \\
\hline Shreveport-Bossier City, Louisiana & 203 & 77.2 & 3.6 & $(70.2-84.2)$ \\
\hline $\begin{array}{l}\text { Silver Spring-Frederick-Rockville, } \\
\text { Maryland* }\end{array}$ & 610 & 82.4 & 2.5 & $(77.5-87.2)$ \\
\hline $\begin{array}{l}\text { Sioux City, lowa-Nebraska- } \\
\text { South Dakota }\end{array}$ & 342 & 71.8 & 5.1 & $(61.8-81.8)$ \\
\hline Sioux Falls, South Dakota & 307 & 82.2 & 3.3 & $(75.8-88.6)$ \\
\hline Spartanburg, South Carolina & 216 & 75.5 & 3.9 & $(67.8-83.2)$ \\
\hline Spokane-Spokane Valley, Washington & 328 & 75.4 & 2.9 & $(69.6-81.1)$ \\
\hline Springfield, Massachusetts & 652 & 88.2 & 1.7 & $(84.8-91.6)$ \\
\hline
\end{tabular}

TABLE 29. (Continued) Estimated prevalence of women aged 50-74 years who have had a mammogram in the past 2 years, by metropolitan and micropolitan statistical area - Behavioral Risk Factor Surveillance System, United States, 2012

\begin{tabular}{|c|c|c|c|c|}
\hline MMSA(s) & $\begin{array}{l}\text { Sample } \\
\text { size }\end{array}$ & $\%$ & SE & $95 \% \mathrm{Cl}$ \\
\hline Tacoma-Lakewood, Washington* & 329 & 77.7 & 2.9 & $(72.0-83.5)$ \\
\hline $\begin{array}{l}\text { Tampa-St. Petersburg-Clearwater, } \\
\text { Florida }\end{array}$ & 219 & 76.5 & 3.6 & $(69.5-83.5)$ \\
\hline Toledo, Ohio & 315 & 73.9 & 3.4 & $(67.2-80.5)$ \\
\hline Topeka, Kansas & 320 & 78.8 & 2.9 & $(73.2-84.5)$ \\
\hline Torrington, Connecticut & 210 & 87.3 & 2.4 & $(82.6-92.0)$ \\
\hline Trenton, New Jersey & 147 & 81.6 & 4.0 & $(73.9-89.4)$ \\
\hline Tucson, Arizona & 281 & 80.5 & 3.0 & $(74.6-86.3)$ \\
\hline Tulsa, Oklahoma & 475 & 70.8 & 2.4 & $(66.1-75.6)$ \\
\hline Tuscaloosa, Alabama & 189 & 85.4 & 3.2 & $(79.1-91.8)$ \\
\hline Urban Honolulu, Hawaii & 896 & 81.8 & 1.9 & $(78.1-85.4)$ \\
\hline Vineland-Bridgeton, New Jersey & 174 & 79.0 & 4.1 & $(71.0-87.0)$ \\
\hline $\begin{array}{l}\text { Virginia Beach-Norfolk-Newport News, } \\
\text { Virginia-North Carolina }\end{array}$ & 440 & 82.8 & 2.3 & $(78.4-87.3)$ \\
\hline $\begin{array}{l}\text { Warren-Troy-Farmington Hills, } \\
\text { Michigan* }\end{array}$ & 624 & 80.9 & 2.0 & $(77.0-84.9)$ \\
\hline $\begin{array}{l}\text { Washington-Arlington-Alexandria, } \\
\text { District of Columbia-Virginia- } \\
\text { Maryland-West Virginia* }\end{array}$ & 2,194 & 85.9 & 1.2 & $(83.5-88.3)$ \\
\hline Wichita, Kansas & 680 & 79.3 & 1.8 & $(75.7-82.8)$ \\
\hline $\begin{array}{l}\text { Wilmington, Delaware-Maryland- } \\
\text { New Jersey* }\end{array}$ & 912 & 83.2 & 1.5 & $(80.2-86.3)$ \\
\hline Winston-Salem, North Carolina & 221 & 80.4 & 3.3 & $(73.9-86.9)$ \\
\hline Worcester, Massachusetts-Connecticut & 746 & 88.7 & 1.7 & $(85.4-91.9)$ \\
\hline Yakima, Washington & 147 & 68.5 & 4.9 & $(58.9-78.0)$ \\
\hline $\begin{array}{l}\text { Youngstown-Warren-Boardman, } \\
\text { Ohio-Pennsylvania }\end{array}$ & 292 & 79.9 & 3.4 & $(73.3-86.5)$ \\
\hline Median & & 79.6 & & \\
\hline Range & & $61.1-91$. & & \\
\hline
\end{tabular}

Abbreviations: $\mathrm{Cl}=$ confidence interval; $\mathrm{MMSA}=$ metropolitan and micropolitan statistical area; $\mathrm{SE}=$ standard error.

* Metropolitan division. 
TABLE 30. Estimated prevalence of women aged 50-74 years who have had a mammogram in the past 2 years, by county - Behavioral Risk Factor Surveillance System, United States, 2012

\begin{tabular}{|c|c|c|c|c|}
\hline County & $\begin{array}{l}\text { Sample } \\
\text { size }\end{array}$ & $\%$ & SE & $95 \% \mathrm{Cl}$ \\
\hline Jefferson County, Alabama & 266 & 83.7 & 2.9 & $(78.0-89.5)$ \\
\hline Madison County, Alabama & 162 & 83.2 & 3.8 & $(75.7-90.7)$ \\
\hline Mobile County, Alabama & 310 & 76.7 & 3.7 & $(69.4-84.0)$ \\
\hline Anchorage Municipality, Alaska & 200 & 77.0 & 3.6 & $(69.9-84.0)$ \\
\hline Fairbanks North Star Borough, Alaska & 113 & 80.5 & 4.4 & $(71.8-89.2)$ \\
\hline Matanuska-Susitna Borough, Alaska & 154 & 64.8 & 4.8 & $(55.4-74.2)$ \\
\hline Maricopa County, Arizona & 511 & 74.0 & 2.5 & $(69.1-79.0)$ \\
\hline Pima County, Arizona & 281 & 80.5 & 3.0 & $(74.6-86.3)$ \\
\hline Pulaski County, Arkansas & 240 & 73.9 & 3.4 & $(67.3-80.5)$ \\
\hline Alameda County, California & 157 & 85.4 & 5.6 & $(74.3-96.4)$ \\
\hline Los Angeles County, California & 657 & 84.3 & 2.0 & $(80.3-88.3)$ \\
\hline Orange County, California & 209 & 82.9 & 4.7 & $(73.8-92.1)$ \\
\hline Riverside County, California & 188 & 77.1 & 3.8 & $(69.7-84.6)$ \\
\hline Sacramento County, California & 159 & 83.8 & 3.6 & $(76.7-91.0)$ \\
\hline San Bernardino County, California & 172 & 78.4 & 3.8 & $(70.9-85.9)$ \\
\hline San Diego County, California & 225 & 82.3 & 3.1 & $(76.2-88.5)$ \\
\hline Santa Clara County, California & 144 & 89.6 & 3.7 & $(82.4-96.8)$ \\
\hline Adams County, Colorado & 217 & 66.2 & 4.2 & $(58.1-74.4)$ \\
\hline Arapahoe County, Colorado & 198 & 79.3 & 3.5 & $(72.4-86.2)$ \\
\hline Boulder County, Colorado & 148 & 70.2 & 4.3 & $(61.8-78.6)$ \\
\hline Denver County, Colorado & 217 & 77.1 & 3.4 & $(70.4-83.8)$ \\
\hline Douglas County, Colorado & 130 & 83.9 & 3.5 & $(77.0-90.7)$ \\
\hline El Paso County, Colorado & 227 & 74.9 & 3.3 & $(68.3-81.4)$ \\
\hline Jefferson County, Colorado & 278 & 76.9 & 3.1 & $(70.9-82.9)$ \\
\hline Larimer County, Colorado & 172 & 76.1 & 3.7 & $(69.0-83.3)$ \\
\hline Weld County, Colorado & 119 & 72.4 & 4.8 & $(63.1-81.8)$ \\
\hline Fairfield County, Connecticut & 555 & 79.7 & 2.4 & $(75.1-84.3)$ \\
\hline Hartford County, Connecticut & 547 & 86.7 & 1.9 & $(83.1-90.4)$ \\
\hline Litchfield County, Connecticut & 210 & 87.3 & 2.4 & $(82.6-92.0)$ \\
\hline New Haven County, Connecticut & 564 & 84.1 & 2.2 & $(79.8-88.3)$ \\
\hline New London County, Connecticut & 285 & 84.7 & 3.4 & $(78.1-91.3)$ \\
\hline Kent County, Delaware & 432 & 79.7 & 2.8 & $(74.2-85.2)$ \\
\hline New Castle County, Delaware & 623 & 84.5 & 1.7 & $(81.1-87.9)$ \\
\hline Sussex County, Delaware & 497 & 87.2 & 1.9 & $(83.5-90.8)$ \\
\hline $\begin{array}{l}\text { District of Columbia, } \\
\text { District of Columbia }\end{array}$ & 1,058 & 85.4 & 1.5 & $(82.5-88.4)$ \\
\hline Broward County, Florida & 141 & 79.5 & 5.2 & $(69.2-89.8)$ \\
\hline Miami-Dade County, Florida & 208 & 78.4 & 4.7 & $(69.1-87.6)$ \\
\hline Hawaii County, Hawaii & 345 & 79.8 & 2.7 & $(74.5-85.1)$ \\
\hline Honolulu County, Hawaii & 896 & 81.8 & 1.9 & $(78.1-85.4)$ \\
\hline Kauai County, Hawaii & 218 & 77.3 & 3.8 & $(69.9-84.7)$ \\
\hline Maui County, Hawaii & 341 & 79.0 & 2.9 & $(73.4-84.6)$ \\
\hline Ada County, Idaho & 199 & 70.8 & 5.0 & $(61.0-80.5)$ \\
\hline Canyon County, Idaho & 149 & 62.8 & 6.2 & $(50.7-74.9)$ \\
\hline Cook County, Illinois & 393 & 81.4 & 2.4 & $(76.7-86.2)$ \\
\hline Lake County, Indiana & 296 & 67.8 & 4.5 & $(58.9-76.7)$ \\
\hline Marion County, Indiana & 354 & 76.2 & 3.0 & $(70.3-82.0)$ \\
\hline Polk County, lowa & 228 & 75.9 & 3.3 & $(69.5-82.3)$ \\
\hline Johnson County, Kansas & 643 & 83.9 & 1.8 & $(80.4-87.4)$ \\
\hline Sedgwick County, Kansas & 498 & 78.8 & 2.1 & $(74.7-83.0)$ \\
\hline Shawnee County, Kansas & 229 & 80.8 & 3.1 & $(74.8-86.8)$ \\
\hline Wyandotte County, Kansas & 286 & 79.4 & 2.9 & $(73.7-85.1)$ \\
\hline Jefferson County, Kentucky & 561 & 79.7 & 3.3 & $(73.4-86.1)$ \\
\hline East Baton Rouge Parish, Louisiana & 172 & 87.3 & 3.4 & $(80.6-94.0)$ \\
\hline Androscoggin County, Maine & 199 & 82.8 & 3.1 & $(76.7-88.9)$ \\
\hline Aroostook County, Maine & 177 & 85.8 & 3.2 & $(79.6-92.1)$ \\
\hline Cumberland County, Maine & 494 & 84.9 & 1.9 & $(81.2-88.7)$ \\
\hline Kennebec County, Maine & 232 & 83.8 & 3.0 & $(77.9-89.7)$ \\
\hline Penobscot County, Maine & 257 & 86.3 & 2.3 & $(81.9-90.8)$ \\
\hline York County, Maine & 364 & 83.2 & 2.4 & (78.4-87.9) \\
\hline
\end{tabular}

See table footnotes on page 65.
TABLE 30. (Continued) Estimated prevalence of women aged 50-74 years who have had a mammogram in the past 2 years, by county - Behavioral Risk Factor Surveillance System, United States, 2012

\begin{tabular}{lcccc}
\hline County & $\begin{array}{c}\text { Sample } \\
\text { size }\end{array}$ & $\%$ & SE & $95 \% \mathrm{Cl}$ \\
\hline Anne Arundel County, Maryland & 281 & 78.1 & 3.4 & $(71.4-84.8)$ \\
Baltimore County, Maryland & 449 & 83.0 & 2.2 & $(78.8-87.3)$ \\
Charles County, Maryland & 140 & 79.9 & 5.3 & $(69.5-90.3)$ \\
Frederick County, Maryland & 215 & 81.2 & 3.5 & $(74.3-88.0)$
\end{tabular}

Montgomery County, Maryland

Prince George's County, Maryland

Washington County, Maryland

Baltimore city, Maryland

Barnstable County, Massachusetts

Bristol County, Massachusetts

Essex County, Massachusetts

Hampden County, Massachusetts

Middlesex County, Massachusetts

Norfolk County, Massachusetts

Plymouth County, Massachusetts

Suffolk County, Massachusetts

Worcester County, Massachusetts

Kent County, Michigan

Macomb County, Michigan

Oakland County, Michigan

Wayne County, Michigan

Anoka County, Minnesota

Dakota County, Minnesota

Hennepin County, Minnesota

Ramsey County, Minnesota

Jackson County, Missouri

St. Louis County, Missouri

Cascade County, Montana

Flathead County, Montana

Hill County, Montana

Lake County, Montana

Missoula County, Montana

Yellowstone County, Montana

Dakota County, Nebraska

Douglas County, Nebraska

Hall County, Nebraska

Lancaster County, Nebraska

Lincoln County, Nebraska

Sarpy County, Nebraska

Scotts Bluff County, Nebraska

Clark County, Nevada

Washoe County, Nevada

Belknap County, New Hampshire

Carroll County, New Hampshire

Cheshire County, New Hampshire

Coos County, New Hampshire

Grafton County, New Hampshire

$\begin{array}{llll}395 & 82.6 & 3.0 & (76.8-88.5)\end{array}$

$\begin{array}{llll}342 & 89.1 & 2.3 & (84.5-93.6)\end{array}$

$\begin{array}{llll}180 & 86.2 & 3.5 & (79.3-93.1)\end{array}$

$\begin{array}{llll}262 & 85.9 & 3.0 & (80.0-91.8)\end{array}$

$\begin{array}{llll}188 & 91.5 & 2.0 & (87.5-95.5)\end{array}$

$\begin{array}{llll}772 & 90.9 & 1.9 & (87.1-94.6)\end{array}$

$\begin{array}{llll}737 & 87.7 & 1.9 & (83.8-91.5)\end{array}$

$\begin{array}{llll}537 & 88.9 & 1.9 & (85.2-92.7)\end{array}$

$\begin{array}{llll}1,090 & 88.9 & 1.3 & (86.3-91.5)\end{array}$

$\begin{array}{llll}454 & 90.4 & 1.8 & (86.8-94.0)\end{array}$

$\begin{array}{llll}534 & 90.6 & 1.6 & (87.4-93.8)\end{array}$

$\begin{array}{llll}622 & 91.6 & 1.5 & (88.7-94.4)\end{array}$

$\begin{array}{llll}681 & 89.6 & 1.7 & (86.3-93.0)\end{array}$

$\begin{array}{llll}159 & 88.5 & 2.8 & (83.1-94.0)\end{array}$

$\begin{array}{llll}170 & 80.2 & 3.5 & (73.3-87.1)\end{array}$

$\begin{array}{llll}346 & 82.8 & 2.8 & (77.3-88.3)\end{array}$

$\begin{array}{llll}700 & 82.7 & 2.3 & (78.3-87.1)\end{array}$

$\begin{array}{llll}140 & 84.1 & 3.4 & (77.4-90.8)\end{array}$

$\begin{array}{llll}153 & 85.0 & 3.7 & (77.7-92.3)\end{array}$

$\begin{array}{llll}863 & 88.3 & 1.4 & (85.6-91.0)\end{array}$

$\begin{array}{llll}585 & 82.0 & 2.4 & (77.2-86.8)\end{array}$

$\begin{array}{llll}272 & 79.6 & 3.9 & (71.9-87.3)\end{array}$

$\begin{array}{llll}301 & 87.9 & 2.5 & (83.1-92.7)\end{array}$

$\begin{array}{llll}192 & 75.2 & 3.8 & (67.7-82.6)\end{array}$

$\begin{array}{llll}144 & 76.1 & 4.1 & (68.1-84.1)\end{array}$

$\begin{array}{llll}178 & 74.0 & 4.3 & (65.5-82.4)\end{array}$

$\begin{array}{llll}296 & 68.2 & 4.4 & (59.5-76.8)\end{array}$

$\begin{array}{llll}210 & 73.7 & 3.4 & (67.0-80.4)\end{array}$

$\begin{array}{llll}181 & 73.6 & 3.7 & (66.4-80.9)\end{array}$

$\begin{array}{llll}221 & 68.0 & 5.5 & (57.2-78.8)\end{array}$

$\begin{array}{llll}870 & 78.2 & 1.7 & (74.8-81.6)\end{array}$

$\begin{array}{llll}143 & 68.4 & 5.7 & (57.3-79.5)\end{array}$

$\begin{array}{llll}298 & 77.4 & 3.0 & (71.5-83.2)\end{array}$

$\begin{array}{llll}173 & 73.8 & 4.4 & (65.2-82.4)\end{array}$

$\begin{array}{llll}288 & 75.6 & 3.3 & (69.1-82.0)\end{array}$

$\begin{array}{llll}175 & 70.3 & 4.6 & (61.3-79.2)\end{array}$

$\begin{array}{llll}490 & 73.9 & 2.9 & (68.1-79.6)\end{array}$

$\begin{array}{llll}402 & 76.6 & 2.5 & (71.7-81.6)\end{array}$

$\begin{array}{llll}185 & 83.3 & 3.3 & (76.8-89.9)\end{array}$

$\begin{array}{llll}174 & 81.5 & 3.8 & (74.0-89.0)\end{array}$

$\begin{array}{llll}173 & 82.5 & 4.2 & (74.3-90.7)\end{array}$

$\begin{array}{llll}153 & 87.5 & 2.8 & (82.0-93.0)\end{array}$

$\begin{array}{llll}160 & 86.1 & 3.7 & (78.9-93.2)\end{array}$

Hillsborough County, New Hampshire $\quad 543 \quad 84.5 \quad 1.8 \quad$ (80.9-88.1)

Merrimack County, New Hampshire $\quad \begin{array}{llll}196 & 82.7 & 3.3 & \text { (76.3-89.1) }\end{array}$

Rockingham County, New Hampshire $\quad 318 \quad 88.3 \quad 2.2 \quad$ (84.1-92.6)

$\begin{array}{lllll}\text { Strafford County, New Hampshire } & 197 & 86.6 & 2.8 & (81.1-92.2)\end{array}$

$\begin{array}{lllll}\text { Atlantic County, New Jersey } & 286 & 77.1 & 3.8 & (69.6-84.6)\end{array}$

$\begin{array}{lllll}\text { Bergen County, New Jersey } & 227 & 80.2 & 3.1 & (74.1-86.4)\end{array}$

$\begin{array}{lllll}\text { Burlington County, New Jersey } & 185 & 84.2 & 3.2 & (77.9-90.6)\end{array}$

$\begin{array}{lllll}\text { Camden County, New Jersey } & 209 & 74.4 & 4.5 & (65.6-83.1)\end{array}$

$\begin{array}{lllll}\text { Cape May County, New Jersey } & 178 & 88.4 & 2.7 & (83.2-93.7)\end{array}$

$\begin{array}{lllll}\text { Cumberland County, New Jersey } & 174 & 79.0 & 4.1 & (71.0-87.0)\end{array}$

$\begin{array}{lllll}\text { Essex County, New Jersey } & 314 & 81.1 & 3.3 & (74.6-87.6)\end{array}$

$\begin{array}{lllll}\text { Gloucester County, New Jersey } & 167 & 76.3 & 3.9 & (68.8-83.9)\end{array}$

Hudson County, New Jersey

$\begin{array}{llll}297 & 81.8 & 2.9 & (76.1-87.5)\end{array}$ 
TABLE 30. (Continued) Estimated prevalence of women aged 50-74 years who have had a mammogram in the past 2 years, by county - Behavioral Risk Factor Surveillance System, United States, 2012

\begin{tabular}{|c|c|c|c|c|}
\hline County & $\begin{array}{l}\text { Sample } \\
\text { size }\end{array}$ & $\%$ & SE & $95 \% \mathrm{Cl}$ \\
\hline Hunterdon County, New Jersey & 173 & 80.8 & 5.5 & (70.0-91.6) \\
\hline Mercer County, New Jersey & 147 & 81.6 & 4.0 & $(73.9-89.4)$ \\
\hline Middlesex County, New Jersey & 186 & 79.8 & 4.2 & $(71.6-88.0)$ \\
\hline Monmouth County, New Jersey & 190 & 79.8 & 3.8 & (72.3-87.3) \\
\hline Morris County, New Jersey & 214 & 77.7 & 3.9 & $(70.1-85.2)$ \\
\hline Ocean County, New Jersey & 162 & 76.4 & 4.1 & $(68.4-84.5)$ \\
\hline Passaic County, New Jersey & 160 & 76.4 & 4.0 & $(68.5-84.4)$ \\
\hline Salem County, New Jersey & 186 & 75.9 & 3.5 & $(69.0-82.8)$ \\
\hline Somerset County, New Jersey & 170 & 88.5 & 2.8 & $(83.0-93.9)$ \\
\hline Sussex County, New Jersey & 179 & 73.4 & 4.5 & $(64.6-82.2)$ \\
\hline Union County, New Jersey & 178 & 77.6 & 4.1 & $(69.5-85.7)$ \\
\hline Warren County, New Jersey & 165 & 78.1 & 3.7 & $(70.8-85.4)$ \\
\hline Bernalillo County, New Mexico & 476 & 73.5 & 2.5 & $(68.7-78.3)$ \\
\hline Dona Ana County, New Mexico & 197 & 81.3 & 2.9 & $(75.6-87.1)$ \\
\hline Sandoval County, New Mexico & 191 & 74.8 & 3.8 & $(67.3-82.3)$ \\
\hline San Juan County, New Mexico & 175 & 73.1 & 4.1 & $(65.1-81.1)$ \\
\hline Santa Fe County, New Mexico & 221 & 71.7 & 3.5 & $(64.8-78.6)$ \\
\hline Kings County, New York & 85 & 78.8 & 5.9 & $(67.3-90.3)$ \\
\hline Guilford County, North Carolina & 126 & 86.8 & 3.3 & $(80.3-93.3)$ \\
\hline Mecklenburg County, North Carolina & 153 & 76.9 & 4.7 & $(67.8-86.1)$ \\
\hline Robeson County, North Carolina & 194 & 76.1 & 4.8 & $(66.7-85.5)$ \\
\hline Wake County, North Carolina & 121 & 82.9 & 4.1 & $(74.8-90.9)$ \\
\hline Burleigh County, North Dakota & 155 & 81.6 & 3.8 & (74.3-89.0) \\
\hline Cass County, North Dakota & 218 & 81.7 & 3.5 & $(74.9-88.4)$ \\
\hline Cuyahoga County, Ohio & 230 & 81.0 & 3.1 & $(74.9-87.0)$ \\
\hline Franklin County, Ohio & 201 & 80.5 & 4.0 & $(72.7-88.3)$ \\
\hline Hamilton County, Ohio & 212 & 76.8 & 3.4 & $(70.0-83.5)$ \\
\hline Lorain County, Ohio & 202 & 87.1 & 2.5 & $(82.2-92.1)$ \\
\hline Lucas County, Ohio & 209 & 72.9 & 4.0 & $(65.1-80.7)$ \\
\hline Mahoning County, Ohio & 186 & 71.5 & 5.0 & $(61.7-81.3)$ \\
\hline Montgomery County, Ohio & 214 & 73.2 & 4.0 & $(65.3-81.1)$ \\
\hline Stark County, Ohio & 205 & 81.8 & 3.6 & $(74.6-88.9)$ \\
\hline Summit County, Ohio & 184 & 75.8 & 4.0 & $(67.9-83.8)$ \\
\hline Oklahoma County, Oklahoma & 277 & 80.1 & 2.6 & $(74.9-85.3)$ \\
\hline Tulsa County, Oklahoma & 330 & 69.4 & 3.1 & $(63.3-75.6)$ \\
\hline Clackamas County, Oregon & 171 & 84.2 & 3.4 & $(77.6-90.8)$ \\
\hline Lane County, Oregon & 172 & 74.1 & 4.0 & $(66.2-82.0)$ \\
\hline Multnomah County, Oregon & 235 & 76.7 & 3.9 & $(69.1-84.3)$ \\
\hline Washington County, Oregon & 170 & 75.4 & 4.0 & $(67.6-83.2)$ \\
\hline Allegheny County, Pennsylvania & 482 & 76.5 & 2.3 & $(72.1-80.9)$ \\
\hline Bradford County, Pennsylvania & 550 & 77.3 & 1.9 & $(73.6-80.9)$ \\
\hline Montgomery County, Pennsylvania & 123 & 75.4 & 4.9 & $(65.8-84.9)$ \\
\hline Philadelphia County, Pennsylvania & 588 & 83.8 & 1.9 & $(80.2-87.5)$ \\
\hline Pike County, Pennsylvania & 583 & 74.3 & 1.9 & $(70.5-78.0)$ \\
\hline Kent County, Rhode Island & 232 & 85.9 & 2.6 & $(80.9-91.0)$ \\
\hline Providence County, Rhode Island & 952 & 85.0 & 1.6 & $(81.9-88.1)$ \\
\hline Washington County, Rhode Island & 207 & 84.8 & 2.9 & $(79.1-90.4)$ \\
\hline Aiken County, South Carolina & 175 & 72.6 & 4.3 & $(64.2-81.0)$ \\
\hline
\end{tabular}

TABLE 30. (Continued) Estimated prevalence of women aged 50-74 years who have had a mammogram in the past 2 years, by county - Behavioral Risk Factor Surveillance System, United States, 2012

\begin{tabular}{|c|c|c|c|c|}
\hline County & $\begin{array}{l}\text { Sample } \\
\text { size }\end{array}$ & $\%$ & SE & $95 \% \mathrm{Cl}$ \\
\hline Beaufort County, South Carolina & 273 & 76.8 & 3.6 & $(69.8-83.9)$ \\
\hline Charleston County, South Carolina & 301 & 82.1 & 3.2 & (76.0-88.3) \\
\hline Greenville County, South Carolina & 223 & 70.9 & 4.9 & $(61.4-80.4)$ \\
\hline Horry County, South Carolina & 245 & 71.7 & 3.7 & $(64.5-79.0)$ \\
\hline Richland County, South Carolina & 282 & 84.1 & 3.3 & (77.5-90.6) \\
\hline Spartanburg County, South Carolina & 195 & 72.8 & 4.5 & $(64.0-81.6)$ \\
\hline Lincoln County, South Dakota & 106 & 85.6 & 3.7 & $(78.3-92.8)$ \\
\hline Minnehaha County, South Dakota & 181 & 80.9 & 4.1 & $(72.7-89.0)$ \\
\hline Pennington County, South Dakota & 131 & 76.4 & 4.6 & $(67.3-85.5)$ \\
\hline Davidson County, Tennessee & 152 & 79.4 & 4.0 & $(71.6-87.3)$ \\
\hline Shelby County, Tennessee & 128 & 78.5 & 5.2 & $(68.2-88.7)$ \\
\hline Bexar County, Texas & 138 & 70.3 & 5.7 & (59.2-81.5) \\
\hline Dallas County, Texas & 108 & 74.9 & 6.1 & $(63.0-86.8)$ \\
\hline El Paso County, Texas & 197 & 74.6 & 4.2 & $(66.3-82.9)$ \\
\hline Harris County, Texas & 142 & 79.0 & 4.4 & $(70.4-87.6)$ \\
\hline Hidalgo County, Texas & 160 & 69.3 & 5.2 & $(59.1-79.5)$ \\
\hline Tarrant County, Texas & 144 & 80.7 & 4.0 & $(72.9-88.4)$ \\
\hline Travis County, Texas & 295 & 70.1 & 5.0 & $(60.3-79.9)$ \\
\hline Davis County, Utah & 232 & 73.1 & 3.3 & $(66.5-79.6)$ \\
\hline Salt Lake County, Utah & 1,000 & 73.3 & 1.7 & $(70.0-76.7)$ \\
\hline Tooele County, Utah & 140 & 67.8 & 5.4 & $(57.2-78.4)$ \\
\hline Utah County, Utah & 300 & 70.9 & 3.0 & $(65.1-76.7)$ \\
\hline Wasatch County, Utah & 133 & 68.8 & 6.1 & $(56.9-80.6)$ \\
\hline Weber County, Utah & 247 & 77.3 & 3.1 & $(71.2-83.4)$ \\
\hline Chittenden County, Vermont & 246 & 80.6 & 3.0 & $(74.7-86.6)$ \\
\hline Rutland County, Vermont & 194 & 75.3 & 3.7 & $(68.0-82.7)$ \\
\hline Washington County, Vermont & 162 & 88.5 & 2.8 & $(83.1-93.9)$ \\
\hline Windsor County, Vermont & 169 & 89.7 & 2.3 & $(85.1-94.2)$ \\
\hline Fairfax County, Virginia & 167 & 85.3 & 3.8 & $(77.8-92.8)$ \\
\hline Clark County, Washington & 250 & 81.9 & 4.1 & $(73.8-90.0)$ \\
\hline King County, Washington & 1,037 & 78.1 & 1.7 & $(74.8-81.5)$ \\
\hline Kitsap County, Washington & 174 & 75.6 & 4.2 & $(67.5-83.7)$ \\
\hline Pierce County, Washington & 329 & 77.7 & 2.9 & $(72.0-83.5)$ \\
\hline Snohomish County, Washington & 316 & 76.8 & 2.8 & $(71.3-82.3)$ \\
\hline Spokane County, Washington & 283 & 78.4 & 3.1 & $(72.3-84.5)$ \\
\hline Thurston County, Washington & 151 & 77.2 & 4.3 & $(68.7-85.7)$ \\
\hline Whatcom County, Washington & 277 & 73.2 & 3.8 & $(65.8-80.7)$ \\
\hline Yakima County, Washington & 147 & 68.5 & 4.9 & $(58.9-78.0)$ \\
\hline Kanawha County, West Virginia & 165 & 82.0 & 3.3 & $(75.6-88.4)$ \\
\hline Milwaukee County, Wisconsin & 284 & 81.5 & 3.9 & $(73.9-89.2)$ \\
\hline Laramie County, Wyoming & 297 & 80.4 & 3.7 & $(73.1-87.7)$ \\
\hline Natrona County, Wyoming & 245 & 61.8 & 4.6 & $(52.7-70.9)$ \\
\hline San Juan Municipio, Puerto Rico & 182 & 77.4 & 3.8 & $(70.0-84.8)$ \\
\hline Median & & 79.7 & & \\
\hline Range & & $61.8-91.6$ & & \\
\hline
\end{tabular}

Abbreviations: $\mathrm{Cl}=$ confidence interval; $\mathrm{SE}=$ standard error. 
TABLE 31. Estimated prevalence of adults aged $\geq 18$ years who reported ever smoking at least 100 cigarettes and who currently smoke, ${ }^{*}$ by state/territory - Behavioral Risk Factor Surveillance System, United States, 2012

\begin{tabular}{|c|c|c|c|c|}
\hline State/Territory & $\begin{array}{l}\text { Sample } \\
\text { size }\end{array}$ & $\%$ & SE & $95 \% \mathrm{Cl}$ \\
\hline Alabama & 8,913 & 23.8 & 0.7 & $(22.3-25.2)$ \\
\hline Alaska & 4,267 & 20.5 & 0.9 & $(18.8-22.3)$ \\
\hline Arizona & 7,140 & 17.1 & 0.7 & $(15.6-18.5)$ \\
\hline Arkansas & 5,079 & 25.0 & 0.9 & $(23.3-26.8)$ \\
\hline California & 14,543 & 12.6 & 0.4 & $(11.8-13.4)$ \\
\hline Colorado & 11,657 & 17.7 & 0.5 & $(16.8-18.7)$ \\
\hline Connecticut & 8,609 & 16.0 & 0.6 & $(14.8-17.2)$ \\
\hline Delaware & 5,146 & 19.7 & 0.8 & $(18.2-21.3)$ \\
\hline District of Columbia & 3,720 & 19.6 & 1.1 & $(17.4-21.9)$ \\
\hline Florida & 7,465 & 17.7 & 0.7 & $(16.3-19.1)$ \\
\hline Georgia & 5,894 & 20.4 & 0.8 & (18.8-21.9) \\
\hline Hawaii & 7,495 & 14.6 & 0.6 & $(13.3-15.8)$ \\
\hline Idaho & 5,794 & 16.4 & 0.9 & $(14.6-18.3)$ \\
\hline Illinois & 5,530 & 18.6 & 0.8 & $(16.9-20.2)$ \\
\hline Indiana & 8,511 & 24.0 & 0.6 & $(22.8-25.2)$ \\
\hline lowa & 7,015 & 18.1 & 0.6 & (16.9-19.3) \\
\hline Kansas & 11,709 & 19.4 & 0.5 & (18.4-20.4) \\
\hline Kentucky & 10,867 & 28.3 & 0.7 & (26.9-29.7) \\
\hline Louisiana & 8,997 & 24.8 & 0.8 & $(23.2-26.3)$ \\
\hline Maine & 9,810 & 20.3 & 0.6 & $(19.2-21.4)$ \\
\hline Maryland & 12,456 & 16.2 & 0.6 & $(15.0-17.4)$ \\
\hline Massachusetts & 20,682 & 16.4 & 0.4 & $(15.5-17.2)$ \\
\hline Michigan & 10,361 & 23.3 & 0.6 & $(22.1-24.6)$ \\
\hline Minnesota & 11,889 & 18.8 & 0.5 & $(17.8-19.8)$ \\
\hline Mississippi & 7,700 & 24.0 & 0.7 & $(22.5-25.4)$ \\
\hline Missouri & 6,661 & 23.9 & 0.8 & $(22.3-25.5)$ \\
\hline Montana & 8,564 & 19.7 & 0.6 & (18.5-20.9) \\
\hline Nebraska & 18,985 & 19.7 & 0.4 & (18.9-20.6) \\
\hline Nevada & 4,778 & 18.1 & 0.8 & $(16.5-19.7)$ \\
\hline New Hampshire & 7,421 & 17.2 & 0.7 & $(15.8-18.6)$ \\
\hline New Jersey & 15,009 & 17.3 & 0.5 & (16.4-18.3) \\
\hline New Mexico & 8,627 & 19.3 & 0.6 & $(18.2-20.4)$ \\
\hline New York & 5,925 & 16.2 & 0.7 & $(14.8-17.6)$ \\
\hline North Carolina & 11,727 & 20.9 & 0.5 & (19.9-21.9) \\
\hline North Dakota & 4,817 & 21.2 & 0.8 & $(19.6-22.9)$ \\
\hline Ohio & 12,645 & 23.3 & 0.6 & $(22.2-24.4)$ \\
\hline Oklahoma & 7,948 & 23.3 & 0.6 & $(22.0-24.5)$ \\
\hline Oregon & 5,170 & 17.9 & 0.8 & (16.4-19.4) \\
\hline Pennsylvania & 19,621 & 21.4 & 0.5 & $(20.4-22.3)$ \\
\hline Rhode Island & 5,383 & 17.4 & 0.7 & $(16.0-18.9)$ \\
\hline South Carolina & 12,535 & 22.5 & 0.6 & (21.4-23.7) \\
\hline South Dakota & 7,778 & 22.0 & 0.7 & $(20.5-23.4)$ \\
\hline Tennessee & 6,780 & 24.9 & 0.8 & $(23.3-26.4)$ \\
\hline Texas & 8,963 & 18.2 & 0.6 & $(17.0-19.3)$ \\
\hline Utah & 12,203 & 10.6 & 0.4 & $(9.8-11.3)$ \\
\hline Vermont & 5,957 & 16.5 & 0.7 & $(15.1-17.8)$ \\
\hline Virginia & 7,154 & 19.0 & 0.7 & (17.7-20.3) \\
\hline Washington & 15,173 & 17.2 & 0.5 & $(16.3-18.1)$ \\
\hline West Virginia & 5,390 & 28.2 & 0.8 & (26.7-29.7) \\
\hline Wisconsin & 5,133 & 20.4 & 0.9 & $(18.7-22.1)$ \\
\hline Wyoming & 6,159 & 21.8 & 1.0 & (19.9-23.7) \\
\hline Guam & 1,981 & 25.8 & 1.4 & $(23.1-28.5)$ \\
\hline Puerto Rico & 6,282 & 12.6 & 0.5 & (11.6-13.7) \\
\hline Median & & 19.6 & & \\
\hline Range & & $10.6-28.3$ & & \\
\hline
\end{tabular}

Abbreviations: $\mathrm{Cl}=$ confidence interval; $\mathrm{SE}=$ standard error.

* Smoked every day or someday during the period of the survey.
TABLE 32. Estimated prevalence of adults aged $\geq 18$ years who reported ever smoking at least 100 cigarettes and who currently smoke, ${ }^{*}$ by metropolitan and micropolitan statistical area Behavioral Risk Factor Surveillance System, United States, 2012

\begin{tabular}{|c|c|c|c|c|}
\hline MMSA(s) & $\begin{array}{l}\text { Sample } \\
\text { size }\end{array}$ & $\%$ & SE & $95 \% \mathrm{Cl}$ \\
\hline Aguadilla-Isabela, Puerto Rico & 548 & 12.7 & 1.9 & $(9.0-16.3)$ \\
\hline Akron, Ohio & 726 & 21.7 & 2.2 & $(17.4-26.1)$ \\
\hline Albuquerque, New Mexico & 3,201 & 18.7 & 0.9 & $(16.9-20.5)$ \\
\hline $\begin{array}{l}\text { Allentown-Bethlehem-Easton, } \\
\text { Pennsylvania-New Jersey }\end{array}$ & 1,312 & 20.8 & 1.8 & $(17.3-24.3)$ \\
\hline Anaheim-Santa Ana-Irvine, California ${ }^{\dagger}$ & 1,036 & 10.0 & 1.4 & $(7.2-12.7)$ \\
\hline Anchorage, Alaska & 1,481 & 18.1 & 1.3 & $(15.6-20.6)$ \\
\hline Asheville, North Carolina & 590 & 23.3 & 2.4 & $(18.6-27.9)$ \\
\hline $\begin{array}{l}\text { Atlanta-Sandy Springs-Roswell, } \\
\text { Georgia }\end{array}$ & 2,460 & 17.3 & 1.1 & $(15.2-19.5)$ \\
\hline Atlantic City-Hammonton, New Jersey & 983 & 22.0 & 2.0 & $(18.1-25.9)$ \\
\hline $\begin{array}{l}\text { Augusta-Richmond County, Georgia- } \\
\text { South Carolina }\end{array}$ & 1,005 & 22.7 & 2.6 & $(17.5-27.8)$ \\
\hline Augusta-Waterville, Maine & 825 & 20.4 & 1.7 & $(17.0-23.8)$ \\
\hline Austin-Round Rock, Texas & 1,363 & 15.2 & 1.8 & $(11.7-18.7)$ \\
\hline $\begin{array}{l}\text { Baltimore-Columbia-Towson, } \\
\text { Maryland }\end{array}$ & 4,607 & 18.5 & 0.9 & $(16.7-20.4)$ \\
\hline Bangor, Maine & 920 & 22.2 & 1.9 & $(18.5-26.0)$ \\
\hline Barnstable Town, Massachusetts & 534 & 15.0 & 2.0 & $(11.1-18.8)$ \\
\hline Barre, Vermont & 507 & 14.3 & 2.3 & $(9.8-18.8)$ \\
\hline Baton Rouge, Louisiana & 1,373 & 21.0 & 1.8 & $(17.5-24.5)$ \\
\hline Bellingham, Washington & 835 & 18.4 & 2.7 & $(13.2-23.6)$ \\
\hline Berlin, New Hampshire-Vermont & 703 & 19.0 & 2.6 & $(13.9-24.2)$ \\
\hline Billings, Montana & 838 & 20.0 & 1.6 & $(16.8-23.1)$ \\
\hline Birmingham-Hoover, Alabama & 1,798 & 21.5 & 1.4 & $(18.7-24.2)$ \\
\hline Bismarck, North Dakota & 813 & 19.0 & 2.0 & $(15.0-23.0)$ \\
\hline Boise City, Idaho & 1,453 & 16.0 & 1.6 & $(12.8-19.2)$ \\
\hline Boston, Massachusetts ${ }^{\dagger}$ & 5,627 & 14.9 & 0.8 & $(13.4-16.5)$ \\
\hline Boulder, Colorado & 494 & 13.5 & 1.9 & $(9.8-17.3)$ \\
\hline Bremerton-Silverdale, Washington & 561 & 19.9 & 2.3 & $(15.3-24.4)$ \\
\hline $\begin{array}{l}\text { Bridgeport-Stamford-Norwalk, } \\
\text { Connecticut }\end{array}$ & 2,135 & 13.2 & 1.2 & $(10.9-15.5)$ \\
\hline Burlington-South Burlington, Vermont & 1,500 & 14.8 & 1.2 & $(12.5-17.2)$ \\
\hline $\begin{array}{l}\text { Cambridge-Newton-Framingham, } \\
\text { Massachusetts }{ }^{\dagger}\end{array}$ & 6,581 & 13.7 & 0.6 & $(12.4-14.9)$ \\
\hline Camden, New Jersey ${ }^{\dagger}$ & 1,921 & 19.1 & 1.4 & $(16.4-21.7)$ \\
\hline Canton-Massillon, Ohio & 665 & 26.7 & 2.6 & $(21.6-31.7)$ \\
\hline Casper, Wyoming & 815 & 25.3 & 2.6 & $(20.2-30.4)$ \\
\hline Cedar Rapids, lowa & 576 & 19.1 & 2.1 & $(14.9-23.2)$ \\
\hline Charleston, West Virginia & 770 & 25.9 & 1.9 & $(22.1-29.7)$ \\
\hline $\begin{array}{l}\text { Charleston-North Charleston, } \\
\text { South Carolina }\end{array}$ & 1,683 & 20.2 & 1.4 & $(17.4-23.0)$ \\
\hline $\begin{array}{l}\text { Charlotte-Concord-Gastonia, } \\
\text { North Carolina-South Carolina }\end{array}$ & 2,495 & 18.6 & 1.0 & $(16.5-20.6)$ \\
\hline Chattanooga, Tennessee-Georgia & 609 & 24.8 & 2.8 & $(19.4-30.3)$ \\
\hline Cheyenne, Wyoming & 938 & 20.1 & 2.3 & $(15.6-24.7)$ \\
\hline $\begin{array}{l}\text { Chicago-Naperville-Elgin, } \\
\text { Illinois-Indiana-Wisconsin }\end{array}$ & 3,685 & 17.7 & 1.0 & $(15.7-19.7)$ \\
\hline Cincinnati, Ohio-Kentucky-Indiana & 2,314 & 23.3 & 1.2 & $(20.9-25.7)$ \\
\hline $\begin{array}{l}\text { Claremont-Lebanon, New } \\
\text { Hampshire-Vermont }\end{array}$ & 1,923 & 17.1 & 1.5 & $(14.3-20.0)$ \\
\hline Cleveland-Elyria, Ohio & 1,692 & 22.2 & 1.5 & $(19.3-25.0)$ \\
\hline Colorado Springs, Colorado & 1,111 & 17.9 & 1.5 & $(14.9-20.9)$ \\
\hline Columbia, South Carolina & 1,766 & 21.2 & 1.5 & $(18.2-24.2)$ \\
\hline Columbus, Ohio & 1,564 & 20.0 & 1.3 & $(17.4-22.6)$ \\
\hline Concord, New Hampshire & 696 & 14.9 & 2.1 & $(10.8-19.0)$ \\
\hline Dallas-Plano-Irving, Texas $^{\dagger}$ & 886 & 15.8 & 1.6 & $(12.7-18.9)$ \\
\hline Dayton, Ohio & 838 & 23.7 & 2.1 & $(19.7-27.8)$ \\
\hline Denver-Aurora-Lakewood, Colorado & 4,616 & 18.0 & 0.7 & $(16.5-19.4)$ \\
\hline Des Moines-West Des Moines, lowa & 1,142 & 18.7 & 1.5 & $(15.8-21.6)$ \\
\hline
\end{tabular}

See table footnotes on page 68. 
TABLE 32. (Continued) Estimated prevalence of adults aged $\geq 18$ years who reported ever smoking at least 100 cigarettes and who currently smoke,* by metropolitan and micropolitan statistical area Behavioral Risk Factor Surveillance System, United States, 2012

\begin{tabular}{|c|c|c|c|c|}
\hline MMSA(s) & $\begin{array}{l}\text { Sample } \\
\text { size }\end{array}$ & $\%$ & SE & $95 \% \mathrm{Cl}$ \\
\hline Detroit-Dearborn-Livonia, Michigan $^{\dagger}$ & 2,177 & 24.4 & 1.6 & $(21.3-27.4)$ \\
\hline Dover, Delaware & 1,432 & 21.1 & 1.7 & $(17.8-24.3)$ \\
\hline Duluth, Minnesota-Wisconsin & 506 & 23.8 & 2.8 & $(18.3-29.3)$ \\
\hline Durham-Chapel Hill, North Carolina & 785 & 17.9 & 1.8 & $(14.4-21.4)$ \\
\hline El Paso, Texas & 616 & 15.1 & 2.3 & $(10.5-19.7)$ \\
\hline Eugene, Oregon & 516 & 17.1 & 2.3 & $(12.5-21.7)$ \\
\hline Fairbanks, Alaska & 590 & 22.7 & 2.4 & $(18.0-27.4)$ \\
\hline Fargo, North Dakota-Minnesota & 928 & 20.3 & 2.2 & $(16.0-24.6)$ \\
\hline Farmington, New Mexico & 636 & 21.0 & 2.1 & $(17.0-25.1)$ \\
\hline Fayetteville, North Carolina & 500 & 22.5 & 2.4 & $(17.8-27.2)$ \\
\hline $\begin{array}{l}\text { Fayetteville-Springdale-Rogers, } \\
\text { Arkansas-Missouri }\end{array}$ & 796 & 20.1 & 2.2 & $(15.8-24.3)$ \\
\hline Fort Collins, Colorado & 570 & 15.7 & 1.9 & $(11.9-19.5)$ \\
\hline Fort Wayne, Indiana & 526 & 26.2 & 2.4 & $(21.5-31.0)$ \\
\hline Fort Worth-Arlington, Texas ${ }^{\dagger}$ & 715 & 20.5 & 2.0 & $(16.5-24.5)$ \\
\hline Grand Island, Nebraska & 847 & 20.2 & 1.9 & $(16.5-23.9)$ \\
\hline Grand Rapids-Wyoming, Michigan & 882 & 19.5 & 1.9 & $(15.7-23.2)$ \\
\hline Great Falls, Montana & 703 & 22.1 & 2.2 & $(17.8-26.4)$ \\
\hline Greeley, Colorado & 514 & 20.6 & 2.2 & $(16.3-24.8)$ \\
\hline $\begin{array}{l}\text { Greensboro-High Point, } \\
\text { North Carolina }\end{array}$ & 800 & 20.0 & 1.9 & $(16.4-23.7)$ \\
\hline $\begin{array}{l}\text { Greenville-Anderson-Mauldin, } \\
\text { South Carolina }\end{array}$ & 1,661 & 23.3 & 1.5 & $(20.5-26.2)$ \\
\hline Gulfport-Biloxi-Pascagoula, Mississippi & 732 & 26.0 & 2.1 & $(21.8-30.2)$ \\
\hline $\begin{array}{l}\text { Hagerstown-Martinsburg, Maryland- } \\
\text { West Virginia }\end{array}$ & 751 & 21.6 & 2.9 & $(15.9-27.3)$ \\
\hline Harrisburg-Carlisle, Pennsylvania & 662 & 18.4 & 1.9 & $(14.6-22.2)$ \\
\hline $\begin{array}{l}\text { Hartford-West Hartford-East Hartford, } \\
\text { Connecticut }\end{array}$ & 2,632 & 15.3 & 1.0 & $(13.4-17.3)$ \\
\hline Heber, Utah & 501 & $\mathrm{~N} / \mathrm{A}^{\S}$ & $\mathrm{N} / \mathrm{A}$ & $(\mathrm{N} / \mathrm{A}-\mathrm{N} / \mathrm{A})$ \\
\hline Hilo, Hawaii & 1,340 & 16.3 & 1.4 & $(13.5-19.1)$ \\
\hline $\begin{array}{l}\text { Hilton Head Island-Bluffton-Beaufort, } \\
\text { South Carolina }\end{array}$ & 913 & 19.0 & 2.7 & $(13.8-24.2)$ \\
\hline $\begin{array}{l}\text { Houston-The Woodlands-Sugar Land, } \\
\text { Texas }\end{array}$ & 1,118 & 16.6 & 1.4 & $(13.9-19.3)$ \\
\hline $\begin{array}{l}\text { Huntington-Ashland, West } \\
\text { Virginia-Kentucky-Ohio }\end{array}$ & 1,100 & 30.1 & 1.9 & $(26.4-33.9)$ \\
\hline Huntsville, Alabama & 605 & 22.0 & 2.4 & $(17.3-26.8)$ \\
\hline Idaho Falls, Idaho & 529 & 9.8 & 2.2 & $(5.6-14.1)$ \\
\hline $\begin{array}{l}\text { Indianapolis-Carmel-Anderson, } \\
\text { Indiana }\end{array}$ & 2,161 & 21.8 & 1.2 & $(19.5-24.1)$ \\
\hline Jackson, Mississippi & 904 & 19.8 & 1.7 & $(16.5-23.2)$ \\
\hline Jacksonville, Florida & 513 & 20.9 & 2.8 & $(15.4-26.4)$ \\
\hline Kahului-Wailuku-Lahaina, Hawaii & 1,207 & 14.0 & 1.4 & $(11.2-16.8)$ \\
\hline Kalispell, Montana & 554 & 17.0 & 2.0 & $(13.2-20.9)$ \\
\hline Kansas City, Missouri-Kansas & 4,691 & 22.0 & 1.2 & $(19.6-24.4)$ \\
\hline Kapaa, Hawaii & 659 & 17.8 & 2.9 & $(12.2-23.4)$ \\
\hline Keene, New Hampshire & 540 & 18.5 & 2.9 & $(12.8-24.1)$ \\
\hline Kennewick-Richland, Washington & 535 & 14.3 & 1.9 & $(10.5-18.1)$ \\
\hline $\begin{array}{l}\text { Kingsport-Bristol-Bristol, } \\
\text { Tennessee-Virginia }\end{array}$ & 548 & 23.4 & 2.8 & $(17.9-28.9)$ \\
\hline Knoxville, Tennessee & 806 & 20.1 & 1.9 & $(16.4-23.7)$ \\
\hline Laconia, New Hampshire & 555 & 21.1 & 3.2 & $(14.8-27.3)$ \\
\hline Lafayette, Louisiana & 556 & 26.0 & 2.5 & $(21.1-30.9)$ \\
\hline Las Cruces, New Mexico & 697 & 18.1 & 2.0 & $(14.1-22.0)$ \\
\hline $\begin{array}{l}\text { Las Vegas-Henderson-Paradise, } \\
\text { Nevada }\end{array}$ & 1,983 & 17.0 & 1.0 & $(15.0-19.0)$ \\
\hline Lewiston-Auburn, Maine & 696 & 23.7 & 2.1 & $(19.5-27.8)$ \\
\hline Lexington-Fayette, Kentucky & 518 & 23.2 & 2.5 & $(18.3-28.0)$ \\
\hline Lincoln, Nebraska & 1,653 & 21.3 & 1.2 & $(18.9-23.7)$ \\
\hline
\end{tabular}

See table footnotes on page 68.
TABLE 32. (Continued) Estimated prevalence of adults aged $\geq 18$ years who reported ever smoking at least 100 cigarettes and who currently smoke,* by metropolitan and micropolitan statistical area Behavioral Risk Factor Surveillance System, United States, 2012

\begin{tabular}{|c|c|c|c|c|}
\hline MMSA(s) & $\begin{array}{l}\text { Sample } \\
\text { size }\end{array}$ & $\%$ & SE & $95 \% \mathrm{Cl}$ \\
\hline $\begin{array}{l}\text { Little Rock-North Little Rock-Conway, } \\
\text { Arkansas }\end{array}$ & 1,145 & 22.2 & 1.7 & $(18.8-25.6)$ \\
\hline Logan, Utah-Idaho & 503 & 6.1 & 1.6 & $(2.9-9.3)$ \\
\hline $\begin{array}{l}\text { Los Angeles-Long Beach-Glendale, } \\
\text { California }^{\dagger}\end{array}$ & 3,490 & 11.8 & 0.8 & $(10.3-13.3)$ \\
\hline $\begin{array}{l}\text { Louisville/Jefferson County, } \\
\text { Kentucky-Indiana }\end{array}$ & 2,092 & 25.9 & 1.6 & $(22.8-29.0)$ \\
\hline Lumberton, North Carolina & 532 & 24.1 & 3.7 & $(16.8-31.4)$ \\
\hline Manchester-Nashua, New Hampshire & 1,882 & 17.7 & 1.3 & $(15.2-20.1)$ \\
\hline McAllen-Edinburg-Mission, Texas & 614 & 14.1 & 2.6 & $(9.0-19.3)$ \\
\hline $\begin{array}{l}\text { Memphis, } \\
\text { Tennessee-Mississippi-Arkansas }\end{array}$ & 1,278 & 23.1 & 1.8 & $(19.6-26.7)$ \\
\hline $\begin{array}{l}\text { Miami-Fort Lauderdale-West Palm } \\
\text { Beach, Florida }\end{array}$ & 1,627 & 13.2 & 1.2 & $(10.8-15.6)$ \\
\hline $\begin{array}{l}\text { Milwaukee-Waukesha-West Allis, } \\
\text { Wisconsin }\end{array}$ & 1,202 & 20.3 & 1.7 & $(16.9-23.7)$ \\
\hline $\begin{array}{l}\text { Minneapolis-St. Paul-Bloomington, } \\
\text { Minnesota-Wisconsin }\end{array}$ & 7,649 & 18.0 & 0.7 & $(16.7-19.3)$ \\
\hline Missoula, Montana & 774 & 20.0 & 1.8 & $(16.4-23.6)$ \\
\hline Mobile, Alabama & 808 & 25.7 & 2.6 & $(20.7-30.8)$ \\
\hline Montgomery, Alabama & 530 & 23.1 & 2.7 & $(17.9-28.4)$ \\
\hline $\begin{array}{l}\text { Montgomery County-Bucks County- } \\
\text { Chester County, Pennsylvania }{ }^{\dagger}\end{array}$ & 1,292 & 16.4 & 1.6 & $(13.3-19.5)$ \\
\hline $\begin{array}{l}\text { Myrtle Beach-Conway-North Myrtle } \\
\text { Beach, South Carolina-North Carolina }\end{array}$ & 937 & 23.8 & 1.9 & $(20.0-27.6)$ \\
\hline $\begin{array}{l}\text { Nashville-Davidson-Murfreesboro- } \\
\text { Franklin, Tennessee }\end{array}$ & 1,295 & 23.7 & 1.6 & $(20.5-26.8)$ \\
\hline $\begin{array}{l}\text { Nassau County-Suffolk County, } \\
\text { New York }^{\dagger}\end{array}$ & 883 & 14.6 & 1.9 & $(11.0-18.3)$ \\
\hline Newark, New Jersey-Pennsylvania ${ }^{\dagger}$ & 6,338 & 16.0 & 0.8 & $(14.4-17.6)$ \\
\hline New Haven-Milford, Connecticut & 1,973 & 18.3 & 1.3 & $(15.8-20.8)$ \\
\hline New Orleans-Metairie, Louisiana & 1,261 & 22.8 & 1.9 & $(19.1-26.4)$ \\
\hline $\begin{array}{l}\text { New York-Jersey City-White Plains, } \\
\text { New York-New Jersey }{ }^{\dagger}\end{array}$ & 7,045 & 14.8 & 0.8 & $(13.3-16.3)$ \\
\hline Norfolk, Nebraska & 565 & 15.1 & 2.1 & $(11.0-19.2)$ \\
\hline North Platte, Nebraska & 608 & 16.2 & 2.3 & $(11.7-20.7)$ \\
\hline Norwich-New London, Connecticut & 980 & 18.2 & 1.9 & $(14.5-22.0)$ \\
\hline $\begin{array}{l}\text { Oakland-Hayward-Berkeley, } \\
\text { California }{ }^{\dagger}\end{array}$ & 976 & 11.2 & 1.7 & $(7.9-14.6)$ \\
\hline Ocean City, New Jersey & 530 & 23.5 & 3.7 & $(16.3-30.8)$ \\
\hline Ogden-Clearfield, Utah & 2,390 & 10.9 & 0.9 & $(9.2-12.6)$ \\
\hline Oklahoma City, Oklahoma & 2,419 & 20.4 & 1.1 & $(18.3-22.5)$ \\
\hline Olympia-Tumwater, Washington & 514 & 23.3 & 2.9 & $(17.7-28.9)$ \\
\hline Omaha-Council Bluffs, Nebraska-lowa & 5,521 & 21.2 & 0.8 & $(19.6-22.8)$ \\
\hline Orlando-Kissimmee-Sanford, Florida & 551 & 18.7 & 2.9 & $(13.1-24.3)$ \\
\hline Philadelphia, Pennsylvania $^{\dagger}$ & 2,402 & 23.2 & 1.2 & $(20.9-25.6)$ \\
\hline Phoenix-Mesa-Scottsdale, Arizona & 2,537 & 16.9 & 1.0 & $(14.8-18.9)$ \\
\hline Pittsburgh, Pennsylvania & 3,306 & 22.4 & 1.0 & $(20.5-24.3)$ \\
\hline Ponce, Puerto Rico & 546 & 13.9 & 1.8 & $(10.4-17.4)$ \\
\hline Portland-South Portland, Maine & 3,295 & 17.6 & 0.9 & $(15.8-19.4)$ \\
\hline $\begin{array}{l}\text { Portland-Vancouver-Hillsboro, } \\
\text { Oregon-Washington }\end{array}$ & 3,069 & 15.9 & 1.0 & $(14.0-17.8)$ \\
\hline $\begin{array}{l}\text { Providence-Warwick, Rhode } \\
\text { Island-Massachusetts }\end{array}$ & 7,924 & 18.4 & 0.7 & $(17.0-19.8)$ \\
\hline Provo-Orem, Utah & 1,716 & 5.1 & 0.7 & $(3.8-6.5)$ \\
\hline Raleigh, North Carolina & 933 & 16.6 & 1.5 & $(13.8-19.5)$ \\
\hline Rapid City, South Dakota & 1,042 & 24.7 & 1.8 & $(21.2-28.3)$ \\
\hline Reno, Nevada & 1,490 & 17.4 & 1.6 & $(14.3-20.5)$ \\
\hline Richmond, Virginia & 981 & 19.4 & 1.8 & $(15.8-23.0)$ \\
\hline
\end{tabular}

See table footnotes on page 68. 
TABLE 32. (Continued) Estimated prevalence of adults aged $\geq 18$ years who reported ever smoking at least 100 cigarettes and who currently smoke, ${ }^{*}$ by metropolitan and micropolitan statistical area Behavioral Risk Factor Surveillance System, United States, 2012

\begin{tabular}{|c|c|c|c|c|}
\hline MMSA(s) & $\begin{array}{l}\text { Sample } \\
\text { size }\end{array}$ & $\%$ & SE & $95 \% \mathrm{Cl}$ \\
\hline $\begin{array}{l}\text { Riverside-San Bernardino-Ontario, } \\
\text { California }\end{array}$ & 1,527 & 15.1 & 1.3 & $(12.6-17.6)$ \\
\hline $\begin{array}{l}\text { Rockingham County-Strafford County, } \\
\text { New Hampshire }\end{array}$ & 1,645 & 16.6 & 1.4 & $(13.8-19.4)$ \\
\hline Rutland, Vermont & 585 & 17.7 & 2.4 & $(13.1-22.4)$ \\
\hline $\begin{array}{l}\text { Sacramento-Roseville-Arden-Arcade, } \\
\text { California }\end{array}$ & 998 & 14.7 & 1.7 & $(11.4-18.0)$ \\
\hline St. Louis, Missouri-Illinois & 2,030 & 20.1 & 1.4 & $(17.3-22.9)$ \\
\hline Salisbury, Maryland-Delaware & 2,093 & 20.5 & 1.7 & $(17.3-23.8)$ \\
\hline Salt Lake City, Utah & 4,536 & 12.8 & 0.7 & $(11.4-14.3)$ \\
\hline San Antonio-New Braunfels, Texas & 767 & 17.7 & 2.0 & $(13.8-21.5)$ \\
\hline San Diego-Carlsbad, California & 1,127 & 10.6 & 1.2 & $(8.2-12.9)$ \\
\hline $\begin{array}{l}\text { San Francisco-Redwood City-South } \\
\text { San Francisco, California }{ }^{\dagger}\end{array}$ & 595 & 11.3 & 2.1 & $(7.2-15.3)$ \\
\hline $\begin{array}{l}\text { San Jose-Sunnyvale-Santa Clara, } \\
\text { California }\end{array}$ & 681 & 9.6 & 1.6 & $(6.5-12.6)$ \\
\hline San Juan-Carolina-Caguas, Puerto Rico & 3,936 & 12.7 & 0.7 & $(11.4-14.1)$ \\
\hline Santa Fe, New Mexico & 689 & 16.8 & 1.9 & $(13.1-20.5)$ \\
\hline Sayre, Pennsylvania & 1,829 & 23.7 & 2.8 & $(18.2-29.2)$ \\
\hline Scottsbluff, Nebraska & 626 & 20.2 & 3.0 & $(14.4-26.0)$ \\
\hline $\begin{array}{l}\text { Scranton-Wilkes-Barre-Hazleton, } \\
\text { Pennsylvania }\end{array}$ & 728 & 26.1 & 2.6 & $(21.0-31.3)$ \\
\hline Seattle-Bellevue-Everett, Washington ${ }^{\dagger}$ & 5,053 & 14.9 & 0.7 & $(13.5-16.3)$ \\
\hline Shreveport-Bossier City, Louisiana & 576 & 22.3 & 2.5 & $(17.4-27.2)$ \\
\hline $\begin{array}{l}\text { Silver Spring-Frederick-Rockville, } \\
\text { Maryland }^{\dagger}\end{array}$ & 2,220 & 9.0 & 1.0 & $(7.1-10.9)$ \\
\hline $\begin{array}{l}\text { Sioux City, lowa-Nebraska- } \\
\text { South Dakota }\end{array}$ & 1,190 & 20.7 & 2.7 & $(15.4-26.0)$ \\
\hline Sioux Falls, South Dakota & 1,453 & 20.1 & 1.4 & $(17.4-22.8)$ \\
\hline Spartanburg, South Carolina & 699 & 22.0 & 2.2 & $(17.8-26.3)$ \\
\hline Spokane-Spokane Valley, Washington & 1,089 & 18.4 & 1.7 & $(15.1-21.6)$ \\
\hline Springfield, Massachusetts & 2,257 & 19.1 & 1.3 & $(16.5-21.7)$ \\
\hline Tacoma-Lakewood, Washington ${ }^{\dagger}$ & 1,174 & 20.8 & 1.7 & $(17.6-24.1)$ \\
\hline
\end{tabular}

TABLE 32. (Continued) Estimated prevalence of adults aged $\geq 18$ years who reported ever smoking at least 100 cigarettes and who currently smoke, ${ }^{*}$ by metropolitan and micropolitan statistical area Behavioral Risk Factor Surveillance System, United States, 2012

\begin{tabular}{|c|c|c|c|c|}
\hline MMSA(s) & $\begin{array}{l}\text { Sample } \\
\text { size }\end{array}$ & $\%$ & SE & $95 \% \mathrm{Cl}$ \\
\hline $\begin{array}{l}\text { Tampa-St. Petersburg-Clearwater, } \\
\text { Florida }\end{array}$ & 810 & 20.3 & 2.1 & $(16.2-24.3)$ \\
\hline Toledo, Ohio & 947 & 22.5 & 2.4 & $(17.8-27.1)$ \\
\hline Topeka, Kansas & 1,078 & 21.1 & 1.8 & $(17.6-24.5)$ \\
\hline Torrington, Connecticut & 650 & 16.6 & 1.9 & $(12.8-20.4)$ \\
\hline Trenton, New Jersey & 558 & 15.0 & 2.0 & $(11.0-19.0)$ \\
\hline Tucson, Arizona & 977 & 16.4 & 1.6 & $(13.2-19.6)$ \\
\hline Tulsa, Oklahoma & 1,728 & 21.9 & 1.4 & $(19.2-24.5)$ \\
\hline Tuscaloosa, Alabama & 576 & 23.9 & 3.3 & $(17.5-30.3)$ \\
\hline Urban Honolulu, Hawaii & 4,289 & 14.0 & 0.8 & $(12.4-15.7)$ \\
\hline Vineland-Bridgeton, New Jersey & 536 & 25.6 & 3.1 & $(19.6-31.6)$ \\
\hline $\begin{array}{l}\text { Virginia Beach-Norfolk-Newport News, } \\
\text { Virginia-North Carolina }\end{array}$ & 1,470 & 20.6 & 1.5 & $(17.7-23.6)$ \\
\hline $\begin{array}{l}\text { Warren-Troy-Farmington Hills, } \\
\text { Michigan }{ }^{\dagger}\end{array}$ & 2,161 & 23.3 & 1.3 & $(20.9-25.8)$ \\
\hline $\begin{array}{l}\text { Washington-Arlington-Alexandria, } \\
\text { District of Columbia-Virginia- } \\
\text { Maryland-West Virginia }{ }^{\dagger}\end{array}$ & 7,958 & 14.6 & 0.7 & $(13.2-16.0)$ \\
\hline Wichita, Kansas & 2,332 & 18.7 & 1.1 & $(16.5-20.9)$ \\
\hline $\begin{array}{l}\text { Wilmington, Delaware-Maryland- } \\
\text { New Jersey }{ }^{\dagger}\end{array}$ & 3,197 & 19.8 & 1.1 & $(17.5-22.0)$ \\
\hline Winston-Salem, North Carolina & 789 & 25.4 & 2.2 & $(21.1-29.7)$ \\
\hline Worcester, Massachusetts-Connecticut & 2,716 & 17.6 & 1.1 & $(15.4-19.8)$ \\
\hline Yakima, Washington & 530 & 20.1 & 2.7 & $(14.8-25.3)$ \\
\hline $\begin{array}{l}\text { Youngstown-Warren-Boardman, } \\
\text { Ohio-Pennsylvania }\end{array}$ & 904 & 26.4 & 2.4 & $(21.6-31.2)$ \\
\hline Median & & 19.5 & & \\
\hline Range & & $5.1-30.1$ & & \\
\hline
\end{tabular}

Abbreviations: $\mathrm{Cl}=$ confidence interval; $\mathrm{MMSA}=$ metropolitan and micropolitan statistical area; $\mathrm{SE}=$ standard error.

* Smoked every day or someday during the period of the survey.

† Metropolitan division.

$\S$ Estimate not available (N/A) if the unweighted sample size for the denominator was $<50$ or if the relative standard error is $>0.3$. 
TABLE 33. Estimated prevalence of adults aged $\geq 18$ years who reported ever smoking at least 100 cigarettes and who currently smoke, ${ }^{*}$ by county - Behavioral Risk Factor Surveillance System, United States, 2012

\begin{tabular}{|c|c|c|c|c|}
\hline County & $\begin{array}{l}\text { Sample } \\
\text { size }\end{array}$ & $\%$ & SE & $95 \% \mathrm{Cl}$ \\
\hline Jefferson County, Alabama & 945 & 20.0 & 1.7 & $(16.6-23.3)$ \\
\hline Madison County, Alabama & 502 & 20.0 & 2.5 & $(15.1-24.8)$ \\
\hline Mobile County, Alabama & 808 & 25.7 & 2.6 & $(20.7-30.8)$ \\
\hline Anchorage Municipality, Alaska & 887 & 16.6 & 1.5 & $(13.7-19.6)$ \\
\hline Fairbanks North Star Borough, Alaska & 590 & 22.7 & 2.4 & $(18.0-27.4)$ \\
\hline Matanuska-Susitna Borough, Alaska & 594 & 22.7 & 2.3 & $(18.2-27.1)$ \\
\hline Maricopa County, Arizona & 2,062 & 16.7 & 1.1 & $(14.5-18.9)$ \\
\hline Pima County, Arizona & 977 & 16.4 & 1.6 & $(13.2-19.6)$ \\
\hline Pulaski County, Arkansas & 737 & 22.7 & 2.3 & $(18.2-27.3)$ \\
\hline Alameda County, California & 619 & 11.0 & 2.0 & $(7.1-14.9)$ \\
\hline Los Angeles County, California & 3,490 & 11.8 & 0.8 & $(10.3-13.4)$ \\
\hline Orange County, California & 1,036 & 10.0 & 1.4 & $(7.2-12.7)$ \\
\hline Riverside County, California & 823 & 14.8 & 1.8 & $(11.3-18.3)$ \\
\hline Sacramento County, California & 609 & 16.3 & 2.1 & $(12.2-20.4)$ \\
\hline San Bernardino County, California & 704 & 15.8 & 1.8 & $(12.2-19.3)$ \\
\hline San Diego County, California & 1,127 & 10.6 & 1.2 & $(8.2-12.9)$ \\
\hline Santa Clara County, California & 662 & 9.2 & 1.6 & $(6.2-12.3)$ \\
\hline Adams County, Colorado & 776 & 23.7 & 2.0 & $(19.9-27.5)$ \\
\hline Arapahoe County, Colorado & 824 & 15.6 & 1.6 & $(12.5-18.6)$ \\
\hline Boulder County, Colorado & 494 & 13.5 & 1.9 & $(9.8-17.3)$ \\
\hline Denver County, Colorado & 922 & 21.2 & 1.7 & $(17.9-24.5)$ \\
\hline Douglas County, Colorado & 519 & 10.3 & 1.9 & $(6.7-14.0)$ \\
\hline El Paso County, Colorado & 977 & 17.7 & 1.6 & $(14.6-20.8)$ \\
\hline Jefferson County, Colorado & 1,071 & 15.5 & 1.4 & $(12.7-18.2)$ \\
\hline Larimer County, Colorado & 570 & 15.7 & 1.9 & (11.9-19.5) \\
\hline Weld County, Colorado & 514 & 20.6 & 2.2 & $(16.3-24.8)$ \\
\hline Fairfield County, Connecticut & 2,135 & 13.2 & 1.2 & $(10.9-15.5)$ \\
\hline Hartford County, Connecticut & 1,963 & 15.7 & 1.1 & $(13.5-17.9)$ \\
\hline Litchfield County, Connecticut & 650 & 16.6 & 1.9 & $(12.8-20.4)$ \\
\hline New Haven County, Connecticut & 1,973 & 18.3 & 1.3 & $(15.8-20.8)$ \\
\hline New London County, Connecticut & 980 & 18.2 & 1.9 & $(14.5-22.0)$ \\
\hline Kent County, Delaware & 1,432 & 21.1 & 1.7 & $(17.9-24.4)$ \\
\hline New Castle County, Delaware & 2,321 & 18.6 & 1.1 & $(16.4-20.7)$ \\
\hline Sussex County, Delaware & 1,393 & 22.1 & 1.7 & $(18.8-25.4)$ \\
\hline $\begin{array}{l}\text { District of Columbia, } \\
\text { District of Columbia }\end{array}$ & 3,720 & 19.6 & 1.2 & $(17.3-21.9)$ \\
\hline Broward County, Florida & 517 & 12.9 & 2.1 & $(8.8-17.0)$ \\
\hline Miami-Dade County, Florida & 791 & 12.7 & 1.9 & $(9.0-16.3)$ \\
\hline Hawaii County, Hawaii & 1,340 & 16.3 & 1.4 & $(13.5-19.2)$ \\
\hline Honolulu County, Hawaii & 4,289 & 14.0 & 0.8 & $(12.4-15.7)$ \\
\hline Kauai County, Hawaii & 659 & 17.8 & 2.8 & $(12.2-23.4)$ \\
\hline Maui County, Hawaii & 1,207 & 14.0 & 1.4 & $(11.2-16.8)$ \\
\hline Ada County, Idaho & 793 & 17.6 & 2.3 & $(13.2-22.1)$ \\
\hline Canyon County, Idaho & 494 & 15.6 & 2.6 & $(10.6-20.6)$ \\
\hline Cook County, Illinois & 1,487 & 17.0 & 1.5 & $(14.2-19.9)$ \\
\hline Lake County, Indiana & 874 & 25.8 & 2.6 & $(20.8-30.8)$ \\
\hline Marion County, Indiana & 1,261 & 22.7 & 1.6 & $(19.5-25.9)$ \\
\hline Polk County, lowa & 795 & 18.9 & 1.7 & $(15.5-22.3)$ \\
\hline Johnson County, Kansas & 2,159 & 15.0 & 1.1 & $(12.9-17.1)$ \\
\hline Sedgwick County, Kansas & 1,776 & 19.3 & 1.3 & $(16.8-21.8)$ \\
\hline Shawnee County, Kansas & 765 & 20.7 & 2.2 & $(16.4-24.9)$ \\
\hline Wyandotte County, Kansas & 869 & 19.9 & 2.3 & $(15.4-24.3)$ \\
\hline Jefferson County, Kentucky & 1,616 & 25.1 & 2.0 & $(21.3-28.9)$ \\
\hline East Baton Rouge Parish, Louisiana & 567 & 18.2 & 2.3 & $(13.7-22.7)$ \\
\hline Androscoggin County, Maine & 696 & 23.7 & 2.1 & $(19.5-27.8)$ \\
\hline Aroostook County, Maine & 531 & 24.9 & 2.4 & $(20.3-29.5)$ \\
\hline Cumberland County, Maine & 1,735 & 16.6 & 1.2 & $(14.3-19.0)$ \\
\hline Kennebec County, Maine & 825 & 20.4 & 1.8 & $(17.0-23.8)$ \\
\hline Penobscot County, Maine & 920 & 22.2 & 1.9 & $(18.5-26.0)$ \\
\hline York County, Maine & 1,180 & 18.1 & 1.5 & $(15.3-21.0)$ \\
\hline
\end{tabular}

See table footnotes on page 70 .
TABLE 33. (Continued) Estimated prevalence of adults aged $\geq 18$ years who reported ever smoking at least 100 cigarettes and who currently smoke, ${ }^{*}$ by county - Behavioral Risk Factor Surveillance System, United States, 2012

\begin{tabular}{|c|c|c|c|c|}
\hline County & $\begin{array}{l}\text { Sample } \\
\text { size }\end{array}$ & $\%$ & SE & $95 \% \mathrm{Cl}$ \\
\hline Anne Arundel County, Maryland & 880 & 18.0 & 2.1 & $(13.9-22.1)$ \\
\hline Baltimore County, Maryland & 1,483 & 18.6 & 1.6 & $(15.4-21.8)$ \\
\hline Charles County, Maryland & 497 & 18.7 & 3.5 & $(11.8-25.5)$ \\
\hline Frederick County, Maryland & 738 & 15.7 & 2.7 & $(10.5-21.0)$ \\
\hline Montgomery County, Maryland & 1,482 & 7.4 & 1.0 & $(5.5-9.3)$ \\
\hline Prince George's County, Maryland & 1,110 & 14.3 & 1.9 & $(10.6-17.9)$ \\
\hline Washington County, Maryland & 529 & 18.9 & 3.7 & $(11.6-26.1)$ \\
\hline Baltimore city, Maryland & 723 & 21.8 & 2.6 & $(16.7-26.9)$ \\
\hline Barnstable County, Massachusetts & 534 & 15.0 & 1.9 & $(11.1-18.8)$ \\
\hline Bristol County, Massachusetts & 2,541 & 20.9 & 1.5 & $(17.9-23.9)$ \\
\hline Essex County, Massachusetts & 2,464 & 15.4 & 1.2 & $(13.0-17.8)$ \\
\hline Hampden County, Massachusetts & 1,885 & 21.5 & 1.6 & $(18.3-24.6)$ \\
\hline Middlesex County, Massachusetts & 4,117 & 12.7 & 0.8 & $(11.2-14.2)$ \\
\hline Norfolk County, Massachusetts & 1,686 & 13.1 & 1.3 & $(10.5-15.6)$ \\
\hline Plymouth County, Massachusetts & 1,742 & 18.6 & 1.6 & $(15.5-21.7)$ \\
\hline Suffolk County, Massachusetts & 2,199 & 13.4 & 1.0 & $(11.4-15.4)$ \\
\hline Worcester County, Massachusetts & 2,477 & 17.3 & 1.2 & $(15.0-19.6)$ \\
\hline Kent County, Michigan & 521 & 18.7 & 2.4 & $(14.0-23.3)$ \\
\hline Macomb County, Michigan & 608 & 26.0 & 2.3 & $(21.6-30.4)$ \\
\hline Oakland County, Michigan & 1,154 & 20.2 & 1.8 & $(16.7-23.6)$ \\
\hline Wayne County, Michigan & 2,177 & 24.4 & 1.6 & $(21.3-27.4)$ \\
\hline Anoka County, Minnesota & 532 & 16.9 & 2.0 & $(13.1-20.8)$ \\
\hline Dakota County, Minnesota & 660 & 17.7 & 1.8 & $(14.2-21.1)$ \\
\hline Hennepin County, Minnesota & 3,171 & 16.4 & 1.0 & $(14.4-18.4)$ \\
\hline Ramsey County, Minnesota & 1,890 & 22.2 & 2.1 & $(18.1-26.3)$ \\
\hline Jackson County, Missouri & 864 & 24.2 & 2.4 & $(19.5-29.0)$ \\
\hline St. Louis County, Missouri & 942 & 16.4 & 1.6 & $(13.2-19.6)$ \\
\hline Cascade County, Montana & 703 & 22.1 & 2.2 & $(17.8-26.4)$ \\
\hline Flathead County, Montana & 554 & 17.0 & 1.9 & $(13.2-20.9)$ \\
\hline Hill County, Montana & 575 & 24.3 & 2.9 & $(18.5-30.0)$ \\
\hline Lake County, Montana & 882 & 23.8 & 2.6 & $(18.7-28.9)$ \\
\hline Missoula County, Montana & 774 & 20.0 & 1.9 & $(16.4-23.7)$ \\
\hline Yellowstone County, Montana & 743 & 20.1 & 1.7 & $(16.8-23.4)$ \\
\hline Dakota County, Nebraska & 728 & 16.8 & 3.3 & $(10.4-23.3)$ \\
\hline Douglas County, Nebraska & 3,529 & 20.6 & 0.9 & $(18.8-22.4)$ \\
\hline Hall County, Nebraska & 529 & 18.7 & 2.2 & $(14.3-23.1)$ \\
\hline Lancaster County, Nebraska & 1,431 & 21.5 & 1.3 & $(19.0-24.0)$ \\
\hline Lincoln County, Nebraska & 585 & 16.6 & 2.4 & $(12.0-21.3)$ \\
\hline Sarpy County, Nebraska & 1,164 & 19.3 & 1.6 & $(16.1-22.4)$ \\
\hline Scotts Bluff County, Nebraska & 574 & 20.9 & 3.0 & $(15.1-26.8)$ \\
\hline Clark County, Nevada & 1,983 & 17.0 & 1.0 & $(15.0-19.0)$ \\
\hline Washoe County, Nevada & 1,471 & 17.6 & 1.6 & $(14.4-20.7)$ \\
\hline Belknap County, New Hampshire & 555 & 21.1 & 3.2 & $(14.8-27.3)$ \\
\hline Carroll County, New Hampshire & 524 & 20.3 & 3.5 & $(13.5-27.2)$ \\
\hline Cheshire County, New Hampshire & 540 & 18.5 & 2.9 & $(12.8-24.1)$ \\
\hline Coos County, New Hampshire & 534 & 19.8 & 3.1 & $(13.6-26.0)$ \\
\hline Grafton County, New Hampshire & 559 & 17.0 & 2.8 & $(11.5-22.5)$ \\
\hline Hillsborough County, New Hampshire & 1,882 & 17.7 & 1.2 & $(15.2-20.1)$ \\
\hline Merrimack County, New Hampshire & 696 & 14.9 & 2.1 & $(10.8-19.0)$ \\
\hline Rockingham County, New Hampshire & 1,032 & 15.6 & 1.7 & $(12.2-18.9)$ \\
\hline Strafford County, New Hampshire & 613 & 18.7 & 2.6 & $(13.6-23.7)$ \\
\hline Atlantic County, New Jersey & 983 & 22.0 & 2.0 & $(18.1-25.9)$ \\
\hline Bergen County, New Jersey & 897 & 17.4 & 1.9 & $(13.6-21.2)$ \\
\hline Burlington County, New Jersey & 662 & 17.9 & 2.2 & $(13.5-22.3)$ \\
\hline Camden County, New Jersey & 720 & 19.3 & 2.0 & $(15.3-23.3)$ \\
\hline Cape May County, New Jersey & 530 & 23.6 & 3.7 & $(16.3-30.8)$ \\
\hline Cumberland County, New Jersey & 536 & 25.6 & 3.1 & $(19.6-31.6)$ \\
\hline Essex County, New Jersey & 1,297 & 17.0 & 1.6 & $(14.0-20.1)$ \\
\hline Gloucester County, New Jersey & 539 & 19.6 & 3.1 & $(13.6-25.5)$ \\
\hline Hudson County, New Jersey & 1,221 & 16.2 & 1.5 & $(13.1-19.2)$ \\
\hline
\end{tabular}

See table footnotes on page 70 . 
TABLE 33. (Continued) Estimated prevalence of adults aged $\geq 18$ years who reported ever smoking at least 100 cigarettes and who currently smoke,* by county - Behavioral Risk Factor Surveillance System, United States, 2012

\begin{tabular}{|c|c|c|c|c|}
\hline County & $\begin{array}{l}\text { Sample } \\
\text { size }\end{array}$ & $\%$ & SE & $95 \% \mathrm{Cl}$ \\
\hline Hunterdon County, New Jersey & 544 & 15.1 & 2.7 & $(9.8-20.5)$ \\
\hline Mercer County, New Jersey & 558 & 15.0 & 2.0 & $(11.0-19.0)$ \\
\hline Middlesex County, New Jersey & 853 & 14.6 & 1.7 & $(11.3-17.9)$ \\
\hline Monmouth County, New Jersey & 681 & 18.8 & 2.2 & $(14.4-23.1)$ \\
\hline Morris County, New Jersey & 817 & 16.1 & 2.0 & $(12.3-20.0)$ \\
\hline Ocean County, New Jersey & 631 & 20.0 & 2.2 & $(15.6-24.4)$ \\
\hline Passaic County, New Jersey & 649 & 16.9 & 1.9 & $(13.1-20.6)$ \\
\hline Salem County, New Jersey & 568 & 25.9 & 4.1 & $(17.8-34.0)$ \\
\hline Somerset County, New Jersey & 609 & 7.6 & 1.4 & $(4.8-10.4)$ \\
\hline Sussex County, New Jersey & 529 & 20.4 & 3.0 & $(14.5-26.2)$ \\
\hline Union County, New Jersey & 675 & 16.9 & 1.9 & $(13.3-20.6)$ \\
\hline Warren County, New Jersey & 510 & 18.6 & 2.4 & $(13.8-23.4)$ \\
\hline Bernalillo County, New Mexico & 2,022 & 18.6 & 1.1 & $(16.5-20.7)$ \\
\hline Dona Ana County, New Mexico & 697 & 18.1 & 2.0 & $(14.1-22.0)$ \\
\hline Sandoval County, New Mexico & 637 & 15.5 & 2.0 & $(11.5-$ \\
\hline San Juan County, New Mexico & 636 & 21.0 & 2.1 & $(17.0-25.1)$ \\
\hline Santa Fe County, New Mexico & 689 & 16.8 & 1.9 & $(13.1-20.5)$ \\
\hline Kings County, New York & 483 & 15.1 & 2.2 & $(10.7-19.5)$ \\
\hline Guilford County, North Carolina & 505 & 17.0 & 2.1 & $(12.9-21.2)$ \\
\hline Mecklenburg County, North Carolina & 721 & 15.1 & 1.7 & $(11.8-18.4)$ \\
\hline Robeson County, North Carolina & 532 & 24.1 & 3.8 & $(16.7-31.5)$ \\
\hline Wake County, North Carolina & 690 & 15.1 & 1.6 & $(12.0-18.2)$ \\
\hline Burleigh County, North Dakota & 563 & 13.8 & 2.1 & $(9.7-17.9)$ \\
\hline Cass County, North Dakota & 832 & 19.4 & 2.1 & $(15.3-23.5)$ \\
\hline Cuyahoga County, Ohio & 805 & 22.4 & 1.9 & $(18.6-26.2)$ \\
\hline Franklin County, Ohio & 815 & 20.2 & 1.8 & $(16.8-23.7)$ \\
\hline Hamilton County, Ohio & 750 & 19.4 & 1.8 & $(15.8-23.0)$ \\
\hline Lorain County, Ohio & 594 & 28.3 & 3.7 & $(20.9-35.6)$ \\
\hline Lucas County, Ohio & 606 & 25.7 & 3.1 & $(19.6-31.8)$ \\
\hline Mahoning County, Ohio & 572 & 27.2 & 3.2 & $(20.9-33.5)$ \\
\hline Montgomery County, Ohio & 648 & 25.4 & 2.5 & $(20.5-30.2)$ \\
\hline Stark County, Ohio & 609 & 27.1 & 2.7 & $(21.8-32.4)$ \\
\hline Summit County, Ohio & 613 & 22.4 & 2.5 & $(17.4-27.4)$ \\
\hline Oklahoma County, Oklahoma & 1,181 & 20.7 & 1.4 & $(17.9-23.4)$ \\
\hline Tulsa County, Oklahoma & 1,192 & 19.3 & 1.5 & $(16.3-22.2)$ \\
\hline Clackamas County, Oregon & 501 & 15.0 & 2.5 & $(10.2-19.9)$ \\
\hline Lane County, Oregon & 516 & 17.1 & 2.3 & $(12.5-21.7)$ \\
\hline Multnomah County, Oregon & 873 & 21.3 & 2.1 & $(17.2-25.3)$ \\
\hline Washington County, Oregon & 607 & 10.6 & 1.5 & $(7.6-13.6)$ \\
\hline Allegheny County, Pennsylvania & 1,856 & 21.9 & 1.3 & $(19.3-24.4)$ \\
\hline Bradford County, Pennsylvania & 1,829 & 23.7 & 2.8 & $(18.2-29.2)$ \\
\hline Montgomery County, Pennsylvania & 538 & 15.5 & 2.2 & $(11.3-19.8)$ \\
\hline Philadelphia County, Pennsylvania & 2,012 & 23.8 & 1.3 & $(21.1-26.4)$ \\
\hline Pike County, Pennsylvania & 1,867 & 20.3 & 1.6 & $(17.2-23.4)$ \\
\hline Kent County, Rhode Island & 802 & 16.1 & 1.8 & $(12.6-19.6)$ \\
\hline Providence County, Rhode Is & 3,282 & 19.3 & 1.0 & $(17.4-21.3)$ \\
\hline Washington County, Rhode Island & 649 & 15.0 & 2.4 & $(10.4-19.7)$ \\
\hline Aiken County, South Carolina & 546 & 22.5 & 3.1 & $(16.4-28.6)$ \\
\hline
\end{tabular}

TABLE 33. (Continued) Estimated prevalence of adults aged $\geq 18$ years who reported ever smoking at least 100 cigarettes and who currently smoke, ${ }^{*}$ by county - Behavioral Risk Factor Surveillance System, United States, 2012

\begin{tabular}{|c|c|c|c|c|}
\hline County & $\begin{array}{l}\text { Sample } \\
\text { size }\end{array}$ & $\%$ & SE & $95 \% \mathrm{Cl}$ \\
\hline Beaufort County, South Carolina & 784 & 18.5 & 2.8 & $(13.0-23.9$ \\
\hline Charleston County, South Carolina & 998 & 20.8 & 2.1 & $(16.8-24.8$ \\
\hline Greenville County, South Carolina & 885 & 22.2 & 2.0 & $(18.3-26.2$ \\
\hline Horry County, South Carolina & 768 & 23.9 & 2.0 & $(20.0-27.8$ \\
\hline Richland County, South Carolina & 945 & 20.3 & 2.1 & $(16.2-24.4$ \\
\hline Spartanburg County, South Carolina & 645 & 21.9 & 2.3 & $(17.4-26.3$ \\
\hline Lincoln County, South Dakota & 495 & 14.4 & 2.0 & $(10.4-18.4$ \\
\hline Minnehaha County, South Dakota & 871 & 22.4 & 1.8 & $(18.9-25.8$ \\
\hline Pennington County, South Dakota & 604 & 24.3 & 2.1 & $(20.2-28.4$ \\
\hline Davidson County, Tennessee & 533 & 17.2 & 2.1 & $(13.2-21.3$ \\
\hline Shelby County, Tennessee & 513 & 21.1 & 2.3 & $(16.5-25.6)$ \\
\hline Bexar County, Texas & 575 & 17.3 & 2.3 & $(12.8-21.8$ \\
\hline Dallas County, Texas & 496 & 16.2 & 2.0 & $(12.4-20.1)$ \\
\hline El Paso County, Texas & 615 & 15.1 & 2.3 & $(10.5-19.7)$ \\
\hline Harris County, Texas & 751 & 17.3 & 1.7 & $(13.9-20.7$ \\
\hline Hidalgo County, Texas & 614 & 14.1 & 2.6 & $(9.0-19.3$ \\
\hline Tarrant County, Texas & 563 & 20.0 & 2.3 & $(15.5-24.5)$ \\
\hline Travis County, Texas & 1,030 & 15.8 & 2.1 & $(11.6-20.0$ \\
\hline Davis County, Utah & 1,127 & 7.8 & 1.1 & $(5.6-10.1)$ \\
\hline Salt Lake County, Utah & 3,983 & 12.7 & 0.7 & $(11.2-14.1)$ \\
\hline Tooele County, Utah & 553 & 15.9 & 3.0 & $(10.1-21.8$ \\
\hline Utah County, Utah & 1,649 & 5.1 & 0.7 & $(3.7-6.5)$ \\
\hline Wasatch County, Utah & 501 & $\mathrm{~N} / \mathrm{A}^{+}$ & $\mathrm{N} / \mathrm{A}$ & $(\mathrm{N} / \mathrm{A}-\mathrm{N} / \mathrm{A})$ \\
\hline Weber County, Utah & 1,031 & 14.4 & 1.5 & $(11.5-17.2)$ \\
\hline Chittenden County, Vermont & 903 & 13.3 & 1.4 & $(10.6-16.1)$ \\
\hline Rutland County, Vermont & 585 & 17.7 & 2.4 & $(13.1-22.4$ \\
\hline Washington County, Vermont & 507 & 14.3 & 2.3 & $(9.8-18.8$ \\
\hline Windsor County, Vermont & 533 & 15.9 & 2.2 & $(11.5-20.3)$ \\
\hline Fairfax County, Virginia & 698 & 11.0 & 1.6 & $(7.9-14.1)$ \\
\hline Clark County, Washington & 785 & 15.7 & 1.9 & $(12.1-19.3$ \\
\hline King County, Washington & 3,895 & 13.6 & 0.8 & $(12.0-15.2$ \\
\hline Kitsap County, Washington & 561 & 19.9 & 2.3 & $(15.3-24.5)$ \\
\hline Pierce County, Washington & 1,174 & 20.8 & 1.6 & $(17.6-24.1)$ \\
\hline Snohomish County, Washington & 1,158 & 19.2 & 1.6 & $(16.0-22.3)$ \\
\hline Spokane County, Washington & 942 & 17.9 & 1.8 & $(14.4-21.4)$ \\
\hline Thurston County, Washington & 514 & 23.3 & 2.9 & $(17.7-28.9)$ \\
\hline Whatcom County, Washington & 835 & 18.4 & 2.6 & $(13.2-23.6)$ \\
\hline Yakima County, Washington & 530 & 20.1 & 2.7 & $(14.8-25.3)$ \\
\hline Kanawha County, West Virginia & 640 & 25.6 & 2.1 & $(21.5-29.7)$ \\
\hline Milwaukee County, Wisconsin & 913 & 23.6 & 2.3 & $(19.2-28.1)$ \\
\hline Laramie County, Wyoming & 938 & 20.1 & 2.3 & $(15.6-24.7)$ \\
\hline Natrona County, Wyoming & 815 & 25.3 & 2.6 & $(20.2-30.4)$ \\
\hline San Juan Municipio, Puerto Rico & 665 & 13.2 & 1.6 & $(10.0-16.4)$ \\
\hline Median & & 18.3 & & \\
\hline Range & & $5.1-28.3$ & & \\
\hline
\end{tabular}

Abbreviations: $\mathrm{Cl}=$ confidence interval; $\mathrm{SE}=$ standard error.

* Smoked everyday or someday during the period of the survey.

† Estimate not available (N/A) if the unweighted sample size for the denominator was $<50$ or if the relative standard error is $>0.3$. 
TABLE 34. Estimated prevalence of adults aged $\geq 18$ years who reported binge drinking* during the preceding month, by state/ territory - Behavioral Risk Factor Surveillance System, United States, 2012

\begin{tabular}{|c|c|c|c|c|}
\hline State/Territory & $\begin{array}{l}\text { Sample } \\
\text { size }\end{array}$ & $\%$ & SE & $95 \% \mathrm{Cl}$ \\
\hline Alabama & 8,662 & 12.3 & 0.6 & $(11.1-13.4)$ \\
\hline Alaska & 4,172 & 16.8 & 0.8 & $(15.2-18.3)$ \\
\hline Arizona & 7,000 & 15.3 & 0.8 & $(13.8-16.8)$ \\
\hline Arkansas & 5,020 & 11.8 & 0.7 & $(10.5-13.2)$ \\
\hline California & 12,552 & 16.9 & 0.5 & $(15.9-17.9)$ \\
\hline Colorado & 11,519 & 19.2 & 0.5 & $(18.2-20.2)$ \\
\hline Connecticut & 8,458 & 17.5 & 0.6 & $(16.3-18.7)$ \\
\hline Delaware & 5,094 & 18.6 & 0.8 & $(17.0-20.2)$ \\
\hline District of Columbia & 3,642 & 23.1 & 1.3 & $(20.6-25.6)$ \\
\hline Florida & 7,329 & 16.5 & 0.7 & $(15.1-18.0)$ \\
\hline Georgia & 5,821 & 14.4 & 0.7 & $(13.0-15.8)$ \\
\hline Hawaii & 7,394 & 18.2 & 0.7 & $(16.9-19.5)$ \\
\hline Idaho & 5,725 & 14.1 & 0.9 & $(12.4-15.9)$ \\
\hline Illinois & 5,477 & 21.6 & 0.9 & $(19.9-23.3)$ \\
\hline Indiana & 8,397 & 15.9 & 0.6 & $(14.8-17.0)$ \\
\hline lowa & 6,926 & 21.7 & 0.7 & $(20.4-23.0)$ \\
\hline Kansas & 11,586 & 15.8 & 0.5 & $(14.8-16.8)$ \\
\hline Kentucky & 10,723 & 14.9 & 0.6 & $(13.7-16.0)$ \\
\hline Louisiana & 8,818 & 16.5 & 0.7 & $(15.1-18.0)$ \\
\hline Maine & 9,707 & 17.7 & 0.5 & $(16.7-18.7)$ \\
\hline Maryland & 12,240 & 16.4 & 0.6 & $(15.2-17.6)$ \\
\hline Massachusetts & 20,391 & 19.7 & 0.5 & $(18.8-20.6)$ \\
\hline Michigan & 10,223 & 19.2 & 0.6 & $(18.0-20.3)$ \\
\hline Minnesota & 11,719 & 21.9 & 0.5 & $(20.9-22.9)$ \\
\hline Mississippi & 7,566 & 12.1 & 0.6 & $(10.9-13.4)$ \\
\hline Missouri & 6,574 & 17.3 & 0.7 & $(15.8-18.7)$ \\
\hline Montana & 8,457 & 21.8 & 0.6 & $(20.5-23.0)$ \\
\hline Nebraska & 18,742 & 22.1 & 0.5 & $(21.2-23.0)$ \\
\hline Nevada & 4,657 & 15.1 & 0.8 & $(13.5-16.6)$ \\
\hline New Hampshire & 7,291 & 17.0 & 0.7 & $(15.7-18.4)$ \\
\hline New Jersey & 14,719 & 17.7 & 0.5 & $(16.7-18.7)$ \\
\hline New Mexico & 8,370 & 14.6 & 0.5 & $(13.5-15.6)$ \\
\hline New York & 5,808 & 17.0 & 0.7 & $(15.6-18.3)$ \\
\hline North Carolina & 11,351 & 13.1 & 0.5 & $(12.2-14.0)$ \\
\hline North Dakota & 4,750 & 24.1 & 0.9 & $(22.3-25.8)$ \\
\hline Ohio & 12,444 & 18.0 & 0.5 & $(17.0-19.0)$ \\
\hline Oklahoma & 7,870 & 14.4 & 0.5 & $(13.3-15.4)$ \\
\hline Oregon & 5,091 & 15.3 & 0.7 & (13.9-16.8) \\
\hline Pennsylvania & 19,270 & 17.9 & 0.4 & $(17.0-18.7)$ \\
\hline Rhode Island & 5,278 & 17.2 & 0.8 & $(15.7-18.7)$ \\
\hline South Carolina & 12,344 & 15.4 & 0.5 & $(14.3-16.4)$ \\
\hline South Dakota & 7,654 & 20.6 & 0.7 & $(19.2-22.0)$ \\
\hline Tennessee & 6,624 & 11.3 & 0.6 & $(10.0-12.5)$ \\
\hline Texas & 8,797 & 16.2 & 0.6 & $(15.1-17.4)$ \\
\hline Utah & 12,103 & 11.2 & 0.4 & $(10.3-12.0)$ \\
\hline Vermont & 5,894 & 19.3 & 0.7 & (17.9-20.8) \\
\hline Virginia & 7,045 & 15.7 & 0.6 & $(14.5-16.8)$ \\
\hline Washington & 14,992 & 16.9 & 0.4 & (16.1-17.8) \\
\hline West Virginia & 5,316 & 10.2 & 0.5 & $(9.1-11.2)$ \\
\hline Wisconsin & 5,041 & 25.2 & 0.9 & $(23.4-27.0)$ \\
\hline Wyoming & 6,062 & 17.0 & 0.9 & $(15.2-18.8)$ \\
\hline Guam & 1,927 & 19.4 & 1.3 & (16.9-21.8) \\
\hline Puerto Rico & 6,161 & 14.2 & 0.6 & $(13.0-15.3)$ \\
\hline Median & & 16.9 & & \\
\hline Range & & $10.2-25.2$ & & \\
\hline
\end{tabular}

Abbreviations: $\mathrm{Cl}=$ confidence interval; $\mathrm{SE}=$ standard error.

* For males: having at least five drinks on at least one occasion; for females: having at least four drinks on at least one occasion.
TABLE 35. Estimated prevalence of adults aged $\geq 18$ years who reported binge drinking* during the preceding month, by metropolitan and micropolitan statistical area - Behavioral Risk Factor Surveillance System, United States, 2012

\begin{tabular}{|c|c|c|c|c|}
\hline MMSA(s) & $\begin{array}{l}\text { Sample } \\
\text { size }\end{array}$ & $\%$ & SE & $95 \% \mathrm{Cl}$ \\
\hline Aguadilla-Isabela, Puerto Rico & 539 & 10.8 & 1.6 & $(7.7-14.0)$ \\
\hline Akron, Ohio & 709 & 17.8 & 2.0 & $(13.9-21.8)$ \\
\hline Albuquerque, New Mexico & 3,115 & 14.1 & 0.8 & $(12.5-15.6)$ \\
\hline $\begin{array}{l}\text { Allentown-Bethlehem-Easton, } \\
\text { Pennsylvania-New Jersey }\end{array}$ & 1,299 & 14.9 & 1.6 & $(11.8-18.0)$ \\
\hline Anaheim-Santa Ana-Irvine, California ${ }^{\dagger}$ & 892 & 19.5 & 2.1 & $(15.4-23.6)$ \\
\hline Anchorage, Alaska & 1,456 & 17.2 & 1.2 & $(14.8-19.6)$ \\
\hline Asheville, North Carolina & 565 & 12.8 & 2.1 & $(8.8-16.9)$ \\
\hline $\begin{array}{l}\text { Atlanta-Sandy Springs-Roswell, } \\
\text { Georgia }\end{array}$ & 2,430 & 14.6 & 1.0 & $(12.6-16.6)$ \\
\hline Atlantic City-Hammonton, New Jersey & 959 & 19.3 & 2.0 & $(15.3-23.3)$ \\
\hline $\begin{array}{l}\text { Augusta-Richmond County, Georgia- } \\
\text { South Carolina }\end{array}$ & 991 & 15.1 & 2.4 & $(10.5-19.8)$ \\
\hline Augusta-Waterville, Maine & 817 & 16.6 & 1.7 & $(13.3-19.8)$ \\
\hline Austin-Round Rock, Texas & 1,350 & 17.6 & 1.7 & $(14.3-20.9)$ \\
\hline $\begin{array}{l}\text { Baltimore-Columbia-Towson, } \\
\text { Maryland }\end{array}$ & 4,524 & 17.7 & 0.9 & $(15.8-19.5)$ \\
\hline Bangor, Maine & 907 & 16.1 & 1.6 & $(12.9-19.2)$ \\
\hline Barnstable Town, Massachusetts & 527 & 14.6 & 2.0 & $(10.6-18.5)$ \\
\hline Barre, Vermont & 509 & 16.8 & 2.1 & $(12.6-21.0)$ \\
\hline Baton Rouge, Louisiana & 1,345 & 17.4 & 1.9 & $(13.8-21.1)$ \\
\hline Bellingham, Washington & 833 & 19.7 & 2.5 & $(14.7-24.6)$ \\
\hline Berlin, New Hampshire-Vermont & 683 & 17.8 & 2.6 & $(12.7-22.9)$ \\
\hline Billings, Montana & 831 & 22.5 & 1.7 & $(19.2-25.9)$ \\
\hline Birmingham-Hoover, Alabama & 1,740 & 11.7 & 1.1 & $(9.5-14.0)$ \\
\hline Bismarck, North Dakota & 806 & 22.4 & 2.1 & $(18.2-26.5)$ \\
\hline Boise City, Idaho & 1,431 & 15.1 & 1.6 & $(12.1-18.2)$ \\
\hline Boston, Massachusetts $^{\dagger}$ & 5,533 & 20.5 & 0.9 & $(18.8-22.2)$ \\
\hline Boulder, Colorado & 491 & 15.4 & 2.0 & $(11.5-19.4)$ \\
\hline Bremerton-Silverdale, Washington & 554 & 15.4 & 2.1 & $(11.2-19.6)$ \\
\hline $\begin{array}{l}\text { Bridgeport-Stamford-Norwalk, } \\
\text { Connecticut }\end{array}$ & 2,089 & 19.5 & 1.4 & $(16.7-22.2)$ \\
\hline Burlington-South Burlington, Vermont & 1,480 & 23.5 & 1.5 & $(20.6-26.4)$ \\
\hline $\begin{array}{l}\text { Cambridge-Newton-Framingham, } \\
\text { Massachusetts }^{\dagger}\end{array}$ & 6,490 & 19.0 & 0.8 & $(17.4-20.5)$ \\
\hline Camden, New Jersey ${ }^{\dagger}$ & 1,892 & 17.2 & 1.3 & $(14.7-19.8)$ \\
\hline Canton-Massillon, Ohio & 654 & 15.0 & 2.0 & $(11.1-19.0)$ \\
\hline Casper, Wyoming & 803 & 17.4 & 2.4 & $(12.7-22.1)$ \\
\hline Cedar Rapids, lowa & 568 & 17.2 & 2.1 & $(13.1-21.3)$ \\
\hline Charleston, West Virginia & 760 & 9.4 & 1.3 & $(6.9-12.0)$ \\
\hline $\begin{array}{l}\text { Charleston-North Charleston, } \\
\text { South Carolina }\end{array}$ & 1,655 & 21.1 & 1.5 & $(18.2-24.0)$ \\
\hline $\begin{array}{l}\text { Charlotte-Concord-Gastonia, } \\
\text { North Carolina-South Carolina }\end{array}$ & 2,401 & 13.1 & 0.9 & $(11.3-14.8)$ \\
\hline Chattanooga, Tennessee-Georgia & 589 & 11.4 & 2.1 & $(7.2-15.5)$ \\
\hline Cheyenne, Wyoming & 922 & 16.0 & 2.2 & $(11.7-20.3)$ \\
\hline $\begin{array}{l}\text { Chicago-Naperville-Elgin, } \\
\text { Illinois-Indiana-Wisconsin }\end{array}$ & 3,634 & 21.1 & 1.1 & $(19.0-23.3)$ \\
\hline Cincinnati, Ohio-Kentucky-Indiana & 2,272 & 19.4 & 1.1 & $(17.1-21.6)$ \\
\hline $\begin{array}{l}\text { Claremont-Lebanon, New } \\
\text { Hampshire-Vermont }\end{array}$ & 1,908 & 15.9 & 1.3 & $(13.4-18.4)$ \\
\hline Cleveland-Elyria, Ohio & 1,659 & 18.6 & 1.3 & $(16.0-21.2)$ \\
\hline Colorado Springs, Colorado & 1,097 & 12.8 & 1.3 & $(10.2-15.4)$ \\
\hline Columbia, South Carolina & 1,734 & 19.0 & 1.5 & $(16.2-21.9)$ \\
\hline Columbus, Ohio & 1,531 & 17.7 & 1.3 & $(15.2-20.1)$ \\
\hline Concord, New Hampshire & 685 & 12.8 & 1.8 & $(9.3-16.3)$ \\
\hline Dallas-Plano-Irving, Texas ${ }^{\dagger}$ & 871 & 16.0 & 1.6 & $(12.9-19.0)$ \\
\hline Dayton, Ohio & 824 & 18.4 & 1.9 & $(14.6-22.1)$ \\
\hline Denver-Aurora-Lakewood, Colorado & 4,562 & 21.6 & 0.8 & $(20.1-23.1)$ \\
\hline Des Moines-West Des Moines, lowa & 1,124 & 23.1 & 1.6 & $(19.9-26.3)$ \\
\hline
\end{tabular}

See table footnotes on page 73. 
TABLE 35. (Continued) Estimated prevalence of adults aged $\geq 18$ years who reported binge drinking* during the preceding month, by metropolitan and micropolitan statistical area - Behavioral Risk Factor Surveillance System, United States, 2012

\begin{tabular}{|c|c|c|c|c|}
\hline MMSA(s) & $\begin{array}{l}\text { Sample } \\
\text { size }\end{array}$ & $\%$ & SE & $95 \% \mathrm{Cl}$ \\
\hline 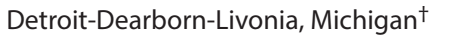 & 2,142 & 18.9 & 1.5 & $(16.0-21.7)$ \\
\hline Dover, Delaware & 1,426 & 15.1 & 1.6 & $(12.0-18.1)$ \\
\hline Duluth, Minnesota-Wisconsin & 501 & 23.1 & 2.7 & $(17.8-28.5)$ \\
\hline Durham-Chapel Hill, North Carolina & 761 & 16.8 & 1.8 & $(13.2-20.3)$ \\
\hline El Paso, Texas & 598 & 21.0 & 2.9 & $(15.3-26.8)$ \\
\hline Eugene, Oregon & 512 & 16.3 & 2.6 & $(11.3-21.3)$ \\
\hline Fairbanks, Alaska & 585 & 15.9 & 2.0 & $(12.0-19.8)$ \\
\hline Fargo, North Dakota-Minnesota & 917 & 28.1 & 2.3 & $(23.6-32.7)$ \\
\hline Farmington, New Mexico & 614 & 10.8 & 1.6 & $(7.8-13.9)$ \\
\hline Fayetteville, North Carolina & 490 & 11.7 & 2.0 & $(7.7-15.7)$ \\
\hline $\begin{array}{l}\text { Fayetteville-Springdale-Rogers, } \\
\text { Arkansas-Missouri }\end{array}$ & 786 & 12.6 & 1.8 & $(9.1-16.1)$ \\
\hline Fort Collins, Colorado & 565 & 18.0 & 2.0 & $(14.0-22.0)$ \\
\hline Fort Wayne, Indiana & 518 & 20.3 & 2.3 & $(15.8-24.8)$ \\
\hline Fort Worth-Arlington, Texas ${ }^{\dagger}$ & 707 & 17.5 & 2.0 & $(13.5-21.5)$ \\
\hline Grand Island, Nebraska & 840 & 18.6 & 1.9 & $(14.9-22.3)$ \\
\hline Grand Rapids-Wyoming, Michigan & 874 & 20.7 & 2.1 & $(16.5-24.8)$ \\
\hline Great Falls, Montana & 691 & 20.0 & 2.1 & $(15.8-24.2)$ \\
\hline Greeley, Colorado & 499 & 17.8 & 2.1 & $(13.6-22.0)$ \\
\hline $\begin{array}{l}\text { Greensboro-High Point, } \\
\text { North Carolina }\end{array}$ & 779 & 13.7 & 1.7 & $(10.4-16.9)$ \\
\hline $\begin{array}{l}\text { Greenville-Anderson-Mauldin, } \\
\text { South Carolina }\end{array}$ & 1,634 & 13.6 & 1.3 & $(11.1-16.1)$ \\
\hline Gulfport-Biloxi-Pascagoula, Mississippi & 723 & 14.9 & 1.9 & $(11.2-18.7)$ \\
\hline $\begin{array}{l}\text { Hagerstown-Martinsburg, Maryland- } \\
\text { West Virginia }\end{array}$ & 739 & 13.4 & 2.8 & $(7.9-18.9)$ \\
\hline Harrisburg-Carlisle, Pennsylvania & 651 & 17.6 & 2.0 & $(13.7-21.5)$ \\
\hline $\begin{array}{l}\text { Hartford-West Hartford-East Hartford, } \\
\text { Connecticut }\end{array}$ & 2,584 & 17.5 & 1.1 & $(15.4-19.6)$ \\
\hline Heber, Utah & 500 & $\mathrm{~N} / \mathrm{A}^{\S}$ & N/A & $(\mathrm{N} / \mathrm{A}-\mathrm{N} / \mathrm{A})$ \\
\hline Hilo, Hawaii & 1,317 & 21.6 & 1.7 & $(18.3-24.9)$ \\
\hline $\begin{array}{l}\text { Hilton Head Island-Bluffton-Beaufort, } \\
\text { South Carolina }\end{array}$ & 901 & 12.3 & 1.7 & $(8.9-15.7)$ \\
\hline $\begin{array}{l}\text { Houston-The Woodlands-Sugar Land, } \\
\text { Texas }\end{array}$ & 1,088 & 15.1 & 1.3 & $(12.6-17.7)$ \\
\hline $\begin{array}{l}\text { Huntington-Ashland, West } \\
\text { Virginia-Kentucky-Ohio }\end{array}$ & 1,089 & 13.6 & 1.6 & $(10.5-16.7)$ \\
\hline Huntsville, Alabama & 589 & 11.4 & 1.8 & $(7.9-14.8)$ \\
\hline Idaho Falls, Idaho & 527 & 9.1 & 2.4 & $(4.5-13.8)$ \\
\hline $\begin{array}{l}\text { Indianapolis-Carmel-Anderson, } \\
\text { Indiana }\end{array}$ & 2,127 & 15.8 & 1.0 & $(13.7-17.8)$ \\
\hline Jackson, Mississippi & 884 & 12.5 & 1.5 & $(9.6-15.5)$ \\
\hline Jacksonville, Florida & 506 & 19.3 & 3.1 & $(13.3-25.4)$ \\
\hline Kahului-Wailuku-Lahaina, Hawaii & 1,191 & 17.7 & 1.7 & $(14.3-21.0)$ \\
\hline Kalispell, Montana & 550 & 21.2 & 2.1 & $(17.0-25.4)$ \\
\hline Kansas City, Missouri-Kansas & 4,632 & 17.3 & 1.1 & $(15.1-19.5)$ \\
\hline Kapaa, Hawaii & 652 & 19.4 & 2.8 & $(13.9-24.8)$ \\
\hline Keene, New Hampshire & 535 & 17.2 & 2.8 & $(11.8-22.6)$ \\
\hline Kennewick-Richland, Washington & 525 & 17.0 & 2.3 & $(12.5-21.4)$ \\
\hline $\begin{array}{l}\text { Kingsport-Bristol-Bristol, } \\
\text { Tennessee-Virginia }\end{array}$ & 536 & N/A & $\mathrm{N} / \mathrm{A}$ & $(\mathrm{N} / \mathrm{A}-\mathrm{N} / \mathrm{A})$ \\
\hline Knoxville, Tennessee & 797 & 10.9 & 1.6 & $(7.9-14.0)$ \\
\hline Laconia, New Hampshire & 544 & 20.6 & 3.3 & $(14.1-27.1)$ \\
\hline Lafayette, Louisiana & 544 & 18.2 & 2.3 & $(13.6-22.8)$ \\
\hline Las Cruces, New Mexico & 682 & 20.0 & 2.3 & $(15.5-24.5)$ \\
\hline $\begin{array}{l}\text { Las Vegas-Henderson-Paradise, } \\
\text { Nevada }\end{array}$ & 1,928 & 13.8 & 1.0 & $(11.8-15.7)$ \\
\hline Lewiston-Auburn, Maine & 686 & 18.0 & 1.8 & $(14.4-21.6)$ \\
\hline Lexington-Fayette, Kentucky & 511 & 17.4 & 2.0 & $(13.5-21.3)$ \\
\hline Lincoln, Nebraska & 1,642 & 23.7 & 1.2 & $(21.3-26.2)$ \\
\hline
\end{tabular}

TABLE 35. (Continued) Estimated prevalence of adults aged $\geq 18$ years who reported binge drinking* during the preceding month, by metropolitan and micropolitan statistical area - Behavioral Risk Factor Surveillance System, United States, 2012

\begin{tabular}{|c|c|c|c|c|}
\hline MMSA(s) & $\begin{array}{l}\text { Sample } \\
\text { size }\end{array}$ & $\%$ & SE & $95 \% \mathrm{Cl}$ \\
\hline $\begin{array}{l}\text { Little Rock-North Little Rock-Conway, } \\
\text { Arkansas }\end{array}$ & 1,130 & 12.5 & 1.4 & $(9.8-15.3)$ \\
\hline Logan, Utah-Idaho & 504 & 6.6 & 1.7 & $(3.2-9.9)$ \\
\hline $\begin{array}{l}\text { Los Angeles-Long Beach-Glendale, } \\
\text { California }^{\dagger}\end{array}$ & 2,859 & 16.3 & 1.0 & $(14.3-18.3)$ \\
\hline $\begin{array}{l}\text { Louisville/Jefferson County, } \\
\text { Kentucky-Indiana }\end{array}$ & 2,050 & 16.8 & 1.3 & $(14.2-19.3)$ \\
\hline Lumberton, North Carolina & 518 & 10.5 & 3.1 & $(4.4-16.6)$ \\
\hline Manchester-Nashua, New Hampshire & 1,840 & 16.1 & 1.3 & $(13.6-18.5)$ \\
\hline McAllen-Edinburg-Mission, Texas & 603 & 17.6 & 2.7 & $(12.4-22.9)$ \\
\hline $\begin{array}{l}\text { Memphis, } \\
\text { Tennessee-Mississippi-Arkansas }\end{array}$ & 1,252 & 11.9 & 1.4 & $(9.1-14.7)$ \\
\hline $\begin{array}{l}\text { Miami-Fort Lauderdale-West Palm } \\
\text { Beach, Florida }\end{array}$ & 1,585 & 15.4 & 1.4 & $(12.6-18.2)$ \\
\hline $\begin{array}{l}\text { Milwaukee-Waukesha-West Allis, } \\
\text { Wisconsin }\end{array}$ & 1,172 & 23.5 & 1.8 & $(20.0-27.0)$ \\
\hline $\begin{array}{l}\text { Minneapolis-St. Paul-Bloomington, } \\
\text { Minnesota-Wisconsin }\end{array}$ & 7,538 & 21.4 & 0.7 & $(20.1-22.8)$ \\
\hline Missoula, Montana & 768 & 27.5 & 2.1 & $(23.3-31.7)$ \\
\hline Mobile, Alabama & 784 & 15.4 & 2.5 & $(10.6-20.3)$ \\
\hline Montgomery, Alabama & 511 & 14.0 & 2.4 & $(9.2-18.7)$ \\
\hline $\begin{array}{l}\text { Montgomery County-Bucks County- } \\
\text { Chester County, Pennsylvania }{ }^{\dagger}\end{array}$ & 1,277 & 18.9 & 1.6 & $(15.8-22.1)$ \\
\hline $\begin{array}{l}\text { Myrtle Beach-Conway-North Myrtle } \\
\text { Beach, South Carolina-North Carolina }\end{array}$ & 917 & 17.1 & 1.8 & $(13.5-20.7)$ \\
\hline $\begin{array}{l}\text { Nashville-Davidson-Murfreesboro- } \\
\text { Franklin, Tennessee }\end{array}$ & 1,254 & 16.0 & 1.5 & $(13.0-19.0)$ \\
\hline $\begin{array}{l}\text { Nassau County-Suffolk County, } \\
\text { New York }^{\dagger}\end{array}$ & 866 & 16.1 & 1.9 & $(12.5-19.8)$ \\
\hline Newark, New Jersey-Pennsylvania ${ }^{\dagger}$ & 6,205 & 17.4 & 0.8 & $(15.8-19.0)$ \\
\hline New Haven-Milford, Connecticut & 1,947 & 15.5 & 1.2 & $(13.2-17.8)$ \\
\hline New Orleans-Metairie, Louisiana & 1,225 & 19.0 & 1.7 & $(15.6-22.3)$ \\
\hline $\begin{array}{l}\text { New York-Jersey City-White Plains, } \\
\text { New York-New Jersey }{ }^{\dagger}\end{array}$ & 6,877 & 16.9 & 0.8 & $(15.4-18.5)$ \\
\hline Norfolk, Nebraska & 557 & 24.7 & 2.8 & $(19.3-30.2)$ \\
\hline North Platte, Nebraska & 599 & 18.5 & 2.8 & $(13.1-23.9)$ \\
\hline Norwich-New London, Connecticut & 961 & 18.5 & 2.0 & $(14.5-22.4)$ \\
\hline $\begin{array}{l}\text { Oakland-Hayward-Berkeley, } \\
\text { California }^{\dagger}\end{array}$ & 865 & 18.9 & 2.0 & $(15.0-22.8)$ \\
\hline Ocean City, New Jersey & 522 & 14.2 & 3.1 & $(8.1-20.2)$ \\
\hline Ogden-Clearfield, Utah & 2,375 & 9.7 & 0.9 & $(7.9-11.4)$ \\
\hline Oklahoma City, Oklahoma & 2,395 & 16.3 & 1.0 & $(14.3-18.3)$ \\
\hline Olympia-Tumwater, Washington & 506 & 18.7 & 2.4 & $(14.0-23.4)$ \\
\hline Omaha-Council Bluffs, Nebraska-lowa & 5,445 & 22.0 & 0.8 & $(20.5-23.6)$ \\
\hline Orlando-Kissimmee-Sanford, Florida & 541 & 17.6 & 2.7 & $(12.3-22.9)$ \\
\hline Philadelphia, Pennsylvania $^{\dagger}$ & 2,341 & 19.3 & 1.1 & $(17.1-21.6)$ \\
\hline Phoenix-Mesa-Scottsdale, Arizona & 2,484 & 15.5 & 1.0 & $(13.5-17.5)$ \\
\hline Pittsburgh, Pennsylvania & 3,241 & 19.0 & 0.9 & $(17.2-20.8)$ \\
\hline Ponce, Puerto Rico & 535 & 14.2 & 1.7 & $(10.9-17.4)$ \\
\hline Portland-South Portland, Maine & 3,265 & 19.9 & 0.9 & $(18.1-21.7)$ \\
\hline $\begin{array}{l}\text { Portland-Vancouver-Hillsboro, } \\
\text { Oregon-Washington }\end{array}$ & 3,029 & 17.4 & 1.0 & $(15.5-19.3)$ \\
\hline $\begin{array}{l}\text { Providence-Warwick, Rhode } \\
\text { Island-Massachusetts }\end{array}$ & 7,782 & 18.1 & 0.8 & $(16.6-19.7)$ \\
\hline Provo-Orem, Utah & 1,710 & 6.2 & 0.8 & $(4.7-7.7)$ \\
\hline Raleigh, North Carolina & 911 & 14.3 & 1.4 & $(11.6-17.0)$ \\
\hline Rapid City, South Dakota & 1,026 & 16.6 & 1.5 & $(13.7-19.4)$ \\
\hline Reno, Nevada & 1,454 & 17.5 & 1.6 & $(14.4-20.6)$ \\
\hline Richmond, Virginia & 972 & 15.4 & 1.6 & $(12.2-18.6)$ \\
\hline
\end{tabular}

See table footnotes on page 73 . 
TABLE 35. (Continued) Estimated prevalence of adults aged $\geq 18$ years who reported binge drinking* during the preceding month, by metropolitan and micropolitan statistical area - Behavioral Risk Factor Surveillance System, United States, 2012

\begin{tabular}{|c|c|c|c|c|}
\hline MMSA(s) & $\begin{array}{l}\text { Sample } \\
\text { size }\end{array}$ & $\%$ & SE & $95 \% \mathrm{Cl}$ \\
\hline $\begin{array}{l}\text { Riverside-San Bernardino-Ontario, } \\
\text { California }\end{array}$ & 1,325 & 17.2 & 1.6 & $(14.1-20.3)$ \\
\hline $\begin{array}{l}\text { Rockingham County-Strafford County, } \\
\text { New Hampshire }\end{array}$ & 1,613 & 19.2 & 1.4 & $(16.4-21.9)$ \\
\hline Rutland, Vermont & 583 & 18.3 & 2.4 & $(13.7-23.0)$ \\
\hline $\begin{array}{l}\text { Sacramento-Roseville-Arden-Arcade, } \\
\text { California }\end{array}$ & 878 & 16.6 & 2.0 & $(12.7-20.4)$ \\
\hline St. Louis, Missouri-Illinois & 2,003 & 22.1 & 1.4 & $(19.3-24.9)$ \\
\hline Salisbury, Maryland-Delaware & 2,060 & 19.2 & 1.9 & $(15.4-23.0)$ \\
\hline Salt Lake City, Utah & 4,479 & 14.6 & 0.8 & $(13.1-16.1)$ \\
\hline San Antonio-New Braunfels, Texas & 753 & 20.7 & 2.3 & $(16.1-25.2)$ \\
\hline San Diego-Carlsbad, California & 996 & 19.0 & 1.7 & $(15.6-22.4)$ \\
\hline $\begin{array}{l}\text { San Francisco-Redwood City-South } \\
\text { San Francisco, California }{ }^{\dagger}\end{array}$ & 517 & 18.9 & 2.6 & $(13.8-24.1)$ \\
\hline $\begin{array}{l}\text { San Jose-Sunnyvale-Santa Clara, } \\
\text { California }\end{array}$ & 592 & 16.4 & 2.0 & $(12.5-20.2)$ \\
\hline San Juan-Carolina-Caguas, Puerto Rico & 3,854 & 14.8 & 0.7 & $(13.3-16.2)$ \\
\hline Santa Fe, New Mexico & 662 & 13.9 & 1.7 & $(10.5-17.2)$ \\
\hline Sayre, Pennsylvania & 1,806 & 22.3 & 3.4 & $(15.6-29.0)$ \\
\hline Scottsbluff, Nebraska & 617 & 14.1 & 2.6 & $(9.1-19.1)$ \\
\hline $\begin{array}{l}\text { Scranton-Wilkes-Barre-Hazleton, } \\
\text { Pennsylvania }\end{array}$ & 719 & 21.6 & 2.1 & $(17.5-25.8)$ \\
\hline Seattle-Bellevue-Everett, Washington ${ }^{\dagger}$ & 4,989 & 17.8 & 0.7 & $(16.4-19.3)$ \\
\hline Shreveport-Bossier City, Louisiana & 565 & 15.5 & 2.4 & $(10.8-20.1)$ \\
\hline $\begin{array}{l}\text { Silver Spring-Frederick-Rockville, } \\
\text { Maryland }\end{array}$ & 2,184 & 15.7 & 1.3 & $(13.2-18.2)$ \\
\hline $\begin{array}{l}\text { Sioux City, lowa-Nebraska- } \\
\text { South Dakota }\end{array}$ & 1,174 & 22.2 & 3.0 & $(16.4-28.0)$ \\
\hline Sioux Falls, South Dakota & 1,431 & 22.5 & 1.5 & $(19.6-25.3)$ \\
\hline Spartanburg, South Carolina & 693 & 11.7 & 1.7 & $(8.5-15.0)$ \\
\hline Spokane-Spokane Valley, Washington & 1,071 & 17.2 & 1.7 & $(13.9-20.5)$ \\
\hline Springfield, Massachusetts & 2,224 & 19.5 & 1.6 & $(16.5-22.6)$ \\
\hline Tacoma-Lakewood, Washington $^{\dagger}$ & 1,160 & 15.6 & 1.4 & $(12.8-18.4)$ \\
\hline $\begin{array}{l}\text { Tampa-St. Petersburg-Clearwater, } \\
\text { Florida }\end{array}$ & 806 & 18.9 & 2.0 & $(15.0-22.8)$ \\
\hline
\end{tabular}

TABLE 35. (Continued) Estimated prevalence of adults aged $\geq 18$ years who reported binge drinking* during the preceding month, by metropolitan and micropolitan statistical area - Behavioral Risk Factor Surveillance System, United States, 2012

\begin{tabular}{|c|c|c|c|c|}
\hline MMSA(s) & $\begin{array}{l}\text { Sample } \\
\text { size }\end{array}$ & $\%$ & SE & $95 \% \mathrm{Cl}$ \\
\hline Toledo, Ohio & 929 & 20.4 & 2.5 & $(15.5-25.3)$ \\
\hline Topeka, Kansas & 1,070 & 15.0 & 1.5 & $(12.0-18.0)$ \\
\hline Torrington, Connecticut & 641 & 19.9 & 2.3 & $(15.4-24.3)$ \\
\hline Trenton, New Jersey & 546 & 15.7 & 2.4 & $(10.9-20.4)$ \\
\hline Tucson, Arizona & 963 & 17.5 & 1.9 & $(13.7-21.2)$ \\
\hline Tulsa, Oklahoma & 1,704 & 14.4 & 1.1 & $(12.2-16.5)$ \\
\hline Tuscaloosa, Alabama & 557 & 15.2 & 2.9 & $(9.5-20.8)$ \\
\hline Urban Honolulu, Hawaii & 4,234 & 17.4 & 0.8 & $(15.8-19.1)$ \\
\hline Vineland-Bridgeton, New Jersey & 530 & 16.9 & 3.1 & $(10.8-23.1)$ \\
\hline $\begin{array}{l}\text { Virginia Beach-Norfolk-Newport News, } \\
\text { Virginia-North Carolina }\end{array}$ & 1,438 & 15.4 & 1.4 & $(12.6-18.1)$ \\
\hline $\begin{array}{l}\text { Warren-Troy-Farmington Hills, } \\
\text { Michigan }{ }^{\dagger}\end{array}$ & 2,133 & 19.3 & 1.2 & $(17.0-21.6)$ \\
\hline $\begin{array}{l}\text { Washington-Arlington-Alexandria, } \\
\text { District of Columbia-Virginia- } \\
\text { Maryland-West Virginia }{ }^{\dagger}\end{array}$ & 7,819 & 17.4 & 0.8 & $(15.9-18.9)$ \\
\hline Wichita, Kansas & 2,311 & 14.2 & 1.1 & $(12.1-16.3)$ \\
\hline $\begin{array}{l}\text { Wilmington, Delaware-Maryland- } \\
\text { New Jersey }{ }^{\dagger}\end{array}$ & 3,157 & 19.4 & 1.1 & $(17.2-21.7)$ \\
\hline Winston-Salem, North Carolina & 761 & 11.9 & 1.8 & $(8.5-15.4)$ \\
\hline Worcester, Massachusetts-Connecticut & t 2,695 & 19.6 & 1.2 & $(17.3-21.9)$ \\
\hline Yakima, Washington & 525 & 16.0 & 2.5 & $(11.2-20.9)$ \\
\hline $\begin{array}{l}\text { Youngstown-Warren-Boardman, } \\
\text { Ohio-Pennsylvania }\end{array}$ & 890 & 19.1 & 2.3 & $(14.5-23.7)$ \\
\hline Median & & 17.2 & & \\
\hline Range & & $6.2-28.1$ & & \\
\hline
\end{tabular}

Abbreviations: $\mathrm{Cl}=$ confidence interval; $\mathrm{MMSA}=$ metropolitan and micropolitan statistical area; $\mathrm{SE}=$ standard error.

* For males: having at least five drinks on at least one occasion; for females: having at least four drinks on at least one occasion.

+ Metropolitan division.

$\$$ Estimate not available (N/A) if the unweighted sample size for the denominator was $<50$ or if the relative standard error is $>0.3$. 
TABLE 36. Estimated prevalence of adults aged $\geq 18$ years who reported binge drinking* during the preceding month, by county - Behavioral Risk Factor Surveillance System, United States, 2012

\begin{tabular}{|c|c|c|c|c|}
\hline County & $\begin{array}{l}\text { Sample } \\
\text { size }\end{array}$ & $\%$ & SE & $95 \% \mathrm{Cl}$ \\
\hline Jefferson County, Alabama & 918 & 12.2 & 1.4 & $(9.4-15.0)$ \\
\hline Madison County, Alabama & 487 & 12.8 & 2.2 & $(8.6-17.0)$ \\
\hline Mobile County, Alabama & 784 & 15.4 & 2.5 & $(10.6-20.3)$ \\
\hline Anchorage Municipality, Alaska & 872 & 16.7 & 1.5 & $(13.8-19.6)$ \\
\hline Fairbanks North Star Borough, Alaska & 585 & 15.9 & 2.0 & $(12.0-19.8)$ \\
\hline Matanuska-Susitna Borough, Alaska & 584 & 18.7 & 2.3 & $(14.2-23.1)$ \\
\hline Maricopa County, Arizona & 2,018 & 15.2 & 1.1 & $(13.1-17.3)$ \\
\hline Pima County, Arizona & 963 & 17.5 & 1.9 & $(13.8-21.2)$ \\
\hline Pulaski County, Arkansas & 727 & 13.5 & 2.0 & $(9.5-17.4)$ \\
\hline Alameda County, California & 553 & 17.3 & 2.3 & $(12.8-21.9)$ \\
\hline Los Angeles County, California & 2,859 & 16.3 & 1.0 & $(14.3-18.3)$ \\
\hline Orange County, California & 892 & 19.5 & 2.1 & $(15.4-23.6)$ \\
\hline Riverside County, California & 707 & 18.4 & 2.3 & $(13.9-22.9)$ \\
\hline Sacramento County, California & 529 & 16.7 & 2.7 & $(11.4-22.0)$ \\
\hline San Bernardino County, California & 618 & 15.7 & 2.1 & $(11.6-19.8)$ \\
\hline San Diego County, California & 996 & 19.0 & 1.7 & $(15.6-22.4)$ \\
\hline Santa Clara County, California & 574 & 16.3 & 2.0 & $(12.4-20.3)$ \\
\hline Adams County, Colorado & 771 & 21.8 & 2.0 & $(17.8-25.7)$ \\
\hline Arapahoe County, Colorado & 814 & 23.2 & 1.8 & $(19.7-26.8)$ \\
\hline Boulder County, Colorado & 491 & 15.4 & 2.0 & $(11.5-19.4)$ \\
\hline Denver County, Colorado & 909 & 27.2 & 1.8 & $(23.8-30.7)$ \\
\hline Douglas County, Colorado & 517 & 15.8 & 2.0 & $(11.8-19.7)$ \\
\hline El Paso County, Colorado & 964 & 12.7 & 1.4 & $(10.0-15.4)$ \\
\hline Jefferson County, Colorado & 1,055 & 19.1 & 1.5 & $(16.1-22.0)$ \\
\hline Larimer County, Colorado & 565 & 18.0 & 2.0 & $(14.0-22.0)$ \\
\hline Weld County, Colorado & 499 & 17.8 & 2.1 & $(13.6-22.0)$ \\
\hline Fairfield County, Connecticut & 2,089 & 19.5 & 1.4 & $(16.7-22.2)$ \\
\hline Hartford County, Connecticut & 1,922 & 16.1 & 1.2 & $(13.8-18.4)$ \\
\hline Litchfield County, Connecticut & 641 & 19.9 & 2.3 & $(15.4-24.3)$ \\
\hline New Haven County, Connecticut & 1,947 & 15.5 & 1.2 & $(13.2-17.8)$ \\
\hline New London County, Connecticut & 961 & 18.5 & 2.0 & $(14.5-22.4)$ \\
\hline Kent County, Delaware & 1,426 & 15.1 & 1.6 & $(12.0-18.1)$ \\
\hline New Castle County, Delaware & 2,297 & 19.9 & 1.1 & $(17.7-22.1)$ \\
\hline Sussex County, Delaware & 1,371 & 18.1 & 1.6 & $(15.0-21.2)$ \\
\hline $\begin{array}{l}\text { District of Columbia, } \\
\text { District of Columbia }\end{array}$ & 3,642 & 23.2 & 1.3 & $(20.7-25.7)$ \\
\hline Broward County, Florida & 505 & 15.9 & 2.6 & $(10.7-21.1)$ \\
\hline Miami-Dade County, Florida & 764 & 14.9 & 2.3 & $(10.4-19.5)$ \\
\hline Hawaii County, Hawaii & 1,317 & 21.6 & 1.7 & $(18.3-24.9)$ \\
\hline Honolulu County, Hawaii & 4,234 & 17.4 & 0.8 & $(15.8-19.1)$ \\
\hline Kauai County, Hawaii & 652 & 19.4 & 2.8 & $(13.9-24.8)$ \\
\hline Maui County, Hawaii & 1,191 & 17.7 & 1.7 & $(14.4-21.0)$ \\
\hline Ada County, Idaho & 778 & 15.6 & 2.0 & $(11.7-19.5)$ \\
\hline Canyon County, Idaho & 489 & 15.6 & 3.0 & $(9.7-21.5)$ \\
\hline Cook County, Illinois & 1,470 & 21.5 & 1.5 & $(18.5-24.5)$ \\
\hline Lake County, Indiana & 852 & 16.6 & 2.2 & $(12.3-20.9)$ \\
\hline Marion County, Indiana & 1,234 & 16.7 & 1.5 & $(13.7-19.6)$ \\
\hline Polk County, lowa & 783 & 23.1 & 1.9 & $(19.4-26.8)$ \\
\hline Johnson County, Kansas & 2,139 & 18.1 & 1.2 & $(15.8-20.4)$ \\
\hline Sedgwick County, Kansas & 1,763 & 14.7 & 1.2 & $(12.3-17.1)$ \\
\hline Shawnee County, Kansas & 758 & 14.2 & 1.8 & $(10.7-17.7)$ \\
\hline Wyandotte County, Kansas & 854 & 14.4 & 2.2 & $(10.1-18.6)$ \\
\hline Jefferson County, Kentucky & 1,576 & 19.9 & 1.7 & $(16.6-23.2)$ \\
\hline East Baton Rouge Parish, Louisiana & 550 & 18.0 & 2.5 & $(13.1-22.9)$ \\
\hline Androscoggin County, Maine & 686 & 18.0 & 1.8 & $(14.4-21.6)$ \\
\hline Aroostook County, Maine & 525 & 15.1 & 2.1 & $(11.1-19.2)$ \\
\hline Cumberland County, Maine & 1,725 & 20.6 & 1.3 & $(18.1-23.1)$ \\
\hline Kennebec County, Maine & 817 & 16.6 & 1.7 & $(13.3-19.8)$ \\
\hline Penobscot County, Maine & 907 & 16.1 & 1.6 & $(12.9-19.2)$ \\
\hline York County, Maine & 1,164 & 19.9 & 1.5 & $(17.0-22.8)$ \\
\hline Anne Arundel County, Maryland & 866 & 18.9 & 2.2 & $(14.6-23.1)$ \\
\hline
\end{tabular}

See table footnotes on page 75 .
TABLE 36. (Continued) Estimated prevalence of adults aged $\geq 18$ years who reported binge drinking* during the preceding month, by county - Behavioral Risk Factor Surveillance System, United States, 2012

\begin{tabular}{|c|c|c|c|c|}
\hline County & $\begin{array}{c}\text { Sample } \\
\text { size }\end{array}$ & $\%$ & SE & $95 \% \mathrm{Cl}$ \\
\hline Baltimore County, Maryland & 1,455 & 17.2 & 1.7 & $(13.8-20.6)$ \\
\hline Charles County, Maryland & 493 & 16.0 & 3.3 & $(9.7-22.4)$ \\
\hline Frederick County, Maryland & 721 & 21.0 & 3.5 & $(14.1-27.9)$ \\
\hline Montgomery County, Maryland & 1,463 & 14.5 & 1.3 & $(11.9-17.1)$ \\
\hline Prince George's County, Maryland & 1,087 & 12.4 & 1.8 & $(8.9-15.9)$ \\
\hline Washington County, Maryland & 522 & 14.4 & 3.4 & $(7.8-21.0)$ \\
\hline Baltimore city, Maryland & 706 & 18.9 & 2.4 & $(14.2-23.5)$ \\
\hline Barnstable County, Massachusetts & 527 & 14.6 & 2.0 & $(10.6-18.5)$ \\
\hline Bristol County, Massachusetts & 2,504 & 19.7 & 1.8 & $(16.3-23.2)$ \\
\hline Essex County, Massachusetts & 2,437 & 18.7 & 1.4 & $(16.0-21.4)$ \\
\hline Hampden County, Massachusetts & 1,851 & 17.2 & 1.6 & (14. \\
\hline Middlesex County, Massachusetts & 4,053 & 19.1 & 0.9 & $(17.3-20.9)$ \\
\hline Norfolk County, Massachusetts & 1,654 & 18.5 & 1.4 & $(15.7-21.3)$ \\
\hline Plymouth County, Massachusetts & 1,711 & 20.4 & 1.8 & $(16.8-24.0)$ \\
\hline Suffolk County, Massachusetts & 2,168 & 22.8 & 1.4 & $(20.1-25.5)$ \\
\hline Worcester County, Massachusetts & 2,459 & 20.8 & 1.3 & $(18.2-23.3)$ \\
\hline Kent County, Michigan & 516 & 19.6 & 2.6 & $(14.4-24.7)$ \\
\hline Macomb County, Michigan & 598 & 20.3 & 2.1 & $(16.3-24.3)$ \\
\hline Oakland County, Michigan & 1,138 & 17.3 & 1.7 & $(14.1-20.6)$ \\
\hline Wayne County, Michigan & 2,142 & 18.9 & 1.4 & $(16.0-21.7)$ \\
\hline Anoka County, Minnesota & 523 & 19.5 & 2.1 & $(15.4-23.7)$ \\
\hline Dakota County, Minnesota & 650 & 21.4 & 1.9 & $(17.6-25.2)$ \\
\hline Hennepin County, Minnesota & 3,121 & 20.9 & 1.1 & $(18.8-22.9)$ \\
\hline Ramsey County, Minnesota & 1,875 & 21.7 & 1.8 & $(18.2-25.2)$ \\
\hline Jackson County, Missouri & 851 & 15.6 & 2.2 & $(11.3-19.9)$ \\
\hline St. Louis County, Missouri & 923 & 18.9 & 1.8 & $(15.5-22.4)$ \\
\hline Cascade County, Montana & 691 & 20.0 & 2.2 & $(15.8-24.2)$ \\
\hline Flathead County, Montana & 550 & 21.2 & 2.1 & $(17.0-25.4)$ \\
\hline Hill County, Montana & 564 & 24.7 & 3.3 & $(18.4-31.1)$ \\
\hline Lake County, Montana & 874 & 17.6 & 2.4 & $(12.9-22.3)$ \\
\hline Missoula County, Montana & 768 & 27.5 & 2.1 & $(23.3-31.7)$ \\
\hline Yellowstone County, Montana & 737 & 22.0 & 1.8 & $(18.6-25.4)$ \\
\hline Dakota County, Nebraska & 721 & 19.9 & 4.4 & $(11.2-28.5)$ \\
\hline Douglas County, Nebraska & 3,483 & 22.6 & 0.9 & $(20.8-24.5)$ \\
\hline Hall County, Nebraska & 525 & 17.7 & 2.3 & $(13.3-22.1)$ \\
\hline Lancaster County, Nebraska & 1,422 & 23.9 & 1.3 & $(21.4-26.5)$ \\
\hline Lincoln County, Nebraska & 575 & 19.0 & 2.8 & $(13.5-24.6)$ \\
\hline Sarpy County, Nebraska & 1,156 & 23.4 & 1.7 & $(20.0-26.7)$ \\
\hline Scotts Bluff County, Nebraska & 567 & 13.7 & 2.6 & $(8.7-18.8)$ \\
\hline Clark County, Nevada & 1,928 & 13.8 & 1.0 & $(11.8-15.7)$ \\
\hline Washoe County, Nevada & 1,435 & 17.6 & 1.6 & $(14.4-20.7)$ \\
\hline Belknap County, New Hampshire & 544 & 20.6 & 3.3 & $(14.1-27.1)$ \\
\hline Carroll County, New Hampshire & 517 & 17.4 & 3.5 & $(10.5-24.2)$ \\
\hline Cheshire County, New Hampshire & 535 & 17.2 & 2.7 & $(11.8-22.6)$ \\
\hline Coos County, New Hampshire & 521 & 19.1 & 3.1 & $(13.0-25.1)$ \\
\hline Grafton County, New Hampshire & 552 & 15.8 & 2.4 & $(11.1-20.4)$ \\
\hline Hillsborough County, New Hampshire & 1,840 & 16.1 & 1.2 & $(13.7-18.5)$ \\
\hline Merrimack County, New Hampshire & 685 & 12.8 & 1.8 & $(9.3-16.3)$ \\
\hline Rockingham County, New Hampshire & 1,012 & 19.9 & 1.7 & $(16.6-23.2)$ \\
\hline Strafford County, New Hampshire & 601 & 17.4 & 2.6 & $(12.3-22.6)$ \\
\hline Atlantic County, New Jersey & 959 & 19.3 & 2.1 & $(15.3-23.3)$ \\
\hline Bergen County, New Jersey & 874 & 17.0 & 1.7 & $(13.6-20.4)$ \\
\hline Burlington County, New Jersey & 654 & 16.4 & 2.1 & $(12.2-20.6)$ \\
\hline Camden County, New Jersey & 708 & 16.9 & 2.2 & $(12.6-21.3)$ \\
\hline Cape May County, New Jersey & 522 & 14.2 & 3.1 & $(8.1-20.2)$ \\
\hline Cumberland County, New Jersey & 530 & 16.9 & 3.1 & $(10.8-23.1)$ \\
\hline Essex County, New Jersey & 1,273 & 18.8 & 1.6 & $(15.6-22.0)$ \\
\hline Gloucester County, New Jersey & 530 & 18.7 & 2.6 & $(13.5-23.8)$ \\
\hline Hudson County, New Jersey & 1,198 & 21.6 & 1.7 & $(18.3-24.9)$ \\
\hline Hunterdon County, New Jersey & 535 & 19.8 & 3.1 & $(13.7-25.8)$ \\
\hline
\end{tabular}

See table footnotes on page 75 . 
TABLE 36. (Continued) Estimated prevalence of adults aged $\geq 18$ years who reported binge drinking* during the preceding month, by county - Behavioral Risk Factor Surveillance System, United States, 2012

\begin{tabular}{|c|c|c|c|c|}
\hline County & $\begin{array}{l}\text { Sample } \\
\text { size }\end{array}$ & $\%$ & SE & $95 \% \mathrm{Cl}$ \\
\hline Mercer County, New Jersey & 546 & 15.7 & 2.4 & $(10.9-20.4)$ \\
\hline Middlesex County, New Jersey & 831 & 14.2 & 1.7 & $(10.9-17.4)$ \\
\hline Monmouth County, New Jersey & 665 & 23.4 & 2.4 & $(18.7-28.0)$ \\
\hline Morris County, New Jersey & 798 & 16.9 & 1.8 & (13.4-20.4) \\
\hline Ocean County, New Jersey & 614 & 18.3 & 2.2 & $(13.9-22.7)$ \\
\hline Passaic County, New Jersey & 642 & 19.1 & 2.1 & $(15.0-23.1)$ \\
\hline Salem County, New Jersey & 559 & 15.2 & 3.5 & $(8.3-22.2)$ \\
\hline Somerset County, New Jersey & 595 & 16.3 & 2.1 & $(12.1-20.4)$ \\
\hline Sussex County, New Jersey & 515 & 18.5 & 2.7 & $(13.3-23.8)$ \\
\hline Union County, New Jersey & 664 & 15.5 & 1.8 & $(12.0-19.0)$ \\
\hline Warren County, New Jersey & 507 & 12.0 & 2.4 & $(7.3-16.7)$ \\
\hline Bernalillo County, New Mexico & 1,965 & 14.5 & 0.9 & $(12.6-16.3)$ \\
\hline Dona Ana County, New Mexico & 682 & 20.0 & 2.3 & $(15.5$ \\
\hline Sandoval County, New Mexico & 618 & 13.2 & 1.8 & $(9$. \\
\hline San Juan County, New Mexico & 614 & 10.9 & 1.6 & $(7.8-14.0)$ \\
\hline Santa Fe County, New Mexico & 662 & 13.9 & 1.7 & $(10.5-17.2)$ \\
\hline Kings County, New York & 465 & 17.0 & 2.4 & $(12.3-21.6)$ \\
\hline Guilford County, North Carolina & 498 & 14.9 & 2.1 & $(10.8-19.1)$ \\
\hline Mecklenburg County, North Carolina & 680 & 12.5 & 1.4 & $(9.8-15.2)$ \\
\hline Robeson County, North Carolina & 518 & 10.5 & 3.1 & $(4.4-16.6)$ \\
\hline Wake County, North Carolina & 669 & 15.1 & 1.6 & $(12.0-18.2)$ \\
\hline Burleigh County, North Dakota & 558 & 22.8 & 2.6 & $(17.7-27.9)$ \\
\hline Cass County, North Dakota & 822 & 26.0 & 2.3 & $(21.5-30.5)$ \\
\hline Cuyahoga County, Ohio & 790 & 18.1 & 1.7 & $(14.8-21.3)$ \\
\hline Frar & 794 & 18.9 & 1.7 & $(15.6-$ \\
\hline Hamilton County, Ohio & 736 & 20.2 & 1.9 & $(16.6-23.9)$ \\
\hline Lorain County, Ohio & 582 & 23.4 & 3.4 & $(16.9$ \\
\hline Lucas County, Ohio & 591 & 20.8 & 3.1 & $(14$. \\
\hline Mahoning County, Ohio & 563 & 25.7 & 3.8 & $(18.2-33.1)$ \\
\hline Montgomery County, Ohio & 637 & 19.1 & 2.3 & $(14.5-23.6)$ \\
\hline Stark County, Ohio & 598 & 15.8 & 2.1 & $(11.6-20.0)$ \\
\hline Summit County, Ohio & 600 & 19.6 & 2.3 & $(15.0-24.1)$ \\
\hline Oklahoma County, Oklahoma & 1,163 & 15.4 & 1.3 & $(12.8-17.9)$ \\
\hline Tulsa County, Oklahoma & 1,175 & 15.6 & 1.4 & $(12.9-18.2)$ \\
\hline Clackamas County, Oregon & 492 & 17.0 & 2.4 & $(12.3-21.7)$ \\
\hline Lane County, Oregon & 512 & 16.3 & 2.5 & $(11.4-21.3)$ \\
\hline Multnomah County, Oregon & 864 & 20.5 & 1.9 & $(16.8-24.3)$ \\
\hline Washington County, Oregon & 599 & 15.3 & 1.9 & $(11.4-19.1)$ \\
\hline Allegheny County, Pennsylvania & 1,822 & 19.9 & 1.3 & $(17.5-22.4)$ \\
\hline Bradford County, Pennsylvania & 1,806 & 22.3 & 3.4 & $(15.6-29.0)$ \\
\hline Montgomery County, Pennsylvania & 532 & 20.0 & 2.2 & $(15.6-24.3)$ \\
\hline Philadelphia County, Pennsylvania & 1,960 & 18.7 & 1.3 & $(16.3-21.2)$ \\
\hline Pike County, Pennsylvania & 1,825 & 12.8 & 1.1 & $(10.6-14.9)$ \\
\hline Kent County, Rhode Island & 789 & 15.9 & 1.9 & $(12.3-19.6)$ \\
\hline Providence County, Rhode Island & 3,213 & 17.1 & 1.0 & $(15.2-19.0)$ \\
\hline Washington County, Rhode Island & 637 & 18.4 & 2.4 & $(13.6-23.1)$ \\
\hline Aiken County, South Carolina & 537 & 12.8 & 2.8 & $(7.2-18.3)$ \\
\hline Beaufort County, South Carolin & 772 & 12.4 & 1.9 & $(8.8-16.1)$ \\
\hline Charleston County, South Carolina & 980 & 24.7 & 2.1 & $(20.5-28.8)$ \\
\hline
\end{tabular}

TABLE 36. (Continued) Estimated prevalence of adults aged $\geq 18$ years who reported binge drinking* during the preceding month, by county - Behavioral Risk Factor Surveillance System, United States, 2012

\begin{tabular}{|c|c|c|c|c|}
\hline County & $\begin{array}{c}\text { Sample } \\
\text { size }\end{array}$ & $\%$ & SE & $95 \% \mathrm{Cl}$ \\
\hline Greenville County, South Carolina & 872 & 15.4 & 1.9 & $(11.6-19.1)$ \\
\hline Horry County, South Carolina & 753 & 14.4 & 1.7 & $(11.1-17.7)$ \\
\hline Richland County, South Carolina & 928 & 22.0 & 2.2 & $(17.7-26.3)$ \\
\hline Spartanburg County, South Carolina & 640 & 12.3 & 1.8 & $(8.8-15.8)$ \\
\hline Lincoln County, South Dakota & 488 & 29.5 & 3.7 & $(22.2-36.7)$ \\
\hline Minnehaha County, South Dakota & 857 & 21.4 & 1.6 & $(18.2-24.7)$ \\
\hline Pennington County, South Dakota & 593 & 15.0 & 1.7 & $(11.7-18.3)$ \\
\hline Davidson County, Tennessee & 516 & 17.2 & 2.1 & $(13.0-21.4)$ \\
\hline Shelby County, Tennessee & 498 & 11.1 & 1.8 & $(7.5-14.7)$ \\
\hline Bexar County, Texas & 566 & 21.1 & 2.7 & $(15.8-26.4)$ \\
\hline Dallas County, Texas & 487 & 16.3 & 1.9 & $(12.5-20.1)$ \\
\hline El Paso County, Texas & 597 & 21.1 & 2.9 & $(15.3-26.8)$ \\
\hline Harris County, Texas & 733 & 15.3 & 1.6 & $(12.2-18.4)$ \\
\hline Hidalgo County, Texas & 603 & 17.6 & 2.7 & $(12.4-22.9)$ \\
\hline Tarrant County, Texas & 556 & 17.7 & 2.3 & $(13.3-22.2)$ \\
\hline Travis County, Texas & 1,022 & 21.1 & 2.2 & $(16.7-25.5)$ \\
\hline Davis County, Utah & 1,122 & 9.6 & 1.3 & $(7.0-12.2)$ \\
\hline Salt Lake County, Utah & 3,930 & 14.8 & 0.8 & $(13.2-16.4)$ \\
\hline Tooele County, Utah & 549 & 10.8 & 2.6 & $(5.7-15.9)$ \\
\hline Utah County, Utah & 1,643 & 6.2 & 0.8 & $(4.7-7.7)$ \\
\hline Wasatch County, Utah & 500 & $\mathrm{~N} / \mathrm{A}^{\dagger}$ & $\mathrm{N} / \mathrm{A}$ & $(\mathrm{N} / \mathrm{A}-\mathrm{N} / \mathrm{A})$ \\
\hline Weber County, Utah & 1,020 & 10.2 & 1.3 & $(7.7-12.6)$ \\
\hline Chittenden County, Vermont & 890 & 24.2 & 1.8 & $(20.6-27.7)$ \\
\hline Rutland County, Vermont & 583 & 18.3 & 2.4 & $(13.7-23.0)$ \\
\hline Washington County, Vermont & 509 & 16.8 & 2.1 & $(12.6-21.0)$ \\
\hline Windsor County, Vermont & 529 & 17.4 & 2.2 & $(13.1-21.6)$ \\
\hline Fairfax County, Virginia & 692 & 13.8 & 1.7 & $(10.5-17.1)$ \\
\hline Clark County, Washington & 776 & 17.7 & 1.9 & $(13.9-21.5)$ \\
\hline King County, Washington & 3,846 & 17.6 & 0.8 & $(16.0-19.3)$ \\
\hline Kitsap County, Washington & 554 & 15.4 & 2.1 & $(11.2-19.6)$ \\
\hline Pierce County, Washington & 1,160 & 15.6 & 1.4 & $(12.9-18.4)$ \\
\hline Snohomish County, Washington & 1,143 & 19.0 & 1.7 & $(15.7-22.3)$ \\
\hline Spokane County, Washington & 928 & 17.1 & 1.8 & $(13.5-20.7)$ \\
\hline Thurston County, Washington & 506 & 18.7 & 2.4 & $(14.0-23.4)$ \\
\hline Whatcom County, Washington & 833 & 19.7 & 2.5 & $(14.7-24.6)$ \\
\hline Yakima County, Washington & 525 & 16.0 & 2.5 & $(11.2-20.9)$ \\
\hline Kanawha County, West Virginia & 631 & 9.4 & 1.4 & $(6.7-12.1)$ \\
\hline Milwaukee County, Wisconsin & 891 & 21.4 & 2.1 & $(17.3-25.5)$ \\
\hline Laramie County, Wyoming & 922 & 16.0 & 2.2 & $(11.7-20.3)$ \\
\hline Natrona County, Wyoming & 803 & 17.4 & 2.4 & $(12.7-22.1)$ \\
\hline San Juan Municipio, Puerto Rico & 652 & 14.9 & 1.7 & $(11.6-18.2)$ \\
\hline Median & & 17.5 & & \\
\hline Range & & $6.2-29.5$ & & \\
\hline
\end{tabular}

Abbreviations: $\mathrm{Cl}=$ confidence interval; $\mathrm{SE}=$ standard error.

* For males: having at least five drinks on at least one occasion; for females: having at least four drinks on at least one occasion.

† Estimate not available (N/A) if the unweighted sample size for the denominator was $<50$ or if the relative standard error is $>0.3$. 
TABLE 37. Estimated prevalence of adults aged $\geq 18$ years who reported heavy drinking* during the preceding month, by state/ territory - Behavioral Risk Factor Surveillance System, United States, 2012

\begin{tabular}{|c|c|c|c|c|}
\hline State/Territory & $\begin{array}{l}\text { Sample } \\
\text { size }\end{array}$ & $\%$ & SE & $95 \% \mathrm{Cl}$ \\
\hline Alabama & 8,663 & 5.2 & 0.4 & $(4.5-6.0)$ \\
\hline Alaska & 4,163 & 6.6 & 0.6 & $(5.5-7.7)$ \\
\hline Arizona & 7,017 & 5.1 & 0.4 & $(4.3-5.9)$ \\
\hline Arkansas & 5,005 & 4.1 & 0.4 & $(3.3-4.9)$ \\
\hline California & 12,564 & 5.8 & 0.3 & $(5.2-6.4)$ \\
\hline Colorado & 11,524 & 6.5 & 0.3 & $(5.9-7.1)$ \\
\hline Connecticut & 8,450 & 6.5 & 0.4 & $(5.8-7.2)$ \\
\hline Delaware & 5,094 & 6.8 & 0.5 & $(5.8-7.8)$ \\
\hline District of Columbia & 3,641 & 8.1 & 0.8 & $(6.4-9.7)$ \\
\hline Florida & 7,323 & 7.1 & 0.5 & $(6.2-8.0)$ \\
\hline Georgia & 5,815 & 5.1 & 0.4 & $(4.2-5.9)$ \\
\hline Hawaii & 7,407 & 7.4 & 0.4 & $(6.5-8.2)$ \\
\hline Idaho & 5,730 & 5.7 & 0.6 & $(4.6-6.9)$ \\
\hline Illinois & 5,484 & 6.1 & 0.5 & $(5.2-7.1)$ \\
\hline Indiana & 8,393 & 5.1 & 0.3 & $(4.4-5.7)$ \\
\hline lowa & 6,929 & 6.2 & 0.4 & $(5.5-6.9)$ \\
\hline Kansas & 11,580 & 5.3 & 0.3 & $(4.7-5.9)$ \\
\hline Kentucky & 10,713 & 5.3 & 0.4 & $(4.6-6.1)$ \\
\hline Louisiana & 8,797 & 6.9 & 0.5 & $(5.9-8.0)$ \\
\hline Maine & 9,708 & 6.7 & 0.3 & $(6.1-7.4)$ \\
\hline Maryland & 12,216 & 5.5 & 0.4 & $(4.7-6.2)$ \\
\hline Massachusetts & 20,401 & 7.4 & 0.3 & $(6.9-8.0)$ \\
\hline Michigan & 10,215 & 6.1 & 0.4 & $(5.4-6.8)$ \\
\hline Minnesota & 11,705 & 6.3 & 0.3 & $(5.7-6.9)$ \\
\hline Mississippi & 7,570 & 4.6 & 0.4 & $(3.8-5.4)$ \\
\hline Missouri & 6,579 & 6.3 & 0.5 & $(5.4-7.2)$ \\
\hline Montana & 8,446 & 8.5 & 0.4 & $(7.7-9.4)$ \\
\hline Nebraska & 18,738 & 7.2 & 0.3 & $(6.6-7.7)$ \\
\hline Nevada & 4,647 & 6.5 & 0.5 & $(5.5-7.5)$ \\
\hline New Hampshire & 7,285 & 7.3 & 0.5 & $(6.3-8.2)$ \\
\hline New Jersey & 14,694 & 5.3 & 0.3 & $(4.8-5.8)$ \\
\hline New Mexico & 8,384 & 5.5 & 0.3 & $(4.9-6.2)$ \\
\hline New York & 5,818 & 5.0 & 0.4 & $(4.3-5.7)$ \\
\hline North Carolina & 11,335 & 4.9 & 0.3 & $(4.4-5.4)$ \\
\hline North Dakota & 4,754 & 6.5 & 0.5 & $(5.4-7.5)$ \\
\hline Ohio & 12,464 & 6.3 & 0.3 & $(5.7-6.9)$ \\
\hline Oklahoma & 7,857 & 4.6 & 0.3 & $(4.0-5.3)$ \\
\hline Oregon & 5,072 & 7.3 & 0.5 & $(6.3-8.2)$ \\
\hline Pennsylvania & 19,271 & 5.8 & 0.2 & $(5.3-6.2)$ \\
\hline Rhode Island & 5,276 & 6.2 & 0.5 & $(5.3-7.1)$ \\
\hline South Carolina & 12,348 & 5.9 & 0.3 & $(5.2-6.5)$ \\
\hline South Dakota & 7,665 & 5.9 & 0.4 & $(5.1-6.7)$ \\
\hline Tennessee & 6,618 & 3.6 & 0.4 & $(2.9-4.3)$ \\
\hline Texas & 8,779 & 6.1 & 0.4 & $(5.3-6.8)$ \\
\hline Utah & 12,091 & 3.5 & 0.2 & $(3.1-4.0)$ \\
\hline Vermont & 5,903 & 7.5 & 0.5 & $(6.6-8.4)$ \\
\hline Virginia & 7,038 & 5.4 & 0.4 & $(4.7-6.1)$ \\
\hline Washington & 14,990 & 6.4 & 0.3 & $(5.8-6.9)$ \\
\hline West Virginia & 5,320 & 3.5 & 0.3 & $(2.9-4.1)$ \\
\hline Wisconsin & 5,055 & 8.5 & 0.6 & $(7.4-9.6)$ \\
\hline Wyoming & 6,056 & 6.0 & 0.6 & $(4.9-7.2)$ \\
\hline Guam & 1,925 & 7.5 & 0.9 & $(5.7-9.3)$ \\
\hline Puerto Rico & 6,140 & 4.9 & 0.4 & $(4.2-5.6)$ \\
\hline Median & & 6.1 & & \\
\hline Range & & $3.5-8.5$ & & \\
\hline
\end{tabular}

Abbreviations: $\mathrm{Cl}=$ confidence interval; $\mathrm{SE}=$ standard error.

* For adult men: having more than two drinks per day; for adult women: having more than one drink per day.
TABLE 38. Estimated prevalence of adults aged $\geq 18$ years who reported heavy drinking* during the preceding month, by metropolitan and micropolitan statistical area - Behavioral Risk Factor Surveillance System, United States, 2012

\begin{tabular}{|c|c|c|c|c|}
\hline MMSA(s) & $\begin{array}{l}\text { Sample } \\
\text { size }\end{array}$ & $\%$ & SE & $95 \% \mathrm{Cl}$ \\
\hline Aguadilla-Isabela, Puerto Rico & 537 & 3.2 & 0.9 & $(1.5-4.9)$ \\
\hline Akron, Ohio & 713 & 7.0 & 1.4 & $(4.3-9.8)$ \\
\hline Albuquerque, New Mexico & 3,112 & 5.4 & 0.5 & $(4.4-6.4)$ \\
\hline $\begin{array}{l}\text { Allentown-Bethlehem-Easton, } \\
\text { Pennsylvania-New Jersey }\end{array}$ & 1,297 & 5.0 & 0.9 & $(3.3-6.7)$ \\
\hline Anaheim-Santa Ana-Irvine, California ${ }^{\dagger}$ & 896 & 5.3 & 1.1 & $(3.1-7.5)$ \\
\hline Anchorage, Alaska & 1,455 & 6.6 & 0.8 & $(5.0-8.3)$ \\
\hline Asheville, North Carolina & 572 & 5.5 & 1.1 & $(3.3-7.7)$ \\
\hline $\begin{array}{l}\text { Atlanta-Sandy Springs-Roswell, } \\
\text { Georgia }\end{array}$ & 2,427 & 4.6 & 0.6 & $(3.5-5.7)$ \\
\hline Atlantic City-Hammonton, New Jersey & 962 & 7.4 & 1.3 & $(4.8-10.0)$ \\
\hline $\begin{array}{l}\text { Augusta-Richmond County, Georgia- } \\
\text { South Carolina }\end{array}$ & 993 & 5.1 & 1.3 & $(2.6-7.6)$ \\
\hline Augusta-Waterville, Maine & 815 & 4.7 & 0.9 & $(3.0-6.4)$ \\
\hline Austin-Round Rock, Texas & 1,347 & 7.5 & 1.1 & $(5.3-9.7)$ \\
\hline $\begin{array}{l}\text { Baltimore-Columbia-Towson, } \\
\text { Maryland }\end{array}$ & 4,513 & 5.3 & 0.5 & $(4.3-6.3)$ \\
\hline Bangor, Maine & 910 & 5.2 & 0.9 & $(3.5-6.9)$ \\
\hline Barnstable Town, Massachusetts & 524 & 6.3 & 1.3 & $(3.9-8.8)$ \\
\hline Barre, Vermont & 507 & 8.7 & 1.5 & $(5.8-11.5)$ \\
\hline Baton Rouge, Louisiana & 1,340 & 6.7 & 1.3 & $(4.3-9.2)$ \\
\hline Bellingham, Washington & 832 & 8.0 & 1.7 & $(4.7-11.3)$ \\
\hline Berlin, New Hampshire-Vermont & 682 & 7.2 & 1.5 & $(4.3-10.0)$ \\
\hline Billings, Montana & 829 & 9.1 & 1.1 & $(6.8-11.3)$ \\
\hline Birmingham-Hoover, Alabama & 1,741 & 4.4 & 0.7 & $(3.1-5.7)$ \\
\hline Bismarck, North Dakota & 807 & 7.5 & 1.6 & $(4.4-10.5)$ \\
\hline Boise City, Idaho & 1,436 & 5.6 & 1.0 & $(3.6-7.5)$ \\
\hline Boston, Massachusetts $^{\dagger}$ & 5,553 & 8.1 & 0.6 & $(6.9-9.3)$ \\
\hline Boulder, Colorado & 489 & 5.8 & 1.3 & $(3.3-8.2)$ \\
\hline Bremerton-Silverdale, Washington & 553 & 5.1 & 1.1 & $(3.0-7.3)$ \\
\hline $\begin{array}{l}\text { Bridgeport-Stamford-Norwalk, } \\
\text { Connecticut }\end{array}$ & 2,087 & 6.0 & 0.6 & $(4.7-7.2)$ \\
\hline Burlington-South Burlington, Vermont & 1,484 & 8.4 & 0.9 & $(6.7-10.2)$ \\
\hline $\begin{array}{l}\text { Cambridge-Newton-Framingham, } \\
\text { Massachusetts }{ }^{\dagger}\end{array}$ & 6,475 & 6.7 & 0.5 & $(5.8-7.7)$ \\
\hline Camden, New Jersey ${ }^{\dagger}$ & 1,879 & 5.4 & 0.7 & $(4.0-6.9)$ \\
\hline Canton-Massillon, Ohio & 651 & 6.8 & 1.6 & $(3.7-9.9)$ \\
\hline Casper, Wyoming & 801 & 4.4 & 1.2 & $(2.1-6.8)$ \\
\hline Cedar Rapids, lowa & 571 & 7.0 & 1.4 & $(4.2-9.8)$ \\
\hline Charleston, West Virginia & 763 & 3.0 & 0.7 & $(1.6-4.5)$ \\
\hline $\begin{array}{l}\text { Charleston-North Charleston, } \\
\text { South Carolina }\end{array}$ & 1,652 & 8.2 & 0.9 & $(6.3-10.0)$ \\
\hline $\begin{array}{l}\text { Charlotte-Concord-Gastonia, } \\
\text { North Carolina-South Carolina }\end{array}$ & 2,394 & 4.5 & 0.5 & $(3.5-5.5)$ \\
\hline Chattanooga, Tennessee-Georgia & 591 & $\mathrm{~N} / \mathrm{A}^{\S}$ & $\mathrm{N} / \mathrm{A}$ & $(\mathrm{N} / \mathrm{A}-\mathrm{N} / \mathrm{A})$ \\
\hline Cheyenne, Wyoming & 921 & 6.6 & 1.5 & $(3.6-9.5)$ \\
\hline $\begin{array}{l}\text { Chicago-Naperville-Elgin, } \\
\text { Illinois-Indiana-Wisconsin }\end{array}$ & 3,638 & 5.8 & 0.5 & $(4.8-6.8)$ \\
\hline Cincinnati, Ohio-Kentucky-Indiana & 2,272 & 5.8 & 0.6 & $(4.5-7.0)$ \\
\hline $\begin{array}{l}\text { Claremont-Lebanon, New } \\
\text { Hampshire-Vermont }\end{array}$ & 1,900 & 5.7 & 0.7 & $(4.4-7.1)$ \\
\hline Cleveland-Elyria, Ohio & 1,664 & 7.0 & 0.9 & $(5.3-8.7)$ \\
\hline Colorado Springs, Colorado & 1,098 & 4.3 & 0.8 & $(2.8-5.8)$ \\
\hline Columbia, South Carolina & 1,735 & 6.8 & 0.9 & $(5.1-8.5)$ \\
\hline Columbus, Ohio & 1,528 & 6.1 & 0.7 & $(4.6-7.5)$ \\
\hline Concord, New Hampshire & 686 & 7.3 & 1.4 & $(4.5-10.1)$ \\
\hline Dallas-Plano-Irving, Texas ${ }^{\dagger}$ & 867 & 5.0 & 0.9 & $(3.3-6.7)$ \\
\hline Dayton, Ohio & 825 & 9.6 & 1.6 & $(6.4-12.7)$ \\
\hline Denver-Aurora-Lakewood, Colorado & 4,577 & 7.5 & 0.5 & $(6.6-8.5)$ \\
\hline Des Moines-West Des Moines, lowa & 1,125 & 6.3 & 0.9 & $(4.4-8.1)$ \\
\hline
\end{tabular}

See table footnotes on page 78 . 
TABLE 38. (Continued) Estimated prevalence of adults aged $\geq 18$ years who reported heavy drinking* during the preceding month, by metropolitan and micropolitan statistical area - Behavioral Risk Factor Surveillance System, United States, 2012

\begin{tabular}{|c|c|c|c|c|}
\hline MMSA(s) & $\begin{array}{l}\text { Sample } \\
\text { size }\end{array}$ & $\%$ & SE & $95 \% \mathrm{Cl}$ \\
\hline 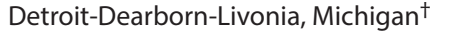 & 2,146 & 5.7 & 0.8 & $(4.2-7.3)$ \\
\hline Dover, Delaware & 1,428 & 7.0 & 1.0 & $(5.0-9.1)$ \\
\hline Duluth, Minnesota-Wisconsin & 500 & 6.6 & 1.4 & $(3.9-9.3)$ \\
\hline Durham-Chapel Hill, North Carolina & 757 & 7.7 & 1.3 & $(5.2-10.2)$ \\
\hline El Paso, Texas & 598 & 5.2 & 1.6 & $(2.2-8.3)$ \\
\hline Eugene, Oregon & 507 & 7.5 & 1.5 & $(4.5-10.5)$ \\
\hline Fairbanks, Alaska & 582 & 7.6 & 1.4 & $(4.9-10.4)$ \\
\hline Fargo, North Dakota-Minnesota & 918 & 3.9 & 0.9 & $(2.2-5.6)$ \\
\hline Farmington, New Mexico & 619 & 4.5 & 1.1 & $(2.4-6.6)$ \\
\hline Fayetteville, North Carolina & 487 & $\mathrm{~N} / \mathrm{A}$ & $\mathrm{N} / \mathrm{A}$ & $(\mathrm{N} / \mathrm{A}-\mathrm{N} / \mathrm{A})$ \\
\hline $\begin{array}{l}\text { Fayetteville-Springdale-Rogers, } \\
\text { Arkansas-Missouri }\end{array}$ & 786 & 4.0 & 1.0 & $(2.1-6.0)$ \\
\hline Fort Collins, Colorado & 567 & 4.1 & 1.0 & $(2.1-6.0)$ \\
\hline Fort Wayne, Indiana & 519 & 4.9 & 1.1 & $(2.6-7.1)$ \\
\hline Fort Worth-Arlington, Texas ${ }^{\dagger}$ & 702 & 7.1 & 1.4 & $(4.4-9.8)$ \\
\hline Grand Island, Nebraska & 840 & 7.0 & 1.2 & $(4.6-9.4)$ \\
\hline Grand Rapids-Wyoming, Michigan & 872 & 6.6 & 1.4 & $(3.8-9.4)$ \\
\hline Great Falls, Montana & 694 & 10.0 & 1.6 & $(6.9-13.0)$ \\
\hline Greeley, Colorado & 497 & 5.5 & 1.4 & $(2.8-8.2)$ \\
\hline $\begin{array}{l}\text { Greensboro-High Point, } \\
\text { North Carolina }\end{array}$ & 779 & 5.0 & 0.9 & $(3.2-6.8)$ \\
\hline $\begin{array}{l}\text { Greenville-Anderson-Mauldin, } \\
\text { South Carolina }\end{array}$ & 1,641 & 5.4 & 0.8 & $(3.9-7.0)$ \\
\hline Gulfport-Biloxi-Pascagoula, Mississippi & 719 & 6.7 & 1.3 & $(4.1-9.2)$ \\
\hline $\begin{array}{l}\text { Hagerstown-Martinsburg, Maryland- } \\
\text { West Virginia }\end{array}$ & 743 & 3.8 & 1.0 & $(1.9-5.7)$ \\
\hline Harrisburg-Carlisle, Pennsylvania & 657 & 3.7 & 0.9 & $(2.0-5.4)$ \\
\hline $\begin{array}{l}\text { Hartford-West Hartford-East Hartford, } \\
\text { Connecticut }\end{array}$ & 2,573 & 6.4 & 0.7 & $(5.1-7.8)$ \\
\hline Heber, Utah & 497 & $\mathrm{~N} / \mathrm{A}$ & N/A & $(\mathrm{N} / \mathrm{A}-\mathrm{N} / \mathrm{A})$ \\
\hline Hilo, Hawaii & 1,321 & 11.0 & 1.2 & $(8.6-13.4)$ \\
\hline $\begin{array}{l}\text { Hilton Head Island-Bluffton-Beaufort, } \\
\text { South Carolina }\end{array}$ & 903 & 8.9 & 1.7 & $(5.5-12.2)$ \\
\hline $\begin{array}{l}\text { Houston-The Woodlands-Sugar Land, } \\
\text { Texas }\end{array}$ & 1,085 & 5.6 & 0.8 & $(4.1-7.2)$ \\
\hline $\begin{array}{l}\text { Huntington-Ashland, West } \\
\text { Virginia-Kentucky-Ohio }\end{array}$ & 1,092 & 5.0 & 1.1 & $(2.8-7.1)$ \\
\hline Huntsville, Alabama & 586 & 4.1 & 1.1 & $(1.9-6.3)$ \\
\hline Idaho Falls, Idaho & 526 & $\mathrm{~N} / \mathrm{A}$ & $\mathrm{N} / \mathrm{A}$ & $(\mathrm{N} / \mathrm{A}-\mathrm{N} / \mathrm{A})$ \\
\hline $\begin{array}{l}\text { Indianapolis-Carmel-Anderson, } \\
\text { Indiana }\end{array}$ & 2,129 & 4.5 & 0.5 & $(3.4-5.5)$ \\
\hline Jackson, Mississippi & 886 & 4.9 & 1.0 & $(3.0-6.8)$ \\
\hline Jacksonville, Florida & 503 & 9.7 & 2.3 & $(5.2-14.1)$ \\
\hline Kahului-Wailuku-Lahaina, Hawaii & 1,193 & 8.2 & 0.9 & $(6.3-10.0)$ \\
\hline Kalispell, Montana & 547 & 10.0 & 1.7 & $(6.7-13.2)$ \\
\hline Kansas City, Missouri-Kansas & 4,639 & 6.2 & 0.7 & $(4.7-7.6)$ \\
\hline Kapaa, Hawaii & 651 & 10.3 & 2.4 & $(5.6-14.9)$ \\
\hline Keene, New Hampshire & 532 & 8.2 & 1.9 & $(4.5-11.9)$ \\
\hline Kennewick-Richland, Washington & 524 & 5.2 & 1.2 & $(2.9-7.5)$ \\
\hline $\begin{array}{l}\text { Kingsport-Bristol-Bristol, } \\
\text { Tennessee-Virginia }\end{array}$ & 539 & $\mathrm{~N} / \mathrm{A}$ & $\mathrm{N} / \mathrm{A}$ & $(\mathrm{N} / \mathrm{A}-\mathrm{N} / \mathrm{A})$ \\
\hline Knoxville, Tennessee & 792 & 4.2 & 1.0 & $(2.2-6.2)$ \\
\hline Laconia, New Hampshire & 542 & 8.6 & 1.9 & $(4.9-12.3)$ \\
\hline Lafayette, Louisiana & 540 & 9.0 & 1.8 & $(5.5-12.6)$ \\
\hline Las Cruces, New Mexico & 681 & 6.6 & 1.3 & $(4.0-9.1)$ \\
\hline $\begin{array}{l}\text { Las Vegas-Henderson-Paradise, } \\
\text { Nevada }\end{array}$ & 1,911 & 5.8 & 0.6 & $(4.5-7.0)$ \\
\hline Lewiston-Auburn, Maine & 685 & 5.6 & 1.0 & $(3.5-7.6)$ \\
\hline Lexington-Fayette, Kentucky & 511 & 6.4 & 1.2 & $(4.1-8.7)$ \\
\hline Lincoln, Nebraska & 1,643 & 6.3 & 0.7 & $(5.0-7.7)$ \\
\hline
\end{tabular}

See table footnotes on page 78.
TABLE 38. (Continued) Estimated prevalence of adults aged $\geq 18$ years who reported heavy drinking* during the preceding month, by metropolitan and micropolitan statistical area - Behavioral Risk Factor Surveillance System, United States, 2012

\begin{tabular}{|c|c|c|c|c|}
\hline MMSA(s) & $\begin{array}{l}\text { Sample } \\
\text { size }\end{array}$ & $\%$ & SE & $95 \% \mathrm{Cl}$ \\
\hline $\begin{array}{l}\text { Little Rock-North Little Rock-Conway, } \\
\text { Arkansas }\end{array}$ & 1,127 & 4.8 & 0.8 & $(3.2-6.4)$ \\
\hline Logan, Utah-Idaho & 502 & N/A & N/A & $(\mathrm{N} / \mathrm{A}-\mathrm{N} / \mathrm{A})$ \\
\hline $\begin{array}{l}\text { Los Angeles-Long Beach-Glendale, } \\
\text { California }^{\dagger}\end{array}$ & 2,858 & 5.0 & 0.5 & $(3.9-6.0)$ \\
\hline $\begin{array}{l}\text { Louisville/Jefferson County, } \\
\text { Kentucky-Indiana }\end{array}$ & 2,045 & 5.9 & 0.8 & $(4.3-7.4)$ \\
\hline Lumberton, North Carolina & 513 & $\mathrm{~N} / \mathrm{A}$ & $\mathrm{N} / \mathrm{A}$ & $(\mathrm{N} / \mathrm{A}-\mathrm{N} / \mathrm{A})$ \\
\hline Manchester-Nashua, New Hampshire & 1,844 & 7.5 & 1.0 & $(5.6-9.4)$ \\
\hline McAllen-Edinburg-Mission, Texas & 600 & $\mathrm{~N} / \mathrm{A}$ & N/A & $(\mathrm{N} / \mathrm{A}-\mathrm{N} / \mathrm{A})$ \\
\hline $\begin{array}{l}\text { Memphis, } \\
\text { Tennessee-Mississippi-Arkansas }\end{array}$ & 1,251 & 3.8 & 0.9 & $(2.1-5.5)$ \\
\hline $\begin{array}{l}\text { Miami-Fort Lauderdale-West Palm } \\
\text { Beach, Florida }\end{array}$ & 1,581 & 4.6 & 0.7 & $(3.3-6.0)$ \\
\hline $\begin{array}{l}\text { Milwaukee-Waukesha-West Allis, } \\
\text { Wisconsin }\end{array}$ & 1,174 & 8.3 & 1.1 & $(6.1-10.6)$ \\
\hline $\begin{array}{l}\text { Minneapolis-St. Paul-Bloomington, } \\
\text { Minnesota-Wisconsin }\end{array}$ & 7,528 & 6.6 & 0.4 & $(5.8-7.4)$ \\
\hline Missoula, Montana & 764 & 8.1 & 1.4 & $(5.5-10.8)$ \\
\hline Mobile, Alabama & 785 & 7.3 & 1.5 & $(4.3-10.3)$ \\
\hline Montgomery, Alabama & 514 & 7.3 & 1.8 & $(3.8-10.9)$ \\
\hline $\begin{array}{l}\text { Montgomery County-Bucks County- } \\
\text { Chester County, Pennsylvania }{ }^{\dagger}\end{array}$ & 1,276 & 4.9 & 0.6 & $(3.7-6.2)$ \\
\hline $\begin{array}{l}\text { Myrtle Beach-Conway-North Myrtle } \\
\text { Beach, South Carolina-North Carolina }\end{array}$ & 922 & 6.9 & 1.2 & $(4.5-9.3)$ \\
\hline $\begin{array}{l}\text { Nashville-Davidson-Murfreesboro- } \\
\text { Franklin, Tennessee }\end{array}$ & 1,259 & 4.5 & 0.8 & $(3.0-6.0)$ \\
\hline $\begin{array}{l}\text { Nassau County-Suffolk County, } \\
\text { New York }^{\dagger}\end{array}$ & 868 & 3.8 & 0.8 & $(2.3-5.3)$ \\
\hline Newark, New Jersey-Pennsylvania ${ }^{\dagger}$ & 6,204 & 4.9 & 0.4 & $(4.1-5.7)$ \\
\hline New Haven-Milford, Connecticut & 1,948 & 6.3 & 0.7 & $(4.9-7.8)$ \\
\hline New Orleans-Metairie, Louisiana & 1,233 & 7.9 & 1.3 & $(5.4-10.4)$ \\
\hline $\begin{array}{l}\text { New York-Jersey City-White Plains, } \\
\text { New York-New Jersey }{ }^{\dagger}\end{array}$ & 6,883 & 4.5 & 0.4 & $(3.8-5.2)$ \\
\hline Norfolk, Nebraska & 562 & 7.6 & 1.6 & $(4.5-10.6)$ \\
\hline North Platte, Nebraska & 598 & 5.1 & 1.3 & $(2.5-7.6)$ \\
\hline Norwich-New London, Connecticut & 963 & 7.1 & 1.3 & $(4.6-9.6)$ \\
\hline $\begin{array}{l}\text { Oakland-Hayward-Berkeley, } \\
\text { California }^{\dagger}\end{array}$ & 862 & 7.6 & 1.2 & $(5.2-10.0)$ \\
\hline Ocean City, New Jersey & 523 & 5.9 & 1.4 & $(3.3-8.6)$ \\
\hline Ogden-Clearfield, Utah & 2,370 & 2.5 & 0.4 & $(1.7-3.3)$ \\
\hline Oklahoma City, Oklahoma & 2,391 & 4.7 & 0.6 & $(3.5-5.8)$ \\
\hline Olympia-Tumwater, Washington & 508 & 6.3 & 1.3 & $(3.7-8.9)$ \\
\hline Omaha-Council Bluffs, Nebraska-lowa & 5,448 & 7.3 & 0.5 & $(6.4-8.2)$ \\
\hline Orlando-Kissimmee-Sanford, Florida & 545 & 5.7 & 1.4 & $(2.9-8.4)$ \\
\hline Philadelphia, Pennsylvania $^{\dagger}$ & 2,331 & 7.0 & 0.8 & $(5.5-8.6)$ \\
\hline Phoenix-Mesa-Scottsdale, Arizona & 2,491 & 5.0 & 0.6 & $(3.8-6.2)$ \\
\hline Pittsburgh, Pennsylvania & 3,255 & 6.9 & 0.6 & $(5.7-8.2)$ \\
\hline Ponce, Puerto Rico & 533 & 4.2 & 1.0 & $(2.3-6.1)$ \\
\hline Portland-South Portland, Maine & 3,270 & 8.6 & 0.6 & $(7.4-9.8)$ \\
\hline $\begin{array}{l}\text { Portland-Vancouver-Hillsboro, } \\
\text { Oregon-Washington }\end{array}$ & 3,019 & 7.4 & 0.7 & $(6.1-8.7)$ \\
\hline $\begin{array}{l}\text { Providence-Warwick, Rhode } \\
\text { Island-Massachusetts }\end{array}$ & 7,781 & 6.4 & 0.5 & $(5.4-7.4)$ \\
\hline Provo-Orem, Utah & 1,707 & 2.0 & 0.5 & $(1.1-2.9)$ \\
\hline Raleigh, North Carolina & 904 & 5.0 & 0.9 & $(3.3-6.7)$ \\
\hline Rapid City, South Dakota & 1,029 & 6.7 & 1.0 & $(4.8-8.6)$ \\
\hline Reno, Nevada & 1,454 & 7.5 & 1.0 & $(5.6-9.4)$ \\
\hline Richmond, Virginia & 967 & 6.1 & 1.0 & $(4.1-8.2)$ \\
\hline
\end{tabular}

See table footnotes on page 78 . 
TABLE 38. (Continued) Estimated prevalence of adults aged $\geq 18$ years who reported heavy drinking* during the preceding month, by metropolitan and micropolitan statistical area - Behavioral Risk Factor Surveillance System, United States, 2012

\begin{tabular}{|c|c|c|c|c|}
\hline MMSA(s) & $\begin{array}{l}\text { Sample } \\
\text { size }\end{array}$ & $\%$ & SE & $95 \% \mathrm{Cl}$ \\
\hline $\begin{array}{l}\text { Riverside-San Bernardino-Ontario, } \\
\text { California }\end{array}$ & 1,327 & 5.2 & 0.9 & $(3.4-7.0)$ \\
\hline $\begin{array}{l}\text { Rockingham County-Strafford County, } \\
\text { New Hampshire }\end{array}$ & 1,616 & 7.0 & 0.9 & $(5.3-8.7)$ \\
\hline Rutland, Vermont & 582 & 5.5 & 1.1 & $(3.2-7.7)$ \\
\hline $\begin{array}{l}\text { Sacramento-Roseville-Arden-Arcade, } \\
\text { California }\end{array}$ & 877 & 6.2 & 1.2 & $(3.8-8.5)$ \\
\hline St. Louis, Missouri-Illinois & 2,007 & 6.9 & 0.8 & $(5.3-8.4)$ \\
\hline Salisbury, Maryland-Delaware & 2,053 & 8.6 & 1.5 & $(5.7-11.5)$ \\
\hline Salt Lake City, Utah & 4,485 & 4.5 & 0.4 & $(3.7-5.3)$ \\
\hline San Antonio-New Braunfels, Texas & 753 & 7.3 & 1.5 & $(4.5-10.2)$ \\
\hline San Diego-Carlsbad, California & 996 & 8.0 & 1.1 & $(5.8-10.3)$ \\
\hline $\begin{array}{l}\text { San Francisco-Redwood City-South } \\
\text { San Francisco, California }\end{array}$ & 519 & 6.0 & 1.4 & $(3.4-8.7)$ \\
\hline $\begin{array}{l}\text { San Jose-Sunnyvale-Santa Clara, } \\
\text { California }\end{array}$ & 594 & 4.3 & 1.0 & $(2.3-6.2)$ \\
\hline San Juan-Carolina-Caguas, Puerto Rico & 3,836 & 5.3 & 0.4 & $(4.5-6.2)$ \\
\hline Santa Fe, New Mexico & 665 & 8.4 & 1.3 & $(5.8-10.9)$ \\
\hline Sayre, Pennsylvania & 1,807 & 4.4 & 0.6 & $(3.3-5.5)$ \\
\hline Scottsbluff, Nebraska & 619 & 6.1 & 1.8 & $(2.6-9.6)$ \\
\hline $\begin{array}{l}\text { Scranton-Wilkes-Barre-Hazleton, } \\
\text { Pennsylvania }\end{array}$ & 719 & 7.8 & 1.5 & $(4.8-10.7)$ \\
\hline Seattle-Bellevue-Everett, Washington ${ }^{\dagger}$ & 4,992 & 6.6 & 0.5 & $(5.7-7.5)$ \\
\hline Shreveport-Bossier City, Louisiana & 567 & 5.5 & 1.4 & $(2.7-8.3)$ \\
\hline $\begin{array}{l}\text { Silver Spring-Frederick-Rockville, } \\
\text { Maryland }{ }^{\dagger}\end{array}$ & 2,180 & 5.2 & 0.7 & $(3.7-6.6)$ \\
\hline $\begin{array}{l}\text { Sioux City, lowa-Nebraska- } \\
\text { South Dakota }\end{array}$ & 1,173 & 8.9 & 2.1 & $(4.7-13.1)$ \\
\hline Sioux Falls, South Dakota & 1,438 & 6.0 & 0.7 & $(4.7-7.4)$ \\
\hline Spartanburg, South Carolina & 689 & 4.0 & 1.0 & $(2.0-6.0)$ \\
\hline Spokane-Spokane Valley, Washington & 1,073 & 8.1 & 1.1 & $(5.9-10.3)$ \\
\hline Springfield, Massachusetts & 2,230 & 8.0 & 1.1 & $(5.8-10.2)$ \\
\hline Tacoma-Lakewood, Washington ${ }^{\dagger}$ & 1,162 & 6.1 & 0.9 & $(4.4-7.8)$ \\
\hline $\begin{array}{l}\text { Tampa-St. Petersburg-Clearwater, } \\
\text { Florida }\end{array}$ & 801 & 8.3 & 1.4 & $(5.7-11.0)$ \\
\hline
\end{tabular}

TABLE 38. (Continued) Estimated prevalence of adults aged $\geq 18$ years who reported heavy drinking* during the preceding month, by metropolitan and micropolitan statistical area - Behavioral Risk Factor Surveillance System, United States, 2012

\begin{tabular}{|c|c|c|c|c|}
\hline MMSA(s) & $\begin{array}{l}\text { Sample } \\
\text { size }\end{array}$ & $\%$ & SE & $95 \% \mathrm{Cl}$ \\
\hline Toledo, Ohio & 931 & 6.4 & 1.5 & $(3.5-9.3)$ \\
\hline Topeka, Kansas & 1,072 & 4.5 & 0.9 & $(2.6-6.3)$ \\
\hline Torrington, Connecticut & 641 & 9.1 & 1.6 & $(5.9-12.3)$ \\
\hline Trenton, New Jersey & 544 & 3.7 & 0.8 & $(2.1-5.3)$ \\
\hline Tucson, Arizona & 961 & 5.6 & 0.9 & $(3.8-7.4)$ \\
\hline Tulsa, Oklahoma & 1,700 & 4.5 & 0.6 & $(3.3-5.8)$ \\
\hline Tuscaloosa, Alabama & 556 & N/A & $\mathrm{N} / \mathrm{A}$ & $(\mathrm{N} / \mathrm{A}-\mathrm{N} / \mathrm{A})$ \\
\hline Urban Honolulu, Hawaii & 4,242 & 6.3 & 0.5 & $(5.3-7.3)$ \\
\hline Vineland-Bridgeton, New Jersey & 529 & 3.2 & 0.8 & $(1.6-4.8)$ \\
\hline $\begin{array}{l}\text { Virginia Beach-Norfolk-Newport News, } \\
\text { Virginia-North Carolina }\end{array}$ & 1,436 & 5.9 & 0.8 & $(4.3-7.5)$ \\
\hline $\begin{array}{l}\text { Warren-Troy-Farmington Hills, } \\
\text { Michigan }\end{array}$ & 2,122 & 5.7 & 0.6 & $(4.5-7.0)$ \\
\hline $\begin{array}{l}\text { Washington-Arlington-Alexandria, } \\
\text { District of Columbia-Virginia- } \\
\text { Maryland-West Virginia }{ }^{\dagger}\end{array}$ & 7,805 & 5.6 & 0.4 & $(4.8-6.5)$ \\
\hline Wichita, Kansas & 2,307 & 4.8 & 0.6 & $(3.6-6.1)$ \\
\hline $\begin{array}{l}\text { Wilmington, Delaware-Maryland- } \\
\text { New Jersey }{ }^{\dagger}\end{array}$ & 3,152 & 6.4 & 0.7 & $(5.0-7.7)$ \\
\hline Winston-Salem, North Carolina & 765 & 2.8 & 0.7 & $(1.4-4.3)$ \\
\hline Worcester, Massachusetts-Connecticut & t 2,698 & 7.4 & 0.7 & $(6.0-8.8)$ \\
\hline Yakima, Washington & 524 & N/A & N/A & $(\mathrm{N} / \mathrm{A}-\mathrm{N} / \mathrm{A})$ \\
\hline $\begin{array}{l}\text { Youngstown-Warren-Boardman, } \\
\text { Ohio-Pennsylvania }\end{array}$ & 891 & 6.6 & 1.4 & $(3.8-9.3)$ \\
\hline Median & & 6.1 & & \\
\hline Range & & $2.0-11.0$ & & \\
\hline
\end{tabular}

Abbreviations: $\mathrm{Cl}=$ confidence interval; $\mathrm{MMSA}=$ metropolitan and micropolitan statistical area; $\mathrm{SE}=$ standard error.

* For adult men: having more than two drinks per day; for adult women: having more than one drink per day.

+ Metropolitan division.

$\$$ Estimate not available (N/A) if the unweighted sample size for the denominator was $<50$ or if the relative standard error is $>0.3$. 
TABLE 39. Estimated prevalence of adults aged $\geq 18$ years who reported heavy drinking* during the preceding month, by county — Behavioral Risk Factor Surveillance System, United States, 2012

\begin{tabular}{|c|c|c|c|c|}
\hline County & $\begin{array}{l}\text { Sample } \\
\text { size }\end{array}$ & $\%$ & SE & $95 \% \mathrm{Cl}$ \\
\hline Jefferson County, Alabama & 919 & 4.7 & 0.8 & $(3.1-6.3)$ \\
\hline Madison County, Alabama & 485 & $\mathrm{~N} / \mathrm{A}^{\dagger}$ & N/A & $(\mathrm{N} / \mathrm{A}-\mathrm{N} / \mathrm{A})$ \\
\hline Mobile County, Alabama & 785 & 7.3 & 1.5 & $(4.3-10.3)$ \\
\hline Anchorage Municipality, Alaska & 872 & 6.0 & 1.0 & $(4.1-7.9)$ \\
\hline Fairbanks North Star Borough, Alaska & 582 & 7.6 & 1.4 & $(4.9-10.4)$ \\
\hline Matanuska-Susitna Borough, Alaska & 583 & 8.5 & 1.7 & $(5.1-11.8)$ \\
\hline Maricopa County, Arizona & 2,024 & 5.0 & 0.6 & $(3.8-6.3)$ \\
\hline Pima County, Arizona & 961 & 5.6 & 0.9 & $(3.8-7.4)$ \\
\hline Pulaski County, Arkansas & 722 & 5.5 & 1.1 & $(3.4-7.7)$ \\
\hline Alameda County, California & 553 & 6.9 & 1.4 & $(4.1-9.7)$ \\
\hline Los Angeles County, California & 2,858 & 5.0 & 0.5 & $(3.9-6.0)$ \\
\hline Orange County, California & 896 & 5.3 & 1.1 & $(3.1-7.5)$ \\
\hline Riverside County, California & 709 & 6.2 & 1.4 & $(3.4-9.0)$ \\
\hline Sacramento County, California & 530 & 5.6 & 1.5 & $(2.7-8.6)$ \\
\hline San Bernardino County, California & 618 & 4.3 & 1.0 & $(2.2-6.3)$ \\
\hline San Diego County, California & 996 & 8.0 & 1.1 & $(5.8-10.3)$ \\
\hline Santa Clara County, California & 576 & 4.1 & 1.0 & $(2.2-6.1)$ \\
\hline Adams County, Colorado & 773 & 6.2 & 1.2 & $(4.0-8.5)$ \\
\hline Arapahoe County, Colorado & 818 & 7.6 & 1.0 & $(5.7-9.6)$ \\
\hline Boulder County, Colorado & 489 & 5.8 & 1.2 & $(3.3-8.2)$ \\
\hline Denver County, Colorado & 918 & 7.7 & 1.0 & $(5.8-9.6)$ \\
\hline Douglas County, Colorado & 513 & 6.7 & 1.4 & $(3.8-9.5)$ \\
\hline El Paso County, Colorado & 965 & 4.0 & 0.8 & $(2.5-5.4)$ \\
\hline Jefferson County, Colorado & 1,058 & 9.1 & 1.1 & $(7.0-11.2)$ \\
\hline Larimer County, Colorado & 567 & 4.1 & 1.0 & $(2.1-6.0)$ \\
\hline Weld County, Colorado & 497 & 5.5 & 1.4 & $(2.8-8.2)$ \\
\hline Fairfield County, Connecticut & 2,087 & 6.0 & 0.6 & $(4.7-7.2)$ \\
\hline Hartford County, Connecticut & 1,914 & 5.0 & 0.6 & $(3.9-6.2)$ \\
\hline Litchfield County, Connecticut & 641 & 9.1 & 1.6 & $(5.9-12.3)$ \\
\hline New Haven County, Connecticut & 1,948 & 6.3 & 0.8 & $(4.9-7.8)$ \\
\hline New London County, Connecticut & 963 & 7.1 & 1.3 & $(4.6-9.6)$ \\
\hline Kent County, Delaware & 1,428 & 7.0 & 1.1 & $(5.0-9.1)$ \\
\hline New Castle County, Delaware & 2,297 & 6.7 & 0.8 & $(5.2-8.1)$ \\
\hline Sussex County, Delaware & 1,369 & 7.4 & 1.0 & $(5.5-9.3)$ \\
\hline $\begin{array}{l}\text { District of Columbia, } \\
\text { District of Columbia }\end{array}$ & 3,641 & 8.0 & 0.8 & $(6.4-9.7)$ \\
\hline Broward County, Florida & 502 & 5.8 & 1.4 & $(3.0-8.7)$ \\
\hline Miami-Dade County, Florida & 764 & 2.9 & 0.8 & $(1.4-4.4)$ \\
\hline Hawaii County, Hawaii & 1,321 & 11.0 & 1.2 & $(8.6-13.4)$ \\
\hline Honolulu County, Hawaii & 4,242 & 6.3 & 0.5 & $(5.3-7.3)$ \\
\hline Kauai County, Hawaii & 651 & 10.3 & 2.4 & $(5.6-15.0)$ \\
\hline Maui County, Hawaii & 1,193 & 8.2 & 0.9 & $(6.3-10.0)$ \\
\hline Ada County, Idaho & 779 & 5.5 & 1.2 & $(3.1-7.9)$ \\
\hline Canyon County, Idaho & 490 & N/A & N/A & $(\mathrm{N} / \mathrm{A}-\mathrm{N} / \mathrm{A})$ \\
\hline Cook County, Illinois & 1,473 & 5.5 & 0.7 & $(4.1-6.9)$ \\
\hline Lake County, Indiana & 851 & 4.5 & 1.2 & $(2.2-6.9)$ \\
\hline Marion County, Indiana & 1,236 & 4.9 & 0.8 & $(3.4-6.4)$ \\
\hline Polk County, lowa & 782 & 7.3 & 1.2 & $(4.9-9.6)$ \\
\hline Johnson County, Kansas & 2,136 & 5.8 & 0.7 & $(4.4-7.3)$ \\
\hline Sedgwick County, Kansas & 1,761 & 4.6 & 0.7 & $(3.3-6.0)$ \\
\hline Shawnee County, Kansas & 760 & 4.0 & 1.0 & $(2.0-5.9)$ \\
\hline Wyandotte County, Kansas & 857 & 3.7 & 1.0 & $(1.9-5.6)$ \\
\hline Jefferson County, Kentucky & 1,574 & 6.4 & 1.0 & $(4.4-8.4)$ \\
\hline East Baton Rouge Parish, Louisiana & 548 & 6.3 & 1.6 & $(3.2-9.4)$ \\
\hline Androscoggin County, Maine & 685 & 5.6 & 1.0 & $(3.5-7.6)$ \\
\hline Aroostook County, Maine & 525 & 3.4 & 0.9 & $(1.7-5.1)$ \\
\hline Cumberland County, Maine & 1,726 & 8.1 & 0.8 & $(6.4-9.7)$ \\
\hline Kennebec County, Maine & 815 & 4.7 & 0.9 & $(3.0-6.4)$ \\
\hline Penobscot County, Maine & 910 & 5.2 & 0.9 & $(3.5-6.9)$ \\
\hline York County, Maine & 1,167 & 9.1 & 1.1 & $(7.0-11.2)$ \\
\hline
\end{tabular}

See table footnotes on page 80 .
TABLE 39. (Continued) Estimated prevalence of adults aged $\geq 18$ years who reported heavy drinking* during the preceding month, by county — Behavioral Risk Factor Surveillance System, United States, 2012

\begin{tabular}{|c|c|c|c|c|}
\hline County & $\begin{array}{l}\text { Sample } \\
\text { size }\end{array}$ & $\%$ & SE & $95 \% \mathrm{Cl}$ \\
\hline Anne Arundel County, Maryland & 863 & 4.6 & 1.1 & $(2.4-6.8)$ \\
\hline Baltimore County, Maryland & 1,455 & 5.9 & 1.0 & $(3.9-7.8)$ \\
\hline Charles County, Maryland & 485 & 8.5 & 2.5 & $(3.6-13.4)$ \\
\hline Frederick County, Maryland & 724 & N/A & $\mathrm{N} / \mathrm{A}$ & $(\mathrm{N} / \mathrm{A}-\mathrm{N} / \mathrm{A})$ \\
\hline Montgomery County, Maryland & 1,456 & 5.3 & 0.8 & $(3.7-7.0)$ \\
\hline Prince George's County, Maryland & 1,084 & 4.9 & 1.2 & $(2.6-7.2)$ \\
\hline Washington County, Maryland & 526 & N/A & $\mathrm{N} / \mathrm{A}$ & $(\mathrm{N} / \mathrm{A}-\mathrm{N} / \mathrm{A})$ \\
\hline Baltimore city, Maryland & 700 & 4.5 & 1.1 & $(2.3-6.7)$ \\
\hline Barnstable County, Massachusetts & 524 & 6.3 & 1.2 & $(3.9-8.8)$ \\
\hline Bristol County, Massachusetts & 2,505 & 6.5 & 1.1 & $(4.3-8.7)$ \\
\hline Essex County, Massachusetts & 2,427 & 6.0 & 0.8 & $(4.5-7.6)$ \\
\hline Hampden County, Massachusetts & 1,860 & 7.8 & 1.3 & $(5.3-10.3)$ \\
\hline Middlesex County, Massachusetts & 4,048 & 7.1 & 0.6 & $(5.9-8.3)$ \\
\hline Norfolk County, Massachusetts & 1,660 & 7.9 & 1.0 & $(5.9-10.0)$ \\
\hline Plymouth County, Massachusetts & 1,723 & 8.0 & 1.2 & $(5.7-10.4)$ \\
\hline Suffolk County, Massachusetts & 2,170 & 8.6 & 0.9 & $(6.7-10.4)$ \\
\hline Worcester County, Massachusetts & 2,460 & 7.8 & 0.8 & $(6.2-9.3)$ \\
\hline Kent County, Michigan & 512 & 4.7 & 1.3 & $(2.1-7.4)$ \\
\hline Macomb County, Michigan & 596 & 5.6 & 1.1 & $(3.4-7.9)$ \\
\hline Oakland County, Michigan & 1,132 & 5.3 & 0.9 & $(3.4-7.1)$ \\
\hline Wayne County, Michigan & 2,146 & 5.7 & 0.8 & $(4.2-7.3)$ \\
\hline Anoka County, Minnesota & 521 & 5.4 & 1.4 & $(2.7-8.1)$ \\
\hline Dakota County, Minnesota & 648 & 9.0 & 1.4 & $(6.3-11.7)$ \\
\hline Hennepin County, Minnesota & 3,121 & 7.2 & 0.7 & $(5.8-8.5)$ \\
\hline Ramsey County, Minnesota & 1,876 & 7.8 & 1.2 & $(5.5-10.1)$ \\
\hline Jackson County, Missouri & 855 & 5.3 & 1.3 & $(2.7-7.8)$ \\
\hline St. Louis County, Missouri & 925 & 7.1 & 1.1 & $(4.9-9.3)$ \\
\hline Cascade County, Montana & 694 & 10.0 & 1.6 & $(6.9-13.0)$ \\
\hline Flathead County, Montana & 547 & 10.0 & 1.7 & $(6.7-13.2)$ \\
\hline Hill County, Montana & 566 & 8.7 & 2.3 & $(4.1-13.2)$ \\
\hline Lake County, Montana & 870 & 8.4 & 1.8 & $(4.8-11.9)$ \\
\hline Missoula County, Montana & 764 & 8.1 & 1.4 & $(5.5-10.8)$ \\
\hline Yellowstone County, Montana & 735 & 8.7 & 1.2 & $(6.4-11.0)$ \\
\hline Dakota County, Nebraska & 720 & 4.7 & 1.3 & $(2.2-7.3)$ \\
\hline Douglas County, Nebraska & 3,486 & 7.6 & 0.6 & $(6.4-8.8)$ \\
\hline Hall County, Nebraska & 526 & 7.1 & 1.5 & $(4.2-10.0)$ \\
\hline Lancaster County, Nebraska & 1,421 & 6.3 & 0.7 & $(4.8-7.7)$ \\
\hline Lincoln County, Nebraska & 574 & 5.3 & 1.3 & $(2.6-7.9)$ \\
\hline Sarpy County, Nebraska & 1,155 & 8.7 & 1.1 & $(6.5-10.9)$ \\
\hline Scotts Bluff County, Nebraska & 570 & N/A & $\mathrm{N} / \mathrm{A}$ & $(\mathrm{N} / \mathrm{A}-\mathrm{N} / \mathrm{A})$ \\
\hline Clark County, Nevada & 1,911 & 5.8 & 0.6 & $(4.5-7.0)$ \\
\hline Washoe County, Nevada & 1,435 & 7.4 & 1.0 & $(5.4-9.4)$ \\
\hline Belknap County, New Hampshire & 542 & 8.6 & 1.9 & $(4.9-12.3)$ \\
\hline Carroll County, New Hampshire & 513 & 8.4 & 1.9 & $(4.7-12.2)$ \\
\hline Cheshire County, New Hampshire & 532 & 8.2 & 1.9 & $(4.5-11.9)$ \\
\hline Coos County, New Hampshire & 520 & 7.3 & 1.7 & $(4.0-10.5)$ \\
\hline Grafton County, New Hampshire & 551 & 6.1 & 1.2 & $(3.6-8.5)$ \\
\hline Hillsborough County, New Hampshire & 1,844 & 7.5 & 1.0 & $(5.6-9.4)$ \\
\hline Merrimack County, New Hampshire & 686 & 7.3 & 1.4 & $(4.6-10.1)$ \\
\hline Rockingham County, New Hampshire & 1,012 & 7.6 & 1.1 & $(5.4-9.7)$ \\
\hline Strafford County, New Hampshire & 604 & 5.6 & 1.5 & $(2.7-8.5)$ \\
\hline Atlantic County, New Jersey & 962 & 7.4 & 1.3 & $(4.8-10.0)$ \\
\hline Bergen County, New Jersey & 869 & 4.5 & 1.0 & $(2.5-6.4)$ \\
\hline Burlington County, New Jersey & 652 & 5.3 & 1.5 & $(2.4-8.2)$ \\
\hline Camden County, New Jersey & 699 & 5.4 & 1.1 & $(3.3-7.5)$ \\
\hline Cape May County, New Jersey & 523 & 5.9 & 1.4 & $(3.3-8.6)$ \\
\hline Cumberland County, New Jersey & 529 & 3.2 & 0.8 & $(1.6-4.8)$ \\
\hline Essex County, New Jersey & 1,262 & 4.5 & 0.8 & $(3.0-6.0)$ \\
\hline Gloucester County, New Jersey & 528 & 6.1 & 1.5 & $(3.2-9.0)$ \\
\hline Hudson County, New Jersey & 1,195 & 4.7 & 0.8 & $(3.1-6.3)$ \\
\hline
\end{tabular}

See table footnotes on page 80. 
TABLE 39. (Continued) Estimated prevalence of adults aged $\geq 18$ years who reported heavy drinking* during the preceding month, by county - Behavioral Risk Factor Surveillance System, United States, 2012

\begin{tabular}{|c|c|c|c|c|}
\hline County & $\begin{array}{l}\text { Sample } \\
\text { size }\end{array}$ & $\%$ & SE & $95 \% \mathrm{Cl}$ \\
\hline Hunterdon County, New Jersey & 532 & 7.2 & 1.6 & $(4.0-10.3)$ \\
\hline Mercer County, New Jersey & 544 & 3.7 & 0.8 & $(2.1-5.3)$ \\
\hline Middlesex County, New Jersey & 835 & 4.5 & 0.8 & $(2.8-6.1)$ \\
\hline Monmouth County, New Jersey & 667 & 7.8 & 1.3 & $(5.3-10.4)$ \\
\hline Morris County, New Jersey & 799 & 6.0 & 1.1 & $(3.8-8.1)$ \\
\hline Ocean County, New Jersey & 617 & 5.6 & 1.1 & $(3.5-7.7)$ \\
\hline Passaic County, New Jersey & 643 & 7.1 & 1.4 & $(4.2-9.9)$ \\
\hline Salem County, New Jersey & 555 & N/A & N/A & $(\mathrm{N} / \mathrm{A}-\mathrm{N} / \mathrm{A})$ \\
\hline Somerset County, New Jersey & 599 & 4.2 & 1.0 & $(2.3-6.1)$ \\
\hline Sussex County, New Jersey & 516 & 7.8 & 2.0 & $(3.8-11.7)$ \\
\hline Union County, New Jersey & 662 & 3.7 & 0.9 & $(2.0-5.4)$ \\
\hline Warren County, New Jersey & 506 & 3.7 & 0.9 & $(2.0-5.4)$ \\
\hline Bernalillo County, New Mexico & 1,964 & 5.4 & 0.6 & $(4.2-6.6)$ \\
\hline Dona Ana County, New Mexico & 681 & 6.6 & 1.3 & $(4.0-9.1)$ \\
\hline Sandoval County, New Mexico & 614 & 5.8 & 1.3 & $(3.3-8.3)$ \\
\hline San Juan County, New Mexico & 619 & 4.5 & 1.1 & $(2.4-6.6)$ \\
\hline Santa Fe County, New Mexico & 665 & 8.4 & 1.3 & $(5.8-10.9)$ \\
\hline Kings County, New York & 465 & $\mathrm{~N} / \mathrm{A}$ & $\mathrm{N} / \mathrm{A}$ & $(\mathrm{N} / \mathrm{A}-\mathrm{N} / \mathrm{A})$ \\
\hline Guilford County, North Carolina & 498 & 6.1 & 1.4 & $(3.5-8.8)$ \\
\hline Mecklenburg County, North Carolina & 677 & 5.4 & 0.9 & $(3.5-7.3)$ \\
\hline Robeson County, North Carolina & 513 & $\mathrm{~N} / \mathrm{A}$ & $\mathrm{N} / \mathrm{A}$ & $(\mathrm{N} / \mathrm{A}-\mathrm{N} / \mathrm{A})$ \\
\hline Wake County, North Carolina & 664 & 5.3 & 1.0 & $(3.4-7.2)$ \\
\hline Burleigh County, North Dakota & 559 & 7.9 & 2.0 & $(4.0-11.9)$ \\
\hline Cass County, North Dakota & 823 & 4.7 & 1.1 & $(2.6-6.8)$ \\
\hline Cuyahoga County, Ohio & 795 & 7.1 & 1.2 & $(4.8-9.3)$ \\
\hline Franklin County, Ohio & 794 & 6.0 & 1.0 & $(4.1-7.9)$ \\
\hline Hamilton County, Ohio & 739 & 6.6 & 1.2 & $(4.3-8.9)$ \\
\hline Lorain County, Ohio & 583 & 8.2 & 2.1 & $(4.2-12.3)$ \\
\hline Lucas County, Ohio & 593 & 6.5 & 1.9 & $(2.9-10.2)$ \\
\hline Mahoning County, Ohio & 564 & 8.6 & 2.5 & $(3.7-13.5)$ \\
\hline Montgomery County, Ohio & 639 & 10.6 & 2.0 & $(6.7-14.5)$ \\
\hline Stark County, Ohio & 595 & 7.0 & 1.6 & $(3.9-10.2)$ \\
\hline Summit County, Ohio & 603 & 7.9 & 1.6 & $(4.7-11.1)$ \\
\hline Oklahoma County, Oklahoma & 1,161 & 4.7 & 0.8 & $(3.2-6.2)$ \\
\hline Tulsa County, Oklahoma & 1,171 & 4.7 & 0.7 & $(3.3-6.2)$ \\
\hline Clackamas County, Oregon & 490 & 6.4 & 1.3 & $(3.8-9.0)$ \\
\hline Lane County, Oregon & 507 & 7.5 & 1.5 & $(4.5-10.5)$ \\
\hline Multnomah County, Oregon & 855 & 9.8 & 1.4 & $(7.0-12.5)$ \\
\hline Washington County, Oregon & 599 & 7.2 & 1.3 & $(4.5-9.8)$ \\
\hline Allegheny County, Pennsylvania & 1,827 & 8.0 & 0.9 & $(6.1-9.8)$ \\
\hline Bradford County, Pennsylvania & 1,807 & 4.4 & 0.6 & $(3.3-5.5)$ \\
\hline Montgomery County, Pennsylvania & 528 & 4.7 & 1.0 & $(2.8-6.5)$ \\
\hline Philadelphia County, Pennsylvania & 1,948 & 7.0 & 0.9 & $(5.2-8.7)$ \\
\hline Pike County, Pennsylvania & 1,834 & 4.0 & 0.5 & $(3.1-4.9)$ \\
\hline Kent County, Rhode Island & 790 & 3.1 & 0.7 & $(1.8-4.4)$ \\
\hline Providence County, Rhode Island & 3,211 & 6.5 & 0.6 & $(5.2-7.7)$ \\
\hline Washington County, Rhode Island & 640 & 5.0 & 1.0 & $(3.0-7.0)$ \\
\hline Aiken County, South Carolina & 538 & 5.7 & 1.4 & $(3.0-8.3)$ \\
\hline Beaufort County, South Carolina & 774 & 9.1 & 1.8 & $(5.6-12.7)$ \\
\hline
\end{tabular}

TABLE 39. (Continued) Estimated prevalence of adults aged $\geq 18$ years who reported heavy drinking* during the preceding month, by county - Behavioral Risk Factor Surveillance System, United States, 2012

\begin{tabular}{|c|c|c|c|c|}
\hline County & $\begin{array}{l}\text { Sample } \\
\text { size }\end{array}$ & $\%$ & SE & $95 \% \mathrm{Cl}$ \\
\hline Charleston County, South Carolina & 977 & 10.3 & 1.4 & $(7.5-13.0)$ \\
\hline Greenville County, South Carolina & 876 & 5.4 & 1.0 & $(3.3-7.4)$ \\
\hline Horry County, South Carolina & 757 & 5.9 & 1.1 & $(3.8-8.0)$ \\
\hline Richland County, South Carolina & 927 & 7.4 & 1.3 & $(4.9-10.0)$ \\
\hline Spartanburg County, South Carolina & 637 & 4.6 & 1.1 & $(2.4-6.8)$ \\
\hline Lincoln County, South Dakota & 489 & 6.6 & 1.6 & $(3.5-9.6)$ \\
\hline Minnehaha County, South Dakota & 862 & 6.0 & 0.8 & $(4.4-7.6)$ \\
\hline Pennington County, South Dakota & 591 & 6.5 & 1.1 & $(4.3-8.7)$ \\
\hline Davidson County, Tennessee & 518 & 4.9 & 1.2 & $(2.5-7.3)$ \\
\hline Shelby County, Tennessee & 494 & N/A & $\mathrm{N} / \mathrm{A}$ & $(\mathrm{N} / \mathrm{A}-\mathrm{N} / \mathrm{A})$ \\
\hline Bexar County, Texas & 566 & 7.4 & 1.7 & $(4.0-10.7)$ \\
\hline Dallas County, Texas & 484 & 4.5 & 1.0 & $(2.5-6.5)$ \\
\hline El Paso County, Texas & 597 & 5.2 & 1.5 & $(2.2-8.3)$ \\
\hline Harris County, Texas & 732 & 5.0 & 0.9 & $(3.3-6.8)$ \\
\hline Hidalgo County, Texas & 600 & N/A & $\mathrm{N} / \mathrm{A}$ & $(\mathrm{N} / \mathrm{A}-\mathrm{N} / \mathrm{A})$ \\
\hline Tarrant County, Texas & 553 & 6.9 & 1.5 & $(3.9-9.9)$ \\
\hline Travis County, Texas & 1,019 & 8.3 & 1.6 & $(5.3-11.4)$ \\
\hline Davis County, Utah & 1,119 & 2.1 & 0.5 & $(1.0-3.2)$ \\
\hline Salt Lake County, Utah & 3,935 & 4.6 & 0.4 & $(3.7-5.4)$ \\
\hline Tooele County, Utah & 550 & N/A & $\mathrm{N} / \mathrm{A}$ & $(\mathrm{N} / \mathrm{A}-\mathrm{N} / \mathrm{A})$ \\
\hline Utah County, Utah & 1,640 & 1.9 & 0.5 & $(1.0-2.9)$ \\
\hline Wasatch County, Utah & 497 & N/A & $\mathrm{N} / \mathrm{A}$ & $(\mathrm{N} / \mathrm{A}-\mathrm{N} / \mathrm{A})$ \\
\hline Weber County, Utah & 1,018 & 3.0 & 0.7 & $(1.6-4.4)$ \\
\hline Chittenden County, Vermont & 892 & 9.1 & 1.1 & $(6.9-11.3)$ \\
\hline Rutland County, Vermont & 582 & 5.5 & 1.2 & $(3.2-7.7)$ \\
\hline Washington County, Vermont & 507 & 8.7 & 1.5 & $(5.8-11.5)$ \\
\hline Windsor County, Vermont & 528 & 6.0 & 1.2 & $(3.6-8.4)$ \\
\hline Fairfax County, Virginia & 693 & 4.3 & 0.9 & $(2.6-6.0)$ \\
\hline Clark County, Washington & 774 & 5.5 & 1.1 & $(3.4-7.6)$ \\
\hline King County, Washington & 3,849 & 6.7 & 0.5 & $(5.7-7.8)$ \\
\hline Kitsap County, Washington & 553 & 5.1 & 1.1 & $(3.0-7.3)$ \\
\hline Pierce County, Washington & 1,162 & 6.1 & 0.9 & $(4.4-7.8)$ \\
\hline Snohomish County, Washington & 1,143 & 6.0 & 0.9 & $(4.3-7.8)$ \\
\hline Spokane County, Washington & 929 & 8.1 & 1.2 & $(5.7-10.5)$ \\
\hline Thurston County, Washington & 508 & 6.3 & 1.3 & $(3.7-8.9)$ \\
\hline Whatcom County, Washington & 832 & 8.0 & 1.7 & $(4.7-11.3)$ \\
\hline Yakima County, Washington & 524 & N/A & $\mathrm{N} / \mathrm{A}$ & $(\mathrm{N} / \mathrm{A}-\mathrm{N} / \mathrm{A})$ \\
\hline Kanawha County, West Virginia & 633 & 3.1 & 0.8 & $(1.5-4.7)$ \\
\hline Milwaukee County, Wisconsin & 891 & 8.2 & 1.4 & $(5.5-10.8)$ \\
\hline Laramie County, Wyoming & 921 & 6.6 & 1.5 & $(3.6-9.6)$ \\
\hline Natrona County, Wyoming & 801 & 4.4 & 1.2 & $(2.1-6.8)$ \\
\hline San Juan Municipio, Puerto Rico & 652 & 5.9 & 1.1 & $(3.8-8.1)$ \\
\hline Median & \multicolumn{4}{|c|}{6.} \\
\hline Range & \multicolumn{4}{|c|}{$1.9-11.0$} \\
\hline
\end{tabular}

Abbreviations: $\mathrm{Cl}=$ confidence interval; $\mathrm{SE}=$ standard error.

* For adult men: having more than two drinks per day; for adult women: having more than one drink per day.

$\dagger$ Estimate not available (N/A) if the unweighted sample size for the denominator was $<50$ or if the relative standard error is $>0.3$. 
TABLE 40 . Estimated prevalence of adults aged $\geq 18$ years who reported no leisure time of physical activity* during the preceding month, by state/territory - Behavioral Risk Factor Surveillance System, United States, 2012

\begin{tabular}{|c|c|c|c|c|}
\hline State/Territory & $\begin{array}{c}\text { Sample } \\
\text { size }\end{array}$ & $\%$ & SE & $95 \% \mathrm{Cl}$ \\
\hline Alabama & 9,014 & 27.2 & 0.7 & (25.9-28.6) \\
\hline Alaska & 4,336 & 18.5 & 0.8 & $(16.8-20.1)$ \\
\hline Arizona & 7,285 & 22.6 & 0.8 & $(20.9-24.2)$ \\
\hline Arkansas & 5,174 & 31.5 & 0.9 & $(29.8-33.2)$ \\
\hline California & 13,671 & 19.2 & 0.5 & $(18.2-20.2)$ \\
\hline Colorado & 12,239 & 17.0 & 0.5 & $(16.1-18.0)$ \\
\hline Connecticut & 8,760 & 22.1 & 0.6 & $(20.9-23.4)$ \\
\hline Delaware & 5,172 & 23.5 & 0.8 & $(21.9-25.1)$ \\
\hline District of Columbia & 3,817 & 17.4 & 0.9 & $(15.6-19.2)$ \\
\hline Florida & 7,613 & 23.3 & 0.8 & $(21.8-24.8)$ \\
\hline Georgia & 6,091 & 23.6 & 0.7 & $(22.1-25.0)$ \\
\hline Hawaii & 7,580 & 18.7 & 0.7 & $(17.3-20.1)$ \\
\hline Idaho & 5,889 & 20.3 & 0.9 & $(18.5-22.1)$ \\
\hline Illinois & 5,578 & 21.8 & 0.8 & $(20.2-23.4)$ \\
\hline Indiana & 8,628 & 25.9 & 0.6 & $(24.7-27.1)$ \\
\hline lowa & 7,160 & 23.1 & 0.6 & $(21.9-24.3)$ \\
\hline Kansas & 11,793 & 22.9 & 0.5 & (21.9-24.0) \\
\hline Kentucky & 11,201 & 29.7 & 0.7 & $(28.4-31.0)$ \\
\hline Louisiana & 9,062 & 29.9 & 0.8 & $(28.3-31.4)$ \\
\hline Maine & 9,905 & 20.9 & 0.5 & $(19.9-21.9)$ \\
\hline Maryland & 12,798 & 23.1 & 0.6 & $(21.8-24.3)$ \\
\hline Massachusetts & 21,691 & 19.8 & 0.4 & $(18.9-20.6)$ \\
\hline Michigan & 10,490 & 23.3 & 0.6 & $(22.2-24.5)$ \\
\hline Minnesota & 12,226 & 17.6 & 0.5 & $(16.6-18.5)$ \\
\hline Mississippi & 7,781 & 30.8 & 0.8 & $(29.3-32.3)$ \\
\hline Missouri & 6,751 & 24.7 & 0.7 & $(23.2-26.1)$ \\
\hline Montana & 8,669 & 20.5 & 0.6 & $(19.4-21.6)$ \\
\hline Nebraska & 19,153 & 21.0 & 0.4 & $(20.2-21.9)$ \\
\hline Nevada & 4,837 & 21.3 & 0.9 & $(19.6-23.0)$ \\
\hline New Hampshire & 7,508 & 20.0 & 0.7 & $(18.7-21.3)$ \\
\hline New Jersey & 15,732 & 24.9 & 0.5 & $(23.9-25.9)$ \\
\hline New Mexico & 8,773 & 21.8 & 0.6 & $(20.7-22.9)$ \\
\hline New York & 6,045 & 24.7 & 0.8 & $(23.1-26.3)$ \\
\hline North Carolina & 11,889 & 24.9 & 0.5 & $(23.9-25.9)$ \\
\hline North Dakota & 4,873 & 23.8 & 0.8 & $(22.2-25.4)$ \\
\hline Ohio & 12,996 & 25.3 & 0.5 & $(24.3-26.3)$ \\
\hline Oklahoma & 8,014 & 28.3 & 0.7 & $(27.0-29.6)$ \\
\hline Oregon & 5,292 & 16.3 & 0.7 & $(14.9-17.6)$ \\
\hline Pennsylvania & 19,912 & 23.5 & 0.4 & $(22.6-24.3)$ \\
\hline Rhode Island & 5,465 & 23.6 & 0.8 & $(22.0-25.1)$ \\
\hline South Carolina & 12,777 & 25.1 & 0.6 & $(24.0-26.2)$ \\
\hline South Dakota & 7,874 & 22.5 & 0.8 & $(21.0-24.1)$ \\
\hline Tennessee & 7,048 & 28.6 & 0.7 & $(27.2-30.1)$ \\
\hline Texas & 9,113 & 27.2 & 0.7 & $(25.9-28.5)$ \\
\hline Utah & 12,403 & 16.5 & 0.5 & $(15.6-17.4)$ \\
\hline Vermont & 6,048 & 17.2 & 0.6 & $(16.0-18.4)$ \\
\hline Virginia & 7,388 & 22.5 & 0.6 & $(21.3-23.7)$ \\
\hline Washington & 15,305 & 19.0 & 0.4 & $(18.1-19.8)$ \\
\hline West Virginia & 5,408 & 31.0 & 0.7 & $(29.5-32.4)$ \\
\hline Wisconsin & 5,290 & 20.4 & 0.8 & $(18.7-22.0)$ \\
\hline Wyoming & 6,260 & 21.1 & 0.8 & $(19.5-22.7)$ \\
\hline Guam & 2,031 & 27.6 & 1.3 & $(24.9-30.2)$ \\
\hline Puerto Rico & 6,320 & 42.4 & 0.7 & $(41.0-43.9)$ \\
\hline Median & & 23.1 & & \\
\hline Range & & $16.3-42.4$ & & \\
\hline
\end{tabular}

Abbreviations: $\mathrm{Cl}=$ confidence interval; $\mathrm{SE}=$ standard error.

* Any physical activities or exercises such as running, calisthenics, golf, gardening, or walking for exercise.
TABLE 41. Estimated prevalence of adults aged $\geq 18$ years who reported no leisure time of physical activity* during the preceding month, by metropolitan and micropolitan statistical area Behavioral Risk Factor Surveillance System, United States, 2012

\begin{tabular}{|c|c|c|c|c|}
\hline MMSA(s) & $\begin{array}{l}\text { Sample } \\
\text { size }\end{array}$ & $\%$ & SE & $95 \% \mathrm{Cl}$ \\
\hline Aguadilla-Isabela, Puerto Rico & 551 & 47.3 & 2.5 & $(42.4-52.1)$ \\
\hline Akron, Ohio & 743 & 22.3 & 2.0 & $(18.3-26.2)$ \\
\hline Albuquerque, New Mexico & 3,269 & 18.9 & 0.8 & $(17.2-20.6)$ \\
\hline $\begin{array}{l}\text { Allentown-Bethlehem-Easton, } \\
\text { Pennsylvania-New Jersey }\end{array}$ & 1,352 & 21.0 & 1.6 & $(17.8-24.2)$ \\
\hline Anaheim-Santa Ana-Irvine, California ${ }^{\dagger}$ & 963 & 15.6 & 1.6 & $(12.4-18.8)$ \\
\hline Anchorage, Alaska & 1,503 & 16.6 & 1.2 & $(14.2-19.0)$ \\
\hline Asheville, North Carolina & 594 & 21.3 & 2.0 & $(17.4-25.2)$ \\
\hline $\begin{array}{l}\text { Atlanta-Sandy Springs-Roswell, } \\
\text { Georgia }\end{array}$ & 2,539 & 19.0 & 1.1 & $(16.9-21.0)$ \\
\hline Atlantic City-Hammonton, New Jersey & 1,023 & 25.9 & 1.9 & $(22.2-29.6)$ \\
\hline $\begin{array}{l}\text { Augusta-Richmond County, Georgia- } \\
\text { South Carolina }\end{array}$ & 1,034 & 24.2 & 2.3 & $(19.6-28.8)$ \\
\hline Augusta-Waterville, Maine & 832 & 23.1 & 1.8 & $(19.5-26.6)$ \\
\hline Austin-Round Rock, Texas & 1,385 & 19.8 & 1.7 & $(16.4-23.2)$ \\
\hline $\begin{array}{l}\text { Baltimore-Columbia-Towson, } \\
\text { Maryland }\end{array}$ & 4,724 & 23.4 & 0.9 & $(21.5-25.2)$ \\
\hline Bangor, Maine & 928 & 24.4 & 1.8 & $(21.0-27.8)$ \\
\hline Barnstable Town, Massachusetts & 559 & 17.3 & 2.1 & $(13.1-21.4)$ \\
\hline Barre, Vermont & 516 & 12.3 & 1.6 & $(9.2-15.4)$ \\
\hline Baton Rouge, Louisiana & 1,390 & 27.2 & 1.9 & $(23.4-31.0)$ \\
\hline Bellingham, Washington & 847 & 18.2 & 2.3 & $(13.8-22.6)$ \\
\hline Berlin, New Hampshire-Vermont & 708 & 28.9 & 2.6 & $(23.8-34.0)$ \\
\hline Billings, Montana & 848 & 21.4 & 1.6 & $(18.3-24.5)$ \\
\hline Birmingham-Hoover, Alabama & 1,823 & 24.6 & 1.4 & $(21.9-27.3)$ \\
\hline Bismarck, North Dakota & 822 & 21.9 & 2.0 & $(18.0-25.9)$ \\
\hline Boise City, Idaho & 1,481 & 18.5 & 1.5 & $(15.5-21.4)$ \\
\hline Boston, Massachusetts ${ }^{\dagger}$ & 5,908 & 19.9 & 0.8 & $(18.3-21.5)$ \\
\hline Boulder, Colorado & 516 & 9.2 & 1.7 & $(6.0-12.5)$ \\
\hline Bremerton-Silverdale, Washington & 563 & 19.2 & 2.0 & $(15.2-23.2)$ \\
\hline $\begin{array}{l}\text { Bridgeport-Stamford-Norwalk, } \\
\text { Connecticut }\end{array}$ & 2,182 & 21.6 & 1.3 & $(19.0-24.1)$ \\
\hline Burlington-South Burlington, Vermont & 1,520 & 14.9 & 1.1 & $(12.8-17.0)$ \\
\hline $\begin{array}{l}\text { Cambridge-Newton-Framingham, } \\
\text { Massachusetts }^{\dagger}\end{array}$ & 6,926 & 18.8 & 0.7 & $(17.4-20.2)$ \\
\hline Camden, New Jersey ${ }^{\dagger}$ & 1,983 & 27.1 & 1.4 & $(24.4-29.8)$ \\
\hline Canton-Massillon, Ohio & 680 & 24.6 & 2.2 & $(20.3-28.9)$ \\
\hline Casper, Wyoming & 830 & 19.5 & 2.0 & $(15.5-23.5)$ \\
\hline Cedar Rapids, lowa & 584 & 21.2 & 2.0 & $(17.2-25.2)$ \\
\hline Charleston, West Virginia & 772 & 29.6 & 1.9 & $(25.9-33.3)$ \\
\hline $\begin{array}{l}\text { Charleston-North Charleston, } \\
\text { South Carolina }\end{array}$ & 1,708 & 20.9 & 1.4 & $(18.2-23.7)$ \\
\hline $\begin{array}{l}\text { Charlotte-Concord-Gastonia, } \\
\text { North Carolina-South Carolina }\end{array}$ & 2,524 & 21.4 & 1.0 & $(19.4-23.4)$ \\
\hline Chattanooga, Tennessee-Georgia & 631 & 26.2 & 2.7 & $(21.0-31.4)$ \\
\hline Cheyenne, Wyoming & 955 & 21.2 & 2.0 & $(17.2-25.2)$ \\
\hline $\begin{array}{l}\text { Chicago-Naperville-Elgin, } \\
\text { Illinois-Indiana-Wisconsin }\end{array}$ & 3,731 & 20.9 & 1.0 & $(18.9-22.9)$ \\
\hline Cincinnati, Ohio-Kentucky-Indiana & 2,362 & 24.1 & 1.2 & $(21.8-26.3)$ \\
\hline $\begin{array}{l}\text { Claremont-Lebanon, New } \\
\text { Hampshire-Vermont }\end{array}$ & 1,948 & 19.1 & 1.3 & $(16.6-21.6)$ \\
\hline Cleveland-Elyria, Ohio & 1,776 & 23.7 & 1.4 & $(21.0-26.4)$ \\
\hline Colorado Springs, Colorado & 1,162 & 16.6 & 1.4 & $(14.0-19.3)$ \\
\hline Columbia, South Carolina & 1,799 & 23.3 & 1.4 & $(20.5-26.1)$ \\
\hline Columbus, Ohio & 1,602 & 22.8 & 1.3 & $(20.2-25.4)$ \\
\hline Concord, New Hampshire & 705 & 17.3 & 1.8 & $(13.8-20.8)$ \\
\hline Dallas-Plano-Irving, Texas ${ }^{\dagger}$ & 908 & 24.9 & 1.8 & $(21.4-28.4)$ \\
\hline Dayton, Ohio & 857 & 24.9 & 1.9 & $(21.2-28.6)$ \\
\hline Denver-Aurora-Lakewood, Colorado & 4,848 & 17.1 & 0.7 & $(15.7-18.5)$ \\
\hline Des Moines-West Des Moines, lowa & 1,160 & 21.7 & 1.5 & $(18.8-24.7)$ \\
\hline
\end{tabular}

See table footnotes on page 83. 
TABLE 41. (Continued) Estimated prevalence of adults aged $\geq 18$ years who reported no leisure time of physical activity* during the preceding month, by metropolitan and micropolitan statistical area — Behavioral Risk Factor Surveillance System, United States, 2012

\begin{tabular}{|c|c|c|c|c|}
\hline MMSA(s) & $\begin{array}{l}\text { Sample } \\
\text { size }\end{array}$ & $\%$ & SE & $95 \% \mathrm{Cl}$ \\
\hline Detroit-Dearborn-Livonia, Michigan $^{\dagger}$ & 2,219 & 28.2 & 1.6 & $(25.1-31.2)$ \\
\hline Dover, Delaware & 1,440 & 26.4 & 1.7 & $(23.1-29.7)$ \\
\hline Duluth, Minnesota-Wisconsin & 514 & 15.9 & 2.2 & $(11.7-20.2)$ \\
\hline Durham-Chapel Hill, North Carolina & 796 & 22.5 & 1.9 & $(18.7-26.2)$ \\
\hline El Paso, Texas & 626 & 32.0 & 3.0 & $(26.1-37.9)$ \\
\hline Eugene, Oregon & 526 & 14.4 & 2.1 & $(10.2-18.5)$ \\
\hline Fairbanks, Alaska & 599 & 18.9 & 2.1 & $(14.8-23.0)$ \\
\hline Fargo, North Dakota-Minnesota & 939 & 18.5 & 1.7 & $(15.2-21.8)$ \\
\hline Farmington, New Mexico & 650 & 23.2 & 2.1 & $(19.1-27.3)$ \\
\hline Fayetteville, North Carolina & 506 & 23.9 & 2.3 & $(19.3-28.4)$ \\
\hline $\begin{array}{l}\text { Fayetteville-Springdale-Rogers, } \\
\text { Arkansas-Missouri }\end{array}$ & 810 & 25.2 & 2.2 & $(20.8-29.5)$ \\
\hline Fort Collins, Colorado & 595 & 12.4 & 1.6 & $(9.2-15.6)$ \\
\hline Fort Wayne, Indiana & 539 & 21.9 & 2.1 & $(17.7-26.1)$ \\
\hline Fort Worth-Arlington, Texas ${ }^{\dagger}$ & 727 & 28.8 & 2.3 & $(24.4-33.3)$ \\
\hline Grand Island, Nebraska & 855 & 23.3 & 1.9 & $(19.5-27.1)$ \\
\hline Grand Rapids-Wyoming, Michigan & 892 & 20.1 & 1.9 & $(16.3-23.9)$ \\
\hline Great Falls, Montana & 707 & 26.9 & 2.1 & $(22.8-31.0)$ \\
\hline Greeley, Colorado & 533 & 23.8 & 2.5 & $(18.9-28.6)$ \\
\hline $\begin{array}{l}\text { Greensboro-High Point, } \\
\text { North Carolina }\end{array}$ & 808 & 27.3 & 1.9 & $(23.5-31.1)$ \\
\hline $\begin{array}{l}\text { Greenville-Anderson-Mauldin, } \\
\text { South Carolina }\end{array}$ & 1,689 & 26.8 & 1.5 & $(23.9-29.7)$ \\
\hline Gulfport-Biloxi-Pascagoula, Mississippi & 742 & 25.0 & 2.0 & $(21.1-28.9)$ \\
\hline $\begin{array}{l}\text { Hagerstown-Martinsburg, Maryland- } \\
\text { West Virginia }\end{array}$ & 759 & 29.2 & 3.0 & $(23.2-35.2)$ \\
\hline Harrisburg-Carlisle, Pennsylvania & 665 & 21.2 & 1.9 & $(17.4-25.0)$ \\
\hline $\begin{array}{l}\text { Hartford-West Hartford-East Hartford, } \\
\text { Connecticut }\end{array}$ & 2,662 & 21.0 & 1.0 & $(18.9-23.0)$ \\
\hline Heber, Utah & 512 & 11.0 & 1.8 & $(7.6-14.5)$ \\
\hline Hilo, Hawaii & 1,349 & 20.5 & 1.6 & $(17.3-23.7)$ \\
\hline $\begin{array}{l}\text { Hilton Head Island-Bluffton-Beaufort, } \\
\text { South Carolina }\end{array}$ & 936 & 17.7 & 2.1 & $(13.6-21.8)$ \\
\hline $\begin{array}{l}\text { Houston-The Woodlands-Sugar Land, } \\
\text { Texas }\end{array}$ & 1,145 & 27.0 & 1.7 & $(23.7-30.4)$ \\
\hline $\begin{array}{l}\text { Huntington-Ashland, West } \\
\text { Virginia-Kentucky-Ohio }\end{array}$ & 1,113 & 34.2 & 1.9 & $(30.5-37.8)$ \\
\hline Huntsville, Alabama & 616 & 26.2 & 2.4 & $(21.5-30.9)$ \\
\hline Idaho Falls, Idaho & 541 & 20.9 & 2.9 & $(15.2-26.6)$ \\
\hline $\begin{array}{l}\text { Indianapolis-Carmel-Anderson, } \\
\text { Indiana }\end{array}$ & 2,196 & 24.1 & 1.2 & $(21.8-26.4)$ \\
\hline Jackson, Mississippi & 922 & 28.2 & 1.8 & $(24.7-31.7)$ \\
\hline Jacksonville, Florida & 517 & 18.5 & 2.2 & $(14.2-22.8)$ \\
\hline Kahului-Wailuku-Lahaina, Hawaii & 1,218 & 18.9 & 1.8 & $(15.4-22.4)$ \\
\hline Kalispell, Montana & 560 & 15.7 & 1.8 & $(12.1-19.4)$ \\
\hline Kansas City, Missouri-Kansas & 4,744 & 20.5 & 1.1 & $(18.4-22.5)$ \\
\hline Kapaa, Hawaii & 670 & 14.9 & 1.9 & $(11.2-18.6)$ \\
\hline Keene, New Hampshire & 547 & 17.1 & 2.1 & $(12.9-21.3)$ \\
\hline Kennewick-Richland, Washington & 535 & 21.3 & 2.3 & $(16.7-25.8)$ \\
\hline $\begin{array}{l}\text { Kingsport-Bristol-Bristol, } \\
\text { Tennessee-Virginia }\end{array}$ & 568 & 33.0 & 2.9 & $(27.4-38.6)$ \\
\hline Knoxville, Tennessee & 834 & 29.6 & 2.1 & $(25.5-33.6)$ \\
\hline Laconia, New Hampshire & 566 & 27.0 & 3.0 & $(21.1-32.9)$ \\
\hline Lafayette, Louisiana & 557 & 26.0 & 2.4 & $(21.3-30.8)$ \\
\hline Las Cruces, New Mexico & 708 & 26.2 & 2.2 & $(21.8-30.6)$ \\
\hline $\begin{array}{l}\text { Las Vegas-Henderson-Paradise, } \\
\text { Nevada }\end{array}$ & 2,015 & 21.9 & 1.1 & $(19.7-24.1)$ \\
\hline Lewiston-Auburn, Maine & 700 & 24.0 & 1.9 & $(20.3-27.8)$ \\
\hline Lexington-Fayette, Kentucky & 531 & 25.5 & 2.3 & $(20.9-30.1)$ \\
\hline Lincoln, Nebraska & 1,668 & 15.9 & 1.1 & $(13.7-18.0)$ \\
\hline
\end{tabular}

See table footnotes on page 83 .
TABLE 41. (Continued) Estimated prevalence of adults aged $\geq 18$ years who reported no leisure time of physical activity* during the preceding month, by metropolitan and micropolitan statistical area — Behavioral Risk Factor Surveillance System, United States, 2012

\begin{tabular}{|c|c|c|c|c|}
\hline MMSA(s) & $\begin{array}{l}\text { Sample } \\
\text { size }\end{array}$ & $\%$ & SE & $95 \% \mathrm{Cl}$ \\
\hline $\begin{array}{l}\text { Little Rock-North Little Rock-Conway, } \\
\text { Arkansas }\end{array}$ & 1,172 & 26.6 & 1.7 & $(23.3-30.0)$ \\
\hline Logan, Utah-Idaho & 505 & 13.3 & 2.0 & $(9.4-17.1)$ \\
\hline $\begin{array}{l}\text { Los Angeles-Long Beach-Glendale, } \\
\text { California }^{\dagger}\end{array}$ & 3,229 & 21.6 & 1.1 & $(19.5-23.6)$ \\
\hline $\begin{array}{l}\text { Louisville/Jefferson County, } \\
\text { Kentucky-Indiana }\end{array}$ & 2,180 & 26.3 & 1.4 & $(23.5-29.1)$ \\
\hline Lumberton, North Carolina & 546 & 39.0 & 4.4 & $(30.3-47.6)$ \\
\hline Manchester-Nashua, New Hampshire & 1,901 & 21.1 & 1.2 & $(18.6-23.5)$ \\
\hline McAllen-Edinburg-Mission, Texas & 622 & 36.6 & 3.1 & $(30.5-42.7)$ \\
\hline $\begin{array}{l}\text { Memphis, } \\
\text { Tennessee-Mississippi-Arkansas }\end{array}$ & 1,309 & 28.1 & 1.7 & $(24.7-31.5)$ \\
\hline $\begin{array}{l}\text { Miami-Fort Lauderdale-West Palm } \\
\text { Beach, Florida }\end{array}$ & 1,668 & 25.3 & 1.6 & $(22.1-28.5)$ \\
\hline $\begin{array}{l}\text { Milwaukee-Waukesha-West Allis, } \\
\text { Wisconsin }\end{array}$ & 1,256 & 17.9 & 1.6 & $(14.8-21.0)$ \\
\hline $\begin{array}{l}\text { Minneapolis-St. Paul-Bloomington, } \\
\text { Minnesota-Wisconsin }\end{array}$ & 7,895 & 16.6 & 0.6 & $(15.3-17.8)$ \\
\hline Missoula, Montana & 782 & 16.8 & 1.6 & $(13.5-20.0)$ \\
\hline Mobile, Alabama & 815 & 26.4 & 2.4 & $(21.8-31.0)$ \\
\hline Montgomery, Alabama & 532 & 27.1 & 2.6 & $(22.0-32.2)$ \\
\hline $\begin{array}{l}\text { Montgomery County-Bucks County- } \\
\text { Chester County, Pennsylvania }{ }^{\dagger}\end{array}$ & 1,315 & 18.3 & 1.3 & $(15.7-21.0)$ \\
\hline $\begin{array}{l}\text { Myrtle Beach-Conway-North Myrtle } \\
\text { Beach, South Carolina-North Carolina }\end{array}$ & 949 & 22.5 & 1.7 & $(19.1-25.9)$ \\
\hline $\begin{array}{l}\text { Nashville-Davidson-Murfreesboro- } \\
\text { Franklin, Tennessee }\end{array}$ & 1,341 & 25.7 & 1.6 & $(22.5-28.8)$ \\
\hline $\begin{array}{l}\text { Nassau County-Suffolk County, } \\
\text { New York }^{\dagger}\end{array}$ & 899 & 21.4 & 1.7 & $(18.0-24.8)$ \\
\hline Newark, New Jersey-Pennsylvania ${ }^{\dagger}$ & 6,548 & 24.9 & 0.9 & $(23.2-26.6)$ \\
\hline New Haven-Milford, Connecticut & 2,008 & 24.1 & 1.4 & $(21.3-26.8)$ \\
\hline New Orleans-Metairie, Louisiana & 1,275 & 26.2 & 1.7 & $(22.8-29.6)$ \\
\hline $\begin{array}{l}\text { New York-Jersey City-White Plains, } \\
\text { New York-New Jersey }{ }^{\dagger}\end{array}$ & 7,435 & 25.9 & 1.0 & $(23.9-27.8)$ \\
\hline Norfolk, Nebraska & 570 & 26.7 & 2.6 & $(21.7-31.7)$ \\
\hline North Platte, Nebraska & 613 & 22.5 & 2.5 & $(17.6-27.4)$ \\
\hline Norwich-New London, Connecticut & 999 & 22.2 & 2.0 & $(18.3-26.2)$ \\
\hline $\begin{array}{l}\text { Oakland-Hayward-Berkeley, } \\
\text { California }^{\dagger}\end{array}$ & 922 & 16.2 & 2.0 & $(12.2-20.1)$ \\
\hline Ocean City, New Jersey & 553 & 25.3 & 3.1 & $(19.3-31.3)$ \\
\hline Ogden-Clearfield, Utah & 2,428 & 16.5 & 1.0 & $(14.5-18.4)$ \\
\hline Oklahoma City, Oklahoma & 2,443 & 25.8 & 1.1 & $(23.7-28.0)$ \\
\hline Olympia-Tumwater, Washington & 517 & 18.8 & 2.4 & $(14.1-23.6)$ \\
\hline Omaha-Council Bluffs, Nebraska-lowa & 5,585 & 21.5 & 0.8 & $(20.0-23.0)$ \\
\hline Orlando-Kissimmee-Sanford, Florida & 569 & 22.3 & 2.6 & $(17.1-27.5)$ \\
\hline Philadelphia, Pennsylvania ${ }^{\dagger}$ & 2,460 & 25.9 & 1.2 & $(23.5-28.3)$ \\
\hline Phoenix-Mesa-Scottsdale, Arizona & 2,600 & 22.7 & 1.2 & $(20.5-25.0)$ \\
\hline Pittsburgh, Pennsylvania & 3,362 & 24.4 & 0.9 & $(22.6-26.2)$ \\
\hline Ponce, Puerto Rico & 549 & 40.7 & 2.5 & $(35.9-45.6)$ \\
\hline Portland-South Portland, Maine & 3,333 & 16.5 & 0.8 & $(15.0-18.0)$ \\
\hline $\begin{array}{l}\text { Portland-Vancouver-Hillsboro, } \\
\text { Oregon-Washington }\end{array}$ & 3,125 & 15.5 & 0.9 & $(13.7-17.2)$ \\
\hline $\begin{array}{l}\text { Providence-Warwick, Rhode } \\
\text { Island-Massachusetts }\end{array}$ & 8,116 & 23.0 & 0.7 & $(21.6-24.4)$ \\
\hline Provo-Orem, Utah & 1,748 & 14.5 & 1.1 & $(12.4-16.6)$ \\
\hline Raleigh, North Carolina & 946 & 19.8 & 1.6 & $(16.7-22.9)$ \\
\hline Rapid City, South Dakota & 1,054 & 21.1 & 1.7 & $(17.9-24.4)$ \\
\hline Reno, Nevada & 1,505 & 17.5 & 1.4 & $(14.7-20.2)$ \\
\hline Richmond, Virginia & 1,012 & 21.5 & 1.7 & $(18.1-24.8)$ \\
\hline
\end{tabular}

See table footnotes on page 83. 
TABLE 41. (Continued) Estimated prevalence of adults aged $\geq 18$ years who reported no leisure time of physical activity* during the preceding month, by metropolitan and micropolitan statistical area — Behavioral Risk Factor Surveillance System, United States, 2012

\begin{tabular}{|c|c|c|c|c|}
\hline MMSA(s) & $\begin{array}{l}\text { Sample } \\
\text { size }\end{array}$ & $\%$ & SE & $95 \% \mathrm{Cl}$ \\
\hline $\begin{array}{l}\text { Riverside-San Bernardino-Ontario, } \\
\text { California }\end{array}$ & 1,432 & 20.8 & 1.5 & $(17.8-23.7)$ \\
\hline $\begin{array}{l}\text { Rockingham County-Strafford County, } \\
\text { New Hampshire }\end{array}$ & 1,671 & 19.3 & 1.3 & $(16.9-21.8)$ \\
\hline Rutland, Vermont & 597 & 21.1 & 2.3 & $(16.5-25.7)$ \\
\hline $\begin{array}{l}\text { Sacramento-Roseville-Arden-Arcade, } \\
\text { California }\end{array}$ & 947 & 15.6 & 1.9 & $(12.0-19.3)$ \\
\hline St. Louis, Missouri-Illinois & 2,070 & 24.4 & 1.3 & $(21.8-27.0)$ \\
\hline Salisbury, Maryland-Delaware & 2,123 & 26.0 & 1.5 & $(23.1-29.0)$ \\
\hline Salt Lake City, Utah & 4,611 & 17.7 & 0.8 & $(16.1-19.2)$ \\
\hline San Antonio-New Braunfels, Texas & 777 & 25.3 & 2.1 & $(21.2-29.5)$ \\
\hline San Diego-Carlsbad, California & 1,063 & 17.1 & 1.8 & $(13.5-20.7)$ \\
\hline $\begin{array}{l}\text { San Francisco-Redwood City-South } \\
\text { San Francisco, California }{ }^{\dagger}\end{array}$ & 569 & 17.4 & 2.5 & $(12.6-22.3)$ \\
\hline $\begin{array}{l}\text { San Jose-Sunnyvale-Santa Clara, } \\
\text { California }\end{array}$ & 648 & 16.4 & 2.0 & $(12.4-20.4)$ \\
\hline San Juan-Carolina-Caguas, Puerto Rico & 3,956 & 41.4 & 0.9 & $(39.6-43.2)$ \\
\hline Santa Fe, New Mexico & 697 & 15.6 & 1.6 & $(12.5-18.8)$ \\
\hline Sayre, Pennsylvania & 1,857 & 27.4 & 2.3 & $(22.8-31.9)$ \\
\hline Scottsbluff, Nebraska & 631 & 22.2 & 2.5 & $(17.2-27.2)$ \\
\hline $\begin{array}{l}\text { Scranton-Wilkes-Barre-Hazleton, } \\
\text { Pennsylvania }\end{array}$ & 741 & 29.5 & 2.3 & $(25.0-34.0)$ \\
\hline Seattle-Bellevue-Everett, Washington ${ }^{\dagger}$ & 5,103 & 15.5 & 0.7 & $(14.1-16.9)$ \\
\hline Shreveport-Bossier City, Louisiana & 579 & 29.1 & 2.4 & $(24.3-33.8)$ \\
\hline $\begin{array}{l}\text { Silver Spring-Frederick-Rockville, } \\
\text { Maryland }{ }^{\dagger}\end{array}$ & 2,301 & 17.7 & 1.3 & $(15.2-20.3)$ \\
\hline $\begin{array}{l}\text { Sioux City, lowa-Nebraska- } \\
\text { South Dakota }\end{array}$ & 1,204 & 25.0 & 2.6 & $(20.0-30.1)$ \\
\hline Sioux Falls, South Dakota & 1,466 & 22.5 & 1.5 & $(19.5-25.4)$ \\
\hline Spartanburg, South Carolina & 710 & 30.3 & 2.7 & $(25.1-35.5)$ \\
\hline Spokane-Spokane Valley, Washington & 1,099 & 20.5 & 1.6 & $(17.4-23.6)$ \\
\hline Springfield, Massachusetts & 2,347 & 22.4 & 1.3 & $(19.8-25.0)$ \\
\hline Tacoma-Lakewood, Washington ${ }^{\dagger}$ & 1,183 & 17.8 & 1.4 & $(15.1-20.5)$ \\
\hline
\end{tabular}

TABLE 41. (Continued) Estimated prevalence of adults aged $\geq 18$ years who reported no leisure time of physical activity* during the preceding month, by metropolitan and micropolitan statistical area - Behavioral Risk Factor Surveillance System, United States, 2012

\begin{tabular}{|c|c|c|c|c|}
\hline MMSA(s) & $\begin{array}{l}\text { Sample } \\
\text { size }\end{array}$ & $\%$ & SE & $95 \% \mathrm{Cl}$ \\
\hline $\begin{array}{l}\text { Tampa-St. Petersburg-Clearwater, } \\
\text { Florida }\end{array}$ & 829 & 24.5 & 2.2 & $(20.2-28.8)$ \\
\hline Toledo, Ohio & 971 & 25.5 & 2.3 & $(21.0-30.0)$ \\
\hline Topeka, Kansas & 1,087 & 23.7 & 1.7 & $(20.5-27.0)$ \\
\hline Torrington, Connecticut & 663 & 21.4 & 2.6 & $(16.4-26.4)$ \\
\hline Trenton, New Jersey & 578 & 21.9 & 2.2 & $(17.5-26.3)$ \\
\hline Tucson, Arizona & 998 & 21.0 & 1.9 & $(17.3-24.6)$ \\
\hline Tulsa, Oklahoma & 1,744 & 26.8 & 1.3 & $(24.2-29.3)$ \\
\hline Tuscaloosa, Alabama & 581 & 25.9 & 2.7 & $(20.6-31.2)$ \\
\hline Urban Honolulu, Hawaii & 4,343 & 18.6 & 0.9 & $(16.8-20.4)$ \\
\hline Vineland-Bridgeton, New Jersey & 558 & 29.1 & 2.9 & $(23.5-34.8)$ \\
\hline $\begin{array}{l}\text { Virginia Beach-Norfolk-Newport News, } \\
\text { Virginia-North Carolina }\end{array}$ & 1,504 & 20.7 & 1.4 & $(18.0-23.5)$ \\
\hline $\begin{array}{l}\text { Warren-Troy-Farmington Hills, } \\
\text { Michigan }\end{array}$ & 2,186 & 20.5 & 1.1 & $(18.3-22.6)$ \\
\hline $\begin{array}{l}\text { Washington-Arlington-Alexandria, } \\
\text { District of Columbia-Virginia- } \\
\text { Maryland-West Virginia }\end{array}$ & 8,195 & 19.2 & 0.8 & $(17.7-20.7)$ \\
\hline Wichita, Kansas & 2,356 & 23.2 & 1.2 & $(20.8-25.6)$ \\
\hline $\begin{array}{l}\text { Wilmington, Delaware-Maryland- } \\
\text { New Jersey }{ }^{\dagger}\end{array}$ & 3,233 & 22.0 & 1.0 & $(20.0-24.0)$ \\
\hline Winston-Salem, North Carolina & 796 & 28.4 & 2.0 & $(24.4-32.4)$ \\
\hline Worcester, Massachusetts-Connecticut & 2,842 & 20.3 & 1.1 & $(18.1-22.4)$ \\
\hline Yakima, Washington & 531 & 32.0 & 3.0 & $(26.1-37.9)$ \\
\hline $\begin{array}{l}\text { Youngstown-Warren-Boardman, } \\
\text { Ohio-Pennsylvania }\end{array}$ & 928 & 31.3 & 2.6 & $(26.3-36.3)$ \\
\hline Median & \multicolumn{2}{|c|}{22.2} & & \\
\hline Range & \multicolumn{2}{|c|}{$9.2-47.3$} & & \\
\hline
\end{tabular}

Abbreviations: $\mathrm{Cl}=$ confidence interval; $\mathrm{MMSA}=$ metropolitan and micropolitan statistical area; $\mathrm{SE}=$ standard error.

* Any physical activities or exercises such as running, calisthenics, golf, gardening, or walking for exercise.

† Metropolitan division. 
TABLE 42. Estimated prevalence of adults aged $\geq 18$ years who reported no leisure time of physical activity* during the preceding month, by county - Behavioral Risk Factor Surveillance System, United States, 2012

\begin{tabular}{|c|c|c|c|c|}
\hline County & $\begin{array}{l}\text { Sample } \\
\text { size }\end{array}$ & $\%$ & SE & $95 \% \mathrm{Cl}$ \\
\hline Jefferson County, Alabama & 960 & 24.5 & 1.8 & $(21.0-28.0)$ \\
\hline Madison County, Alabama & 513 & 22.2 & 2.4 & $(17.6-26.9)$ \\
\hline Mobile County, Alabama & 815 & 26.4 & 2.4 & $(21.8-31.0)$ \\
\hline Anchorage Municipality, Alaska & 904 & 15.8 & 1.5 & $(12.9-18.8)$ \\
\hline Fairbanks North Star Borough, Alaska & 599 & 18.9 & 2.1 & $(14.8-23.0)$ \\
\hline Matanuska-Susitna Borough, Alaska & 599 & 19.9 & 2.0 & $(16.0-23.8)$ \\
\hline Maricopa County, Arizona & 2,121 & 22.6 & 1.2 & $(20.2-25.0)$ \\
\hline Pima County, Arizona & 998 & 21.0 & 1.9 & $(17.3-24.6)$ \\
\hline Pulaski County, Arkansas & 748 & 25.1 & 2.3 & $(20.7-29.5)$ \\
\hline Alameda County, California & 593 & 13.8 & 2.5 & $(9.0-18.6)$ \\
\hline Los Angeles County, California & 3,229 & 21.6 & 1.1 & $(19.5-23.6)$ \\
\hline Orange County, California & 963 & 15.6 & 1.6 & $(12.4$ \\
\hline Riverside County, California & 760 & 21.5 & 2.1 & (17. \\
\hline Sacramento County, California & 575 & 15.0 & 2.3 & $(10.6-19.5)$ \\
\hline San Bernardino County, California & 672 & 20.1 & 2.1 & $(15.9-24.2)$ \\
\hline San Diego County, California & 1,063 & 17.1 & 1.8 & $(13.5-20.7)$ \\
\hline Santa Clara County, California & 630 & 16.6 & 2.1 & $(12.6-20.7)$ \\
\hline Adams County, Colorado & 809 & 24.3 & 2.1 & $(20.2-28.4)$ \\
\hline Arapahoe County, Colorado & 858 & 18.9 & 1.6 & $(15.6-22.1)$ \\
\hline Boulder County, Colorado & 516 & 9.2 & 1.7 & $(6.0-12.5)$ \\
\hline Denver County, Colorado & 997 & 17.9 & 1.5 & $(15.0-20.9)$ \\
\hline Douglas County, Colorado & 547 & 10.5 & 1.5 & $(7.7-13.4)$ \\
\hline El Paso County, Colorado & 1,022 & 16.5 & 1.4 & $(13.7-19.2)$ \\
\hline Jefferson County, Colorado & 1,116 & 12.5 & 1.2 & $(10.2-14.8)$ \\
\hline Larimer County, Colorado & 595 & 12.4 & 1.6 & $(9.2-15.6)$ \\
\hline Weld County, Colorado & 533 & 23.8 & 2.5 & $(18.9-28.6)$ \\
\hline Fairfield County, Connecticut & 2,182 & 21.6 & 1.3 & $(19$ \\
\hline Hartford County, Connecticut & 1,980 & 22.4 & 1.3 & $(19.9-24.8)$ \\
\hline Litchfield County, Connecticut & 663 & 21.4 & 2.6 & $(16.4-26.4)$ \\
\hline New Haven County, Connecticut & 2,008 & 24.1 & 1.4 & $(21.3-26.8)$ \\
\hline New London County, Connecticut & 999 & 22.2 & 2.0 & $(18.3-26.2)$ \\
\hline Kent County, Delaware & 1,440 & 26.4 & 1.7 & $(23.1-29.7)$ \\
\hline New Castle County, Delaware & 2,332 & 21.0 & 1.1 & $(18.9-23.2)$ \\
\hline Sussex County, Delaware & 1,400 & 27.6 & 1.6 & $(24.4-30.8)$ \\
\hline $\begin{array}{l}\text { District of Columbia, } \\
\text { District of Columbia }\end{array}$ & 3,817 & 17.4 & 0.9 & $(15.6-19.2)$ \\
\hline Broward County, Florida & 527 & 20.5 & 2.6 & $(15.5-25.6)$ \\
\hline Miami-Dade County, Florida & 814 & 28.4 & 2.6 & $(23.3-33.5)$ \\
\hline Hawaii County, Hawaii & 1,349 & 20.5 & 1.6 & $(17.3-23.7)$ \\
\hline Honolulu County, Hawaii & 4,343 & 18.6 & .9 & $(16.8-20.4)$ \\
\hline Kauai County, Hawaii & 670 & 14.9 & 1.9 & $(11.2-18.6)$ \\
\hline Maui County, Hawaii & 1,218 & 18.9 & 1.8 & $(15.4-22.4)$ \\
\hline Ada County, Idaho & 808 & 15.1 & 1.7 & $(11.7-18.6)$ \\
\hline Canyon County, Idaho & 503 & 23.2 & 3.1 & $(17.1-29.4)$ \\
\hline Cook County, Illinois & 1,504 & 20.5 & 1.4 & $(17.7-23.3)$ \\
\hline Lake County, Indiana & 888 & 31.8 & 2.6 & $(26.6-36.9)$ \\
\hline Marion County, Indiana & 1,280 & 25.4 & 1.6 & $(22.3-28.5)$ \\
\hline Polk County, lowa & 809 & 19.7 & 1.7 & $(16.5-23.0)$ \\
\hline Johnson County, Kansas & 2,178 & 14.2 & 1.0 & $(12.1-16.2)$ \\
\hline Sedgwick County, Kansas & 1,795 & 23.2 & 1.4 & $(20.5-25.9)$ \\
\hline Shawnee County, Kansas & 769 & 24.0 & 2.1 & $(20.0-28.0)$ \\
\hline Wyandotte County, Kansas & 876 & 30.3 & 2.7 & $(25.1-35.6)$ \\
\hline Jefferson County, Kentucky & 1,694 & 26.0 & 1.8 & $(22.5-29.4)$ \\
\hline East Baton Rouge Parish, Louisiana & 571 & 24.5 & 2.5 & $(19.7-29.4)$ \\
\hline Androscoggin County, Maine & 700 & 24.0 & 1.9 & $(20.3-27.8)$ \\
\hline Aroostook County, Maine & 538 & 28.8 & 2.4 & $(24.1-33.4)$ \\
\hline Cumberland County, Maine & 1,758 & 16.1 & 1.1 & $(14.0-18.2)$ \\
\hline Kennebec County, Maine & 832 & 23.1 & 1.8 & $(19.5-26.6)$ \\
\hline Penobscot County, Maine & 928 & 24.4 & 1.8 & $(21.0-27.8)$ \\
\hline
\end{tabular}

See table footnotes on page 85 .
TABLE 42. (Continued) Estimated prevalence of adults aged $\geq 18$ years who reported no leisure time of physical activity* during the preceding month, by county - Behavioral Risk Factor Surveillance System, United States, 2012

\begin{tabular}{|c|c|c|c|c|}
\hline County & $\begin{array}{c}\text { Sample } \\
\text { size }\end{array}$ & $\%$ & SE & $95 \% \mathrm{Cl}$ \\
\hline York County, Maine & 1,194 & 16.3 & 1.2 & $(13.9-18.7)$ \\
\hline Anne Arundel County, Maryland & 896 & 20.2 & 1.9 & $(16.4-23.9)$ \\
\hline Baltimore County, Maryland & 1,521 & 25.5 & 1.7 & $(22.2-28.8)$ \\
\hline Charles County, Maryland & 514 & 23.1 & 2.7 & $(17.7-28.4)$ \\
\hline Frederick County, Maryland & 755 & 21.2 & 3.1 & $(15.1-27.3)$ \\
\hline Montgomery County, Maryland & 1,546 & 16.8 & 1.4 & $(14.0-19.7)$ \\
\hline Prince George's County, Maryland & 1,142 & 25.1 & 2.1 & $(20.9-29.3)$ \\
\hline Washington County, Maryland & 537 & 30.6 & 4.0 & $(22.8-38.5)$ \\
\hline Baltimore city, Maryland & 745 & 30.1 & 2.7 & $(24.9-35.3)$ \\
\hline Barnstable County, Massachusetts & 559 & 17.3 & 2.1 & $(13.1-21.4)$ \\
\hline Bristol County, Massachusetts & 2,651 & 22.6 & 1.5 & $(19.6-25.6)$ \\
\hline Essex County, Massachusetts & 2,566 & 20.4 & 1.3 & $(17.9-22.9)$ \\
\hline Hampden County, Massachusetts & 1,964 & 26.7 & 1.6 & $(23.5-29.8)$ \\
\hline Middlesex County, Massachusetts & 4,360 & 18.0 & 0.9 & $(16.3-19.7)$ \\
\hline Norfolk County, Massachusetts & 1,760 & 17.1 & 1.4 & $(14.3-19.9)$ \\
\hline Plymouth County, Massachusetts & 1,835 & 21.0 & 1.5 & $(18.0-23.9)$ \\
\hline Suffolk County, Massachusetts & 2,313 & 22.0 & 1.3 & $(19.4-24.6)$ \\
\hline Worcester County, Massachusetts & 2,596 & 19.9 & 1.2 & $(17.6-22.2)$ \\
\hline Kent County, Michigan & 526 & 19.7 & 2.5 & $(14.9-24.6)$ \\
\hline Macomb County, Michigan & 613 & 23.1 & 2.0 & $(19.1-27.1)$ \\
\hline Oakland County, Michigan & 1,170 & 19.0 & 1.5 & $(16.1-21.9)$ \\
\hline Wayne County, Michigan & 2,219 & 28.2 & 1.6 & $(25.1-31.2)$ \\
\hline Anoka County, Minnesota & 544 & 13.9 & 1.8 & $(10.4-17.3)$ \\
\hline Dakota County, Minnesota & 671 & 13.9 & 1.7 & $(10.7-17.1)$ \\
\hline Hennepin County, Minnesota & 3,300 & 16.4 & 1.0 & $(14.5-18.4)$ \\
\hline Ramsey County, Minnesota & 1,949 & 18.8 & 1.7 & $(15.4-22.1)$ \\
\hline Jackson County, Missouri & 884 & 22.8 & 2.3 & $(18.4-27.3)$ \\
\hline St. Louis County, Missouri & 954 & 23.1 & 1.9 & $(19.4-26.8)$ \\
\hline Cascade County, Montana & 707 & 26.9 & 2.1 & $(22.8-31.0)$ \\
\hline Flathead County, Montana & 560 & 15.7 & 1.8 & $(12.1-19.4)$ \\
\hline Hill County, Montana & 584 & 28.2 & 3.0 & $(22.3-34.1)$ \\
\hline Lake County, Montana & 899 & 20.2 & 1.9 & $(16.4-24.0)$ \\
\hline Missoula County, Montana & 782 & 16.8 & 1.6 & $(13.5-20.0)$ \\
\hline Yellowstone County, Montana & 751 & 21.1 & 1.6 & $(17.9-24.3)$ \\
\hline Dakota County, Nebraska & 732 & 33.2 & 4.6 & $(24.2-42.2)$ \\
\hline Douglas County, Nebraska & 3,572 & 20.6 & 0.9 & $(18.8-22.3)$ \\
\hline Hall County, Nebraska & 535 & 24.7 & 2.6 & $(19.6-29.9)$ \\
\hline Lancaster County, Nebraska & 1,446 & 15.5 & 1.1 & $(13.3-17.7)$ \\
\hline Lincoln County, Nebraska & 589 & 22.1 & 2.5 & $(17.1-27.0)$ \\
\hline Sarpy County, Nebraska & 1,175 & 19.3 & 1.5 & $(16.4-22.2)$ \\
\hline Scotts Bluff County, Nebraska & 578 & 21.6 & 2.5 & $(16.7-26.5)$ \\
\hline Clark County, Nevada & 2,015 & 21.9 & 1.1 & $(19.7-24.1)$ \\
\hline Washoe County, Nevada & 1,486 & 17.5 & 1.4 & $(14.7-20.2)$ \\
\hline Belknap County, New Hampshire & 566 & 27.0 & 3.0 & $(21.1-32.9)$ \\
\hline Carroll County, New Hampshire & 529 & 17.1 & 2.1 & $(12.9-21.3)$ \\
\hline Cheshire County, New Hampshire & 547 & 17.1 & 2.1 & $(12.9-21.3)$ \\
\hline Coos County, New Hampshire & 536 & 29.4 & 3.1 & $(23.4-35.5)$ \\
\hline Grafton County, New Hampshire & 566 & 19.5 & 2.5 & $(14.6-24.4)$ \\
\hline Hillsborough County, New Hampshire & 1,901 & 21.1 & 1.2 & $(18.6-23.5)$ \\
\hline Merrimack County, New Hampshire & 705 & 17.3 & 1.8 & $(13.8-20.8)$ \\
\hline Rockingham County, New Hampshire & 1,048 & 18.3 & 1.5 & $(15.3-21.3)$ \\
\hline Strafford County, New Hampshire & 623 & 21.4 & 2.2 & $(17.2-25.7)$ \\
\hline Atlantic County, New Jersey & 1,023 & 25.9 & 1.9 & $(22.2-29.6)$ \\
\hline Bergen County, New Jersey & 1,010 & 23.7 & 1.8 & $(20.2-27.3)$ \\
\hline Burlington County, New Jersey & 686 & 24.7 & 2.3 & $(20.1-29.2)$ \\
\hline Camden County, New Jersey & 744 & 28.8 & 2.3 & $(24.3-33.2)$ \\
\hline Cape May County, New Jersey & 553 & 25.3 & 3.1 & $(19.3-31.3)$ \\
\hline Cumberland County, New Jersey & 558 & 29.1 & 2.9 & $(23.5-34.8)$ \\
\hline Essex County, New Jersey & 1,339 & 27.7 & 1.7 & $(24.4-30.9)$ \\
\hline
\end{tabular}

See table footnotes on page 85 . 
TABLE 42. (Continued) Estimated prevalence of adults aged $\geq 18$ years who reported no leisure time of physical activity* during the preceding month, by county - Behavioral Risk Factor Surveillance System, United States, 2012

\begin{tabular}{|c|c|c|c|c|}
\hline County & $\begin{array}{l}\text { Sample } \\
\text { size }\end{array}$ & $\%$ & SE & $95 \% \mathrm{Cl}$ \\
\hline Gloucester County, New Jersey & 553 & 27.9 & 2.6 & $(22.8-33.0)$ \\
\hline Hudson County, New Jersey & 1,304 & 29.5 & 1.7 & $(26.2-32.8)$ \\
\hline Hunterdon County, New Jersey & 570 & 17.1 & 2.1 & $(12.9-21.3)$ \\
\hline Mercer County, New Jersey & 578 & 21.9 & 2.2 & $(17.5-26.3)$ \\
\hline Middlesex County, New Jersey & 882 & 20.4 & 1.8 & $(17.0-23.9)$ \\
\hline Monmouth County, New Jersey & 714 & 19.8 & 2.0 & $(15.8-23.8)$ \\
\hline Morris County, New Jersey & 847 & 21.5 & 2.0 & $(17.6-25.3)$ \\
\hline Ocean County, New Jersey & 663 & 29.1 & 2.3 & $(24.7-33.6)$ \\
\hline Passaic County, New Jersey & 686 & 26.5 & 2.2 & $(22.2-30.8)$ \\
\hline Salem County, New Jersey & 587 & 26.2 & 2.9 & $(20.6-31.8)$ \\
\hline Somerset County, New Jersey & 640 & 19.0 & 2.1 & $(14.9-23.1)$ \\
\hline Sussex County, New Jersey & 550 & 23.8 & 2.7 & $(18.5-29.0)$ \\
\hline Union County, New Jersey & 713 & 29.2 & 2.2 & $(24.9-33.5)$ \\
\hline Warren County, New Jersey & 532 & 23.3 & 2.8 & $(17.8-28.7)$ \\
\hline Bernalillo County, New Mexico & 2,063 & 18.0 & 1.0 & $(16.0-19.9)$ \\
\hline Dona Ana County, New Mexico & 708 & 26.2 & 2.2 & 0.6) \\
\hline Sandoval County, New Mexico & 657 & 18.0 & 1.9 & 1.7) \\
\hline San Juan County, New Mexico & 650 & 23.2 & 2.1 & $(19.1-27.3)$ \\
\hline Santa Fe County, New Mexico & 697 & 15.6 & 1.6 & $(12.5-18.8)$ \\
\hline Kings County, New York & 501 & 27.0 & 2.9 & $(21.4-32.6)$ \\
\hline Guilford County, North Carolina & 512 & 21.7 & 2.2 & $(17.3-26.1)$ \\
\hline Mecklenburg County, North Carolina & 727 & 20.2 & 1.8 & $(16.6-23.8)$ \\
\hline Robeson County, North Carolina & 546 & 39.0 & 4.4 & $(30.3-47.6)$ \\
\hline Wake County, North Carolina & 702 & 18.9 & 1.8 & $(15.3-22.5)$ \\
\hline Burleigh County, North Dakota & 568 & 20.7 & 2.5 & $(15.9-25.6)$ \\
\hline Cass County, North Dakota & 843 & 19.3 & 1.8 & $(15.7-23.0)$ \\
\hline Cuyahoga County, Ohio & 866 & 24.7 & 1.8 & $(21.2-28.3)$ \\
\hline Frar & 838 & 22.5 & 1.8 & $(1$ \\
\hline Hamilton County, Ohio & 772 & 23.3 & 1.9 & $(19.5-27.0)$ \\
\hline Lorain County, Ohio & 611 & 21.4 & 2.8 & $(16.0-26.8)$ \\
\hline Lucas County, Ohio & 622 & 25.5 & 2.8 & $(20.0-31.0)$ \\
\hline Mahoning County, Ohio & 591 & 28.6 & 2.9 & $(23.0-34.3)$ \\
\hline Montgomery County, Ohio & 664 & 24.5 & 2.1 & $(20.3-28.8)$ \\
\hline Stark County, Ohio & 622 & 24.2 & 2.3 & $(19.7-28.7)$ \\
\hline Summit County, Ohio & 630 & 22.4 & 2.2 & $(18.1-26.6)$ \\
\hline Oklahoma County, Oklahoma & 1,193 & 25.6 & 1.5 & $(22.6-28.5)$ \\
\hline Tulsa County, Oklahoma & 1,204 & 26.9 & 1.6 & $(23.8-30.0)$ \\
\hline Clackamas County, Oregon & 510 & 14.2 & 2.0 & $(10.2-18.2)$ \\
\hline Lane County, Oregon & 526 & 14.4 & 2.1 & $(10.2-18.5)$ \\
\hline Multnomah County, Oregon & 896 & 12.8 & 1.5 & $(9.9-15.6)$ \\
\hline Washington County, Oregon & 616 & 15.6 & 2.0 & $(11.7-19.4)$ \\
\hline Allegheny County, Pennsylvania & 1,887 & 22.4 & 1.2 & $(20.0-24.8)$ \\
\hline Bradford County, Pennsylvania & 1,857 & 27.4 & 2.3 & $(22.8-31.9)$ \\
\hline Montgomery County, Pennsylvania & 547 & 18.0 & 2.0 & $(14.0-22.0)$ \\
\hline Philadelphia County, Pennsylvania & 2,065 & 26.9 & 1.3 & $(24.3-29.6)$ \\
\hline Pike County, Pennsylvania & 1,889 & 24.0 & 2.9 & $(18.3-29.7)$ \\
\hline Kent County, Rhode Island & 813 & 22.7 & 2.0 & $(18.7-26.6)$ \\
\hline Providence County, Rhode Island & 3,336 & 27.0 & 1.0 & $(25.0-29.1)$ \\
\hline Washington County, Rhode Island & 656 & 13.9 & 1.7 & $(10.5-17.2)$ \\
\hline
\end{tabular}

TABLE 42. (Continued) Estimated prevalence of adults aged $\geq 18$ years who reported no leisure time of physical activity* during the preceding month, by county - Behavioral Risk Factor Surveillance System, United States, 2012

\begin{tabular}{|c|c|c|c|c|}
\hline County & $\begin{array}{l}\text { Sample } \\
\text { size }\end{array}$ & $\%$ & SE & $95 \% \mathrm{Cl}$ \\
\hline iken County, South Carolina & 555 & 26.6 & 2.5 & $(21.7-31.6)$ \\
\hline Beaufort County, South Carolina & 803 & 16.1 & 2.1 & $(12.0-20.2)$ \\
\hline Charleston County, South Carolina & 1,016 & 20.4 & 1.8 & $(16.7-24.0)$ \\
\hline Greenville County, South Carolina & 906 & 25.4 & 2.1 & $(21.3-29.4)$ \\
\hline Horry County, South Carolina & 779 & 22.4 & 1.9 & $(18.7-26.1)$ \\
\hline Richland County, South Carolina & 964 & 21.7 & 1.9 & $(17.9-25.5)$ \\
\hline Spartanburg County, South Carolina & 656 & 27.3 & 2.7 & $(22.0-32.6)$ \\
\hline Lincoln County, South Dakota & 500 & 19.0 & 2.3 & $(14.4-23.6)$ \\
\hline Minnehaha County, South Dakota & 878 & 22.5 & 1.7 & $(19.1-25.9)$ \\
\hline Pennington County, South Dakota & 605 & 19.9 & 1.9 & $(16.2-23.5)$ \\
\hline Davidson County, Tennessee & 556 & 24.7 & 2.6 & $(19.5-29.9)$ \\
\hline Shelby County, Tennessee & 536 & 24.4 & 2.2 & $(20.2-28.7)$ \\
\hline Bexar County, Texas & 585 & 27.0 & 2.5 & $(22.1-31.9)$ \\
\hline Dallas County, Texas & 512 & 27.6 & 2.4 & $(22.8-32.3)$ \\
\hline El Paso County, Texas & 625 & 32.0 & 3.0 & $(26.2-37.9)$ \\
\hline Harris County, Texas & 773 & 27.3 & 2.0 & $(23.3-31.3)$ \\
\hline Hidalgo County, Texas & 622 & 36.6 & 3.1 & $(30.5-42.7)$ \\
\hline Tarrant County, Texas & 572 & 28.7 & 2.5 & $(23.7-33.6)$ \\
\hline Travis County, Texas & 1,048 & 21.4 & 2.2 & $(17.2-25.7)$ \\
\hline Davis County, Utah & 1,147 & 15.2 & 1.4 & $(12.4-17.9)$ \\
\hline Salt Lake County, Utah & 4,046 & 17.3 & 0.8 & $(15.8-18.9)$ \\
\hline Tooele County, Utah & 565 & 24.0 & 3.1 & $(18.0-30.1)$ \\
\hline Utah County, Utah & 1,681 & 14.6 & 1.1 & $(12.4-16.7)$ \\
\hline Wasatch County, Utah & 512 & 11.0 & 1.8 & $(7.6-14.5)$ \\
\hline Weber County, Utah & 1,048 & 17.8 & 1.6 & $(14.6-20.9)$ \\
\hline Chittenden County, Vermont & 917 & 12.1 & 1.2 & $(9.8-14.4)$ \\
\hline Rutland County, Vermont & 597 & 21.1 & 2.3 & $(16.5-25.7)$ \\
\hline Washington County, Vermont & 516 & 12.3 & 1.6 & $(9.2-15.4)$ \\
\hline Windsor County, Vermont & 546 & 17.9 & 1.9 & $(14.1-21.7)$ \\
\hline Fairfax County, Virginia & 743 & 14.2 & 1.7 & $(10.9-17.5)$ \\
\hline Clark County, Washington & 789 & 20.2 & 1.9 & $(16.5-23.8)$ \\
\hline King County, Washington & 3,928 & 14.4 & 0.8 & $(12.9-15.9)$ \\
\hline Kitsap County, Washington & 563 & 19.2 & 2.0 & $(15.2-23.2)$ \\
\hline Pierce County, Washington & 1,183 & 17.8 & 1.4 & $(15.1-20.5)$ \\
\hline Snohomish County, Washington & 1,175 & 18.5 & 1.5 & $(15.5-21.5)$ \\
\hline Spokane County, Washington & 952 & 21.0 & 1.7 & $(17.6-24.4)$ \\
\hline Thurston County, Washington & 517 & 18.8 & 2.4 & $(14.1-23.6)$ \\
\hline Whatcom County, Washington & 847 & 18.2 & 2.3 & $(13.8-22.6)$ \\
\hline Yakima County, Washington & 531 & 32.0 & 3.0 & $(26.1-37.9)$ \\
\hline Kanawha County, West Virginia & 641 & 29.0 & 2.1 & $(24.9-33.0)$ \\
\hline Milwaukee County, Wisconsin & 963 & 19.2 & 2.0 & $(15.4-23.1)$ \\
\hline Laramie County, Wyoming & 955 & 21.2 & 2.0 & $(17.2-25.2)$ \\
\hline Natrona County, Wyoming & 830 & 19.5 & 2.0 & $(15.5-23.5)$ \\
\hline San Juan Municipio, Puerto Rico & 667 & 37.6 & 2.2 & $(33.3-41.9)$ \\
\hline Median & & 21.5 & & \\
\hline Range & & $9.2-39$. & & \\
\hline
\end{tabular}

Abbreviations: $\mathrm{Cl}=$ confidence interval; $\mathrm{SE}=$ standard error.

* Any physical activities or exercises such as running, calisthenics, golf, gardening, or walking for exercise. 
TABLE 43. Estimated prevalence of adults aged $\geq 18$ years who reported they always use a seat belt when they ride or drive in a car, by state/territory - Behavioral Risk Factor Surveillance System, United States, 2012

\begin{tabular}{|c|c|c|c|c|}
\hline State/Territory & $\begin{array}{c}\text { Sample } \\
\text { size }\end{array}$ & $\%$ & SE & $95 \% \mathrm{Cl}$ \\
\hline Alabama & 8,880 & 85.8 & 0.6 & $(84.7-87.0)$ \\
\hline Alaska & 4,249 & 82.3 & 0.8 & $(80.7-83.9)$ \\
\hline Arizona & 7,027 & 84.7 & 0.8 & $(83.2-86.2)$ \\
\hline Arkansas & 5,019 & 76.2 & 0.9 & $(74.5-78.0)$ \\
\hline California & 12,369 & 93.7 & 0.3 & $(93.0-94.4)$ \\
\hline Colorado & 11,605 & 83.1 & 0.5 & $(82.2-84.1)$ \\
\hline Connecticut & 8,513 & 87.6 & 0.5 & $(86.5-88.6)$ \\
\hline Delaware & 5,137 & 91.9 & 0.5 & (90.8-92.9) \\
\hline District of Columbia & 3,666 & 86.1 & 1.1 & $(83.9-88.3)$ \\
\hline Florida & 7,358 & 86.2 & 0.7 & $(84.9-87.6)$ \\
\hline Georgia & 5,833 & 87.5 & 0.7 & $(86.2-88.8)$ \\
\hline Hawaii & 7,422 & 91.3 & 0.5 & $(90.3-92.4)$ \\
\hline Idaho & 5,745 & 73.5 & 1.1 & $(71.5-75.6)$ \\
\hline Illinois & 5,459 & 88.0 & 0.7 & $(86.6-89.4)$ \\
\hline Indiana & 8,416 & 84.7 & 0.6 & $(83.6-85.8)$ \\
\hline lowa & 6,938 & 83.4 & 0.6 & $(82.3-84.6)$ \\
\hline Kansas & 11,692 & 79.6 & 0.5 & $(78.6-80.7)$ \\
\hline Kentucky & 10,744 & 80.3 & 0.6 & (79.0-81.5) \\
\hline Louisiana & 8,955 & 89.2 & 0.6 & (88.0-90.4) \\
\hline Maine & 9,772 & 82.3 & 0.5 & (81.3-83.4) \\
\hline Maryland & 12,384 & 90.6 & 0.5 & $(89.6-91.5)$ \\
\hline Massachusetts & 20,463 & 78.4 & 0.5 & (77.5-79.4) \\
\hline Michigan & 10,302 & 88.4 & 0.5 & (87.4-89.3) \\
\hline Minnesota & 11,811 & 86.9 & 0.4 & (86.1-87.8) \\
\hline Mississippi & 7,665 & 80.0 & 0.7 & $(78.6-81.5)$ \\
\hline Missouri & 6,600 & 75.5 & 0.8 & (73.9-77.1) \\
\hline Montana & 8,493 & 70.2 & 0.7 & (68.9-71.5) \\
\hline Nebraska & 18,851 & 69.7 & 0.5 & (68.7-70.6) \\
\hline Nevada & 4,719 & 88.6 & 0.7 & (87.3-89.9) \\
\hline New Hampshire & 7,332 & 67.4 & 0.8 & $(65.8-69.1)$ \\
\hline New Jersey & 14,895 & 88.8 & 0.4 & $(88.0-89.6)$ \\
\hline New Mexico & 8,352 & 89.0 & 0.5 & (88.1-89.9) \\
\hline New York & 5,820 & 87.1 & 0.6 & $(85.8-88.3)$ \\
\hline North Carolina & 11,644 & 90.6 & 0.4 & (89.8-91.3) \\
\hline North Dakota & 4,778 & 65.2 & 0.9 & $(63.3-67.0)$ \\
\hline Ohio & 12,540 & 79.7 & 0.5 & (78.7-80.8) \\
\hline Oklahoma & 7,863 & 82.3 & 0.6 & $(81.1-83.5)$ \\
\hline Oregon & 5,119 & 92.6 & 0.5 & (91.6-93.6) \\
\hline Pennsylvania & 19,384 & 75.9 & 0.5 & (74.9-76.9) \\
\hline Rhode Island & 5,285 & 83.8 & 0.8 & $(82.3-85.4)$ \\
\hline South Carolina & 12,409 & 85.4 & 0.5 & $(84.4-86.4)$ \\
\hline South Dakota & 7,744 & 62.0 & 0.9 & $(60.2-63.7)$ \\
\hline Tennessee & 6,703 & 86.0 & 0.6 & $(84.8-87.3)$ \\
\hline Texas & 8,874 & 91.6 & 0.4 & (90.7-92.4) \\
\hline Utah & 12,093 & 78.9 & 0.5 & $(77.9-80.0)$ \\
\hline Vermont & 5,927 & 81.7 & 0.7 & $(80.3-83.1)$ \\
\hline Virginia & 7,104 & 84.5 & 0.6 & $(83.3-85.7)$ \\
\hline Washington & 15,084 & 91.4 & 0.3 & (90.7-92.0) \\
\hline West Virginia & 5,387 & 80.7 & 0.7 & $(79.3-82.0)$ \\
\hline Wisconsin & 5,032 & 78.0 & 0.9 & (76.3-79.8) \\
\hline Wyoming & 6,062 & 68.1 & 1.0 & $(66.1-70.1)$ \\
\hline Guam & 1,953 & 89.2 & 0.9 & (87.4-91.1) \\
\hline Puerto Rico & 6,274 & 90.8 & 0.5 & (89.9-91.8) \\
\hline Median & \multicolumn{4}{|c|}{84.7} \\
\hline Range & \multicolumn{4}{|c|}{$62.0-93.7$} \\
\hline
\end{tabular}

Abbreviations: $\mathrm{Cl}=$ confidence interval; $\mathrm{SE}=$ standard error.
TABLE 44. Estimated prevalence of adults aged $\geq 18$ years who reported they always use a seat belt when they ride in or drive a car, by metropolitan and micropolitan statistical area - Behavioral Risk Factor Surveillance System, United States, 2012

\begin{tabular}{|c|c|c|c|c|}
\hline MMSA(s) & $\begin{array}{l}\text { Sample } \\
\text { size }\end{array}$ & $\%$ & SE & $95 \% \mathrm{Cl}$ \\
\hline Aguadilla-Isabela, Puerto Rico & 548 & 88.6 & 1.8 & $(85.1-92.1)$ \\
\hline Akron, Ohio & 717 & 80.8 & 2.1 & $(76.7-85.0)$ \\
\hline Albuquerque, New Mexico & 3,095 & 91.2 & 0.6 & $(90.0-92.5)$ \\
\hline $\begin{array}{l}\text { Allentown-Bethlehem-Easton, } \\
\text { Pennsylvania-New Jersey }\end{array}$ & 1,303 & 81.6 & 1.6 & $(78.4-84.8)$ \\
\hline Anaheim-Santa Ana-Irvine, California* & 872 & 94.8 & 1.0 & $(92.8-96.8)$ \\
\hline Anchorage, Alaska & 1,470 & 87.9 & 1.1 & $(85.7-90.1)$ \\
\hline Asheville, North Carolina & 584 & 92.3 & 1.6 & $(89.2-95.3)$ \\
\hline $\begin{array}{l}\text { Atlanta-Sandy Springs-Roswell, } \\
\text { Georgia }\end{array}$ & 2,432 & 91.6 & 0.8 & $(89.9-93.2)$ \\
\hline Atlantic City-Hammonton, New Jersey & 975 & 89.6 & 1.5 & $(86.6-92.6)$ \\
\hline $\begin{array}{l}\text { Augusta-Richmond County, Georgia- } \\
\text { South Carolina }\end{array}$ & 993 & 81.7 & 2.6 & $(76.5-86.8)$ \\
\hline Augusta-Waterville, Maine & 819 & 81.4 & 1.8 & $(77.8-85.0)$ \\
\hline Austin-Round Rock, Texas & 1,359 & 93.2 & 1.1 & $(91.0-95.3)$ \\
\hline $\begin{array}{l}\text { Baltimore-Columbia-Towson, } \\
\text { Maryland }\end{array}$ & 4,589 & 90.5 & 0.8 & $(89.0-91.9)$ \\
\hline Bangor, Maine & 911 & 79.0 & 1.8 & $(75.4-82.5)$ \\
\hline Barnstable Town, Massachusetts & 529 & 79.0 & 2.4 & $(74.2-83.8)$ \\
\hline Barre, Vermont & 510 & 81.7 & 2.6 & $(76.6-86.8)$ \\
\hline Baton Rouge, Louisiana & 1,374 & 90.5 & 1.4 & $(87.8-93.3)$ \\
\hline Bellingham, Washington & 836 & 93.3 & 1.3 & $(90.7-95.9)$ \\
\hline Berlin, New Hampshire-Vermont & 691 & 54.1 & 3.0 & $(48.3-60.0)$ \\
\hline Billings, Montana & 836 & 74.2 & 1.8 & $(70.8-77.7)$ \\
\hline Birmingham-Hoover, Alabama & 1,792 & 85.7 & 1.4 & $(82.9-88.4)$ \\
\hline Bismarck, North Dakota & 813 & 69.5 & 2.2 & $(65.2-73.8)$ \\
\hline Boise City, Idaho & 1,445 & 79.2 & 1.7 & $(75.9-82.6)$ \\
\hline Boston, Massachusetts* & 5,540 & 79.5 & 0.9 & $(77.8-81.3)$ \\
\hline Boulder, Colorado & 489 & 83.4 & 2.1 & $(79.2-87.6)$ \\
\hline Bremerton-Silverdale, Washington & 559 & 94.0 & 1.4 & $(91.2-96.8)$ \\
\hline $\begin{array}{l}\text { Bridgeport-Stamford-Norwalk, } \\
\text { Connecticut }\end{array}$ & 2,104 & 88.8 & 0.9 & $(87.0-90.6)$ \\
\hline Burlington-South Burlington, Vermont & 1,491 & 84.0 & 1.2 & $(81.6-86.4)$ \\
\hline $\begin{array}{l}\text { Cambridge-Newton-Framingham, } \\
\text { Massachusetts* }\end{array}$ & 6,519 & 80.8 & 0.8 & $(79.3-82.4)$ \\
\hline Camden, New Jersey* & 1,904 & 86.9 & 1.3 & $(84.4-89.4)$ \\
\hline Canton-Massillon, Ohio & 652 & 79.4 & 2.3 & $(74.9-83.8)$ \\
\hline Casper, Wyoming & 800 & 73.8 & 2.6 & $(68.7-78.8)$ \\
\hline Cedar Rapids, lowa & 567 & 85.4 & 2.2 & $(81.2-89.7)$ \\
\hline Charleston, West Virginia & 769 & 84.5 & 1.6 & $(81.4-87.7)$ \\
\hline $\begin{array}{l}\text { Charleston-North Charleston, } \\
\text { South Carolina }\end{array}$ & 1,670 & 85.8 & 1.2 & $(83.3-88.2)$ \\
\hline $\begin{array}{l}\text { Charlotte-Concord-Gastonia, } \\
\text { North Carolina-South Carolina }\end{array}$ & 2,464 & 91.1 & 0.8 & $(89.5-92.6)$ \\
\hline Chattanooga, Tennessee-Georgia & 603 & 85.7 & 2.3 & $(81.2-90.2)$ \\
\hline Cheyenne, Wyoming & 926 & 76.6 & 2.4 & $(71.8-81.4)$ \\
\hline $\begin{array}{l}\text { Chicago-Naperville-Elgin, } \\
\text { Illinois-Indiana-Wisconsin }\end{array}$ & 3,640 & 88.4 & 0.9 & $(86.7-90.2)$ \\
\hline Cincinnati, Ohio-Kentucky-Indiana & 2,280 & 82.5 & 1.1 & $(80.4-84.7)$ \\
\hline $\begin{array}{l}\text { Claremont-Lebanon, New } \\
\text { Hampshire-Vermont }\end{array}$ & 1,911 & 72.5 & 1.6 & $(69.4-75.6)$ \\
\hline Cleveland-Elyria, Ohio & 1,677 & 81.5 & 1.4 & $(78.8-84.2)$ \\
\hline Colorado Springs, Colorado & 1,107 & 84.7 & 1.4 & $(81.9-87.5)$ \\
\hline Columbia, South Carolina & 1,747 & 86.1 & 1.3 & $(83.7-88.6)$ \\
\hline Columbus, Ohio & 1,548 & 80.9 & 1.3 & $(78.3-83.5)$ \\
\hline Concord, New Hampshire & 685 & 64.1 & 2.6 & $(59.0-69.2)$ \\
\hline Dallas-Plano-Irving, Texas* & 877 & 94.9 & 1.0 & $(93.0-96.8)$ \\
\hline Dayton, Ohio & 832 & 82.6 & 1.9 & $(79.0-86.3)$ \\
\hline Denver-Aurora-Lakewood, Colorado & 4,599 & 85.2 & 0.7 & $(83.8-86.5)$ \\
\hline Des Moines-West Des Moines, lowa & 1,128 & 90.4 & 1.1 & $(88.3-92.5)$ \\
\hline
\end{tabular}

See table footnotes on page 88 . 
TABLE 44. (Continued) Estimated prevalence of adults aged $\geq 18$ years who reported they always use a seat belt when they ride in or drive a car, by metropolitan and micropolitan statistical area - Behavioral Risk Factor Surveillance System, United States, 2012

\begin{tabular}{|c|c|c|c|c|}
\hline MMSA(s) & $\begin{array}{l}\text { Sample } \\
\text { size }\end{array}$ & $\%$ & SE & $95 \% \mathrm{Cl}$ \\
\hline Detroit-Dearborn-Livonia, Michigan* & 2,153 & 88.6 & 1.2 & $(86.3-90.9)$ \\
\hline Dover, Delaware & 1,432 & 91.7 & 1.2 & $(89.3-94.0)$ \\
\hline Duluth, Minnesota-Wisconsin & 502 & 84.2 & 2.1 & $(80.0-88.3)$ \\
\hline Durham-Chapel Hill, North Carolina & 779 & 90.7 & 1.4 & $(88.0-93.4)$ \\
\hline El Paso, Texas & 608 & 90.2 & 2.3 & $(85.7-94.7)$ \\
\hline Eugene, Oregon & 509 & 93.1 & 1.7 & $(89.8-96.5)$ \\
\hline Fairbanks, Alaska & 591 & 86.8 & 2.1 & $(82.8-90.9)$ \\
\hline Fargo, North Dakota-Minnesota & 921 & 79.7 & 2.0 & $(75.7-83.7)$ \\
\hline Farmington, New Mexico & 623 & 89.6 & 1.5 & $(86.6-92.6)$ \\
\hline Fayetteville, North Carolina & 495 & 90.4 & 1.9 & $(86.7-94.1)$ \\
\hline $\begin{array}{l}\text { Fayetteville-Springdale-Rogers, } \\
\text { Arkansas-Missouri }\end{array}$ & 788 & 82.0 & 2.4 & $(77.3-86.7)$ \\
\hline Fort Collins, Colorado & 564 & 85.3 & 1.9 & $(81.6-88.9)$ \\
\hline Fort Wayne, Indiana & 523 & 85.1 & 2.2 & $(80.8-89.4)$ \\
\hline Fort Worth-Arlington, Texas* & 715 & 92.2 & 1.4 & $(89.4-94.9)$ \\
\hline Grand Island, Nebraska & 842 & 60.9 & 2.4 & $(56.2-65.5)$ \\
\hline Grand Rapids-Wyoming, Michigan & 879 & 88.9 & 1.4 & $(86.1-91.7)$ \\
\hline Great Falls, Montana & 698 & 72.7 & 2.2 & $(68.3-77.0)$ \\
\hline Greeley, Colorado & 509 & 84.1 & 1.9 & $(80.3-87.8)$ \\
\hline $\begin{array}{l}\text { Greensboro-High Point, } \\
\text { North Carolina }\end{array}$ & 795 & 88.5 & 1.4 & $(85.7-91.3)$ \\
\hline $\begin{array}{l}\text { Greenville-Anderson-Mauldin, } \\
\text { South Carolina }\end{array}$ & 1,638 & 86.1 & 1.3 & $(83.5-88.6)$ \\
\hline Gulfport-Biloxi-Pascagoula, Mississippi & 731 & 88.8 & 1.5 & $(85.8-91.8)$ \\
\hline $\begin{array}{l}\text { Hagerstown-Martinsburg, Maryland- } \\
\text { West Virginia }\end{array}$ & 749 & 84.5 & 2.9 & $(78.8-90.3)$ \\
\hline Harrisburg-Carlisle, Pennsylvania & 655 & 79.4 & 2.0 & $(75.4-83.4)$ \\
\hline $\begin{array}{l}\text { Hartford-West Hartford-East Hartford, } \\
\text { Connecticut }\end{array}$ & 2,604 & 87.6 & 0.9 & $(85.9-89.4)$ \\
\hline Heber, Utah & 501 & 73.3 & 4.2 & $(65.0-81.5)$ \\
\hline Hilo, Hawaii & 1,319 & 93.0 & 1.1 & $(90.9-95.1)$ \\
\hline $\begin{array}{l}\text { Hilton Head Island-Bluffton-Beaufort, } \\
\text { South Carolina }\end{array}$ & 908 & 84.9 & 3.3 & $(78.5-91.2)$ \\
\hline $\begin{array}{l}\text { Houston-The Woodlands-Sugar Land, } \\
\text { Texas }\end{array}$ & 1,106 & 92.8 & 1.0 & $(90.9-94.7)$ \\
\hline $\begin{array}{l}\text { Huntington-Ashland, West } \\
\text { Virginia-Kentucky-Ohio }\end{array}$ & 1,096 & 81.5 & 1.6 & $(78.5-84.6)$ \\
\hline Huntsville, Alabama & 608 & 86.6 & 1.9 & $(82.9-90.4)$ \\
\hline Idaho Falls, Idaho & 525 & 68.5 & 3.5 & $(61.6-75.4)$ \\
\hline $\begin{array}{l}\text { Indianapolis-Carmel-Anderson, } \\
\text { Indiana }\end{array}$ & 2,132 & 87.3 & 1.0 & $(85.3-89.2)$ \\
\hline Jackson, Mississippi & 900 & 84.6 & 1.6 & 81.5-87.7) \\
\hline Jacksonville, Florida & 502 & 85.8 & 2.5 & $(81.0-90.7)$ \\
\hline Kahului-Wailuku-Lahaina, Hawaii & 1,199 & 89.8 & 1.5 & $(87.0-92.7)$ \\
\hline Kalispell, Montana & 548 & 76.8 & 2.2 & $(72.5-81.2)$ \\
\hline Kansas City, Missouri-Kansas & 4,667 & 79.9 & 1.2 & $(77.5-82.4)$ \\
\hline Kapaa, Hawaii & 656 & 94.3 & 1.4 & $(91.6-96.9)$ \\
\hline Keene, New Hampshire & 534 & 61.7 & 3.4 & $(55.0-68.3)$ \\
\hline Kennewick-Richland, Washington & 526 & 91.3 & 1.8 & $(87.6-94.9)$ \\
\hline $\begin{array}{l}\text { Kingsport-Bristol-Bristol, } \\
\text { Tennessee-Virginia }\end{array}$ & 539 & 80.5 & 2.9 & $(74.8-86.3)$ \\
\hline Knoxville, Tennessee & 800 & 89.3 & 1.7 & $(86.0-92.7)$ \\
\hline Laconia, New Hampshire & 553 & 56.4 & 3.6 & $(49.3-63.4)$ \\
\hline Lafayette, Louisiana & 551 & 91.1 & 1.7 & $(87.7-94.5)$ \\
\hline Las Cruces, New Mexico & 677 & 91.4 & 1.5 & $(88.5-94.3)$ \\
\hline $\begin{array}{l}\text { Las Vegas-Henderson-Paradise, } \\
\text { Nevada }\end{array}$ & 1,960 & 89.6 & 0.8 & $(88.0-91.3)$ \\
\hline Lewiston-Auburn, Maine & 693 & 85.5 & 1.8 & $(82.0-89.1)$ \\
\hline Lexington-Fayette, Kentucky & 511 & 81.4 & 2.3 & $(77.0-85.9)$ \\
\hline Lincoln, Nebraska & 1,649 & 74.1 & 1.3 & $(71.6-76.7)$ \\
\hline
\end{tabular}

See table footnotes on page 88 .
TABLE 44. (Continued) Estimated prevalence of adults aged $\geq 18$ years who reported they always use a seat belt when they ride in or drive a car, by metropolitan and micropolitan statistical area - Behavioral Risk Factor Surveillance System, United States, 2012

\begin{tabular}{|c|c|c|c|c|}
\hline MMSA(s) & $\begin{array}{l}\text { Sample } \\
\text { size }\end{array}$ & $\%$ & SE & $95 \% \mathrm{Cl}$ \\
\hline $\begin{array}{l}\text { Little Rock-North Little Rock-Conway, } \\
\text { Arkansas }\end{array}$ & 1,127 & 81.3 & 1.7 & $(78.0-84.6)$ \\
\hline Logan, Utah-Idaho & 499 & 77.1 & 2.6 & $(72.0-82.2)$ \\
\hline $\begin{array}{l}\text { Los Angeles-Long Beach-Glendale, } \\
\text { California* }\end{array}$ & 2,812 & 92.7 & 0.7 & $(91.3-94.1)$ \\
\hline $\begin{array}{l}\text { Louisville/Jefferson County, } \\
\text { Kentucky-Indiana }\end{array}$ & 2,064 & 83.3 & 1.3 & $(80.7-85.9)$ \\
\hline Lumberton, North Carolina & 525 & 74.6 & 4.5 & $(65.7-83.6)$ \\
\hline Manchester-Nashua, New Hampshire & 1,860 & 70.3 & 1.5 & $(67.4-73.2)$ \\
\hline McAllen-Edinburg-Mission, Texas & 607 & 90.8 & 1.9 & $(87.0-94.6)$ \\
\hline $\begin{array}{l}\text { Memphis, } \\
\text { Tennessee-Mississippi-Arkansas }\end{array}$ & 1,255 & 86.7 & 1.5 & $(83.7-89.6)$ \\
\hline $\begin{array}{l}\text { Miami-Fort Lauderdale-West Palm } \\
\text { Beach, Florida }\end{array}$ & 1,592 & 84.9 & 1.5 & $(81.9-87.9)$ \\
\hline $\begin{array}{l}\text { Milwaukee-Waukesha-West Allis, } \\
\text { Wisconsin }\end{array}$ & 1,170 & 77.0 & 2.0 & $(73.1-80.8)$ \\
\hline $\begin{array}{l}\text { Minneapolis-St. Paul-Bloomington, } \\
\text { Minnesota-Wisconsin }\end{array}$ & 7,589 & 90.8 & 0.5 & $(89.8-91.8)$ \\
\hline Missoula, Montana & 772 & 75.4 & 2.1 & $(71.4-79.5)$ \\
\hline Mobile, Alabama & 806 & 84.9 & 2.3 & $(80.3-89.4)$ \\
\hline Montgomery, Alabama & 526 & 88.4 & 2.3 & $(83.8-92.9)$ \\
\hline $\begin{array}{l}\text { Montgomery County-Bucks County- } \\
\text { Chester County, Pennsylvania* }\end{array}$ & 1,273 & 81.9 & 1.7 & $(78.7-85.2)$ \\
\hline $\begin{array}{l}\text { Myrtle Beach-Conway-North Myrtle } \\
\text { Beach, South Carolina-North Carolina }\end{array}$ & 923 & 89.2 & 1.5 & $(86.2-92.2)$ \\
\hline $\begin{array}{l}\text { Nashville-Davidson-Murfreesboro- } \\
\text { Franklin, Tennessee }\end{array}$ & 1,278 & 89.0 & 1.2 & $(86.8-91.3)$ \\
\hline $\begin{array}{l}\text { Nassau County-Suffolk County, } \\
\text { New York* }\end{array}$ & 874 & 87.4 & 1.6 & $(84.2-90.6)$ \\
\hline Newark, New Jersey-Pennsylvania* & 6,280 & 89.5 & 0.7 & $(88.1-90.9)$ \\
\hline New Haven-Milford, Connecticut & 1,944 & 86.0 & 1.2 & $(83.5-88.4)$ \\
\hline New Orleans-Metairie, Louisiana & 1,256 & 90.1 & 1.2 & $(87.8-92.5)$ \\
\hline $\begin{array}{l}\text { New York-Jersey City-White Plains, } \\
\text { New York-New Jersey* }\end{array}$ & 6,953 & 86.1 & 0.7 & $(84.7-87.6)$ \\
\hline Norfolk, Nebraska & 561 & 59.7 & 2.9 & $(54.0-65.4)$ \\
\hline North Platte, Nebraska & 603 & 59.1 & 3.0 & $(53.2-65.0)$ \\
\hline Norwich-New London, Connecticut & 973 & 91.9 & 1.3 & $(89.2-94.5)$ \\
\hline $\begin{array}{l}\text { Oakland-Hayward-Berkeley, } \\
\text { California* }\end{array}$ & 859 & 93.5 & 1.4 & $(90.8-96.2)$ \\
\hline Ocean City, New Jersey & 532 & 81.5 & 3.8 & $(74.0-89.0)$ \\
\hline Ogden-Clearfield, Utah & 2,373 & 83.0 & 1.0 & $(81.1-85.0)$ \\
\hline Oklahoma City, Oklahoma & 2,393 & 85.4 & 1.0 & $(83.3-87.4)$ \\
\hline Olympia-Tumwater, Washington & 508 & 91.2 & 1.8 & $(87.6-94.7)$ \\
\hline Omaha-Council Bluffs, Nebraska-lowa & 5,477 & 79.5 & 0.7 & $(78.0-80.9)$ \\
\hline Orlando-Kissimmee-Sanford, Florida & 546 & 88.3 & 2.1 & $(84.1-92.4)$ \\
\hline Philadelphia, Pennsylvania* & 2,347 & 73.2 & 1.3 & $(70.6-75.8)$ \\
\hline Phoenix-Mesa-Scottsdale, Arizona & 2,490 & 85.6 & 1.0 & $(83.7-87.6)$ \\
\hline Pittsburgh, Pennsylvania & 3,280 & 76.4 & 1.0 & $(74.3-78.4)$ \\
\hline Ponce, Puerto Rico & 546 & 91.6 & 1.4 & $(88.8-94.5)$ \\
\hline Portland-South Portland, Maine & 3,283 & 85.9 & 0.8 & $(84.4-87.5)$ \\
\hline $\begin{array}{l}\text { Portland-Vancouver-Hillsboro, } \\
\text { Oregon-Washington }\end{array}$ & 3,041 & 94.4 & 0.6 & $(93.2-95.5)$ \\
\hline $\begin{array}{l}\text { Providence-Warwick, Rhode } \\
\text { Island-Massachusetts }\end{array}$ & 7,798 & 80.7 & 0.8 & $(79.1-82.3)$ \\
\hline Provo-Orem, Utah & 1,699 & 76.0 & 1.3 & $(73.4-78.6)$ \\
\hline Raleigh, North Carolina & 928 & 93.7 & 1.0 & $(91.8-95.5)$ \\
\hline Rapid City, South Dakota & 1,039 & 67.9 & 2.0 & $(64.0-71.8)$ \\
\hline Reno, Nevada & 1,463 & 88.2 & 1.5 & $(85.3-91.0)$ \\
\hline Richmond, Virginia & 970 & 83.0 & 1.8 & $(79.6-86.4)$ \\
\hline
\end{tabular}

See table footnotes on page 88. 
TABLE 44. (Continued) Estimated prevalence of adults aged $\geq 18$ years who reported they always use a seat belt when they ride in or drive a car, by metropolitan and micropolitan statistical area - Behavioral Risk Factor Surveillance System, United States, 2012

\begin{tabular}{|c|c|c|c|c|}
\hline MMSA(s) & $\begin{array}{l}\text { Sample } \\
\text { size }\end{array}$ & $\%$ & SE & $95 \% \mathrm{Cl}$ \\
\hline $\begin{array}{l}\text { Riverside-San Bernardino-Ontario, } \\
\text { California }\end{array}$ & 1,300 & 94.1 & 1.0 & $(92.2-96.0)$ \\
\hline $\begin{array}{l}\text { Rockingham County-Strafford County, } \\
\text { New Hampshire* }\end{array}$ & 1,623 & 70.5 & 1.6 & $(67.3-73.6)$ \\
\hline Rutland, Vermont & 582 & 84.6 & 2.1 & $(80.5-88.7)$ \\
\hline $\begin{array}{l}\text { Sacramento-Roseville-Arden-Arcade, } \\
\text { California }\end{array}$ & 875 & 94.8 & 1.0 & $(92.8-96.8)$ \\
\hline St. Louis, Missouri-Illinois & 2,013 & 82.0 & 1.2 & $(79.6-84.5)$ \\
\hline Salisbury, Maryland-Delaware & 2,083 & 92.3 & 0.9 & $(90.6-94.1)$ \\
\hline Salt Lake City, Utah & 4,492 & 83.1 & 0.8 & $(81.5-84.6)$ \\
\hline San Antonio-New Braunfels, Texas & 756 & 91.2 & 1.8 & (87.6-94.8) \\
\hline San Diego-Carlsbad, California & 982 & 95.5 & 0.9 & $(93.8-97.3)$ \\
\hline $\begin{array}{l}\text { San Francisco-Redwood City-South } \\
\text { San Francisco, California** }\end{array}$ & 510 & 91.7 & 2.0 & $(87.9-95.6)$ \\
\hline $\begin{array}{l}\text { San Jose-Sunnyvale-Santa Clara, } \\
\text { California }\end{array}$ & 581 & 97.1 & 1.1 & $(94.9-99.2)$ \\
\hline San Juan-Carolina-Caguas, Puerto Rico & 3,930 & 91.3 & 0.6 & $(90.2-92.4)$ \\
\hline Santa Fe, New Mexico & 662 & 87.1 & 1.9 & (83.4-90.7) \\
\hline Sayre, Pennsylvania & 1,818 & 79.6 & 1.9 & $(76.0-83.3)$ \\
\hline Scottsbluff, Nebraska & 622 & 60.2 & 3.2 & $(54.0-66.4)$ \\
\hline $\begin{array}{l}\text { Scranton-Wilkes-Barre-Hazleton, } \\
\text { Pennsylvania }\end{array}$ & 724 & 74.4 & 2.2 & $(70.1-78.8)$ \\
\hline Seattle-Bellevue-Everett, Washington* & 5,019 & 91.6 & 0.6 & $(90.5-92.7)$ \\
\hline Shreveport-Bossier City, Louisiana & 574 & 89.0 & 1.9 & $(85.3-92.7)$ \\
\hline $\begin{array}{l}\text { Silver Spring-Frederick-Rockville, } \\
\text { Maryland** }\end{array}$ & 2,212 & 93.2 & 0.9 & $(91.4-95.1)$ \\
\hline $\begin{array}{l}\text { Sioux City, lowa-Nebraska- } \\
\text { South Dakota }\end{array}$ & 1,177 & 81.5 & 2.4 & $(76.9-86.2)$ \\
\hline Sioux Falls, South Dakota & 1,440 & 68.4 & 1.6 & $(65.2-71.6)$ \\
\hline Spartanburg, South Carolina & 692 & 78.8 & 2.4 & $(74.0-83.5)$ \\
\hline Spokane-Spokane Valley, Washington & 1,082 & 90.0 & 1.4 & $(87.3-92.7)$ \\
\hline Springfield, Massachusetts & 2,236 & 74.5 & 1.6 & (71.4-77.6) \\
\hline
\end{tabular}

TABLE 44. (Continued) Estimated prevalence of adults aged $\geq 18$ years who reported they always use a seat belt when they ride in or drive a car, by metropolitan and micropolitan statistical area - Behavioral Risk Factor Surveillance System, United States, 2012

\begin{tabular}{|c|c|c|c|c|}
\hline MMSA(s) & $\begin{array}{l}\text { Sample } \\
\text { size }\end{array}$ & $\%$ & SE & $95 \% \mathrm{Cl}$ \\
\hline Tacoma-Lakewood, Washington* & 1,166 & 92.0 & 1.1 & $(89.9-94.2)$ \\
\hline $\begin{array}{l}\text { Tampa-St. Petersburg-Clearwater, } \\
\text { Florida }\end{array}$ & 804 & 85.6 & 2.1 & $(81.4-89.7)$ \\
\hline Toledo, Ohio & 941 & 79.9 & 2.4 & $(75.1-84.6)$ \\
\hline Topeka, Kansas & 1,079 & 82.0 & 1.5 & $(79.0-85.0)$ \\
\hline Torrington, Connecticut & 649 & 85.9 & 1.9 & $(82.1-89.7)$ \\
\hline Trenton, New Jersey & 551 & 91.7 & 1.7 & $(88.3-95.0)$ \\
\hline Tucson, Arizona & 958 & 85.1 & 1.9 & $(81.4-88.7)$ \\
\hline Tulsa, Oklahoma & 1,704 & 87.0 & 1.1 & $(84.9-89.1)$ \\
\hline Tuscaloosa, Alabama & 569 & 90.9 & 2.1 & $(86.7-95.0)$ \\
\hline Urban Honolulu, Hawaii & 4,248 & 91.1 & 0.7 & $(89.7-92.4)$ \\
\hline Vineland-Bridgeton, New Jersey & 535 & 86.0 & 2.5 & $(81.2-90.8)$ \\
\hline $\begin{array}{l}\text { Virginia Beach-Norfolk-Newport News, } \\
\text { Virginia-North Carolina }\end{array}$ & ; 1,455 & 87.0 & 1.3 & $(84.5-89.5)$ \\
\hline $\begin{array}{l}\text { Warren-Troy-Farmington Hills, } \\
\text { Michigan* }\end{array}$ & 2,146 & 90.0 & 0.9 & $(88.3-91.7)$ \\
\hline $\begin{array}{l}\text { Washington-Arlington-Alexandria, } \\
\text { District of Columbia-Virginia- } \\
\text { Maryland-West Virginia** }\end{array}$ & 7,876 & 89.4 & 0.6 & $(88.1-90.6)$ \\
\hline Wichita, Kansas & 2,327 & 82.9 & 1.1 & $(80.8-85.1)$ \\
\hline $\begin{array}{l}\text { Wilmington, Delaware-Maryland- } \\
\text { New Jersey* }\end{array}$ & 3,186 & 90.8 & 0.8 & $(89.3-92.3)$ \\
\hline Winston-Salem, North Carolina & 778 & 89.6 & 1.5 & $(86.6-92.5)$ \\
\hline Worcester, Massachusetts-Connecticut & $t \quad 2,707$ & 80.1 & 1.2 & $(77.8-82.4)$ \\
\hline Yakima, Washington & 525 & 92.7 & 1.7 & $(89.4-95.9)$ \\
\hline $\begin{array}{l}\text { Youngstown-Warren-Boardman, } \\
\text { Ohio-Pennsylvania }\end{array}$ & 900 & 75.6 & 2.6 & $(70.4-80.7)$ \\
\hline Median & & 85.7 & & \\
\hline Range & & $54.1-97.1$ & & \\
\hline
\end{tabular}

Abbreviations: $\mathrm{Cl}=$ confidence interval; $\mathrm{MMSA}=$ metropolitan and micropolitan statistical area; $\mathrm{SE}=$ standard error.

* Metropolitan division. 
TABLE 45. Estimated prevalence of adults aged $\geq 18$ years who reported they always use a seat belt when they ride in or drive a car, by county - Behavioral Risk Factor Surveillance System, United States, 2012

\begin{tabular}{|c|c|c|c|c|}
\hline County & $\begin{array}{l}\text { Sample } \\
\text { size }\end{array}$ & $\%$ & SE & $95 \% \mathrm{Cl}$ \\
\hline Jefferson County, Alabama & 941 & 85.9 & 1.7 & $(82.6-89.1)$ \\
\hline Madison County, Alabama & 505 & 86.6 & 2.3 & $(82.1-91.1)$ \\
\hline Mobile County, Alabama & 806 & 84.9 & 2.3 & $(80.3-89.4)$ \\
\hline Anchorage Municipality, Alaska & 881 & 87.3 & 1.4 & $(84.6-90.1)$ \\
\hline Fairbanks North Star Borough, Alaska & 591 & 86.8 & 2.1 & $(82.8-90.9)$ \\
\hline Matanuska-Susitna Borough, Alaska & 589 & 89.5 & 1.7 & $(86.2-92.7)$ \\
\hline Maricopa County, Arizona & 2,021 & 85.8 & 1.0 & $(83.7-87.8)$ \\
\hline Pima County, Arizona & 958 & 85.1 & 1.9 & $(81.4-88.7)$ \\
\hline Pulaski County, Arkansas & 726 & 82.9 & 2.1 & $(78.8-87.0)$ \\
\hline Alameda County, California & 550 & 92.4 & 1.9 & $(88.7-96.1)$ \\
\hline Los Angeles County, California & 2,812 & 92.7 & 0.7 & $(91.3-94.1)$ \\
\hline Orange County, California & 872 & 94.8 & 1.0 & $(92.8-96.8)$ \\
\hline Riverside County, California & 696 & 95.1 & 1.0 & $(93.1-97.1)$ \\
\hline Sacramento County, California & 525 & 96.3 & 1.1 & $(94.2$ \\
\hline San Bernardino County, California & 604 & 93.4 & 1.5 & $(90.5-96.4)$ \\
\hline San Diego County, California & 982 & 95.5 & 0.9 & $(93.8-97.3)$ \\
\hline Santa Clara County, California & 564 & 97.3 & 1.1 & $(95.1-99.5)$ \\
\hline Adams County, Colorado & 772 & 82.0 & 1.8 & $(78.4-85.6)$ \\
\hline Arapahoe County, Colorado & 819 & 85.1 & 1.5 & $(82.1-88.1)$ \\
\hline Boulder County, Colorado & 489 & 83.4 & 2.1 & $(79.2-87.6)$ \\
\hline Denver County, Colorado & 925 & 85.1 & 1.5 & $(82.0-88.1)$ \\
\hline Douglas County, Colorado & 519 & 91.7 & 1.6 & $(88.5-94.8)$ \\
\hline El Paso County, Colorado & 972 & 84.7 & 1.5 & $(81.8-87.6)$ \\
\hline Jefferson County, Colorado & 1,064 & 84.4 & 1.5 & $(81.6-87.3)$ \\
\hline Larimer County, Colorado & 564 & 85.3 & 1.9 & $(81.6-88.9)$ \\
\hline Weld County, Colorado & 509 & 84.1 & 1.9 & $(80.3-87.8)$ \\
\hline Fairfield County, Connecticut & 2,104 & 88.8 & 0.9 & $(87.0-90.6)$ \\
\hline Hartford County, Connecticut & 1,936 & 86.9 & 1.1 & $(84.8-89.1)$ \\
\hline Litchfield County, Connecticut & 649 & 85.9 & 1.9 & $(82.1-89.7)$ \\
\hline New Haven County, Connecticut & 1,944 & 86.0 & 1.2 & $(83.5-88.4)$ \\
\hline New London County, Connecticut & 973 & 91.9 & 1.3 & $(89.2-94.5)$ \\
\hline Kent County, Delaware & 1,432 & 91.7 & 1.2 & $(89.3-94.0)$ \\
\hline New Castle County, Delaware & 2,317 & 92.0 & 0.7 & $(90.6-93.4)$ \\
\hline Sussex County, Delaware & 1,388 & 92.2 & 1.1 & $(90.1-94.4)$ \\
\hline $\begin{array}{l}\text { District of Columbia, } \\
\text { District of Columbia }\end{array}$ & 3,666 & 86.0 & 1.1 & $(83.8-88.2)$ \\
\hline Broward County, Florida & 506 & 84.4 & 2.8 & $(79.0-89.8)$ \\
\hline Miami-Dade County, Florida & 768 & 83.6 & 2.4 & $(79.0-88.3)$ \\
\hline Hawaii County, Hawaii & 1,319 & 93.0 & 1.1 & $(90.9-95.1)$ \\
\hline Honolulu County, Hawaii & 4,248 & 91.1 & 0.7 & $(89.7-92.4)$ \\
\hline Kauai County, Hawaii & 656 & 94.3 & 1.4 & $(91.6-96.9)$ \\
\hline Maui County, Hawaii & 1,199 & 89.8 & 1.5 & $(87.0-92.7)$ \\
\hline Ada County, Idaho & 788 & 82.6 & 2.0 & $(78.6-86.6)$ \\
\hline Canyon County, Idaho & 493 & 72.9 & 3.5 & $(66.0-79.8)$ \\
\hline Cook County, Illinois & 1,462 & 87.6 & 1.3 & $(85.1-90.2)$ \\
\hline Lake County, Indiana & 865 & 87.6 & 1.9 & $(83.8-91.4)$ \\
\hline Marion County, Indiana & 1,238 & 86.9 & 1.4 & $(84.1-89.6)$ \\
\hline Polk County, lowa & 787 & 90.1 & 1.3 & $(87.7-92.6)$ \\
\hline Johnson County, Kansas & 2,157 & 89.5 & 1.0 & $(87.5-91.5)$ \\
\hline Sedgwick County, Kansas & 1,773 & 84.9 & 1.2 & $(82.6-87.2)$ \\
\hline Shawnee County, Kansas & 763 & 83.7 & 1.7 & $(80.3-87.2)$ \\
\hline Wyandotte County, Kansas & 867 & 81.3 & 2.6 & $(76.1-86.5)$ \\
\hline Jefferson County, Kentucky & 1,592 & 83.6 & 1.7 & $(80.3-86.8)$ \\
\hline East Baton Rouge Parish, Louisiana & 567 & 91.0 & 1.6 & $(87.8-94.2)$ \\
\hline Androscoggin County, Maine & 693 & 85.5 & 1.8 & $(82.0-89.1)$ \\
\hline Aroostook County, Maine & 533 & 77.2 & 2.3 & $(72.7-81.8)$ \\
\hline Cumberland County, Maine & 1,731 & 86.4 & 1.1 & $(84.3-88.5)$ \\
\hline Kennebec County, Maine & 819 & 81.4 & 1.8 & $(77.8-85.0)$ \\
\hline Penobscot County, Maine & 911 & 79.0 & 1.8 & $(75.4-82.5)$ \\
\hline
\end{tabular}

See table footnotes on page 90.
TABLE 45. (Continued) Estimated prevalence of adults aged $\geq 18$ years who reported they always use a seat belt when they ride in or drive a car, by county - Behavioral Risk Factor Surveillance System, United States, 2012

\begin{tabular}{|c|c|c|c|c|}
\hline County & $\begin{array}{c}\text { Sample } \\
\text { size }\end{array}$ & $\%$ & SE & $95 \% \mathrm{Cl}$ \\
\hline York County, Maine & 1,172 & 85.8 & 1.3 & $(83.2-88.5)$ \\
\hline Anne Arundel County, Maryland & 877 & 94.4 & 1.2 & $(92.1-96.7)$ \\
\hline Baltimore County, Maryland & 1,479 & 88.3 & 1.4 & $(85.5-91.0)$ \\
\hline Charles County, Maryland & 499 & 90.4 & 2.4 & $(85.7-95.1)$ \\
\hline Frederick County, Maryland & 740 & 89.1 & 2.7 & $(83.8-94.3)$ \\
\hline Montgomery County, Maryland & 1,472 & 94.0 & 1.0 & $(92.0-96.0)$ \\
\hline Prince George's County, Maryland & 1,090 & 89.3 & 1.8 & $(85.9-92.8)$ \\
\hline Washington County, Maryland & 529 & 84.2 & 3.8 & (76.9-91.6) \\
\hline Baltimore city, Maryland & 715 & 87.7 & 2.1 & $(83.5-91.9)$ \\
\hline Barnstable County, Massachusetts & 529 & 79.0 & 2.4 & $(74.2-83.8)$ \\
\hline Bristol County, Massachusetts & 2,513 & 74.3 & 1.8 & $(70.7-77.9)$ \\
\hline Essex County, Massachusetts & 2,441 & 76.9 & 1.5 & (73.9-79.9) \\
\hline Hampden County, Massachusetts & 1,864 & 73.3 & 1.8 & $(69.7-76.8)$ \\
\hline Middlesex County, Massachusetts & 4,078 & 82.6 & 0.9 & $(80.8-84.4)$ \\
\hline Norfolk County, Massachusetts & 1,655 & 85.0 & 1.3 & $(82.5-87.6)$ \\
\hline Plymouth County, Massachusetts & 1,713 & 75.4 & 1.8 & $(72.0-78.9)$ \\
\hline Suffolk County, Massachusetts & 2,172 & 78.0 & 1.4 & $(75.1-80.8)$ \\
\hline Worcester County, Massachusetts & 2,468 & 79.7 & 1.3 & $(77.2-82.2)$ \\
\hline Kent County, Michigan & 516 & 88.1 & 2.2 & $(83.7-92.4)$ \\
\hline Macomb County, Michigan & 602 & 89.1 & 1.7 & $(85.7-92.5)$ \\
\hline Oakland County, Michigan & 1,146 & 90.2 & 1.3 & $(87.6-92.9)$ \\
\hline Wayne County, Michigan & 2,153 & 88.6 & 1.2 & $(86.3-90.9)$ \\
\hline Anoka County, Minnesota & 526 & 91.6 & 1.6 & $(88.5-94.8)$ \\
\hline Dakota County, Minnesota & 650 & 92.0 & 1.2 & $(89.6-94.3)$ \\
\hline Hennepin County, Minnesota & 3,145 & 91.8 & 0.7 & $(90.4-$ \\
\hline Ramsey County, Minnesota & 1,884 & 91.4 & 1.4 & $(88.7-94.0)$ \\
\hline Jackson County, Missouri & 848 & 73.2 & 2.7 & $(68.0-78.4)$ \\
\hline St. Louis County, Missouri & 929 & 81.9 & 1.9 & $(78.3-85.6)$ \\
\hline Cascade County, Montana & 698 & 72.7 & 2.2 & $(68.3-77.0)$ \\
\hline Flathead County, Montana & 548 & 76.8 & 2.2 & $(72.5-81.2)$ \\
\hline Hill County, Montana & 570 & 56.1 & 3.5 & $(49.2-63.0)$ \\
\hline Lake County, Montana & 879 & 68.8 & 2.8 & $(63.3-74.2)$ \\
\hline Missoula County, Montana & 772 & 75.4 & 2.1 & (71.4-79.5) \\
\hline Yellowstone County, Montana & 741 & 74.8 & 1.8 & $(71.2-78.4)$ \\
\hline Dakota County, Nebraska & 719 & 76.7 & 4.0 & $(68.8-84.5)$ \\
\hline Douglas County, Nebraska & 3,503 & 78.3 & 0.9 & $(76.5-80.1)$ \\
\hline Hall County, Nebraska & 529 & 60.5 & 3.0 & $(54.5-66.4)$ \\
\hline Lancaster County, Nebraska & 1,427 & 74.8 & 1.3 & $(72.2-77.5)$ \\
\hline Lincoln County, Nebraska & 579 & 59.7 & 3.1 & $(53.7-65.8)$ \\
\hline Sarpy County, Nebraska & 1,161 & 81.8 & 1.6 & $(78.7-84.9)$ \\
\hline Scotts Bluff County, Nebraska & 571 & 59.4 & 3.3 & $(52.9-65.8)$ \\
\hline Clark County, Nevada & 1,960 & 89.6 & 0.8 & $(88.0-91.3)$ \\
\hline Washoe County, Nevada & 1,444 & 88.1 & 1.5 & $(85.2-91.0)$ \\
\hline Belknap County, New Hampshire & 553 & 56.4 & 3.6 & $(49.3-63.4)$ \\
\hline Carroll County, New Hampshire & 521 & 60.9 & 3.7 & $(53.7-68.1)$ \\
\hline Cheshire County, New Hampshire & 534 & 61.7 & 3.4 & $(55.0-68.3)$ \\
\hline Coos County, New Hampshire & 524 & 50.1 & 3.4 & $(43.4-56.8)$ \\
\hline Grafton County, New Hampshire & 552 & 72.1 & 3.0 & $(66.2-78.0)$ \\
\hline Hillsborough County, New Hampshire & 1,860 & 70.3 & 1.5 & $(67.4-73.2)$ \\
\hline Merrimack County, New Hampshire & 685 & 64.1 & 2.6 & $(59.0-69.2)$ \\
\hline Rockingham County, New Hampshire & 1,009 & 74.5 & 1.9 & $(70.9-78.2)$ \\
\hline Strafford County, New Hampshire & 614 & 60.9 & 3.0 & $(54.9-66.8)$ \\
\hline Atlantic County, New Jersey & 975 & 89.6 & 1.5 & $(86.6-92.6)$ \\
\hline Bergen County, New Jersey & 891 & 90.3 & 1.5 & $(87.2-93.3)$ \\
\hline Burlington County, New Jersey & 659 & 89.9 & 2.1 & $(85.8-94.0)$ \\
\hline Camden County, New Jersey & 711 & 84.1 & 2.1 & $(79.9-88.3)$ \\
\hline Cape May County, New Jersey & 532 & 81.5 & 3.8 & $(74.0-89.0)$ \\
\hline Cumberland County, New Jersey & 535 & 86.0 & 2.5 & $(81.2-90.8)$ \\
\hline Essex County, New Jersey & 1,285 & 88.7 & 1.3 & $(86.3-91.2)$ \\
\hline
\end{tabular}

See table footnotes on page 90 . 
TABLE 45. (Continued) Estimated prevalence of adults aged $\geq 18$ years who reported they always use a seat belt when they ride in or drive a car, by county - Behavioral Risk Factor Surveillance System, United States, 2012

\begin{tabular}{|c|c|c|c|c|}
\hline County & $\begin{array}{l}\text { Sample } \\
\text { size }\end{array}$ & $\%$ & SE & $95 \% \mathrm{Cl}$ \\
\hline Gloucester County, New Jersey & 534 & 86.8 & 2.5 & $(82.0-91.6)$ \\
\hline Hudson County, New Jersey & 1,214 & 86.9 & 1.4 & $(84.1-89.7)$ \\
\hline Hunterdon County, New Jersey & 540 & 89.7 & 2.0 & $(85.9-93.6)$ \\
\hline Mercer County, New Jersey & 551 & 91.7 & 1.7 & $(88.3-95.0)$ \\
\hline Middlesex County, New Jersey & 843 & 91.5 & 1.4 & $(88.7-94.4)$ \\
\hline Monmouth County, New Jersey & 679 & 88.9 & 1.6 & $(85.8-92.0)$ \\
\hline Morris County, New Jersey & 809 & 90.9 & 1.7 & $(87.6-94.2)$ \\
\hline Ocean County, New Jersey & 626 & 87.8 & 1.9 & $(84.2-91.5)$ \\
\hline Passaic County, New Jersey & 640 & 86.3 & 2.1 & $(82.3-90.4)$ \\
\hline Salem County, New Jersey & 567 & 84.2 & 4.0 & $(76.3-92.0)$ \\
\hline Somerset County, New Jersey & 601 & 93.5 & 1.4 & $(90.8-96.2)$ \\
\hline Sussex County, New Jersey & 524 & 89.0 & 2.5 & $(84.2-93.9)$ \\
\hline Union County, New Jersey & 670 & 87.6 & 1.8 & $(84.0-91.2)$ \\
\hline Warren County, New Jersey & 509 & 91.8 & 1.6 & $(88.7-94.9)$ \\
\hline Bernalillo County, New Mexico & 1,951 & 91.3 & 0.8 & $(89.8-92.8)$ \\
\hline Dona Ana County, New Mexico & 677 & 91.4 & 1.5 & $(88.5-$ \\
\hline Sandoval County, New Mexico & 617 & 90.7 & 1.8 & $(87.2-$ \\
\hline San Juan County, New Mexico & 623 & 89.6 & 1.5 & $(86.6-92.6)$ \\
\hline Santa Fe County, New Mexico & 662 & 87.1 & 1.9 & $(83.4-90.7)$ \\
\hline Kings County, New York & 472 & 85.4 & 2.0 & $(81.5-89.4)$ \\
\hline Guilford County, North Carolina & 502 & 89.9 & 1.6 & $(86.7-93.1)$ \\
\hline Mecklenburg County, North Carolina & 705 & 90.4 & 1.3 & $(87.8-93.1)$ \\
\hline Robeson County, North Carolina & 525 & 74.6 & 4.6 & $(65.7-83.6)$ \\
\hline Wake County, North Carolina & 687 & 93.7 & 1.1 & $(91.6-95.8)$ \\
\hline Burleigh County, North Dakota & 563 & 69.9 & 2.7 & $(64.6-75.2)$ \\
\hline Cass County, North Dakota & 826 & 79.4 & 2.0 & $(75.4-83.3)$ \\
\hline Cuyahoga County, Ohio & 798 & 79.0 & 1.8 & $(75.4-82.6)$ \\
\hline Franklin County, Ohio & 802 & 82.4 & 1.7 & $(78.9-85.8)$ \\
\hline Hamilton County, Ohio & 743 & 85.0 & 1.6 & $(81.8-88.2)$ \\
\hline Lorain County, Ohio & 588 & 80.2 & 3.3 & $(73.8-86.6)$ \\
\hline Lucas County, Ohio & 605 & 82.0 & 2.9 & $(76.4-87.6)$ \\
\hline Mahoning County, Ohio & 572 & 81.3 & 2.8 & $(75.8-86.9)$ \\
\hline Montgomery County, Ohio & 643 & 81.8 & 2.1 & $(77.6-85.9)$ \\
\hline Stark County, Ohio & 596 & 79.1 & 2.4 & $(74.4-83.8)$ \\
\hline Summit County, Ohio & 606 & 79.4 & 2.4 & $(74.6-84.2)$ \\
\hline Oklahoma County, Oklahoma & 1,162 & 86.5 & 1.4 & $(83.8-89.2)$ \\
\hline Tulsa County, Oklahoma & 1,179 & 87.8 & 1.2 & $(85.4-90.3)$ \\
\hline Clackamas County, Oregon & 497 & 97.4 & 0.8 & $(95.9-98.9)$ \\
\hline Lane County, Oregon & 509 & 93.1 & 1.7 & $(89.8-96.5)$ \\
\hline Multnomah County, Oregon & 864 & 92.4 & 1.3 & $(89.9-94.9)$ \\
\hline Washington County, Oregon & 601 & 95.6 & 1.1 & $(93.6-97.7)$ \\
\hline Allegheny County, Pennsylvania & 1,842 & 77.9 & 1.4 & $(75.1-80.6)$ \\
\hline Bradford County, Pennsylvania & 1,818 & 79.6 & 1.9 & $(76.0-83.3)$ \\
\hline Montgomery County, Pennsylvania & 533 & 81.3 & 2.3 & $(76.8-85.8)$ \\
\hline Philadelphia County, Pennsylvania & 1,965 & 72.1 & 1.5 & $(69.2-75.0)$ \\
\hline Pike County, Pennsylvania & 1,851 & 82.2 & 3.3 & $(75.7-88.6)$ \\
\hline Kent County, Rhode Island & 789 & 84.7 & 2.0 & $(80.9-88.5)$ \\
\hline Providence County, Rhode Is & 3,217 & 82.9 & 1.0 & $(80.9-84.9)$ \\
\hline Washington County, Rhode Island & 637 & 90.4 & 1.6 & $(87.2-93.5)$ \\
\hline
\end{tabular}

TABLE 45. (Continued) Estimated prevalence of adults aged $\geq 18$ years who reported they always use a seat belt when they ride in or drive a car, by county - Behavioral Risk Factor Surveillance System, United States, 2012

\begin{tabular}{|c|c|c|c|c|}
\hline County & $\begin{array}{l}\text { Sample } \\
\text { size }\end{array}$ & $\%$ & SE & $95 \% \mathrm{Cl}$ \\
\hline iken County, South Carolina & 535 & 86.6 & 2.8 & $(81.1-92.1)$ \\
\hline Beaufort County, South Carolina & 777 & 85.2 & 3.3 & $(78.6-91.7)$ \\
\hline Charleston County, South Carolina & 992 & 84.6 & 1.7 & $(81.2-87.9)$ \\
\hline Greenville County, South Carolina & 878 & 86.5 & 1.8 & $(82.9-90.1)$ \\
\hline Horry County, South Carolina & 757 & 89.1 & 1.4 & $(86.4-91.9)$ \\
\hline Richland County, South Carolina & 936 & 81.7 & 2.1 & $(77.6-85.7)$ \\
\hline Spartanburg County, South Carolina & 639 & 80.2 & 2.4 & $(75.4-85.0)$ \\
\hline Lincoln County, South Dakota & 490 & 72.6 & 2.9 & $(66.9-78.3)$ \\
\hline Minnehaha County, South Dakota & 863 & 68.2 & 1.9 & $(64.5-71.9)$ \\
\hline Pennington County, South Dakota & 599 & 68.6 & 2.3 & $(64.1-73.2)$ \\
\hline Davidson County, Tennessee & 527 & 90.5 & 1.7 & $(87.3-93.8)$ \\
\hline Shelby County, Tennessee & 497 & 89.2 & 1.9 & $(85.4-92.9)$ \\
\hline Bexar County, Texas & 567 & 90.4 & 2.2 & $(86.2-94.7)$ \\
\hline Dallas County, Texas & 489 & 95.2 & 1.2 & $(92.9-97.6)$ \\
\hline El Paso County, Texas & 607 & 90.2 & 2.3 & $(85.7-94.6)$ \\
\hline Harris County, Texas & 742 & 92.2 & 1.3 & $(89.7-94.7)$ \\
\hline Hidalgo County, Texas & 607 & 90.8 & 1.9 & $(87.0-94.6)$ \\
\hline Tarrant County, Texas & 564 & 92.6 & 1.6 & $(89.5-95.7)$ \\
\hline Travis County, Texas & 1,030 & 93.1 & 1.3 & $(90.5-95.8)$ \\
\hline Davis County, Utah & 1,120 & 85.5 & 1.4 & $(82.8-88.2)$ \\
\hline Salt Lake County, Utah & 3,945 & 83.4 & 0.8 & $(81.8-85.0)$ \\
\hline Tooele County, Utah & 547 & 76.4 & 3.2 & $(70.1-82.7)$ \\
\hline Utah County, Utah & 1,632 & 76.2 & 1.4 & $(73.6-78.9)$ \\
\hline Wasatch County, Utah & 501 & 73.3 & 4.2 & $(65.0-81.5)$ \\
\hline Weber County, Utah & 1,020 & 82.2 & 1.5 & $(79.2-85.2)$ \\
\hline Chittenden County, Vermont & 897 & 85.5 & 1.4 & $(82.7-88.3)$ \\
\hline Rutland County, Vermont & 582 & 84.6 & 2.1 & $(80.5-88.7)$ \\
\hline Washington County, Vermont & 510 & 81.7 & 2.6 & $(76.6-86.8)$ \\
\hline Windsor County, Vermont & 533 & 79.1 & 2.3 & $(74.6-83.7)$ \\
\hline Fairfax County, Virginia & 700 & 92.1 & 1.4 & $(89.5-94.8)$ \\
\hline Clark County, Washington & 778 & 93.9 & 1.2 & $(91.6-96.1)$ \\
\hline King County, Washington & 3,872 & 91.5 & 0.7 & $(90.2-92.8)$ \\
\hline Kitsap County, Washington & 559 & 94.0 & 1.4 & $(91.2-96.8)$ \\
\hline Pierce County, Washington & 1,166 & 92.0 & 1.1 & $(89.9-94.2)$ \\
\hline Snohomish County, Washington & 1,147 & 91.5 & 1.1 & $(89.3-93.8)$ \\
\hline Spokane County, Washington & 935 & 91.1 & 1.4 & $(88.3-93.9)$ \\
\hline Thurston County, Washington & 508 & 91.2 & 1.8 & $(87.6-94.7)$ \\
\hline Whatcom County, Washington & 836 & 93.3 & 1.3 & $(90.7-95.9)$ \\
\hline Yakima County, Washington & 525 & 92.7 & 1.7 & $(89.4-95.9)$ \\
\hline Kanawha County, West Virginia & 639 & 84.7 & 1.7 & $(81.3-88.1)$ \\
\hline Milwaukee County, Wisconsin & 888 & 75.2 & 2.5 & $(70.4-80.0)$ \\
\hline Laramie County, Wyoming & 926 & 76.6 & 2.4 & $(71.8-81.4)$ \\
\hline Natrona County, Wyoming & 800 & 73.8 & 2.6 & $(68.7-78.8)$ \\
\hline San Juan Municipio, Puerto Rico & 664 & 93.1 & 1.2 & $(90.6-95.5)$ \\
\hline Median & & 86.0 & & \\
\hline Range & & $50.1-97.4$ & & \\
\hline
\end{tabular}

Abbreviations: $\mathrm{Cl}=$ confidence interval; $\mathrm{SE}=$ standard error. 
TABLE 46. Estimated prevalence of adults aged $\geq 18$ years who are obese, ${ }^{*}$ by state/territory - Behavioral Risk Factor Surveillance System, United States, 2012

\begin{tabular}{|c|c|c|c|c|}
\hline State/Territory & $\begin{array}{l}\text { Sample } \\
\text { size }\end{array}$ & $\%$ & SE & $95 \% \mathrm{Cl}$ \\
\hline Alabama & 8,651 & 33.0 & 0.7 & $(31.5-34.4)$ \\
\hline Alaska & 4,093 & 25.7 & 0.9 & (23.9-27.5) \\
\hline Arizona & 6,951 & 26.0 & 0.9 & $(24.3-27.7)$ \\
\hline Arkansas & 4,943 & 34.5 & 0.9 & $(32.7-36.4)$ \\
\hline California & 13,646 & 25.0 & 0.5 & $(23.9-26.0)$ \\
\hline Colorado & 11,643 & 20.5 & 0.5 & $(19.5-21.4)$ \\
\hline Connecticut & 8,312 & 25.6 & 0.7 & $(24.3-26.9)$ \\
\hline Delaware & 4,944 & 26.9 & 0.9 & $(25.2-28.6)$ \\
\hline District of Columbia & 3,644 & 21.9 & 1.1 & $(19.8-24.0)$ \\
\hline Florida & 7,282 & 25.2 & 0.8 & $(23.6-26.7)$ \\
\hline Georgia & 5,737 & 29.1 & 0.8 & $(27.4-30.8)$ \\
\hline Hawaii & 7,371 & 23.6 & 0.8 & $(22.0-25.1)$ \\
\hline Idaho & 5,578 & 26.8 & 1.0 & $(24.8-28.8)$ \\
\hline Illinois & 5,452 & 28.1 & 0.9 & (26.4-29.9) \\
\hline Indiana & 8,225 & 31.4 & 0.7 & $(30.1-32.7)$ \\
\hline lowa & 6,769 & 30.4 & 0.7 & $(29.1-31.8)$ \\
\hline Kansas & 11,238 & 29.8 & 0.6 & $(28.7-31.0)$ \\
\hline Kentucky & 10,570 & 31.3 & 0.7 & $(29.9-32.6)$ \\
\hline Louisiana & 8,656 & 34.7 & 0.8 & $(33.1-36.4)$ \\
\hline Maine & 9,512 & 28.3 & 0.6 & $(27.2-29.5)$ \\
\hline Maryland & 11,947 & 27.6 & 0.7 & (26.3-28.9) \\
\hline Massachusetts & 19,904 & 22.9 & 0.5 & $(22.0-23.8)$ \\
\hline Michigan & 10,052 & 31.1 & 0.6 & $(29.8-32.3)$ \\
\hline Minnesota & 11,429 & 25.7 & 0.5 & $(24.7-26.8)$ \\
\hline Mississippi & 7,429 & 34.6 & 0.8 & $(33.0-36.2)$ \\
\hline Missouri & 6,446 & 29.6 & 0.8 & $(28.0-31.2)$ \\
\hline Montana & 8,357 & 24.3 & 0.6 & $(23.1-25.5)$ \\
\hline Nebraska & 18,385 & 28.6 & 0.5 & $(27.7-29.6)$ \\
\hline Nevada & 4,592 & 26.2 & 1.0 & $(24.3-28.1)$ \\
\hline New Hampshire & 7,182 & 27.3 & 0.8 & $(25.8-28.8)$ \\
\hline New Jersey & 14,477 & 24.6 & 0.5 & $(23.6-25.6)$ \\
\hline New Mexico & 8,385 & 27.1 & 0.6 & $(25.9-28.3)$ \\
\hline New York & 5,700 & 23.6 & 0.8 & $(22.0-25.1)$ \\
\hline North Carolina & 11,113 & 29.6 & 0.6 & $(28.5-30.7)$ \\
\hline North Dakota & 4,625 & 29.7 & 0.9 & $(27.9-31.4)$ \\
\hline Ohio & 12,319 & 30.1 & 0.6 & $(29.0-31.2)$ \\
\hline Oklahoma & 7,719 & 32.2 & 0.7 & $(30.8-33.6)$ \\
\hline Oregon & 5,056 & 27.3 & 0.8 & $(25.7-29.0)$ \\
\hline Pennsylvania & 18,893 & 29.1 & 0.5 & $(28.1-30.0)$ \\
\hline Rhode Island & 5,188 & 25.7 & 0.8 & $(24.1-27.4)$ \\
\hline South Carolina & 12,210 & 31.6 & 0.6 & (30.4-32.8) \\
\hline South Dakota & 7,433 & 28.1 & 0.8 & $(26.5-29.8)$ \\
\hline Tennessee & 6,613 & 31.1 & 0.8 & $(29.6-32.7)$ \\
\hline Texas & 8,530 & 29.2 & 0.7 & $(27.8-30.5)$ \\
\hline Utah & 11,683 & 24.3 & 0.5 & $(23.3-25.3)$ \\
\hline Vermont & 5,791 & 23.7 & 0.7 & $(22.3-25.1)$ \\
\hline Virginia & 6,948 & 27.4 & 0.7 & $(26.0-28.7)$ \\
\hline Washington & 14,540 & 26.8 & 0.5 & $(25.8-27.8)$ \\
\hline West Virginia & 5,125 & 33.8 & 0.8 & $(32.2-35.4)$ \\
\hline Wisconsin & 4,966 & 29.7 & 1.0 & $(27.8-31.6)$ \\
\hline Wyoming & 5,976 & 24.6 & 0.9 & $(22.8-26.4)$ \\
\hline Guam & 1,926 & 29.1 & 1.4 & $(26.3-31.9)$ \\
\hline Puerto Rico & 6,056 & 28.4 & 0.7 & $(27.0-29.7)$ \\
\hline Median & & 28.1 & & \\
\hline Range & & $20.5-34.7$ & & \\
\hline
\end{tabular}

Abbreviations: $\mathrm{Cl}=$ confidence interval; $\mathrm{SE}=$ standard error.

* Body mass index $\geq 30.0 \mathrm{~kg} / \mathrm{m}^{2}$.
TABLE 47. Estimated prevalence of adults aged $\geq 18$ years who are obese, ${ }^{*}$ by metropolitan and micropolitan statistical area Behavioral Risk Factor Surveillance System, United States, 2012

\begin{tabular}{|c|c|c|c|c|}
\hline MMSA(s) & $\begin{array}{l}\text { Sample } \\
\text { size }\end{array}$ & $\%$ & SE & $95 \% \mathrm{Cl}$ \\
\hline Aguadilla-Isabela, Puerto Rico & 519 & 23.8 & 2.2 & $(19.6-28.0)$ \\
\hline Akron, Ohio & 698 & 29.7 & 2.4 & $(25.0-34.4)$ \\
\hline Albuquerque, New Mexico & 3,137 & 25.1 & 1.0 & $(23.2-27.0)$ \\
\hline $\begin{array}{l}\text { Allentown-Bethlehem-Easton, } \\
\text { Pennsylvania-New Jersey }\end{array}$ & 1,270 & 28.8 & 1.9 & $(25.1-32.6)$ \\
\hline Anaheim-Santa Ana-Irvine, California ${ }^{\dagger}$ & 971 & 21.5 & 2.0 & $(17.7-25.4)$ \\
\hline Anchorage, Alaska & 1,426 & 25.3 & 1.4 & $(22.5-28.0)$ \\
\hline Asheville, North Carolina & 557 & 19.4 & 2.0 & $(15.5-23.3)$ \\
\hline $\begin{array}{l}\text { Atlanta-Sandy Springs-Roswell, } \\
\text { Georgia }\end{array}$ & 2,399 & 26.5 & 1.2 & $(24.1-28.9)$ \\
\hline Atlantic City-Hammonton, New Jersey & 957 & 26.5 & 2.1 & $(22.4-30.6)$ \\
\hline $\begin{array}{l}\text { Augusta-Richmond County, Georgia- } \\
\text { South Carolina }\end{array}$ & 980 & 30.9 & 2.8 & $(25.3-36.4)$ \\
\hline Augusta-Waterville, Maine & 794 & 33.7 & 2.0 & $(29.7-37.6)$ \\
\hline Austin-Round Rock, Texas & 1,321 & 25.5 & 1.9 & $(21.8-29.2)$ \\
\hline $\begin{array}{l}\text { Baltimore-Columbia-Towson, } \\
\text { Maryland }\end{array}$ & 4,428 & 27.4 & 1.0 & $(25.5-29.3)$ \\
\hline Bangor, Maine & 892 & 30.0 & 1.9 & $(26.2-33.8)$ \\
\hline Barnstable Town, Massachusetts & 531 & 18.0 & 2.0 & $(14.0-21.9)$ \\
\hline Barre, Vermont & 495 & 23.1 & 2.4 & $(18.4-27.7)$ \\
\hline Baton Rouge, Louisiana & 1,336 & 35.6 & 2.1 & $(31.6-39.7)$ \\
\hline Bellingham, Washington & 812 & 20.5 & 2.1 & $(16.5-24.6)$ \\
\hline Berlin, New Hampshire-Vermont & 671 & 29.6 & 2.8 & $(24.2-35.0)$ \\
\hline Billings, Montana & 815 & 23.3 & 1.7 & $(20.0-26.6)$ \\
\hline Birmingham-Hoover, Alabama & 1,748 & 34.3 & 1.5 & $(31.3-37.3)$ \\
\hline Bismarck, North Dakota & 782 & 33.0 & 2.3 & $(28.5-37.5)$ \\
\hline Boise City, Idaho & 1,399 & 28.1 & 1.8 & $(24.5-31.6)$ \\
\hline Boston, Massachusetts ${ }^{\dagger}$ & 5,400 & 21.1 & 0.9 & $(19.4-22.8)$ \\
\hline Boulder, Colorado & 493 & 14.8 & 2.0 & $(11.0-18.6)$ \\
\hline Bremerton-Silverdale, Washington & 551 & 30.0 & 2.6 & $(24.9-35.1)$ \\
\hline $\begin{array}{l}\text { Bridgeport-Stamford-Norwalk, } \\
\text { Connecticut }\end{array}$ & 2,048 & 21.6 & 1.3 & $(19.1-24.0)$ \\
\hline Burlington-South Burlington, Vermont & 1,465 & 21.3 & 1.3 & $(18.8-23.8)$ \\
\hline $\begin{array}{l}\text { Cambridge-Newton-Framingham, } \\
\text { Massachusetts }{ }^{\dagger}\end{array}$ & 6,328 & 20.1 & 0.7 & $(18.6-21.5)$ \\
\hline Camden, New Jersey ${ }^{\dagger}$ & 1,858 & 29.1 & 1.5 & $(26.2-32.0)$ \\
\hline Canton-Massillon, Ohio & 657 & 31.7 & 2.5 & $(26.8-36.5)$ \\
\hline Casper, Wyoming & 793 & 23.1 & 2.3 & $(18.7-27.5)$ \\
\hline Cedar Rapids, lowa & 567 & 30.3 & 2.5 & $(25.4-35.3)$ \\
\hline Charleston, West Virginia & 730 & 31.5 & 2.0 & $(27.6-35.5)$ \\
\hline $\begin{array}{l}\text { Charleston-North Charleston, } \\
\text { South Carolina }\end{array}$ & 1,650 & 28.5 & 1.6 & $(25.4-31.6)$ \\
\hline $\begin{array}{l}\text { Charlotte-Concord-Gastonia, } \\
\text { North Carolina-South Carolina }\end{array}$ & 2,339 & 27.9 & 1.2 & $(25.5-30.3)$ \\
\hline Chattanooga, Tennessee-Georgia & 597 & 28.8 & 2.9 & $(23.0-34.6)$ \\
\hline Cheyenne, Wyoming & 906 & 27.3 & 2.3 & $(22.7-31.9)$ \\
\hline $\begin{array}{l}\text { Chicago-Naperville-Elgin, } \\
\text { Illinois-Indiana-Wisconsin }\end{array}$ & 3,620 & 26.6 & 1.1 & $(24.4-28.7)$ \\
\hline Cincinnati, Ohio-Kentucky-Indiana & 2,249 & 28.3 & 1.2 & $(25.9-30.7)$ \\
\hline $\begin{array}{l}\text { Claremont-Lebanon, New } \\
\text { Hampshire-Vermont }\end{array}$ & 1,865 & 25.2 & 1.4 & $(22.5-27.9)$ \\
\hline Cleveland-Elyria, Ohio & 1,665 & 27.7 & 1.6 & $(24.6-30.7)$ \\
\hline Colorado Springs, Colorado & 1,094 & 21.1 & 1.5 & $(18.1-24.1)$ \\
\hline Columbia, South Carolina & 1,712 & 31.6 & 1.6 & $(28.5-34.8)$ \\
\hline Columbus, Ohio & 1,525 & 30.7 & 1.6 & $(27.6-33.7)$ \\
\hline Concord, New Hampshire & 672 & 29.6 & 2.3 & $(25.0-34.2)$ \\
\hline Dallas-Plano-Irving, Texas ${ }^{\dagger}$ & 852 & 26.1 & 1.8 & $(22.6-29.7)$ \\
\hline Dayton, Ohio & 818 & 29.5 & 2.1 & $(25.3-33.7)$ \\
\hline Denver-Aurora-Lakewood, Colorado & 4,616 & 20.1 & 0.7 & $(18.7-21.5)$ \\
\hline Des Moines-West Des Moines, lowa & 1,100 & 29.1 & 1.6 & $(25.9-32.3)$ \\
\hline 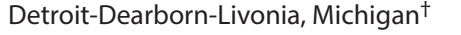 & 2,121 & 34.2 & 1.6 & (31.1-37.4) \\
\hline
\end{tabular}

See table footnotes on page 93. 
TABLE 47. (Continued) Estimated prevalence of adults aged $\geq 18$ years who are obese,* by metropolitan and micropolitan statistical area — Behavioral Risk Factor Surveillance System, United States, 2012

\begin{tabular}{|c|c|c|c|c|}
\hline MMSA(s) & $\begin{array}{l}\text { Sample } \\
\text { size }\end{array}$ & $\%$ & SE & $95 \% \mathrm{Cl}$ \\
\hline Dover, Delaware & 1,369 & 29.1 & 1.7 & $(25.7-32.5)$ \\
\hline Duluth, Minnesota-Wisconsin & 479 & 30.2 & 2.8 & $(24.8-35.6)$ \\
\hline Durham-Chapel Hill, North Carolina & 738 & 26.9 & 2.0 & $(23.0-30.8)$ \\
\hline El Paso, Texas & 576 & 29.4 & 3.1 & $(23.4-35.5)$ \\
\hline Eugene, Oregon & 507 & 30.6 & 2.8 & $(25.2-36.1)$ \\
\hline Fairbanks, Alaska & 564 & 23.5 & 2.2 & $(19.3-27.7)$ \\
\hline Fargo, North Dakota-Minnesota & 898 & 25.1 & 2.0 & $(21.2-28.9)$ \\
\hline Farmington, New Mexico & 622 & 29.9 & 2.4 & $(25.3-34.5)$ \\
\hline Fayetteville, North Carolina & 481 & 30.7 & 2.6 & $(25.5-35.9)$ \\
\hline $\begin{array}{l}\text { Fayetteville-Springdale-Rogers, } \\
\text { Arkansas-Missouri }\end{array}$ & 781 & 29.1 & 2.5 & $(24.1-34.0)$ \\
\hline Fort Collins, Colorado & 572 & 18.3 & 1.9 & $(14.6-22.0)$ \\
\hline Fort Wayne, Indiana & 505 & 33.5 & 2.6 & $(28.3-38.6)$ \\
\hline Fort Worth-Arlington, Texas ${ }^{\dagger}$ & 679 & 30.0 & 2.4 & $(25.4-34.7)$ \\
\hline Grand Island, Nebraska & 819 & 33.0 & 2.3 & $(28.6-37.5)$ \\
\hline Grand Rapids-Wyoming, Michigan & 849 & 24.4 & 2.0 & $(20.4-28.4)$ \\
\hline Great Falls, Montana & 683 & 31.4 & 2.3 & $(26.8-36.0)$ \\
\hline Greeley, Colorado & 508 & 26.7 & 2.5 & $(21.8-31.7)$ \\
\hline $\begin{array}{l}\text { Greensboro-High Point, } \\
\text { North Carolina }\end{array}$ & 754 & 32.2 & 2.2 & $(28.0-36.5)$ \\
\hline $\begin{array}{l}\text { Greenville-Anderson-Mauldin, } \\
\text { South Carolina }\end{array}$ & 1,602 & 31.2 & 1.5 & $(28.2-34.2)$ \\
\hline Gulfport-Biloxi-Pascagoula, Mississippi & 722 & 28.8 & 2.2 & $(24.6-33.1)$ \\
\hline $\begin{array}{l}\text { Hagerstown-Martinsburg, Maryland- } \\
\text { West Virginia }\end{array}$ & 709 & 34.3 & 2.8 & $(28.8-$ \\
\hline Harrisburg-Carlisle, Pennsylvania & 622 & 33.2 & 2.3 & $(28.7-37.8)$ \\
\hline $\begin{array}{l}\text { Hartford-West Hartford-East Hartford, } \\
\text { Connecticut }\end{array}$ & 2,527 & 26.7 & 1.2 & $(24.4-29.1)$ \\
\hline Heber, Utah & 491 & 17.6 & 2.8 & $(12.1-23.1)$ \\
\hline Hilo, Hawaii & 1,320 & 24.9 & 1.7 & $(21.5-28.2)$ \\
\hline $\begin{array}{l}\text { Hilton Head Island-Bluffton-Beaufort, } \\
\text { South Carolina }\end{array}$ & 894 & 23.0 & 2.5 & $(18.0-27.9)$ \\
\hline $\begin{array}{l}\text { Houston-The Woodlands-Sugar Land, } \\
\text { Texas }\end{array}$ & 1,054 & 26.6 & 1.7 & $(23.3-29.8)$ \\
\hline $\begin{array}{l}\text { Huntington-Ashland, West } \\
\text { Virginia-Kentucky-Ohio }\end{array}$ & 2 & 35.1 & 1.9 & $(31.3-38.9)$ \\
\hline Huntsville, Alabama & 595 & 29.4 & 2.4 & $(24.7-34.1)$ \\
\hline Idaho Falls, Idaho & 515 & 29.7 & 3.3 & $(23.2-36.2)$ \\
\hline $\begin{array}{l}\text { Indianapolis-Carmel-Anderson, } \\
\text { Indiana }\end{array}$ & 2,096 & 30.1 & 1.3 & $(27.6-32.6)$ \\
\hline Jackson, Mississippi & 879 & 33.6 & 2.0 & $(29.8-37.5)$ \\
\hline Jacksonville, Florida & 494 & 29.2 & 3.1 & $(23.1-35.2)$ \\
\hline Kahului-Wailuku-Lahaina, Hawaii & 1,190 & 21.4 & 1.8 & $(17.8-24.9)$ \\
\hline Kalispell, Montana & 539 & 26.2 & 2.3 & $(21.7-30.6)$ \\
\hline Kansas City, Missouri-Kansas & 4,507 & 28.3 & 1.2 & $(25.8-30.7)$ \\
\hline Kapaa, Hawaii & 656 & 21.0 & 2.5 & $(16.1-25.9)$ \\
\hline Keene, New Hampshire & 527 & 24.5 & 2.7 & $(19.2-29.8)$ \\
\hline Kennewick-Richland, Washington & 501 & 31.7 & 2.7 & $(26.4-36.9)$ \\
\hline $\begin{array}{l}\text { Kingsport-Bristol-Bristol, } \\
\text { Tennessee-Virginia }\end{array}$ & 528 & 32.9 & 3.1 & $(26.8-39.1)$ \\
\hline Knoxville, Tennessee & 763 & 29.9 & 2.2 & $(25.6-34.2)$ \\
\hline Laconia, New Hampshire & 542 & 34.0 & 3.3 & $(27.5-40.5)$ \\
\hline Lafayette, Louisiana & 527 & 29.6 & 2.7 & $(24.4-34.8)$ \\
\hline Las Cruces, New Mexico & 667 & 26.7 & 2.1 & $(22.5-31.0)$ \\
\hline $\begin{array}{l}\text { Las Vegas-Henderson-Paradise, } \\
\text { Nevada }\end{array}$ & 1,884 & 27.4 & 1.3 & $(24.9-29.9)$ \\
\hline Lewiston-Auburn, Maine & 657 & 33.6 & 2.2 & $(29.3-38.0)$ \\
\hline Lexington-Fayette, Kentucky & 497 & 22.8 & 2.2 & $(18.5-27.1)$ \\
\hline Lincoln, Nebraska & 1,614 & 24.5 & 1.3 & $(22.0-27.1)$ \\
\hline $\begin{array}{l}\text { Little Rock-North Little Rock-Conway, } \\
\text { Arkansas }\end{array}$ & 1,112 & 32.7 & 1.9 & $(29.0-36.4)$ \\
\hline
\end{tabular}

See table footnotes on page 93.
TABLE 47. (Continued) Estimated prevalence of adults aged $\geq 18$ years who are obese, ${ }^{*}$ by metropolitan and micropolitan statistical area — Behavioral Risk Factor Surveillance System, United States, 2012

\begin{tabular}{|c|c|c|c|c|}
\hline MMSA(s) & $\begin{array}{l}\text { Sample } \\
\text { size }\end{array}$ & $\%$ & SE & $95 \% \mathrm{Cl}$ \\
\hline Logan, Utah-Idaho & 480 & 18.7 & 2.1 & $(14.7-22.8)$ \\
\hline $\begin{array}{l}\text { Los Angeles-Long Beach-Glendale, } \\
\text { California }^{\dagger}\end{array}$ & 3,204 & 25.0 & 1.1 & $(22.9-27.0)$ \\
\hline $\begin{array}{l}\text { Louisville/Jefferson County, } \\
\text { Kentucky-Indiana }\end{array}$ & 2,054 & 31.8 & 1.6 & $(28.6-34.9)$ \\
\hline Lumberton, North Carolina & 512 & 38.7 & 4.3 & $(30.3-47.2)$ \\
\hline Manchester-Nashua, New Hampshire & 1,827 & 26.9 & 1.3 & $(24.3-29.4)$ \\
\hline McAllen-Edinburg-Mission, Texas & 559 & 44.5 & 3.3 & $(38.0-51.0)$ \\
\hline $\begin{array}{l}\text { Memphis, } \\
\text { Tennessee-Mississippi-Arkansas }\end{array}$ & 1,225 & 35.1 & 2.0 & $(31.2-39.0)$ \\
\hline $\begin{array}{l}\text { Miami-Fort Lauderdale-West Palm } \\
\text { Beach, Florida }\end{array}$ & 1,580 & 23.1 & 1.6 & $(20.0-26.3)$ \\
\hline $\begin{array}{l}\text { Milwaukee-Waukesha-West Allis, } \\
\text { Wisconsin }\end{array}$ & 1,176 & 30.9 & 2.1 & $(26.9-34.9)$ \\
\hline $\begin{array}{l}\text { Minneapolis-St. Paul-Bloomington, } \\
\text { Minnesota-Wisconsin }\end{array}$ & 7,351 & 24.0 & 0.7 & $(22.5-25.4)$ \\
\hline Missoula, Montana & 771 & 18.6 & 1.7 & $(15.3-21.9)$ \\
\hline Mobile, Alabama & 791 & 34.2 & 2.6 & $(29.2-39.3)$ \\
\hline Montgomery, Alabama & 507 & 33.2 & 2.8 & $(27.7-38.8)$ \\
\hline $\begin{array}{l}\text { Montgomery County-Bucks County- } \\
\text { Chester County, Pennsylvania }^{\dagger}\end{array}$ & 1,270 & 23.3 & 1.5 & $(20.4-26.2)$ \\
\hline $\begin{array}{l}\text { Myrtle Beach-Conway-North Myrtle } \\
\text { Beach, South Carolina-North Carolina }\end{array}$ & 915 & 26.7 & 2.0 & $(22.8-30.5)$ \\
\hline $\begin{array}{l}\text { Nashville-Davidson-Murfreesboro- } \\
\text { Franklin, Tennessee }\end{array}$ & 1,271 & 29.1 & 1.6 & $(26.0-32.2)$ \\
\hline $\begin{array}{l}\text { Nassau County-Suffolk County, } \\
\text { New York }^{\dagger}\end{array}$ & 854 & 22.2 & 1.9 & $(18.5-25.8)$ \\
\hline Newark, New Jersey-Pennsylvania ${ }^{\dagger}$ & 6,075 & 24.8 & 0.9 & $(23.1-26.5)$ \\
\hline New Haven-Milford, Connecticut & 1,905 & 26.0 & 1.4 & $(23.2-28.8)$ \\
\hline New Orleans-Metairie, Louisiana & 1,219 & 28.7 & 1.7 & $(25.3-32.1)$ \\
\hline $\begin{array}{l}\text { New York-Jersey City-White Plains, } \\
\text { New York-New Jersey }{ }^{\dagger}\end{array}$ & 6,806 & 21.7 & 0.9 & $(20.0-23.5)$ \\
\hline Norfolk, Nebraska & 547 & 30.5 & 2.6 & $(25.5-35.5)$ \\
\hline North Platte, Nebraska & 597 & 32.1 & 2.8 & $(26.7-37.6)$ \\
\hline Norwich-New London, Connecticut & 957 & 27.9 & 2.2 & $(23.5-32.3)$ \\
\hline $\begin{array}{l}\text { Oakland-Hayward-Berkeley, } \\
\text { California }^{\dagger}\end{array}$ & 931 & 22.6 & 2.2 & $(18.3-26.9)$ \\
\hline Ocean City, New Jersey & 522 & 29.9 & 3.5 & $(22.9-36.8)$ \\
\hline Ogden-Clearfield, Utah & 2,295 & 28.0 & 1.2 & $(25.7-30.3)$ \\
\hline Oklahoma City, Oklahoma & 2,324 & 32.0 & 1.2 & $(29.6-34.5)$ \\
\hline Olympia-Tumwater, Washington & 492 & 29.5 & 2.8 & $(24.1-34.9)$ \\
\hline Omaha-Council Bluffs, Nebraska-lowa & 5,296 & 28.6 & 0.9 & $(26.9-30.3)$ \\
\hline Orlando-Kissimmee-Sanford, Florida & 547 & 28.1 & 3.0 & $(22.2-34.0)$ \\
\hline Philadelphia, Pennsylvania ${ }^{\dagger}$ & 2,309 & 26.9 & 1.2 & $(24.5-29.3)$ \\
\hline Phoenix-Mesa-Scottsdale, Arizona & 2,467 & 25.3 & 1.2 & $(22.9-27.7)$ \\
\hline Pittsburgh, Pennsylvania & 3,207 & 26.9 & 1.0 & $(25.0-28.8)$ \\
\hline Ponce, Puerto Rico & 526 & 31.7 & 2.4 & $(27.0-36.4)$ \\
\hline Portland-South Portland, Maine & 3,193 & 24.2 & 0.9 & $(22.4-26.1)$ \\
\hline $\begin{array}{l}\text { Portland-Vancouver-Hillsboro, } \\
\text { Oregon-Washington }\end{array}$ & 2,973 & 25.9 & 1.1 & $(23.8-28.1)$ \\
\hline $\begin{array}{l}\text { Providence-Warwick, Rhode } \\
\text { Island-Massachusetts }\end{array}$ & 7,632 & 25.8 & 0.8 & $(24.2-27.3)$ \\
\hline Provo-Orem, Utah & 1,635 & 22.5 & 1.2 & $(20.1-24.8)$ \\
\hline Raleigh, North Carolina & 889 & 24.3 & 1.7 & $(21.1-27.6)$ \\
\hline Rapid City, South Dakota & 1,000 & 28.4 & 1.9 & $(24.6-32.2)$ \\
\hline Reno, Nevada & 1,435 & 21.9 & 1.6 & $(18.8-25.0)$ \\
\hline Richmond, Virginia & 949 & 27.5 & 1.9 & $(23.8-31.2)$ \\
\hline $\begin{array}{l}\text { Riverside-San Bernardino-Ontario, } \\
\text { California }\end{array}$ & 1,457 & 29.1 & 1.7 & $(25.9-32.4)$ \\
\hline $\begin{array}{l}\text { Rockingham County-Strafford County, } \\
\text { New Hampshire }\end{array}$ & 1,592 & 27.1 & 1.5 & $(24.1-30.1)$ \\
\hline
\end{tabular}

See table footnotes on page 93 . 
TABLE 47. (Continued) Estimated prevalence of adults aged $\geq 18$ years who are obese, ${ }^{*}$ by metropolitan and micropolitan statistical area - Behavioral Risk Factor Surveillance System, United States, 2012

\begin{tabular}{|c|c|c|c|c|}
\hline MMSA(s) & $\begin{array}{l}\text { Sample } \\
\text { size }\end{array}$ & $\%$ & SE & $95 \% \mathrm{Cl}$ \\
\hline Rutland, Vermont & 562 & 29.1 & 2.6 & $(23.9-34.2)$ \\
\hline $\begin{array}{l}\text { Sacramento-Roseville-Arden-Arcade, } \\
\text { California }\end{array}$ & 940 & 25.4 & 2.1 & $(21.3-29.5)$ \\
\hline St. Louis, Missouri-Illinois & 1,990 & 31.1 & 1.5 & $(28.0-34.1)$ \\
\hline Salisbury, Maryland-Delaware & 1,998 & 30.9 & 1.7 & $(27.5-34.2)$ \\
\hline Salt Lake City, Utah & 4,306 & 24.3 & 0.8 & $(22.6-25.9)$ \\
\hline San Antonio-New Braunfels, Texas & 745 & 28.5 & 2.1 & $(24.3-32.6)$ \\
\hline San Diego-Carlsbad, California & 1,058 & 22.3 & 1.8 & $(18.8-25.9)$ \\
\hline $\begin{array}{l}\text { San Francisco-Redwood City-South } \\
\text { San Francisco, California }{ }^{\dagger}\end{array}$ & 566 & 19.1 & 2.4 & $(14.5-23.8)$ \\
\hline $\begin{array}{l}\text { San Jose-Sunnyvale-Santa Clara, } \\
\text { California }\end{array}$ & 643 & 15.9 & 1.8 & $(12.3-19.5)$ \\
\hline San Juan-Carolina-Caguas, Puerto Rico & 3,809 & 28.5 & 0.9 & $(26.8-30.2)$ \\
\hline Santa Fe, New Mexico & 660 & 17.6 & 1.8 & $(14.2-21.1)$ \\
\hline Sayre, Pennsylvania & 1,753 & 33.6 & 2.6 & $(28.4-38.7)$ \\
\hline Scottsbluff, Nebraska & 602 & 39.2 & 3.3 & $(32.8-45.6)$ \\
\hline $\begin{array}{l}\text { Scranton-Wilkes-Barre-Hazleton, } \\
\text { Pennsylvania }\end{array}$ & 701 & 31.9 & 2.4 & $(27.2-36.7)$ \\
\hline Seattle-Bellevue-Everett, Washington ${ }^{\dagger}$ & 4,856 & 23.8 & 0.8 & $(22.2-25.3)$ \\
\hline Shreveport-Bossier City, Louisiana & 554 & 38.5 & 2.8 & $(33.0-44.1)$ \\
\hline $\begin{array}{l}\text { Silver Spring-Frederick-Rockville, } \\
\text { Maryland }^{\dagger}\end{array}$ & 2,120 & 21.1 & 1.4 & $(18.3-23.8)$ \\
\hline $\begin{array}{l}\text { Sioux City, lowa-Nebraska- } \\
\text { South Dakota }\end{array}$ & 1,160 & 35.9 & 3.1 & $(29.9-42.0)$ \\
\hline Sioux Falls, South Dakota & 1,381 & 25.3 & 1.5 & $(22.3-28.2)$ \\
\hline Spartanburg, Sout & 669 & 32.8 & 2.7 & $(27.5$ \\
\hline Spokane-Spokane Valley, Washington & 1,059 & 28.9 & 1.9 & $(25.2$ \\
\hline Springfield, Mas & 2,152 & 26.4 & 1.4 & $(23.6-29.2)$ \\
\hline Tacoma-Lakewood, Washington ${ }^{\dagger}$ & 1,134 & 26.7 & 1.6 & $(23.5-29.9)$ \\
\hline $\begin{array}{l}\text { Tampa-St. Petersburg-Clearwater, } \\
\text { Florida }\end{array}$ & 786 & 25.1 & 2.1 & $(21.0-29.3)$ \\
\hline Toledo, Ohio & 928 & 31.2 & 2.4 & $(26.4-35.9)$ \\
\hline Topeka, Kansas & 1,047 & 34.3 & 1.9 & $(30.5-38.1)$ \\
\hline Torrington, Connecticut & 640 & 24.0 & 2.3 & $(19.5-28.6)$ \\
\hline Trenton, New Jersey & 533 & 24.0 & 2.6 & $(18.9-29.1)$ \\
\hline Tucson, Arizona & 956 & 22.9 & 1.8 & $(19.4-26.4)$ \\
\hline Tulsa, Oklahoma & 1,689 & 32.1 & 1.5 & $(29.2-35.0)$ \\
\hline Tuscaloosa, Alabama & 561 & 34.8 & 3.3 & $(28.4-41.2)$ \\
\hline Urban Honolulu, Hawaii & 4,205 & 23.8 & 1.0 & $(21.8-25.8)$ \\
\hline Vineland-Bridgeto & 519 & 33.7 & 3.2 & $(27.4-40.0)$ \\
\hline $\begin{array}{l}\text { Virginia Beach-Norfolk-Newport News, } \\
\text { Virginia-North Carolina }\end{array}$ & 1,443 & 29.4 & 1.6 & $(26.3-32.6)$ \\
\hline $\begin{array}{l}\text { Warren-Troy-Farmington Hills, } \\
\text { Michigan }^{\dagger}\end{array}$ & 2,100 & 29.4 & 1.3 & $(26.9-31.9)$ \\
\hline $\begin{array}{l}\text { Washington-Arlington-Alexandria, } \\
\text { District of Columbia-Virginia- } \\
\text { Maryland-West Virginia }\end{array}$ & 7,706 & 24.2 & 0.8 & $(22.6-25.8)$ \\
\hline Wichita, Kansas & 2,257 & 29.0 & 1.3 & $(26.5-31.5)$ \\
\hline $\begin{array}{l}\text { Wilmington, Delaware-Maryland- } \\
\text { New Jersey }{ }^{\dagger}\end{array}$ & 3,076 & 26.5 & 1.2 & $(24.2-28.7)$ \\
\hline Winston-Salem, North Carolina & 737 & 29.4 & 2.1 & $(25.4-33.5)$ \\
\hline Worcester, Massachusetts-Connecticut & 2,628 & 28.5 & 1.3 & $(26.0-31.1)$ \\
\hline Yakima, Washington & 472 & 31.4 & 3.0 & $(25.5-37.3)$ \\
\hline $\begin{array}{l}\text { Youngstown-Warren-Boardman, } \\
\text { Ohio-Pennsylvania }\end{array}$ & 876 & 32.2 & 2.5 & $(27.2-37.1)$ \\
\hline Median & & 28.3 & & \\
\hline Range & & $4.8-44$. & & \\
\hline
\end{tabular}

Abbreviations: $\mathrm{Cl}=$ confidence interval; $\mathrm{MMSA}=$ metropolitan and micropolitan statistical area; $\mathrm{SE}=$ standard error.

* Body mass index $\geq 30.0 \mathrm{~kg} / \mathrm{m}^{2}$.

+ Metropolitan division.
TABLE 48. Estimated prevalence of adults aged $\geq 18$ years who are obese, ${ }^{*}$ by county - Behavioral Risk Factor Surveillance System, United States, 2012

\begin{tabular}{|c|c|c|c|c|}
\hline County & $\begin{array}{l}\text { Sample } \\
\text { size }\end{array}$ & $\%$ & SE & $95 \% \mathrm{Cl}$ \\
\hline Jefferson County, Alabama & 921 & 34.8 & 2.0 & $(30.9-38.6)$ \\
\hline Madison County, Alabama & 497 & 29.1 & 2.7 & $(23.9-34.3)$ \\
\hline Mobile County, Alabama & 791 & 34.2 & 2.6 & $(29.2-39.3)$ \\
\hline Anchorage Municipality, Alaska & 852 & 25.3 & 1.7 & $(21.9-28.6)$ \\
\hline Fairbanks North Star Borough, Alaska & 564 & 23.5 & 2.2 & $(19.3-27.7)$ \\
\hline Matanuska-Susitna Borough, Alaska & 574 & 26.2 & 2.2 & $(21.9-30.5)$ \\
\hline Maricopa County, Arizona & 1,998 & 25.3 & 1.3 & $(22.7-27.9)$ \\
\hline Pima County, Arizona & 956 & 22.9 & 1.8 & $(19.4-26.4)$ \\
\hline Pulaski County, Arkansas & 709 & 33.2 & 2.5 & $(28.3-38.1)$ \\
\hline Alameda County, California & 595 & 20.4 & 2.8 & $(15.0-25.8)$ \\
\hline Los Angeles County, California & 3,204 & 25.0 & 1.1 & $(22.9-27.0)$ \\
\hline Orange County, California & 971 & 21.5 & 2.0 & $(17.7-25.4)$ \\
\hline Riverside County, California & 784 & 27.7 & 2.3 & $(23.2-32.2)$ \\
\hline Sacramento County, California & 572 & 27.5 & 2.8 & $(22.1-33.0)$ \\
\hline San Bernardino County, California & 673 & 31.4 & 2.4 & $(26.7-36.1)$ \\
\hline San Diego County, California & 1,058 & 22.3 & 1.8 & $(18.8-25.9)$ \\
\hline Santa Clara County, California & 626 & 15.8 & 1.8 & $(12.2-19.4)$ \\
\hline Adams County, Colorado & 764 & 25.2 & 2.0 & $(21.2-29.1)$ \\
\hline Arapahoe County, Colorado & 818 & 19.1 & 1.6 & $(15.9-22.3)$ \\
\hline Boulder County, Colorado & 493 & 14.8 & 2.0 & $(11.0-18.6)$ \\
\hline Denver County, Colorado & 928 & 19.0 & 1.5 & $(16.1-22.0)$ \\
\hline Douglas County, Colorado & 525 & 15.7 & 1.9 & $(12.1-19.4)$ \\
\hline El Paso County, Colorado & 957 & 21.3 & 1.6 & $(18.2-24.4)$ \\
\hline Jefferson County, Colorado & 1,082 & 20.2 & 1.5 & $(17.3-23.1)$ \\
\hline Larimer County, Colorado & 572 & 18.3 & 1.9 & $(14.6-22.0)$ \\
\hline Weld County, Colorado & 508 & 26.7 & 2.5 & $(21.8-31.7)$ \\
\hline Fairfield County, Connecticut & 2,048 & 21.6 & 1.3 & $(19.1-24.0)$ \\
\hline Hartford County, Connecticut & 1,874 & 28.3 & 1.4 & $(25.4-31.1)$ \\
\hline Litchfield County, Connecticut & 640 & 24.0 & 2.3 & $(19.5-28.6)$ \\
\hline New Haven County, Connecticut & 1,905 & 26.0 & 1.4 & $(23.2-28.8)$ \\
\hline New London County, Connecticut & 957 & 27.9 & 2.2 & $(23.5-32.3)$ \\
\hline Kent County, Delaware & 1,369 & 29.1 & 1.7 & $(25.7-32.5)$ \\
\hline New Castle County, Delaware & 2,239 & 25.2 & 1.2 & $(22.9-27.6)$ \\
\hline Sussex County, Delaware & 1,336 & 29.4 & 1.7 & $(26.1-32.7)$ \\
\hline $\begin{array}{l}\text { District of Columbia, District of } \\
\text { Columbia }\end{array}$ & 3,644 & 21.9 & 1.1 & $(19.8-24.0)$ \\
\hline Broward County, Florida & 497 & 22.6 & 2.7 & $(17.3-27.9)$ \\
\hline Miami-Dade County, Florida & 770 & 23.9 & 2.5 & $(19.0-28.7)$ \\
\hline Hawaii County, Hawaii & 1,320 & 24.9 & 1.7 & $(21.5-28.2)$ \\
\hline Honolulu County, Hawaii & 4,205 & 23.8 & 1.0 & $(21.8-25.8)$ \\
\hline Kauai County, Hawaii & 656 & 21.0 & 2.5 & $(16.1-25.9)$ \\
\hline Maui County, Hawaii & 1,190 & 21.4 & 1.8 & $(17.8-24.9)$ \\
\hline Ada County, Idaho & 764 & 25.1 & 2.2 & $(20.7-29.4)$ \\
\hline Canyon County, Idaho & 474 & 35.7 & 3.6 & $(28.6-42.7)$ \\
\hline Cook County, Illinois & 1,471 & 25.5 & 1.5 & $(22.6-28.4)$ \\
\hline Lake County, Indiana & 849 & 34.0 & 2.8 & $(28.6-39.4)$ \\
\hline Marion County, Indiana & 1,222 & 33.2 & 1.8 & $(29.6-36.9)$ \\
\hline Polk County, lowa & 760 & 28.5 & 1.9 & $(24.8-32.3)$ \\
\hline Johnson County, Kansas & 2,071 & 22.6 & 1.2 & $(20.2-25.0)$ \\
\hline Sedgwick County, Kansas & 1,721 & 28.4 & 1.4 & $(25.5-31.2)$ \\
\hline Shawnee County, Kansas & 735 & 31.3 & 2.2 & $(26.9-35.7)$ \\
\hline Wyandotte County, Kansas & 826 & 39.8 & 3.1 & $(33.7-45.9)$ \\
\hline Jefferson County, Kentucky & 1,587 & 33.2 & 2.0 & $(29.2-37.1)$ \\
\hline East Baton Rouge Parish, Louisiana & 553 & 33.3 & 2.7 & $(28.0-38.6)$ \\
\hline Androscoggin County, Maine & 657 & 33.6 & 2.2 & $(29.3-38.0)$ \\
\hline Aroostook County, Maine & 523 & 32.8 & 2.5 & $(27.9-37.7)$ \\
\hline Cumberland County, Maine & 1,695 & 21.6 & 1.2 & $(19.2-24.1)$ \\
\hline Kennebec County, Maine & 794 & 33.7 & 2.0 & $(29.7-37.6)$ \\
\hline Penobscot County, Maine & 892 & 30.0 & 1.9 & $(26.2-33.8)$ \\
\hline York County, Maine & 1,130 & 27.8 & 1.6 & $(24.7-31.0)$ \\
\hline
\end{tabular}


TABLE 48. (Continued) Estimated prevalence of adults aged $\geq 18$ years who are obese,* by county - Behavioral Risk Factor Surveillance System, United States, 2012

\begin{tabular}{|c|c|c|c|c|}
\hline County & $\begin{array}{l}\text { Sample } \\
\text { size }\end{array}$ & $\%$ & SE & $95 \% \mathrm{Cl}$ \\
\hline Anne Arundel County, Maryland & 845 & 26.5 & 2.2 & $(22.2-30.8)$ \\
\hline Baltimore County, Maryland & 1,424 & 28.1 & 1.7 & $(24.8-31.5)$ \\
\hline Charles County, Maryland & 476 & 33.7 & 3.7 & $(26.5-41.0)$ \\
\hline Frederick County, Maryland & 701 & 25.2 & 2.7 & $(19.8-30.6)$ \\
\hline Montgomery County, Maryland & 1,419 & 20.1 & 1.6 & $(16.9-23.2)$ \\
\hline Prince George's County, Maryland & 1,055 & 32.1 & 2.2 & $(27.7-36.4)$ \\
\hline Washington County, Maryland & 505 & 33.4 & 3.6 & $(26.3-40.4)$ \\
\hline Baltimore city, Maryland & 692 & 30.7 & 2.7 & $(25.6-35.9)$ \\
\hline Barnstable County, Massachusetts & 531 & 18.0 & 2.0 & $(14.0-21.9)$ \\
\hline Bristol County, Massachusetts & 2,444 & 26.6 & 1.7 & $(23.3-29.9)$ \\
\hline Essex County, Massachusetts & 2,353 & 22.9 & 1.4 & $(20.2-25.6)$ \\
\hline Hampden County, Massachusetts & 1,799 & 28.9 & 1.7 & $(25.6-32.2)$ \\
\hline Middlesex County, Massachusetts & 3,975 & 18.8 & 0.9 & $(17$. \\
\hline Norfolk County, Massachusetts & 1,638 & 18.2 & 1.4 & 21.0) \\
\hline Plymouth County, Massachusetts & 1,645 & 22.8 & 1.7 & $(19.5-26.1)$ \\
\hline Suffolk County, Massachusetts & 2,117 & 22.2 & 1.3 & $(19.6-24.8)$ \\
\hline Worcester County, Massachusetts & 2,393 & 27.9 & 1.4 & $(25.2-30.7)$ \\
\hline Kent County, Michigan & 502 & 25.7 & 2.7 & $(20.5-31.0)$ \\
\hline Macomb County, Michigan & 590 & 35.2 & 2.4 & $(30.5-39.9)$ \\
\hline Oakland County, Michigan & 1,121 & 25.1 & 1.7 & $(21.8-28.5)$ \\
\hline Wayne County, Michigan & 2,121 & 34.2 & 1.6 & $(31.1-37.4)$ \\
\hline Anoka County, Minnesota & 515 & 25.6 & 2.3 & $(21.0-30.2)$ \\
\hline Dakota County, Minnesota & 625 & 22.6 & 1.9 & $(18.7-26.4)$ \\
\hline Hennepin County, Minnesota & 3,045 & 21.7 & 1.1 & $(19.5-23.9)$ \\
\hline Ramsey County, Minnesota & 1,827 & 21.8 & 1.7 & 5.0) \\
\hline Jackson County, Missouri & 837 & 30.5 & 2.5 & 35.4) \\
\hline St. Louis County, Missouri & 907 & 27.7 & 2.1 & $(23$ \\
\hline Cascade County, Montana & 683 & 31.4 & 2.3 & 6.0) \\
\hline Flathead County, Montana & 539 & 26.2 & 2.3 & $(21.7-30.6)$ \\
\hline Hill County, Montana & 553 & 29.7 & 3.0 & $(23.8-35.5)$ \\
\hline Lake County, Montana & 864 & 25.0 & 2.4 & $(20.4-29.6)$ \\
\hline Missoula County, Montana & 771 & 18.6 & 1.7 & $(15.3-21.9)$ \\
\hline Yellowstone County, Montana & 722 & 23.4 & 1.8 & $(20.0-26.9)$ \\
\hline Dakota County, Nebraska & 708 & 34.1 & 4.0 & $(26.3-41.9)$ \\
\hline Douglas County, Nebraska & 3,389 & 27.9 & 1.0 & $(25.9-29.8)$ \\
\hline Hall County, Nebraska & 508 & 33.3 & 2.8 & $(27.7-38.9)$ \\
\hline Lancaster County, Nebraska & 1,397 & 24.4 & 1.3 & $(21.8-27.0)$ \\
\hline Lincoln County, Nebraska & 574 & 32.3 & 2.9 & $(26.7-38.0)$ \\
\hline y, Nebraska & 1,111 & 26.9 & 1.6 & $(23$ \\
\hline Scotts Bluff County, Nebraska & 552 & 39.1 & 3.3 & $(32.5-45.6)$ \\
\hline Clark County, Nevada & 1,884 & 27.4 & 1.3 & $(24.9-29.9)$ \\
\hline Washoe County, Nevada & 1,418 & 21.9 & 1.6 & $(18.7-25.0)$ \\
\hline Belknap County, New Hampshire & 542 & 34.0 & 3.3 & $(27.5-40.5)$ \\
\hline Carroll County, New Hampshire & 509 & 24.2 & 3.4 & $(17.6-30.8)$ \\
\hline Cheshire County, New Hampshire & 527 & 24.5 & 2.7 & $(19.2-29.8)$ \\
\hline Coos County, New Hampshire & 510 & 31.9 & 3.3 & $(25.4-38.3)$ \\
\hline Grafton County, New Hampshire & 539 & 25.0 & 2.5 & $(20.2-29.9)$ \\
\hline Hillsborough County, New Hampshire & 1,827 & 26.9 & 1.3 & $(24.3-29.4)$ \\
\hline Merrimack County, New Hampshire & 672 & 29.6 & 2.3 & $(25.0-34.2)$ \\
\hline Rockingham County, New Hampshire & 1,001 & 26.9 & 1.9 & $(23.2-30.6)$ \\
\hline Strafford County, New Hampshire & 591 & 27.7 & 2.6 & $(22.6-32.9)$ \\
\hline Atlantic County, New Jersey & 957 & 26.5 & 2.1 & $(22.4-30.6)$ \\
\hline Bergen County, New Jersey & 864 & 18.4 & 1.7 & $(15.0-21.7)$ \\
\hline igton County, New Jersey & 634 & 28.6 & 2.4 & $(23.9-33.3)$ \\
\hline Camden County, New Jersey & 702 & 30.4 & 2.5 & $(25.5-35.3)$ \\
\hline Cape May County, New Jersey & 522 & 29.9 & 3.5 & $(22.9-36.8)$ \\
\hline Cumberland County, New Jersey & 519 & 33.7 & 3.2 & $(27.4-40.0)$ \\
\hline Essex County, New Jersey & 1,244 & 29.3 & 1.7 & $(26.0-32.6)$ \\
\hline Gloucester County, New Jersey & 522 & 26.5 & 2.7 & $(21.2-31.8)$ \\
\hline Hudson County, New Jersey & 1,184 & 23.2 & 1.6 & $(20.0-26.4)$ \\
\hline
\end{tabular}

See table footnotes on page 95.
TABLE 48. (Continued) Estimated prevalence of adults aged $\geq 18$ years who are obese,* by county - Behavioral Risk Factor Surveillance System, United States, 2012

\begin{tabular}{|c|c|c|c|c|}
\hline County & $\begin{array}{c}\text { Sample } \\
\text { size }\end{array}$ & $\%$ & SE & $95 \% \mathrm{Cl}$ \\
\hline Hunterdon County, New Jersey & 526 & 24.0 & 2.8 & $(18.5-29.6)$ \\
\hline Mercer County, New Jersey & 533 & 24.0 & 2.6 & $(18.9-29.1)$ \\
\hline Middlesex County, New Jersey & 809 & 23.7 & 2.1 & $(19.5-27.9)$ \\
\hline Monmouth County, New Jersey & 663 & 23.8 & 2.2 & $(19.6-28.1)$ \\
\hline Morris County, New Jersey & 791 & 18.1 & 1.8 & $(14.6-21.5)$ \\
\hline Ocean County, New Jersey & 612 & 26.3 & 2.3 & $(21.9-30.8)$ \\
\hline Passaic County, New Jersey & 629 & 21.2 & 2.0 & $(17.3-25.1)$ \\
\hline Salem County, New Jersey & 541 & 30.7 & 3.2 & $(24.3-37.0)$ \\
\hline Somerset County, New Jersey & 593 & 24.5 & 2.5 & $(19.5-29.5)$ \\
\hline Sussex County, New Jersey & 508 & 24.6 & 2.6 & $(19.5-29.8)$ \\
\hline Union County, New Jersey & 631 & 23.5 & 2.1 & $(19.4-27.5)$ \\
\hline Warren County, New Jersey & 493 & 32.4 & 3.3 & $(25.8-38.9)$ \\
\hline Bernalillo County, New Mexico & 1,987 & 23.9 & 1.2 & $(21.6-26.2)$ \\
\hline Dona Ana County, New Mexico & 667 & 26.7 & 2.1 & $(22.5-31.0)$ \\
\hline Sandoval County, New Mexico & 623 & 26.9 & 2.4 & $(22.2-31.5)$ \\
\hline San Juan County, New Mexico & 622 & 29.9 & 2.4 & $(25.3-34.5)$ \\
\hline Santa Fe County, New Mexico & 660 & 17.6 & 1.8 & $(14.2-21.1)$ \\
\hline Kings County, New York & 469 & 24.3 & 2.8 & $(18.8-29.9)$ \\
\hline Guilford County, North Carolina & 479 & 29.5 & 2.6 & $(24.5-34.6)$ \\
\hline Mecklenburg County, North Carolina & 662 & 26.4 & 2.3 & $(22.0-30.9)$ \\
\hline Robeson County, North Carolina & 512 & 38.7 & 4.3 & $(30.4-47.1)$ \\
\hline Wake County, North Carolina & 659 & 22.7 & 1.9 & $(19.0-26.4)$ \\
\hline Burleigh County, North Dakota & 536 & 32.1 & 2.8 & $(26.6-37.6)$ \\
\hline Cass County, North Dakota & 805 & 26.0 & 2.1 & $(22$. \\
\hline Cuyahoga County, Ohio & 791 & 30.1 & 2.1 & $(25.9-34.2)$ \\
\hline Franklin County, Ohio & 799 & 30.7 & 2.1 & $(26.5-34.9)$ \\
\hline Hamilton County, Ohio & 730 & 28.8 & 2.1 & $(24.8-32.8)$ \\
\hline Lorain County, Ohio & 587 & 26.7 & 2.8 & $(21.2-32.2)$ \\
\hline Lucas County, Ohio & 595 & 32.1 & 3.1 & $(26.1-38.2)$ \\
\hline Mahoning County, Ohio & 555 & 35.8 & 3.5 & $(28.9-42.8)$ \\
\hline Montgomery County, Ohio & 632 & 29.1 & 2.5 & $(24.3-34.0)$ \\
\hline Stark County, Ohio & 600 & 30.5 & 2.6 & $(25.4-35.5)$ \\
\hline Summit County, Ohio & 589 & 31.9 & 2.7 & $(26.6-37.3)$ \\
\hline Oklahoma County, Oklahoma & 1,119 & 31.6 & 1.7 & $(28.3-34.9)$ \\
\hline Tulsa County, Oklahoma & 1,163 & 29.7 & 1.7 & $(26.4-33.1)$ \\
\hline Clackamas County, Oregon & 488 & 23.6 & 2.5 & $(18.7-28.6)$ \\
\hline Lane County, Oregon & 507 & 30.6 & 2.8 & $(25.2-36.1)$ \\
\hline Multnomah County, Oregon & 854 & 22.7 & 2.0 & $(18.9-26.6)$ \\
\hline Washington County, Oregon & 586 & 25.5 & 2.3 & $(21.0-30.0)$ \\
\hline Allegheny County, Pennsylvania & 1,800 & 24.4 & 1.2 & $(22.0-26.8)$ \\
\hline Bradford County, Pennsylvania & 1,753 & 33.6 & 2.6 & $(28.4-38.7)$ \\
\hline Montgomery County, Pennsylvania & 525 & 23.8 & 2.4 & $(19.2-28.4)$ \\
\hline Philadelphia County, Pennsylvania & 1,935 & 29.0 & 1.4 & $(26.3-31.8)$ \\
\hline Pike County, Pennsylvania & 1,782 & 31.4 & 2.9 & $(25.8-37.1)$ \\
\hline Kent County, Rhode Island & 780 & 26.5 & 2.1 & $(22.4-30.7)$ \\
\hline Providence County, Rhode Island & 3,140 & 26.9 & 1.1 & $(24.8-29.0)$ \\
\hline Washington County, Rhode Island & 630 & 21.0 & 2.1 & $(16.8-25.2)$ \\
\hline Aiken County, South Carolina & 530 & 32.7 & 3.1 & $(26.6-38.8)$ \\
\hline Beaufort County, South Carolina & 770 & 21.9 & 2.6 & $(16.8-27.0)$ \\
\hline Charleston County, South Carolina & 975 & 24.1 & 2.0 & $(20.1-28.1)$ \\
\hline Greenville County, South Carolina & 855 & 27.5 & 2.1 & $(23.5-31.6)$ \\
\hline Horry County, South Carolina & 751 & 25.2 & 2.0 & $(21.2-29.2)$ \\
\hline Richland County, South Carolina & 918 & 31.6 & 2.3 & $(27.2-36.1)$ \\
\hline Spartanburg County, South Carolina & 618 & 33.2 & 2.8 & $(27.6-38.7)$ \\
\hline Lincoln County, South Dakota & 474 & 23.2 & 2.5 & $(18.2-28.2)$ \\
\hline Minnehaha County, South Dakota & 826 & 26.5 & 1.9 & $(22.8-30.1)$ \\
\hline Pennington County, South Dakota & 572 & 30.2 & 2.3 & $(25.6-34.7)$ \\
\hline Davidson County, Tennessee & 528 & 29.7 & 2.5 & $(24.8-34.5)$ \\
\hline Shelby County, Tennessee & 497 & 33.4 & 2.7 & $(28.1-38.6)$ \\
\hline Bexar County, Texas & 561 & 29.3 & 2.4 & $(24.6-34.0)$ \\
\hline
\end{tabular}

See table footnotes on page 95. 
TABLE 48. (Continued) Estimated prevalence of adults aged $\geq 18$ years who are obese, ${ }^{*}$ by county - Behavioral Risk Factor Surveillance System, United States, 2012

\begin{tabular}{|c|c|c|c|c|}
\hline County & $\begin{array}{l}\text { Sample } \\
\text { size }\end{array}$ & $\%$ & SE & $95 \% \mathrm{Cl}$ \\
\hline Dallas County, Texas & 474 & 30.5 & 2.6 & $(25.5-35.6)$ \\
\hline El Paso County, Texas & 576 & 29.5 & 3.1 & $(23.4-35.5)$ \\
\hline Harris County, Texas & 700 & 28.2 & 2.1 & $(24.2-32.3)$ \\
\hline Hidalgo County, Texas & 559 & 44.5 & 3.3 & $(38.0-51.0)$ \\
\hline Tarrant County, Texas & 532 & 29.4 & 2.6 & $(24.3-34.5)$ \\
\hline Travis County, Texas & 1,000 & 21.9 & 2.0 & $(18.0-25.8)$ \\
\hline Davis County, Utah & 1,078 & 25.5 & 1.6 & $(22.3-28.7)$ \\
\hline Salt Lake County, Utah & 3,787 & 24.0 & 0.9 & $(22.3-25.7)$ \\
\hline Tooele County, Utah & 519 & 28.1 & 3.1 & $(22.0-34.1)$ \\
\hline Utah County, Utah & 1,571 & 22.3 & 1.2 & $(19.9-24.7)$ \\
\hline Wasatch County, Utah & 491 & 17.6 & 2.8 & $(12.1-23.1)$ \\
\hline Weber County, Utah & 991 & 29.5 & 1.9 & $(25.8-33.2)$ \\
\hline Chittenden County, Vermont & 889 & 19.6 & 1.6 & $(16.6-22.6)$ \\
\hline Rutland County, Vermont & 562 & 29.1 & 2.6 & $(23.9-34.2)$ \\
\hline Washington County, Vermont & 495 & 23.1 & 2.4 & $(18.4-27.7)$ \\
\hline Windsor County, Vermont & 526 & 23.6 & 2.4 & $(19.0-28.3)$ \\
\hline Fairfax County, Virginia & 674 & 16.5 & 1.8 & $(13.0-20.0)$ \\
\hline Clark County, Washington & 748 & 30.7 & 2.2 & $(26.4-34.9)$ \\
\hline King County, Washington & 3,737 & 22.4 & 0.9 & $(20.6-24.2)$ \\
\hline Kitsap County, Washington & 551 & 30.0 & 2.6 & $(24.9-35.1)$ \\
\hline Pierce County, Washington & 1,134 & 26.7 & 1.6 & $(23.5-29.9)$ \\
\hline Snohomish County, Washington & 1,119 & 28.4 & 1.7 & $(25.0-31.7)$ \\
\hline Spokane County, Washington & 914 & 28.6 & 2.0 & $(24.6-32.6)$ \\
\hline Thurston County, Washington & 492 & 29.5 & 2.8 & $(24.1-34.9)$ \\
\hline Whatcom County, Washington & 812 & 20.5 & 2.1 & $(16.5-24.6)$ \\
\hline Yakima County, Washington & 472 & 31.4 & 3.0 & $(25.5-37.3)$ \\
\hline Kanawha County, West Virginia & 605 & 31.5 & 2.2 & $(27.2-35.9)$ \\
\hline Milwaukee County, Wisconsin & 891 & 30.9 & 2.5 & $(26.0-35.7)$ \\
\hline Laramie County, Wyoming & 906 & 27.3 & 2.3 & $(22.7-31.9)$ \\
\hline Natrona County, Wyoming & 793 & 23.1 & 2.3 & $(18.7-27.5)$ \\
\hline San Juan Municipio, Puerto Rico & 641 & 24.8 & 2.0 & $(20.9-28.7)$ \\
\hline Median & & 26.7 & & \\
\hline Range & & $14.8-44.5$ & & \\
\hline
\end{tabular}

Abbreviation: $\mathrm{Cl}=$ confidence interval; $\mathrm{SE}=$ standard error.

* Body mass index $\geq 30 \mathrm{~kg} / \mathrm{m}^{2}$.
TABLE 49. Estimated prevalence of adults aged $\geq 18$ years who were ever told by a doctor that they have diabetes, ${ }^{*}$ by state/territory —Behavioral Risk Factor Surveillance System, United States, 2012

\begin{tabular}{|c|c|c|c|c|}
\hline State/Territory & $\begin{array}{c}\text { Sample } \\
\text { size }\end{array}$ & $\%$ & SE & $95 \% \mathrm{Cl}$ \\
\hline Alabama & 9,003 & 12.3 & 0.4 & $(11.4-13.1)$ \\
\hline Alaska & 4,339 & 7.0 & 0.5 & $(5.9-8.0)$ \\
\hline Arizona & 7,293 & 10.6 & 0.6 & $(9.4-11.8)$ \\
\hline Arkansas & 5,168 & 11.3 & 0.5 & $(10.3-12.3)$ \\
\hline California & 14,564 & 9.8 & 0.4 & $(9.1-10.5)$ \\
\hline Colorado & 12,230 & 7.4 & 0.3 & $(6.8-8.0)$ \\
\hline Connecticut & 8,768 & 9.2 & 0.4 & $(8.3-10.0)$ \\
\hline Delaware & 5,167 & 9.6 & 0.5 & $(8.6-10.7)$ \\
\hline District of Columbia & 3,819 & 8.2 & 0.6 & $(7.1-9.4)$ \\
\hline Florida & 7,609 & 11.4 & 0.5 & $(10.3-12.4)$ \\
\hline Georgia & 6,081 & 9.9 & 0.5 & $(9.0-10.8)$ \\
\hline Hawaii & 7,566 & 7.8 & 0.5 & $(6.8-8.7)$ \\
\hline Idaho & 5,884 & 8.5 & 0.5 & $(7.4-9.5)$ \\
\hline Illinois & 5,578 & 9.4 & 0.5 & $(8.4-10.4)$ \\
\hline Indiana & 8,632 & 10.9 & 0.4 & $(10.2-11.7)$ \\
\hline lowa & 7,162 & 9.7 & 0.4 & $(8.9-10.4)$ \\
\hline Kansas & 11,787 & 9.4 & 0.3 & $(8.8-10.1)$ \\
\hline Kentucky & 11,197 & 10.7 & 0.4 & $(9.9-11.5)$ \\
\hline Louisiana & 9,051 & 12.3 & 0.5 & $(11.3-13.2)$ \\
\hline Maine & 9,909 & 9.7 & 0.3 & $(9.0-10.3)$ \\
\hline Maryland & 12,757 & 10.2 & 0.4 & $(9.5-11.0)$ \\
\hline Massachusetts & 21,681 & 8.3 & 0.3 & $(7.8-8.9)$ \\
\hline Michigan & 10,485 & 10.5 & 0.4 & $(9.7-11.2)$ \\
\hline Minnesota & 12,235 & 7.3 & 0.3 & $(6.7-7.9)$ \\
\hline Mississippi & 7,779 & 12.5 & 0.5 & $(11.6-13.4)$ \\
\hline Missouri & 6,745 & 10.7 & 0.5 & $(9.7-11.7)$ \\
\hline Montana & 8,666 & 7.2 & 0.3 & $(6.6-7.9)$ \\
\hline Nebraska & 19,156 & 8.1 & 0.3 & $(7.6-8.6)$ \\
\hline Nevada & 4,830 & 8.9 & 0.6 & $(7.7-10.1)$ \\
\hline New Hampshire & 7,518 & 9.1 & 0.4 & $(8.4-9.9)$ \\
\hline New Jersey & 15,730 & 9.3 & 0.3 & $(8.7-9.9)$ \\
\hline New Mexico & 8,770 & 10.3 & 0.4 & $(9.5-11.0)$ \\
\hline New York & 6,046 & 9.7 & 0.5 & $(8.7-10.8)$ \\
\hline North Carolina & 11,879 & 10.4 & 0.3 & $(9.8-11.1)$ \\
\hline North Dakota & 4,873 & 8.6 & 0.5 & $(7.7-9.5)$ \\
\hline Ohio & 13,004 & 11.7 & 0.4 & $(11.0-12.4)$ \\
\hline Oklahoma & 8,001 & 11.5 & 0.4 & $(10.7-12.3)$ \\
\hline Oregon & 5,292 & 9.9 & 0.5 & $(8.9-10.9)$ \\
\hline Pennsylvania & 19,916 & 10.2 & 0.3 & $(9.7-10.8)$ \\
\hline Rhode Island & 5,471 & 9.8 & 0.5 & $(8.8-10.7)$ \\
\hline South Carolina & 12,767 & 11.6 & 0.4 & $(10.8-12.3)$ \\
\hline South Dakota & 7,873 & 7.8 & 0.5 & $(6.9-8.7)$ \\
\hline Tennessee & 7,031 & 11.9 & 0.5 & $(11.0-12.9)$ \\
\hline Texas & 9,112 & 10.6 & 0.4 & $(9.8-11.4)$ \\
\hline Utah & 12,419 & 7.2 & 0.3 & $(6.7-7.7)$ \\
\hline Vermont & 6,049 & 7.3 & 0.4 & $(6.5-8.0)$ \\
\hline Virginia & 7,388 & 10.6 & 0.4 & $(9.7-11.4)$ \\
\hline Washington & 15,292 & 8.8 & 0.3 & $(8.2-9.4)$ \\
\hline West Virginia & 5,405 & 13.0 & 0.5 & $(12.0-14.0)$ \\
\hline Wisconsin & 5,294 & 8.3 & 0.5 & $(7.3-9.3)$ \\
\hline Wyoming & 6,263 & 9.1 & 0.5 & $(8.0-10.1)$ \\
\hline Guam & 2,016 & 9.6 & 0.8 & $(8.0-11.3)$ \\
\hline Puerto Rico & 6,314 & 16.4 & 0.5 & $(15.3-17.4)$ \\
\hline Median & & 9.7 & & \\
\hline Range & & $7.0-16.4$ & & \\
\hline
\end{tabular}

Abbreviations: $\mathrm{Cl}=$ confidence interval; $\mathrm{SE}=$ standard error.

* Excluding diabetes during pregnancy in females, or prediabetes or borderline diabetes in adults. 
TABLE 50. Estimated prevalence of adults aged $\geq 18$ years who were ever told by a doctor that they have diabetes, ${ }^{*}$ by metropolitan and micropolitan statistical area - Behavioral Risk Factor Surveillance System, United States, 2012

\begin{tabular}{|c|c|c|c|c|}
\hline MMSA(s) & $\begin{array}{l}\text { Sample } \\
\text { size }\end{array}$ & $\%$ & SE & $95 \% \mathrm{Cl}$ \\
\hline Aguadilla-Isabela, Puerto Rico & 551 & 15.2 & 1.7 & $(11.9-18.5)$ \\
\hline Akron, Ohio & 743 & 8.4 & 1.1 & $(6.2-10.7)$ \\
\hline Albuquerque, New Mexico & 3,268 & 8.7 & 0.6 & $(7.6-9.8)$ \\
\hline $\begin{array}{l}\text { Allentown-Bethlehem-Easton, } \\
\text { Pennsylvania-New Jersey }\end{array}$ & 1,350 & 10.0 & 1.2 & $(7.7-12.4)$ \\
\hline Anaheim-Santa Ana-Irvine, California ${ }^{\dagger}$ & 1,036 & 9.4 & 1.3 & $(7.0-11.9)$ \\
\hline Anchorage, Alaska & 1,503 & 7.8 & 0.9 & $(6.1-9.5)$ \\
\hline Asheville, North Carolina & 594 & 8.3 & 1.3 & $(5.9-10.8)$ \\
\hline $\begin{array}{l}\text { Atlanta-Sandy Springs-Roswell, } \\
\text { Georgia }\end{array}$ & 2,531 & 8.9 & 0.7 & $(7.6-10.2)$ \\
\hline Atlantic City-Hammonton, New Jersey & 1,022 & 9.8 & 1.1 & $(7.6-12.0)$ \\
\hline $\begin{array}{l}\text { Augusta-Richmond County, Georgia- } \\
\text { South Carolina }\end{array}$ & 1,033 & 11.1 & 1.4 & $(8.4-13.8)$ \\
\hline Augusta-Waterville, Maine & 833 & 10.8 & 1.1 & $(8.6-13.1)$ \\
\hline Austin-Round Rock, Texas & 1,384 & 7.4 & 1.0 & $(5.5-9.3)$ \\
\hline $\begin{array}{l}\text { Baltimore-Columbia-Towson, } \\
\text { Maryland }\end{array}$ & 4,700 & 10.0 & 0.6 & $(8.9-11.1)$ \\
\hline Bangor, Maine & 929 & 8.2 & 0.9 & $(6.5-9.9)$ \\
\hline Barnstable Town, Massachusetts & 559 & 9.6 & 1.5 & $(6.7-12.6)$ \\
\hline Barre, Vermont & 517 & 7.5 & 1.4 & $(4.8-10.1)$ \\
\hline Baton Rouge, Louisiana & 1,386 & 11.8 & 1.1 & $(9.6-14.0)$ \\
\hline Bellingham, Washington & 846 & 6.7 & 0.9 & $(4.9-8.6)$ \\
\hline Berlin, New Hampshire-Vermont & 708 & 13.6 & 2.0 & $(9.7-17.4)$ \\
\hline Billings, Montana & 847 & 8.1 & 1.0 & $(6.2-10.1)$ \\
\hline Birmingham-Hoover, Alabama & 1,825 & 11.3 & 0.8 & $(9.6-12.9)$ \\
\hline Bismarck, North Dakota & 822 & 9.5 & 1.2 & $(7.1-11.8)$ \\
\hline Boise City, Idaho & 1,480 & 7.3 & 0.9 & $(5.5-9.0)$ \\
\hline Boston, Massachusetts ${ }^{\dagger}$ & 5,907 & 7.3 & 0.5 & $(6.3-8.2)$ \\
\hline Boulder, Colorado & 517 & 3.4 & 0.8 & $(1.8-5.1)$ \\
\hline Bremerton-Silverdale, Washington & 565 & 8.7 & 1.4 & $(6.0-11.4)$ \\
\hline $\begin{array}{l}\text { Bridgeport-Stamford-Norwalk, } \\
\text { Connecticut }\end{array}$ & 2,186 & 7.4 & 0.8 & $(5.9-8.9)$ \\
\hline Burlington-South Burlington, Vermont & 1,520 & 6.7 & 0.7 & $(5.3-8.0)$ \\
\hline $\begin{array}{l}\text { Cambridge-Newton-Framingham, } \\
\text { Massachusetts }{ }^{\dagger}\end{array}$ & 6,924 & 7.9 & 0.5 & $(7.0-8.8)$ \\
\hline Camden, New Jersey ${ }^{\dagger}$ & 1,976 & 11.5 & 0.9 & $(9.7-13.2)$ \\
\hline Canton-Massillon, Ohio & 682 & 15.4 & 1.8 & $(11.9-18.8)$ \\
\hline Casper, Wyoming & 830 & 10.7 & 1.4 & $(8.0-13.4)$ \\
\hline Cedar Rapids, lowa & 585 & 9.8 & 1.3 & $(7.2-12.3)$ \\
\hline Charleston, West Virginia & 772 & 12.6 & 1.3 & $(10.1-15.0)$ \\
\hline $\begin{array}{l}\text { Charleston-North Charleston, } \\
\text { South Carolina }\end{array}$ & 1,709 & 11.7 & 1.0 & $(9.6-13.7)$ \\
\hline $\begin{array}{l}\text { Charlotte-Concord-Gastonia, } \\
\text { North Carolina-South Carolina }\end{array}$ & 2,523 & 10.0 & 0.7 & $(8.5-11.4)$ \\
\hline Chattanooga, Tennessee-Georgia & 628 & 12.9 & 2.0 & $(8.9-16.8)$ \\
\hline Cheyenne, Wyoming & 959 & 11.9 & 1.5 & $(8.9-14.9)$ \\
\hline $\begin{array}{l}\text { Chicago-Naperville-Elgin, } \\
\text { Illinois-Indiana-Wisconsin }\end{array}$ & 3,732 & 8.0 & 0.6 & $(6.8-9.2)$ \\
\hline Cincinnati, Ohio-Kentucky-Indiana & 2,364 & 11.9 & 0.8 & $(10.3-13.5)$ \\
\hline $\begin{array}{l}\text { Claremont-Lebanon, New } \\
\text { Hampshire-Vermont }\end{array}$ & 1,949 & 8.5 & 0.7 & $(7.1-9.9)$ \\
\hline Cleveland-Elyria, Ohio & 1,778 & 11.5 & 1.0 & $(9.5-13.5)$ \\
\hline Colorado Springs, Colorado & 1,160 & 8.6 & 0.9 & $(6.8-10.5)$ \\
\hline Columbia, South Carolina & 1,799 & 10.4 & 0.9 & $(8.6-12.2)$ \\
\hline Columbus, Ohio & 1,602 & 9.3 & 0.8 & $(7.7-11.0)$ \\
\hline Concord, New Hampshire & 704 & 8.8 & 1.2 & $(6.5-11.2)$ \\
\hline Dallas-Plano-Irving, Texas $^{\dagger}$ & 907 & 9.9 & 1.1 & $(7.6-12.1)$ \\
\hline Dayton, Ohio & 857 & 12.7 & 1.3 & $(10.1-15.2)$ \\
\hline
\end{tabular}

See table footnotes on page 98.
TABLE 50. (Continued) Estimated prevalence of adults aged $\geq 18$ years who were ever told by a doctor that they have diabetes, ${ }^{*}$ by metropolitan and micropolitan statistical area - Behavioral Risk Factor Surveillance System, United States, 2012

\begin{tabular}{|c|c|c|c|c|}
\hline $\operatorname{MMSA}(\mathrm{s})$ & $\begin{array}{l}\text { Sample } \\
\text { size }\end{array}$ & $\%$ & SE & $95 \% \mathrm{Cl}$ \\
\hline Denver-Aurora-Lakewood, Colorado & 4,845 & 7.4 & 0.5 & $(6.5-8.3)$ \\
\hline Des Moines-West Des Moines, lowa & 1,159 & 8.9 & 0.9 & $(7.2-10.7)$ \\
\hline 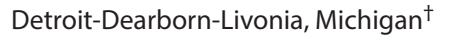 & 2,217 & 12.6 & 1.1 & $(10.4-14.7)$ \\
\hline Dover, Delaware & 1,441 & 10.9 & 1.1 & $(8.7-13.1)$ \\
\hline Duluth, Minnesota-Wisconsin & 514 & 12.2 & 1.7 & $(8.9-15.4)$ \\
\hline Durham-Chapel Hill, North Carolina & 794 & 8.1 & 1.0 & $(6.1-10.1)$ \\
\hline El Paso, Texas & 625 & 14.1 & 2.0 & $(10.3-18.0)$ \\
\hline Eugene, Oregon & 526 & 8.7 & 1.4 & $(6.0-11.4)$ \\
\hline Fairbanks, Alaska & 599 & 5.0 & 1.1 & $(2.9-7.1)$ \\
\hline Fargo, North Dakota-Minnesota & 940 & 6.5 & 1.0 & $(4.6-8.4)$ \\
\hline Farmington, New Mexico & 650 & 10.9 & 1.7 & $(7.6-14.2)$ \\
\hline Fayetteville, North Carolina & 506 & 13.4 & 1.8 & $(9.9-16.9)$ \\
\hline $\begin{array}{l}\text { Fayetteville-Springdale-Rogers, } \\
\text { Arkansas-Missouri }\end{array}$ & 812 & 9.4 & 1.3 & $(6.8-11.9)$ \\
\hline Fort Collins, Colorado & 593 & 5.4 & 0.9 & $(3.6-7.2)$ \\
\hline Fort Wayne, Indiana & 539 & 10.0 & 1.5 & $(7.1-12.8)$ \\
\hline Fort Worth-Arlington, Texas ${ }^{\dagger}$ & 725 & 9.7 & 1.2 & $(7.4-12.0)$ \\
\hline Grand Island, Nebraska & 855 & 7.7 & 1.1 & $(5.5-9.9)$ \\
\hline Grand Rapids-Wyoming, Michigan & 892 & 7.3 & 0.9 & $(5.6-9.0)$ \\
\hline Great Falls, Montana & 709 & 7.8 & 1.0 & $(5.7-9.8)$ \\
\hline Greeley, Colorado & 535 & 8.0 & 1.4 & $(5.3-10.6)$ \\
\hline $\begin{array}{l}\text { Greensboro-High Point, } \\
\text { North Carolina }\end{array}$ & 807 & 11.0 & 1.3 & $(8.6-13.5)$ \\
\hline $\begin{array}{l}\text { Greenville-Anderson-Mauldin, } \\
\text { South Carolina }\end{array}$ & 1,690 & 11.0 & 0.9 & $(9.1-12.8)$ \\
\hline Gulfport-Biloxi-Pascagoula, Mississippi & i 744 & 11.3 & 1.2 & $(9.0-13.6)$ \\
\hline $\begin{array}{l}\text { Hagerstown-Martinsburg, Maryland- } \\
\text { West Virginia }\end{array}$ & 758 & 12.4 & 1.7 & $(9.1-15.8)$ \\
\hline Harrisburg-Carlisle, Pennsylvania & 663 & 10.9 & 1.3 & $(8.3-13.5)$ \\
\hline $\begin{array}{l}\text { Hartford-West Hartford-East Hartford, } \\
\text { Connecticut }\end{array}$ & 2,667 & 9.4 & 0.8 & $(7.9-11.0)$ \\
\hline Heber, Utah & 511 & 4.5 & 0.8 & $(3.0-6.1)$ \\
\hline Hilo, Hawaii & 1,346 & 8.6 & 1.1 & $(6.4-10.8)$ \\
\hline $\begin{array}{l}\text { Hilton Head Island-Bluffton-Beaufort, } \\
\text { South Carolina }\end{array}$ & 935 & 8.4 & 1.1 & $(6.2-10.7)$ \\
\hline $\begin{array}{l}\text { Houston-The Woodlands-Sugar Land, } \\
\text { Texas }\end{array}$ & 1,147 & 10.5 & 1.0 & $(8.5-12.5)$ \\
\hline $\begin{array}{l}\text { Huntington-Ashland, West } \\
\text { Virginia-Kentucky-Ohio }\end{array}$ & 1,115 & 13.4 & 1.2 & $(11.1-15.7)$ \\
\hline Huntsville, Alabama & 613 & 8.8 & 1.2 & $(6.4-11.2)$ \\
\hline Idaho Falls, Idaho & 541 & 11.5 & 2.2 & $(7.1-15.9)$ \\
\hline $\begin{array}{l}\text { Indianapolis-Carmel-Anderson, } \\
\text { Indiana }\end{array}$ & 2,194 & 9.8 & 0.7 & $(8.4-11.2)$ \\
\hline Jackson, Mississippi & 921 & 11.8 & 1.2 & $(9.4-14.1)$ \\
\hline Jacksonville, Florida & 519 & 11.6 & 2.1 & $(7.5-15.6)$ \\
\hline Kahului-Wailuku-Lahaina, Hawaii & 1,216 & 7.0 & 1.1 & $(4.8-9.1)$ \\
\hline Kalispell, Montana & 560 & 6.2 & 1.1 & $(4.1-8.3)$ \\
\hline Kansas City, Missouri-Kansas & 4,742 & 10.4 & 0.7 & $(9.0-11.8)$ \\
\hline Kapaa, Hawaii & 667 & 7.3 & 1.3 & $(4.9-9.8)$ \\
\hline Keene, New Hampshire & 548 & 8.4 & 1.5 & $(5.4-11.4)$ \\
\hline Kennewick-Richland, Washington & 533 & 11.2 & 1.9 & $(7.4-15.0)$ \\
\hline $\begin{array}{l}\text { Kingsport-Bristol-Bristol, } \\
\text { Tennessee-Virginia }\end{array}$ & 568 & 15.0 & 2.2 & $(10.7-19.4)$ \\
\hline Knoxville, Tennessee & 831 & 9.6 & 1.1 & $(7.4-11.7)$ \\
\hline Laconia, New Hampshire & 567 & 9.5 & 1.7 & $(6.2-12.9)$ \\
\hline Lafayette, Louisiana & 557 & 10.3 & 1.6 & $(7.3-13.4)$ \\
\hline Las Cruces, New Mexico & 707 & 11.6 & 1.3 & $(9.0-14.1)$ \\
\hline
\end{tabular}

See table footnotes on page 98. 
TABLE 50. (Continued) Estimated prevalence of adults aged $\geq 18$ years who were ever told by a doctor that they have diabetes, ${ }^{*}$ by metropolitan and micropolitan statistical area - Behavioral Risk Factor Surveillance System, United States, 2012

\begin{tabular}{|c|c|c|c|c|}
\hline MMSA(s) & $\begin{array}{l}\text { Sample } \\
\text { size }\end{array}$ & $\%$ & SE & $95 \% \mathrm{Cl}$ \\
\hline $\begin{array}{l}\text { Las Vegas-Henderson-Paradise, } \\
\text { Nevada }\end{array}$ & 2,007 & 9.5 & 0.8 & $(7.9-11.1)$ \\
\hline Lewiston-Auburn, Maine & 699 & 11.8 & 1.4 & $(9.1-14.5)$ \\
\hline Lexington-Fayette, Kentucky & 530 & 7.5 & 1.2 & $(5.2-9.9)$ \\
\hline Lincoln, Nebraska & 1,667 & 6.4 & 0.7 & $(5.1-7.7)$ \\
\hline $\begin{array}{l}\text { Little Rock-North Little Rock-Conway, } \\
\text { Arkansas }\end{array}$ & 1,168 & 9.3 & 1.0 & $(7.4-11.2)$ \\
\hline Logan, Utah-Idaho & 505 & 5.1 & 0.9 & $(3.4-6.9)$ \\
\hline $\begin{array}{l}\text { Los Angeles-Long Beach-Glendale, } \\
\text { California }^{\dagger}\end{array}$ & 3,504 & 10.6 & 0.7 & $(9.2-12.0)$ \\
\hline $\begin{array}{l}\text { Louisville/Jefferson County, } \\
\text { Kentucky-Indiana }\end{array}$ & 2,178 & 10.1 & 0.9 & $(8.3-11.9)$ \\
\hline Lumberton, North Carolina & 545 & 17.4 & 3.2 & $(11.1-23.7)$ \\
\hline Manchester-Nashua, New Hampshire & 1,905 & 8.7 & 0.7 & $(7.3-10.1)$ \\
\hline McAllen-Edinburg-Mission, Texas & 621 & 13.4 & 1.9 & $(9.6-17.2)$ \\
\hline $\begin{array}{l}\text { Memphis, } \\
\text { Tennessee-Mississippi-Arkansas }\end{array}$ & 1,307 & 14.1 & 1.3 & $(11.5-16.6)$ \\
\hline $\begin{array}{l}\text { Miami-Fort Lauderdale-West Palm } \\
\text { Beach, Florida }\end{array}$ & 1,666 & 10.5 & 1.1 & $(8.3-12.7)$ \\
\hline $\begin{array}{l}\text { Milwaukee-Waukesha-West Allis, } \\
\text { Wisconsin }\end{array}$ & 1,256 & 8.9 & 1.2 & $(6.5-11.2)$ \\
\hline $\begin{array}{l}\text { Minneapolis-St. Paul-Bloomington, } \\
\text { Minnesota-Wisconsin }\end{array}$ & 7,896 & 6.7 & 0.4 & $(5.8-7.5)$ \\
\hline Missoula, Montana & 782 & 3.4 & 0.6 & $(2.2-4.7)$ \\
\hline Mobile, Alabama & 816 & 12.4 & 1.4 & $(9.6-15.2)$ \\
\hline Montgomery, Alabama & 534 & 14.4 & 1.8 & $(10.9-18.0)$ \\
\hline $\begin{array}{l}\text { Montgomery County-Bucks County- } \\
\text { Chester County, Pennsylvania }{ }^{\dagger}\end{array}$ & 1,309 & 8.1 & 1.0 & $(6.1-10.1)$ \\
\hline $\begin{array}{l}\text { Myrtle Beach-Conway-North Myrtle } \\
\text { Beach, South Carolina-North Carolina }\end{array}$ & 946 & 9.7 & 1.1 & $(7.6-11.9)$ \\
\hline $\begin{array}{l}\text { Nashville-Davidson-Murfreesboro- } \\
\text { Franklin, Tennessee }\end{array}$ & 1,337 & 9.4 & 0.9 & $(7.7-11.2)$ \\
\hline $\begin{array}{l}\text { Nassau County-Suffolk County, } \\
\text { New York }{ }^{\dagger}\end{array}$ & 900 & 6.3 & 0.9 & $(4.4-8.1)$ \\
\hline Newark, New Jersey-Pennsylvania ${ }^{\dagger}$ & 6,550 & 8.5 & 0.5 & $(7.5-9.5)$ \\
\hline New Haven-Milford, Connecticut & 2,006 & 10.2 & 0.9 & $(8.3-12.0)$ \\
\hline New Orleans-Metairie, Louisiana & 1,274 & 12.3 & 1.1 & $(10.1-14.4)$ \\
\hline $\begin{array}{l}\text { New York-Jersey City-White Plains, } \\
\text { New York-New Jersey }{ }^{\dagger}\end{array}$ & 7,436 & 9.9 & 0.7 & $(8.6-11.3)$ \\
\hline Norfolk, Nebraska & 571 & 8.1 & 1.3 & $(5.6-10.6)$ \\
\hline North Platte, Nebraska & 613 & 8.9 & 1.4 & $(6.1-11.6)$ \\
\hline Norwich-New London, Connecticut & 1,000 & 10.0 & 1.3 & $(7.5-12.4)$ \\
\hline $\begin{array}{l}\text { Oakland-Hayward-Berkeley, } \\
\text { California }^{\dagger}\end{array}$ & 974 & 8.6 & 1.3 & $(6.0-11.1)$ \\
\hline Ocean City, New Jersey & 554 & 9.6 & 1.6 & $(6.6-12.7)$ \\
\hline Ogden-Clearfield, Utah & 2,432 & 8.7 & 0.7 & $(7.4-10.0)$ \\
\hline Oklahoma City, Oklahoma & 2,438 & 10.6 & 0.7 & $(9.2-12.0)$ \\
\hline Olympia-Tumwater, Washington & 517 & 13.7 & 2.1 & $(9.6-17.8)$ \\
\hline Omaha-Council Bluffs, Nebraska-lowa & 5,584 & 8.1 & 0.5 & $(7.2-9.0)$ \\
\hline Orlando-Kissimmee-Sanford, Florida & 570 & 10.2 & 1.7 & $(6.9-13.6)$ \\
\hline Philadelphia, Pennsylvania $^{\dagger}$ & 2,455 & 11.2 & 0.8 & $(9.6-12.9)$ \\
\hline Phoenix-Mesa-Scottsdale, Arizona & 2,599 & 9.8 & 0.8 & $(8.2-11.4)$ \\
\hline Pittsburgh, Pennsylvania & 3,365 & 10.9 & 0.6 & $(9.6-12.1)$ \\
\hline Ponce, Puerto Rico & 548 & 17.3 & 1.8 & $(13.8-20.7)$ \\
\hline Portland-South Portland, Maine & 3,329 & 8.9 & 0.6 & $(7.8-10.1)$ \\
\hline $\begin{array}{l}\text { Portland-Vancouver-Hillsboro, } \\
\text { Oregon-Washington }\end{array}$ & 3,126 & 8.9 & 0.6 & $(7.7-10.1)$ \\
\hline
\end{tabular}

See table footnotes on page 98.
TABLE 50. (Continued) Estimated prevalence of adults aged $\geq 18$ years who were ever told by a doctor that they have diabetes, ${ }^{*}$ by metropolitan and micropolitan statistical area - Behavioral Risk Factor Surveillance System, United States, 2012

\begin{tabular}{|c|c|c|c|c|}
\hline MMSA(s) & $\begin{array}{l}\text { Sample } \\
\text { size }\end{array}$ & $\%$ & SE & $95 \% \mathrm{Cl}$ \\
\hline $\begin{array}{l}\text { Providence-Warwick, Rhode } \\
\text { Island-Massachusetts }\end{array}$ & 8,124 & 10.5 & 0.5 & $(9.5-11.4)$ \\
\hline Provo-Orem, Utah & 1,748 & 5.0 & 0.5 & $(4.0-6.0)$ \\
\hline Raleigh, North Carolina & 946 & 8.1 & 1.0 & $(6.1-10.0)$ \\
\hline Rapid City, South Dakota & 1,055 & 7.0 & 0.9 & $(5.1-8.8)$ \\
\hline Reno, Nevada & 1,508 & 6.4 & 0.8 & $(4.9-8.0)$ \\
\hline Richmond, Virginia & 1,014 & 10.9 & 1.1 & $(8.7-13.1)$ \\
\hline $\begin{array}{l}\text { Riverside-San Bernardino-Ontario, } \\
\text { California }\end{array}$ & 1,529 & 10.6 & 1.0 & $(8.6-12.7)$ \\
\hline $\begin{array}{l}\text { Rockingham County-Strafford County, } \\
\text { New Hampshire }{ }^{\dagger}\end{array}$ & 1,671 & 9.6 & 0.8 & $(8.0-11.2)$ \\
\hline Rutland, Vermont & 596 & 8.1 & 1.2 & $(5.8-10.5)$ \\
\hline $\begin{array}{l}\text { Sacramento-Roseville-Arden-Arcade, } \\
\text { California }\end{array}$ & 996 & 9.5 & 1.5 & $(6.5-12.5)$ \\
\hline St. Louis, Missouri-Illinois & 2,069 & 10.6 & 1.0 & $(8.7-12.5)$ \\
\hline Salisbury, Maryland-Delaware & 2,119 & 12.8 & 1.0 & $(10.8-14.9)$ \\
\hline Salt Lake City, Utah & 4,611 & 7.2 & 0.4 & $(6.3-8.0)$ \\
\hline San Antonio-New Braunfels, Texas & 777 & 10.3 & 1.3 & $(7.8-12.7)$ \\
\hline San Diego-Carlsbad, California & 1,128 & 9.3 & 1.2 & $(6.9-11.6)$ \\
\hline $\begin{array}{l}\text { San Francisco-Redwood City-South } \\
\text { San Francisco, California }{ }^{\dagger}\end{array}$ & 596 & 8.2 & 2.0 & $(4.3-12.0)$ \\
\hline $\begin{array}{l}\text { San Jose-Sunnyvale-Santa Clara, } \\
\text { California }\end{array}$ & 685 & 7.4 & 1.3 & $(4.9-10.0)$ \\
\hline San Juan-Carolina-Caguas, Puerto Rico & 3,952 & 15.8 & 0.6 & $(14.6-17.1)$ \\
\hline Santa Fe, New Mexico & 697 & 6.8 & 1.1 & $(4.6-9.0)$ \\
\hline Sayre, Pennsylvania & 1,862 & 11.0 & 1.0 & $(8.9-13.0)$ \\
\hline Scottsbluff, Nebraska & 631 & 12.3 & 1.7 & $(9.0-15.5)$ \\
\hline $\begin{array}{l}\text { Scranton-Wilkes-Barre-Hazleton, } \\
\text { Pennsylvania }\end{array}$ & 743 & 9.8 & 1.1 & $(7.7-11.9)$ \\
\hline Seattle-Bellevue-Everett, Washington ${ }^{\dagger}$ & 5,095 & 7.8 & 0.5 & $(6.9-8.8)$ \\
\hline Shreveport-Bossier City, Louisiana & 578 & 13.2 & 1.7 & $(9.9-16.4)$ \\
\hline $\begin{array}{l}\text { Silver Spring-Frederick-Rockville, } \\
\text { Maryland }^{\dagger}\end{array}$ & 2,298 & 7.7 & 0.8 & $(6.1-9.4)$ \\
\hline $\begin{array}{l}\text { Sioux City, lowa-Nebraska- } \\
\text { South Dakota }\end{array}$ & 1,204 & 8.8 & 1.4 & $(6.0-11.6)$ \\
\hline Sioux Falls, South Dakota & 1,466 & 6.2 & 0.8 & $(4.7-7.7)$ \\
\hline Spartanburg, South Carolina & 707 & 12.3 & 1.7 & $(8.9-15.6)$ \\
\hline Spokane-Spokane Valley, Washington & 1,099 & 9.2 & 1.0 & $(7.2-11.3)$ \\
\hline Springfield, Massachusetts & 2,343 & 9.4 & 0.8 & $(7.9-11.0)$ \\
\hline 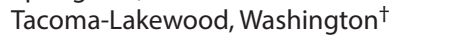 & 1,180 & 8.1 & 0.9 & $(6.4-9.8)$ \\
\hline $\begin{array}{l}\text { Tampa-St. Petersburg-Clearwater, } \\
\text { Florida }\end{array}$ & 827 & 11.5 & 1.4 & $(8.8-14.2)$ \\
\hline Toledo, Ohio & 968 & 12.8 & 1.4 & $(10.1-15.5)$ \\
\hline Topeka, Kansas & 1,087 & 9.2 & 1.0 & $(7.2-11.1)$ \\
\hline Torrington, Connecticut & 663 & 6.9 & 1.2 & $(4.6-9.2)$ \\
\hline Trenton, New Jersey & 579 & 9.9 & 1.4 & $(7.1-12.6)$ \\
\hline Tucson, Arizona & 998 & 11.8 & 1.5 & $(8.9-14.7)$ \\
\hline Tulsa, Oklahoma & 1,740 & 11.1 & 0.9 & $(9.4-12.8)$ \\
\hline Tuscaloosa, Alabama & 581 & 9.8 & 1.4 & $(7.1-12.4)$ \\
\hline Urban Honolulu, Hawaii & 4,337 & 7.8 & 0.6 & $(6.5-9.0)$ \\
\hline Vineland-Bridgeton, New Jersey & 557 & 13.4 & 2.2 & $(9.1-17.8)$ \\
\hline $\begin{array}{l}\text { Virginia Beach-Norfolk-Newport News, } \\
\text { Virginia-North Carolina }\end{array}$ & 1,503 & 10.1 & 0.9 & $(8.4-11.9)$ \\
\hline $\begin{array}{l}\text { Warren-Troy-Farmington Hills, } \\
\text { Michigan }{ }^{\dagger}\end{array}$ & 2,185 & 8.2 & 0.7 & $(6.9-9.5)$ \\
\hline $\begin{array}{l}\text { Washington-Arlington-Alexandria, } \\
\text { District of Columbia-Virginia- } \\
\text { Maryland-West Virginia }{ }^{\dagger}\end{array}$ & 8,194 & 8.7 & 0.5 & $(7.7-9.6)$ \\
\hline
\end{tabular}

See table footnotes on page 98. 
TABLE 50. (Continued) Estimated prevalence of adults aged $\geq 18$ years who were ever told by a doctor that they have diabetes,* by metropolitan and micropolitan statistical area - Behavioral Risk Factor Surveillance System, United States, 2012

\begin{tabular}{|c|c|c|c|c|}
\hline MMSA(s) & $\begin{array}{l}\text { Sample } \\
\text { size }\end{array}$ & $\%$ & SE & $95 \% \mathrm{Cl}$ \\
\hline Wichita, Kansas & 2,353 & 10.3 & 0.7 & $(8.8-11.7)$ \\
\hline $\begin{array}{l}\text { Wilmington, Delaware-Maryland- } \\
\text { New Jersey }{ }^{\dagger}\end{array}$ & 3,229 & 8.5 & 0.6 & $(7.3-9.7)$ \\
\hline Winston-Salem, North Carolina & 794 & 9.8 & 1.1 & $(7.5-12.0)$ \\
\hline Worcester, Massachusetts-Connecticut & 2,838 & 9.0 & 0.7 & $(7.5-10.4)$ \\
\hline Yakima, Washington & 531 & 10.3 & 1.6 & $(7.2-13.4)$ \\
\hline $\begin{array}{l}\text { Youngstown-Warren-Boardman, } \\
\text { Ohio-Pennsylvania }\end{array}$ & 930 & 12.8 & 1.6 & $(9.7-15.8)$ \\
\hline Median & & 9.6 & & \\
\hline Range & & $3.4-17.4$ & & \\
\hline
\end{tabular}

Abbreviations: $\mathrm{Cl}=$ confidence interval; $\mathrm{MMSA}=$ metropolitan and micropolitan statistical area; $\mathrm{SE}=$ standard error.

* Excluding diabetes during pregnancy in females, or prediabetes or borderline diabetes in adults.

† Metropolitan division.
TABLE 51. Estimated prevalence of adults aged $\geq 18$ years who were ever told by a doctor that they have diabetes, ${ }^{*}$ by county Behavioral Risk Factor Surveillance System, United States, 2012

\begin{tabular}{|c|c|c|c|c|}
\hline County & $\begin{array}{l}\text { Sample } \\
\text { size }\end{array}$ & $\%$ & SE & $95 \% \mathrm{Cl}$ \\
\hline Jefferson County, Alabama & 962 & 12.3 & 1.1 & $(10.1-14.6)$ \\
\hline Madison County, Alabama & 511 & 7.3 & 1.2 & $(5.0-9.6)$ \\
\hline Mobile County, Alabama & 816 & 12.4 & 1.4 & $(9.6-15.2)$ \\
\hline Anchorage Municipality, Alaska & 903 & 7.4 & 1.0 & $(5.4-9.4)$ \\
\hline Fairbanks North Star Borough, Alaska & 599 & 5.0 & 1.1 & $(2.9-7.1)$ \\
\hline Matanuska-Susitna Borough, Alaska & 600 & 9.6 & 1.4 & $(6.8-12.5)$ \\
\hline Maricopa County, Arizona & 2,118 & 9.4 & 0.9 & $(7.7-11.1)$ \\
\hline Pima County, Arizona & 998 & 11.8 & 1.5 & $(8.9-14.7)$ \\
\hline Pulaski County, Arkansas & 745 & 8.8 & 1.2 & $(6.4-11.2)$ \\
\hline Alameda County, California & 617 & 9.1 & 1.8 & $(5.6-12.6)$ \\
\hline Los Angeles County, California & 3,504 & 10.6 & 0.7 & $(9.3-12.0)$ \\
\hline Orange County, California & 1,036 & 9.5 & 1.2 & $(7.0-11.9)$ \\
\hline Riverside County, California & 824 & 10.5 & 1.5 & $(7.6-13.3)$ \\
\hline Sacramento County, California & 609 & 8.4 & 1.8 & $(4.8-12.0)$ \\
\hline San Bernardino County, California & 705 & 11.1 & 1.5 & $(8.1-14.0)$ \\
\hline San Diego County, California & 1,128 & 9.3 & 1.2 & $(6.9-11.6)$ \\
\hline Santa Clara County, California & 666 & 7.4 & 1.3 & $(4.9-10.0)$ \\
\hline Adams County, Colorado & 808 & 8.6 & 1.3 & $(6.1-11.0)$ \\
\hline Arapahoe County, Colorado & 856 & 6.4 & 0.9 & $(4.6-8.3)$ \\
\hline Boulder County, Colorado & 517 & 3.4 & 0.8 & $(1.8-5.1)$ \\
\hline Denver County, Colorado & 996 & 9.6 & 1.0 & $(7.6-11.6)$ \\
\hline Douglas County, Colorado & 546 & 3.1 & 0.7 & $(1.8-4.4)$ \\
\hline El Paso County, Colorado & 1,021 & 8.6 & 1.0 & $(6.7-10.5)$ \\
\hline Jefferson County, Colorado & 1,117 & 6.2 & 0.8 & $(4.7-7.8)$ \\
\hline Larimer County, Colorado & 593 & 5.4 & 0.9 & $(3.6-7.2)$ \\
\hline Weld County, Colorado & 535 & 8.0 & 1.4 & $(5.3-10.6)$ \\
\hline Fairfield County, Connecticut & 2,186 & 7.4 & 0.8 & $(5.9-8.9)$ \\
\hline Hartford County, Connecticut & 1,985 & 10.0 & 1.0 & $(7.9-12.0)$ \\
\hline Litchfield County, Connecticut & 663 & 6.9 & 1.2 & $(4.6-9.2)$ \\
\hline New Haven County, Connecticut & 2,006 & 10.2 & 0.9 & $(8.3-12.0)$ \\
\hline New London County, Connecticut & 1,000 & 10.0 & 1.3 & $(7.5-12.4)$ \\
\hline Kent County, Delaware & 1,441 & 10.9 & 1.1 & $(8.7-13.1)$ \\
\hline New Castle County, Delaware & 2,329 & 8.3 & 0.7 & $(6.9-9.7)$ \\
\hline Sussex County, Delaware & 1,397 & 12.2 & 1.1 & $(10.1-14.3)$ \\
\hline $\begin{array}{l}\text { District of Columbia, } \\
\text { District of Columbia }\end{array}$ & 3,819 & 8.2 & 0.6 & $(7.1-9.4)$ \\
\hline Broward County, Florida & 526 & 9.2 & 1.5 & $(6.2-12.2)$ \\
\hline Miami-Dade County, Florida & 813 & 10.9 & 1.6 & $(7.7-14.1)$ \\
\hline Hawaii County, Hawaii & 1,346 & 8.6 & 1.1 & $(6.4-10.8)$ \\
\hline Honolulu County, Hawaii & 4,337 & 7.8 & 0.6 & $(6.5-9.0)$ \\
\hline Kauai County, Hawaii & 667 & 7.4 & 1.2 & $(4.9-9.8)$ \\
\hline Maui County, Hawaii & 1,216 & 7.0 & 1.1 & $(4.8-9.1)$ \\
\hline Ada County, Idaho & 807 & 6.2 & 0.9 & $(4.5-7.9)$ \\
\hline Canyon County, Idaho & 504 & 10.4 & 2.2 & $(6.2-14.7)$ \\
\hline Cook County, Illinois & 1,504 & 7.7 & 0.9 & $(5.9-9.4)$ \\
\hline Lake County, Indiana & 889 & 12.1 & 1.7 & $(8.8-15.5)$ \\
\hline Marion County, Indiana & 1,279 & 11.5 & 1.1 & $(9.4-13.6)$ \\
\hline Polk County, lowa & 808 & 9.2 & 1.1 & $(7.1-11.3)$ \\
\hline Johnson County, Kansas & 2,176 & 7.2 & 0.6 & $(5.9-8.4)$ \\
\hline Sedgwick County, Kansas & 1,794 & 10.0 & 0.8 & $(8.3-11.6)$ \\
\hline Shawnee County, Kansas & 769 & 8.7 & 1.2 & $(6.4-11.0)$ \\
\hline Wyandotte County, Kansas & 876 & 14.8 & 2.1 & $(10.6-19.0)$ \\
\hline Jefferson County, Kentucky & 1,694 & 10.6 & 1.2 & $(8.3-13.0)$ \\
\hline East Baton Rouge Parish, Louisiana & 571 & 9.2 & 1.2 & $(6.7-11.6)$ \\
\hline Androscoggin County, Maine & 699 & 11.8 & 1.4 & $(9.1-14.5)$ \\
\hline Aroostook County, Maine & 539 & 13.9 & 1.6 & $(10.7-17.1)$ \\
\hline Cumberland County, Maine & 1,757 & 7.9 & 0.8 & $(6.4-9.4)$ \\
\hline Kennebec County, Maine & 833 & 10.8 & 1.1 & $(8.6-13.1)$ \\
\hline Penobscot County, Maine & 929 & 8.2 & 0.9 & $(6.5-9.9)$ \\
\hline York County, Maine & 1,192 & 10.5 & 1.0 & $(8.5-12.4)$ \\
\hline
\end{tabular}

See table footnotes on page 100. 
TABLE 51. (Continued) Estimated prevalence of adults aged $\geq 18$ years who were ever told by a doctor that they have diabetes, ${ }^{*}$ by county — Behavioral Risk Factor Surveillance System, United States, 2012

\begin{tabular}{|c|c|c|c|c|}
\hline County & $\begin{array}{l}\text { Sample } \\
\text { size }\end{array}$ & $\%$ & SE & $95 \% \mathrm{Cl}$ \\
\hline Anne Arundel County, Maryland & 892 & 10.6 & 1.3 & $(8.1-13.2)$ \\
\hline Baltimore County, Maryland & 1,515 & 9.4 & 0.9 & $(7.7-11.1)$ \\
\hline Charles County, Maryland & 510 & 10.7 & 2.2 & $(6.3-15.1)$ \\
\hline Frederick County, Maryland & 753 & 10.1 & 2.1 & $(6.0-14.1)$ \\
\hline Montgomery County, Maryland & 1,545 & 7.2 & 0.9 & $(5.4-9.0)$ \\
\hline Prince George's County, Maryland & 1,141 & 12.5 & 1.3 & $(9.9-15.1)$ \\
\hline Washington County, Maryland & 536 & 13.9 & 2.4 & $(9.2-18.6)$ \\
\hline Baltimore city, Maryland & 740 & 13.4 & 1.8 & $(9.9-16.9)$ \\
\hline Barnstable County, Massachusetts & 559 & 9.6 & 1.5 & $(6.7-12.6)$ \\
\hline Bristol County, Massachusetts & 2,653 & 12.0 & 1.1 & $(9.8-14.2)$ \\
\hline Essex County, Massachusetts & 2,564 & 9.3 & 0.8 & $(7.7-10.9)$ \\
\hline Hampden County, Massachusetts & 1,961 & 11.4 & 1.0 & $(9.4-13.4)$ \\
\hline Middlesex County, Massachusetts & 4,360 & 7.2 & 0.6 & $(6.1-8.3)$ \\
\hline Norfolk County, Massachusetts & 1,759 & 6.3 & 0.8 & $(4.7-8.0)$ \\
\hline Plymouth County, Massachusetts & 1,836 & 8.8 & 1.1 & $(6.6-10.9)$ \\
\hline Suffolk County, Massachusetts & 2,312 & 7.1 & 0.7 & $(5.7-8.4)$ \\
\hline Worcester County, Massachusetts & 2,592 & 8.4 & 0.8 & $(6.9-9.8)$ \\
\hline Kent County, Michigan & 526 & 7.9 & 1.1 & $(5.6-10.1)$ \\
\hline Macomb County, Michigan & 613 & 9.5 & 1.3 & $(7.0-12.1)$ \\
\hline Oakland County, Michigan & 1,169 & 7.8 & 0.9 & $(6.1-9.5)$ \\
\hline Wayne County, Michigan & 2,217 & 12.6 & 1.1 & $(10.4-14.7)$ \\
\hline Anoka County, Minnesota & 545 & 5.8 & 1.1 & $(3.6-7.9)$ \\
\hline Dakota County, Minnesota & 673 & 4.7 & 1.0 & $(2.7-6.6)$ \\
\hline Hennepin County, Minnesota & 3,299 & 5.6 & 0.6 & $(4.4-6.9)$ \\
\hline Ramsey County, Minnesota & 1,947 & 7.6 & 1.1 & $(5.4-9.8)$ \\
\hline Jackson County, Missouri & 883 & 9.6 & 1.3 & $(7.1-12.2)$ \\
\hline St. Louis County, Missouri & 953 & 9.1 & 1.3 & $(6.5-11.7)$ \\
\hline Cascade County, Montana & 709 & 7.8 & 1.0 & $(5.7-9.8)$ \\
\hline Flathead County, Montana & 560 & 6.2 & 1.1 & $(4.1-8.3)$ \\
\hline Hill County, Montana & 583 & 11.4 & 1.8 & $(8.0-14.9)$ \\
\hline Lake County, Montana & 899 & 8.2 & 1.1 & $(6.0-10.5)$ \\
\hline Missoula County, Montana & 782 & 3.4 & 0.6 & $(2.2-4.7)$ \\
\hline Yellowstone County, Montana & 750 & 8.1 & 1.0 & $(6.0-10.1)$ \\
\hline Dakota County, Nebraska & 733 & 9.2 & 2.4 & $(4.6-13.9)$ \\
\hline Douglas County, Nebraska & 3,569 & 8.1 & 0.5 & $(7.0-9.1)$ \\
\hline Hall County, Nebraska & 535 & 8.7 & 1.6 & $(5.6-11.7)$ \\
\hline Lancaster County, Nebraska & 1,445 & 6.4 & 0.7 & $(5.0-7.7)$ \\
\hline Lincoln County, Nebraska & 589 & 8.8 & 1.4 & $(6.0-11.6)$ \\
\hline Sarpy County, Nebraska & 1,176 & 8.0 & 0.9 & $(6.2-9.7)$ \\
\hline Scotts Bluff County, Nebraska & 578 & 13.1 & 1.8 & $(9.6-16.6)$ \\
\hline Clark County, Nevada & 2,007 & 9.5 & 0.8 & $(7.9-11.1)$ \\
\hline Washoe County, Nevada & 1,489 & 6.5 & 0.8 & $(4.9-8.0)$ \\
\hline Belknap County, New Hampshire & 567 & 9.5 & 1.7 & $(6.2-12.9)$ \\
\hline Carroll County, New Hampshire & 532 & 8.1 & 1.4 & $(5.5-10.8)$ \\
\hline Cheshire County, New Hampshire & 548 & 8.4 & 1.5 & $(5.4-11.4)$ \\
\hline Coos County, New Hampshire & 537 & 15.1 & 2.4 & $(10.3-19.8)$ \\
\hline Grafton County, New Hampshire & 565 & 7.9 & 1.2 & $(5.5-10.2)$ \\
\hline Hillsborough County, New Hampshire & 1,905 & 8.7 & 0.7 & $(7.3-10.1)$ \\
\hline Merrimack County, New Hampshire & 704 & 8.8 & 1.2 & $(6.5-11.2)$ \\
\hline Rockingham County, New Hampshire & 1,049 & 9.4 & 1.0 & $(7.4-11.5)$ \\
\hline Strafford County, New Hampshire & 622 & 9.7 & 1.3 & $(7.2-12.2)$ \\
\hline Atlantic County, New Jersey & 1,022 & 9.8 & 1.1 & $(7.6-12.0)$ \\
\hline Bergen County, New Jersey & 1,011 & 6.7 & 1.0 & $(4.8-8.6)$ \\
\hline Burlington County, New Jersey & 682 & 11.4 & 1.6 & $(8.3-14.5)$ \\
\hline Camden County, New Jersey & 742 & 12.0 & 1.5 & $(9.0-14.9)$ \\
\hline Cape May County, New Jersey & 554 & 9.6 & 1.6 & $(6.6-12.7)$ \\
\hline Cumberland County, New Jersey & 557 & 13.4 & 2.2 & $(9.1-17.8)$ \\
\hline Essex County, New Jersey & 1,339 & 9.3 & 1.0 & $(7.4-11.3)$ \\
\hline Gloucester County, New Jersey & 552 & 11.2 & 1.5 & $(8.2-14.2)$ \\
\hline Hudson County, New Jersey & 1,305 & 8.1 & 0.9 & $(6.3-9.9)$ \\
\hline
\end{tabular}

See table footnotes on page 100.
TABLE 51. (Continued) Estimated prevalence of adults aged $\geq 18$ years who were ever told by a doctor that they have diabetes, ${ }^{*}$ by county - Behavioral Risk Factor Surveillance System, United States, 2012

\begin{tabular}{lcccr}
\hline County & $\begin{array}{c}\text { Sample } \\
\text { size }\end{array}$ & $\%$ & SE & \multicolumn{1}{c}{$95 \% \mathrm{Cl}$} \\
\hline Hunterdon County, New Jersey & 572 & 5.8 & 1.1 & $(3.6-8.0)$ \\
Mercer County, New Jersey & 579 & 9.9 & 1.4 & $(7.1-12.6)$ \\
Middlesex County, New Jersey & 882 & 9.1 & 1.2 & $(6.7-11.4)$ \\
Monmouth County, New Jersey & 713 & 9.7 & 1.3 & $(7.0-12.3)$
\end{tabular}

Morris County, New Jersey

Ocean County, New Jersey

Passaic County, New Jersey

Salem County, New Jersey

Somerset County, New Jersey

Sussex County, New Jersey

Union County, New Jersey

Warren County, New Jersey

Bernalillo County, New Mexico

Dona Ana County, New Mexico

Sandoval County, New Mexico

San Juan County, New Mexico

Santa Fe County, New Mexico

Kings County, New York

Guilford County, North Carolina

Mecklenburg County, North Carolina

Robeson County, North Carolina

Wake County, North Carolina

Burleigh County, North Dakota

Cass County, North Dakota

Cuyahoga County, Ohio

Franklin County, Ohio

Hamilton County, Ohio

Lorain County, Ohio

Lucas County, Ohio

Mahoning County, Ohio

Montgomery County, Ohio

Stark County, Ohio

Summit County, Ohio

Oklahoma County, Oklahoma

Tulsa County, Oklahoma

Clackamas County, Oregon

Lane County, Oregon

Multnomah County, Oregon

Washington County, Oregon

Allegheny County, Pennsylvania

Bradford County, Pennsylvania

Montgomery County, Pennsylvania

Philadelphia County, Pennsylvania

Pike County, Pennsylvania

Kent County, Rhode Island

Providence County, Rhode Island

Washington County, Rhode Island

Aiken County, South Carolina

Beaufort County, South Carolina

Charleston County, South Carolina

Greenville County, South Carolina

Horry County, South Carolina

Richland County, South Carolina

Spartanburg County, South Carolina

Lincoln County, South Dakota

Minnehaha County, South Dakota

Pennington County, South Dakota

Davidson County, Tennessee

Shelby County, Tennessee

Bexar County, Texas

See table footnotes on page 100
$(5.8-10.2)$

$(8.5-14.5)$

$(6.9-12.7)$

(8.6-15.1)

$(5.6-11.5)$

$(7.3-13.2)$

$(5.7-9.8)$

$(5.5-10.9)$

$(6.9-9.5)$

$(9.0-14.1)$

(6.4-11.4)

(7.6-14.2)

(4.6-9.0)

(6.3-13.7)

(6.4-12.4)

(6.6-11.6)

(11.1-23.7)

(4.8-9.3)

(6.6-12.2)

(4.4-8.5)

(10.2-15.7)

(7.0-11.4)

(9.5-15.0)

(6.9-12.5)

(10.1-16.9)

(10.4-19.6)

(10.1-16.1)

(11.8-19.1)

(7.1-12.5)

(8.0-11.8)

(7.9-11.6)

(5.7-11.8)

(6.0-11.4)

(6.2-11.1)

(7.2-12.5)

(8.1-11.2)

(8.9-13.0)

(4.1-8.5)

(10.7-14.4)

(8.9-13.9)

(9.0-14.2)

(8.7-11.2)

(5.2-9.5)

(10.5-17.9)

(5.6-10.0)

(6.7-11.7)

(7.6-12.7)

(7.3-11.8)

(7.9-13.2)

(8.4-15.2)

(2.4-7.1)

$(5.0-8.7)$

(4.9-9.4)

(7.0-12.7)

(9.9-17.3)

(7.7-13.3) 
TABLE 51. (Continued) Estimated prevalence of adults aged $\geq 18$ years who were ever told by a doctor that they have diabetes, ${ }^{*}$ by county - Behavioral Risk Factor Surveillance System, United States, 2012

\begin{tabular}{lrrrr}
\hline & $\begin{array}{c}\text { Sample } \\
\text { Size }\end{array}$ & \multicolumn{1}{c}{$\%$} & \multicolumn{1}{c}{ SE } & \multicolumn{1}{c}{$95 \% \mathrm{Cl}$} \\
\hline County & 512 & 12.1 & 1.7 & $(8.7-15.4)$ \\
Dallas County, Texas & 624 & 14.2 & 2.0 & $(10.3-18.0)$ \\
El Paso County, Texas & 774 & 11.3 & 1.3 & $(8.7-13.8)$ \\
Harris County, Texas & 621 & 13.4 & 1.9 & $(9.6-17.2)$ \\
Hidalgo County, Texas & 570 & 9.6 & 1.3 & $(7.1-12.2)$ \\
Tarrant County, Texas & 1,048 & 6.6 & 1.0 & $(4.6-8.5)$ \\
Travis County, Texas & 1,149 & 7.8 & 0.8 & $(6.2-9.3)$ \\
Davis County, Utah & 4,045 & 7.2 & 0.5 & $(6.3-8.1)$ \\
Salt Lake County, Utah & 566 & 6.6 & 1.1 & $(4.4-8.9)$ \\
Tooele County, Utah & 1,680 & 4.9 & 0.5 & $(3.8-5.9)$ \\
Utah County, Utah & 511 & 4.5 & 0.8 & $(3.0-6.1)$ \\
Wasatch County, Utah & 1,048 & 9.9 & 1.2 & $(7.6-12.2)$ \\
Weber County, Utah & 917 & 5.9 & 0.9 & $(4.2-7.5)$ \\
Chittenden County, Vermont & 596 & 8.1 & 1.2 & $(5.8-10.5)$ \\
Rutland County, Vermont & 517 & 7.5 & 1.4 & $(4.8-10.1)$ \\
Washington County, Vermont & 545 & 7.1 & 1.2 & $(4.8-9.5)$ \\
Windsor County, Vermont & 741 & 5.2 & 1.0 & $(3.2-7.1)$ \\
Fairfax County, Virginia & 791 & 9.2 & 1.1 & $(7.0-11.3)$ \\
Clark County, Washington & 3,920 & 7.3 & 0.6 & $(6.2-8.4)$ \\
King County, Washington & 565 & 8.7 & 1.4 & $(6.0-11.4)$ \\
Kitsap County, Washington & 1,180 & 8.1 & 0.9 & $(6.4-9.8)$ \\
Pierce County, Washington & 1,175 & 9.6 & 1.0 & $(7.7-11.5)$ \\
Snohomish County, Washington & 952 & 9.2 & 1.1 & $(7.0-11.4)$ \\
Spokane County, Washington & 517 & 13.7 & 2.1 & $(9.6-17.8)$ \\
Thurston County, Washington & 846 & 6.7 & 0.9 & $(4.9-8.6)$ \\
Whatcom County, Washington & 531 & 10.3 & 1.6 & $(7.2-13.4)$ \\
Yakima County, Washington & 641 & 13.1 & 1.4 & $(10.4-15.9)$ \\
Kanawha County, West Virginia & 962 & 8.9 & 1.4 & $(6.1-11.7)$ \\
Milwaukee County, Wisconsin & 959 & 11.9 & 1.5 & $(8.9-14.9)$ \\
Laramie County, Wyoming & 830 & 10.7 & 1.4 & $(8.0-13.4)$ \\
Natrona County, Wyoming & 666 & 14.2 & 1.5 & $(11.2-17.2)$ \\
San Juan Municipio, Puerto Rico & & 9.3 & & \\
Median & & $3.1-17.4$ & & \\
Range & & & & \\
\hline Abbrevation & & & & \\
& & & &
\end{tabular}

Abbreviations: $\mathrm{Cl}=$ confidence interval; $\mathrm{SE}=$ standard error.

* Excluding diabetes during pregnancy in females, or prediabetes or borderline diabetes in adults.
TABLE 52. Estimated prevalence of adults aged $\geq 18$ years who were ever told they had any type of cancer, by state/territory - Behavioral Risk Factor Surveillance System, United States, 2012

\begin{tabular}{|c|c|c|c|c|}
\hline State/Territory & $\begin{array}{l}\text { Sample } \\
\text { size }\end{array}$ & $\%$ & SE & $95 \% \mathrm{Cl}$ \\
\hline Alabama & 9,023 & 13.1 & 0.4 & $(12.2-14.0)$ \\
\hline Alaska & 4,341 & 7.0 & 0.4 & $(6.2-7.9)$ \\
\hline Arizona & 7,302 & 12.5 & 0.5 & $(11.5-13.5)$ \\
\hline Arkansas & 5,181 & 12.5 & 0.5 & $(11.5-13.5)$ \\
\hline California & 14,574 & 9.9 & 0.3 & (9.3-10.5) \\
\hline Colorado & 12,254 & 10.9 & 0.3 & $(10.3-11.5)$ \\
\hline Connecticut & 8,776 & 11.3 & 0.4 & $(10.5-12.1)$ \\
\hline Delaware & 5,174 & 12.4 & 0.5 & $(11.4-13.5)$ \\
\hline District of Columbia & 3,821 & 6.4 & 0.4 & $(5.6-7.3)$ \\
\hline Florida & 7,617 & 13.7 & 0.5 & $(12.8-14.7)$ \\
\hline Georgia & 6,096 & 10.6 & 0.4 & $(9.7-11.4)$ \\
\hline Hawaii & 7,581 & 9.0 & 0.4 & $(8.1-9.8)$ \\
\hline Idaho & 5,892 & 12.0 & 0.6 & $(10.9-13.2)$ \\
\hline Illinois & 5,577 & 9.5 & 0.5 & $(8.6-10.4)$ \\
\hline Indiana & 8,642 & 10.5 & 0.4 & $(9.7-11.2)$ \\
\hline lowa & 7,165 & 11.5 & 0.4 & $(10.7-12.2)$ \\
\hline Kansas & 11,795 & 11.2 & 0.3 & (10.6-11.8) \\
\hline Kentucky & 11,220 & 13.0 & 0.5 & $(12.2-13.9)$ \\
\hline Louisiana & 9,067 & 10.3 & 0.4 & $(9.5-11.1)$ \\
\hline Maine & 9,915 & 11.7 & 0.3 & $(11.1-12.4)$ \\
\hline Maryland & 12,804 & 9.2 & 0.3 & $(8.6-9.9)$ \\
\hline Massachusetts & 21,701 & 10.9 & 0.3 & (10.4-11.5) \\
\hline Michigan & 10,495 & 12.2 & 0.4 & $(11.5-13.0)$ \\
\hline Minnesota & 12,239 & 10.3 & 0.3 & $(9.6-10.9)$ \\
\hline Mississippi & 7,785 & 10.9 & 0.4 & $(10.1-11.7)$ \\
\hline Missouri & 6,753 & 12.6 & 0.5 & (11.7-13.6) \\
\hline Montana & 8,677 & 13.4 & 0.4 & $(12.5-14.2)$ \\
\hline Nebraska & 19,169 & 10.8 & 0.3 & $(10.3-11.4)$ \\
\hline Nevada & 4,844 & 9.6 & 0.5 & $(8.6-10.5)$ \\
\hline New Hampshire & 7,525 & 11.7 & 0.4 & $(10.8-12.5)$ \\
\hline New Jersey & 15,751 & 9.1 & 0.3 & (8.5-9.6) \\
\hline New Mexico & 8,775 & 10.4 & 0.3 & $(9.7-11.1)$ \\
\hline New York & 6,056 & 8.9 & 0.4 & $(8.1-9.7)$ \\
\hline North Carolina & 11,895 & 12.1 & 0.3 & $(11.4-12.7)$ \\
\hline North Dakota & 4,878 & 10.0 & 0.5 & (9.1-10.9) \\
\hline Ohio & 13,024 & 11.1 & 0.3 & $(10.5-11.7)$ \\
\hline Oklahoma & 8,010 & 11.2 & 0.4 & $(10.4-11.9)$ \\
\hline Oregon & 5,298 & 12.7 & 0.5 & $(11.7-13.7)$ \\
\hline Pennsylvania & 19,947 & 11.0 & 0.3 & $(10.4-11.6)$ \\
\hline Rhode Island & 5,478 & 11.3 & 0.5 & $(10.4-12.2)$ \\
\hline South Carolina & 12,791 & 11.9 & 0.3 & $(11.3-12.6)$ \\
\hline South Dakota & 7,878 & 10.7 & 0.5 & $(9.8-11.7)$ \\
\hline Tennessee & 7,047 & 12.9 & 0.5 & $(12.0-13.9)$ \\
\hline Texas & 9,125 & 9.3 & 0.3 & $(8.6-10.0)$ \\
\hline Utah & 12,431 & 10.0 & 0.3 & $(9.4-10.5)$ \\
\hline Vermont & 6,055 & 11.6 & 0.5 & $(10.7-12.4)$ \\
\hline Virginia & 7,390 & 10.1 & 0.4 & $(9.4-10.8)$ \\
\hline Washington & 15,312 & 11.6 & 0.3 & $(11.0-12.1)$ \\
\hline West Virginia & 5,409 & 13.2 & 0.5 & $(12.3-14.2)$ \\
\hline Wisconsin & 5,297 & 9.6 & 0.5 & $(8.6-10.7)$ \\
\hline Wyoming & 6,270 & 11.3 & 0.5 & $(10.3-12.3)$ \\
\hline Guam & 2,030 & 3.0 & 0.4 & $(2.2-3.8)$ \\
\hline Puerto Rico & 6,320 & 4.8 & 0.3 & $(4.3-5.4)$ \\
\hline Median & & 11.0 & & \\
\hline Range & & $3.0-13.7$ & & \\
\hline
\end{tabular}

Abbreviations: $\mathrm{Cl}=$ confidence interval; $\mathrm{SE}=$ standard error. 
TABLE 53. Estimated prevalence of adults aged $\geq 18$ years who were ever told they had any type of cancer, by metropolitan and micropolitan statistical area - Behavioral Risk Factor Surveillance System, United States, 2012

\begin{tabular}{|c|c|c|c|c|}
\hline MMSA(s) & $\begin{array}{l}\text { Sample } \\
\text { size }\end{array}$ & $\%$ & SE & $95 \% \mathrm{Cl}$ \\
\hline Aguadilla-Isabela, Puerto Rico & 551 & 3.8 & 0.8 & $(2.2-5.4)$ \\
\hline Akron, Ohio & 744 & 11.6 & 1.3 & $(9.1-14.1)$ \\
\hline Albuquerque, New Mexico & 3,270 & 10.1 & 0.6 & $(9.0-11.2)$ \\
\hline $\begin{array}{l}\text { Allentown-Bethlehem-Easton, } \\
\text { Pennsylvania-New Jersey }\end{array}$ & 1,352 & 10.1 & 1.1 & $(8.0-12.2)$ \\
\hline Anaheim-Santa Ana-Irvine, California* & 1,037 & 13.4 & 1.7 & $(10.2-16.7)$ \\
\hline Anchorage, Alaska & 1,506 & 6.4 & 0.6 & $(5.1-7.6)$ \\
\hline Asheville, North Carolina & 593 & 17.7 & 1.8 & $(14.2-21.2)$ \\
\hline $\begin{array}{l}\text { Atlanta-Sandy Springs-Roswell, } \\
\text { Georgia }\end{array}$ & 2,537 & 9.3 & 0.6 & $(8.0-10.5)$ \\
\hline Atlantic City-Hammonton, New Jersey & 1,024 & 10.9 & 1.0 & $(8.9-13.0)$ \\
\hline $\begin{array}{l}\text { Augusta-Richmond County, Georgia- } \\
\text { South Carolina }\end{array}$ & 1,036 & 10.6 & 1.3 & $(8.0-13.1)$ \\
\hline Augusta-Waterville, Maine & 833 & 10.5 & 1.2 & $(8.3-12.8)$ \\
\hline Austin-Round Rock, Texas & 1,387 & 8.8 & 0.9 & $(7.0-10.5)$ \\
\hline $\begin{array}{l}\text { Baltimore-Columbia-Towson, } \\
\text { Maryland }\end{array}$ & 4,724 & 9.3 & 0.5 & $(8.4-10.2)$ \\
\hline Bangor, Maine & 929 & 11.1 & 1.1 & $(8.9-13.3)$ \\
\hline Barnstable Town, Massachusetts & 559 & 18.5 & 1.9 & $(14.7-22.3)$ \\
\hline Barre, Vermont & 517 & 10.7 & 1.5 & $(7.8-13.6)$ \\
\hline Baton Rouge, Louisiana & 1,391 & 11.0 & 1.2 & $(8.7-13.2)$ \\
\hline Bellingham, Washington & 847 & 13.6 & 1.5 & $(10.6-16.6)$ \\
\hline Berlin, New Hampshire-Vermont & 711 & 13.1 & 1.8 & $(9.6-16.6)$ \\
\hline Billings, Montana & 849 & 13.2 & 1.2 & $(10.9-15.6)$ \\
\hline Birmingham-Hoover, Alabama & 1,825 & 11.4 & 0.8 & $(9.8-13.0)$ \\
\hline Bismarck, North Dakota & 823 & 9.8 & 1.1 & $(7.7-11.9)$ \\
\hline Boise City, Idaho & 1,483 & 12.7 & 1.1 & $(10.6-14.8)$ \\
\hline Boston, Massachusetts* & 5,914 & 10.0 & 0.5 & $(9.0-11.0)$ \\
\hline Boulder, Colorado & 517 & 11.8 & 1.5 & $(8.9-14.6)$ \\
\hline Bremerton-Silverdale, Washington & 566 & 13.3 & 1.4 & $(10.5-16.0)$ \\
\hline $\begin{array}{l}\text { Bridgeport-Stamford-Norwalk, } \\
\text { Connecticut }\end{array}$ & 2,187 & 10.7 & 0.8 & $(9.2-12.3)$ \\
\hline Burlington-South Burlington, Vermont & 1,522 & 10.4 & 0.8 & $(8.9-12.0)$ \\
\hline $\begin{array}{l}\text { Cambridge-Newton-Framingham, } \\
\text { Massachusetts* }\end{array}$ & 6,930 & 10.5 & 0.5 & $(9.6-11.5)$ \\
\hline Camden, New Jersey* & 1,983 & 9.8 & 0.7 & $(8.3-11.2)$ \\
\hline Canton-Massillon, Ohio & 682 & 12.5 & 1.4 & $(9.7-15.3)$ \\
\hline Casper, Wyoming & 830 & 11.3 & 1.3 & $(8.8-13.8)$ \\
\hline Cedar Rapids, lowa & 585 & 11.4 & 1.4 & $(8.5-14.2)$ \\
\hline Charleston, West Virginia & 772 & 11.5 & 1.1 & $(9.3-13.8)$ \\
\hline $\begin{array}{l}\text { Charleston-North Charleston, } \\
\text { South Carolina }\end{array}$ & 1,711 & 11.4 & 0.9 & $(9.6-13.2)$ \\
\hline $\begin{array}{l}\text { Charlotte-Concord-Gastonia, } \\
\text { North Carolina-South Carolina }\end{array}$ & 2,528 & 10.9 & 0.7 & $(9.5-12.3)$ \\
\hline Chattanooga, Tennessee-Georgia & 632 & 13.7 & 1.7 & $(10.4-17.1)$ \\
\hline Cheyenne, Wyoming & 959 & 12.6 & 1.4 & $(9.9-15.3)$ \\
\hline $\begin{array}{l}\text { Chicago-Naperville-Elgin, } \\
\text { Illinois-Indiana-Wisconsin }\end{array}$ & 3,731 & 8.4 & 0.6 & $(7.3-9.5)$ \\
\hline Cincinnati, Ohio-Kentucky-Indiana & 2,365 & 11.6 & 0.8 & $(10.1-13.1)$ \\
\hline $\begin{array}{l}\text { Claremont-Lebanon, New } \\
\text { Hampshire-Vermont }\end{array}$ & 1,951 & 13.2 & 0.9 & $(11.3-15.0)$ \\
\hline Cleveland-Elyria, Ohio & 1,782 & 9.8 & 0.9 & $(8.1-11.5)$ \\
\hline Colorado Springs, Colorado & 1,162 & 9.4 & 0.9 & $(7.7-11.2)$ \\
\hline Columbia, South Carolina & 1,801 & 9.8 & 0.9 & $(8.1-11.5)$ \\
\hline Columbus, Ohio & 1,604 & 10.4 & 0.9 & $(8.7-12.1)$ \\
\hline Concord, New Hampshire & 704 & 13.2 & 1.4 & $(10.5-16.0)$ \\
\hline Dallas-Plano-Irving, Texas* & 908 & 9.9 & 1.0 & $(7.9-11.8)$ \\
\hline Dayton, Ohio & 857 & 12.5 & 1.3 & $(10.0-15.0)$ \\
\hline Denver-Aurora-Lakewood, Colorado & 4,854 & 10.7 & 0.5 & $(9.8-11.6)$ \\
\hline Des Moines-West Des Moines, lowa & 1,160 & 10.8 & 1.0 & $(8.9-12.7)$ \\
\hline
\end{tabular}

See table footnotes on page 103.
TABLE 53. (Continued) Estimated prevalence of adults aged $\geq 18$ years who were ever told they had any type of cancer, by metropolitan and micropolitan statistical area - Behavioral Risk Factor Surveillance System, United States, 2012

\begin{tabular}{|c|c|c|c|c|}
\hline $\operatorname{MMSA}(\mathrm{s})$ & $\begin{array}{l}\text { Sample } \\
\text { size }\end{array}$ & $\%$ & SE & $95 \% \mathrm{Cl}$ \\
\hline Detroit-Dearborn-Livonia, Michigan* & 2,222 & 10.0 & 0.8 & $(8.4-11.6)$ \\
\hline Dover, Delaware & 1,441 & 11.8 & 1.0 & $(9.8-13.7)$ \\
\hline Duluth, Minnesota-Wisconsin & 514 & 9.3 & 1.3 & $(6.8-11.9)$ \\
\hline Durham-Chapel Hill, North Carolina & 796 & 10.8 & 1.1 & $(8.6-13.0)$ \\
\hline El Paso, Texas & 626 & 7.5 & 1.5 & $(4.6-10.4)$ \\
\hline Eugene, Oregon & 526 & 11.8 & 1.4 & $(8.9-14.6)$ \\
\hline Fairbanks, Alaska & 599 & 9.8 & 1.5 & $(6.9-12.8)$ \\
\hline Fargo, North Dakota-Minnesota & 940 & 8.0 & 1.2 & $(5.8-10.3)$ \\
\hline Farmington, New Mexico & 650 & 8.9 & 1.2 & $(6.6-11.3)$ \\
\hline Fayetteville, North Carolina & 506 & 7.6 & 1.1 & $(5.4-9.8)$ \\
\hline $\begin{array}{l}\text { Fayetteville-Springdale-Rogers, } \\
\text { Arkansas-Missouri }\end{array}$ & 812 & 10.5 & 1.2 & $(8.2-12.7)$ \\
\hline Fort Collins, Colorado & 596 & 10.7 & 1.2 & $(8.3-13.1)$ \\
\hline Fort Wayne, Indiana & 538 & 9.8 & 1.3 & $(7.3-12.3)$ \\
\hline Fort Worth-Arlington, Texas* & 727 & 10.1 & 1.1 & $(7.9-12.2)$ \\
\hline Grand Island, Nebraska & 855 & 11.0 & 1.1 & $(8.9-13.2)$ \\
\hline Grand Rapids-Wyoming, Michigan & 893 & 14.0 & 1.5 & $(11.1-17.0)$ \\
\hline Great Falls, Montana & 709 & 13.8 & 1.4 & $(11.0-16.6)$ \\
\hline Greeley, Colorado & 535 & 11.0 & 1.4 & $(8.2-13.9)$ \\
\hline $\begin{array}{l}\text { Greensboro-High Point, } \\
\text { North Carolina }\end{array}$ & 807 & 12.1 & 1.2 & $(9.7-14.5)$ \\
\hline $\begin{array}{l}\text { Greenville-Anderson-Mauldin, } \\
\text { South Carolina }\end{array}$ & 1,693 & 13.0 & 0.9 & $(11.2-14.9)$ \\
\hline Gulfport-Biloxi-Pascagoula, Mississippi & 744 & 11.0 & 1.2 & $(8.7-13.3)$ \\
\hline $\begin{array}{l}\text { Hagerstown-Martinsburg, Maryland- } \\
\text { West Virginia }\end{array}$ & 760 & 11.6 & 1.4 & $(8.8-14.4)$ \\
\hline Harrisburg-Carlisle, Pennsylvania & 665 & 10.5 & 1.3 & $(7.9-13.0)$ \\
\hline $\begin{array}{l}\text { Hartford-West Hartford-East Hartford, } \\
\text { Connecticut }\end{array}$ & 2,670 & 11.2 & 0.7 & $(9.9-12.5)$ \\
\hline Heber, Utah & 512 & 10.5 & 1.9 & $(6.7-14.3)$ \\
\hline Hilo, Hawaii & 1,349 & 12.4 & 1.1 & $(10.1-14.6)$ \\
\hline $\begin{array}{l}\text { Hilton Head Island-Bluffton-Beaufort, } \\
\text { South Carolina }\end{array}$ & 936 & 16.0 & 1.5 & $(13.1-18.9)$ \\
\hline $\begin{array}{l}\text { Houston-The Woodlands-Sugar Land, } \\
\text { Texas }\end{array}$ & 1,148 & 8.3 & 0.9 & $(6.6-10.0)$ \\
\hline $\begin{array}{l}\text { Huntington-Ashland, West } \\
\text { Virginia-Kentucky-Ohio }\end{array}$ & 1,116 & 15.2 & 1.2 & $(12.8-17.7)$ \\
\hline Huntsville, Alabama & 616 & 12.9 & 1.7 & $(9.6-16.2)$ \\
\hline Idaho Falls, Idaho & 540 & 13.5 & 2.0 & $(9.4-17.5)$ \\
\hline $\begin{array}{l}\text { Indianapolis-Carmel-Anderson, } \\
\text { Indiana }\end{array}$ & 2,197 & 10.5 & 0.7 & $(9.1-11.9)$ \\
\hline Jackson, Mississippi & 921 & 9.2 & 1.0 & $(7.1-11.2)$ \\
\hline Jacksonville, Florida & 519 & 14.1 & 1.8 & $(10.5-17.7)$ \\
\hline Kahului-Wailuku-Lahaina, Hawaii & 1,219 & 11.4 & 1.4 & $(8.6-14.2)$ \\
\hline Kalispell, Montana & 560 & 11.7 & 1.5 & $(8.7-14.6)$ \\
\hline Kansas City, Missouri-Kansas & 4,744 & 10.9 & 0.7 & $(9.5-12.3)$ \\
\hline Kapaa, Hawaii & 670 & 10.0 & 2.1 & $(5.8-14.2)$ \\
\hline Keene, New Hampshire & 548 & 10.9 & 1.4 & $(8.1-13.6)$ \\
\hline Kennewick-Richland, Washington & 535 & 10.8 & 1.4 & $(8.0-13.6)$ \\
\hline $\begin{array}{l}\text { Kingsport-Bristol-Bristol, } \\
\text { Tennessee-Virginia }\end{array}$ & 569 & 14.8 & 1.8 & $(11.3-18.3)$ \\
\hline Knoxville, Tennessee & 834 & 16.4 & 1.6 & $(13.4-19.5)$ \\
\hline Laconia, New Hampshire & 567 & 12.9 & 1.8 & $(9.4-16.4)$ \\
\hline Lafayette, Louisiana & 557 & 12.2 & 1.7 & $(8.9-15.6)$ \\
\hline Las Cruces, New Mexico & 708 & 10.3 & 1.1 & $(8.2-12.5)$ \\
\hline $\begin{array}{l}\text { Las Vegas-Henderson-Paradise, } \\
\text { Nevada }\end{array}$ & 2,016 & 8.6 & 0.6 & $(7.4-9.9)$ \\
\hline Lewiston-Auburn, Maine & 700 & 8.0 & 1.1 & $(5.9-10.1)$ \\
\hline Lexington-Fayette, Kentucky & 531 & 13.7 & 1.8 & $(10.2-17.3)$ \\
\hline Lincoln, Nebraska & 1,668 & 9.8 & 0.8 & $(8.2-11.4)$ \\
\hline
\end{tabular}

See table footnotes on page 103. 
TABLE 53. (Continued) Estimated prevalence of adults aged $\geq 18$ years who were ever told they had any type of cancer, by metropolitan and micropolitan statistical area - Behavioral Risk Factor Surveillance System, United States, 2012

\begin{tabular}{|c|c|c|c|c|}
\hline MMSA(s) & $\begin{array}{l}\text { Sample } \\
\text { size }\end{array}$ & $\%$ & SE & $95 \% \mathrm{Cl}$ \\
\hline $\begin{array}{l}\text { Little Rock-North Little Rock-Conway, } \\
\text { Arkansas }\end{array}$ & 1,171 & 12.0 & 1.1 & $(9.8-14.1)$ \\
\hline Logan, Utah-Idaho & 505 & 7.9 & 1.2 & $(5.6-10.3)$ \\
\hline $\begin{array}{l}\text { Los Angeles-Long Beach-Glendale, } \\
\text { California* }\end{array}$ & 3,504 & 7.0 & 0.5 & $(6.1-7.9)$ \\
\hline $\begin{array}{l}\text { Louisville/Jefferson County, } \\
\text { Kentucky-Indiana }\end{array}$ & 2,181 & 12.8 & 1.1 & $(10.6-14.9)$ \\
\hline Lumberton, North Carolina & 546 & 13.4 & 2.9 & $(7.7-19.1)$ \\
\hline Manchester-Nashua, New Hampshire & 1,906 & 10.7 & 0.7 & $(9.3-12.2)$ \\
\hline McAllen-Edinburg-Mission, Texas & 623 & 5.6 & 0.9 & $(3.8-7.4)$ \\
\hline $\begin{array}{l}\text { Memphis, } \\
\text { Tennessee-Mississippi-Arkansas }\end{array}$ & 1,310 & 8.2 & 0.9 & $(6.5-9.9)$ \\
\hline $\begin{array}{l}\text { Miami-Fort Lauderdale-West Palm } \\
\text { Beach, Florida }\end{array}$ & 1,670 & 10.1 & 0.9 & $(8.4-11.8)$ \\
\hline $\begin{array}{l}\text { Milwaukee-Waukesha-West Allis, } \\
\text { Wisconsin }\end{array}$ & 1,258 & 9.6 & 1.2 & $(7.3-11.9)$ \\
\hline $\begin{array}{l}\text { Minneapolis-St. Paul-Bloomington, } \\
\text { Minnesota-Wisconsin }\end{array}$ & 7,899 & 9.8 & 0.4 & $(9.0-10.7)$ \\
\hline Missoula, Montana & 782 & 12.5 & 1.3 & $(9.9-15.0)$ \\
\hline Mobile, Alabama & 818 & 11.7 & 1.4 & $(8.9-14.5)$ \\
\hline Montgomery, Alabama & 534 & 10.6 & 1.4 & $(7.8-13.4)$ \\
\hline $\begin{array}{l}\text { Montgomery County-Bucks County- } \\
\text { Chester County, Pennsylvania* }\end{array}$ & 1,314 & 11.6 & 1.0 & $(9.7-13.5)$ \\
\hline $\begin{array}{l}\text { Myrtle Beach-Conway-North Myrtle } \\
\text { Beach, South Carolina-North Carolina }\end{array}$ & 948 & 13.9 & 1.3 & $(11.3-16.5)$ \\
\hline $\begin{array}{l}\text { Nashville-Davidson-Murfreesboro- } \\
\text { Franklin, Tennessee }\end{array}$ & 1,341 & 11.3 & 1.0 & $(9.4-13.2)$ \\
\hline $\begin{array}{l}\text { Nassau County-Suffolk County, } \\
\text { New York* }\end{array}$ & 900 & 10.5 & 1.1 & $(8.4-12.6)$ \\
\hline Newark, New Jersey-Pennsylvania* & 6,557 & 8.7 & 0.5 & $(7.8-9.6)$ \\
\hline New Haven-Milford, Connecticut & 2,010 & 11.5 & 0.8 & $(9.9-13.2)$ \\
\hline New Orleans-Metairie, Louisiana & 1,277 & 9.9 & 0.8 & $(8.2-11.5)$ \\
\hline $\begin{array}{l}\text { New York-Jersey City-White Plains, } \\
\text { New York-New Jersey* }\end{array}$ & 7,447 & 7.6 & 0.5 & $(6.7-8.6)$ \\
\hline Norfolk, Nebraska & 571 & 10.5 & 1.5 & $(7.6-13.4)$ \\
\hline North Platte, Nebraska & 613 & 12.1 & 1.5 & $(9.1-15.1)$ \\
\hline Norwich-New London, Connecticut & 1,000 & 11.8 & 1.2 & $(9.5-14.1)$ \\
\hline $\begin{array}{l}\text { Oakland-Hayward-Berkeley, } \\
\text { California* }\end{array}$ & 976 & 10.7 & 1.5 & $(7.7-13.6)$ \\
\hline Ocean City, New Jersey & 554 & 19.2 & 2.4 & $(14.5-23.9)$ \\
\hline Ogden-Clearfield, Utah & 2,433 & 11.2 & 0.7 & $(9.9-12.5)$ \\
\hline Oklahoma City, Oklahoma & 2,440 & 9.6 & 0.6 & $(8.3-10.8)$ \\
\hline Olympia-Tumwater, Washington & 517 & 10.9 & 1.3 & $(8.3-13.5)$ \\
\hline Omaha-Council Bluffs, Nebraska-lowa & 5,590 & 9.7 & 0.5 & $(8.8-10.6)$ \\
\hline Orlando-Kissimmee-Sanford, Florida & 569 & 11.7 & 1.5 & $(8.7-14.7)$ \\
\hline Philadelphia, Pennsylvania* & 2,461 & 9.0 & 0.7 & $(7.7-10.4)$ \\
\hline Phoenix-Mesa-Scottsdale, Arizona & 2,602 & 11.2 & 0.7 & $(9.9-12.6)$ \\
\hline Pittsburgh, Pennsylvania & 3,368 & 12.5 & 0.7 & $(11.2-13.8)$ \\
\hline Ponce, Puerto Rico & 549 & 4.1 & 1.0 & $(2.2-5.9)$ \\
\hline Portland-South Portland, Maine & 3,334 & 12.3 & 0.6 & $(11.1-13.5)$ \\
\hline $\begin{array}{l}\text { Portland-Vancouver-Hillsboro, } \\
\text { Oregon-Washington }\end{array}$ & 3,129 & 11.0 & 0.6 & $(9.8-12.1)$ \\
\hline $\begin{array}{l}\text { Providence-Warwick, Rhode } \\
\text { Island-Massachusetts }\end{array}$ & 8,134 & 11.1 & 0.5 & $(10.2-11.9)$ \\
\hline Provo-Orem, Utah & 1,752 & 8.2 & 0.6 & $(6.9-9.5)$ \\
\hline Raleigh, North Carolina & 946 & 10.6 & 1.1 & $(8.4-12.8)$ \\
\hline Rapid City, South Dakota & 1,055 & 12.4 & 1.2 & $(10.0-14.7)$ \\
\hline Reno, Nevada & 1,509 & 10.6 & 0.9 & $(8.9-12.4)$ \\
\hline Richmond, Virginia & 1,014 & 10.1 & 1.0 & $(8.2-12.0)$ \\
\hline
\end{tabular}

See table footnotes on page 103.
TABLE 53. (Continued) Estimated prevalence of adults aged $\geq 18$ years who were ever told they had any type of cancer, by metropolitan and micropolitan statistical area - Behavioral Risk Factor Surveillance System, United States, 2012

\begin{tabular}{|c|c|c|c|c|}
\hline MMSA(s) & $\begin{array}{l}\text { Sample } \\
\text { size }\end{array}$ & $\%$ & SE & $95 \% \mathrm{Cl}$ \\
\hline $\begin{array}{l}\text { Riverside-San Bernardino-Ontario, } \\
\text { California }\end{array}$ & 1,529 & 10.7 & 0.9 & $(9.0-12.5)$ \\
\hline $\begin{array}{l}\text { Rockingham County-Strafford County, } \\
\text { New Hampshire* }\end{array}$ & 1,673 & 11.2 & 0.8 & $(9.6-12.9)$ \\
\hline Rutland, Vermont & 597 & 11.0 & 1.5 & $(8.1-13.9)$ \\
\hline $\begin{array}{l}\text { Sacramento-Roseville-Arden-Arcade, } \\
\text { California }\end{array}$ & 998 & 10.3 & 1.2 & $(8.0-12.6)$ \\
\hline St. Louis, Missouri-Illinois & 2,070 & 11.2 & 0.8 & $(9.6-12.8)$ \\
\hline Salisbury, Maryland-Delaware & 2,124 & 13.8 & 0.9 & $(12.0-15.5)$ \\
\hline Salt Lake City, Utah & 4,617 & 9.5 & 0.5 & $(8.6-10.4)$ \\
\hline San Antonio-New Braunfels, Texas & 779 & 10.1 & 1.1 & $(8.0-12.3)$ \\
\hline San Diego-Carlsbad, California & 1,128 & 11.3 & 1.0 & $(9.3-13.3)$ \\
\hline $\begin{array}{l}\text { San Francisco-Redwood City-South } \\
\text { San Francisco, California* }\end{array}$ & 596 & 10.0 & 1.7 & $(6.6-13.3)$ \\
\hline $\begin{array}{l}\text { San Jose-Sunnyvale-Santa Clara, } \\
\text { California }\end{array}$ & 685 & 7.4 & 1.1 & $(5.3-9.5)$ \\
\hline San Juan-Carolina-Caguas, Puerto Rico & 3,957 & 5.1 & 0.4 & $(4.4-5.8)$ \\
\hline Santa Fe, New Mexico & 697 & 13.0 & 1.4 & $(10.3-15.6)$ \\
\hline Sayre, Pennsylvania & 1,864 & 13.4 & 1.5 & $(10.4-16.3)$ \\
\hline Scottsbluff, Nebraska & 631 & 13.9 & 1.9 & $(10.3-17.6)$ \\
\hline $\begin{array}{l}\text { Scranton-Wilkes-Barre-Hazleton, } \\
\text { Pennsylvania }\end{array}$ & 744 & 10.3 & 1.4 & $(7.7-13.0)$ \\
\hline Seattle-Bellevue-Everett, Washington* & 5,099 & 10.6 & 0.5 & $(9.7-11.5)$ \\
\hline Shreveport-Bossier City, Louisiana & 579 & 9.5 & 1.3 & $(7.0-12.0)$ \\
\hline $\begin{array}{l}\text { Silver Spring-Frederick-Rockville, } \\
\text { Maryland* }\end{array}$ & 2,302 & 10.0 & 0.8 & $(8.5-11.6)$ \\
\hline $\begin{array}{l}\text { Sioux City, lowa-Nebraska- } \\
\text { South Dakota }\end{array}$ & 1,204 & 7.2 & 1.2 & $(4.8-9.5)$ \\
\hline Sioux Falls, South Dakota & 1,466 & 10.5 & 1.0 & $(8.7-12.4)$ \\
\hline Spartanburg, South Carolina & 710 & 10.5 & 1.3 & $(8.0-13.0)$ \\
\hline Spokane-Spokane Valley, Washington & 1,099 & 11.3 & 1.0 & $(9.4-13.2)$ \\
\hline Springfield, Massachusetts & 2,343 & 10.9 & 1.0 & $(9.0-12.9)$ \\
\hline
\end{tabular}

See table footnotes on page 103. 
TABLE 53. (Continued) Estimated prevalence of adults aged $\geq 18$ years who were ever told they had any type of cancer, by metropolitan and micropolitan statistical area - Behavioral Risk Factor Surveillance System, United States, 2012

\begin{tabular}{|c|c|c|c|c|}
\hline MMSA(s) & $\begin{array}{l}\text { Sample } \\
\text { size }\end{array}$ & $\%$ & SE & $95 \% \mathrm{Cl}$ \\
\hline Tacoma-Lakewood, Washington* & 1,183 & 11.9 & 1.1 & $(9.7-14.0)$ \\
\hline $\begin{array}{l}\text { Tampa-St. Petersburg-Clearwater, } \\
\text { Florida }\end{array}$ & 829 & 14.0 & 1.4 & $(11.2-16.8)$ \\
\hline Toledo, Ohio & 972 & 9.8 & 1.0 & $(7.8-11.8)$ \\
\hline Topeka, Kansas & 1,088 & 13.4 & 1.1 & $(11.2-15.7)$ \\
\hline Torrington, Connecticut & 663 & 12.6 & 1.4 & $(9.8-15.3)$ \\
\hline Trenton, New Jersey & 579 & 9.1 & 1.2 & $(6.8-11.4)$ \\
\hline Tucson, Arizona & 998 & 14.5 & 1.2 & $(12.2-16.8)$ \\
\hline Tulsa, Oklahoma & 1,742 & 11.1 & 0.8 & $(9.5-12.7)$ \\
\hline Tuscaloosa, Alabama & 582 & 9.8 & 1.4 & $(7.0-12.6)$ \\
\hline Urban Honolulu, Hawaii & 4,343 & 7.8 & 0.5 & $(6.8-8.8)$ \\
\hline Vineland-Bridgeton, New Jersey & 558 & 7.9 & 1.2 & $(5.5-10.2)$ \\
\hline $\begin{array}{l}\text { Virginia Beach-Norfolk-Newport News, } \\
\text { Virginia-North Carolina }\end{array}$ & 1,502 & 9.1 & 0.8 & $(7.6-10.6)$ \\
\hline $\begin{array}{l}\text { Warren-Troy-Farmington Hills, } \\
\text { Michigan* }\end{array}$ & 2,186 & 12.3 & 0.7 & $(10.9-13.8)$ \\
\hline $\begin{array}{l}\text { Washington-Arlington-Alexandria, } \\
\text { District of Columbia-Virginia- } \\
\text { Maryland-West Virginia* }\end{array}$ & 8,199 & 7.8 & 0.4 & $(7.1-8.6)$ \\
\hline Wichita, Kansas & 2,355 & 10.8 & 0.7 & $(9.5-12.1)$ \\
\hline $\begin{array}{l}\text { Wilmington, Delaware-Maryland- } \\
\text { New Jersey* }\end{array}$ & 3,237 & 10.9 & 0.6 & $(9.7-12.2)$ \\
\hline Winston-Salem, North Carolina & 796 & 12.3 & 1.3 & $(9.8-14.9)$ \\
\hline Worcester, Massachusetts-Connecticut & 2,841 & 10.0 & 0.7 & $(8.7-11.4)$ \\
\hline Yakima, Washington & 533 & 9.1 & 1.4 & $(6.2-11.9)$ \\
\hline $\begin{array}{l}\text { Youngstown-Warren-Boardman, } \\
\text { Ohio-Pennsylvania }\end{array}$ & 930 & 10.0 & 1.2 & $(7.6-12.4)$ \\
\hline Median & & 10.8 & & \\
\hline Range & & $3.8-19.2$ & & \\
\hline
\end{tabular}

Abbreviations: $\mathrm{Cl}=$ confidence interval; $\mathrm{MMSA}=$ metropolitan and micropolitan statistical area; $\mathrm{SE}=$ standard error.

* Metropolitan division.
TABLE 54. Estimated prevalence of adults aged $\geq 18$ years who were ever told they had any type of cancer, by county - Behavioral Risk Factor Surveillance System, United States, 2012

\begin{tabular}{|c|c|c|c|c|}
\hline County & $\begin{array}{l}\text { Sample } \\
\text { size }\end{array}$ & $\%$ & SE & $95 \% \mathrm{Cl}$ \\
\hline Jefferson County, Alabama & 962 & 10.4 & 1.0 & $(8.4-12.5)$ \\
\hline Madison County, Alabama & 513 & 12.6 & 1.9 & $(8.8-16.4)$ \\
\hline Mobile County, Alabama & 818 & 11.7 & 1.4 & $(8.9-14.5)$ \\
\hline Anchorage Municipality, Alaska & 905 & 5.5 & 0.7 & $(4.1-7.0)$ \\
\hline Fairbanks North Star Borough, Alaska & 599 & 9.8 & 1.5 & $(6.9-12.8)$ \\
\hline Matanuska-Susitna Borough, Alaska & 601 & 8.8 & 1.2 & $(6.4-11.3)$ \\
\hline Maricopa County, Arizona & 2,121 & 11.4 & 0.7 & $(9.9-12.8)$ \\
\hline Pima County, Arizona & 998 & 14.5 & 1.2 & $(12.2-16.8)$ \\
\hline Pulaski County, Arkansas & 747 & 10.2 & 1.2 & $(7.7-12.6)$ \\
\hline Alameda County, California & 619 & 10.5 & 2.2 & $(6.2-14.8)$ \\
\hline Los Angeles County, California & 3,504 & 7.0 & 0.5 & $(6.1-7.9)$ \\
\hline Orange County, California & 1,037 & 13.4 & 1.7 & $(10.2-16.7)$ \\
\hline Riverside County, California & 824 & 12.6 & 1.4 & $(9.9-15.3)$ \\
\hline Sacramento County, California & 609 & 9.2 & 1.5 & $(6.2-12.2)$ \\
\hline San Bernardino County, California & 705 & 8.8 & 1.2 & $(6.6-11.1)$ \\
\hline San Diego County, California & 1,128 & 11.3 & 1.0 & $(9.3-13.3)$ \\
\hline Santa Clara County, California & 666 & 7.5 & 1.1 & $(5.4-9.7)$ \\
\hline Adams County, Colorado & 810 & 8.4 & 1.0 & $(6.5-10.3)$ \\
\hline Arapahoe County, Colorado & 858 & 9.9 & 1.0 & $(7.9-11.8)$ \\
\hline Boulder County, Colorado & 517 & 11.8 & 1.5 & $(8.9-14.6)$ \\
\hline Denver County, Colorado & 999 & 9.7 & 0.9 & $(7.9-11.5)$ \\
\hline Douglas County, Colorado & 547 & 12.0 & 1.5 & $(9.0-15.0)$ \\
\hline El Paso County, Colorado & 1,022 & 9.2 & 0.9 & $(7.4-11.0)$ \\
\hline Jefferson County, Colorado & 1,118 & 13.6 & 1.1 & $(11.4-15.7)$ \\
\hline Larimer County, Colorado & 596 & 10.7 & 1.2 & $(8.3-13.1)$ \\
\hline Weld County, Colorado & 535 & 11.0 & 1.4 & $(8.2-13.9)$ \\
\hline Fairfield County, Connecticut & 2,187 & 10.7 & 0.8 & $(9.2-12.3)$ \\
\hline Hartford County, Connecticut & 1,987 & 11.7 & 0.8 & $(10.1-13.3)$ \\
\hline Litchfield County, Connecticut & 663 & 12.6 & 1.4 & $(9.8-15.3)$ \\
\hline New Haven County, Connecticut & 2,010 & 11.5 & 0.8 & $(9.9-13.2)$ \\
\hline New London County, Connecticut & 1,000 & 11.8 & 1.2 & $(9.5-14.1)$ \\
\hline Kent County, Delaware & 1,441 & 11.8 & 1.0 & $(9.8-13.7)$ \\
\hline New Castle County, Delaware & 2,333 & 11.4 & 0.7 & $(10.0-12.8)$ \\
\hline Sussex County, Delaware & 1,400 & 15.6 & 1.1 & $(13.5-17.7)$ \\
\hline $\begin{array}{l}\text { District of Columbia, } \\
\text { District of Columbia }\end{array}$ & 3,821 & 6.4 & 0.4 & $(5.6-7.2)$ \\
\hline Broward County, Florida & 528 & 11.2 & 1.6 & $(8.1-14.3)$ \\
\hline Miami-Dade County, Florida & 814 & 7.1 & 1.1 & $(4.9-9.3)$ \\
\hline Hawaii County, Hawaii & 1,349 & 12.4 & 1.1 & $(10.1-14.6)$ \\
\hline Honolulu County, Hawaii & 4,343 & 7.8 & 0.5 & $(6.8-8.8)$ \\
\hline Kauai County, Hawaii & 670 & 10.0 & 2.1 & $(5.8-14.2)$ \\
\hline Maui County, Hawaii & 1,219 & 11.4 & 1.4 & $(8.6-14.2)$ \\
\hline Ada County, Idaho & 809 & 12.1 & 1.3 & $(9.5-14.7)$ \\
\hline Canyon County, Idaho & 504 & 13.3 & 2.0 & $(9.3-17.2)$ \\
\hline Cook County, Illinois & 1,504 & 8.1 & 0.8 & $(6.5-9.7)$ \\
\hline Lake County, Indiana & 889 & 8.2 & 1.3 & $(5.7-10.6)$ \\
\hline Marion County, Indiana & 1,280 & 10.2 & 0.9 & $(8.4-12.0)$ \\
\hline Polk County, lowa & 809 & 11.0 & 1.2 & $(8.7-13.3)$ \\
\hline Johnson County, Kansas & 2,176 & 11.7 & 0.7 & $(10.3-13.2)$ \\
\hline Sedgwick County, Kansas & 1,796 & 9.8 & 0.7 & $(8.4-11.2)$ \\
\hline Shawnee County, Kansas & 770 & 13.3 & 1.3 & $(10.7-15.8)$ \\
\hline Wyandotte County, Kansas & 877 & 9.1 & 1.2 & $(6.7-11.5)$ \\
\hline Jefferson County, Kentucky & 1,695 & 12.4 & 1.3 & $(9.8-15.0)$ \\
\hline East Baton Rouge Parish, Louisiana & 572 & 9.6 & 1.4 & $(6.9-12.3)$ \\
\hline Androscoggin County, Maine & 700 & 8.0 & 1.1 & $(5.9-10.1)$ \\
\hline Aroostook County, Maine & 539 & 11.9 & 1.5 & $(8.8-14.9)$ \\
\hline Cumberland County, Maine & 1,760 & 11.2 & 0.8 & $(9.7-12.8)$ \\
\hline Kennebec County, Maine & 833 & 10.5 & 1.2 & $(8.3-12.8)$ \\
\hline Penobscot County, Maine & 929 & 11.1 & 1.1 & $(8.9-13.3)$ \\
\hline York County, Maine & 1,193 & 13.0 & 1.0 & $(11.0-15.0)$ \\
\hline
\end{tabular}

See table footnotes on page 105. 
TABLE 54. (Continued) Estimated prevalence of adults aged $\geq 18$ years who were ever told they had any type of cancer, by county Behavioral Risk Factor Surveillance System, United States, 2012

\begin{tabular}{|c|c|c|c|c|}
\hline County & $\begin{array}{c}\text { Sample } \\
\text { size }\end{array}$ & $\%$ & SE & $95 \% \mathrm{Cl}$ \\
\hline Anne Arundel County, Maryland & 896 & 9.9 & 1.0 & $(7.9-12.0)$ \\
\hline Baltimore County, Maryland & 1,521 & 9.4 & 0.8 & $(7.8-11.1)$ \\
\hline Charles County, Maryland & 513 & 9.1 & 1.5 & $(6.2-12.0)$ \\
\hline Frederick County, Maryland & 755 & 13.1 & 1.9 & $(9.4-16.8)$ \\
\hline Montgomery County, Maryland & 1,547 & 9.2 & 0.9 & $(7.6-10.9)$ \\
\hline Prince George's County, Maryland & 1,140 & 4.6 & 0.7 & $(3.2-6.1)$ \\
\hline Washington County, Maryland & 538 & 12.6 & 1.9 & $(8.9-16.4)$ \\
\hline Baltimore city, Maryland & 744 & 6.1 & 1.0 & $(4.2-8.0)$ \\
\hline Barnstable County, Massachusetts & 559 & 18.5 & 1.9 & $(14.7-22.3)$ \\
\hline Bristol County, Massachusetts & 2,656 & 10.5 & 1.0 & $(8.6-12.4)$ \\
\hline Essex County, Massachusetts & 2,567 & 11.0 & 0.9 & $(9.3-12.6)$ \\
\hline Hampden County, Massachusetts & 1,962 & 11.0 & 1.1 & $(8.9-13.1)$ \\
\hline Middlesex County, Massachusetts & 4,363 & 10.3 & 0.6 & \\
\hline Norfolk County, Massachusetts & 1,761 & 11.4 & 1.0 & $(9.4$ \\
\hline Plymouth County, Massachusetts & 1,838 & 11.7 & 1.1 & \\
\hline Suffolk County, Massachusetts & 2,315 & 8.2 & 0.7 & \\
\hline Worcester County, Massachusetts & 2,595 & 10.2 & 0.8 & $(8.7-$ \\
\hline Kent County, Michigan & 526 & 11.7 & 1.6 & $(8.7-14.8)$ \\
\hline Macomb County, Michigan & 614 & 14.8 & 1.5 & $(11.9-17.7)$ \\
\hline Oakland County, Michigan & 1,169 & 11.7 & 1.0 & $(9.7-13.7)$ \\
\hline Wayne County, Michigan & 2,222 & 10.0 & 0.8 & $(8.4-11.6)$ \\
\hline Anoka County, Minnesota & 544 & 10.1 & 1.6 & $(6.9-13.4)$ \\
\hline Dakota County, Minnesota & 673 & 8.4 & 1.2 & $(6.1-10.8)$ \\
\hline Hennepin County, Minnesota & 3,300 & 9.6 & 0.6 & $(8.4-10.8)$ \\
\hline Ramsey Coun & 1,949 & 10.0 & 0.9 & $(8.1-11.8)$ \\
\hline Jackson County, Missouri & 884 & 10.5 & 1.5 & $(7.5$ \\
\hline County, Missouri & 954 & 12.7 & 1.4 & 5.4) \\
\hline Cascade County, Montana & 709 & 13.8 & 1.4 & $(11.0$ \\
\hline Flathead County, Montana & 560 & 11.7 & 1.5 & 4.6) \\
\hline Hill County, Montana & 584 & 8.3 & 1.4 & $.9)$ \\
\hline Lake County, Montana & 900 & 14.1 & 1.6 & $(11.1$ \\
\hline Missoula County, Montana & 782 & 12.5 & 1.3 & $(9.9-15.1)$ \\
\hline Yellowstone County, Montana & 752 & 13.6 & 1.3 & $(11.1-16.2)$ \\
\hline Dakota County, Nebraska & 732 & 9.0 & 1.5 & $(6.0-12.0)$ \\
\hline Douglas County, Nebraska & 3,575 & 9.9 & 0.5 & $(8.8-11.0)$ \\
\hline Hall County, Nebraska & 535 & 11.2 & 1.4 & $(8.4-13.9)$ \\
\hline Lancaster County, Nebraska & 1,446 & 9.9 & 0.9 & $(8.2-11.6)$ \\
\hline Lincoln County, Nebraska & 589 & 11.6 & 1.5 & $(8.6-14.6)$ \\
\hline Sarpy County, Nebraska & 1,176 & 8.8 & 1.0 & $(6.9-10.6)$ \\
\hline Scotts Bluff County, Nebraska & 578 & 14.8 & 1.9 & $(11.0-18.6)$ \\
\hline evada & 016 & 8.6 & 0.6 & \\
\hline Washoe County, Nevada & 1,490 & 10.6 & 0.9 & $(8.8-12.4)$ \\
\hline Belknap County, New Ham & 567 & 12.9 & 1.8 & $(9.4-16.4)$ \\
\hline Carroll County, New Hampshire & 532 & 13.2 & 1.6 & $(10.1-16.3)$ \\
\hline Cheshire County, New Hampshire & 548 & 10.9 & 1.4 & $(8.1-13.6)$ \\
\hline Coos County, New Hampshire & 539 & 14.0 & 2.2 & $(9.7-18.3)$ \\
\hline Grafton County, New Hampshire & 567 & 13.6 & 1.8 & $(10.1-17.1)$ \\
\hline Hillsborough County, New Hampshire & 1,906 & 10.7 & 0.7 & $(9.3-12.2)$ \\
\hline Merrimack County, New Hampshire & 704 & 13.2 & 1.4 & $(10.5-16.0)$ \\
\hline Rockingham County, New Hampshire & 1,049 & 11.6 & 1.1 & $(9.5-13.7)$ \\
\hline Strafford County, New Hampshire & 624 & 10.4 & 1.4 & $(7.7-13.0)$ \\
\hline Atlantic County, New Jersey & 1,024 & 10.9 & 1.0 & $(8.9-13.0)$ \\
\hline Bergen County, New Jersey & 1,011 & 9.2 & 1.0 & $(7.3-11.1)$ \\
\hline Burlington County, New Jersey & 685 & 9.7 & 1.3 & $(7.1-12.2)$ \\
\hline Camden County, New Jersey & 745 & 9.6 & 1.1 & $(7.4-11.8)$ \\
\hline Cape May County, New Jersey & 554 & 19.2 & 2.4 & $(14.5-23.9)$ \\
\hline Cumberland County, New Jersey & 558 & 7.9 & 1.2 & $(5.5-10.2)$ \\
\hline Essex County, New Jersey & 1,342 & 6.2 & 0.9 & $(4.5-7.9)$ \\
\hline Gloucester County, New Jersey & 553 & 10.2 & 1.4 & $(7.4-13.0)$ \\
\hline Hudson County, New Jersey & 1,305 & 4.5 & 0.6 & $(3.3-5.8)$ \\
\hline
\end{tabular}

See table footnotes on page 105.
TABLE 54. (Continued) Estimated prevalence of adults aged $\geq 18$ years who were ever told they had any type of cancer, by county Behavioral Risk Factor Surveillance System, United States, 2012

\begin{tabular}{|c|c|c|c|c|}
\hline County & $\begin{array}{l}\text { Sample } \\
\text { size }\end{array}$ & $\%$ & SE & $95 \% \mathrm{Cl}$ \\
\hline Hunterdon County, New Jersey & 572 & 12.5 & 1.6 & $(9.5-15.6)$ \\
\hline Mercer County, New Jersey & 579 & 9.1 & 1.2 & $(6.8-11.4)$ \\
\hline Middlesex County, New Jersey & 884 & 8.5 & 1.1 & $(6.3-10.8)$ \\
\hline Monmouth County, New Jersey & 714 & 11.7 & 1.4 & $(9.0-14.4)$ \\
\hline Morris County, New Jersey & 849 & 12.7 & 1.2 & $(10.3-15.2)$ \\
\hline Ocean County, New Jersey & 664 & 11.4 & 1.3 & $(8.8-14.0)$ \\
\hline Passaic County, New Jersey & 690 & 6.6 & 1.0 & $(4.6-8.6)$ \\
\hline Salem County, New Jersey & 588 & 11.4 & 1.4 & $(8.7-14.0)$ \\
\hline Somerset County, New Jersey & 640 & 7.3 & 1.0 & $(5.3-9.3)$ \\
\hline Sussex County, New Jersey & 549 & 13.5 & 2.2 & $(9.1-17.8)$ \\
\hline Union County, New Jersey & 713 & 7.2 & 0.9 & $(5.3-9.0)$ \\
\hline Warren County, New Jersey & 532 & 11.7 & 1.7 & $(8.5-15.0)$ \\
\hline Bernalillo County, New Mexico & 2,064 & 9.7 & 0.7 & $(8.4-11.0)$ \\
\hline Dona Ana County, New Mexico & 708 & 10.3 & 1.1 & $(8.2-12.5)$ \\
\hline Sandoval County, New Mexico & 657 & 11.1 & 1.3 & $(8.6-13.7)$ \\
\hline San Juan County, New Mexico & 650 & 8.9 & 1.2 & $(6.6-11.3)$ \\
\hline Santa Fe County, New Mexico & 697 & 13.0 & 1.4 & $(10.3-15.6)$ \\
\hline Kings County, New York & 498 & 7.1 & 1.5 & $(4.2-10.0)$ \\
\hline Guilford County, North Carolina & 511 & 12.0 & 1.5 & $(9.0-15.0)$ \\
\hline Mecklenburg County, North Carolina & 729 & 8.7 & 1.1 & $(6.4-10.9)$ \\
\hline Robeson County, North Carolina & 546 & 13.4 & 2.9 & $(7.7-19.0)$ \\
\hline Wake County, North Carolina & 702 & 10.0 & 1.2 & $(7.6-12.4)$ \\
\hline Burleigh County, North Dakota & 569 & 10.1 & 1.4 & $(7.4-12.8)$ \\
\hline Cass County, North Dakota & 844 & 7.4 & 1.0 & $(5.5-9.3)$ \\
\hline Cuyahoga County, Ohio & 867 & 9.3 & 1.1 & $(7.2-11.5)$ \\
\hline Franklin County, Ohio & 839 & 9.7 & 1.1 & $(7.6-11.9)$ \\
\hline Hamilton County, Ohio & 772 & 12.3 & 1.4 & $(9.6-15.0)$ \\
\hline Lorain County, Ohio & 615 & 11.5 & 1.6 & $(8.4-14.7)$ \\
\hline Lucas County, Ohio & 623 & 10.0 & 1.2 & $(7.6-12.4)$ \\
\hline Mahoning County, Ohio & 592 & 11.3 & 1.6 & $(8.3-14.4)$ \\
\hline Montgomery County, Ohio & 664 & 12.2 & 1.4 & $(9.5-14.9)$ \\
\hline Stark County, Ohio & 624 & 12.4 & 1.5 & $(9.6-15.3)$ \\
\hline Summit County, Ohio & 631 & 12.2 & 1.4 & $(9.5-14.8)$ \\
\hline Oklahoma County, Oklahoma & 1,190 & 9.1 & 0.8 & $(7.4-10.7)$ \\
\hline Tulsa County, Oklahoma & 1,203 & 10.0 & 0.9 & $(8.2-11.7)$ \\
\hline Clackamas County, Oregon & 511 & 11.3 & 1.4 & $(8.5-14.1)$ \\
\hline Lane County, Oregon & 526 & 11.8 & 1.4 & $(8.9-14.6)$ \\
\hline Multnomah County, Oregon & 894 & 10.9 & 1.2 & $(8.6-13.2)$ \\
\hline Washington County, Oregon & 619 & 8.7 & 1.0 & $(6.7-10.8)$ \\
\hline Allegheny County, Pennsylvania & 1,890 & 11.6 & 0.8 & $(9.9-13.2)$ \\
\hline Bradford County, Pennsylvania & 1,864 & 13.4 & 1.5 & $(10.4-16.3)$ \\
\hline Montgomery County, Pennsylvania & 546 & 13.2 & 1.6 & $(10.0-16.3)$ \\
\hline Philadelphia County, Pennsylvania & 2,066 & 8.1 & 0.7 & $(6.8-9.4)$ \\
\hline Pike County, Pennsylvania & 1,892 & 11.6 & 0.8 & $(9.9-13.2)$ \\
\hline Kent County, Rhode Island & 813 & 13.0 & 1.3 & $(10.5-15.6)$ \\
\hline Providence County, Rhode Island & 3,347 & 9.5 & 0.5 & $(8.5-10.6)$ \\
\hline Washington County, Rhode Island & 657 & 13.4 & 1.5 & $(10.5-16.3)$ \\
\hline
\end{tabular}

See table footnotes on page 105. 
TABLE 54. (Continued) Estimated prevalence of adults aged $\geq 18$ years who were ever told they had any type of cancer, by county Behavioral Risk Factor Surveillance System, United States, 2012

\begin{tabular}{|c|c|c|c|c|}
\hline County & $\begin{array}{l}\text { Sample } \\
\text { size }\end{array}$ & $\%$ & SE & $95 \% \mathrm{Cl}$ \\
\hline Aiken County, South Carolina & 555 & 12.4 & 1.4 & $(9.6-15.2)$ \\
\hline Beaufort County, South Carolina & 803 & 17.2 & 1.7 & $(14.0-20.5)$ \\
\hline Charleston County, South Carolina & 1,016 & 10.9 & 1.2 & $(8.6-13.2)$ \\
\hline Greenville County, South Carolina & 908 & 12.4 & 1.3 & $(9.8-15.0)$ \\
\hline Horry County, South Carolina & 778 & 12.2 & 1.2 & $(9.7-14.6)$ \\
\hline Richland County, South Carolina & 964 & 8.5 & 1.1 & $(6.3-10.7)$ \\
\hline Spartanburg County, South Carolina & 656 & 10.2 & 1.3 & $(7.6-12.8)$ \\
\hline Lincoln County, South Dakota & 500 & 10.2 & 1.9 & $(6.5-13.9)$ \\
\hline Minnehaha County, South Dakota & 878 & 10.3 & 1.1 & $(8.0-12.5)$ \\
\hline Pennington County, South Dakota & 606 & 12.1 & 1.4 & $(9.5-14.8)$ \\
\hline Davidson County, Tennessee & 556 & 10.8 & 1.5 & $(7.8-13.7)$ \\
\hline Shelby County, Tennessee & 536 & 7.1 & 1.1 & $(4.9-9.3)$ \\
\hline Bexar County, Texas & 586 & 8.7 & 1.1 & $(6.4-10.9)$ \\
\hline Dallas County, Texas & 513 & 8.9 & 1.2 & $(6.5-11.3)$ \\
\hline El Paso County, Texas & 625 & 7.5 & 1.5 & $(4.6-10.4)$ \\
\hline Harris County, Texas & 775 & 7.2 & 0.9 & $(5.4-9.0)$ \\
\hline Hidalgo County, Texas & 623 & 5.6 & 0.9 & $(3.8-7.4)$ \\
\hline Tarrant County, Texas & 572 & 9.8 & 1.2 & $(7.4-12.1)$ \\
\hline Travis County, Texas & 1,049 & 7.8 & 0.8 & $(6.1-9.4)$ \\
\hline Davis County, Utah & 1,149 & 10.5 & 0.9 & $(8.7-12.3)$ \\
\hline Salt Lake County, Utah & 4,051 & 9.5 & 0.5 & $(8.6-10.5)$ \\
\hline Tooele County, Utah & 566 & 9.7 & 1.6 & $(6.4-12.9)$ \\
\hline Utah County, Utah & 1,684 & 8.1 & 0.7 & $(6.8-9.4)$ \\
\hline Wasatch County, Utah & 512 & 10.5 & 1.9 & $(6.7-14.3)$ \\
\hline Weber County, Utah & 1,049 & 11.5 & 1.0 & $(9.5-13.5)$ \\
\hline Chittenden County, Vermont & 918 & 10.7 & 1.0 & $(8.7-12.7)$ \\
\hline Rutland County, Vermont & 597 & 11.0 & 1.5 & $(8.1-13.9)$ \\
\hline Washington County, Vermont & 517 & 10.7 & 1.5 & $(7.8-13.6)$ \\
\hline Windsor County, Vermont & 546 & 12.7 & 1.4 & $(9.9-15.5)$ \\
\hline Fairfax County, Virginia & 741 & 9.9 & 1.1 & $(7.8-12.1)$ \\
\hline Clark County, Washington & 791 & 12.5 & 1.1 & $(10.2-14.7)$ \\
\hline King County, Washington & 3,924 & 10.3 & 0.5 & $(9.3-11.3)$ \\
\hline Kitsap County, Washington & 566 & 13.3 & 1.4 & $(10.5-16.0)$ \\
\hline Pierce County, Washington & 1,183 & 11.9 & 1.1 & $(9.7-14.0)$ \\
\hline Snohomish County, Washington & 1,175 & 11.3 & 1.0 & $(9.4-13.2)$ \\
\hline Spokane County, Washington & 952 & 11.4 & 1.1 & $(9.3-13.4)$ \\
\hline Thurston County, Washington & 517 & 10.9 & 1.3 & $(8.3-13.5)$ \\
\hline Whatcom County, Washington & 847 & 13.6 & 1.5 & $(10.6-16.6)$ \\
\hline Yakima County, Washington & 533 & 9.1 & 1.4 & $(6.2-11.9)$ \\
\hline Kanawha County, West Virginia & 641 & 11.5 & 1.2 & $(9.1-13.9)$ \\
\hline Milwaukee County, Wisconsin & 964 & 7.7 & 1.1 & $(5.6-9.8)$ \\
\hline Laramie County, Wyoming & 959 & 12.6 & 1.4 & $(9.9-15.3)$ \\
\hline Natrona County, Wyoming & 830 & 11.3 & 1.3 & $(8.8-13.8)$ \\
\hline San Juan Municipio, Puerto Rico & 666 & 4.8 & 0.9 & $(3.1-6.5)$ \\
\hline Median & & 10.8 & & \\
\hline Range & & $4.5-19.2$ & & \\
\hline
\end{tabular}

Abbreviations: $\mathrm{Cl}=$ confidence interval; $\mathrm{SE}=$ standard error.
TABLE 55. Estimated prevalence of adults aged $\geq 18$ years told by a health care professional that they currently have asthma, ${ }^{*}$ by state/ territory - Behavioral Risk Factor Surveillance System, United States, 2012

\begin{tabular}{|c|c|c|c|c|}
\hline State/Territory & $\begin{array}{l}\text { Sample } \\
\text { size }\end{array}$ & $\%$ & SE & $95 \% \mathrm{Cl}$ \\
\hline Alabama & 8,935 & 8.6 & 0.4 & $(7.7-9.4)$ \\
\hline Alaska & 4,302 & 9.0 & 0.6 & $(7.8-10.2)$ \\
\hline Arizona & 7,241 & 8.6 & 0.5 & $(7.6-9.6)$ \\
\hline Arkansas & 5,140 & 8.8 & 0.5 & $(7.7-9.8)$ \\
\hline California & 14,538 & 8.8 & 0.3 & $(8.2-9.5)$ \\
\hline Colorado & 12,184 & 8.9 & 0.4 & $(8.2-9.6)$ \\
\hline Connecticut & 8,705 & 9.9 & 0.5 & $(9.0-10.9)$ \\
\hline Delaware & 5,150 & 9.9 & 0.6 & $(8.8-11.1)$ \\
\hline District of Columbia & 3,787 & 10.3 & 0.8 & $(8.6-11.9)$ \\
\hline Florida & 7,579 & 8.2 & 0.5 & $(7.2-9.2)$ \\
\hline Georgia & 6,068 & 8.2 & 0.5 & $(7.2-9.2)$ \\
\hline Hawaii & 7,523 & 8.9 & 0.5 & $(7.9-9.9)$ \\
\hline Idaho & 5,838 & 8.5 & 0.6 & $(7.3-9.7)$ \\
\hline Illinois & 5,563 & 8.5 & 0.6 & $(7.4-9.6)$ \\
\hline Indiana & 8,577 & 9.1 & 0.4 & $(8.3-9.8)$ \\
\hline lowa & 7,132 & 8.1 & 0.4 & $(7.2-8.9)$ \\
\hline Kansas & 11,740 & 8.4 & 0.4 & $(7.7-9.1)$ \\
\hline Kentucky & 11,127 & 11.1 & 0.5 & $(10.1-12.0)$ \\
\hline Louisiana & 9,027 & 7.4 & 0.4 & $(6.6-8.3)$ \\
\hline Maine & 9,843 & 11.1 & 0.4 & $(10.3-11.9)$ \\
\hline Maryland & 12,747 & 9.0 & 0.4 & $(8.2-9.9)$ \\
\hline Massachusetts & 21,550 & 10.8 & 0.3 & $(10.2-11.5)$ \\
\hline Michigan & 10,421 & 10.5 & 0.4 & $(9.6-11.3)$ \\
\hline Minnesota & 12,173 & 8.0 & 0.3 & $(7.3-8.6)$ \\
\hline Mississippi & 7,735 & 8.1 & 0.4 & $(7.3-9.0)$ \\
\hline Missouri & 6,710 & 10.4 & 0.6 & $(9.3-11.5)$ \\
\hline Montana & 8,624 & 9.5 & 0.4 & $(8.6-10.3)$ \\
\hline Nebraska & 19,053 & 7.4 & 0.3 & $(6.9-7.9)$ \\
\hline Nevada & 4,804 & 7.4 & 0.5 & $(6.3-8.4)$ \\
\hline New Hampshire & 7,477 & 10.2 & 0.5 & $(9.2-11.3)$ \\
\hline New Jersey & 15,661 & 8.7 & 0.4 & $(8.0-9.4)$ \\
\hline New Mexico & 8,723 & 9.2 & 0.4 & $(8.5-10.0)$ \\
\hline New York & 6,006 & 9.3 & 0.5 & $(8.3-10.3)$ \\
\hline North Carolina & 11,823 & 7.7 & 0.3 & $(7.0-8.3)$ \\
\hline North Dakota & 4,850 & 7.6 & 0.5 & $(6.6-8.6)$ \\
\hline Ohio & 12,963 & 10.5 & 0.4 & $(9.7-11.2)$ \\
\hline Oklahoma & 7,974 & 10.2 & 0.4 & $(9.3-11.0)$ \\
\hline Oregon & 5,235 & 10.6 & 0.6 & $(9.5-11.8)$ \\
\hline Pennsylvania & 19,818 & 10.1 & 0.4 & $(9.4-10.8)$ \\
\hline Rhode Island & 5,449 & 10.8 & 0.6 & $(9.6-11.9)$ \\
\hline South Carolina & 12,695 & 8.7 & 0.4 & $(7.9-9.4)$ \\
\hline South Dakota & 7,825 & 7.5 & 0.5 & $(6.6-8.4)$ \\
\hline Tennessee & 7,023 & 7.7 & 0.5 & $(6.8-8.6)$ \\
\hline Texas & 9,073 & 6.8 & 0.4 & $(6.1-7.6)$ \\
\hline Utah & 12,366 & 8.9 & 0.3 & $(8.2-9.5)$ \\
\hline Vermont & 6,022 & 10.9 & 0.6 & $(9.8-12.0)$ \\
\hline Virginia & 7,348 & 8.8 & 0.4 & $(7.9-9.6)$ \\
\hline Washington & 15,174 & 9.7 & 0.3 & $(9.1-10.4)$ \\
\hline West Virginia & 5,380 & 10.2 & 0.5 & $(9.3-11.2)$ \\
\hline Wisconsin & 5,284 & 8.6 & 0.6 & $(7.4-9.7)$ \\
\hline Wyoming & 6,229 & 9.0 & 0.6 & $(7.8-10.2)$ \\
\hline Guam & 2,020 & 5.8 & 0.8 & $(4.3-7.3)$ \\
\hline Puerto Rico & 6,313 & 8.9 & 0.4 & $(8.0-9.7)$ \\
\hline Median & & 8.9 & & \\
\hline Range & & $5.8-11.1$ & & \\
\hline
\end{tabular}

Abbreviations: $\mathrm{Cl}=$ confidence interval; $\mathrm{SE}=$ standard error.

* Defined as ever been told by a health care professional that the respondent had asthma and reporting that they still have asthma. 
TABLE 56. Estimated prevalence of adults aged $\geq 18$ years told by a health care professional that they currently have asthma,* by metropolitan and micropolitan statistical area - Behavioral Risk Factor Surveillance System, United States, 2012

\begin{tabular}{|c|c|c|c|c|}
\hline MMSA(s) & $\begin{array}{l}\text { Sample } \\
\text { size }\end{array}$ & $\%$ & SE & $95 \% \mathrm{Cl}$ \\
\hline Aguadilla-Isabela, Puerto Rico & 550 & 10.4 & 1.5 & $(7.4-13.3)$ \\
\hline Akron, Ohio & 741 & 9.5 & 1.5 & $(6.6-12.5)$ \\
\hline Albuquerque, New Mexico & 3,247 & 9.8 & 0.7 & $(8.5-11.1)$ \\
\hline $\begin{array}{l}\text { Allentown-Bethlehem-Easton, } \\
\text { Pennsylvania-New Jersey }\end{array}$ & 1,343 & 10.4 & 1.2 & $(8.0-12.8)$ \\
\hline Anaheim-Santa Ana-Irvine, California ${ }^{\dagger}$ & 1,032 & 5.7 & 1.0 & $(3.7-7.7)$ \\
\hline Anchorage, Alaska & 1,495 & 10.0 & 0.9 & $(8.2-11.9)$ \\
\hline Asheville, North Carolina & 592 & 9.4 & 1.6 & $(6.3-12.4)$ \\
\hline $\begin{array}{l}\text { Atlanta-Sandy Springs-Roswell, } \\
\text { Georgia }\end{array}$ & 2,526 & 8.0 & 0.7 & $(6.5-9.4)$ \\
\hline Atlantic City-Hammonton, New Jersey & 1,020 & 12.7 & 1.8 & $(9.2-16.3)$ \\
\hline $\begin{array}{l}\text { Augusta-Richmond County, Georgia- } \\
\text { South Carolina }\end{array}$ & 1,031 & 7.1 & 1.6 & $(4.1-10.2)$ \\
\hline Augusta-Waterville, Maine & 828 & 12.6 & 1.4 & $(9.9-15.3)$ \\
\hline Austin-Round Rock, Texas & 1,380 & 8.4 & 1.3 & $(5.8-11.0)$ \\
\hline $\begin{array}{l}\text { Baltimore-Columbia-Towson, } \\
\text { Maryland }\end{array}$ & 4,698 & 10.0 & 0.7 & $(8.6-11.4)$ \\
\hline Bangor, Maine & 924 & 12.6 & 1.5 & $(9.7-15.5)$ \\
\hline Barnstable Town, Massachusetts & 556 & 10.1 & 1.9 & $(6.5-13.8)$ \\
\hline Barre, Vermont & 516 & 8.8 & 1.8 & $(5.3-12.2)$ \\
\hline Baton Rouge, Louisiana & 1,384 & 7.7 & 1.1 & $(5.5-10.0)$ \\
\hline Bellingham, Washington & 840 & 7.5 & 1.3 & $(5.0-10.0)$ \\
\hline Berlin, New Hampshire-Vermont & 707 & 14.0 & 1.9 & $(10.3-17.8)$ \\
\hline Billings, Montana & 843 & 8.3 & 1.1 & $(6.1-10.5)$ \\
\hline Birmingham-Hoover, Alabama & 1,803 & 7.8 & 0.8 & $(6.3-9.4)$ \\
\hline Bismarck, North Dakota & 822 & 6.1 & 1.0 & $(4.1-8.1)$ \\
\hline Boise City, Idaho & 1,464 & 7.3 & 1.0 & $(5.4-9.2)$ \\
\hline Boston, Massachusetts $^{\dagger}$ & 5,866 & 9.2 & 0.6 & $(8.0-10.4)$ \\
\hline Boulder, Colorado & 514 & 6.3 & 1.2 & $(3.9-8.7)$ \\
\hline Bremerton-Silverdale, Washington & 559 & 12.9 & 1.9 & $(9.2-16.6)$ \\
\hline $\begin{array}{l}\text { Bridgeport-Stamford-Norwalk, } \\
\text { Connecticut }\end{array}$ & 2,178 & 7.8 & 0.9 & $(6.0-9.6)$ \\
\hline Burlington-South Burlington, Vermont & 1,516 & 10.0 & 1.0 & $(8.1-11.9)$ \\
\hline $\begin{array}{l}\text { Cambridge-Newton-Framingham, } \\
\text { Massachusetts }{ }^{\dagger}\end{array}$ & 6,885 & 9.9 & 0.6 & $(8.8-11.0)$ \\
\hline Camden, New Jersey ${ }^{\dagger}$ & 1,967 & 10.5 & 1.0 & $(8.5-12.4)$ \\
\hline Canton-Massillon, Ohio & 678 & 12.2 & 1.8 & $(8.8-15.7)$ \\
\hline Casper, Wyoming & 827 & 8.8 & 1.6 & $(5.8-11.9)$ \\
\hline Cedar Rapids, lowa & 582 & 9.8 & 1.7 & $(6.6-13.1)$ \\
\hline Charleston, West Virginia & 765 & 9.8 & 1.3 & $(7.3-12.2)$ \\
\hline $\begin{array}{l}\text { Charleston-North Charleston, } \\
\text { South Carolina }\end{array}$ & 1,701 & 8.0 & 0.9 & $(6.3-9.7)$ \\
\hline $\begin{array}{l}\text { Charlotte-Concord-Gastonia, } \\
\text { North Carolina-South Carolina }\end{array}$ & 2,514 & 7.3 & 0.7 & $(5.9-8.8)$ \\
\hline Chattanooga, Tennessee-Georgia & 629 & 4.7 & 1.1 & $(2.6-6.9)$ \\
\hline Cheyenne, Wyoming & 946 & 9.7 & 1.5 & $(6.7-12.6)$ \\
\hline $\begin{array}{l}\text { Chicago-Naperville-Elgin, } \\
\text { Illinois-Indiana-Wisconsin }\end{array}$ & 3,711 & 8.0 & 0.7 & $(6.7-9.3)$ \\
\hline Cincinnati, Ohio-Kentucky-Indiana & 2,355 & 10.3 & 0.8 & $(8.7-12.0)$ \\
\hline $\begin{array}{l}\text { Claremont-Lebanon, New } \\
\text { Hampshire-Vermont }\end{array}$ & 1,939 & 10.5 & 1.1 & $(8.4-12.6)$ \\
\hline Cleveland-Elyria, Ohio & 1,777 & 11.4 & 1.1 & $(9.2-13.5)$ \\
\hline Colorado Springs, Colorado & 1,149 & 7.8 & 1.1 & $(5.7-9.9)$ \\
\hline Columbia, South Carolina & 1,785 & 7.8 & 0.9 & $(6.0-9.6)$ \\
\hline Columbus, Ohio & 1,595 & 9.3 & 0.9 & $(7.4-11.1)$ \\
\hline Concord, New Hampshire & 699 & 11.9 & 2.0 & $(7.9-15.9)$ \\
\hline Dallas-Plano-Irving, Texas ${ }^{\dagger}$ & 907 & 8.0 & 1.3 & $(5.5-10.5)$ \\
\hline Dayton, Ohio & 856 & 11.0 & 1.5 & $(8.1-13.8)$ \\
\hline Denver-Aurora-Lakewood, Colorado & 4,827 & 9.6 & 0.5 & $(8.5-10.6)$ \\
\hline Des Moines-West Des Moines, lowa & 1,156 & 8.4 & 1.0 & $(6.4-10.3)$ \\
\hline Detroit-Dearborn-Livonia, Michigan ${ }^{\dagger}$ & 2,207 & 11.8 & 1.1 & $(9.6-13.9)$ \\
\hline
\end{tabular}

See table footnotes on page 107.
TABLE 56. (Continued) Estimated prevalence of adults aged $\geq 18$ years told by a health care professional that they currently have asthma,* by metropolitan and micropolitan statistical area - Behavioral Risk Factor Surveillance System, United States, 2012

\begin{tabular}{|c|c|c|c|c|}
\hline MMSA(s) & $\begin{array}{l}\text { Sample } \\
\text { size }\end{array}$ & $\%$ & SE & $95 \% \mathrm{Cl}$ \\
\hline Dover, Delaware & 1,431 & 11.1 & 1.3 & $(8.6-13.7)$ \\
\hline Duluth, Minnesota-Wisconsin & 508 & 8.9 & 1.6 & $(5.9-12.0)$ \\
\hline Durham-Chapel Hill, North Carolina & 792 & 7.4 & 1.1 & $(5.3-9.5)$ \\
\hline El Paso, Texas & 624 & 7.5 & 1.7 & $(4.3-10.8)$ \\
\hline Eugene, Oregon & 522 & 13.8 & 2.2 & $(9.4-18.1)$ \\
\hline Fairbanks, Alaska & 586 & 9.8 & 1.6 & $(6.6-13.0)$ \\
\hline Fargo, North Dakota-Minnesota & 933 & 6.6 & 1.1 & $(4.5-8.7)$ \\
\hline Farmington, New Mexico & 646 & 6.9 & 1.1 & $(4.8-9.1)$ \\
\hline Fayetteville, North Carolina & 503 & 5.6 & 1.1 & $(3.4-7.8)$ \\
\hline $\begin{array}{l}\text { Fayetteville-Springdale-Rogers, } \\
\text { Arkansas-Missouri }\end{array}$ & 803 & 7.7 & 1.2 & $(5.4-10.1)$ \\
\hline Fort Collins, Colorado & 595 & 6.1 & 1.1 & $(3.8-8.3)$ \\
\hline Fort Wayne, Indiana & 535 & 10.3 & 1.7 & $(6.9-13.6)$ \\
\hline Fort Worth-Arlington, Texas ${ }^{\dagger}$ & 720 & 7.2 & 1.2 & $(4.9-9.6)$ \\
\hline Grand Island, Nebraska & 850 & 6.8 & 1.1 & $(4.6-9.1)$ \\
\hline Grand Rapids-Wyoming, Michigan & 886 & 9.6 & 1.2 & $(7.2-12.0)$ \\
\hline Great Falls, Montana & 707 & 12.6 & 1.7 & $(9.4-15.9)$ \\
\hline Greeley, Colorado & 530 & 7.1 & 1.3 & $(4.6-9.7)$ \\
\hline $\begin{array}{l}\text { Greensboro-High Point, } \\
\text { North Carolina }\end{array}$ & 798 & 7.2 & 1.1 & $(5.0-9.4)$ \\
\hline $\begin{array}{l}\text { Greenville-Anderson-Mauldin, } \\
\text { South Carolina }\end{array}$ & 1,680 & 8.8 & 0.9 & $(7.0-10.6)$ \\
\hline Gulfport-Biloxi-Pascagoula, Mississippi & 738 & 6.9 & 1.1 & $(4.7-9.1)$ \\
\hline $\begin{array}{l}\text { Hagerstown-Martinsburg, Maryland- } \\
\text { West Virginia }\end{array}$ & 757 & 11.1 & 1.9 & $(7.4-14.8)$ \\
\hline Harrisburg-Carlisle, Pennsylvania & 659 & 10.4 & 1.6 & $(7.3-13.5)$ \\
\hline $\begin{array}{l}\text { Hartford-West Hartford-East Hartford, } \\
\text { Connecticut }\end{array}$ & 2,650 & 10.4 & 0.8 & $(8.8-12.1)$ \\
\hline Heber, Utah & 511 & 4.2 & 0.8 & $(2.5-5.8)$ \\
\hline Hilo, Hawaii & 1,338 & 9.7 & 1.2 & $(7.3-12.0)$ \\
\hline $\begin{array}{l}\text { Hilton Head Island-Bluffton-Beaufort, } \\
\text { South Carolina }\end{array}$ & 929 & 6.8 & 1.8 & $(3.2-10.3)$ \\
\hline Houston-The Woodlands-Sugar Land, & 1,141 & 5.1 & 0.7 & $(3.6-6.5)$ \\
\hline
\end{tabular}

Texas

Huntington-Ashland, West

$1,112 \quad 13.3 \quad 1.4 \quad(10.6-16.0)$

Virginia-Kentucky-Ohio

Huntsville, Alabama

Idaho Falls, Idaho

Indianapolis-Carmel-Anderson,

Indiana

Jackson, Mississippi

Jacksonville, Florida

Kahului-Wailuku-Lahaina, Hawaii

Kalispell, Montana

Kansas City, Missouri-Kansas

Kapaa, Hawaii

Keene, New Hampshire

Kennewick-Richland, Washington

Kingsport-Bristol-Bristol,

Tennessee-Virginia

Knoxville, Tennessee

Laconia, New Hampshire

Lafayette, Louisiana

Las Cruces, New Mexico

Las Vegas-Henderson-Paradise,

Nevada

Lewiston-Auburn, Maine

Lexington-Fayette, Kentucky

613

8.6

1.5

(5.6-11.6)

539

2,179

8.7

2.3

$(4.1-13.3)$

(7.9-11.1)

918

517

8.6

1.1

(6.4-10.8)

1,211

555

4,717

666

543

532

566

832

832
566
553

553

704

1,999

10.3

8.9

9.0

9.7

6.8

9.7

10.8

10.8
7.6

7.6

8.4

10.4

6.8

1.3

$\begin{array}{ll}7.1 & 0.7\end{array}$

(6.4-14.1)

(6.5-11.4)

(6.0-11.9)

(8.2-11.2)

(4.8-8.8)

(5.9-13.6)

(7.6-14.0)

(5.0-10.1)

Lincoln, Nebraska

691
526

$12.3 \quad 1.6$

1.6
1.6

$\begin{array}{ll}9.9 & 1.6 \\ 6.7 & 0.7\end{array}$

$8.8 \quad 1.1$

(6.0-10.8)

(5.6-15.3)

(4.3-9.3)

(5.1-9.1)

(5.8-8.4)

(9.3-15.4)

(6.8-13.0)

(5.4-8.1)

Little Rock-North Little Rock-Conway, 1,161 Arkansas 
TABLE 56. (Continued) Estimated prevalence of adults aged $\geq 18$ years told by a health care professional that they currently have asthma,* by metropolitan and micropolitan statistical area - Behavioral Risk Factor Surveillance System, United States, 2012

\begin{tabular}{|c|c|c|c|c|}
\hline MMSA(s) & $\begin{array}{l}\text { Sample } \\
\text { size }\end{array}$ & $\%$ & SE & $95 \% \mathrm{Cl}$ \\
\hline Logan, Utah-Idaho & 503 & 10.4 & 1.8 & $(6.8-14.0)$ \\
\hline $\begin{array}{l}\text { Los Angeles-Long Beach-Glendale, } \\
\text { California }^{\dagger}\end{array}$ & 3,496 & 7.2 & 0.6 & $(6.0-8.3)$ \\
\hline $\begin{array}{l}\text { Louisville/Jefferson County, } \\
\text { Kentucky-Indiana }\end{array}$ & 2,162 & 12.0 & 1.1 & $(9.8-14.2)$ \\
\hline Lumberton, North Carolina & 545 & 8.9 & 2.4 & $(4.3-13.6)$ \\
\hline Manchester-Nashua, New Hampshire & 1,894 & 10.6 & 1.0 & $(8.6-12.5)$ \\
\hline McAllen-Edinburg-Mission, Texas & 618 & 3.1 & 0.8 & $(1.5-4.8)$ \\
\hline $\begin{array}{l}\text { Memphis, } \\
\text { Tennessee-Mississippi-Arkansas }\end{array}$ & 1,305 & 7.4 & 1.1 & $(5.1-9.6)$ \\
\hline $\begin{array}{l}\text { Miami-Fort Lauderdale-West Palm } \\
\text { Beach, Florida }\end{array}$ & 1,664 & 5.3 & 0.9 & $(3.6-7.0)$ \\
\hline $\begin{array}{l}\text { Milwaukee-Waukesha-West Allis, } \\
\text { Wisconsin }\end{array}$ & 1,253 & 8.2 & 1.1 & $(6.0-10.4)$ \\
\hline $\begin{array}{l}\text { Minneapolis-St. Paul-Bloomington, } \\
\text { Minnesota-Wisconsin }\end{array}$ & 7,860 & 8.7 & 0.5 & $(7.8-9.6)$ \\
\hline Missoula, Montana & 778 & 8.7 & 1.3 & $(6.1-11.2)$ \\
\hline Mobile, Alabama & 810 & 10.1 & 1.7 & $(6.8-13.4)$ \\
\hline Montgomery, Alabama & 530 & 7.2 & 1.5 & $(4.2-10.2)$ \\
\hline $\begin{array}{l}\text { Montgomery County-Bucks County- } \\
\text { Chester County, Pennsylvania }{ }^{\dagger}\end{array}$ & 1,307 & 7.6 & 1.0 & $(5.7-9.5)$ \\
\hline $\begin{array}{l}\text { Myrtle Beach-Conway-North Myrtle } \\
\text { Beach, South Carolina-North Carolina }\end{array}$ & 944 & 6.5 & 1.0 & $(4.5-8.6)$ \\
\hline $\begin{array}{l}\text { Nashville-Davidson-Murfreesboro- } \\
\text { Franklin, Tennessee }\end{array}$ & 1,338 & 6.7 & 1.0 & $(4.8-8.6)$ \\
\hline $\begin{array}{l}\text { Nassau County-Suffolk County, } \\
\text { New York }{ }^{\dagger}\end{array}$ & 895 & 8.5 & 1.1 & $(6.3-10.7)$ \\
\hline Newark, New Jersey-Pennsylvania ${ }^{\dagger}$ & 6,516 & 8.7 & 0.6 & $(7.6-9.9)$ \\
\hline New Haven-Milford, Connecticut & 1,988 & 11.8 & 1.1 & $(9.7-13.9)$ \\
\hline New Orleans-Metairie, Louisiana & 1,272 & 6.1 & 1.0 & $(4.1-8.0)$ \\
\hline $\begin{array}{l}\text { New York-Jersey City-White Plains, } \\
\text { New York-New Jersey }{ }^{\dagger}\end{array}$ & 7,390 & 8.0 & 0.6 & $(6.9-9.1)$ \\
\hline Norfolk, Nebraska & 570 & 6.2 & 1.2 & $(3.8-8.6)$ \\
\hline North Platte, Nebraska & 609 & 9.2 & 1.8 & $(5.6-12.8)$ \\
\hline Norwich-New London, Connecticut & 991 & 10.6 & 1.8 & $(7.1-14.1)$ \\
\hline $\begin{array}{l}\text { Oakland-Hayward-Berkeley, } \\
\text { California }^{\dagger}\end{array}$ & 975 & 10.9 & 1.8 & $(7.5-14.4)$ \\
\hline Ocean City, New Jersey & 551 & 9.4 & 2.4 & $(4.7-14.0)$ \\
\hline Ogden-Clearfield, Utah & 2,422 & 9.3 & 0.8 & $(7.8-10.8)$ \\
\hline Oklahoma City, Oklahoma & 2,428 & 10.7 & 0.8 & $(9.1-12.2)$ \\
\hline Olympia-Tumwater, Washington & 511 & 11.6 & 2.0 & (7.7-15.6) \\
\hline Omaha-Council Bluffs, Nebraska-lowa & 5,553 & 7.6 & 0.5 & $(6.7-8.5)$ \\
\hline Orlando-Kissimmee-Sanford, Florida & 564 & 9.5 & 1.9 & $(5.9-13.2)$ \\
\hline Philadelphia, Pennsylvania ${ }^{\dagger}$ & 2,442 & 10.8 & 0.9 & $(9.1-12.5)$ \\
\hline Phoenix-Mesa-Scottsdale, Arizona & 2,577 & 8.6 & 0.7 & $(7.2-10.0)$ \\
\hline Pittsburgh, Pennsylvania & 3,348 & 9.3 & 0.6 & $(8.1-10.5)$ \\
\hline Ponce, Puerto Rico & 549 & 7.2 & 1.4 & $(4.6-9.9)$ \\
\hline Portland-South Portland, Maine & 3,306 & 10.4 & 0.7 & $(9.0-11.7)$ \\
\hline $\begin{array}{l}\text { Portland-Vancouver-Hillsboro, } \\
\text { Oregon-Washington }\end{array}$ & 3,086 & 9.6 & 0.7 & $(8.2-11.0)$ \\
\hline $\begin{array}{l}\text { Providence-Warwick, Rhode } \\
\text { Island-Massachusetts }\end{array}$ & 8,088 & 11.0 & 0.5 & $(10.0-12.0)$ \\
\hline Provo-Orem, Utah & 1,742 & 8.0 & 0.8 & $(6.4-9.5)$ \\
\hline Raleigh, North Carolina & 945 & 7.1 & 1.1 & $(4.9-9.3)$ \\
\hline Rapid City, South Dakota & 1,050 & 9.4 & 1.3 & $(6.8-11.9)$ \\
\hline Reno, Nevada & 1,492 & 7.8 & 1.0 & $(5.8-9.8)$ \\
\hline Richmond, Virginia & 1,008 & 8.9 & 1.2 & $(6.5-11.3)$ \\
\hline $\begin{array}{l}\text { Riverside-San Bernardino-Ontario, } \\
\text { California }\end{array}$ & 1,527 & 7.9 & 0.9 & $(6.2-9.7)$ \\
\hline $\begin{array}{l}\text { Rockingham County-Strafford County, } \\
\text { New Hampshire }{ }^{\dagger}\end{array}$ & 1,661 & 9.3 & 0.9 & $-11.2)$ \\
\hline
\end{tabular}

TABLE 56. (Continued) Estimated prevalence of adults aged $\geq 18$ years told by a health care professional that they currently have asthma,* by metropolitan and micropolitan statistical area - Behavioral Risk Factor Surveillance System, United States, 2012

\begin{tabular}{|c|c|c|c|c|}
\hline MMSA(s) & $\begin{array}{l}\text { Sample } \\
\text { size }\end{array}$ & $\%$ & SE & $95 \% \mathrm{Cl}$ \\
\hline Rutland, Vermont & 592 & 14.2 & 2.0 & $(10.3-18.2)$ \\
\hline $\begin{array}{l}\text { Sacramento-Roseville-Arden-Arcade, } \\
\text { California }\end{array}$ & 993 & 10.0 & 1.3 & $(7.5-12.5)$ \\
\hline St. Louis, Missouri-Illinois & 2,060 & 10.2 & 0.9 & $(8.3-12.0)$ \\
\hline Salisbury, Maryland-Delaware & 2,118 & 8.7 & 0.9 & $(7.0-10.4)$ \\
\hline Salt Lake City, Utah & 4,599 & 9.6 & 0.6 & $(8.4-10.7)$ \\
\hline San Antonio-New Braunfels, Texas & 771 & 6.2 & 1.0 & $(4.1-8.2)$ \\
\hline San Diego-Carlsbad, California & 1,123 & 6.9 & 1.0 & $(5.0-8.9)$ \\
\hline $\begin{array}{l}\text { San Francisco-Redwood City-South } \\
\text { San Francisco, California }{ }^{\dagger}\end{array}$ & 594 & 10.7 & 1.7 & $(7.4-13.9)$ \\
\hline $\begin{array}{l}\text { San Jose-Sunnyvale-Santa Clara, } \\
\text { California }\end{array}$ & 684 & 8.4 & 1.6 & $(5.2-11.6)$ \\
\hline San Juan-Carolina-Caguas, Puerto Rico & 3,952 & 8.9 & 0.6 & $(7.8-10.0)$ \\
\hline Santa Fe, New Mexico & 697 & 9.6 & 1.4 & $(6.9-12.4)$ \\
\hline Sayre, Pennsylvania & 1,852 & 8.4 & 1.5 & $(5.4-11.4)$ \\
\hline Scottsbluff, Nebraska & 627 & 8.8 & 2.2 & $(4.5-13.2)$ \\
\hline $\begin{array}{l}\text { Scranton-Wilkes-Barre-Hazleton, } \\
\text { Pennsylvania }\end{array}$ & 738 & 10.1 & 1.6 & $(7.0-13.2)$ \\
\hline Seattle-Bellevue-Everett, Washington ${ }^{\dagger}$ & 5,053 & 8.3 & 0.5 & $(7.3-9.3)$ \\
\hline Shreveport-Bossier City, Louisiana & 578 & 9.0 & 1.7 & $(5.8-12.3)$ \\
\hline $\begin{array}{l}\text { Silver Spring-Frederick-Rockville, } \\
\text { Maryland }\end{array}$ & 2,288 & 8.1 & 0.9 & $(6.4-9.9)$ \\
\hline $\begin{array}{l}\text { Sioux City, lowa-Nebraska- } \\
\text { South Dakota }\end{array}$ & 1,203 & 6.4 & 1.3 & $(3.9-8.9)$ \\
\hline Sioux Falls, South Dakota & 1,456 & 6.2 & 0.9 & $(4.5-7.9)$ \\
\hline Spartanburg, South Carolina & 706 & 12.6 & 2.1 & $(8.5-16.6)$ \\
\hline Spokane-Spokane Valley, Washington & 1,087 & 10.8 & 1.3 & $(8.4-13.3)$ \\
\hline Springfield, Massachusetts & 2,323 & 14.1 & 1.1 & $(11.8-16.3)$ \\
\hline Tacoma-Lakewood, Washington ${ }^{\dagger}$ & 1,175 & 10.2 & 1.1 & $(8.1-12.3)$ \\
\hline $\begin{array}{l}\text { Tampa-St. Petersburg-Clearwater, } \\
\text { Florida }\end{array}$ & 824 & 8.8 & 1.7 & $(5.5-12.2)$ \\
\hline Toledo, Ohio & 968 & 12.5 & 1.8 & $(9.0-16.1)$ \\
\hline Topeka, Kansas & 1,084 & 9.0 & 1.1 & $(6.8-11.2)$ \\
\hline Torrington, Connecticut & 656 & 8.8 & 1.5 & $(6.0-11.7)$ \\
\hline Trenton, New Jersey & 578 & 7.4 & 1.3 & $(4.8-9.9)$ \\
\hline Tucson, Arizona & 988 & 8.7 & 1.1 & $(6.6-10.8)$ \\
\hline Tulsa, Oklahoma & 1,739 & 9.2 & 1.0 & $(7.3-11.1)$ \\
\hline Tuscaloosa, Alabama & 576 & 9.3 & 1.9 & $(5.6-13.0)$ \\
\hline Urban Honolulu, Hawaii & 4,308 & 8.9 & 0.6 & $(7.6-10.1)$ \\
\hline Vineland-Bridgeton, New Jersey & 555 & 15.0 & 3.1 & $(8.9-21.2)$ \\
\hline $\begin{array}{l}\text { Virginia Beach-Norfolk-Newport News, } \\
\text { Virginia-North Carolina }\end{array}$ & 1,493 & 7.7 & 0.9 & $(5.9-9.4)$ \\
\hline $\begin{array}{l}\text { Warren-Troy-Farmington Hills, } \\
\text { Michigan }{ }^{\dagger}\end{array}$ & 2,175 & 8.8 & 0.8 & $(7.3-10.3)$ \\
\hline $\begin{array}{l}\text { Washington-Arlington-Alexandria, } \\
\text { District of Columbia-Virginia- } \\
\text { Maryland-West Virginia }\end{array}$ & 8,150 & 8.0 & 0.5 & $(7.1-8.9)$ \\
\hline Wichita, Kansas & 2,352 & 9.4 & 0.8 & $(7.7-11.0)$ \\
\hline $\begin{array}{l}\text { Wilmington, Delaware-Maryland- } \\
\text { New Jersey }{ }^{\dagger}\end{array}$ & 3,223 & 10.3 & 0.9 & $(8.6-12.1)$ \\
\hline Winston-Salem, North Carolina & 788 & 9.0 & 1.3 & $(6.6-11.5)$ \\
\hline Worcester, Massachusetts-Connecticut & 2,823 & 11.8 & 0.9 & $(10.0-13.6)$ \\
\hline Yakima, Washington & 530 & 12.5 & 2.1 & $(8.3-16.7)$ \\
\hline $\begin{array}{l}\text { Youngstown-Warren-Boardman, } \\
\text { Ohio-Pennsylvania }\end{array}$ & 925 & 8.6 & 1.6 & $(5.6-11.7)$ \\
\hline Median & \multicolumn{2}{|c|}{8.9} & & \\
\hline Range & \multicolumn{2}{|c|}{$3.1-15.0$} & & \\
\hline
\end{tabular}

Abbreviations: $\mathrm{Cl}=$ confidence interval; $\mathrm{MMSA}=$ metropolitan and micropolitan statistical area; $\mathrm{SE}=$ standard error.

* Defined as ever been told by a health care professional that the respondent had asthma and reporting that they still have asthma.

${ }^{\dagger}$ Metropolitan division. 
TABLE 57. Estimated prevalence of adults aged $\geq 18$ years told by a health care professional that they currently have asthma,* by county - Behavioral Risk Factor Surveillance System, United States, 2012

\begin{tabular}{|c|c|c|c|c|}
\hline County & $\begin{array}{l}\text { Sample } \\
\text { size }\end{array}$ & $\%$ & SE & $95 \% \mathrm{Cl}$ \\
\hline Jefferson County, Alabama & 951 & 7.0 & 0.9 & $(5.1-8.8)$ \\
\hline Madison County, Alabama & 510 & 9.2 & 1.9 & $(5.5-12.9)$ \\
\hline Mobile County, Alabama & 810 & 10.1 & 1.7 & $(6.8-13.4)$ \\
\hline Anchorage Municipality, Alaska & 895 & 10.5 & 1.2 & $(8.2-12.8)$ \\
\hline Fairbanks North Star Borough, Alaska & 586 & 9.8 & 1.6 & $(6.6-13.0)$ \\
\hline Matanuska-Susitna Borough, Alaska & 600 & 8.7 & 1.4 & $(5.9-11.6)$ \\
\hline Maricopa County, Arizona & 2,102 & 8.6 & 0.8 & $(7.1-10.0)$ \\
\hline Pima County, Arizona & 988 & 8.7 & 1.1 & $(6.6-10.8)$ \\
\hline Pulaski County, Arkansas & 738 & 9.3 & 1.5 & $(6.3-12.3)$ \\
\hline Alameda County, California & 619 & 11.1 & 2.4 & $(6.4-15.9)$ \\
\hline Los Angeles County, California & 3,496 & 7.2 & 0.6 & $(6.0-8.3)$ \\
\hline Orange County, California & 1,032 & 5.7 & 1.0 & $(3.7-7.7)$ \\
\hline Riverside County, California & 823 & 6.3 & 1.0 & $(4.4-8.3)$ \\
\hline Sacramento County, California & 607 & 11.4 & 1.7 & $(8.1-14.7)$ \\
\hline San Bernardino County, California & 704 & 8.9 & 1.3 & $(6.3-11.4)$ \\
\hline San Diego County, California & 1,123 & 6.9 & 1.0 & $(5.0-8.9)$ \\
\hline Santa Clara County, California & 665 & 8.1 & 1.6 & $(5.0-11.3)$ \\
\hline Adams County, Colorado & 806 & 9.3 & 1.4 & $(6.6-12.1)$ \\
\hline Arapahoe County, Colorado & 853 & 12.6 & 1.4 & $(10.0-15.3)$ \\
\hline Boulder County, Colorado & 514 & 6.3 & 1.2 & $(3.9-8.7)$ \\
\hline Denver County, Colorado & 989 & 8.3 & 1.0 & $(6.4-10.3)$ \\
\hline Douglas County, Colorado & 546 & 7.7 & 1.6 & $(4.5-10.8)$ \\
\hline El Paso County, Colorado & 1,011 & 7.8 & 1.1 & $(5.7-10.0)$ \\
\hline Jefferson County, Colorado & 1,113 & 9.7 & 1.1 & $(7.6-11.8)$ \\
\hline Larimer County, Colorado & 595 & 6.1 & 1.1 & $(3.8-8.3)$ \\
\hline Weld County, Colorado & 530 & 7.2 & 1.3 & $(4.6-9.7)$ \\
\hline Fairfield County, Connecticut & 2,178 & 7.8 & 0.9 & $(6.0-9.6)$ \\
\hline Hartford County, Connecticut & 1,977 & 10.6 & 1.0 & $(8.6-12.5)$ \\
\hline Litchfield County, Connecticut & 656 & 8.8 & 1.4 & $(6.0-11.7)$ \\
\hline New Haven County, Connecticut & 1,988 & 11.8 & 1.1 & $(9.7-13.9)$ \\
\hline New London County, Connecticut & 991 & 10.6 & 1.8 & $(7.1-14.1)$ \\
\hline Kent County, Delaware & 1,431 & 11.1 & 1.3 & $(8.6-13.7)$ \\
\hline New Castle County, Delaware & 2,324 & 9.6 & 0.8 & $(8.1-11.2)$ \\
\hline Sussex County, Delaware & 1,395 & 9.5 & 1.1 & $(7.4-11.6)$ \\
\hline $\begin{array}{l}\text { District of Columbia, } \\
\text { District of Columbia }\end{array}$ & 3,787 & 10.3 & 0.9 & $(8.7-12.0)$ \\
\hline Broward County, Florida & 526 & 4.7 & 1.0 & $(2.7-6.7)$ \\
\hline Miami-Dade County, Florida & 811 & 6.1 & 1.7 & $(2.7-9.4)$ \\
\hline Hawaii County, Hawaii & 1,338 & 9.7 & 1.2 & $(7.4-12.0)$ \\
\hline Honolulu County, Hawaii & 4,308 & 8.9 & 0.6 & $(7.6-10.1)$ \\
\hline Kauai County, Hawaii & 666 & 6.8 & 1.0 & $(4.8-8.8)$ \\
\hline Maui County, Hawaii & 1,211 & 8.9 & 1.3 & $(6.5-11.4)$ \\
\hline Ada County, Idaho & 797 & 7.7 & 1.3 & $(5.1-10.3)$ \\
\hline Canyon County, Idaho & 497 & 7.5 & 1.9 & $(3.7-11.3)$ \\
\hline Cook County, Illinois & 1,499 & 7.4 & 0.9 & $(5.6-9.1)$ \\
\hline Lake County, Indiana & 880 & 8.3 & 1.2 & $(5.9-10.7)$ \\
\hline Marion County, Indiana & 1,268 & 9.3 & 1.1 & $(7.1-11.4)$ \\
\hline Polk County, lowa & 806 & 8.5 & 1.2 & $(6.1-10.9)$ \\
\hline Johnson County, Kansas & 2,167 & 7.5 & 0.8 & $(6.0-9.1)$ \\
\hline Sedgwick County, Kansas & 1,792 & 10.1 & 1.0 & $(8.2-12.1)$ \\
\hline Shawnee County, Kansas & 767 & 10.7 & 1.5 & $(7.8-13.7)$ \\
\hline Wyandotte County, Kansas & 872 & 11.1 & 1.9 & $(7.3-14.9)$ \\
\hline Jefferson County, Kentucky & 1,682 & 11.3 & 1.3 & $(8.8-13.8)$ \\
\hline East Baton Rouge Parish, Louisiana & 571 & 8.0 & 1.6 & $(4.8-11.2)$ \\
\hline Androscoggin County, Maine & 691 & 12.3 & 1.6 & $(9.3-15.4)$ \\
\hline Aroostook County, Maine & 536 & 13.5 & 1.8 & $(10.0-17.0)$ \\
\hline Cumberland County, Maine & 1,745 & 9.7 & 0.9 & $(8.0-11.3)$ \\
\hline Kennebec County, Maine & 828 & 12.6 & 1.4 & $(9.9-15.3)$ \\
\hline Penobscot County, Maine & 924 & 12.6 & 1.5 & $(9.7-15.5)$ \\
\hline York County, Maine & 1,186 & 10.7 & 1.1 & $(8.5-12.9)$ \\
\hline Anne Arundel County, Maryland & 890 & 7.6 & 1.3 & $(5.0-10.2)$ \\
\hline
\end{tabular}

See table footnotes on page 109.
TABLE 57. (Continued) Estimated prevalence of adults aged $\geq 18$ years told by a health care professional that they currently have asthma,* by county - Behavioral Risk Factor Surveillance System, United States, 2012

\begin{tabular}{|c|c|c|c|c|}
\hline County & $\begin{array}{l}\text { Sample } \\
\text { size }\end{array}$ & $\%$ & SE & $95 \% \mathrm{Cl}$ \\
\hline Baltimore County, Maryland & 1,508 & 9.0 & 1.1 & $(7.0-11.1)$ \\
\hline Charles County, Maryland & 511 & 9.9 & 2.0 & $(6.0-13.8)$ \\
\hline Frederick County, Maryland & 751 & 13.2 & 3.1 & $(7.2-19.2)$ \\
\hline Montgomery County, Maryland & 1,537 & 7.3 & 0.9 & $(5.5-9.2)$ \\
\hline Prince George's County, Maryland & 1,138 & 6.1 & 0.8 & $(4.5-7.7)$ \\
\hline Washington County, Maryland & 536 & 12.0 & 2.7 & $(6.7-17.4)$ \\
\hline Baltimore city, Maryland & 741 & 13.2 & 2.0 & $(9.3-17.2)$ \\
\hline Barnstable County, Massachusetts & 556 & 10.1 & 1.8 & $(6.5-13.8)$ \\
\hline Bristol County, Massachusetts & 2,639 & 12.2 & 1.1 & $(10.0-14.4)$ \\
\hline Essex County, Massachusetts & 2,549 & 10.1 & 1.0 & $(8.2-12.0)$ \\
\hline Hampden County, Massachusetts & 1,947 & 15.7 & 1.4 & $(13.0-18.4)$ \\
\hline Middlesex County, Massachusetts & 4,336 & 9.8 & 0.7 & $(8.4-11.1)$ \\
\hline Norfolk County, Massachusetts & 1,749 & 9.0 & 1.2 & $(6.7-11.2)$ \\
\hline Plymouth County, Massachusetts & 1,820 & 9.0 & 1.0 & $(7.0-11.0)$ \\
\hline Suffolk County, Massachusetts & 2,297 & 9.7 & 0.9 & $(7.9-11.6)$ \\
\hline Worcester County, Massachusetts & 2,581 & 12.1 & 1.0 & $(10.1-14.1)$ \\
\hline Kent County, Michigan & 522 & 9.7 & 1.6 & $(6.6-12.8)$ \\
\hline Macomb County, Michigan & 607 & 9.8 & 1.5 & $(6.8-12.8)$ \\
\hline Oakland County, Michigan & 1,166 & 7.5 & 0.9 & $(5.6-9.3)$ \\
\hline Wayne County, Michigan & 2,207 & 11.8 & 1.1 & $(9.6-13.9)$ \\
\hline Anoka County, Minnesota & 542 & 9.9 & 1.4 & $(7.1-12.8)$ \\
\hline Dakota County, Minnesota & 668 & 7.5 & 1.2 & $(5.2-9.8)$ \\
\hline Hennepin County, Minnesota & 3,282 & 9.3 & 0.8 & $(7.7-10.9)$ \\
\hline Ramsey County, Minnesota & 1,940 & 10.2 & 1.2 & $(7.8-12.6)$ \\
\hline Jackson County, Missouri & 879 & 12.0 & 1.8 & 5.5) \\
\hline St. Louis County, Missouri & 950 & 9.5 & 1.2 & $(7.1-11.9)$ \\
\hline Cascade County, Montana & 707 & 12.6 & 1.7 & $(9.4-15.9)$ \\
\hline Flathead County, Montana & 555 & 9.0 & 1.5 & $(6.0-11.9)$ \\
\hline Hill County, Montana & 582 & 9.5 & 1.9 & $(5.8-13.2)$ \\
\hline Lake County, Montana & 894 & 8.9 & 1.6 & $(5.8-12.0)$ \\
\hline Missoula County, Montana & 778 & 8.7 & 1.3 & $(6.1-11.2)$ \\
\hline Yellowstone County, Montana & 746 & 8.3 & 1.2 & $(6.0-10.6)$ \\
\hline Dakota County, Nebraska & 731 & 5.5 & 1.2 & $(3.1-7.9)$ \\
\hline Douglas County, Nebraska & 3,545 & 7.7 & 0.6 & $(6.6-8.8)$ \\
\hline Hall County, Nebraska & 532 & 5.2 & 1.2 & $(2.8-7.7)$ \\
\hline Lancaster County, Nebraska & 1,437 & 6.6 & 0.7 & $(5.2-8.0)$ \\
\hline Lincoln County, Nebraska & 585 & 9.2 & 1.9 & $(5.5-13.0)$ \\
\hline Sarpy County, Nebraska & 1,172 & 7.7 & 1.0 & $(5.8-9.6)$ \\
\hline Scotts Bluff County, Nebraska & 574 & 8.2 & 2.0 & $(4.3-12.1)$ \\
\hline Clark County, Nevada & 1,999 & 7.1 & 0.7 & $(5.8-8.4)$ \\
\hline Washoe County, Nevada & 1,473 & 7.8 & 1.0 & $(5.8-9.9)$ \\
\hline Belknap County, New Hampshire & 566 & 10.4 & 2.5 & $(5.6-15.3)$ \\
\hline Carroll County, New Hampshire & 531 & 7.5 & 1.4 & $(4.9-10.2)$ \\
\hline Cheshire County, New Hampshire & 543 & 9.7 & 2.0 & $(5.9-13.6)$ \\
\hline Coos County, New Hampshire & 535 & 15.5 & 2.3 & $(10.9-20.1)$ \\
\hline Grafton County, New Hampshire & 564 & 11.5 & 2.1 & $(7.4-15.7)$ \\
\hline Hillsborough County, New Hampshire & 1,894 & 10.6 & 1.0 & $(8.6-12.5)$ \\
\hline Merrimack County, New Hampshire & 699 & 11.9 & 2.0 & $(7.9-15.9)$ \\
\hline Rockingham County, New Hampshire & 1,043 & 9.4 & 1.2 & $(7.0-11.8)$ \\
\hline Strafford County, New Hampshire & 618 & 9.3 & 1.5 & $(6.4-12.2)$ \\
\hline Atlantic County, New Jersey & 1,020 & 12.7 & 1.8 & $(9.2-16.3)$ \\
\hline Bergen County, New Jersey & 1,004 & 6.5 & 1.2 & $(4.3-8.8)$ \\
\hline Burlington County, New Jersey & 681 & 10.5 & 2.0 & $(6.6-14.4)$ \\
\hline Camden County, New Jersey & 738 & 11.3 & 1.7 & $(8.0-14.7)$ \\
\hline Cape May County, New Jersey & 551 & 9.4 & 2.4 & $(4.7-14.0)$ \\
\hline Cumberland County, New Jersey & 555 & 15.0 & 3.1 & $(8.9-21.2)$ \\
\hline Essex County, New Jersey & 1,328 & 11.3 & 1.3 & $(8.8-13.9)$ \\
\hline Gloucester County, New Jersey & 548 & 10.3 & 1.7 & $(7.0-13.7)$ \\
\hline Hudson County, New Jersey & 1,296 & 7.9 & 0.9 & $(6.2-9.7)$ \\
\hline Hunterdon County, New Jersey & 572 & 8.4 & 1.7 & $(5.1-11.6)$ \\
\hline
\end{tabular}

See table footnotes on page 109. 
TABLE 57. (Continued) Estimated prevalence of adults aged $\geq 18$ years told by a health care professional that they currently have asthma,* by county - Behavioral Risk Factor Surveillance System, United States, 2012

\begin{tabular}{|c|c|c|c|c|}
\hline County & $\begin{array}{l}\text { Sample } \\
\text { size }\end{array}$ & $\%$ & SE & $95 \% \mathrm{Cl}$ \\
\hline Mercer County, New Jersey & 578 & 7.4 & 1.3 & $(4.8-9.9)$ \\
\hline Middlesex County, New Jersey & 881 & 7.8 & 1.4 & $(5.1-10.5)$ \\
\hline Monmouth County, New Jersey & 712 & 9.6 & 1.5 & $(6.6-12.5)$ \\
\hline Morris County, New Jersey & 846 & 6.9 & 1.2 & $(4.6-9.2)$ \\
\hline Ocean County, New Jersey & 656 & 6.2 & 1.1 & $(4.1-8.4)$ \\
\hline Passaic County, New Jersey & 690 & 7.2 & 1.2 & $(4.8-9.5)$ \\
\hline Salem County, New Jersey & 583 & 15.2 & 2.6 & $(10.1-20.3)$ \\
\hline Somerset County, New Jersey & 639 & 7.8 & 1.7 & $(4.5-11.0)$ \\
\hline Sussex County, New Jersey & 548 & 9.9 & 2.1 & $(5.8-14.1)$ \\
\hline Union County, New Jersey & 706 & 7.7 & 1.4 & $(5.0-10.5)$ \\
\hline Warren County, New Jersey & 529 & 11.6 & 2.9 & $(6.0-17.2)$ \\
\hline Bernalillo County, New Mexico & 2,050 & 9.9 & 0.8 & $(8.3-11.4)$ \\
\hline Dona Ana County, New Mexico & 704 & 7.1 & 1.0 & $(5.1-9.1)$ \\
\hline Sandoval County, New Mexico & 652 & 8.5 & 1.3 & $(5.9-11.2)$ \\
\hline San Juan County, New Mexico & 646 & 6.9 & 1.1 & $(4.8-9.0)$ \\
\hline Santa Fe County, New Mexico & 697 & 9.6 & 1.4 & $(6.9-12.4)$ \\
\hline Kings County, New York & 489 & 8.2 & 1.5 & $(5.3-11.1)$ \\
\hline Guilford County, North Carolina & 510 & 7.5 & 1.5 & $(4.6-10.3)$ \\
\hline Mecklenburg County, North Carolina & 726 & 6.4 & 1.2 & $(4.0-8.8)$ \\
\hline Robeson County, North Carolina & 545 & 8.9 & 2.4 & $(4.3-13.6)$ \\
\hline Wake County, North Carolina & 702 & 6.4 & 1.3 & $(3.9-8.9)$ \\
\hline Burleigh County, North Dakota & 568 & 6.9 & 1.3 & $(4.4-9.4)$ \\
\hline Cass County, North Dakota & 837 & 7.4 & 1.2 & $(5.0-9.9)$ \\
\hline Cuyahoga County, Ohio & 865 & 12.2 & 1.5 & $(9.3-15.1)$ \\
\hline Franklin County, Ohio & 834 & 10.4 & 1.3 & $(7.8-13.0)$ \\
\hline Hamilton County, Ohio & 771 & 11.0 & 1.5 & $(8.0-13.9)$ \\
\hline Lorain County, Ohio & 612 & 10.7 & 2.1 & $(6.7-14.7)$ \\
\hline Lucas County, Ohio & 620 & 14.1 & 2.4 & $(9.5-18.8)$ \\
\hline Mahoning County, Ohio & 590 & 8.4 & 1.6 & $(5.2-11.5)$ \\
\hline Montgomery County, Ohio & 663 & 10.9 & 1.7 & $(7.6-14.2)$ \\
\hline Stark County, Ohio & 620 & 12.6 & 1.8 & $(9.0-16.2)$ \\
\hline Summit County, Ohio & 628 & 11.1 & 1.9 & $(7.4-14.7)$ \\
\hline Oklahoma County, Oklahoma & 1,184 & 9.9 & 1.0 & $(8.0-11.7)$ \\
\hline Tulsa County, Oklahoma & 1,201 & 8.2 & 1.0 & $(6.3-10.1)$ \\
\hline Clackamas County, Oregon & 504 & 9.9 & 2.0 & $(5.9-13.9)$ \\
\hline Lane County, Oregon & 522 & 13.8 & 2.2 & $(9.4-18.1)$ \\
\hline Multnomah County, Oregon & 879 & 10.4 & 1.3 & $(7.8-13.0)$ \\
\hline Washington County, Oregon & 612 & 7.8 & 1.4 & $(5.0-10.6)$ \\
\hline Allegheny County, Pennsylvania & 1,876 & 9.3 & 0.9 & $(7.6-11.0)$ \\
\hline Bradford County, Pennsylvania & 1,852 & 8.4 & 1.5 & $(5.4-11.4)$ \\
\hline Montgomery County, Pennsylvania & 542 & 9.3 & 1.4 & $(6.5-12.1)$ \\
\hline Philadelphia County, Pennsylvania & 2,048 & 11.6 & 1.0 & $(9.6-13.5)$ \\
\hline Pike County, Pennsylvania & 1,877 & 8.8 & 1.5 & $(5.9-11.7)$ \\
\hline Kent County, Rhode Island & 809 & 13.1 & 1.5 & $(10.1-16.1)$ \\
\hline Providence County, Rhode Island & 3,331 & 10.8 & 0.7 & $(9.4-12.2)$ \\
\hline Washington County, Rhode Island & 652 & 8.9 & 1.5 & $(5.9-11.8)$ \\
\hline Aiken County, South Carolina & 553 & $\mathrm{~N} / \mathrm{A}^{\dagger}$ & N/A & $(\mathrm{N} / \mathrm{A}-\mathrm{N} / \mathrm{A})$ \\
\hline Beaufort County, South Carolina & 797 & 5.8 & 1.6 & $(2.7-8.9)$ \\
\hline Charleston County, South Carolina & 1,008 & 6.3 & 1.0 & $(4.3-8.3)$ \\
\hline
\end{tabular}

TABLE 57. (Continued) Estimated prevalence of adults aged $\geq 18$ years told by a health care professional that they currently have asthma,* by county - Behavioral Risk Factor Surveillance System, United States, 2012

\begin{tabular}{|c|c|c|c|c|}
\hline County & $\begin{array}{l}\text { Sample } \\
\text { size }\end{array}$ & $\%$ & SE & $95 \% \mathrm{Cl}$ \\
\hline Greenville County, South Carolina & 900 & 7.7 & 1.2 & $(5.4-10.0)$ \\
\hline Horry County, South Carolina & 774 & 7.7 & 1.4 & $(4.9-10.5)$ \\
\hline Richland County, South Carolina & 955 & 5.7 & 1.0 & $(3.7-7.7)$ \\
\hline Spartanburg County, South Carolina & 653 & 13.3 & 2.3 & $(8.8-17.7)$ \\
\hline Lincoln County, South Dakota & 498 & 3.7 & 0.9 & $(1.9-5.5)$ \\
\hline Minnehaha County, South Dakota & 871 & 7.4 & 1.1 & $(5.2-9.6)$ \\
\hline Pennington County, South Dakota & 603 & 9.7 & 1.5 & $(6.7-12.7)$ \\
\hline Davidson County, Tennessee & 555 & 5.3 & 1.1 & $(3.2-7.4)$ \\
\hline Shelby County, Tennessee & 535 & 5.9 & 1.2 & $(3.5-8.3)$ \\
\hline Bexar County, Texas & 582 & 5.1 & 1.1 & $(2.9-7.3)$ \\
\hline Dallas County, Texas & 512 & 7.3 & 1.7 & $(4.0-10.6)$ \\
\hline El Paso County, Texas & 623 & 7.5 & 1.7 & $(4.3-10.8)$ \\
\hline Harris County, Texas & 768 & 4.6 & 0.8 & $(3.0-6.3)$ \\
\hline Hidalgo County, Texas & 618 & 3.1 & 0.8 & $(1.5-4.8)$ \\
\hline Tarrant County, Texas & 567 & 7.8 & 1.4 & $(5.0-10.5)$ \\
\hline Travis County, Texas & 1,044 & 9.7 & 1.7 & $(6.3-13.1)$ \\
\hline Davis County, Utah & 1,144 & 8.9 & 1.0 & $(6.8-10.9)$ \\
\hline Salt Lake County, Utah & 4,036 & 9.5 & 0.6 & $(8.3-10.6)$ \\
\hline Tooele County, Utah & 563 & 11.1 & 2.6 & $(6.0-16.1)$ \\
\hline Utah County, Utah & 1,674 & 7.8 & 0.8 & $(6.2-9.3)$ \\
\hline Wasatch County, Utah & 511 & 4.2 & 0.8 & $(2.5-5.8)$ \\
\hline Weber County, Utah & 1,043 & 9.8 & 1.2 & $(7.4-12.3)$ \\
\hline Chittenden County, Vermont & 914 & 10.1 & 1.2 & $(7.7-12.4)$ \\
\hline Rutland County, Vermont & 592 & 14.3 & 2.0 & $(10.3-18.2)$ \\
\hline Washington County, Vermont & 516 & 8.8 & 1.8 & $(5.3-12.2)$ \\
\hline Windsor County, Vermont & 544 & 9.0 & 1.8 & $(5.6-12.5)$ \\
\hline Fairfax County, Virginia & 736 & 7.2 & 1.1 & $(5.0-9.4)$ \\
\hline Clark County, Washington & 784 & 10.3 & 1.3 & $(7.7-12.9)$ \\
\hline King County, Washington & 3,886 & 7.8 & 0.6 & $(6.7-8.9)$ \\
\hline Kitsap County, Washington & 559 & 12.9 & 1.9 & $(9.2-16.6)$ \\
\hline Pierce County, Washington & 1,175 & 10.2 & 1.1 & $(8.1-12.3)$ \\
\hline Snohomish County, Washington & 1,167 & 9.5 & 1.1 & $(7.4-11.6)$ \\
\hline Spokane County, Washington & 941 & 10.8 & 1.3 & $(8.1-13.4)$ \\
\hline Thurston County, Washington & 511 & 11.6 & 2.0 & $(7.7-15.6)$ \\
\hline Whatcom County, Washington & 840 & 7.5 & 1.3 & $(5.0-10.0)$ \\
\hline Yakima County, Washington & 530 & 12.5 & 2.1 & $(8.3-16.7)$ \\
\hline Kanawha County, West Virginia & 637 & 10.0 & 1.4 & $(7.3-12.6)$ \\
\hline Milwaukee County, Wisconsin & 960 & 9.1 & 1.4 & $(6.4-11.7)$ \\
\hline Laramie County, Wyoming & 946 & 9.7 & 1.5 & $(6.7-12.7)$ \\
\hline Natrona County, Wyoming & 827 & 8.8 & 1.6 & $(5.8-11.9)$ \\
\hline San Juan Municipio, Puerto Rico & 666 & 8.7 & 1.3 & $(6.2-11.2)$ \\
\hline Median & & 9.2 & & \\
\hline Range & & $3.1-15.7$ & & \\
\hline
\end{tabular}

Abbreviations: $\mathrm{Cl}=$ confidence interval; $\mathrm{SE}=$ standard error.

* Defined as ever been told by a health care professional that the respondent had asthma and reporting that they still have asthma.

+ Estimate not available (N/A) if the unweighted sample size for the denominator was $<50$ or if the relative standard error is $>0.3$. 
TABLE 58. Estimated prevalence of adults aged $\geq 18$ years told by a health care professional that they have some form of arthritis, rheumatoid arthritis, gout, lupus, or fibromyalgia, by state/territory — Behavioral Risk Factor Surveillance System, United States, 2012

\begin{tabular}{|c|c|c|c|c|}
\hline State/Territory & $\begin{array}{l}\text { Sample } \\
\text { size }\end{array}$ & $\%$ & SE & $95 \% \mathrm{Cl}$ \\
\hline Alabama & 8,981 & 33.9 & 0.7 & $(32.6-35.3)$ \\
\hline Alaska & 4,313 & 22.5 & 0.8 & $(20.9-24.1)$ \\
\hline Arizona & 7,249 & 25.0 & 0.7 & $(23.5-26.4)$ \\
\hline Arkansas & 5,140 & 30.3 & 0.8 & $(28.7-31.8)$ \\
\hline California & 14,550 & 22.0 & 0.4 & $(21.2-22.9)$ \\
\hline Colorado & 12,188 & 23.2 & 0.5 & $(22.3-24.1)$ \\
\hline Connecticut & 8,715 & 24.3 & 0.6 & $(23.1-25.4)$ \\
\hline Delaware & 5,166 & 28.0 & 0.8 & $(26.4-29.5)$ \\
\hline District of Columbia & 3,801 & 18.2 & 0.8 & $(16.5-19.8)$ \\
\hline Florida & 7,581 & 26.4 & 0.7 & $(25.0-27.8)$ \\
\hline Georgia & 6,073 & 24.0 & 0.7 & $(22.6-25.3)$ \\
\hline Hawaii & 7,541 & 20.3 & 0.7 & $(19.0-21.7)$ \\
\hline Idaho & 5,859 & 26.0 & 0.9 & $(24.2-27.8)$ \\
\hline Illinois & 5,566 & 25.4 & 0.7 & $(24.0-26.9)$ \\
\hline Indiana & 8,587 & 27.8 & 0.6 & $(26.7-29.0)$ \\
\hline lowa & 7,131 & 25.9 & 0.6 & $(24.8-27.1)$ \\
\hline Kansas & 11,749 & 24.0 & 0.5 & $(23.1-24.9)$ \\
\hline Kentucky & 11,154 & 35.0 & 0.7 & $(33.7-36.4)$ \\
\hline Louisiana & 9,041 & 28.0 & 0.7 & $(26.6-29.3)$ \\
\hline Maine & 9,859 & 29.6 & 0.5 & $(28.5-30.6)$ \\
\hline Maryland & 12,754 & 23.5 & 0.5 & $(22.5-24.6)$ \\
\hline Massachusetts & 21,588 & 23.1 & 0.4 & $(22.3-23.9)$ \\
\hline Michigan & 10,453 & 31.8 & 0.6 & $(30.7-33.0)$ \\
\hline Minnesota & 12,194 & 21.5 & 0.5 & $(20.6-22.4)$ \\
\hline Mississippi & 7,725 & 30.8 & 0.7 & $(29.4-32.1)$ \\
\hline Missouri & 6,728 & 29.6 & 0.8 & $(28.1-31.1)$ \\
\hline Montana & 8,637 & 27.8 & 0.6 & $(26.7-29.0)$ \\
\hline Nebraska & 19,089 & 24.6 & 0.4 & $(23.9-25.4)$ \\
\hline Nevada & 4,807 & 24.0 & 0.8 & $(22.4-25.7)$ \\
\hline New Hampshire & 7,490 & 27.3 & 0.6 & $(26.0-28.5)$ \\
\hline New Jersey & 15,668 & 21.8 & 0.4 & $(21.0-22.7)$ \\
\hline New Mexico & 8,757 & 23.9 & 0.5 & $(22.9-25.0)$ \\
\hline New York & 6,017 & 25.0 & 0.8 & $(23.5-26.5)$ \\
\hline North Carolina & 11,847 & 26.1 & 0.5 & $(25.2-27.1)$ \\
\hline North Dakota & 4,854 & 24.5 & 0.7 & $(23.1-26.0)$ \\
\hline Ohio & 12,975 & 30.0 & 0.5 & $(29.0-31.1)$ \\
\hline Oklahoma & 7,979 & 28.0 & 0.6 & $(26.8-29.1)$ \\
\hline Oregon & 5,266 & 28.0 & 0.7 & $(26.6-29.5)$ \\
\hline Pennsylvania & 19,857 & 29.1 & 0.5 & $(28.2-30.0)$ \\
\hline Rhode Island & 5,459 & 26.8 & 0.7 & $(25.3-28.2)$ \\
\hline South Carolina & 12,709 & 28.9 & 0.5 & $(27.9-30.0)$ \\
\hline South Dakota & 7,838 & 23.7 & 0.7 & $(22.2-25.1)$ \\
\hline Tennessee & 7,031 & 29.8 & 0.7 & $(28.4-31.1)$ \\
\hline Texas & 9,079 & 21.3 & 0.6 & $(20.2-22.4)$ \\
\hline Utah & 12,386 & 20.0 & 0.4 & $(19.2-20.8)$ \\
\hline Vermont & 6,028 & 27.2 & 0.7 & $(25.9-28.5)$ \\
\hline Virginia & 7,355 & 25.5 & 0.6 & $(24.3-26.6)$ \\
\hline Washington & 15,198 & 25.2 & 0.4 & $(24.4-26.1)$ \\
\hline West Virginia & 5,370 & 36.4 & 0.7 & $(35.0-37.9)$ \\
\hline Wisconsin & 5,263 & 25.7 & 0.8 & $(24.1-27.4)$ \\
\hline Wyoming & 6,245 & 24.8 & 0.8 & $(23.2-26.3)$ \\
\hline Guam & 2,013 & 15.6 & 1.0 & $(13.5-17.6)$ \\
\hline Puerto Rico & 6,278 & 24.6 & 0.6 & $(23.5-25.8)$ \\
\hline Median & & 25.5 & & \\
\hline Range & & $15.6-36.4$ & & \\
\hline
\end{tabular}

Abbreviations: $\mathrm{SE}=$ standard error, $\mathrm{Cl}=$ confidence interval.
TABLE 59. Estimated prevalence of adults aged $\geq 18$ years told by a health care professional that they have some form of arthritis, rheumatoid arthritis, gout, lupus, or fibromyalgia, by metropolitan and micropolitan statistical area - Behavioral Risk Factor Surveillance System, United States, 2012

\begin{tabular}{|c|c|c|c|c|}
\hline MMSA(s) & $\begin{array}{l}\text { Sample } \\
\text { size }\end{array}$ & $\%$ & SE & $95 \% \mathrm{Cl}$ \\
\hline Aguadilla-Isabela, Puerto Rico & 548 & 25.5 & 1.9 & $(21.7-29.3)$ \\
\hline Akron, Ohio & 743 & 27.1 & 2.1 & $(23.0-31.1)$ \\
\hline Albuquerque, New Mexico & 3,260 & 22.9 & 0.8 & $(21.3-24.6)$ \\
\hline $\begin{array}{l}\text { Allentown-Bethlehem-Easton, } \\
\text { Pennsylvania-New Jersey }\end{array}$ & 1,347 & 29.6 & 1.8 & $(26.1-33.0)$ \\
\hline Anaheim-Santa Ana-Irvine, California* & 1,035 & 18.1 & 1.5 & $(15.1-21.1)$ \\
\hline Anchorage, Alaska & 1,495 & 21.7 & 1.3 & $(19.2-24.2)$ \\
\hline Asheville, North Carolina & 594 & 30.2 & 2.2 & $(25.8-34.6)$ \\
\hline $\begin{array}{l}\text { Atlanta-Sandy Springs-Roswell, } \\
\text { Georgia }\end{array}$ & 2,528 & 20.7 & 1.0 & $(18.8-22.7)$ \\
\hline Atlantic City-Hammonton, New Jersey & 1,016 & 28.8 & 1.9 & $(25.1-32.5)$ \\
\hline $\begin{array}{l}\text { Augusta-Richmond County, Georgia- } \\
\text { South Carolina }\end{array}$ & 1,033 & 29.4 & 2.5 & $(24.5-34.3)$ \\
\hline Augusta-Waterville, Maine & 831 & 30.0 & 1.8 & $(26.4-33.5)$ \\
\hline Austin-Round Rock, Texas & 1,384 & 17.6 & 1.5 & $(14.7-20.4)$ \\
\hline $\begin{array}{l}\text { Baltimore-Columbia-Towson, } \\
\text { Maryland }\end{array}$ & 4,709 & 25.3 & 0.8 & $(23.6-26.9)$ \\
\hline Bangor, Maine & 924 & 27.3 & 1.7 & $(24.0-30.7)$ \\
\hline Barnstable Town, Massachusetts & 558 & 29.3 & 2.4 & $(24.7-34.0)$ \\
\hline Barre, Vermont & 517 & 25.5 & 2.1 & $(21.3-29.7)$ \\
\hline Baton Rouge, Louisiana & 1,388 & 26.2 & 1.6 & $(23.1-29.3)$ \\
\hline Bellingham, Washington & 841 & 22.8 & 1.9 & $(19.1-26.4)$ \\
\hline Berlin, New Hampshire-Vermont & 708 & 33.1 & 2.6 & $(28.1-38.1)$ \\
\hline Billings, Montana & 845 & 30.1 & 8 & $(26.6$ \\
\hline Birmingham-Hoover, Alabama & 1,820 & 31.1 & .4 & $(28$. \\
\hline Bismarck, North Dakota & 820 & 26.0 & 1.9 & $(22.3-29.7)$ \\
\hline Boise City, Idaho & 1,478 & 24.3 & 1.5 & $(21.3-27.3)$ \\
\hline Boston, Massachusetts* & 5,876 & 21.1 & 0.7 & $(19.6-22.5)$ \\
\hline Boulder, Colorado & 517 & 23.0 & 2.1 & $(18.9-27.1)$ \\
\hline Bremerton-Silverdale, Washington & 563 & 31.1 & 2.4 & $(26.4-35.8)$ \\
\hline $\begin{array}{l}\text { Bridgeport-Stamford-Norwalk, } \\
\text { Connecticut }\end{array}$ & 2,175 & 21.6 & 1.1 & $(19.4-23.8)$ \\
\hline Burlington-South Burlington, Vermont & 1,518 & 23.5 & 1.2 & $(21.1-25.9)$ \\
\hline $\begin{array}{l}\text { Cambridge-Newton-Framingham, } \\
\text { Massachusetts* }\end{array}$ & 6,895 & 21.1 & 0.7 & $(19.8-22.5)$ \\
\hline Camden, New Jersey* & 1,973 & 25.2 & 1.2 & $(22.8-27.5)$ \\
\hline Canton-Massillon, Ohio & 678 & 35.4 & 2.4 & $(30.6-40.1)$ \\
\hline Casper, Wyoming & 827 & 24.4 & 2.0 & $(20.4-28.4)$ \\
\hline Cedar Rapids, lowa & 580 & 23.9 & 1.9 & $(20.3-27.6)$ \\
\hline Charleston, West Virginia & 766 & 33.4 & 1.9 & $(29.7-37.0)$ \\
\hline $\begin{array}{l}\text { Charleston-North Charleston, } \\
\text { South Carolina }\end{array}$ & 1,698 & 24.8 & 1.4 & $(22.1-27.5)$ \\
\hline $\begin{array}{l}\text { Charlotte-Concord-Gastonia, } \\
\text { North Carolina-South Carolina }\end{array}$ & 2,523 & 23.0 & 1.0 & $(21.1-25.0)$ \\
\hline Chattanooga, Tennessee-Georgia & 629 & 29.6 & 2.5 & $(24$ \\
\hline Cheyenne, Wyoming & 951 & 25.0 & 2.0 & $(21.1-28.8)$ \\
\hline $\begin{array}{l}\text { Chicago-Naperville-Elgin, } \\
\text { Illinois-Indiana-Wisconsin }\end{array}$ & 3,716 & 23.1 & 0.9 & $(21.4-24.9)$ \\
\hline Cincinnati, Ohio-Kentucky-Indiana & 2,356 & 27.6 & 1.1 & $(25.4-29.8)$ \\
\hline $\begin{array}{l}\text { Claremont-Lebanon, New } \\
\text { Hampshire-Vermont }\end{array}$ & 1,944 & 28.7 & 1.3 & $(26.1-31.3)$ \\
\hline Cleveland-Elyria, Ohio & 1,775 & 29.7 & 1.4 & $(26.9-32.5)$ \\
\hline Colorado Springs, Colorado & 1,155 & 23.5 & 1.4 & $(20.7-26.3)$ \\
\hline Columbia, South Carolina & 1,792 & 28.1 & 1.4 & $(25.3-30.9)$ \\
\hline Columbus, Ohio & 1,598 & 27.4 & 1.4 & $(24.7-30.0)$ \\
\hline Concord, New Ham & 701 & 30.1 & 2.1 & $(25.9-34.2)$ \\
\hline Dallas-Plano-Irving, Texas* & 905 & 19.3 & 1.6 & $(16.3-22.4)$ \\
\hline Dayton, Ohio & 855 & 30.2 & 1.9 & $(26.5-34.0)$ \\
\hline Denver-Aurora-Lakewood, Colorado & 4,825 & 21.6 & 0.7 & $(20.3-23.0)$ \\
\hline
\end{tabular}

See table footnotes on page 112 . 
TABLE 59. (Continued) Estimated prevalence of adults aged $\geq 18$ years told by a health care professional that they have some form of arthritis, rheumatoid arthritis, gout, lupus, or fibromyalgia, by metropolitan and micropolitan statistical area - Behavioral Risk Factor Surveillance System, United States, 2012

\begin{tabular}{|c|c|c|c|c|}
\hline MMSA(s) & $\begin{array}{l}\text { Sample } \\
\text { size }\end{array}$ & $\%$ & SE & $95 \% \mathrm{Cl}$ \\
\hline Des Moines-West Des Moines, lowa & 1,156 & 24.3 & 1.4 & $(21.6-26.9)$ \\
\hline Detroit-Dearborn-Livonia, Michigan* & 2,210 & 32.7 & 1.5 & $(29.9-35.6)$ \\
\hline Dover, Delaware & 1,439 & 28.3 & 1.6 & $(25.1-31.4)$ \\
\hline Duluth, Minnesota-Wisconsin & 511 & 27.2 & 2.3 & $(22.7-31.6)$ \\
\hline Durham-Chapel Hill, North Carolina & 793 & 18.9 & 1.5 & $(16.0-21.9)$ \\
\hline El Paso, Texas & 621 & 19.6 & 2.1 & $(15.4-23.8)$ \\
\hline Eugene, Oregon & 522 & 29.0 & 2.4 & $(24.3-33.7)$ \\
\hline Fairbanks, Alaska & 597 & 22.9 & 1.9 & $(19.1-26.7)$ \\
\hline Fargo, North Dakota-Minnesota & 936 & 20.3 & 1.7 & $(16.9-23.6)$ \\
\hline Farmington, New Mexico & 649 & 21.2 & 1.8 & $(17.6-24.7)$ \\
\hline Fayetteville, North Carolina & 505 & 26.2 & 2.3 & $(21.7-30.7)$ \\
\hline $\begin{array}{l}\text { Fayetteville-Springdale-Rogers, } \\
\text { Arkansas-Missouri }\end{array}$ & 807 & 20.6 & 1.7 & $(17.2-24.0)$ \\
\hline Fort Collins, Colorado & 591 & 19.8 & 1.7 & $(16.4-23.2)$ \\
\hline Fort Wayne, Indiana & 533 & 28.3 & 2.3 & $(23.8-32.9)$ \\
\hline Fort Worth-Arlington, Texas* & 721 & 21.3 & 1.7 & $(17.9-24.7)$ \\
\hline Grand Island, Nebraska & 853 & 24.6 & 1.8 & $(21.2-28.1)$ \\
\hline Grand Rapids-Wyoming, Michigan & 889 & 24.1 & 1.7 & $(20.8-27.3)$ \\
\hline Great Falls, Montana & 706 & 33.4 & 2.1 & $(29.2-37.6)$ \\
\hline Greeley, Colorado & 533 & 25.2 & 2.3 & $(20.6-29.7)$ \\
\hline $\begin{array}{l}\text { Greensboro-High Point, } \\
\text { North Carolina }\end{array}$ & 805 & 25.8 & 1.8 & $(22.3-29.2)$ \\
\hline $\begin{array}{l}\text { Greenville-Anderson-Mauldin, } \\
\text { South Carolina }\end{array}$ & 1,673 & 28.2 & 1.4 & $(25.6-30.9)$ \\
\hline Gulfport-Biloxi-Pascagoula, Mississippi & 738 & 34.1 & 2.2 & $(29.8-38.3)$ \\
\hline $\begin{array}{l}\text { Hagerstown-Martinsburg, Maryland- } \\
\text { West Virginia }\end{array}$ & 755 & 31.9 & 2.5 & $(27.0-36.8)$ \\
\hline Harrisburg-Carlisle, Pennsylvania & 662 & 31.3 & 2.1 & $(27.2-35.5)$ \\
\hline $\begin{array}{l}\text { Hartford-West Hartford-East Hartford, } \\
\text { Connecticut }\end{array}$ & 2,654 & 23.6 & 1.0 & $(21.7-25.6)$ \\
\hline Heber, Utah & 512 & 16.8 & 2.4 & $(12.1-21.6)$ \\
\hline Hilo, Hawaii & 1,343 & 23.2 & 1.6 & $(20.1-26.3)$ \\
\hline $\begin{array}{l}\text { Hilton Head Island-Bluffton-Beaufort, } \\
\text { South Carolina }\end{array}$ & 934 & 21.8 & 1.8 & $(18.2-25.3)$ \\
\hline $\begin{array}{l}\text { Houston-The Woodlands-Sugar Land, } \\
\text { Texas }\end{array}$ & 1,146 & 19.5 & 1.3 & $(16.9-22.1)$ \\
\hline $\begin{array}{l}\text { Huntington-Ashland, West } \\
\text { Virginia-Kentucky-Ohio }\end{array}$ & 1,104 & 38.3 & 1.9 & $(34.7-42.0)$ \\
\hline Huntsville, Alabama & 616 & 29.9 & 2.3 & $(25.5-34.4)$ \\
\hline Idaho Falls, Idaho & 539 & 24.4 & 2.8 & $(18.9-30.0)$ \\
\hline $\begin{array}{l}\text { Indianapolis-Carmel-Anderson, } \\
\text { Indiana }\end{array}$ & 2,184 & 25.3 & 1.1 & $(23.1-27.4)$ \\
\hline Jackson, Mississippi & 916 & 28.7 & 1.7 & $(25.3-32.1)$ \\
\hline Jacksonville, Florida & 519 & 26.6 & 2.6 & $(21.5-31.8)$ \\
\hline Kahului-Wailuku-Lahaina, Hawaii & 1,209 & 19.3 & 1.6 & $(16.1-22.4)$ \\
\hline Kalispell, Montana & 560 & 27.9 & 2.2 & $(23.6-32.1)$ \\
\hline Kansas City, Missouri-Kansas & 4,730 & 25.8 & 1.1 & $(23.7-28.0)$ \\
\hline Kapaa, Hawaii & 665 & 23.4 & 2.5 & $(18.5-28.3)$ \\
\hline Keene, New Hampshire & 546 & 25.1 & 2.3 & $(20.7-29.6)$ \\
\hline Kennewick-Richland, Washington & 530 & 24.7 & 2.1 & $(20.6-28.8)$ \\
\hline $\begin{array}{l}\text { Kingsport-Bristol-Bristol, } \\
\text { Tennessee-Virginia }\end{array}$ & 568 & 45.8 & 3.2 & $(39.5-52.1)$ \\
\hline Knoxville, Tennessee & 833 & 26.9 & 1.9 & $(23.2-30.5)$ \\
\hline Laconia, New Hampshire & 562 & 31.4 & 2.8 & $(25.9-37.0)$ \\
\hline Lafayette, Louisiana & 555 & 25.1 & 2.3 & $(20.5-29.6)$ \\
\hline Las Cruces, New Mexico & 708 & 23.6 & 1.8 & $(20.1-27.2)$ \\
\hline $\begin{array}{l}\text { Las Vegas-Henderson-Paradise, } \\
\text { Nevada }\end{array}$ & 2,003 & 23.5 & 1.1 & $(21.3-25.7)$ \\
\hline Lewiston-Auburn, Maine & 697 & 29.3 & 2.0 & $(25.3-33.3)$ \\
\hline
\end{tabular}

See table footnotes on page 112.
TABLE 59. (Continued) Estimated prevalence of adults aged $\geq 18$ years told by a health care professional that they have some form of arthritis, rheumatoid arthritis, gout, lupus, or fibromyalgia, by metropolitan and micropolitan statistical area - Behavioral Risk Factor Surveillance System, United States, 2012

\begin{tabular}{|c|c|c|c|c|}
\hline MMSA(s) & $\begin{array}{l}\text { Sample } \\
\text { size }\end{array}$ & $\%$ & SE & $95 \% \mathrm{Cl}$ \\
\hline Lexington-Fayette, Kentucky & 526 & 26.7 & 2.3 & $(22.3-31.2)$ \\
\hline Lincoln, Nebraska & 1,659 & 21.9 & 1.1 & $(19.7-24.2)$ \\
\hline $\begin{array}{l}\text { Little Rock-North Little Rock-Conway, } \\
\text { Arkansas }\end{array}$ & 1,165 & 27.7 & 1.6 & $(24.7-30.8)$ \\
\hline Logan, Utah-Idaho & 502 & 18.0 & 2.0 & $(14.1-21.8)$ \\
\hline $\begin{array}{l}\text { Los Angeles-Long Beach-Glendale, } \\
\text { California* }\end{array}$ & 3,494 & 19.5 & 0.9 & $(17.8-21.2)$ \\
\hline $\begin{array}{l}\text { Louisville/Jefferson County, } \\
\text { Kentucky-Indiana }\end{array}$ & 2,176 & 32.3 & 1.5 & $(29.3-35.2)$ \\
\hline Lumberton, North Carolina & 546 & 34.1 & 3.8 & $(26.7-41.4)$ \\
\hline Manchester-Nashua, New Hampshire & 1,894 & 26.4 & 1.2 & $(24.1-28.8)$ \\
\hline McAllen-Edinburg-Mission, Texas & 623 & 21.6 & 2.3 & $(17.0-26.2)$ \\
\hline $\begin{array}{l}\text { Memphis, } \\
\text { Tennessee-Mississippi-Arkansas }\end{array}$ & 1,301 & 24.5 & 1.5 & $(21.5-27.5)$ \\
\hline $\begin{array}{l}\text { Miami-Fort Lauderdale-West Palm } \\
\text { Beach, Florida }\end{array}$ & 1,662 & 21.4 & 1.4 & $(18.6-24.1)$ \\
\hline $\begin{array}{l}\text { Milwaukee-Waukesha-West Allis, } \\
\text { Wisconsin }\end{array}$ & 1,249 & 24.4 & 1.7 & $(21.1-27.7)$ \\
\hline $\begin{array}{l}\text { Minneapolis-St. Paul-Bloomington, } \\
\text { Minnesota-Wisconsin }\end{array}$ & 7,867 & 19.6 & 0.6 & $(18.4-20.8)$ \\
\hline Missoula, Montana & 781 & 21.0 & 1.6 & $(17.9-24.1)$ \\
\hline Mobile, Alabama & 812 & 35.9 & 2.5 & $(31.0-40.8)$ \\
\hline Montgomery, Alabama & 532 & 30.4 & 2.6 & $(25.4-35.5)$ \\
\hline $\begin{array}{l}\text { Montgomery County-Bucks County- } \\
\text { Chester County, Pennsylvania* }\end{array}$ & 1,307 & 25.6 & 1.5 & $(22.7-28.5)$ \\
\hline $\begin{array}{l}\text { Myrtle Beach-Conway-North Myrtle } \\
\text { Beach, South Carolina-North Carolina }\end{array}$ & 939 & 33.5 & 1.9 & $(29.8-37.3)$ \\
\hline $\begin{array}{l}\text { Nashville-Davidson-Murfreesboro- } \\
\text { Franklin, Tennessee }\end{array}$ & 1,337 & 25.2 & 1.4 & $(22.5-28.0)$ \\
\hline $\begin{array}{l}\text { Nassau County-Suffolk County, } \\
\text { New York* }\end{array}$ & 892 & 22.2 & 1.6 & $(19.0-25.4)$ \\
\hline Newark, New Jersey-Pennsylvania* & 6,538 & 19.6 & 0.7 & $(18.2-21.0)$ \\
\hline New Haven-Milford, Connecticut & 1,996 & 25.6 & 1.3 & $(23.0-28.1)$ \\
\hline New Orleans-Metairie, Louisiana & 1,273 & 25.3 & 1.5 & $(22.3-28.2)$ \\
\hline $\begin{array}{l}\text { New York-Jersey City-White Plains, } \\
\text { New York-New Jersey* }\end{array}$ & 7,399 & 22.2 & 0.9 & $(20.4-24.0)$ \\
\hline Norfolk, Nebraska & 570 & 24.1 & 2.0 & $(20.1-28.0)$ \\
\hline North Platte, Nebraska & 609 & 28.1 & 2.4 & $(23.3-32.9)$ \\
\hline Norwich-New London, Connecticut & 989 & 27.1 & 2.1 & $(23.0-31.1)$ \\
\hline $\begin{array}{l}\text { Oakland-Hayward-Berkeley, } \\
\text { California* }\end{array}$ & 976 & 21.9 & 2.0 & $(18.0-25.9)$ \\
\hline Ocean City, New Jersey & 552 & 33.5 & 3.2 & $(27.3-39.8)$ \\
\hline Ogden-Clearfield, Utah & 2,424 & 22.9 & 0.9 & $(21.0-24.7)$ \\
\hline Oklahoma City, Oklahoma & 2,429 & 25.8 & 1.1 & $(23.7-27.9)$ \\
\hline Olympia-Tumwater, Washington & 515 & 30.0 & 2.7 & $(24.8-35.2)$ \\
\hline Omaha-Council Bluffs, Nebraska-lowa & 5,569 & 23.1 & 0.7 & $(21.7-24.5)$ \\
\hline Orlando-Kissimmee-Sanford, Florida & 567 & 21.9 & 2.4 & $(17.2-26.6)$ \\
\hline Philadelphia, Pennsylvania* & 2,452 & 24.8 & 1.1 & $(22.7-26.9)$ \\
\hline Phoenix-Mesa-Scottsdale, Arizona & 2,582 & 23.8 & 1.0 & $(21.8-25.8)$ \\
\hline Pittsburgh, Pennsylvania & 3,356 & 31.2 & 0.9 & $(29.3-33.0)$ \\
\hline Ponce, Puerto Rico & 545 & 23.6 & 2.0 & $(19.6-27.6)$ \\
\hline Portland-South Portland, Maine & 3,311 & 27.8 & 0.9 & $(26.1-29.6)$ \\
\hline $\begin{array}{l}\text { Portland-Vancouver-Hillsboro, } \\
\text { Oregon-Washington }\end{array}$ & 3,110 & 24.4 & 0.9 & $(22.5-26.2)$ \\
\hline $\begin{array}{l}\text { Providence-Warwick, Rhode } \\
\text { Island-Massachusetts }\end{array}$ & 8,106 & 26.9 & 0.7 & $(25.5-28.3)$ \\
\hline Provo-Orem, Utah & 1,745 & 17.2 & 1.0 & $(15.3-19.1)$ \\
\hline Raleigh, North Carolina & 940 & 19.8 & 1.5 & $(16.9-22.8)$ \\
\hline Rapid City, South Dakota & 1,049 & 25.2 & 1.7 & $(21.9-28.5)$ \\
\hline
\end{tabular}

See table footnotes on page 112 . 
TABLE 59. (Continued) Estimated prevalence of adults aged $\geq 18$ years told by a health care professional that they have some form of arthritis, rheumatoid arthritis, gout, lupus, or fibromyalgia, by metropolitan and micropolitan statistical area - Behavioral Risk Factor Surveillance System, United States, 2012

\begin{tabular}{|c|c|c|c|c|}
\hline MMSA(s) & $\begin{array}{l}\text { Sample } \\
\text { size }\end{array}$ & $\%$ & SE & $95 \% \mathrm{Cl}$ \\
\hline Reno, Nevada & 1,500 & 24.2 & 1.5 & $(21.3-27.1)$ \\
\hline Richmond, Virginia & 1,013 & 25.9 & 1.7 & $(22.5-29.3)$ \\
\hline $\begin{array}{l}\text { Riverside-San Bernardino-Ontario, } \\
\text { California }\end{array}$ & 1,529 & 23.8 & 1.4 & $(21.1-26.6)$ \\
\hline $\begin{array}{l}\text { Rockingham County-Strafford County, } \\
\text { New Hampshire* }\end{array}$ & 1,665 & 25.5 & 1.3 & $(23.0-28.0)$ \\
\hline Rutland, Vermont & 592 & 31.8 & 2.3 & $(27.3-36.2)$ \\
\hline $\begin{array}{l}\text { Sacramento-Roseville-Arden-Arcade, } \\
\text { California }\end{array}$ & 997 & 24.5 & 1.7 & $(21.2-27.8)$ \\
\hline St. Louis, Missouri-Illinois & 2,066 & 27.0 & 1.3 & $(24.5-29.6)$ \\
\hline Salisbury, Maryland-Delaware & 2,117 & 31.8 & 1.6 & $(28.7-34.8)$ \\
\hline Salt Lake City, Utah & 4,601 & 19.1 & 0.7 & $(17.8-20.4)$ \\
\hline San Antonio-New Braunfels, Texas & 771 & 22.1 & 1.8 & $(18.6-25.6)$ \\
\hline San Diego-Carlsbad, California & 1,125 & 22.1 & 1.5 & $(19.1-25.1)$ \\
\hline $\begin{array}{l}\text { San Francisco-Redwood City-South } \\
\text { San Francisco, California* }\end{array}$ & 593 & 21.3 & 2.3 & $(16.8-25.7)$ \\
\hline $\begin{array}{l}\text { San Jose-Sunnyvale-Santa Clara, } \\
\text { California }\end{array}$ & 683 & 17.2 & 1.8 & $(13.7-20.6)$ \\
\hline San Juan-Carolina-Caguas, Puerto Rico & 3,929 & 24.1 & 0.7 & $(22.7-25.6)$ \\
\hline Santa Fe, New Mexico & 695 & 24.4 & 1.8 & $(20.8-28.0)$ \\
\hline Sayre, Pennsylvania & 1,862 & 30.2 & 1.9 & $(26.6-33.9)$ \\
\hline Scottsbluff, Nebraska & 628 & 30.2 & 2.8 & $(24.7-35.7)$ \\
\hline $\begin{array}{l}\text { Scranton-Wilkes-Barre-Hazleton, } \\
\text { Pennsylvania }\end{array}$ & 742 & 34.7 & 2.5 & $(29.9-39.6)$ \\
\hline Seattle-Bellevue-Everett, Washington* & 5,060 & 21.9 & 0.7 & $(20.5-23.3)$ \\
\hline Shreveport-Bossier City, Louisiana & 578 & 27.8 & 2.3 & $(23.3-32.4)$ \\
\hline $\begin{array}{l}\text { Silver Spring-Frederick-Rockville, } \\
\text { Maryland* }\end{array}$ & 2,296 & 18.3 & 1.0 & $(16.3-20.4)$ \\
\hline $\begin{array}{l}\text { Sioux City, lowa-Nebraska- } \\
\text { South Dakota }\end{array}$ & 1,202 & 23.8 & 2.5 & $(18.9-28.6)$ \\
\hline Sioux Falls, South Dakota & 1,460 & 19.6 & 1.3 & $(17.0-22.2)$ \\
\hline Spartanburg, South Carolina & 704 & 34.6 & 2.6 & $(29.6-39.6)$ \\
\hline Spokane-Spokane Valley, Washington & 1,087 & 30.0 & 1.7 & $(26.7-33.4)$ \\
\hline
\end{tabular}

TABLE 59. (Continued) Estimated prevalence of adults aged $\geq 18$ years told by a health care professional that they have some form of arthritis, rheumatoid arthritis, gout, lupus, or fibromyalgia, by metropolitan and micropolitan statistical area - Behavioral Risk Factor Surveillance System, United States, 2012

\begin{tabular}{|c|c|c|c|c|}
\hline MMSA(s) & $\begin{array}{l}\text { Sample } \\
\text { size }\end{array}$ & $\%$ & SE & $95 \% \mathrm{Cl}$ \\
\hline Springfield, Massachusetts & 2,327 & 26.0 & 1.3 & $(23.4-28.6)$ \\
\hline Tacoma-Lakewood, Washington* & 1,175 & 23.5 & 1.4 & $(20.7-26.3)$ \\
\hline $\begin{array}{l}\text { Tampa-St. Petersburg-Clearwater, } \\
\text { Florida }\end{array}$ & 824 & 28.2 & 2.0 & $(24.3-32.1)$ \\
\hline Toledo, Ohio & 966 & 30.5 & 2.1 & $(26.4-34.6)$ \\
\hline Topeka, Kansas & 1,084 & 25.9 & 1.5 & $(23.0-28.9)$ \\
\hline Torrington, Connecticut & 658 & 25.9 & 2.2 & $(21.6-30.3)$ \\
\hline Trenton, New Jersey & 570 & 23.1 & 2.0 & $(19.1-27.1)$ \\
\hline Tucson, Arizona & 992 & 25.7 & 1.7 & $(22.4-29.0)$ \\
\hline Tulsa, Oklahoma & 1,739 & 25.4 & 1.2 & $(23.1-27.8)$ \\
\hline Tuscaloosa, Alabama & 578 & 30.2 & 2.8 & $(24.8-35.7)$ \\
\hline Urban Honolulu, Hawaii & 4,324 & 19.8 & 0.9 & $(18.1-21.5)$ \\
\hline Vineland-Bridgeton, New Jersey & 554 & 26.1 & 2.6 & $(21.0-31.2)$ \\
\hline $\begin{array}{l}\text { Virginia Beach-Norfolk-Newport News, } \\
\text { Virginia-North Carolina }\end{array}$ & 1,492 & 25.4 & 1.4 & $(22.7-28.1)$ \\
\hline $\begin{array}{l}\text { Warren-Troy-Farmington Hills, } \\
\text { Michigan* }\end{array}$ & 2,183 & 30.0 & 1.2 & $(27.7-32.3)$ \\
\hline $\begin{array}{l}\text { Washington-Arlington-Alexandria, } \\
\text { District of Columbia-Virginia- } \\
\text { Maryland-West Virginia** }\end{array}$ & 8,153 & 18.6 & 0.6 & $(17.3-19.8)$ \\
\hline Wichita, Kansas & 2,349 & 23.6 & 1.0 & $(21.5-25.6)$ \\
\hline $\begin{array}{l}\text { Wilmington, Delaware-Maryland- } \\
\text { New Jersey* }\end{array}$ & 3,229 & 26.4 & 1.0 & $(24.4-28.5)$ \\
\hline Winston-Salem, North Carolina & 790 & 25.8 & 1.8 & $(22.3-29.3)$ \\
\hline Worcester, Massachusetts-Connecticut & 2,827 & 24.5 & 1.1 & $(22.4-26.6)$ \\
\hline Yakima, Washington & 525 & 25.1 & 2.5 & $(20.2-30.1)$ \\
\hline $\begin{array}{l}\text { Youngstown-Warren-Boardman, } \\
\text { Ohio-Pennsylvania }\end{array}$ & 928 & 34.9 & 2.5 & $(30.1-39.8)$ \\
\hline Median & & 25.3 & & \\
\hline Range & & $16.8-45.8$ & & \\
\hline
\end{tabular}

Abbreviations: $\mathrm{Cl}=$ confidence interval; $\mathrm{MMSA}=$ metropolitan and micropolitan statistical area; $\mathrm{SE}=$ standard error..

* Metropolitan division. 
TABLE 60. Estimated prevalence of adults aged $\geq 18$ years told by a health care professional that they have some form of arthritis, rheumatoid arthritis, gout, lupus, or fibromyalgia, by county Behavioral Risk Factor Surveillance System, United States, 2012

\begin{tabular}{|c|c|c|c|c|}
\hline County & $\begin{array}{l}\text { Sample } \\
\text { size }\end{array}$ & $\%$ & SE & $95 \% \mathrm{Cl}$ \\
\hline Jefferson County, Alabama & 958 & 27.3 & 1.6 & $(24.2-30.5)$ \\
\hline Madison County, Alabama & 513 & 30.2 & 2.6 & $(25.2-35.2)$ \\
\hline Mobile County, Alabama & 812 & 35.9 & 2.5 & $(31.0-40.8)$ \\
\hline Anchorage Municipality, Alaska & 899 & 20.5 & 1.5 & $(17.5-23.5)$ \\
\hline Fairbanks North Star Borough, Alaska & 597 & 22.9 & 1.9 & $(19.1-26.7)$ \\
\hline Matanuska-Susitna Borough, Alaska & 596 & 25.2 & 2.1 & $(21.1-29.4)$ \\
\hline Maricopa County, Arizona & 2,102 & 23.6 & 1.1 & $(21.4-25.7)$ \\
\hline Pima County, Arizona & 992 & 25.7 & 1.7 & $(22.5-29.0)$ \\
\hline Pulaski County, Arkansas & 743 & 26.2 & 2.0 & $(22.3-30.1)$ \\
\hline Alameda County, California & 619 & 20.2 & 2.6 & $(15.2-25.2)$ \\
\hline Los Angeles County, California & 3,494 & 19.5 & 0.9 & $(17.8-21.2)$ \\
\hline Orange County, California & 1,035 & 18.1 & 1.6 & $(15.1-21.2)$ \\
\hline Riverside County, California & 824 & 23.7 & 1.8 & $(20.1-27.3)$ \\
\hline Sacramento County, California & 608 & 23.4 & 2.2 & $(19.1-27.6)$ \\
\hline San Bernardino County, California & 705 & 24.1 & 2.1 & $(20.0-28.1)$ \\
\hline San Diego County, California & 1,125 & 22.1 & 1.5 & $(19.1-25.1)$ \\
\hline Santa Clara County, California & 664 & 17.4 & 1.8 & $(13.9-20.9)$ \\
\hline Adams County, Colorado & 803 & 23.5 & 1.7 & $(20.1-26.9)$ \\
\hline Arapahoe County, Colorado & 854 & 24.9 & 1.6 & $(21.7-28.1)$ \\
\hline Boulder County, Colorado & 517 & 23.0 & 2.1 & $(18.9-27.1)$ \\
\hline Denver County, Colorado & 992 & 19.0 & 1.3 & $(16.5-21.6)$ \\
\hline Douglas County, Colorado & 546 & 15.8 & 1.8 & $(12.2-19.5)$ \\
\hline El Paso County, Colorado & 1,016 & 23.6 & 1.5 & $(20.7-26.5)$ \\
\hline Jefferson County, Colorado & 1,110 & 22.3 & 1.4 & $(19.6-25.1)$ \\
\hline Larimer County, Colorado & 591 & 19.8 & 1.8 & $(16.4-23.2)$ \\
\hline Weld County, Colorado & 533 & 25.2 & 2.3 & $(20.6-29.7)$ \\
\hline Fairfield County, Connecticut & 2,175 & 21.6 & 1.1 & $(19.4-23.8)$ \\
\hline Hartford County, Connecticut & 1,976 & 23.4 & 1.1 & $(21.2-25.6)$ \\
\hline Litchfield County, Connecticut & 658 & 25.9 & 2.2 & $(21.6-30.3)$ \\
\hline New Haven County, Connecticut & 1,996 & 25.6 & 1.3 & $(23.0-28.1)$ \\
\hline New London County, Connecticut & 989 & 27.1 & 2.1 & $(23.0-31.1)$ \\
\hline Kent County, Delaware & 1,439 & 28.3 & 1.6 & $(25.2-31.4)$ \\
\hline New Castle County, Delaware & 2,328 & 25.6 & 1.1 & $(23.4-27.8)$ \\
\hline Sussex County, Delaware & 1,399 & 34.0 & 1.6 & $(30.8-37.2)$ \\
\hline $\begin{array}{l}\text { District of Columbia, } \\
\text { District of Columbia }\end{array}$ & 3,801 & 18.1 & 0.8 & $(16.5-19.8)$ \\
\hline Broward County, Florida & 524 & 19.7 & 2.3 & $(15.2-24.2)$ \\
\hline Miami-Dade County, Florida & 811 & 19.3 & 2.0 & $(15.4-23.3)$ \\
\hline Hawaii County, Hawaii & 1,343 & 23.2 & 1.6 & $(20.1-26.3)$ \\
\hline Honolulu County, Hawaii & 4,324 & 19.8 & 0.9 & $(18.1-21.5)$ \\
\hline Kauai County, Hawaii & 665 & 23.4 & 2.5 & $(18.5-28.3)$ \\
\hline Maui County, Hawaii & 1,209 & 19.3 & 1.6 & $(16.1-22.4)$ \\
\hline Ada County, Idaho & 806 & 24.6 & 2.0 & $(20.8-28.5)$ \\
\hline Canyon County, Idaho & 502 & 23.0 & 2.7 & $(17.7-28.3)$ \\
\hline Cook County, Illinois & 1,498 & 21.4 & 1.2 & $(19.0-23.8)$ \\
\hline Lake County, Indiana & 883 & 32.3 & 2.6 & $(27.2-37.5)$ \\
\hline Marion County, Indiana & 1,272 & 22.9 & 1.4 & $(20.3-25.6)$ \\
\hline Polk County, lowa & 807 & 24.9 & 1.6 & $(21.7-28.2)$ \\
\hline Johnson County, Kansas & 2,171 & 20.3 & 1.0 & $(18.4-22.2)$ \\
\hline Sedgwick County, Kansas & 1,791 & 22.6 & 1.1 & (20.4-24.9) \\
\hline Shawnee County, Kansas & 767 & 26.3 & 1.8 & $(22.7-29.9)$ \\
\hline Wyandotte County, Kansas & 873 & 23.1 & 2.0 & $(19.2-26.9)$ \\
\hline Jefferson County, Kentucky & 1,690 & 32.8 & 1.9 & $(29.2-36.4)$ \\
\hline East Baton Rouge Parish, Louisiana & 571 & 23.5 & 2.0 & $(19.6-27.4)$ \\
\hline Androscoggin County, Maine & 697 & 29.3 & 2.0 & $(25.3-33.3)$ \\
\hline Aroostook County, Maine & 534 & 35.9 & 2.5 & $(31.1-40.8)$ \\
\hline Cumberland County, Maine & 1,748 & 26.2 & 1.2 & $(23.9-28.5)$ \\
\hline Kennebec County, Maine & 831 & 30.0 & 1.8 & $(26.4-33.6)$ \\
\hline Penobscot County, Maine & 924 & 27.3 & 1.7 & $(24.0-30.7)$ \\
\hline York County, Maine & 1,188 & 30.1 & 1.5 & $(27.2-33.1)$ \\
\hline
\end{tabular}

See table footnotes on page 114.
TABLE 60. (Continued) Estimated prevalence of adults aged $\geq 18$ years told by a health care professional that they have some form of arthritis, rheumatoid arthritis, gout, lupus, or fibromyalgia, by county — Behavioral Risk Factor Surveillance System, United States, 2012

\begin{tabular}{|c|c|c|c|c|}
\hline County & $\begin{array}{l}\text { Sample } \\
\text { size }\end{array}$ & $\%$ & SE & $95 \% \mathrm{Cl}$ \\
\hline Anne Arundel County, Maryland & 894 & 25.8 & 1.9 & $(22.1-29.6)$ \\
\hline Baltimore County, Maryland & 1,514 & 25.4 & 1.5 & $(22.5-28.3)$ \\
\hline Charles County, Maryland & 509 & 28.5 & 3.1 & $(22.4-34.7)$ \\
\hline Frederick County, Maryland & 752 & 23.4 & 2.4 & $(18.7-28.0)$ \\
\hline Montgomery County, Maryland & 1,544 & 17.2 & 1.2 & $(14.9-19.5)$ \\
\hline Prince George's County, Maryland & 1,134 & 19.3 & 1.5 & $(16.3-22.3)$ \\
\hline Washington County, Maryland & 534 & 31.3 & 3.4 & $(24.7-37.9)$ \\
\hline Baltimore city, Maryland & 742 & 26.4 & 2.3 & $(21.9-30.9)$ \\
\hline Barnstable County, Massachusetts & 558 & 29.3 & 2.4 & $(24.7-34.0)$ \\
\hline Bristol County, Massachusetts & 2,647 & 28.2 & 1.5 & $(25.2-31.1)$ \\
\hline Essex County, Massachusetts & 2,550 & 23.2 & 1.3 & $(20.6-25.7)$ \\
\hline Hampden County, Massachusetts & 1,952 & 28.1 & 1.5 & $(25.1-31.1)$ \\
\hline Middlesex County, Massachusetts & 4,345 & 20.2 & 0.8 & $(18.6-21.7)$ \\
\hline Norfolk County, Massachusetts & 1,749 & 21.4 & 1.3 & $(18.8-23.9)$ \\
\hline Plymouth County, Massachusetts & 1,827 & 26.5 & 1.6 & $(23.2-29.7)$ \\
\hline Suffolk County, Massachusetts & 2,300 & 17.4 & 1.0 & $(15.4-19.3)$ \\
\hline Worcester County, Massachusetts & 2,584 & 23.5 & 1.1 & $(21.3-25.7)$ \\
\hline Kent County, Michigan & 523 & 23.8 & 2.2 & $(19.5-28.0)$ \\
\hline Macomb County, Michigan & 614 & 30.4 & 2.1 & $(26.3-34.5)$ \\
\hline Oakland County, Michigan & 1,166 & 27.1 & 1.6 & $(24.0-30.2)$ \\
\hline Wayne County, Michigan & 2,210 & 32.7 & 1.5 & $(29.9-35.6)$ \\
\hline Anoka County, Minnesota & 543 & 21.5 & 2.3 & $(17.1-26.0)$ \\
\hline Dakota County, Minnesota & 670 & 19.5 & 1.8 & $(16.0-23.0)$ \\
\hline Hennepin County, Minnesota & 3,289 & 17.4 & 0.9 & $(15.7-19.2)$ \\
\hline Ramsey County, Minnesota & 1,936 & 20.1 & 1.5 & $(17.2-23.0)$ \\
\hline Jackson County, Missouri & 881 & 27.1 & 2.3 & $(22.6-31.5)$ \\
\hline St. Louis County, Missouri & 951 & 26.9 & 1.9 & $(23.2-30.6)$ \\
\hline Cascade County, Montana & 706 & 33.4 & 2.1 & $(29.2-37.6)$ \\
\hline Flathead County, Montana & 560 & 27.9 & 2.2 & $(23.6-32.1)$ \\
\hline Hill County, Montana & 579 & 22.5 & 2.3 & $(18.0-26.9)$ \\
\hline Lake County, Montana & 892 & 29.9 & 2.3 & $(25.5-34.4)$ \\
\hline Missoula County, Montana & 781 & 21.0 & 1.6 & $(17.9-24.2)$ \\
\hline Yellowstone County, Montana & 749 & 28.9 & 1.8 & $(25.3-32.4)$ \\
\hline Dakota County, Nebraska & 732 & 21.1 & 2.6 & $(16.1-26.2)$ \\
\hline Douglas County, Nebraska & 3,561 & 22.6 & 0.8 & $(20.9-24.2)$ \\
\hline Hall County, Nebraska & 534 & 22.0 & 2.1 & $(18.0-26.0)$ \\
\hline Lancaster County, Nebraska & 1,439 & 21.7 & 1.2 & $(19.4-24.1)$ \\
\hline Lincoln County, Nebraska & 585 & 27.9 & 2.5 & $(23.0-32.8)$ \\
\hline Sarpy County, Nebraska & 1,172 & 20.6 & 1.4 & $(18.0-23.3)$ \\
\hline Scotts Bluff County, Nebraska & 576 & 29.4 & 2.8 & $(24.0-34.8)$ \\
\hline Clark County, Nevada & 2,003 & 23.5 & 1.1 & $(21.3-25.7)$ \\
\hline Washoe County, Nevada & 1,481 & 24.2 & 1.5 & $(21.3-27.1)$ \\
\hline Belknap County, New Hampshire & 562 & 31.5 & 2.8 & $(25.9-37.0)$ \\
\hline Carroll County, New Hampshire & 532 & 30.7 & 2.7 & $(25.4-36.0)$ \\
\hline Cheshire County, New Hampshire & 546 & 25.1 & 2.3 & $(20.7-29.6)$ \\
\hline Coos County, New Hampshire & 536 & 33.9 & 3.0 & $(28.1-39.8)$ \\
\hline Grafton County, New Hampshire & 565 & 28.9 & 2.5 & $(24.0-33.8)$ \\
\hline Hillsborough County, New Hampshire & 1,894 & 26.4 & 1.2 & $(24.1-28.8)$ \\
\hline Merrimack County, New Hampshire & 701 & 30.1 & 2.1 & $(25.9-34.2)$ \\
\hline Rockingham County, New Hampshire & 1,045 & 25.3 & 1.6 & $(22.2-28.4)$ \\
\hline Strafford County, New Hampshire & 620 & 25.8 & 2.2 & $(21.6-30.1)$ \\
\hline Atlantic County, New Jersey & 1,016 & 28.8 & 1.9 & $(25.1-32.5)$ \\
\hline Bergen County, New Jersey & 1,002 & 19.6 & 1.5 & $(16.6-22.6)$ \\
\hline Burlington County, New Jersey & 681 & 25.8 & 2.1 & $(21.6-30.0)$ \\
\hline Camden County, New Jersey & 742 & 24.5 & 1.9 & $(20.9-28.2)$ \\
\hline Cape May County, New Jersey & 552 & 33.5 & 3.2 & $(27.3-39.8)$ \\
\hline Cumberland County, New Jersey & 554 & 26.1 & 2.6 & $(21.0-31.2)$ \\
\hline Essex County, New Jersey & 1,340 & 20.3 & 1.4 & $(17.7-23.0)$ \\
\hline Gloucester County, New Jersey & 550 & 25.6 & 2.2 & $(21.2-29.9)$ \\
\hline Hudson County, New Jersey & 1,298 & 19.6 & 1.4 & $(16.9-22.3)$ \\
\hline
\end{tabular}

See table footnotes on page 114 . 
TABLE 60. (Continued) Estimated prevalence of adults aged $\geq 18$ years told by a health care professional that they have some form of arthritis, rheumatoid arthritis, gout, lupus, or fibromyalgia, by county — Behavioral Risk Factor Surveillance System, United States, 2012

\begin{tabular}{|c|c|c|c|c|}
\hline County & $\begin{array}{l}\text { Sample } \\
\text { size }\end{array}$ & $\%$ & SE & $95 \% \mathrm{Cl}$ \\
\hline Hunterdon County, New Jersey & 569 & 21.3 & 2.1 & $(17.1-25.5)$ \\
\hline Mercer County, New Jersey & 570 & 23.1 & 2.0 & $(19.1-27.1)$ \\
\hline Middlesex County, New Jersey & 879 & 16.0 & 1.5 & $(13.1-18.9)$ \\
\hline Monmouth County, New Jersey & 712 & 23.5 & 1.9 & $(19.7-27.2)$ \\
\hline Morris County, New Jersey & 845 & 19.7 & 1.6 & $(16.4-22.9)$ \\
\hline Ocean County, New Jersey & 661 & 28.2 & 2.1 & $(24.0-32.4)$ \\
\hline Passaic County, New Jersey & 684 & 20.0 & 1.8 & $(16.4-23.6)$ \\
\hline Salem County, New Jersey & 586 & 35.7 & 3.2 & $(29.4-41.9)$ \\
\hline Somerset County, New Jersey & 638 & 17.5 & 1.8 & $(14.0-21.1)$ \\
\hline Sussex County, New Jersey & 546 & 25.0 & 2.5 & $(20.1-29.9)$ \\
\hline Union County, New Jersey & 712 & 16.5 & 1.5 & $(13.5-19.5)$ \\
\hline Warren County, New Jersey & 531 & 26.3 & 2.4 & $(21.5-31.0)$ \\
\hline Bernalillo County, New Mexico & 2,057 & 21.5 & 1.0 & $(19.5$ \\
\hline Dona Ana County, New Mexico & 708 & 23.6 & 1.8 & 27.2) \\
\hline Sandoval County, New Mexico & 655 & 26.8 & 2.2 & $(22.5-31.1)$ \\
\hline San Juan County, New Mexico & 649 & 21.2 & 1.8 & $(17.6-24.7)$ \\
\hline Santa Fe County, New Mexico & 695 & 24.4 & 1.8 & $(20.8-28.0)$ \\
\hline Kings County, New York & 495 & 25.1 & 2.8 & $(19.6-30.7)$ \\
\hline Guilford County, North Carolina & 510 & 21.1 & 2.0 & $(17.2-25.1)$ \\
\hline Mecklenburg County, North Carolina & 728 & 17.7 & 1.6 & $(14.6-20.8)$ \\
\hline Robeson County, North Carolina & 546 & 34.1 & 3.7 & $(26.7-41.4)$ \\
\hline Wake County, North Carolina & 697 & 18.4 & 1.7 & $(15.1-21.8)$ \\
\hline Burleigh County, North Dakota & 567 & 25.8 & 2.3 & $(21.3-30.3)$ \\
\hline Cass County, North Dakota & 841 & 19.2 & 1.6 & $(16.0-$ \\
\hline Cuyahoga County, Ohio & 862 & 29.0 & 1.8 & $(25.4-32.6)$ \\
\hline Franklin County, Ohio & 836 & 25.7 & 1.8 & $(22.2-29.1)$ \\
\hline Hamilton County, Ohio & 768 & 25.7 & 1.8 & $(22.1-29.3)$ \\
\hline Lorain County, Ohio & 613 & 31.3 & 2.7 & $(25.9-36.6)$ \\
\hline Lucas County, Ohio & 620 & 31.6 & 2.6 & $(26.5-36.8)$ \\
\hline Mahoning County, Ohio & 590 & 35.7 & 3.1 & $(29.5-41.9)$ \\
\hline Montgomery County, Ohio & 663 & 30.0 & 2.2 & $(25.8-34.3)$ \\
\hline Stark County, Ohio & 620 & 35.3 & 2.6 & $(30.3-40.3)$ \\
\hline Summit County, Ohio & 630 & 26.3 & 2.2 & $(22.0-30.6)$ \\
\hline Oklahoma County, Oklahoma & 1,186 & 25.6 & 1.4 & $(22.8-28.4)$ \\
\hline Tulsa County, Oklahoma & 1,200 & 22.4 & 1.3 & $(19.8-25.0)$ \\
\hline Clackamas County, Oregon & 507 & 27.9 & 2.5 & $(23.0-32.7)$ \\
\hline Lane County, Oregon & 522 & 29.0 & 2.4 & $(24.4-33.7)$ \\
\hline Multnomah County, Oregon & 888 & 22.7 & 1.8 & $(19.2-26.2)$ \\
\hline Washington County, Oregon & 617 & 22.9 & 2.0 & $(18.9-26.9)$ \\
\hline Allegheny County, Pennsylvania & 1,880 & 28.8 & 1.2 & $(26.4-31.2)$ \\
\hline Bradford County, Pennsylvania & 1,862 & 30.2 & 1.9 & $(26.6-33.9)$ \\
\hline Montgomery County, Pennsylvania & 542 & 22.0 & 2.1 & $(17.9-26.0)$ \\
\hline Philadelphia County, Pennsylvania & 2,058 & 24.9 & 1.2 & $(22.6-27.2)$ \\
\hline Pike County, Pennsylvania & 1,888 & 29.4 & 1.7 & $(26.1-32.7)$ \\
\hline Kent County, Rhode Island & 810 & 30.5 & 2.0 & $(26.5-34.4)$ \\
\hline Providence County, Rhode Is & 3,335 & 26.4 & 0.9 & $(24.5-28.2)$ \\
\hline Washington County, Rhode Island & 655 & 25.0 & 2.1 & $(20.8-29.2)$ \\
\hline
\end{tabular}

TABLE 60. (Continued) Estimated prevalence of adults aged $\geq 18$ years told by a health care professional that they have some form of arthritis, rheumatoid arthritis, gout, lupus, or fibromyalgia, by county — Behavioral Risk Factor Surveillance System, United States, 2012

\begin{tabular}{|c|c|c|c|c|}
\hline County & $\begin{array}{l}\text { Sample } \\
\text { size }\end{array}$ & $\%$ & SE & $95 \% \mathrm{Cl}$ \\
\hline Aiken County, South Carolina & 555 & 26.8 & 2.3 & $(22.3-31.4)$ \\
\hline Beaufort County, South Carolina & 801 & 22.4 & 2.0 & $(18.5-26.3)$ \\
\hline Charleston County, South Carolina & 1,009 & 24.9 & 1.8 & $(21.3-28.4)$ \\
\hline Greenville County, South Carolina & 901 & 27.3 & 1.9 & $(23.6-30.9)$ \\
\hline Horry County, South Carolina & 771 & 33.4 & 2.1 & $(29.3-37.4)$ \\
\hline Richland County, South Carolina & 957 & 26.6 & 2.0 & $(22.7-30.4)$ \\
\hline Spartanburg County, South Carolina & 650 & 34.0 & 2.7 & $(28.8-39.3)$ \\
\hline Lincoln County, South Dakota & 499 & 16.4 & 1.9 & $(12.7-20.0)$ \\
\hline Minnehaha County, South Dakota & 873 & 19.4 & 1.5 & $(16.4-22.4)$ \\
\hline Pennington County, South Dakota & 602 & 24.3 & 2.0 & $(20.5-28.2)$ \\
\hline Davidson County, Tennessee & 556 & 24.8 & 2.2 & $(20.6-29.1)$ \\
\hline Shelby County, Tennessee & 533 & 22.2 & 2.0 & $(18.3-26.1)$ \\
\hline Bexar County, Texas & 583 & 23.0 & 2.1 & $(18.9-27.1)$ \\
\hline Dallas County, Texas & 512 & 19.3 & 2.1 & $(15.1-23.6)$ \\
\hline El Paso County, Texas & 620 & 19.6 & 2.1 & $(15.4-23.8)$ \\
\hline Harris County, Texas & 775 & 19.6 & 1.6 & $(16.5-22.8)$ \\
\hline Hidalgo County, Texas & 623 & 21.6 & 2.4 & $(17.0-26.2)$ \\
\hline Tarrant County, Texas & 568 & 20.2 & 1.9 & $(16.5-24.0)$ \\
\hline Travis County, Texas & 1,047 & 14.8 & 1.5 & $(11.8-17.8)$ \\
\hline Davis County, Utah & 1,146 & 21.1 & 1.3 & $(18.5-23.6)$ \\
\hline Salt Lake County, Utah & 4,037 & 19.2 & 0.7 & $(17.8-20.5)$ \\
\hline Tooele County, Utah & 564 & 17.5 & 2.1 & $(13.5-21.5)$ \\
\hline Utah County, Utah & 1,677 & 16.9 & 1.0 & $(15.0-18.9)$ \\
\hline Wasatch County, Utah & 512 & 16.8 & 2.4 & $(12.1-21.6)$ \\
\hline Weber County, Utah & 1,044 & 23.8 & 1.5 & $(20.9-26.7)$ \\
\hline Chittenden County, Vermont & 916 & 21.5 & 1.5 & $(18.6-24.4)$ \\
\hline Rutland County, Vermont & 592 & 31.8 & 2.3 & $(27.3-36.2)$ \\
\hline Washington County, Vermont & 517 & 25.5 & 2.2 & $(21.3-29.7)$ \\
\hline Windsor County, Vermont & 544 & 28.2 & 2.3 & $(23.8-32.7)$ \\
\hline Fairfax County, Virginia & 737 & 18.0 & 1.6 & $(14.8-21.2)$ \\
\hline Clark County, Washington & 787 & 25.1 & 1.8 & $(21.7-28.6)$ \\
\hline King County, Washington & 3,890 & 21.2 & 0.8 & $(19.7-22.8)$ \\
\hline Kitsap County, Washington & 563 & 31.1 & 2.4 & $(26.4-35.8)$ \\
\hline Pierce County, Washington & 1,175 & 23.5 & 1.4 & $(20.7-26.3)$ \\
\hline Snohomish County, Washington & 1,170 & 23.9 & 1.5 & $(21.0-26.9)$ \\
\hline Spokane County, Washington & 941 & 28.1 & 1.8 & $(24.6-31.7)$ \\
\hline Thurston County, Washington & 515 & 30.0 & 2.6 & $(24.8-35.2)$ \\
\hline Whatcom County, Washington & 841 & 22.8 & 1.9 & $(19.1-26.4)$ \\
\hline Yakima County, Washington & 525 & 25.1 & 2.5 & $(20.2-30.1)$ \\
\hline Kanawha County, West Virginia & 635 & 33.0 & 2.0 & $(29.1-36.9)$ \\
\hline Milwaukee County, Wisconsin & 957 & 25.6 & 1.9 & $(21.8-29.4)$ \\
\hline Laramie County, Wyoming & 951 & 25.0 & 2.0 & $(21.1-28.8)$ \\
\hline Natrona County, Wyoming & 827 & 24.4 & 2.0 & $(20.4-28.4)$ \\
\hline San Juan Municipio, Puerto Rico & 663 & 21.1 & 1.7 & $(17.7-24.4)$ \\
\hline Median & & 24.5 & & \\
\hline Range & & $14.8-35.9$ & & \\
\hline
\end{tabular}

Abbreviations: $\mathrm{Cl}=$ confidence interval; $\mathrm{SE}=$ standard error. 
TABLE 61. Estimated prevalence of adults aged $\geq 18$ years told by a health care professional that they have a depressive disorder, ${ }^{*}$ by state/territory - Behavioral Risk Factor Surveillance System, United States, 2012

\begin{tabular}{|c|c|c|c|c|}
\hline State/Territory & $\begin{array}{l}\text { Sample } \\
\text { size }\end{array}$ & $\%$ & SE & $95 \% \mathrm{Cl}$ \\
\hline Alabama & 8,983 & 21.9 & 0.6 & $(20.7-23.1)$ \\
\hline Alaska & 4,313 & 16.9 & 0.8 & $(15.4-18.5)$ \\
\hline Arizona & 7,266 & 18.8 & 0.8 & $(17.3-20.3)$ \\
\hline Arkansas & 5,157 & 22.2 & 0.8 & $(20.6-23.7)$ \\
\hline California & 14,558 & 11.7 & 0.4 & $(10.9-12.4)$ \\
\hline Colorado & 12,215 & 17.4 & 0.5 & $(16.4-18.3)$ \\
\hline Connecticut & 8,738 & 16.7 & 0.6 & $(15.6-17.8)$ \\
\hline Delaware & 5,165 & 14.8 & 0.7 & $(13.4-16.1)$ \\
\hline District of Columbia & 3,802 & 16.3 & 1.0 & $(14.4-18.3)$ \\
\hline Florida & 7,575 & 16.9 & 0.7 & $(15.6-18.2)$ \\
\hline Georgia & 6,069 & 16.7 & 0.7 & $(15.4-18.0)$ \\
\hline Hawaii & 7,552 & 11.5 & 0.6 & $(10.4-12.6)$ \\
\hline Idaho & 5,876 & 19.9 & 0.9 & $(18.1-21.8)$ \\
\hline Illinois & 5,567 & 15.0 & 0.7 & $(13.7-16.4)$ \\
\hline Indiana & 8,585 & 19.5 & 0.6 & $(18.4-20.6)$ \\
\hline lowa & 7,148 & 17.0 & 0.6 & $(15.9-18.1)$ \\
\hline Kansas & 11,746 & 16.5 & 0.5 & $(15.6-17.4)$ \\
\hline Kentucky & 11,176 & 23.5 & 0.6 & $(22.2-24.7)$ \\
\hline Louisiana & 9,044 & 17.2 & 0.6 & $(15.9-18.4)$ \\
\hline Maine & 9,884 & 22.7 & 0.5 & $(21.7-23.8)$ \\
\hline Maryland & 12,763 & 14.2 & 0.5 & $(13.2-15.1)$ \\
\hline Massachusetts & 21,598 & 18.9 & 0.4 & $(18.1-19.7)$ \\
\hline Michigan & 10,464 & 20.6 & 0.6 & $(19.5-21.7)$ \\
\hline Minnesota & 12,204 & 17.1 & 0.4 & $(16.2-17.9)$ \\
\hline Mississippi & 7,749 & 18.4 & 0.6 & $(17.2-19.7)$ \\
\hline Missouri & 6,731 & 20.1 & 0.7 & $(18.7-21.4)$ \\
\hline Montana & 8,638 & 20.0 & 0.6 & $(18.9-21.1)$ \\
\hline Nebraska & 19,115 & 16.8 & 0.4 & $(16.0-17.5)$ \\
\hline Nevada & 4,825 & 16.3 & 0.7 & $(14.8-17.7)$ \\
\hline New Hampshire & 7,500 & 19.7 & 0.7 & $(18.3-21.0)$ \\
\hline New Jersey & 15,683 & 13.0 & 0.4 & $(12.3-13.8)$ \\
\hline New Mexico & 8,747 & 19.9 & 0.5 & $(18.8-20.9)$ \\
\hline New York & 6,016 & 14.8 & 0.6 & $(13.5-16.0)$ \\
\hline North Carolina & 11,848 & 17.6 & 0.5 & $(16.7-18.5)$ \\
\hline North Dakota & 4,850 & 15.0 & 0.7 & $(13.6-16.4)$ \\
\hline Ohio & 12,987 & 18.9 & 0.5 & $(18.0-19.9)$ \\
\hline Oklahoma & 7,985 & 21.9 & 0.6 & $(20.7-23.1)$ \\
\hline Oregon & 5,276 & 23.4 & 0.8 & $(21.8-24.9)$ \\
\hline Pennsylvania & 19,868 & 18.0 & 0.4 & $(17.2-18.9)$ \\
\hline Rhode Island & 5,447 & 20.3 & 0.7 & $(18.9-21.8)$ \\
\hline South Carolina & 12,713 & 18.0 & 0.5 & $(17.0-19.0)$ \\
\hline South Dakota & 7,841 & 15.3 & 0.6 & $(14.1-16.5)$ \\
\hline Tennessee & 7,023 & 20.2 & 0.7 & (18.9-21.5) \\
\hline Texas & 9,076 & 15.5 & 0.5 & $(14.5-16.5)$ \\
\hline Utah & 12,387 & 21.0 & 0.5 & $(20.0-21.9)$ \\
\hline Vermont & 6,028 & 21.9 & 0.7 & $(20.5-23.2)$ \\
\hline Virginia & 7,360 & 16.6 & 0.6 & $(15.5-17.8)$ \\
\hline Washington & 15,244 & 22.3 & 0.5 & (21.4-23.3) \\
\hline West Virginia & 5,382 & 20.9 & 0.7 & $(19.6-22.2)$ \\
\hline Wisconsin & 5,275 & 16.1 & 0.8 & $(14.6-17.6)$ \\
\hline Wyoming & 6,243 & 18.0 & 0.8 & $(16.3-19.6)$ \\
\hline Guam & 2,008 & 9.0 & 0.9 & $(7.2-10.8)$ \\
\hline Puerto Rico & 6,311 & 16.3 & 0.6 & $(15.2-17.4)$ \\
\hline Median & & 17.6 & & \\
\hline Range & & $9.0-23.5$ & & \\
\hline
\end{tabular}

Abbreviations: $\mathrm{Cl}=$ confidence interval; $\mathrm{SE}=$ standard error.

* Including depression, major depression, dysthymia, or minor depression.
TABLE 62. Estimated prevalence of adults aged $\geq 18$ years told by a health care professional that they have a depressive disorder,* by metropolitan and micropolitan statistical area - Behavioral Risk Factor Surveillance System, United States, 2012

\begin{tabular}{|c|c|c|c|c|}
\hline MMSA(s) & $\begin{array}{l}\text { Sample } \\
\text { size }\end{array}$ & $\%$ & SE & $95 \% \mathrm{Cl}$ \\
\hline Aguadilla-Isabela, Puerto Rico & 551 & 17.7 & 1.8 & $(14.1-21.3)$ \\
\hline Akron, Ohio & 743 & 17.2 & 1.8 & $(13.6-20.8)$ \\
\hline Albuquerque, New Mexico & 3,258 & 21.2 & 0.9 & $(19.5-23.0)$ \\
\hline $\begin{array}{l}\text { Allentown-Bethlehem-Easton, } \\
\text { Pennsylvania-New Jersey }\end{array}$ & 1,347 & 18.2 & 1.5 & $(15.3-21.2)$ \\
\hline Anaheim-Santa Ana-Irvine, California ${ }^{\dagger}$ & 1,036 & 9.2 & 1.2 & $(6.9-11.5)$ \\
\hline Anchorage, Alaska & 1,496 & 18.0 & 1.2 & $(15.5-20.4)$ \\
\hline Asheville, North Carolina & 590 & 19.8 & 2.0 & $(15.8-23.8)$ \\
\hline $\begin{array}{l}\text { Atlanta-Sandy Springs-Roswell, } \\
\text { Georgia }\end{array}$ & 2,527 & 14.5 & 0.9 & $(12.7-16.4)$ \\
\hline Atlantic City-Hammonton, New Jersey & 1,016 & 16.4 & 1.8 & $(12.8-19.9)$ \\
\hline $\begin{array}{l}\text { Augusta-Richmond County, Georgia- } \\
\text { South Carolina }\end{array}$ & 1,030 & 19.1 & 2.2 & $(14.7-23.5)$ \\
\hline Augusta-Waterville, Maine & 830 & 23.4 & 1.8 & $(19.9-26.8)$ \\
\hline Austin-Round Rock, Texas & 1,380 & 15.6 & 1.6 & $(12.5-18.6)$ \\
\hline $\begin{array}{l}\text { Baltimore-Columbia-Towson, } \\
\text { Maryland }\end{array}$ & 4,706 & 16.3 & 0.8 & $(14.7-17.9)$ \\
\hline Bangor, Maine & 926 & 23.1 & 1.7 & $(19.7-26.5)$ \\
\hline Barnstable Town, Massachusetts & 558 & 18.1 & 2.1 & $(13.9-22.2)$ \\
\hline Barre, Vermont & 514 & 25.2 & 2.5 & $(20.3-30.0)$ \\
\hline Baton Rouge, Louisiana & 1,387 & 18.8 & 1.7 & $(15.5-22.0)$ \\
\hline Bellingham, Washington & 840 & 23.1 & 2.4 & $(18.3-27.8)$ \\
\hline Berlin, New Hampshire-Vermont & 706 & 21.0 & 2.6 & $(15.9-26.2)$ \\
\hline Billings, Montana & 848 & 22.5 & 1.7 & $(19.1-25.8)$ \\
\hline Birmingham-Hoover, Alabama & 1,817 & 20.4 & 1.2 & $(18.1-22.8)$ \\
\hline Bismarck, North Dakota & 817 & 15.4 & 1.8 & $(11.8-18.9)$ \\
\hline Boise City, Idaho & 1,481 & 20.0 & 1.6 & $(16.9-23.1)$ \\
\hline Boston, Massachusetts ${ }^{\dagger}$ & 5,882 & 16.6 & 0.8 & $(15.1-18.1)$ \\
\hline Boulder, Colorado & 515 & 18.3 & 2.2 & $(14.0-22.5)$ \\
\hline Bremerton-Silverdale, Washington & 565 & 24.6 & 2.3 & $(20.1-29.1)$ \\
\hline $\begin{array}{l}\text { Bridgeport-Stamford-Norwalk, } \\
\text { Connecticut }\end{array}$ & 2,176 & 15.2 & 1.2 & $(12.9-17.5)$ \\
\hline Burlington-South Burlington, Vermont & 1,520 & 20.3 & 1.3 & $(17.8-22.8)$ \\
\hline $\begin{array}{l}\text { Cambridge-Newton-Framingham, } \\
\text { Massachusetts }{ }^{\dagger}\end{array}$ & 6,896 & 17.4 & 0.7 & $(16.0-18.7)$ \\
\hline Camden, New Jersey ${ }^{\dagger}$ & 1,974 & 16.9 & 1.1 & $(14.7-19.1)$ \\
\hline Canton-Massillon, Ohio & 681 & 21.4 & 2.3 & $(16.9-26.0)$ \\
\hline Casper, Wyoming & 826 & 21.8 & 2.2 & $(17.5-26.1)$ \\
\hline Cedar Rapids, lowa & 583 & 15.4 & 1.9 & $(11.7-19.1)$ \\
\hline Charleston, West Virginia & 770 & 20.5 & 1.7 & $(17.3-23.8)$ \\
\hline $\begin{array}{l}\text { Charleston-North Charleston, } \\
\text { South Carolina }\end{array}$ & 1,706 & 17.3 & 1.3 & $(14.7-19.8)$ \\
\hline $\begin{array}{l}\text { Charlotte-Concord-Gastonia, } \\
\text { North Carolina-South Carolina }\end{array}$ & 2,520 & 14.6 & 0.9 & $(12.9-16.4)$ \\
\hline Chattanooga, Tennessee-Georgia & 630 & 22.4 & 2.6 & $(17.3-27.4)$ \\
\hline Cheyenne, Wyoming & 953 & 17.4 & 1.9 & $(13.7-21.1)$ \\
\hline $\begin{array}{l}\text { Chicago-Naperville-Elgin, } \\
\text { Illinois-Indiana-Wisconsin }\end{array}$ & 3,718 & 14.0 & 0.9 & $(12.3-15.7)$ \\
\hline Cincinnati, Ohio-Kentucky-Indiana & 2,361 & 20.2 & 1.1 & $(18.1-22.3)$ \\
\hline $\begin{array}{l}\text { Claremont-Lebanon, New } \\
\text { Hampshire-Vermont }\end{array}$ & 1,947 & 21.8 & 1.4 & $(19.1-24.5)$ \\
\hline Cleveland-Elyria, Ohio & 1,779 & 19.2 & 1.4 & $(16.4-22.0)$ \\
\hline Colorado Springs, Colorado & 1,157 & 17.7 & 1.5 & $(14.9-20.6)$ \\
\hline Columbia, South Carolina & 1,791 & 15.1 & 1.2 & $(12.8-17.4)$ \\
\hline Columbus, Ohio & 1,599 & 18.5 & 1.2 & $(16.1-20.9)$ \\
\hline Concord, New Hampshire & 702 & 21.7 & 2.2 & $(17.3-26.0)$ \\
\hline Dallas-Plano-Irving, Texas $^{\dagger}$ & 903 & 13.3 & 1.3 & $(10.7-15.9)$ \\
\hline Dayton, Ohio & 853 & 17.4 & 1.7 & $(14.1-20.7)$ \\
\hline Denver-Aurora-Lakewood, Colorado & 4,844 & 17.3 & 0.7 & $(16.0-18.7)$ \\
\hline Des Moines-West Des Moines, lowa & 1,156 & 19.4 & 1.5 & $(16.5-22.3)$ \\
\hline
\end{tabular}

See table footnotes on page 117. 
TABLE 62. (Continued) Estimated prevalence of adults aged $\geq 18$ years told by a health care professional that they have a depressive disorder,* by metropolitan and micropolitan statistical area Behavioral Risk Factor Surveillance System, United States, 2012

\begin{tabular}{|c|c|c|c|c|}
\hline MMSA(s) & $\begin{array}{l}\text { Sample } \\
\text { size }\end{array}$ & $\%$ & SE & $95 \% \mathrm{Cl}$ \\
\hline Detroit-Dearborn-Livonia, Michigan $^{\dagger}$ & 2,217 & 19.9 & 1.4 & $(17.2-22.6)$ \\
\hline Dover, Delaware & 1,437 & 14.2 & 1.3 & $(11.6-16.7)$ \\
\hline Duluth, Minnesota-Wisconsin & 512 & 21.9 & 2.6 & $(16.8-26.9)$ \\
\hline Durham-Chapel Hill, North Carolina & 792 & 15.4 & 1.5 & $(12.4-18.4)$ \\
\hline El Paso, Texas & 621 & 17.2 & 2.3 & $(12.7-21.6)$ \\
\hline Eugene, Oregon & 523 & 24.2 & 2.4 & $(19.4-29.0)$ \\
\hline Fairbanks, Alaska & 594 & 17.8 & 1.9 & $(14.0-21.6)$ \\
\hline Fargo, North Dakota-Minnesota & 934 & 20.0 & 2.1 & $(15.9-24.1)$ \\
\hline Farmington, New Mexico & 647 & 19.1 & 1.9 & $(15.4-22.7)$ \\
\hline Fayetteville, North Carolina & 505 & 16.1 & 1.9 & $(12.3-19.9)$ \\
\hline $\begin{array}{l}\text { Fayetteville-Springdale-Rogers, } \\
\text { Arkansas-Missouri }\end{array}$ & 810 & 20.0 & 2.1 & $(16.0-24.1)$ \\
\hline Fort Collins, Colorado & 593 & 17.6 & 1.9 & $(13.8-21.4)$ \\
\hline Fort Wayne, Indiana & 537 & 19.4 & 2.1 & $(15.2-23.5)$ \\
\hline Fort Worth-Arlington, Texas ${ }^{\dagger}$ & 725 & 13.8 & 1.7 & $(10.5-17.1)$ \\
\hline Grand Island, Nebraska & 850 & 16.1 & 1.6 & $(13.0-19.3)$ \\
\hline Grand Rapids-Wyoming, Michigan & 886 & 18.6 & 1.8 & $(15.2-22.1)$ \\
\hline Great Falls, Montana & 704 & 21.6 & 1.9 & $(17.8-25.4)$ \\
\hline Greeley, Colorado & 534 & 18.6 & 2.3 & $(14.1-23.0)$ \\
\hline $\begin{array}{l}\text { Greensboro-High Point, } \\
\text { North Carolina }\end{array}$ & 806 & 18.6 & 1.7 & $(15.2-21.9)$ \\
\hline $\begin{array}{l}\text { Greenville-Anderson-Mauldin, } \\
\text { South Carolina }\end{array}$ & 1,678 & 19.9 & 1.2 & $(17.5-22.4)$ \\
\hline Gulfport-Biloxi-Pascagoula, Mississippi & 743 & 24.3 & 2.1 & $(20.1-28.4)$ \\
\hline $\begin{array}{l}\text { Hagerstown-Martinsburg, Maryland- } \\
\text { West Virginia }\end{array}$ & 759 & 20.7 & 2.3 & $(16.3-25.2)$ \\
\hline Harrisburg-Carlisle, Pennsylvania & 663 & 18.3 & 1.9 & $(14.6-21.9)$ \\
\hline $\begin{array}{l}\text { Hartford-West Hartford-East Hartford, } \\
\text { Connecticut }\end{array}$ & 2,653 & 17.5 & 1.0 & $(15.6-19.5)$ \\
\hline Heber, Utah & 510 & 20.4 & 4.7 & $(11.2-29.7)$ \\
\hline Hilo, Hawaii & 1,344 & 14.2 & 1.4 & $(11.4-17.0)$ \\
\hline $\begin{array}{l}\text { Hilton Head Island-Bluffton-Beaufort, } \\
\text { South Carolina }\end{array}$ & 931 & 11.2 & 1.7 & $(8.0-14.5)$ \\
\hline $\begin{array}{l}\text { Houston-The Woodlands-Sugar Land, } \\
\text { Texas }\end{array}$ & 1,142 & 15.6 & 1.3 & $(13.0-18.1)$ \\
\hline $\begin{array}{l}\text { Huntington-Ashland, West } \\
\text { Virginia-Kentucky-Ohio }\end{array}$ & 1,112 & 23.8 & 1.7 & $(20.5-27.0)$ \\
\hline Huntsville, Alabama & 616 & 20.8 & 2.0 & $(16.8-24.7)$ \\
\hline Idaho Falls, Idaho & 538 & 16.5 & 2.7 & $(11.2-21.8)$ \\
\hline $\begin{array}{l}\text { Indianapolis-Carmel-Anderson, } \\
\text { Indiana }\end{array}$ & 2,183 & 19.6 & 1.1 & $(17.4-21.7)$ \\
\hline Jackson, Mississippi & 918 & 17.4 & 1.5 & $(14.4-20.4)$ \\
\hline Jacksonville, Florida & 517 & 18.7 & 2.7 & $(13.3-24.0)$ \\
\hline Kahului-Wailuku-Lahaina, Hawaii & 1,214 & 13.1 & 1.3 & $(10.6-15.6)$ \\
\hline Kalispell, Montana & 559 & 19.5 & 2.0 & $(15.5-23.5)$ \\
\hline Kansas City, Missouri-Kansas & 4,725 & 16.7 & 1.0 & $(14.7-18.6)$ \\
\hline Kapaa, Hawaii & 666 & 11.3 & 1.5 & $(8.4-14.2)$ \\
\hline Keene, New Hampshire & 546 & 15.1 & 2.0 & $(11.2-19.0)$ \\
\hline Kennewick-Richland, Washington & 533 & 19.3 & 2.2 & $(15.1-23.6)$ \\
\hline $\begin{array}{l}\text { Kingsport-Bristol-Bristol, } \\
\text { Tennessee-Virginia }\end{array}$ & 565 & 22.2 & 2.5 & $(17.2-27.1)$ \\
\hline Knoxville, Tennessee & 833 & 20.8 & 1.9 & $(17.1-24.4)$ \\
\hline Laconia, New Hampshire & 565 & 20.7 & 2.7 & $(15.4-25.9)$ \\
\hline Lafayette, Louisiana & 557 & 13.8 & 1.8 & $(10.3-17.2)$ \\
\hline Las Cruces, New Mexico & 705 & 20.8 & 1.9 & $(17.1-24.4)$ \\
\hline $\begin{array}{l}\text { Las Vegas-Henderson-Paradise, } \\
\text { Nevada }\end{array}$ & 2,007 & 15.4 & 0.9 & $(13.6-17.3)$ \\
\hline Lewiston-Auburn, Maine & 697 & 28.3 & 2.1 & $(24.2-32.5)$ \\
\hline Lexington-Fayette, Kentucky & 530 & 20.8 & 2.2 & $(16.4-25.1)$ \\
\hline
\end{tabular}

See table footnotes on page 117.
TABLE 62. (Continued) Estimated prevalence of adults aged $\geq 18$ years told by a health care professional that they have a depressive disorder,* by metropolitan and micropolitan statistical area Behavioral Risk Factor Surveillance System, United States, 2012

\begin{tabular}{|c|c|c|c|c|}
\hline MMSA(s) & $\begin{array}{l}\text { Sample } \\
\text { size }\end{array}$ & $\%$ & SE & $95 \% \mathrm{Cl}$ \\
\hline Lincoln, Nebraska & 1,664 & 18.6 & 1.1 & $(16.4-20.8)$ \\
\hline $\begin{array}{l}\text { Little Rock-North Little Rock-Conway, } \\
\text { Arkansas }\end{array}$ & 1,169 & 22.6 & 1.6 & $(19.5-25.8)$ \\
\hline Logan, Utah-Idaho & 505 & 17.3 & 2.2 & $(12.9-21.7)$ \\
\hline $\begin{array}{l}\text { Los Angeles-Long Beach-Glendale, } \\
\text { California }^{\dagger}\end{array}$ & 3,499 & 10.6 & 0.7 & $(9.2-12.0)$ \\
\hline $\begin{array}{l}\text { Louisville/Jefferson County, } \\
\text { Kentucky-Indiana }\end{array}$ & 2,175 & 22.2 & 1.4 & $(19.4-25.0)$ \\
\hline Lumberton, North Carolina & 545 & 19.1 & 3.3 & $(12.7-25.5)$ \\
\hline Manchester-Nashua, New Hampshire & 1,901 & 19.0 & 1.2 & $(16.7-21.3)$ \\
\hline McAllen-Edinburg-Mission, Texas & 621 & 14.4 & 2.1 & $(10.2-18.5)$ \\
\hline $\begin{array}{l}\text { Memphis, } \\
\text { Tennessee-Mississippi-Arkansas }\end{array}$ & 1,303 & 18.7 & 1.6 & $(15.5-21.9)$ \\
\hline $\begin{array}{l}\text { Miami-Fort Lauderdale-West Palm } \\
\text { Beach, Florida }\end{array}$ & 1,655 & 13.4 & 1.2 & $(11.1-15.7)$ \\
\hline $\begin{array}{l}\text { Milwaukee-Waukesha-West Allis, } \\
\text { Wisconsin }\end{array}$ & 1,249 & 15.9 & 1.5 & $(12.9-18.8)$ \\
\hline $\begin{array}{l}\text { Minneapolis-St. Paul-Bloomington, } \\
\text { Minnesota-Wisconsin }\end{array}$ & 7,871 & 16.5 & 0.6 & $(15.4-17.7)$ \\
\hline Missoula, Montana & 781 & 23.9 & 1.9 & $(20.2-27.6)$ \\
\hline Mobile, Alabama & 814 & 21.3 & 2.2 & $(17.0-25.5)$ \\
\hline Montgomery, Alabama & 531 & 19.7 & 2.3 & $(15.3-24.2)$ \\
\hline $\begin{array}{l}\text { Montgomery County-Bucks County- } \\
\text { Chester County, Pennsylvania }^{\dagger}\end{array}$ & 1,311 & 15.1 & 1.4 & $(12.5-17.8)$ \\
\hline $\begin{array}{l}\text { Myrtle Beach-Conway-North Myrtle } \\
\text { Beach, South Carolina-North Carolina }\end{array}$ & 945 & 17.6 & 1.6 & $(14.4-20.8)$ \\
\hline $\begin{array}{l}\text { Nashville-Davidson-Murfreesboro- } \\
\text { Franklin, Tennessee }\end{array}$ & 1,332 & 16.6 & 1.2 & $(14.2-19.0)$ \\
\hline $\begin{array}{l}\text { Nassau County-Suffolk County, } \\
\text { New York }\end{array}$ & 895 & 12.2 & 1.4 & $(9.5-15.0)$ \\
\hline Newark, New Jersey-Pennsylvania ${ }^{\dagger}$ & 6,537 & 10.9 & 0.6 & $(9.8-12.1)$ \\
\hline New Haven-Milford, Connecticut & 2,007 & 16.4 & 1.1 & $(14.1-18.6)$ \\
\hline New Orleans-Metairie, Louisiana & 1,275 & 15.3 & 1.4 & $(12.6-17.9)$ \\
\hline $\begin{array}{l}\text { New York-Jersey City-White Plains, } \\
\text { New York-New Jersey }{ }^{\dagger}\end{array}$ & 7,406 & 13.3 & 0.8 & $(11.8-14.8)$ \\
\hline Norfolk, Nebraska & 568 & 12.1 & 1.7 & $(8.8-15.4)$ \\
\hline North Platte, Nebraska & 612 & 19.7 & 2.4 & $(14.9-24.5)$ \\
\hline Norwich-New London, Connecticut & 997 & 16.9 & 1.8 & $(13.3-20.5)$ \\
\hline $\begin{array}{l}\text { Oakland-Hayward-Berkeley, } \\
\text { California }^{\dagger}\end{array}$ & 974 & 11.8 & 1.3 & $(9.3-14.4)$ \\
\hline Ocean City, New Jersey & 551 & 19.1 & 3.1 & $(12.9-25.2)$ \\
\hline Ogden-Clearfield, Utah & 2,424 & 21.4 & 1.0 & $(19.4-23.4)$ \\
\hline Oklahoma City, Oklahoma & 2,427 & 21.0 & 1.1 & $(18.9-23.2)$ \\
\hline Olympia-Tumwater, Washington & 514 & 23.9 & 2.5 & $(19.0-28.9)$ \\
\hline Omaha-Council Bluffs, Nebraska-lowa & 5,580 & 18.0 & 0.7 & $(16.5-19.4)$ \\
\hline Orlando-Kissimmee-Sanford, Florida & 570 & 18.2 & 2.4 & $(13.5-22.9)$ \\
\hline Philadelphia, Pennsylvania ${ }^{\dagger}$ & 2,451 & 17.8 & 1.0 & $(15.8-19.9)$ \\
\hline Phoenix-Mesa-Scottsdale, Arizona & 2,593 & 18.2 & 1.0 & $(16.1-20.2)$ \\
\hline Pittsburgh, Pennsylvania & 3,354 & 18.2 & 0.8 & $(16.5-19.8)$ \\
\hline Ponce, Puerto Rico & 547 & 14.6 & 1.8 & $(11.1-18.2)$ \\
\hline Portland-South Portland, Maine & 3,323 & 22.4 & 0.9 & $(20.7-24.2)$ \\
\hline $\begin{array}{l}\text { Portland-Vancouver-Hillsboro, } \\
\text { Oregon-Washington }\end{array}$ & 3,117 & 21.9 & 1.0 & $(20.0-23.8)$ \\
\hline $\begin{array}{l}\text { Providence-Warwick, Rhode } \\
\text { Island-Massachusetts }\end{array}$ & 8,090 & 20.1 & 0.7 & $(18.8-21.4)$ \\
\hline Provo-Orem, Utah & 1,744 & 19.1 & 1.1 & $(16.8-21.3)$ \\
\hline Raleigh, North Carolina & 943 & 15.2 & 1.4 & $(12.5-17.9)$ \\
\hline Rapid City, South Dakota & 1,052 & 17.4 & 1.6 & $(14.3-20.5)$ \\
\hline Reno, Nevada & 1,505 & 18.8 & 1.5 & $(15.8-21.8)$ \\
\hline
\end{tabular}

See table footnotes on page 117. 
TABLE 62. (Continued) Estimated prevalence of adults aged $\geq 18$ years told by a health care professional that they have a depressive disorder, ${ }^{*}$ by metropolitan and micropolitan statistical area Behavioral Risk Factor Surveillance System, United States, 2012

\begin{tabular}{|c|c|c|c|c|}
\hline MMSA(s) & $\begin{array}{l}\text { Sample } \\
\text { size }\end{array}$ & $\%$ & SE & $95 \% \mathrm{Cl}$ \\
\hline Richmond, Virginia & 1,011 & 13.9 & 1.6 & $(10.8-16.9)$ \\
\hline $\begin{array}{l}\text { Riverside-San Bernardino-Ontario, } \\
\text { California }\end{array}$ & 1,527 & 11.6 & 1.0 & $(9.5-13.6)$ \\
\hline $\begin{array}{l}\text { Rockingham County-Strafford County, } \\
\text { New Hampshire }\end{array}$ & 1,662 & 19.9 & 1.4 & $(17.2-22.5)$ \\
\hline Rutland, Vermont & 597 & 25.1 & 2.4 & $(20.3-29.9)$ \\
\hline $\begin{array}{l}\text { Sacramento-Roseville-Arden-Arcade, } \\
\text { California }\end{array}$ & 998 & 10.6 & 1.4 & $(7.9-13.3)$ \\
\hline St. Louis, Missouri-Illinois & 2,061 & 16.9 & 1.1 & $(14.7-19.1)$ \\
\hline Salisbury, Maryland-Delaware & 2,120 & 15.4 & 1.3 & $(12.8-18.0)$ \\
\hline Salt Lake City, Utah & 4,602 & 22.6 & 0.8 & $(21.0-24.2)$ \\
\hline San Antonio-New Braunfels, Texas & 776 & 16.0 & 1.7 & $(12.7-19.3)$ \\
\hline San Diego-Carlsbad, California & 1,127 & 11.5 & 1.3 & $(8.9-14.1)$ \\
\hline $\begin{array}{l}\text { San Francisco-Redwood City-South } \\
\text { San Francisco, California }{ }^{\dagger}\end{array}$ & 595 & 10.9 & 1.6 & $(7.7-14.0)$ \\
\hline $\begin{array}{l}\text { San Jose-Sunnyvale-Santa Clara, } \\
\text { California }\end{array}$ & 684 & 11.3 & 1.6 & $(8.2-14.5)$ \\
\hline San Juan-Carolina-Caguas, Puerto Rico & 3,951 & 16.1 & 0.7 & $(14.8-17.4)$ \\
\hline Santa Fe, New Mexico & 696 & 19.4 & 1.7 & $(16.0-22.8)$ \\
\hline Sayre, Pennsylvania & 1,859 & 15.6 & 2.2 & $(11.3-20.0)$ \\
\hline Scottsbluff, Nebraska & 630 & 17.3 & 2.4 & $(12.5-22.0)$ \\
\hline $\begin{array}{l}\text { Scranton-Wilkes-Barre-Hazleton, } \\
\text { Pennsylvania }\end{array}$ & 742 & 19.5 & 2.0 & $(15.6-23.3)$ \\
\hline Seattle-Bellevue-Everett, Washington ${ }^{\dagger}$ & 5,077 & 21.3 & 0.8 & $(19.8-22.8)$ \\
\hline Shreveport-Bossier City, Louisiana & 578 & 13.9 & 1.9 & $(10.3-17.6)$ \\
\hline $\begin{array}{l}\text { Silver Spring-Frederick-Rockville, } \\
\text { Maryland }{ }^{\dagger}\end{array}$ & 2,296 & 11.4 & 0.9 & $(9.6-13.1)$ \\
\hline $\begin{array}{l}\text { Sioux City, lowa-Nebraska- } \\
\text { South Dakota }\end{array}$ & 1,198 & 16.9 & 2.5 & $(11.9-21.8)$ \\
\hline Sioux Falls, South Dakota & 1,454 & 15.5 & 1.2 & $(13.1-17.8)$ \\
\hline Spartanburg, South Carolina & 706 & 25.2 & 2.3 & $(20.8-29.7)$ \\
\hline Spokane-Spokane Valley, Washington & 1,095 & 21.6 & 1.6 & $(18.4-24.8)$ \\
\hline Springfield, Massachusetts & 2,330 & 25.1 & 1.4 & $(22.3-27.8)$ \\
\hline
\end{tabular}

TABLE 62. (Continued) Estimated prevalence of adults aged $\geq 18$ years told by a health care professional that they have a depressive disorder,* by metropolitan and micropolitan statistical area Behavioral Risk Factor Surveillance System, United States, 2012

\begin{tabular}{|c|c|c|c|c|}
\hline MMSA(s) & $\begin{array}{l}\text { Sample } \\
\text { size }\end{array}$ & $\%$ & SE & $95 \% \mathrm{Cl}$ \\
\hline Tacoma-Lakewood, Washington ${ }^{\dagger}$ & 1,176 & 24.9 & 1.7 & $(21.6-28.2)$ \\
\hline $\begin{array}{l}\text { Tampa-St. Petersburg-Clearwater, } \\
\text { Florida }\end{array}$ & 821 & 16.6 & 1.8 & $(13.0-20.2)$ \\
\hline Toledo, Ohio & 969 & 19.4 & 2.2 & $(15.0-23.8)$ \\
\hline Topeka, Kansas & 1,080 & 18.9 & 1.5 & $(15.9-21.9)$ \\
\hline Torrington, Connecticut & 660 & 16.8 & 2.0 & $(12.9-20.6)$ \\
\hline Trenton, New Jersey & 575 & 16.2 & 2.3 & $(11.8-20.7)$ \\
\hline Tucson, Arizona & 994 & 20.9 & 1.8 & $(17.4-24.4)$ \\
\hline Tulsa, Oklahoma & 1,741 & 22.4 & 1.3 & $(19.8-24.9)$ \\
\hline Tuscaloosa, Alabama & 581 & 22.1 & 2.8 & $(16.5-27.6)$ \\
\hline Urban Honolulu, Hawaii & 4,328 & 10.7 & 0.7 & $(9.4-12.1)$ \\
\hline Vineland-Bridgeton, New Jersey & 556 & 15.2 & 2.1 & $(11.0-19.4)$ \\
\hline $\begin{array}{l}\text { Virginia Beach-Norfolk-Newport News, } \\
\text { Virginia-North Carolina }\end{array}$ & 1,494 & 16.7 & 1.4 & $(13.9-19.4)$ \\
\hline $\begin{array}{l}\text { Warren-Troy-Farmington Hills, } \\
\text { Michigan }{ }^{\dagger}\end{array}$ & 2,182 & 16.3 & 1.0 & $(14.4-18.3)$ \\
\hline $\begin{array}{l}\text { Washington-Arlington-Alexandria, } \\
\text { District of Columbia-Virginia- } \\
\text { Maryland-West Virginia }\end{array}$ & 8,166 & 13.3 & 0.6 & $(12.0-14.5)$ \\
\hline Wichita, Kansas & 2,350 & 16.8 & 1.0 & $(14.9-18.8)$ \\
\hline $\begin{array}{l}\text { Wilmington, Delaware-Maryland- } \\
\text { New Jersey }{ }^{\dagger}\end{array}$ & 3,230 & 15.6 & 0.9 & $(13.8-17.4)$ \\
\hline Winston-Salem, North Carolina & 791 & 21.6 & 2.0 & $(17.8-25.5)$ \\
\hline Worcester, Massachusetts-Connecticut & 2,832 & 19.3 & 1.1 & $(17.2-21.4)$ \\
\hline Yakima, Washington & 532 & 26.5 & 2.8 & $(21.0-32.0)$ \\
\hline $\begin{array}{l}\text { Youngstown-Warren-Boardman, } \\
\text { Ohio-Pennsylvania }\end{array}$ & 925 & 17.7 & 1.9 & $(14.0-21.4)$ \\
\hline Median & & 18.2 & & \\
\hline Range & & $9.2-28.3$ & & \\
\hline
\end{tabular}

Abbreviations: $\mathrm{Cl}=$ confidence interval; $\mathrm{MMSA}=$ metropolitan and micropolitan statistical area; $\mathrm{SE}=$ standard error.

* Including depression, major depression, dysthymia, or minor depression.

${ }^{\dagger}$ Metropolitan division. 
TABLE 63. Estimated prevalence of adults aged $\geq 18$ years told by a health care professional that they have a depressive disorder,* by county - Behavioral Risk Factor Surveillance System, United States, 2012

\begin{tabular}{|c|c|c|c|c|}
\hline County & $\begin{array}{l}\text { Sample } \\
\text { size }\end{array}$ & $\%$ & SE & $95 \% \mathrm{Cl}$ \\
\hline Jefferson County, Alabama & 956 & 17.7 & 1.4 & $(14.9-20.5)$ \\
\hline Madison County, Alabama & 513 & 21.4 & 2.3 & $(16.9-25.9)$ \\
\hline Mobile County, Alabama & 814 & 21.3 & 2.2 & $(17.0-25.5)$ \\
\hline Anchorage Municipality, Alaska & 895 & 18.1 & 1.5 & $(15.1-21.1)$ \\
\hline Fairbanks North Star Borough, Alaska & 594 & 17.8 & 1.9 & $(14.0-21.6)$ \\
\hline Matanuska-Susitna Borough, Alaska & 601 & 17.6 & 1.9 & $(13.9-21.4)$ \\
\hline Maricopa County, Arizona & 2,113 & 18.0 & 1.1 & $(15.9-20.2)$ \\
\hline Pima County, Arizona & 994 & 20.9 & 1.8 & $(17.4-24.4)$ \\
\hline Pulaski County, Arkansas & 747 & 22.7 & 2.1 & $(18.5-26.9)$ \\
\hline Alameda County, California & 618 & 12.7 & 1.8 & $(9.1-16.3)$ \\
\hline Los Angeles County, California & 3,499 & 10.6 & 0.7 & $(9.2-12.0)$ \\
\hline Orange County, California & 1,036 & 9.2 & 1.2 & $(6.9-11.5)$ \\
\hline Riverside County, California & 823 & 11.2 & 1.3 & $(8.6-13.8)$ \\
\hline Sacramento County, California & 609 & 10.3 & 1.7 & $(7.0-13.6)$ \\
\hline San Bernardino County, California & 704 & 11.9 & 1.6 & $(8.7-15.0)$ \\
\hline San Diego County, California & 1,127 & 11.5 & 1.3 & $(8.9-14.1)$ \\
\hline Santa Clara County, California & 665 & 11.6 & 1.6 & $(8.4-14.8)$ \\
\hline Adams County, Colorado & 808 & 19.4 & 1.8 & $(15.9-22.9)$ \\
\hline Arapahoe County, Colorado & 856 & 16.5 & 1.5 & $(13.7-19.4)$ \\
\hline Boulder County, Colorado & 515 & 18.3 & 2.2 & $(14.0-22.5)$ \\
\hline Denver County, Colorado & 994 & 17.9 & 1.4 & $(15.1-20.7)$ \\
\hline Douglas County, Colorado & 547 & 14.5 & 2.0 & $(10.7-18.3)$ \\
\hline El Paso County, Colorado & 1,019 & 18.0 & 1.5 & $(15.0-20.9)$ \\
\hline Jefferson County, Colorado & 1,117 & 16.4 & 1.3 & $(13.9-19.0)$ \\
\hline Larimer County, Colorado & 593 & 17.6 & 2.0 & $(13.8-21.4)$ \\
\hline Weld County, Colorado & 534 & 18.6 & 2.3 & $(14.1-23.0)$ \\
\hline Fairfield County, Connecticut & 2,176 & 15.2 & 1.2 & $(12.9-17.5)$ \\
\hline Hartford County, Connecticut & 1,977 & 18.6 & 1.1 & (16.4-20.7) \\
\hline Litchfield County, Connecticut & 660 & 16.8 & 1.9 & $(13.0-20.6)$ \\
\hline New Haven County, Connecticut & 2,007 & 16.4 & 1.1 & $(14.1-18.6)$ \\
\hline New London County, Connecticut & 997 & 16.9 & 1.9 & $(13.3-20.5)$ \\
\hline Kent County, Delaware & 1,437 & 14.2 & 1.3 & $(11.6-16.7)$ \\
\hline New Castle County, Delaware & 2,331 & 14.8 & 0.9 & (12.9-16.6) \\
\hline Sussex County, Delaware & 1,397 & 14.9 & 1.3 & $(12.4-17.4)$ \\
\hline $\begin{array}{l}\text { District of Columbia, } \\
\text { District of Columbia }\end{array}$ & 3,802 & 16.3 & 1.0 & $(14.3-18.3)$ \\
\hline Broward County, Florida & 525 & 13.6 & 2.1 & $(9.5-17.8)$ \\
\hline Miami-Dade County, Florida & 807 & 12.9 & 1.7 & $(9.6-16.1)$ \\
\hline Hawaii County, Hawaii & 1,344 & 14.2 & 1.4 & $(11.4-17.0)$ \\
\hline Honolulu County, Hawaii & 4,328 & 10.7 & 0.7 & $(9.4-12.1)$ \\
\hline Kauai County, Hawaii & 666 & 11.3 & 1.5 & $(8.4-14.2)$ \\
\hline Maui County, Hawaii & 1,214 & 13.1 & 1.3 & $(10.6-15.6)$ \\
\hline Ada County, Idaho & 809 & 20.2 & 2.1 & $(16.1-24.2)$ \\
\hline Canyon County, Idaho & 503 & 21.6 & 3.0 & $(15.8-27.4)$ \\
\hline Cook County, Illinois & 1,501 & 14.5 & 1.2 & $(12.1-16.9)$ \\
\hline Lake County, Indiana & 882 & 12.2 & 1.6 & $(9.0-15.4)$ \\
\hline Marion County, Indiana & 1,273 & 17.6 & 1.3 & $(15.0-20.2)$ \\
\hline Polk County, lowa & 806 & 19.9 & 1.8 & $(16.4-23.4)$ \\
\hline Johnson County, Kansas & 2,169 & 13.8 & 1.0 & $(11.8-15.7)$ \\
\hline Sedgwick County, Kansas & 1,791 & 16.4 & 1.1 & $(14.2-18.5)$ \\
\hline Shawnee County, Kansas & 762 & 21.6 & 1.9 & $(17.9-25.3)$ \\
\hline Wyandotte County, Kansas & 870 & 16.8 & 2.2 & $(12.4-21.1)$ \\
\hline Jefferson County, Kentucky & 1,690 & 23.8 & 1.9 & $(20.1-27.4)$ \\
\hline East Baton Rouge Parish, Louisiana & 570 & 18.6 & 2.3 & $(14.1-23.1)$ \\
\hline Androscoggin County, Maine & 697 & 28.4 & 2.1 & $(24.2-32.5)$ \\
\hline Aroostook County, Maine & 536 & 19.0 & 2.0 & $(15.2-22.9)$ \\
\hline Cumberland County, Maine & 1,754 & 23.5 & 1.3 & $(21.0-26.0)$ \\
\hline Kennebec County, Maine & 830 & 23.4 & 1.8 & $(19.9-26.8)$ \\
\hline Penobscot County, Maine & 926 & 23.1 & 1.7 & $(19.7-26.5)$ \\
\hline
\end{tabular}

See table footnotes on page 119.
TABLE 63. (Continued) Estimated prevalence of adults aged $\geq 18$ years told by a health care professional that they have a depressive disorder, ${ }^{*}$ by county - Behavioral Risk Factor Surveillance System, United States, 2012

\begin{tabular}{|c|c|c|c|c|}
\hline County & $\begin{array}{c}\text { Sample } \\
\text { size }\end{array}$ & $\%$ & SE & $95 \% \mathrm{Cl}$ \\
\hline York County, Maine & 1,189 & 20.8 & 1.4 & $(18.0-23.6)$ \\
\hline Anne Arundel County, Maryland & 892 & 16.1 & 1.8 & $(12.6-19.6)$ \\
\hline Baltimore County, Maryland & 1,518 & 15.8 & 1.4 & $(13.1-18.6)$ \\
\hline Charles County, Maryland & 512 & 11.1 & 1.9 & $(7.4-14.8)$ \\
\hline Frederick County, Maryland & 751 & 14.6 & 2.2 & $(10.2-19.0)$ \\
\hline Montgomery County, Maryland & 1,545 & 10.9 & 1.0 & $(9.0-12.9)$ \\
\hline Prince George's County, Maryland & 1,137 & 9.0 & 1.3 & $(6.4-11.6)$ \\
\hline Washington County, Maryland & 537 & 21.3 & 3.0 & $(15.4-27.2)$ \\
\hline Baltimore city, Maryland & 737 & 15.5 & 2.0 & $(11.6-19.3)$ \\
\hline Barnstable County, Massachusetts & 558 & 18.1 & 2.1 & $(13.9-22.2)$ \\
\hline Bristol County, Massachusetts & 2,643 & 20.8 & 1.4 & $(18.1-23.6)$ \\
\hline Essex County, Massachusetts & 2,557 & 17.8 & 1.3 & $(15.3-20.3)$ \\
\hline Hampden County, Massachusetts & 1,952 & 25.6 & 1.5 & $(22.6-28.6)$ \\
\hline Middlesex County, Massachusetts & 4,339 & 17.2 & 0.8 & $(15.6-18.9)$ \\
\hline Norfolk County, Massachusetts & 1,753 & 14.2 & 1.2 & $(11.8-16.7)$ \\
\hline Plymouth County, Massachusetts & 1,829 & 19.1 & 1.6 & $(16.0-22.3)$ \\
\hline Suffolk County, Massachusetts & 2,300 & 16.8 & 1.1 & $(14.6-19.0)$ \\
\hline Worcester County, Massachusetts & 2,587 & 19.1 & 1.1 & $(16.9-21.3)$ \\
\hline Kent County, Michigan & 524 & 18.0 & 2.4 & $(13.3-22.7)$ \\
\hline Macomb County, Michigan & 613 & 15.3 & 1.6 & $(12.2-18.5)$ \\
\hline Oakland County, Michigan & 1,166 & 15.9 & 1.4 & $(13.1-18.8)$ \\
\hline Wayne County, Michigan & 2,217 & 19.9 & 1.4 & $(17.2-22.6)$ \\
\hline Anoka County, Minnesota & 543 & 18.0 & 2.0 & $(14.0-21.9)$ \\
\hline Dakota County, Minnesota & 669 & 13.1 & 1.5 & $(10.2-16.1)$ \\
\hline Hennepin County, Minnesota & 3,292 & 17.5 & 0.9 & $(15.7-19.3)$ \\
\hline Ramsey County, Minnesota & 1,938 & 19.5 & 1.8 & $(15.9-23.0)$ \\
\hline Jackson County, Missouri & 881 & 17.9 & 2.1 & $(13.8-21.9)$ \\
\hline St. Louis County, Missouri & 948 & 14.8 & 1.5 & $(11.8-17.7)$ \\
\hline Cascade County, Montana & 704 & 21.6 & 1.9 & $(17.8-25.4)$ \\
\hline Flathead County, Montana & 559 & 19.5 & 2.0 & $(15.5-23.5)$ \\
\hline Hill County, Montana & 578 & 17.8 & 2.4 & $(13.1-22.5)$ \\
\hline Lake County, Montana & 896 & 20.8 & 2.1 & $(16.7-24.9)$ \\
\hline Missoula County, Montana & 781 & 23.9 & 1.9 & $(20.2-27.6)$ \\
\hline Yellowstone County, Montana & 751 & 22.6 & 1.8 & $(19.2-26.1)$ \\
\hline Dakota County, Nebraska & 729 & 23.2 & 4.7 & $(14.0-32.5)$ \\
\hline Douglas County, Nebraska & 3,566 & 18.0 & 0.9 & $(16.3-19.7)$ \\
\hline Hall County, Nebraska & 532 & 14.0 & 1.9 & $(10.4-17.7)$ \\
\hline Lancaster County, Nebraska & 1,444 & 18.7 & 1.2 & $(16.5-21.0)$ \\
\hline Lincoln County, Nebraska & 588 & 19.8 & 2.5 & $(14.8-24.7)$ \\
\hline Sarpy County, Nebraska & 1,175 & 16.8 & 1.4 & $(14.1-19.6)$ \\
\hline Scotts Bluff County, Nebraska & 577 & 17.2 & 2.5 & $(12.3-22.1)$ \\
\hline Clark County, Nevada & 2,007 & 15.4 & 0.9 & $(13.6-17.3)$ \\
\hline Washoe County, Nevada & 1,486 & 18.7 & 1.5 & $(15.7-21.8)$ \\
\hline Belknap County, New Hampshire & 565 & 20.7 & 2.7 & $(15.4-25.9)$ \\
\hline Carroll County, New Hampshire & 532 & 16.1 & 2.3 & $(11.6-20.5)$ \\
\hline Cheshire County, New Hampshire & 546 & 15.1 & 2.0 & $(11.2-19.0)$ \\
\hline Coos County, New Hampshire & 537 & 22.0 & 3.2 & $(15.8-28.2)$ \\
\hline Grafton County, New Hampshire & 565 & 22.1 & 2.6 & $(17.0-27.3)$ \\
\hline Hillsborough County, New Hampshire & 1,901 & 19.0 & 1.2 & $(16.7-21.3)$ \\
\hline Merrimack County, New Hampshire & 702 & 21.7 & 2.2 & $(17.3-26.0)$ \\
\hline Rockingham County, New Hampshire & 1,042 & 19.9 & 1.7 & $(16.6-23.3)$ \\
\hline Strafford County, New Hampshire & 620 & 19.6 & 2.2 & $(15.4-23.9)$ \\
\hline Atlantic County, New Jersey & 1,016 & 16.4 & 1.8 & $(12.8-19.9)$ \\
\hline Bergen County, New Jersey & 1,006 & 12.2 & 1.4 & $(9.5-14.8)$ \\
\hline Burlington County, New Jersey & 684 & 15.6 & 1.9 & $(11.9-19.3)$ \\
\hline Camden County, New Jersey & 741 & 19.0 & 2.0 & $(15.1-23.0)$ \\
\hline Cape May County, New Jersey & 551 & 19.1 & 3.1 & $(12.9-25.2)$ \\
\hline Cumberland County, New Jersey & 556 & 15.2 & 2.1 & $(11.0-19.4)$ \\
\hline Essex County, New Jersey & 1,335 & 11.8 & 1.1 & $(9.6-14.0)$ \\
\hline
\end{tabular}

See table footnotes on page 119. 
TABLE 63. (Continued) Estimated prevalence of adults aged $\geq 18$ years told by a health care professional that they have a depressive disorder, ${ }^{*}$ by county - Behavioral Risk Factor Surveillance System, United States, 2012

\begin{tabular}{|c|c|c|c|c|}
\hline County & $\begin{array}{l}\text { Sample } \\
\text { size }\end{array}$ & $\%$ & SE & $95 \% \mathrm{Cl}$ \\
\hline Gloucester County, New Jersey & 549 & 15.7 & 1.9 & $(11.9-19.4)$ \\
\hline Hudson County, New Jersey & 1,300 & 13.2 & 1.3 & -15.7) \\
\hline Hunterdon County, New Jersey & 570 & 12.8 & 1.9 & $(9.0$ \\
\hline Mercer County, New Jersey & 575 & 16.2 & 2.3 & $(11.8-20.7)$ \\
\hline Middlesex County, New Jersey & 882 & 10.2 & 1.3 & $(7.7-12.7)$ \\
\hline Monmouth County, New Jersey & 712 & 12.1 & 1.6 & $(9.0-15.2)$ \\
\hline Morris County, New Jersey & 847 & 10.3 & 1.4 & $(7.7-13.0)$ \\
\hline Ocean County, New Jersey & 662 & 13.4 & 1.8 & $(9.9-16.9)$ \\
\hline Passaic County, New Jersey & 683 & 12.9 & 1.6 & $(9.8-16.0)$ \\
\hline Salem County, New Jersey & 585 & 17.9 & 2.2 & $(13.6-22.3)$ \\
\hline Somerset County, New Jersey & 639 & 9.0 & 1.4 & $(6.2-11.8)$ \\
\hline Sussex County, New Jersey & 546 & 17.8 & 2.5 & $(12.9-22.8)$ \\
\hline Union County, New Jersey & 711 & 8.5 & 1.2 & $(6.2-10.8)$ \\
\hline Warren County, New Jersey & 533 & 17.6 & 2.4 & $(12.8-22.3)$ \\
\hline Bernalillo County, New Mexico & 2,057 & 22.5 & 1.1 & $(20.4-24.7)$ \\
\hline Dona Ana County, New Mexico & 705 & 20.8 & 1.9 & $(17.1-24.4)$ \\
\hline Sandoval County, New Mexico & 653 & 16.5 & 1.8 & $(13$. \\
\hline San Juan County, New Mexico & 647 & 19.1 & 1.9 & $(15$ \\
\hline Santa Fe County, New Mexico & 696 & 19.4 & 1.7 & $(16$. \\
\hline Kings County, New York & 495 & 16.1 & 2.3 & $(11.5-20.6)$ \\
\hline Guilford County, North Carolina & 510 & 17.0 & 2.1 & $(12.8-21.1)$ \\
\hline Mecklenburg County, North Carolina & 728 & 11.8 & 1.4 & $(8.9-14.6)$ \\
\hline Robeson County, North Carolina & 545 & 19.1 & 3.3 & $(12.7-25.6)$ \\
\hline Wake County, North Carolina & 701 & 14.1 & 1.6 & $(11.1-17.1)$ \\
\hline Burleigh County, North Dakota & 564 & 15.4 & 2.1 & $(11.2-19.6)$ \\
\hline Cass County, North Dakota & 838 & 16.5 & 1.9 & $(12.8-20.3)$ \\
\hline Cuyahoga County, Ohio & 864 & 18.9 & 1.8 & $(15.5-22.4)$ \\
\hline Franklin County, Ohio & 837 & 19.0 & 1.7 & $(15.7-22.2)$ \\
\hline Har & 771 & 19.3 & 1.8 & $(15.9-$ \\
\hline Lorain County, Ohio & 615 & 18.3 & 2.5 & $(13.5-23.2)$ \\
\hline Lucas County, Ohio & 621 & 18.7 & 2.8 & 13.3-24.2) \\
\hline Mahoning County, Ohio & 589 & 19.7 & 2.8 & $(14.2-25.2)$ \\
\hline Montgomery County, Ohio & 660 & 18.9 & 2.0 & $(14.9-22.8)$ \\
\hline Stark County, Ohio & 623 & 20.3 & 2.4 & $(15.6-24.9)$ \\
\hline Summit County, Ohio & 630 & 20.2 & 2.3 & $(15.7-24.7)$ \\
\hline Oklahoma County, Oklahoma & 1,183 & 20.5 & 1.4 & $(17.7-23.4)$ \\
\hline Tulsa County, Oklahoma & 1,201 & 20.6 & 1.4 & $(17.7-23.4)$ \\
\hline Clackamas County, Oregon & 508 & 24.3 & 2.5 & $(19.4-29.2)$ \\
\hline Lane County, Oregon & 523 & 24.2 & 2.4 & $(19.4-29.0)$ \\
\hline Multnomah County, Oregon & 890 & 22.0 & 1.8 & $(18.4-25.5)$ \\
\hline Washington County, Oregon & 615 & 21.9 & 2.1 & $(17.8-26.0)$ \\
\hline Allegheny County, Pennsylvania & 1,881 & 18.0 & 1.2 & $(15.8-20.3)$ \\
\hline Bradford County, Pennsylvania & 1,859 & 15.6 & 2.2 & $(11.3-20.0)$ \\
\hline Montgomery County, Pennsylvania & 544 & 12.2 & 1.5 & $(9.3-15.1)$ \\
\hline Philadelphia County, Pennsylvania & 2,056 & 19.2 & 1.2 & $(16.9-21.6)$ \\
\hline Pike County, Pennsylvania & 1,889 & 13.3 & 1.0 & $(11.3-15.4)$ \\
\hline Kent County, Rhode Island & 809 & 21.1 & 2.0 & $(17.1-25.1)$ \\
\hline Providence County, Rhode Islan & 3,325 & 21.9 & 0.9 & $(20.1-23.8)$ \\
\hline Washington County, Rhode Island & 653 & 12.3 & 1.6 & $(9.2-15.4)$ \\
\hline
\end{tabular}

TABLE 63. (Continued) Estimated prevalence of adults aged $\geq 18$ years told by a health care professional that they have a depressive disorder, ${ }^{*}$ by county - Behavioral Risk Factor Surveillance System, United States, 2012

\begin{tabular}{|c|c|c|c|c|}
\hline County & $\begin{array}{l}\text { Sample } \\
\text { size }\end{array}$ & $\%$ & SE & $95 \% \mathrm{Cl}$ \\
\hline Aiken County, South Carolina & 552 & 20.9 & 2.4 & $(16.2-25.6)$ \\
\hline Beaufort County, South Carolina & 799 & 12.0 & 1.9 & $(8.4-15.7)$ \\
\hline Charleston County, South Carolina & 1,012 & 16.7 & 1.7 & $(13.3-20.1)$ \\
\hline Greenville County, South Carolina & 898 & 17.8 & 1.6 & $(14.7-21.0)$ \\
\hline Horry County, South Carolina & 776 & 15.9 & 1.6 & $(12.8-19.1)$ \\
\hline Richland County, South Carolina & 958 & 16.0 & 1.8 & $(12.5-19.5)$ \\
\hline Spartanburg County, South Carolina & 652 & 24.8 & 2.3 & $(20.2-29.4)$ \\
\hline Lincoln County, South Dakota & 496 & 12.0 & 1.8 & $(8.4-15.5)$ \\
\hline Minnehaha County, South Dakota & 870 & 16.4 & 1.4 & $(13.6-19.3)$ \\
\hline Pennington County, South Dakota & 603 & 17.5 & 1.8 & $(13.9-21.0)$ \\
\hline Davidson County, Tennessee & 553 & 15.4 & 1.8 & $(12.0-18.9)$ \\
\hline Shelby County, Tennessee & 535 & 18.2 & 2.1 & $(14.1-22.4)$ \\
\hline Bexar County, Texas & 584 & 16.4 & 2.0 & $(12.5-20.3)$ \\
\hline Dallas County, Texas & 510 & 13.4 & 1.8 & $(9.9-16.9)$ \\
\hline El Paso County, Texas & 620 & 17.2 & 2.3 & $(12.7-21.7)$ \\
\hline Harris County, Texas & 771 & 17.2 & 1.7 & $(13.9-20.4)$ \\
\hline Hidalgo County, Texas & 621 & 14.4 & 2.1 & $(10.2-18.5)$ \\
\hline Tarrant County, Texas & 572 & 13.8 & 1.9 & $(10.1-17.5)$ \\
\hline Travis County, Texas & 1,045 & 14.9 & 1.7 & $(11.6-18.1)$ \\
\hline Davis County, Utah & 1,145 & 20.7 & 1.4 & $(18.0-23.4)$ \\
\hline Salt Lake County, Utah & 4,040 & 22.7 & 0.9 & $(21.0-24.4)$ \\
\hline Tooele County, Utah & 562 & 20.2 & 2.7 & $(14.8-25.6)$ \\
\hline Utah County, Utah & 1,676 & 19.1 & 1.2 & $(16.8-21.4)$ \\
\hline Wasatch County, Utah & 510 & 20.4 & 4.7 & $(11.2-29.7)$ \\
\hline Weber County, Utah & 1,046 & 22.9 & 1.7 & $(19.6-26.1)$ \\
\hline Chittenden County, Vermont & 917 & 20.4 & 1.6 & $(17.3-23.5)$ \\
\hline Rutland County, Vermont & 597 & 25.1 & 2.4 & $(20.3-29.9)$ \\
\hline Washington County, Vermont & 514 & 25.2 & 2.5 & $(20.3-30.0)$ \\
\hline Windsor County, Vermont & 543 & 21.7 & 2.2 & $(17.4-26.0)$ \\
\hline Fairfax County, Virginia & 740 & 12.5 & 1.6 & $(9.4-15.6)$ \\
\hline Clark County, Washington & 790 & 22.3 & 1.9 & $(18.7-25.9)$ \\
\hline King County, Washington & 3,910 & 20.3 & 0.9 & $(18.6-22.0)$ \\
\hline Kitsap County, Washington & 565 & 24.6 & 2.3 & $(20.1-29.1)$ \\
\hline Pierce County, Washington & 1,176 & 24.9 & 1.7 & $(21.6-28.2)$ \\
\hline Snohomish County, Washington & 1,167 & 24.1 & 1.6 & $(20.9-27.3)$ \\
\hline Spokane County, Washington & 949 & 22.0 & 1.8 & $(18.5-25.6)$ \\
\hline Thurston County, Washington & 514 & 23.9 & 2.5 & $(19.0-28.9)$ \\
\hline Whatcom County, Washington & 840 & 23.1 & 2.4 & $(18.3-27.8)$ \\
\hline Yakima County, Washington & 532 & 26.5 & 2.8 & $(21.0-32.0)$ \\
\hline Kanawha County, West Virginia & 640 & 20.1 & 1.8 & $(16.5-23.7)$ \\
\hline Milwaukee County, Wisconsin & 957 & 18.8 & 2.0 & $(15.0-22.7)$ \\
\hline Laramie County, Wyoming & 953 & 17.4 & 1.9 & $(13.7-21.1)$ \\
\hline Natrona County, Wyoming & 826 & 21.8 & 2.2 & $(17.5-26.2)$ \\
\hline San Juan Municipio, Puerto Rico & 667 & 10.8 & 1.4 & $(8.0-13.5)$ \\
\hline Median & & 17.8 & & \\
\hline Range & & $8.5-28.4$ & & \\
\hline
\end{tabular}

Abbreviations: $\mathrm{Cl}=$ confidence interval; $\mathrm{SE}=$ standard error.

* Including depression, major depression, dysthymia, or minor depression. 
TABLE 64. Estimated prevalence of adults aged $\geq 45$ years who have ever been told by a health care professional that they have coronary heart disease,* by state/territory - Behavioral Risk Factor Surveillance System, United States, 2012

\begin{tabular}{|c|c|c|c|c|}
\hline State/Territory & $\begin{array}{l}\text { Sample } \\
\text { size }\end{array}$ & $\%$ & SE & $95 \% \mathrm{Cl}$ \\
\hline Alabama & 6,783 & 14.8 & 0.6 & $(13.6-15.9)$ \\
\hline Alaska & 2,624 & 8.9 & 0.7 & $(7.4-10.3)$ \\
\hline Arizona & 5,489 & 11.4 & 0.7 & $(10.1-12.8)$ \\
\hline Arkansas & 3,924 & 14.6 & 0.7 & $(13.2-16.0)$ \\
\hline California & 9,640 & 9.0 & 0.4 & $(8.2-9.8)$ \\
\hline Colorado & 8,646 & 7.7 & 0.3 & $(7.0-8.4)$ \\
\hline Connecticut & 6,189 & 9.2 & 0.5 & $(8.3-10.2)$ \\
\hline Delaware & 3,640 & 12.3 & 0.7 & $(10.8-13.8)$ \\
\hline District of Columbia & 2,665 & 9.8 & 0.8 & $(8.2-11.3)$ \\
\hline Florida & 5,776 & 13.1 & 0.7 & $(11.8-14.4)$ \\
\hline Georgia & 4,402 & 12.3 & 0.7 & (11.0-13.6) \\
\hline Hawaii & 4,841 & 7.4 & 0.5 & $(6.3-8.4)$ \\
\hline Idaho & 4,497 & 9.7 & 0.7 & $(8.4-11.0)$ \\
\hline Illinois & 4,069 & 10.8 & 0.6 & $(9.6-12.1)$ \\
\hline Indiana & 6,006 & 13.1 & 0.5 & $(12.0-14.2)$ \\
\hline lowa & 5,176 & 11.8 & 0.5 & $(10.8-12.8)$ \\
\hline Kansas & 8,580 & 11.6 & 0.4 & $(10.8-12.4)$ \\
\hline Kentucky & 8,212 & 15.6 & 0.6 & $(14.4-16.8)$ \\
\hline Louisiana & 6,899 & 13.3 & 0.6 & $(12.2-14.5)$ \\
\hline Maine & 7,302 & 11.6 & 0.5 & $(10.7-12.4)$ \\
\hline Maryland & 9,615 & 10.4 & 0.4 & $(9.5-11.3)$ \\
\hline Massachusetts & 15,204 & 10.1 & 0.4 & $(9.4-10.8)$ \\
\hline Michigan & 7,743 & 12.9 & 0.5 & (11.9-13.9) \\
\hline Minnesota & 8,405 & 9.3 & 0.4 & $(8.5-10.1)$ \\
\hline Mississippi & 5,917 & 13.5 & 0.6 & $(12.4-14.7)$ \\
\hline Missouri & 4,997 & 12.1 & 0.6 & (10.9-13.3) \\
\hline Montana & 6,267 & 10.4 & 0.5 & $(9.5-11.3)$ \\
\hline Nebraska & 13,948 & 10.8 & 0.4 & $(10.1-11.5)$ \\
\hline Nevada & 3,349 & 11.1 & 0.8 & $(9.5-12.7)$ \\
\hline New Hampshire & 5,780 & 9.9 & 0.5 & $(8.9-10.8)$ \\
\hline New Jersey & 10,740 & 10.3 & 0.4 & $(9.5-11.0)$ \\
\hline New Mexico & 6,059 & 10.5 & 0.5 & $(9.6-11.5)$ \\
\hline New York & 3,978 & 12.0 & 0.8 & (10.4-13.7) \\
\hline North Carolina & 8,106 & 11.5 & 0.4 & $(10.7-12.4)$ \\
\hline North Dakota & 3,509 & 11.5 & 0.7 & $(10.2-12.8)$ \\
\hline Ohio & 9,504 & 12.9 & 0.5 & $(12.0-13.8)$ \\
\hline Oklahoma & 5,679 & 14.1 & 0.6 & $(13.0-15.2)$ \\
\hline Oregon & 3,908 & 11.4 & 0.6 & $(10.1-12.7)$ \\
\hline Pennsylvania & 14,406 & 11.7 & 0.4 & $(11.0-12.5)$ \\
\hline Rhode Island & 3,905 & 10.9 & 0.6 & $(9.7-12.0)$ \\
\hline South Carolina & 9,362 & 13.3 & 0.5 & $(12.2-14.3)$ \\
\hline South Dakota & 5,386 & 10.7 & 0.7 & $(9.4-12.0)$ \\
\hline Tennessee & 5,232 & 17.4 & 0.8 & (15.9-18.9) \\
\hline Texas & 6,006 & 10.4 & 0.5 & $(9.4-11.4)$ \\
\hline Utah & 7,723 & 9.0 & 0.4 & $(8.2-9.8)$ \\
\hline Vermont & 4,533 & 10.0 & 0.5 & $(9.0-11.1)$ \\
\hline Virginia & 5,168 & 11.8 & 0.5 & $(10.7-12.8)$ \\
\hline Washington & 11,070 & 9.6 & 0.3 & $(8.9-10.3)$ \\
\hline West Virginia & 3,900 & 19.0 & 0.7 & (17.6-20.4) \\
\hline Wisconsin & 3,851 & 10.5 & 0.8 & $(9.0-12.0)$ \\
\hline Wyoming & 4,914 & 10.1 & 0.6 & $(8.9-11.2)$ \\
\hline Guam & 890 & 11.8 & 1.6 & $(8.7-15.0)$ \\
\hline Puerto Rico & 3,986 & 16.7 & 0.7 & $(15.3-18.1)$ \\
\hline Median & & 11.4 & & \\
\hline Range & & $7.4-19.0$ & & \\
\hline
\end{tabular}

Abbreviations: $\mathrm{Cl}=$ confidence interval; $\mathrm{SE}=$ standard error.

* Including heart attack, also known as myocardial infarction, and angina.
TABLE 65. Estimated prevalence of adults aged $\geq 45$ years who have ever been told by a health care professional that they have coronary heart disease,* by metropolitan and micropolitan statistical area — Behavioral Risk Factor Surveillance System, United States, 2012

\begin{tabular}{|c|c|c|c|c|}
\hline $\operatorname{MMSA}(\mathrm{s})$ & $\begin{array}{l}\text { Sample } \\
\text { size }\end{array}$ & $\%$ & SE & $95 \% \mathrm{Cl}$ \\
\hline Aguadilla-Isabela, Puerto Rico & 345 & 14.9 & 2.1 & $(10.9-19.0)$ \\
\hline Akron, Ohio & 557 & 9.9 & 1.6 & $(6.8-12.9)$ \\
\hline Albuquerque, New Mexico & 2,178 & 9.2 & 0.8 & $(7.7-10.7)$ \\
\hline $\begin{array}{l}\text { Allentown-Bethlehem-Easton, } \\
\text { Pennsylvania-New Jersey }\end{array}$ & 995 & 13.4 & 1.7 & $(10.1-16.6)$ \\
\hline Anaheim-Santa Ana-Irvine, California ${ }^{\dagger}$ & 677 & 7.2 & 1.1 & $(5.1-9.3)$ \\
\hline Anchorage, Alaska & 864 & 8.4 & 1.1 & $(6.1-10.6)$ \\
\hline Asheville, North Carolina & 439 & 13.4 & 2.1 & $(9.2-17.6)$ \\
\hline $\begin{array}{l}\text { Atlanta-Sandy Springs-Roswell, } \\
\text { Georgia }\end{array}$ & 1,737 & 10.5 & 1.0 & $(8.5-12.5)$ \\
\hline Atlantic City-Hammonton, New Jersey & 787 & 13.4 & 1.5 & $(10.4-16.4)$ \\
\hline $\begin{array}{l}\text { Augusta-Richmond County, Georgia- } \\
\text { South Carolina }\end{array}$ & 795 & 7.8 & 1.3 & $(5.3-10.3)$ \\
\hline Augusta-Waterville, Maine & 580 & 12.4 & 1.6 & $(9.2-15.6)$ \\
\hline Austin-Round Rock, Texas & 961 & 7.8 & 1.1 & $(5.5-10.0)$ \\
\hline $\begin{array}{l}\text { Baltimore-Columbia-Towson, } \\
\text { Maryland }\end{array}$ & 3,429 & 11.3 & 0.7 & $(9.9-12.7)$ \\
\hline Bangor, Maine & 659 & 12.5 & 1.4 & $(9.6-15.3)$ \\
\hline Barnstable Town, Massachusetts & 468 & 11.1 & 1.6 & $(8.0-14.3)$ \\
\hline Barre, Vermont & 386 & 10.7 & 1.9 & $(7.0-14.5)$ \\
\hline Baton Rouge, Louisiana & 1,043 & 12.2 & 1.4 & $(9.5-14.9)$ \\
\hline Bellingham, Washington & 619 & 8.4 & 1.4 & $(5.6-11.3)$ \\
\hline Berlin, New Hampshire-Vermont & 570 & 13.5 & 1.9 & $(9.7-17.3)$ \\
\hline Billings, Montana & 549 & 11.1 & 1.4 & $(8.3-13.9)$ \\
\hline Birmingham-Hoover, Alabama & 1,301 & 12.0 & 1.1 & $(9.8-14.2)$ \\
\hline Bismarck, North Dakota & 582 & 14.0 & 1.8 & $(10.5-17.6)$ \\
\hline Boise City, Idaho & 1,078 & 7.9 & 1.1 & $(5.7-10.1)$ \\
\hline Boston, Massachusetts ${ }^{\dagger}$ & 4,015 & 10.4 & 0.8 & $(8.9-11.9)$ \\
\hline Boulder, Colorado & 355 & 6.1 & 1.3 & $(3.5-8.7)$ \\
\hline Bremerton-Silverdale, Washington & 425 & 8.8 & 1.5 & $(5.9-11.8)$ \\
\hline $\begin{array}{l}\text { Bridgeport-Stamford-Norwalk, } \\
\text { Connecticut }\end{array}$ & 1,450 & 8.1 & 0.9 & $(6.4-9.8)$ \\
\hline Burlington-South Burlington, Vermont & 1,024 & 8.4 & 1.0 & $(6.4-10.3)$ \\
\hline $\begin{array}{l}\text { Cambridge-Newton-Framingham, } \\
\text { Massachusetts }{ }^{\dagger}\end{array}$ & 4,741 & 9.5 & 0.6 & $(8.3-10.7)$ \\
\hline Camden, New Jersey ${ }^{\dagger}$ & 1,408 & 11.6 & 1.1 & $(9.4-13.8)$ \\
\hline Canton-Massillon, Ohio & 531 & 14.0 & 2.0 & $(10.2-17.9)$ \\
\hline Casper, Wyoming & 647 & 10.2 & 1.6 & $(7.0-13.3)$ \\
\hline Cedar Rapids, lowa & 409 & 8.0 & 1.4 & $(5.2-10.7)$ \\
\hline Charleston, West Virginia & 536 & 23.3 & 2.1 & $(19.1-27.4)$ \\
\hline $\begin{array}{l}\text { Charleston-North Charleston, } \\
\text { South Carolina }\end{array}$ & 1,209 & 12.1 & 1.3 & $(9.5-14.7)$ \\
\hline $\begin{array}{l}\text { Charlotte-Concord-Gastonia, } \\
\text { North Carolina-South Carolina }\end{array}$ & 1,607 & 10.2 & 0.9 & $(8.4-12.0)$ \\
\hline Chattanooga, Tennessee-Georgia & 497 & 17.6 & 2.2 & $(13.2-22.0)$ \\
\hline Cheyenne, Wyoming & 753 & 10.1 & 1.7 & $(6.8-13.4)$ \\
\hline $\begin{array}{l}\text { Chicago-Naperville-Elgin, } \\
\text { Illinois-Indiana-Wisconsin }\end{array}$ & 2,572 & 8.6 & 0.8 & $(7.0-10.2)$ \\
\hline Cincinnati, Ohio-Kentucky-Indiana & 1,638 & 12.9 & 1.1 & $(10.8-14.9)$ \\
\hline $\begin{array}{l}\text { Claremont-Lebanon, New } \\
\text { Hampshire-Vermont }\end{array}$ & 1,505 & 9.3 & 0.8 & $(7.7-11.0)$ \\
\hline Cleveland-Elyria, Ohio & 1,295 & 11.4 & 1.2 & $(9.0-13.7)$ \\
\hline Colorado Springs, Colorado & 763 & 7.3 & 1.0 & $(5.3-9.4)$ \\
\hline Columbia, South Carolina & 1,240 & 14.6 & 1.6 & $(11.5-17.8)$ \\
\hline Columbus, Ohio & 1,021 & 13.4 & 1.4 & $(10.8-16.1)$ \\
\hline Concord, New Hampshire & 525 & 9.0 & 1.5 & $(6.0-11.9)$ \\
\hline Dallas-Plano-Irving, Texas $^{\dagger}$ & 525 & 10.2 & 1.4 & $(7.4-13.0)$ \\
\hline Dayton, Ohio & 613 & 12.5 & 1.7 & $(9.2-15.9)$ \\
\hline Denver-Aurora-Lakewood, Colorado & 3,175 & 7.0 & 0.5 & $(6.0-8.0)$ \\
\hline Des Moines-West Des Moines, lowa & 782 & 9.6 & 1.2 & $(7.2-12.0)$ \\
\hline
\end{tabular}

See table footnotes on page 122. 
TABLE 65. (Continued) Estimated prevalence of adults aged $\geq 45$ years who have ever been told by a health care professional that they have coronary heart disease, ${ }^{*}$ by metropolitan and micropolitan statistical area - Behavioral Risk Factor Surveillance System, United States, 2012

\begin{tabular}{|c|c|c|c|c|}
\hline MMSA(s) & $\begin{array}{l}\text { Sample } \\
\text { size }\end{array}$ & $\%$ & SE & $95 \% \mathrm{Cl}$ \\
\hline Detroit-Dearborn-Livonia, Michigan ${ }^{\dagger}$ & 1,608 & 13.2 & 1.4 & $(10.5-15.9)$ \\
\hline Dover, Delaware & 1,030 & 11.8 & 1.3 & $(9.3-14.3)$ \\
\hline Duluth, Minnesota-Wisconsin & 380 & 12.3 & 2.1 & $(8.2-16.4)$ \\
\hline Durham-Chapel Hill, North Carolina & 512 & 6.7 & 1.2 & $(4.3-9.1)$ \\
\hline El Paso, Texas & 440 & 10.2 & 2.6 & $(5.1-15.3)$ \\
\hline Eugene, Oregon & 391 & 9.3 & 1.7 & $(6.0-12.6)$ \\
\hline Fairbanks, Alaska & 329 & 12.2 & 2.3 & $(7.7-16.7)$ \\
\hline Fargo, North Dakota-Minnesota & 630 & 9.2 & 1.6 & $(6.0-12.3)$ \\
\hline Farmington, New Mexico & 425 & 10.1 & 1.7 & $(6.8-13.4)$ \\
\hline Fayetteville, North Carolina & 319 & 14.2 & 2.5 & $(9.2-19.1)$ \\
\hline $\begin{array}{l}\text { Fayetteville-Springdale-Rogers, } \\
\text { Arkansas-Missouri }\end{array}$ & 588 & 14.6 & 2.0 & $(10.7-18.5)$ \\
\hline Fort Collins, Colorado & 414 & 9.0 & 1.7 & $(5.7-12.3)$ \\
\hline Fort Wayne, Indiana & 353 & 13.4 & 2.5 & $(8.5-18.4)$ \\
\hline Fort Worth-Arlington, Texas ${ }^{\dagger}$ & 502 & 10.6 & 1.6 & $(7.4-13.7)$ \\
\hline Grand Island, Nebraska & 633 & 9.8 & 1.3 & $(7.2-12.3)$ \\
\hline Grand Rapids-Wyoming, Michigan & 654 & 11.0 & 1.4 & $(8.2-13.8)$ \\
\hline Great Falls, Montana & 542 & 11.9 & 1.6 & $(8.7-15.1)$ \\
\hline Greeley, Colorado & 338 & 9.2 & 1.7 & $(5.9-12.5)$ \\
\hline $\begin{array}{l}\text { Greensboro-High Point, } \\
\text { North Carolina }\end{array}$ & 555 & 12.6 & 1.6 & $(9.4-15.8)$ \\
\hline $\begin{array}{l}\text { Greenville-Anderson-Mauldin, } \\
\text { South Carolina }\end{array}$ & 1,142 & 14.5 & 1.4 & $(11.9-17.2)$ \\
\hline Gulfport-Biloxi-Pascagoula, Mississippi & i 547 & 16.5 & 1.9 & $(12.9-20.2)$ \\
\hline $\begin{array}{l}\text { Hagerstown-Martinsburg, Maryland- } \\
\text { West Virginia }\end{array}$ & 571 & 15.1 & 2.3 & $(10.6-19.6)$ \\
\hline Harrisburg-Carlisle, Pennsylvania & 444 & 12.3 & 1.8 & $(8.8-15.9)$ \\
\hline $\begin{array}{l}\text { Hartford-West Hartford-East Hartford, } \\
\text { Connecticut }\end{array}$ & 1,869 & 8.9 & 0.8 & $(7.3-10.5)$ \\
\hline Heber, Utah & 358 & 8.0 & 1.4 & $(5.3-10.8)$ \\
\hline Hilo, Hawaii & 941 & 9.4 & 1.5 & $(6.6-12.3)$ \\
\hline $\begin{array}{l}\text { Hilton Head Island-Bluffton-Beaufort, } \\
\text { South Carolina }\end{array}$ & 792 & 9.6 & 1.4 & $(6.8-12.5)$ \\
\hline $\begin{array}{l}\text { Houston-The Woodlands-Sugar Land, } \\
\text { Texas }\end{array}$ & 619 & 8.9 & 1.3 & $(6.4-11.4)$ \\
\hline $\begin{array}{l}\text { Huntington-Ashland, West } \\
\text { Virginia-Kentucky-Ohio }\end{array}$ & 809 & 20.0 & 1.7 & $(16.7-23.4)$ \\
\hline Huntsville, Alabama & 416 & 13.6 & 2.2 & $(9.3-17.8)$ \\
\hline Idaho Falls, Idaho & 393 & 11.4 & 2.8 & $(5.9-16.9)$ \\
\hline $\begin{array}{l}\text { Indianapolis-Carmel-Anderson, } \\
\text { Indiana }\end{array}$ & 1,465 & 11.0 & 1.0 & $(9.1-12.9)$ \\
\hline Jackson, Mississippi & 637 & 11.6 & 1.5 & $(8.7-14.5)$ \\
\hline Jacksonville, Florida & 379 & 12.0 & 2.1 & $(7.8-16.2)$ \\
\hline Kahului-Wailuku-Lahaina, Hawaii & 857 & 6.3 & 1.0 & $(4.4-8.3)$ \\
\hline Kalispell, Montana & 398 & 8.8 & 1.6 & $(5.7-11.9)$ \\
\hline Kansas City, Missouri-Kansas & 3,497 & 10.7 & 1.0 & $(8.7-12.6)$ \\
\hline Kapaa, Hawaii & 534 & 8.1 & 1.5 & $(5.1-11.1)$ \\
\hline Keene, New Hampshire & 440 & 11.0 & 1.8 & $(7.4-14.6)$ \\
\hline Kennewick-Richland, Washington & 370 & 11.3 & 2.0 & $(7.4-15.2)$ \\
\hline $\begin{array}{l}\text { Kingsport-Bristol-Bristol, } \\
\text { Tennessee-Virginia }\end{array}$ & 457 & 21.4 & 2.9 & $(15.7-27.2)$ \\
\hline Knoxville, Tennessee & 625 & 17.0 & 2.0 & $(13.2-20.8)$ \\
\hline Laconia, New Hampshire & 471 & 10.7 & 2.0 & $(6.8-14.7)$ \\
\hline Lafayette, Louisiana & 396 & 13.9 & 2.3 & $(9.5-18.4)$ \\
\hline Las Cruces, New Mexico & 475 & 12.4 & 1.8 & $(8.9-15.9)$ \\
\hline $\begin{array}{l}\text { Las Vegas-Henderson-Paradise, } \\
\text { Nevada }\end{array}$ & 1,210 & 11.3 & 1.1 & $(9.2-13.4)$ \\
\hline Lewiston-Auburn, Maine & 466 & 11.8 & 1.8 & $(8.2-15.4)$ \\
\hline Lexington-Fayette, Kentucky & 320 & 11.3 & 2.0 & $(7.4-15.3)$ \\
\hline Lincoln, Nebraska & 950 & 9.2 & 1.1 & $(7.0-11.5)$ \\
\hline
\end{tabular}

See table footnotes on page 122 .
TABLE 65. (Continued) Estimated prevalence of adults aged $\geq 45$ years who have ever been told by a health care professional that they have coronary heart disease, ${ }^{*}$ by metropolitan and micropolitan statistical area - Behavioral Risk Factor Surveillance System, United States, 2012

\begin{tabular}{|c|c|c|c|c|}
\hline MMSA(s) & $\begin{array}{l}\text { Sample } \\
\text { size }\end{array}$ & $\%$ & SE & $95 \% \mathrm{Cl}$ \\
\hline $\begin{array}{l}\text { Little Rock-North Little Rock-Conway, } \\
\text { Arkansas }\end{array}$ & 857 & 12.3 & 1.4 & $(9.7-15.0)$ \\
\hline Logan, Utah-Idaho & 294 & 7.7 & 1.7 & $(4.3-11.1)$ \\
\hline $\begin{array}{l}\text { Los Angeles-Long Beach-Glendale, } \\
\text { California }^{\dagger}\end{array}$ & 2,126 & 8.5 & 0.9 & $(6.7-10.4)$ \\
\hline $\begin{array}{l}\text { Louisville/Jefferson County, } \\
\text { Kentucky-Indiana }\end{array}$ & 1,567 & 15.0 & 1.4 & $(12.3-17.7)$ \\
\hline Lumberton, North Carolina & 402 & 18.0 & 4.1 & $(10.0-26.0)$ \\
\hline Manchester-Nashua, New Hampshire & 1,367 & 9.8 & 1.0 & $(7.9-11.7)$ \\
\hline McAllen-Edinburg-Mission, Texas & 386 & 12.9 & 2.4 & $(8.2-17.5)$ \\
\hline $\begin{array}{l}\text { Memphis, } \\
\text { Tennessee-Mississippi-Arkansas }\end{array}$ & 930 & 16.2 & 1.8 & $(12.7-19.8)$ \\
\hline $\begin{array}{l}\text { Miami-Fort Lauderdale-West Palm } \\
\text { Beach, Florida }\end{array}$ & 1,183 & 8.8 & 1.1 & $(6.7-11.0)$ \\
\hline $\begin{array}{l}\text { Milwaukee-Waukesha-West Allis, } \\
\text { Wisconsin }\end{array}$ & 862 & 12.0 & 1.8 & $(8.5-15.6)$ \\
\hline $\begin{array}{l}\text { Minneapolis-St. Paul-Bloomington, } \\
\text { Minnesota-Wisconsin }\end{array}$ & 5,349 & 8.6 & 0.6 & $(7.4-9.7)$ \\
\hline Missoula, Montana & 540 & 8.1 & 1.3 & $(5.5-10.6)$ \\
\hline Mobile, Alabama & 641 & 17.1 & 2.2 & $(12.8-21.3)$ \\
\hline Montgomery, Alabama & 379 & 14.3 & 2.2 & $(10.1-18.6)$ \\
\hline $\begin{array}{l}\text { Montgomery County-Bucks County- } \\
\text { Chester County, Pennsylvania }{ }^{\dagger}\end{array}$ & 842 & 8.9 & 1.2 & $(6.6-11.2)$ \\
\hline $\begin{array}{l}\text { Myrtle Beach-Conway-North Myrtle } \\
\text { Beach, South Carolina-North Carolina }\end{array}$ & 716 & 14.7 & 1.7 & $(11.4-18.0)$ \\
\hline $\begin{array}{l}\text { Nashville-Davidson-Murfreesboro- } \\
\text { Franklin, Tennessee }\end{array}$ & 868 & 14.6 & 1.5 & $(11.6-17.7)$ \\
\hline $\begin{array}{l}\text { Nassau County-Suffolk County, } \\
\text { New York }^{\dagger}\end{array}$ & 622 & 10.3 & 1.4 & $(7.5-13.1)$ \\
\hline Newark, New Jersey-Pennsylvania ${ }^{\dagger}$ & 4,700 & 9.1 & 0.6 & $(7.9-10.3)$ \\
\hline New Haven-Milford, Connecticut & 1,439 & 10.9 & 1.1 & $(8.8-13.0)$ \\
\hline New Orleans-Metairie, Louisiana & 946 & 11.2 & 1.2 & $(8.8-13.6)$ \\
\hline $\begin{array}{l}\text { New York-Jersey City-White Plains, } \\
\text { New York-New Jersey }{ }^{\dagger}\end{array}$ & 4,408 & 11.1 & 1.1 & $(9.0-13.2)$ \\
\hline Norfolk, Nebraska & 444 & 9.2 & 1.5 & $(6.2-12.2)$ \\
\hline North Platte, Nebraska & 491 & 10.3 & 1.6 & $(7.2-13.4)$ \\
\hline Norwich-New London, Connecticut & 758 & 9.8 & 1.3 & $(7.2-12.4)$ \\
\hline $\begin{array}{l}\text { Oakland-Hayward-Berkeley, } \\
\text { California }^{\dagger}\end{array}$ & 676 & 6.8 & 1.3 & $(4.2-9.4)$ \\
\hline Ocean City, New Jersey & 456 & 11.1 & 1.9 & $(7.4-14.9)$ \\
\hline Ogden-Clearfield, Utah & 1,495 & 8.6 & 0.9 & $(6.9-10.4)$ \\
\hline Oklahoma City, Oklahoma & 1,556 & 13.2 & 1.0 & $(11.2-15.2)$ \\
\hline Olympia-Tumwater, Washington & 383 & 10.3 & 1.8 & $(6.9-13.8)$ \\
\hline Omaha-Council Bluffs, Nebraska-lowa & 3,576 & 9.9 & 0.6 & $(8.7-11.2)$ \\
\hline Orlando-Kissimmee-Sanford, Florida & 408 & 14.1 & 2.6 & $(9.1-19.1)$ \\
\hline Philadelphia, Pennsylvania $^{\dagger}$ & 1,619 & 11.9 & 1.1 & $(9.7-14.0)$ \\
\hline Phoenix-Mesa-Scottsdale, Arizona & 1,764 & 11.3 & 1.0 & $(9.4-13.3)$ \\
\hline Pittsburgh, Pennsylvania & 2,453 & 12.1 & 0.8 & $(10.6-13.6)$ \\
\hline Ponce, Puerto Rico & 347 & 15.8 & 2.5 & $(11.0-20.6)$ \\
\hline Portland-South Portland, Maine & 2,419 & 10.0 & 0.7 & $(8.5-11.5)$ \\
\hline $\begin{array}{l}\text { Portland-Vancouver-Hillsboro, } \\
\text { Oregon-Washington }\end{array}$ & 2,200 & 9.9 & 0.8 & $(8.3-11.5)$ \\
\hline $\begin{array}{l}\text { Providence-Warwick, Rhode } \\
\text { Island-Massachusetts }\end{array}$ & 5,847 & 11.6 & 0.6 & $(10.4-12.7)$ \\
\hline Provo-Orem, Utah & 894 & 8.4 & 1.0 & $(6.5-10.4)$ \\
\hline Raleigh, North Carolina & 511 & 9.3 & 1.5 & $(6.4-12.3)$ \\
\hline Rapid City, South Dakota & 700 & 10.0 & 1.4 & $(7.3-12.8)$ \\
\hline Reno, Nevada & 1,094 & 8.7 & 1.2 & $(6.4-11.0)$ \\
\hline Richmond, Virginia & 708 & 8.2 & 1.0 & $(6.2-10.2)$ \\
\hline
\end{tabular}

See table footnotes on page 122. 
TABLE 65. (Continued) Estimated prevalence of adults aged $\geq 45$ years who have ever been told by a health care professional that they have coronary heart disease, ${ }^{*}$ by metropolitan and micropolitan statistical area - Behavioral Risk Factor Surveillance System, United States, 2012

\begin{tabular}{|c|c|c|c|c|}
\hline MMSA(s) & $\begin{array}{l}\text { Sample } \\
\text { size }\end{array}$ & $\%$ & SE & $95 \% \mathrm{Cl}$ \\
\hline $\begin{array}{l}\text { Riverside-San Bernardino-Ontario, } \\
\text { California }\end{array}$ & 1,000 & 11.8 & 1.3 & $(9.2-14.5)$ \\
\hline $\begin{array}{l}\text { Rockingham County-Strafford County, } \\
\text { New Hampshire }{ }^{\dagger}\end{array}$ & 1,264 & 9.6 & 1.0 & $(7.7-11.5)$ \\
\hline Rutland, Vermont & 471 & 13.1 & 1.9 & $(9.4-16.7)$ \\
\hline $\begin{array}{l}\text { Sacramento-Roseville-Arden-Arcade, } \\
\text { California }\end{array}$ & 700 & 9.5 & 1.3 & $(6.9-12.1)$ \\
\hline St. Louis, Missouri-Illinois & 1,465 & 12.7 & 1.2 & $(10.4-15.1)$ \\
\hline Salisbury, Maryland-Delaware & 1,720 & 16.6 & 1.4 & $(13.9-19.4)$ \\
\hline Salt Lake City, Utah & 2,903 & 8.2 & 0.6 & $(7.0-9.3)$ \\
\hline San Antonio-New Braunfels, Texas & 522 & 9.1 & 1.5 & $(6.2-12.0)$ \\
\hline San Diego-Carlsbad, California & 730 & 9.5 & 1.3 & $(6.9-12.1)$ \\
\hline $\begin{array}{l}\text { San Francisco-Redwood City-South } \\
\text { San Francisco, California }{ }^{\dagger}\end{array}$ & 389 & 6.5 & 1.6 & $(3.3-9.7)$ \\
\hline $\begin{array}{l}\text { San Jose-Sunnyvale-Santa Clara, } \\
\text { California }\end{array}$ & 418 & 8.8 & 1.9 & $(5.1-12.5)$ \\
\hline San Juan-Carolina-Caguas, Puerto Rico & 2,482 & 16.2 & 0.9 & $(14.4-18.0)$ \\
\hline Santa Fe, New Mexico & 506 & 8.4 & 1.6 & $(5.2-11.6)$ \\
\hline Sayre, Pennsylvania & 1,463 & 11.6 & 0.9 & $(9.9-13.4)$ \\
\hline Scottsbluff, Nebraska & 518 & 13.2 & 2.2 & $(8.9-17.5)$ \\
\hline $\begin{array}{l}\text { Scranton-Wilkes-Barre-Hazleton, } \\
\text { Pennsylvania }\end{array}$ & 552 & 13.3 & 1.7 & $(10.0-16.7)$ \\
\hline Seattle-Bellevue-Everett, Washington ${ }^{\dagger}$ & 3,452 & 8.5 & 0.6 & $(7.3-9.6)$ \\
\hline Shreveport-Bossier City, Louisiana & 421 & 10.9 & 1.7 & $(7.6-14.1)$ \\
\hline $\begin{array}{l}\text { Silver Spring-Frederick-Rockville, } \\
\text { Maryland }^{\dagger}\end{array}$ & 1,700 & 8.3 & 0.8 & $(6.6-9.9)$ \\
\hline $\begin{array}{l}\text { Sioux City, lowa-Nebraska- } \\
\text { South Dakota }\end{array}$ & 926 & 14.6 & 2.4 & $(9.9-19.3)$ \\
\hline Sioux Falls, South Dakota & 836 & 11.3 & 1.5 & $(8.5-14.2)$ \\
\hline Spartanburg, South Carolina & 509 & 14.1 & 2.2 & $(9.8-18.5)$ \\
\hline Spokane-Spokane Valley, Washington & 834 & 8.5 & 1.1 & $(6.4-10.6)$ \\
\hline Springfield, Massachusetts & 1,671 & 11.4 & 1.2 & $(9.1-13.7)$ \\
\hline Tacoma-Lakewood, Washington $^{\dagger}$ & 809 & 9.0 & 1.1 & $(6.8-11.1)$ \\
\hline
\end{tabular}

TABLE 65. (Continued) Estimated prevalence of adults aged $\geq 45$ years who have ever been told by a health care professional that they have coronary heart disease, ${ }^{*}$ by metropolitan and micropolitan statistical area - Behavioral Risk Factor Surveillance System, United States, 2012

\begin{tabular}{|c|c|c|c|c|}
\hline MMSA(s) & $\begin{array}{l}\text { Sample } \\
\text { size }\end{array}$ & $\%$ & SE & $95 \% \mathrm{Cl}$ \\
\hline $\begin{array}{l}\text { Tampa-St. Petersburg-Clearwater, } \\
\text { Florida }\end{array}$ & 610 & 13.4 & 1.7 & $(10.0-16.8)$ \\
\hline Toledo, Ohio & 716 & 11.4 & 1.5 & $(8.6-14.3)$ \\
\hline Topeka, Kansas & 794 & 11.0 & 1.3 & $(8.4-13.6)$ \\
\hline Torrington, Connecticut & 506 & 7.4 & 1.4 & $(4.8-10.1)$ \\
\hline Trenton, New Jersey & 408 & 8.3 & 1.4 & $(5.6-11.0)$ \\
\hline Tucson, Arizona & 729 & 9.3 & 1.5 & $(6.4-12.2)$ \\
\hline Tulsa, Oklahoma & 1,200 & 13.7 & 1.2 & $(11.3-16.1)$ \\
\hline Tuscaloosa, Alabama & 428 & 13.7 & 2.0 & $(9.9-17.5)$ \\
\hline Urban Honolulu, Hawaii & 2,509 & 7.1 & 0.7 & $(5.7-8.5)$ \\
\hline Vineland-Bridgeton, New Jersey & 401 & 12.1 & 2.0 & $(8.2-16.0)$ \\
\hline $\begin{array}{l}\text { Virginia Beach-Norfolk-Newport News, } \\
\text { Virginia-North Carolina }\end{array}$ & 1,077 & 11.3 & 1.2 & $(9.0-13.6)$ \\
\hline $\begin{array}{l}\text { Warren-Troy-Farmington Hills, } \\
\text { Michigan }{ }^{\dagger}\end{array}$ & 1,560 & 10.4 & 0.9 & $(8.6-12.1)$ \\
\hline $\begin{array}{l}\text { Washington-Arlington-Alexandria, } \\
\text { District of Columbia-Virginia- } \\
\text { Maryland-West Virginia }\end{array}$ & 5,565 & 8.4 & 0.6 & (7.3-9.6) \\
\hline Wichita, Kansas & 1,675 & 12.1 & 1.0 & $(10.1-14.1)$ \\
\hline $\begin{array}{l}\text { Wilmington, Delaware-Maryland- } \\
\text { New Jersey }{ }^{\dagger}\end{array}$ & 2,186 & 11.3 & 1.0 & $(9.4-13.2)$ \\
\hline Winston-Salem, North Carolina & 548 & 12.2 & 1.6 & $(9.0-15.3)$ \\
\hline Worcester, Massachusetts-Connecticut & 1,990 & 10.0 & 1.0 & $(8.2-11.9)$ \\
\hline Yakima, Washington & 364 & 9.4 & 1.8 & $(5.8-13.0)$ \\
\hline $\begin{array}{l}\text { Youngstown-Warren-Boardman, } \\
\text { Ohio-Pennsylvania }\end{array}$ & 741 & 13.2 & 2.2 & $(8.8-17.6)$ \\
\hline Median & & 11.0 & & \\
\hline Range & & $6.1-23.3$ & & \\
\hline
\end{tabular}

Abbreviations: $\mathrm{Cl}=$ confidence interval; $\mathrm{MMSA}=$ metropolitan and micropolitan statistical area; $\mathrm{SE}=$ standard error.

* Including heart attack, also known as myocardial infarction, and angina.

† Metropolitan division. 
TABLE 66. Estimated prevalence of adults aged $\geq 45$ years who have ever been told by a health care professional that they have coronary heart disease,* by county - Behavioral Risk Factor Surveillance System, United States, 2012

\begin{tabular}{|c|c|c|c|c|}
\hline County & $\begin{array}{l}\text { Sample } \\
\text { size }\end{array}$ & $\%$ & SE & $95 \% \mathrm{Cl}$ \\
\hline Jefferson County, Alabama & 646 & 12.4 & 1.6 & $(9.3-15.4)$ \\
\hline Madison County, Alabama & 355 & 14.1 & 2.4 & $(9.3-18.8)$ \\
\hline Mobile County, Alabama & 641 & 17.1 & 2.2 & $(12.8-21.3)$ \\
\hline Anchorage Municipality, Alaska & 485 & 7.7 & 1.4 & $(5.0-10.5)$ \\
\hline Fairbanks North Star Borough, Alaska & 329 & 12.2 & 2.3 & $(7.7-16.7)$ \\
\hline Matanuska-Susitna Borough, Alaska & 379 & 9.9 & 1.7 & $(6.5-13.3)$ \\
\hline Maricopa County, Arizona & 1,389 & 11.0 & 1.0 & $(9.0-13.0)$ \\
\hline Pima County, Arizona & 729 & 9.3 & 1.5 & $(6.4-12.2)$ \\
\hline Pulaski County, Arkansas & 561 & 11.7 & 1.7 & $(8.5-15.0)$ \\
\hline Alameda County, California & 408 & 6.4 & 1.7 & $(3.1-9.7)$ \\
\hline Los Angeles County, California & 2,126 & 8.5 & 0.9 & $(6.7-10.4)$ \\
\hline Orange County, California & 677 & 7.2 & 1.1 & $(5.1-9.3)$ \\
\hline Riverside County, California & 562 & 12.8 & 2.0 & $(8.9-16.6)$ \\
\hline Sacramento County, California & 425 & 10.2 & 1.8 & $(6.7-13.7)$ \\
\hline San Bernardino County, California & 438 & 10.6 & 1.7 & $(7.3-14.0)$ \\
\hline San Diego County, California & 730 & 9.5 & 1.3 & $(6.9-12.1)$ \\
\hline Santa Clara County, California & 405 & 8.5 & 1.9 & $(4.7-12.2)$ \\
\hline Adams County, Colorado & 505 & 9.8 & 1.6 & $(6.7-12.9)$ \\
\hline Arapahoe County, Colorado & 557 & 7.0 & 1.1 & $(4.8-9.1)$ \\
\hline Boulder County, Colorado & 355 & 6.1 & 1.3 & $(3.5-8.7)$ \\
\hline Denver County, Colorado & 575 & 7.2 & 1.1 & $(4.9-9.4)$ \\
\hline Douglas County, Colorado & 336 & $\mathrm{~N} / \mathrm{A}^{\dagger}$ & N/A & $(\mathrm{N} / \mathrm{A})$ \\
\hline El Paso County, Colorado & 641 & 7.3 & 1.1 & $(5.2-9.4)$ \\
\hline Jefferson County, Colorado & 795 & 6.6 & 1.0 & $(4.7-8.5)$ \\
\hline Larimer County, Colorado & 414 & 9.0 & 1.7 & $(5.7-12.3)$ \\
\hline Weld County, Colorado & 338 & 9.2 & 1.7 & $(5.9-12.5)$ \\
\hline Fairfield County, Connecticut & 1,450 & 8.1 & 0.9 & $(6.4-9.8)$ \\
\hline Hartford County, Connecticut & 1,391 & 8.5 & 0.9 & $(6.7-10.2)$ \\
\hline Litchfield County, Connecticut & 506 & 7.4 & 1.4 & $(4.8-10.1)$ \\
\hline New Haven County, Connecticut & 1,439 & 10.9 & 1.1 & $(8.8-13.0)$ \\
\hline New London County, Connecticut & 758 & 9.8 & 1.3 & $(7.2-12.4)$ \\
\hline Kent County, Delaware & 1,030 & 11.8 & 1.3 & $(9.3-14.3)$ \\
\hline New Castle County, Delaware & 1,495 & 11.2 & 1.1 & $(9.0-13.4)$ \\
\hline Sussex County, Delaware & 1,115 & 15.4 & 1.4 & $(12.7-18.1)$ \\
\hline $\begin{array}{l}\text { District of Columbia, } \\
\text { District of Columbia }\end{array}$ & 2,665 & 9.8 & 0.8 & $(8.2-11.3)$ \\
\hline Broward County, Florida & 382 & 6.5 & 1.5 & $(3.6-9.4)$ \\
\hline Miami-Dade County, Florida & 554 & 8.9 & 1.7 & $(5.5-12.3)$ \\
\hline Hawaii County, Hawaii & 941 & 9.4 & 1.5 & $(6.6-12.3)$ \\
\hline Honolulu County, Hawaii & 2,509 & 7.1 & 0.7 & $(5.7-8.5)$ \\
\hline Kauai County, Hawaii & 534 & 8.1 & 1.5 & $(5.1-11.1)$ \\
\hline Maui County, Hawaii & 857 & 6.3 & 1.0 & $(4.4-8.3)$ \\
\hline Ada County, Idaho & 578 & 6.7 & 1.2 & $(4.4-9.0)$ \\
\hline Canyon County, Idaho & 369 & 10.7 & 2.8 & $(5.2-16.2)$ \\
\hline Cook County, Illinois & 984 & 8.9 & 1.2 & $(6.5-11.3)$ \\
\hline Lake County, Indiana & 665 & 11.8 & 1.8 & $(8.3-15.3)$ \\
\hline Marion County, Indiana & 858 & 11.3 & 1.4 & $(8.6-14.1)$ \\
\hline Polk County, lowa & 543 & 9.9 & 1.5 & $(6.9-12.8)$ \\
\hline Johnson County, Kansas & 1,587 & 6.8 & 0.6 & $(5.5-8.0)$ \\
\hline Sedgwick County, Kansas & 1,234 & 12.3 & 1.0 & $(10.4-14.3)$ \\
\hline Shawnee County, Kansas & 541 & 11.8 & 1.7 & $(8.6-15.0)$ \\
\hline Wyandotte County, Kansas & 672 & 12.8 & 1.6 & $(9.6-15.9)$ \\
\hline Jefferson County, Kentucky & 1,246 & 15.2 & 1.8 & $(11.8-18.7)$ \\
\hline East Baton Rouge Parish, Louisiana & 398 & 12.2 & 2.0 & $(8.4-16.0)$ \\
\hline Androscoggin County, Maine & 466 & 11.8 & 1.8 & $(8.2-15.4)$ \\
\hline Aroostook County, Maine & 409 & 20.0 & 2.4 & $(15.3-24.7)$ \\
\hline Cumberland County, Maine & 1,257 & 10.2 & 1.1 & $(8.1-12.4)$ \\
\hline Kennebec County, Maine & 580 & 12.4 & 1.6 & $(9.2-15.6)$ \\
\hline Penobscot County, Maine & 659 & 12.5 & 1.4 & $(9.6-15.3)$ \\
\hline
\end{tabular}

See table footnotes on page 124.
TABLE 66. (Continued) Estimated prevalence of adults aged $\geq 45$ years who have ever been told by a health care professional that they have coronary heart disease, * by county - Behavioral Risk Factor Surveillance System, United States, 2012

\begin{tabular}{|c|c|c|c|c|}
\hline County & $\begin{array}{l}\text { Sample } \\
\text { size }\end{array}$ & $\%$ & SE & $95 \% \mathrm{Cl}$ \\
\hline York County, Maine & 867 & 9.6 & 1.1 & $(7.5-11.8)$ \\
\hline Anne Arundel County, Maryland & 641 & 9.6 & 1.4 & $(6.9-12.2)$ \\
\hline Baltimore County, Maryland & 1,095 & 11.9 & 1.2 & $(9.6-14.3)$ \\
\hline Charles County, Maryland & 357 & 10.4 & 2.1 & $(6.3-14.4)$ \\
\hline Frederick County, Maryland & 577 & 9.2 & 1.7 & $(5.9-12.6)$ \\
\hline Montgomery County, Maryland & 1,123 & 8.2 & 1.0 & $(6.2-10.1)$ \\
\hline Prince George's County, Maryland & 806 & 6.8 & 1.0 & $(4.9-8.7)$ \\
\hline Washington County, Maryland & 420 & 15.9 & 3.3 & $(9.4-22.4)$ \\
\hline Baltimore city, Maryland & 536 & 14.4 & 2.4 & $(9.7-19.1)$ \\
\hline Barnstable County, Massachusetts & 468 & 11.1 & 1.6 & $(8.0-14.3)$ \\
\hline Bristol County, Massachusetts & 1,942 & 13.3 & 1.4 & $(10.5-16.0)$ \\
\hline Essex County, Massachusetts & 1,889 & 10.1 & 1.1 & $(7.9-12.4)$ \\
\hline Hampden County, Massachusetts & 1,400 & 12.5 & 1.4 & $(9.8-15.1)$ \\
\hline Middlesex County, Massachusetts & 2,852 & 9.2 & 0.8 & $(7.7-10.7)$ \\
\hline Norfolk County, Massachusetts & 1,255 & 9.3 & 1.3 & $(6.8-11.8)$ \\
\hline Plymouth County, Massachusetts & 1,327 & 9.8 & 1.3 & $(7.4-12.3)$ \\
\hline Suffolk County, Massachusetts & 1,433 & 11.8 & 1.4 & $(9.1-14.4)$ \\
\hline Worcester County, Massachusetts & 1,823 & 9.8 & 1.0 & $(7.8-11.8)$ \\
\hline Kent County, Michigan & 389 & 11.4 & 1.9 & $(7.7-15.1)$ \\
\hline Macomb County, Michigan & 420 & 11.6 & 1.7 & $(8.3-15.0)$ \\
\hline Oakland County, Michigan & 858 & 9.6 & 1.2 & $(7.3-11.9)$ \\
\hline Wayne County, Michigan & 1,608 & 13.2 & 1.4 & $(10.5-15.9)$ \\
\hline Anoka County, Minnesota & 364 & 7.3 & 1.7 & $(4.0-10.6)$ \\
\hline Dakota County, Minnesota & 422 & 7.8 & 1.6 & $(4.7-10.9)$ \\
\hline Hennepin County, Minnesota & 2,196 & 7.0 & 1.0 & $(5.1-8.9)$ \\
\hline Ramsey County, Minnesota & 1,447 & 11.6 & 1.5 & $(8.6-14.5)$ \\
\hline Jackson County, Missouri & 669 & 9.1 & 1.5 & $(6.2-12.1)$ \\
\hline St. Louis County, Missouri & 669 & 9.0 & 1.5 & $(6.1-11.9)$ \\
\hline Cascade County, Montana & 542 & 11.9 & 1.6 & $(8.7-15.1)$ \\
\hline Flathead County, Montana & 398 & 8.8 & 1.6 & $(5.7-11.9)$ \\
\hline Hill County, Montana & 430 & 9.6 & 1.9 & $(6.0-13.3)$ \\
\hline Lake County, Montana & 701 & 11.3 & 1.8 & $(7.8-14.8)$ \\
\hline Missoula County, Montana & 540 & 8.1 & 1.3 & $(5.5-10.6)$ \\
\hline Yellowstone County, Montana & 475 & 11.7 & 1.5 & $(8.7-14.7)$ \\
\hline Dakota County, Nebraska & 574 & 11.7 & 2.2 & $(7.4-16.1)$ \\
\hline Douglas County, Nebraska & 2,285 & 9.9 & 0.8 & $(8.5-11.4)$ \\
\hline Hall County, Nebraska & 379 & 9.6 & 1.6 & $(6.5-12.8)$ \\
\hline Lancaster County, Nebraska & 781 & 9.5 & 1.2 & $(7.1-11.9)$ \\
\hline Lincoln County, Nebraska & 475 & 10.4 & 1.6 & $(7.2-13.6)$ \\
\hline Sarpy County, Nebraska & 732 & 9.6 & 1.4 & $(6.9-12.3)$ \\
\hline Scotts Bluff County, Nebraska & 475 & 13.0 & 2.3 & $(8.5-17.6)$ \\
\hline Clark County, Nevada & 1,210 & 11.3 & 1.1 & $(9.2-13.4)$ \\
\hline Washoe County, Nevada & 1,081 & 8.8 & 1.2 & $(6.5-11.1)$ \\
\hline Belknap County, New Hampshire & 471 & 10.7 & 2.0 & $(6.8-14.7)$ \\
\hline Carroll County, New Hampshire & 461 & 8.7 & 1.9 & $(5.0-12.4)$ \\
\hline Cheshire County, New Hampshire & 440 & 11.0 & 1.8 & $(7.4-14.6)$ \\
\hline Coos County, New Hampshire & 434 & 12.7 & 2.1 & $(8.5-16.8)$ \\
\hline Grafton County, New Hampshire & 439 & 9.3 & 1.5 & $(6.4-12.2)$ \\
\hline Hillsborough County, New Hampshire & 1,367 & 9.8 & 1.0 & $(7.9-11.7)$ \\
\hline Merrimack County, New Hampshire & 525 & 9.0 & 1.5 & $(6.0-11.9)$ \\
\hline Rockingham County, New Hampshire & 785 & 8.0 & 1.1 & $(5.7-10.2)$ \\
\hline Strafford County, New Hampshire & 479 & 13.8 & 1.9 & $(10.2-17.5)$ \\
\hline Atlantic County, New Jersey & 787 & 13.4 & 1.5 & $(10.4-16.4)$ \\
\hline Bergen County, New Jersey & 606 & 7.3 & 1.2 & $(4.9-9.6)$ \\
\hline Burlington County, New Jersey & 488 & 8.5 & 1.5 & $(5.6-11.5)$ \\
\hline Camden County, New Jersey & 516 & 14.2 & 2.1 & $(10.1-18.3)$ \\
\hline Cape May County, New Jersey & 456 & 11.1 & 1.9 & $(7.4-14.9)$ \\
\hline Cumberland County, New Jersey & 401 & 12.1 & 2.0 & $(8.2-16.0)$ \\
\hline Essex County, New Jersey & 793 & 9.3 & 1.2 & $(7.0-11.6)$ \\
\hline
\end{tabular}

See table footnotes on page 124. 
TABLE 66. (Continued) Estimated prevalence of adults aged $\geq 45$ years who have ever been told by a health care professional that they have coronary heart disease,* by county - Behavioral Risk Factor Surveillance System, United States, 2012

\begin{tabular}{|c|c|c|c|c|}
\hline County & $\begin{array}{l}\text { Sample } \\
\text { size }\end{array}$ & $\%$ & SE & $95 \% \mathrm{Cl}$ \\
\hline Gloucester County, New Jersey & 404 & 11.2 & 1.9 & $(7.4-14.9)$ \\
\hline Hudson County, New Jersey & 741 & 14.7 & 1.9 & $(11.0-18.3)$ \\
\hline Hunterdon County, New Jersey & 437 & 9.7 & 2.1 & $(5.6-13.9)$ \\
\hline Mercer County, New Jersey & 408 & 8.3 & 1.4 & $(5.6-11.0)$ \\
\hline Middlesex County, New Jersey & 518 & 9.5 & 1.5 & $(6.5-12.5)$ \\
\hline Monmouth County, New Jersey & 507 & 10.0 & 1.6 & $(6.9-13.1)$ \\
\hline Morris County, New Jersey & 610 & 10.2 & 1.5 & $(7.2-13.1)$ \\
\hline Ocean County, New Jersey & 477 & 14.6 & 2.0 & $(10.6-18.5)$ \\
\hline Passaic County, New Jersey & 407 & 9.3 & 1.7 & $(6.0-12.5)$ \\
\hline Salem County, New Jersey & 447 & 13.6 & 2.0 & $(9.8-17.4)$ \\
\hline Somerset County, New Jersey & 453 & 8.4 & 1.6 & $(5.2-11.6)$ \\
\hline Sussex County, New Jersey & 424 & 8.0 & 1.7 & $(4.7-11.3)$ \\
\hline Union County, New Jersey & 440 & 7.9 & 1.5 & $(5.1-10.8)$ \\
\hline Warren County, New Jersey & 420 & 11.2 & 1.8 & $(7.7-14.8)$ \\
\hline Bernalillo County, New Mexico & 1,286 & 8.5 & 0.9 & $(6.7-10.3)$ \\
\hline Dona Ana County, New Mexico & 475 & 12.4 & 1.8 & $(8.9-15.9)$ \\
\hline Sandoval County, New Mexico & 483 & 10.8 & 2.0 & $(7.0-14.7)$ \\
\hline San Juan County, New Mexico & 425 & 10.1 & 1.7 & $(6.8-13.4)$ \\
\hline Santa Fe County, New Mexico & 506 & 8.4 & 1.6 & $(5.2-11.6)$ \\
\hline Kings County, New York & 208 & 10.0 & 2.6 & $(4.8-15.1)$ \\
\hline Guilford County, North Carolina & 332 & 9.4 & 1.7 & $(6.0-12.7)$ \\
\hline Mecklenburg County, North Carolina & 361 & 7.6 & 1.7 & $(4.3-10.8)$ \\
\hline Robeson County, North Carolina & 402 & 18.0 & 4.1 & $(10.0-26.0)$ \\
\hline Wake County, North Carolina & 358 & 7.4 & 1.6 & $(4.4-10.5)$ \\
\hline Burleigh County, North Dakota & 398 & 16.2 & 2.4 & $(11.5-20.9)$ \\
\hline Cass County, North Dakota & 579 & 7.5 & 1.4 & $(4.9-10.2)$ \\
\hline Cuyahoga County, Ohio & 585 & 11.0 & 1.5 & $(8.2-13.9)$ \\
\hline Franklin County, Ohio & 478 & 13.1 & 1.9 & $(9.3-16.8)$ \\
\hline Hamilton County, Ohio & 512 & 12.8 & 1.8 & $(9.2-16.4)$ \\
\hline Lorain County, Ohio & 498 & 12.1 & 1.7 & $(8.7-15.5)$ \\
\hline Lucas County, Ohio & 465 & 11.0 & 1.9 & $(7.3-14.6)$ \\
\hline Mahoning County, Ohio & 489 & 12.6 & 2.0 & $(8.6-16.6)$ \\
\hline Montgomery County, Ohio & 490 & 12.4 & 1.8 & $(8.9-15.9)$ \\
\hline Stark County, Ohio & 483 & 13.9 & 2.1 & $(9.9-18.0)$ \\
\hline Summit County, Ohio & 481 & 9.8 & 1.6 & $(6.7-12.9)$ \\
\hline Oklahoma County, Oklahoma & 732 & 13.4 & 1.4 & $(10.7-16.2)$ \\
\hline Tulsa County, Oklahoma & 805 & 10.9 & 1.2 & $(8.5-13.3)$ \\
\hline Clackamas County, Oregon & 388 & 8.9 & 1.9 & $(5.1-12.6)$ \\
\hline Lane County, Oregon & 391 & 9.3 & 1.7 & $(6.0-12.6)$ \\
\hline Multnomah County, Oregon & 602 & 10.4 & 1.7 & $(7.1-13.7)$ \\
\hline Washington County, Oregon & 416 & 10.5 & 2.0 & $(6.6-14.3)$ \\
\hline Allegheny County, Pennsylvania & 1,371 & 10.7 & 1.0 & $(8.7-12.7)$ \\
\hline Bradford County, Pennsylvania & 1,463 & 11.6 & 0.9 & $(9.9-13.4)$ \\
\hline Montgomery County, Pennsylvania & 347 & 6.2 & 1.4 & $(3.5-8.9)$ \\
\hline Philadelphia County, Pennsylvania & 1,378 & 12.2 & 1.2 & $(9.8-14.6)$ \\
\hline Pike County, Pennsylvania & 1,543 & 10.8 & 0.8 & $(9.1-12.4)$ \\
\hline Kent County, Rhode Island & 584 & 8.8 & 1.3 & $(6.3-11.4)$ \\
\hline Providence County, Rhode Islar & 2,299 & 12.2 & 0.9 & $(10.4-13.9)$ \\
\hline Washington County, Rhode Island & 519 & 9.5 & 1.4 & $(6.8-12.3)$ \\
\hline Aiken County, South Carolina & 429 & 12.7 & 2.0 & $(8.8-16.6)$ \\
\hline
\end{tabular}

TABLE 66. (Continued) Estimated prevalence of adults aged $\geq 45$ years who have ever been told by a health care professional that they have coronary heart disease, * by county - Behavioral Risk Factor Surveillance System, United States, 2012

\begin{tabular}{|c|c|c|c|c|}
\hline County & $\begin{array}{c}\text { Sample } \\
\text { size }\end{array}$ & $\%$ & SE & $95 \% \mathrm{Cl}$ \\
\hline Beaufort County, South Carolina & 689 & 8.8 & 1.4 & $(6.1-11.5)$ \\
\hline Charleston County, South Carolina & 735 & 9.7 & 1.5 & $(6.8-12.6)$ \\
\hline Greenville County, South Carolina & 573 & 14.0 & 2.0 & $(10.2-17.9)$ \\
\hline Horry County, South Carolina & 579 & 14.2 & 1.7 & $(10.9-17.6)$ \\
\hline Richland County, South Carolina & 655 & 14.0 & 2.4 & $(9.3-18.6)$ \\
\hline Spartanburg County, South Carolina & 467 & 12.9 & 2.1 & $(8.8-17.1)$ \\
\hline Lincoln County, South Dakota & 313 & 7.7 & 1.8 & $(4.2-11.3)$ \\
\hline Minnehaha County, South Dakota & 469 & 12.4 & 1.8 & $(8.8-15.9)$ \\
\hline Pennington County, South Dakota & 358 & 9.8 & 1.7 & $(6.4-13.1)$ \\
\hline Davidson County, Tennessee & 365 & 12.7 & 2.2 & $(8.3-17.1)$ \\
\hline Shelby County, Tennessee & 329 & 14.7 & 2.5 & $(9.8-19.6)$ \\
\hline Bexar County, Texas & 374 & 9.1 & 1.7 & $(5.8-12.4)$ \\
\hline Dallas County, Texas & 287 & 10.3 & 2.0 & $(6.3-14.3)$ \\
\hline El Paso County, Texas & 439 & 10.2 & 2.6 & $(5.1-15.3)$ \\
\hline Harris County, Texas & 386 & 7.8 & 1.4 & $(5.0-10.6)$ \\
\hline Hidalgo County, Texas & 386 & 12.9 & 2.4 & $(8.2-17.5)$ \\
\hline Tarrant County, Texas & 386 & 9.4 & 1.7 & $(6.2-12.7)$ \\
\hline Travis County, Texas & 738 & 8.7 & 1.2 & $(6.4-11.1)$ \\
\hline Davis County, Utah & 652 & 7.4 & 1.1 & $(5.2-9.5)$ \\
\hline Salt Lake County, Utah & 2,544 & 8.0 & 0.6 & $(6.9-9.2)$ \\
\hline Tooele County, Utah & 359 & 10.6 & 2.9 & $(5.0-16.2)$ \\
\hline Utah County, Utah & 849 & 8.5 & 1.0 & $(6.5-10.5)$ \\
\hline Wasatch County, Utah & 358 & 8.0 & 1.4 & $(5.3-10.8)$ \\
\hline Weber County, Utah & 679 & 11.1 & 1.7 & $(7.7-14.4)$ \\
\hline Chittenden County, Vermont & 589 & 7.3 & 1.3 & $(4.6-9.9)$ \\
\hline Rutland County, Vermont & 471 & 13.1 & 1.9 & $(9.4-16.7)$ \\
\hline Washington County, Vermont & 386 & 10.7 & 1.9 & $(7.0-14.5)$ \\
\hline Windsor County, Vermont & 423 & 8.1 & 1.4 & $(5.4-10.8)$ \\
\hline Fairfax County, Virginia & 461 & 6.8 & 1.4 & $(4.1-9.5)$ \\
\hline Clark County, Washington & 558 & 11.5 & 1.5 & $(8.5-14.6)$ \\
\hline King County, Washington & 2,647 & 8.2 & 0.6 & $(7.0-9.5)$ \\
\hline Kitsap County, Washington & 425 & 8.8 & 1.5 & $(5.9-11.8)$ \\
\hline Pierce County, Washington & 809 & 9.0 & 1.1 & $(6.8-11.1)$ \\
\hline Snohomish County, Washington & 805 & 9.2 & 1.3 & $(6.6-11.9)$ \\
\hline Spokane County, Washington & 718 & 9.0 & 1.2 & $(6.7-11.3)$ \\
\hline Thurston County, Washington & 383 & 10.3 & 1.8 & $(6.9-13.8)$ \\
\hline Whatcom County, Washington & 619 & 8.4 & 1.4 & $(5.6-11.3)$ \\
\hline Yakima County, Washington & 364 & 9.4 & 1.8 & $(5.8-13.0)$ \\
\hline Kanawha County, West Virginia & 441 & 20.6 & 2.3 & $(16.1-25.0)$ \\
\hline Milwaukee County, Wisconsin & 657 & 11.9 & 2.0 & $(8.1-15.8)$ \\
\hline Laramie County, Wyoming & 753 & 10.1 & 1.7 & $(6.8-13.4)$ \\
\hline Natrona County, Wyoming & 647 & 10.2 & 1.6 & $(7.0-13.3)$ \\
\hline San Juan Municipio, Puerto Rico & 423 & 11.4 & 1.9 & $(7.6-15.2)$ \\
\hline Median & & 10.0 & & \\
\hline Range & & $6.1-20.6$ & & \\
\hline
\end{tabular}

Abbreviations: $\mathrm{Cl}=$ confidence interval; $\mathrm{SE}=$ standard error.

* Including heart attack also known as myocardial infarction, and angina.

${ }^{\dagger}$ Estimate not available (N/A) if the unweighted sample size for the denominator was $<50$ or if the relative standard error is $>0.3$. 
TABLE 67. Estimated prevalence of adults aged $\geq 45$ years who have ever been told by a health care professional that they had a stroke, by state/territory - Behavioral Risk Factor Surveillance System, United States, 2012

\begin{tabular}{|c|c|c|c|c|}
\hline State/Territory & $\begin{array}{l}\text { Sample } \\
\text { size }\end{array}$ & $\%$ & SE & $95 \% \mathrm{Cl}$ \\
\hline Alabama & 6,780 & 6.6 & 0.4 & $(5.8-7.4)$ \\
\hline Alaska & 2,611 & 4.7 & 0.5 & $(3.6-5.8)$ \\
\hline Arizona & 5,467 & 4.7 & 0.4 & $(3.9-5.6)$ \\
\hline Arkansas & 3,915 & 6.1 & 0.5 & $(5.2-6.9)$ \\
\hline California & 9,634 & 3.9 & 0.3 & $(3.3-4.4)$ \\
\hline Colorado & 8,629 & 3.1 & 0.2 & $(2.7-3.5)$ \\
\hline Connecticut & 6,173 & 3.4 & 0.3 & $(2.9-3.9)$ \\
\hline Delaware & 3,634 & 5.3 & 0.5 & $(4.4-6.3)$ \\
\hline District of Columbia & 2,662 & 7.1 & 1.0 & $(5.2-8.9)$ \\
\hline Florida & 5,767 & 4.6 & 0.4 & $(3.9-5.3)$ \\
\hline Georgia & 4,393 & 5.8 & 0.5 & $(4.9-6.8)$ \\
\hline Hawaii & 4,827 & 4.5 & 0.4 & $(3.7-5.4)$ \\
\hline Idaho & 4,486 & 4.4 & 0.5 & $(3.4-5.3)$ \\
\hline Illinois & 4,069 & 4.2 & 0.4 & $(3.5-5.0)$ \\
\hline Indiana & 5,993 & 5.7 & 0.4 & $(4.9-6.4)$ \\
\hline lowa & 5,167 & 5.2 & 0.4 & $(4.5-5.9)$ \\
\hline Kansas & 8,558 & 4.9 & 0.3 & $(4.3-5.4)$ \\
\hline Kentucky & 8,192 & 6.7 & 0.4 & $(5.9-7.6)$ \\
\hline Louisiana & 6,887 & 6.5 & 0.5 & $(5.6-7.4)$ \\
\hline Maine & 7,285 & 4.3 & 0.3 & $(3.7-4.8)$ \\
\hline Maryland & 9,600 & 4.1 & 0.3 & $(3.5-4.7)$ \\
\hline Massachusetts & 15,161 & 3.4 & 0.2 & $(3.0-3.9)$ \\
\hline Michigan & 7,710 & 5.5 & 0.3 & $(4.8-6.2)$ \\
\hline Minnesota & 8,384 & 3.8 & 0.3 & $(3.2-4.4)$ \\
\hline Mississippi & 5,908 & 7.3 & 0.4 & $(6.5-8.2)$ \\
\hline Missouri & 4,979 & 6.0 & 0.5 & $(5.1-6.9)$ \\
\hline Montana & 6,247 & 4.5 & 0.3 & $(3.9-5.1)$ \\
\hline Nebraska & 13,912 & 4.0 & 0.2 & $(3.6-4.4)$ \\
\hline Nevada & 3,342 & 5.1 & 0.7 & $(3.8-6.4)$ \\
\hline New Hampshire & 5,768 & 3.6 & 0.3 & $(3.0-4.2)$ \\
\hline New Jersey & 10,727 & 3.8 & 0.2 & $(3.3-4.2)$ \\
\hline New Mexico & 6,046 & 4.8 & 0.3 & $(4.1-5.5)$ \\
\hline New York & 3,969 & 4.5 & 0.6 & $(3.4-5.6)$ \\
\hline North Carolina & 8,090 & 5.2 & 0.3 & $(4.6-5.7)$ \\
\hline North Dakota & 3,503 & 4.2 & 0.4 & $(3.3-5.0)$ \\
\hline Ohio & 9,490 & 5.1 & 0.3 & $(4.5-5.7)$ \\
\hline Oklahoma & 5,659 & 6.1 & 0.4 & $(5.3-6.8)$ \\
\hline Oregon & 3,885 & 4.8 & 0.4 & $(4.0-5.6)$ \\
\hline Pennsylvania & 14,370 & 4.6 & 0.2 & $(4.1-5.1)$ \\
\hline Rhode Island & 3,899 & 3.9 & 0.4 & $(3.2-4.7)$ \\
\hline South Carolina & 9,361 & 6.0 & 0.3 & $(5.4-6.7)$ \\
\hline South Dakota & 5,368 & 4.0 & 0.4 & $(3.1-4.8)$ \\
\hline Tennessee & 5,221 & 6.6 & 0.5 & $(5.7-7.5)$ \\
\hline Texas & 5,991 & 4.7 & 0.3 & $(4.0-5.4)$ \\
\hline Utah & 7,710 & 4.0 & 0.3 & $(3.5-4.5)$ \\
\hline Vermont & 4,528 & 3.7 & 0.3 & $(3.1-4.3)$ \\
\hline Virginia & 5,156 & 5.5 & 0.4 & $(4.7-6.2)$ \\
\hline Washington & 11,037 & 4.0 & 0.2 & $(3.5-4.4)$ \\
\hline West Virginia & 3,895 & 5.8 & 0.4 & $(5.0-6.6)$ \\
\hline Wisconsin & 3,846 & 3.7 & 0.5 & $(2.8-4.6)$ \\
\hline Wyoming & 4,901 & 3.7 & 0.4 & $(3.0-4.4)$ \\
\hline Guam & 893 & 5.6 & 1.0 & (3.7-7.5) \\
\hline Puerto Rico & 3,982 & 3.4 & 0.3 & $(2.8-4.1)$ \\
\hline Median & & 4.7 & & \\
\hline Range & & $3.1-7.3$ & & \\
\hline
\end{tabular}

Abbreviations: $\mathrm{Cl}=$ confidence interval; $\mathrm{SE}=$ standard error.
TABLE 68. Estimated prevalence of adults aged $\geq 45$ years who have ever been told by a health care professional that they had a stroke, by metropolitan and micropolitan statistical area - Behavioral Risk Factor Surveillance System, United States, 2012

\begin{tabular}{|c|c|c|c|c|}
\hline MMSA(s) & $\begin{array}{l}\text { Sample } \\
\text { size }\end{array}$ & $\%$ & SE & $95 \% \mathrm{Cl}$ \\
\hline Aguadilla-Isabela, Puerto Rico & 344 & $N / A^{\dagger}$ & N/A & $(\mathrm{N} / \mathrm{A}-\mathrm{N} / \mathrm{A})$ \\
\hline Akron, Ohio & 555 & 5.1 & 1.2 & $(2.8-7.4)$ \\
\hline Albuquerque, New Mexico & 2,175 & 4.6 & 0.6 & $(3.5-5.7)$ \\
\hline $\begin{array}{l}\text { Allentown-Bethlehem-Easton, } \\
\text { Pennsylvania-New Jersey }\end{array}$ & 993 & 3.0 & 0.8 & $(1.4-4.5)$ \\
\hline Anaheim-Santa Ana-Irvine, California* & 677 & N/A & N/A & $(\mathrm{N} / \mathrm{A}-\mathrm{N} / \mathrm{A})$ \\
\hline Anchorage, Alaska & 861 & 3.9 & 0.7 & $(2.4-5.3)$ \\
\hline Asheville, North Carolina & 439 & 6.8 & 1.6 & $(3.7-9.9)$ \\
\hline $\begin{array}{l}\text { Atlanta-Sandy Springs-Roswell, } \\
\text { Georgia }\end{array}$ & 1,733 & 5.0 & 0.7 & $(3.6-6.5)$ \\
\hline Atlantic City-Hammonton, New Jersey & 786 & 4.8 & 1.0 & $(2.8-6.7)$ \\
\hline $\begin{array}{l}\text { Augusta-Richmond County, Georgia- } \\
\text { South Carolina }\end{array}$ & 792 & 5.7 & 1.1 & $(3.5-7.9)$ \\
\hline Augusta-Waterville, Maine & 578 & 3.4 & 0.9 & $(1.8-5.1)$ \\
\hline Austin-Round Rock, Texas & 960 & 3.6 & 0.8 & $(2.0-5.1)$ \\
\hline $\begin{array}{l}\text { Baltimore-Columbia-Towson, } \\
\text { Maryland }\end{array}$ & 3,424 & 4.6 & 0.5 & $(3.7-5.6)$ \\
\hline Bangor, Maine & 658 & 4.4 & 1.0 & $(2.5-6.4)$ \\
\hline Barnstable Town, Massachusetts & 466 & 3.8 & 1.0 & $(1.8-5.9)$ \\
\hline Barre, Vermont & 386 & N/A & N/A & $(\mathrm{N} / \mathrm{A}-\mathrm{N} / \mathrm{A})$ \\
\hline Baton Rouge, Louisiana & 1,044 & 4.8 & 0.9 & $(3.1-6.6)$ \\
\hline Bellingham, Washington & 612 & 3.4 & 0.7 & $(2.0-4.8)$ \\
\hline Berlin, New Hampshire-Vermont & 568 & 5.4 & 1.2 & $(3.0-7.8)$ \\
\hline Billings, Montana & 548 & 4.0 & 0.9 & $(2.3-5.7)$ \\
\hline Birmingham-Hoover, Alabama & 1,301 & 6.0 & 0.8 & $(4.4-7.6)$ \\
\hline Bismarck, North Dakota & 582 & 6.6 & 1.4 & $(3.9-9.3)$ \\
\hline Boise City, Idaho & 1,076 & 4.8 & 0.9 & $(3.0-6.6)$ \\
\hline Boston, Massachusetts* & 3,999 & 2.6 & 0.4 & $(1.9-3.4)$ \\
\hline Boulder, Colorado & 354 & N/A & N/A & $(\mathrm{N} / \mathrm{A}-\mathrm{N} / \mathrm{A})$ \\
\hline Bremerton-Silverdale, Washington & 425 & 5.7 & 1.2 & $(3.5-8.0)$ \\
\hline $\begin{array}{l}\text { Bridgeport-Stamford-Norwalk, } \\
\text { Connecticut }\end{array}$ & 1,446 & 2.8 & 0.5 & $(1.8-3.9)$ \\
\hline Burlington-South Burlington, Vermont & 1,025 & 3.8 & 0.6 & $(2.5-5.0)$ \\
\hline $\begin{array}{l}\text { Cambridge-Newton-Framingham, } \\
\text { Massachusetts* }\end{array}$ & 4,731 & 3.0 & 0.3 & $(2.4-3.6)$ \\
\hline Camden, New Jersey* & 1,403 & 4.3 & 0.7 & $(2.8-5.7)$ \\
\hline Canton-Massillon, Ohio & 530 & 6.0 & 1.2 & $(3.6-8.5)$ \\
\hline Casper, Wyoming & 646 & 4.8 & 1.1 & $(2.5-7.0)$ \\
\hline Cedar Rapids, lowa & 410 & 3.6 & 1.0 & $(1.6-5.5)$ \\
\hline Charleston, West Virginia & 535 & 6.4 & 1.1 & $(4.3-8.5)$ \\
\hline $\begin{array}{l}\text { Charleston-North Charleston, } \\
\text { South Carolina }\end{array}$ & 1,211 & 6.3 & 0.9 & $(4.5-8.0)$ \\
\hline $\begin{array}{l}\text { Charlotte-Concord-Gastonia, } \\
\text { North Carolina-South Carolina }\end{array}$ & 1,604 & 4.4 & 0.6 & $(3.3-5.6)$ \\
\hline Chattanooga, Tennessee-Georgia & 496 & 5.3 & 1.2 & $(2.9-7.6)$ \\
\hline Cheyenne, Wyoming & 748 & 3.5 & 0.7 & $(2.1-4.9)$ \\
\hline $\begin{array}{l}\text { Chicago-Naperville-Elgin, } \\
\text { Illinois-Indiana-Wisconsin }\end{array}$ & 2,569 & 3.6 & 0.4 & $(2.7-4.4)$ \\
\hline Cincinnati, Ohio-Kentucky-Indiana & 1,633 & 7.1 & 0.9 & $(5.3-8.8)$ \\
\hline $\begin{array}{l}\text { Claremont-Lebanon, New } \\
\text { Hampshire-Vermont }\end{array}$ & 1,505 & 3.5 & 0.5 & $(2.6-4.4)$ \\
\hline Cleveland-Elyria, Ohio & 1,292 & 4.8 & 0.8 & $(3.1-6.4)$ \\
\hline Colorado Springs, Colorado & 760 & 3.0 & 0.7 & $(1.7-4.2)$ \\
\hline Columbia, South Carolina & 1,240 & 4.2 & 0.7 & $(2.8-5.6)$ \\
\hline Columbus, Ohio & 1,022 & 5.2 & 0.8 & $(3.7-6.8)$ \\
\hline Concord, New Hampshire & 522 & 3.9 & 1.1 & $(1.7-6.1)$ \\
\hline Dallas-Plano-Irving, Texas* & 525 & 3.5 & 0.8 & $(1.9-5.1)$ \\
\hline Dayton, Ohio & 613 & 4.4 & 0.9 & $(2.6-6.2)$ \\
\hline Denver-Aurora-Lakewood, Colorado & 3,167 & 3.1 & 0.3 & $(2.4-3.7)$ \\
\hline Des Moines-West Des Moines, lowa & 780 & 4.4 & 0.8 & $(2.8-6.0)$ \\
\hline
\end{tabular}

See table footnotes on page 127. 
TABLE 68. (Continued) Estimated prevalence of adults aged $\geq 45$ years who have ever been told by a health care professional that they had a stroke, by metropolitan and micropolitan statistical area Behavioral Risk Factor Surveillance System, United States, 2012

\begin{tabular}{|c|c|c|c|c|}
\hline MMSA(s) & $\begin{array}{l}\text { Sample } \\
\text { size }\end{array}$ & $\%$ & SE & $95 \% \mathrm{Cl}$ \\
\hline Detroit-Dearborn-Livonia, Michigan* & 1,599 & 5.8 & 0.8 & $(4.3-7.4)$ \\
\hline Dover, Delaware & 1,030 & 6.9 & 1.2 & $(4.5-9.3)$ \\
\hline Duluth, Minnesota-Wisconsin & 381 & 3.8 & 1.1 & $(1.7-5.9)$ \\
\hline Durham-Chapel Hill, North Carolina & 512 & 4.7 & 1.0 & $(2.7-6.7)$ \\
\hline El Paso, Texas & 438 & $\mathrm{~N} / \mathrm{A}$ & $\mathrm{N} / \mathrm{A}$ & $(\mathrm{N} / \mathrm{A}-\mathrm{N} / \mathrm{A})$ \\
\hline Eugene, Oregon & 391 & 4.3 & 1.1 & $(2.1-6.5)$ \\
\hline Fairbanks, Alaska & 328 & $\mathrm{~N} / \mathrm{A}$ & N/A & $(\mathrm{N} / \mathrm{A}-\mathrm{N} / \mathrm{A})$ \\
\hline Fargo, North Dakota-Minnesota & 628 & 3.1 & 0.9 & $(1.3-4.9)$ \\
\hline Farmington, New Mexico & 424 & 4.6 & 1.3 & $(2.2-7.1)$ \\
\hline Fayetteville, North Carolina & 318 & 4.8 & 1.4 & $(2.1-7.6)$ \\
\hline $\begin{array}{l}\text { Fayetteville-Springdale-Rogers, } \\
\text { Arkansas-Missouri }\end{array}$ & 586 & 4.4 & 1.1 & $(2.1-6.6)$ \\
\hline Fort Collins, Colorado & 412 & 4.4 & 1.1 & $(2.2-6.5)$ \\
\hline Fort Wayne, Indiana & 353 & 4.4 & 1.2 & $(2.0-6.8)$ \\
\hline Fort Worth-Arlington, Texas* & 500 & 4.9 & 1.3 & $(2.3-7.5)$ \\
\hline Grand Island, Nebraska & 633 & 3.8 & 0.8 & $(2.3-5.4)$ \\
\hline Grand Rapids-Wyoming, Michigan & 653 & 4.2 & 0.9 & $(2.5-6.0)$ \\
\hline Great Falls, Montana & 539 & 6.6 & 1.2 & $(4.2-9.1)$ \\
\hline Greeley, Colorado & 337 & 4.0 & 1.2 & $(1.7-6.3)$ \\
\hline $\begin{array}{l}\text { Greensboro-High Point, } \\
\text { North Carolina }\end{array}$ & 552 & 5.0 & 1.1 & $(2.9-7.1)$ \\
\hline $\begin{array}{l}\text { Greenville-Anderson-Mauldin, } \\
\text { South Carolina }\end{array}$ & 1,143 & 6.2 & 0.9 & $(4.4-7.9)$ \\
\hline Gulfport-Biloxi-Pascagoula, Mississippi & 547 & 7.6 & 1.4 & $(4.9-10.2)$ \\
\hline $\begin{array}{l}\text { Hagerstown-Martinsburg, Maryland- } \\
\text { West Virginia }\end{array}$ & 569 & 3.9 & 0.9 & $(2.1-5.6)$ \\
\hline Harrisburg-Carlisle, Pennsylvania & 441 & 3.5 & 1.0 & $(1.7-5.4)$ \\
\hline $\begin{array}{l}\text { Hartford-West Hartford-East Hartford, } \\
\text { Connecticut }\end{array}$ & 1,865 & 3.2 & 0.4 & $(2.4-4.0)$ \\
\hline Heber, Utah & 356 & $\mathrm{~N} / \mathrm{A}$ & $\mathrm{N} / \mathrm{A}$ & $(\mathrm{N} / \mathrm{A}-\mathrm{N} / \mathrm{A})$ \\
\hline Hilo, Hawaii & 939 & 5.2 & 1.2 & $(2.9-7.4)$ \\
\hline $\begin{array}{l}\text { Hilton Head Island-Bluffton-Beaufort, } \\
\text { South Carolina }\end{array}$ & 791 & 2.3 & 0.5 & $(1.3-3.2)$ \\
\hline $\begin{array}{l}\text { Houston-The Woodlands-Sugar Land, } \\
\text { Texas }\end{array}$ & 619 & 4.8 & 0.9 & $(3.1-6.5)$ \\
\hline $\begin{array}{l}\text { Huntington-Ashland, West } \\
\text { Virginia-Kentucky-Ohio }\end{array}$ & 808 & 5.8 & 0.9 & $(4.0-7.6)$ \\
\hline Huntsville, Alabama & 416 & N/A & $\mathrm{N} / \mathrm{A}$ & $(\mathrm{N} / \mathrm{A}-\mathrm{N} / \mathrm{A})$ \\
\hline Idaho Falls, Idaho & 394 & $\mathrm{~N} / \mathrm{A}$ & $\mathrm{N} / \mathrm{A}$ & $(\mathrm{N} / \mathrm{A}-\mathrm{N} / \mathrm{A})$ \\
\hline $\begin{array}{l}\text { Indianapolis-Carmel-Anderson, } \\
\text { Indiana }\end{array}$ & 1,462 & 5.1 & 0.8 & $(3.6-6.7)$ \\
\hline Jackson, Mississippi & 636 & 7.8 & 1.1 & $(5.5-10.0)$ \\
\hline Jacksonville, Florida & 380 & 5.3 & 1.3 & $(2.8-7.8)$ \\
\hline Kahului-Wailuku-Lahaina, Hawaii & 853 & 3.4 & 0.7 & $(2.0-4.8)$ \\
\hline Kalispell, Montana & 398 & 4.2 & 1.1 & $(2.0-6.5)$ \\
\hline Kansas City, Missouri-Kansas & 3,492 & 6.0 & 0.8 & $(4.3-7.6)$ \\
\hline Kapaa, Hawaii & 532 & 2.8 & 0.8 & $(1.2-4.4)$ \\
\hline Keene, New Hampshire & 439 & $\mathrm{~N} / \mathrm{A}$ & $\mathrm{N} / \mathrm{A}$ & $(\mathrm{N} / \mathrm{A}-\mathrm{N} / \mathrm{A}$ \\
\hline Kennewick-Richland, Washington & 369 & 2.7 & 0.8 & $(1.2-4.2)$ \\
\hline $\begin{array}{l}\text { Kingsport-Bristol-Bristol, } \\
\text { Tennessee-Virginia }\end{array}$ & 457 & 8.0 & 1.6 & $(4.8-11.2)$ \\
\hline Knoxville, Tennessee & 623 & 5.6 & 1.0 & $(3.6-7.6)$ \\
\hline Laconia, New Hampshire & 469 & 4.4 & 1.3 & $(1.9-6.9)$ \\
\hline Lafayette, Louisiana & 396 & 5.1 & 1.5 & $(2.2-8.0)$ \\
\hline Las Cruces, New Mexico & 474 & 4.7 & 1.1 & $(2.5-6.9)$ \\
\hline $\begin{array}{l}\text { Las Vegas-Henderson-Paradise, } \\
\text { Nevada }\end{array}$ & 1,206 & 5.9 & 1.0 & $(4.0-7.8)$ \\
\hline Lewiston-Auburn, Maine & 466 & 8.0 & 1.6 & $(4.9-11.1)$ \\
\hline Lexington-Fayette, Kentucky & 321 & 3.6 & 1.1 & $(1.5-5.7)$ \\
\hline Lincoln, Nebraska & 947 & 3.5 & 0.7 & $(2.1-4.8)$ \\
\hline
\end{tabular}

See table footnotes on page 127.
TABLE 68. (Continued) Estimated prevalence of adults aged $\geq 45$ years who have ever been told by a health care professional that they had a stroke, by metropolitan and micropolitan statistical area Behavioral Risk Factor Surveillance System, United States, 2012

\begin{tabular}{|c|c|c|c|c|}
\hline MMSA(s) & $\begin{array}{l}\text { Sample } \\
\text { size }\end{array}$ & $\%$ & SE & $95 \% \mathrm{Cl}$ \\
\hline $\begin{array}{l}\text { Little Rock-North Little Rock-Conway, } \\
\text { Arkansas }\end{array}$ & 856 & 4.3 & 0.8 & $(2.7-5.8)$ \\
\hline Logan, Utah-Idaho & 292 & 2.7 & 0.8 & $(1.1-4.2)$ \\
\hline $\begin{array}{l}\text { Los Angeles-Long Beach-Glendale, } \\
\text { California* }\end{array}$ & 2,125 & 3.4 & 0.5 & $(2.4-4.4)$ \\
\hline $\begin{array}{l}\text { Louisville/Jefferson County, } \\
\text { Kentucky-Indiana }\end{array}$ & 1,557 & 6.3 & 1.0 & $(4.3-8.3)$ \\
\hline Lumberton, North Carolina & 402 & N/A & N/A & $(\mathrm{N} / \mathrm{A}-\mathrm{N} / \mathrm{A})$ \\
\hline Manchester-Nashua, New Hampshire & 1,366 & 3.5 & 0.6 & $(2.3-4.7)$ \\
\hline McAllen-Edinburg-Mission, Texas & 384 & N/A & N/A & $(\mathrm{N} / \mathrm{A}-\mathrm{N} / \mathrm{A})$ \\
\hline $\begin{array}{l}\text { Memphis, } \\
\text { Tennessee-Mississippi-Arkansas }\end{array}$ & 929 & 8.7 & 1.4 & $(6.0-11.4)$ \\
\hline $\begin{array}{l}\text { Miami-Fort Lauderdale-West Palm } \\
\text { Beach, Florida }\end{array}$ & 1,181 & 3.6 & 0.8 & $(2.1-5.2)$ \\
\hline $\begin{array}{l}\text { Milwaukee-Waukesha-West Allis, } \\
\text { Wisconsin }\end{array}$ & 861 & 5.5 & 1.4 & $(2.8-8.2)$ \\
\hline $\begin{array}{l}\text { Minneapolis-St. Paul-Bloomington, } \\
\text { Minnesota-Wisconsin }\end{array}$ & 5,333 & 4.3 & 0.5 & $(3.3-5.3)$ \\
\hline Missoula, Montana & 539 & 5.1 & 1.1 & $(3.0-7.3)$ \\
\hline Mobile, Alabama & 641 & 9.3 & 1.7 & $(6.0-12.6)$ \\
\hline Montgomery, Alabama & 378 & 8.2 & 1.8 & $(4.7-11.7)$ \\
\hline $\begin{array}{l}\text { Montgomery County-Bucks County- } \\
\text { Chester County, Pennsylvania* }\end{array}$ & 840 & 3.7 & 0.7 & $(2.4-5.1)$ \\
\hline $\begin{array}{l}\text { Myrtle Beach-Conway-North Myrtle } \\
\text { Beach, South Carolina-North Carolina }\end{array}$ & 714 & 5.4 & 0.9 & $(3.6-7.2)$ \\
\hline $\begin{array}{l}\text { Nashville-Davidson-Murfreesboro- } \\
\text { Franklin, Tennessee }\end{array}$ & 867 & 5.1 & 0.9 & $(3.3-6.8)$ \\
\hline $\begin{array}{l}\text { Nassau County-Suffolk County, } \\
\text { New York* }\end{array}$ & 621 & 2.7 & 0.7 & $(1.3-4.1)$ \\
\hline Newark, New Jersey-Pennsylvania* & 4,691 & 3.9 & 0.5 & $(3.1-4.8)$ \\
\hline New Haven-Milford, Connecticut & 1,435 & 3.7 & 0.6 & $(2.6-4.9)$ \\
\hline New Orleans-Metairie, Louisiana & 945 & 4.8 & 0.8 & $(3.1-6.4)$ \\
\hline $\begin{array}{l}\text { New York-Jersey City-White Plains, } \\
\text { New York-New Jersey* }\end{array}$ & 4,405 & 4.7 & 0.8 & $(3.2-6.2)$ \\
\hline Norfolk, Nebraska & 444 & $\mathrm{~N} / \mathrm{A}$ & $\mathrm{N} / \mathrm{A}$ & $(\mathrm{N} / \mathrm{A}-\mathrm{N} / \mathrm{A})$ \\
\hline North Platte, Nebraska & 489 & 4.7 & 1.1 & $(2.6-6.7)$ \\
\hline Norwich-New London, Connecticut & 757 & 3.3 & 0.8 & $(1.8-4.8)$ \\
\hline $\begin{array}{l}\text { Oakland-Hayward-Berkeley, } \\
\text { California* }\end{array}$ & 676 & $\mathrm{~N} / \mathrm{A}$ & $\mathrm{N} / \mathrm{A}$ & $(\mathrm{N} / \mathrm{A}-\mathrm{N} / \mathrm{A})$ \\
\hline Ocean City, New Jersey & 454 & 6.0 & 1.1 & $(3.8-8.2)$ \\
\hline Ogden-Clearfield, Utah & 1,499 & 4.2 & 0.6 & $(3.0-5.4)$ \\
\hline Oklahoma City, Oklahoma & 1,550 & 5.5 & 0.6 & $(4.2-6.7)$ \\
\hline Olympia-Tumwater, Washington & 382 & 4.1 & 1.1 & $(1.9-6.3)$ \\
\hline Omaha-Council Bluffs, Nebraska-lowa & 3,556 & 4.6 & 0.5 & $(3.6-5.5)$ \\
\hline Orlando-Kissimmee-Sanford, Florida & 408 & N/A & N/A & $(\mathrm{N} / \mathrm{A}-\mathrm{N} / \mathrm{A})$ \\
\hline Philadelphia, Pennsylvania* & 1,614 & 5.6 & 0.7 & $(4.3-7.0)$ \\
\hline Phoenix-Mesa-Scottsdale, Arizona & 1,758 & 4.4 & 0.6 & $(3.2-5.6)$ \\
\hline Pittsburgh, Pennsylvania & 2,453 & 5.1 & 0.5 & $(4.1-6.2)$ \\
\hline Ponce, Puerto Rico & 347 & N/A & $\mathrm{N} / \mathrm{A}$ & $(\mathrm{N} / \mathrm{A}-\mathrm{N} / \mathrm{A})$ \\
\hline Portland-South Portland, Maine & 2,411 & 3.7 & 0.5 & $(2.7-4.7)$ \\
\hline $\begin{array}{l}\text { Portland-Vancouver-Hillsboro, } \\
\text { Oregon-Washington }\end{array}$ & 2,190 & 4.3 & 0.5 & $(3.3-5.3)$ \\
\hline $\begin{array}{l}\text { Providence-Warwick, Rhode } \\
\text { Island-Massachusetts }\end{array}$ & 5,836 & 3.8 & 0.3 & $(3.1-4.4)$ \\
\hline Provo-Orem, Utah & 893 & 4.1 & 0.7 & $(2.7-5.4)$ \\
\hline Raleigh, North Carolina & 509 & $\mathrm{~N} / \mathrm{A}$ & $\mathrm{N} / \mathrm{A}$ & $(\mathrm{N} / \mathrm{A}-\mathrm{N} / \mathrm{A})$ \\
\hline Rapid City, South Dakota & 697 & 4.5 & 0.9 & $(2.7-6.3)$ \\
\hline Reno, Nevada & 1,093 & 2.2 & 0.4 & $(1.4-3.1)$ \\
\hline Richmond, Virginia & 708 & 4.3 & 0.8 & $(2.8-5.8)$ \\
\hline
\end{tabular}

See table footnotes on page 127. 
TABLE 68. (Continued) Estimated prevalence of adults aged $\geq 45$ years who have ever been told by a health care professional that they had a stroke, by metropolitan and micropolitan statistical area Behavioral Risk Factor Surveillance System, United States, 2012

\begin{tabular}{|c|c|c|c|c|}
\hline MMSA(s) & $\begin{array}{l}\text { Sample } \\
\text { size }\end{array}$ & $\%$ & SE & $95 \% \mathrm{Cl}$ \\
\hline $\begin{array}{l}\text { Riverside-San Bernardino-Ontario, } \\
\text { California }\end{array}$ & 999 & 3.7 & 0.8 & $(2.3-5.2)$ \\
\hline $\begin{array}{l}\text { Rockingham County-Strafford County, } \\
\text { New Hampshire* }\end{array}$ & 1,262 & 3.2 & 0.5 & $(2.2-4.2)$ \\
\hline Rutland, Vermont & 470 & 3.8 & 1.0 & $(1.9-5.7)$ \\
\hline $\begin{array}{l}\text { Sacramento-Roseville-Arden-Arcade, } \\
\text { California }\end{array}$ & 699 & 4.4 & 1.2 & $(2.0-6.8)$ \\
\hline St. Louis, Missouri-Illinois & 1,462 & 5.4 & 0.8 & $(3.8-7.0)$ \\
\hline Salisbury, Maryland-Delaware & 1,718 & 5.8 & 0.7 & $(4.3-7.2)$ \\
\hline Salt Lake City, Utah & 2,899 & 3.9 & 0.4 & $(3.1-4.7)$ \\
\hline San Antonio-New Braunfels, Texas & 522 & 4.0 & 0.9 & $(2.2-5.9)$ \\
\hline San Diego-Carlsbad, California & 728 & 3.2 & 0.8 & $(1.6-4.8)$ \\
\hline $\begin{array}{l}\text { San Francisco-Redwood City-South } \\
\text { San Francisco, California* }\end{array}$ & 389 & $\mathrm{~N} / \mathrm{A}$ & $\mathrm{N} / \mathrm{A}$ & $(\mathrm{N} / \mathrm{A}-\mathrm{N} / \mathrm{A})$ \\
\hline $\begin{array}{l}\text { San Jose-Sunnyvale-Santa Clara, } \\
\text { California }\end{array}$ & 418 & $\mathrm{~N} / \mathrm{A}$ & $\mathrm{N} / \mathrm{A}$ & $(\mathrm{N} / \mathrm{A}-\mathrm{N} / \mathrm{A})$ \\
\hline San Juan-Carolina-Caguas, Puerto Rico & 2,482 & 3.6 & 0.4 & $(2.8-4.5)$ \\
\hline Santa Fe, New Mexico & 505 & $\mathrm{~N} / \mathrm{A}$ & $\mathrm{N} / \mathrm{A}$ & $(\mathrm{N} / \mathrm{A}-\mathrm{N} / \mathrm{A})$ \\
\hline Sayre, Pennsylvania & 1,457 & 4.2 & 0.6 & $(3.0-5.4)$ \\
\hline Scottsbluff, Nebraska & 518 & 3.4 & 0.8 & $(1.8-4.9)$ \\
\hline $\begin{array}{l}\text { Scranton-Wilkes-Barre-Hazleton, } \\
\text { Pennsylvania }\end{array}$ & 552 & 4.2 & 1.0 & $(2.3-6.1)$ \\
\hline Seattle-Bellevue-Everett, Washington* & 3,445 & 3.5 & 0.4 & $(2.7-4.3)$ \\
\hline Shreveport-Bossier City, Louisiana & 419 & 4.8 & 1.2 & $(2.4-7.2)$ \\
\hline $\begin{array}{l}\text { Silver Spring-Frederick-Rockville, } \\
\text { Maryland* }\end{array}$ & 1,699 & 2.1 & 0.3 & $(1.4-2.8)$ \\
\hline $\begin{array}{l}\text { Sioux City, lowa-Nebraska- } \\
\text { South Dakota }\end{array}$ & 923 & 4.7 & 1.2 & $(2.3-7.0)$ \\
\hline Sioux Falls, South Dakota & 833 & 3.8 & 0.8 & $(2.2-5.4)$ \\
\hline Spartanburg, South Carolina & 509 & 8.0 & 1.7 & $(4.7-11.3)$ \\
\hline Spokane-Spokane Valley, Washington & 830 & 4.6 & 0.8 & $(3.1-6.0)$ \\
\hline Springfield, Massachusetts & 1,667 & 5.1 & 0.9 & $(3.5-6.8)$ \\
\hline Tacoma-Lakewood, Washington* & 809 & 4.4 & 0.8 & $(2.8-6.0)$ \\
\hline
\end{tabular}

TABLE 68. (Continued) Estimated prevalence of adults aged $\geq 45$ years who have ever been told by a health care professional that they had a stroke, by metropolitan and micropolitan statistical area Behavioral Risk Factor Surveillance System, United States, 2012

\begin{tabular}{|c|c|c|c|c|}
\hline MMSA(s) & $\begin{array}{l}\text { Sample } \\
\text { size }\end{array}$ & $\%$ & SE & $95 \% \mathrm{Cl}$ \\
\hline $\begin{array}{l}\text { Tampa-St. Petersburg-Clearwater, } \\
\text { Florida }\end{array}$ & 609 & 4.1 & 0.8 & $(2.4-5.7)$ \\
\hline Toledo, Ohio & 717 & 5.1 & 1.1 & $(3.0-7.2$ \\
\hline Topeka, Kansas & 791 & 3.8 & 0.7 & $(2.4-5.2$ \\
\hline Torrington, Connecticut & 506 & 4.1 & 1.0 & $(2.1-6.0)$ \\
\hline Trenton, New Jersey & 407 & N/A & N/A & $(\mathrm{N} / \mathrm{A}-\mathrm{N} / \mathrm{A})$ \\
\hline Tucson, Arizona & 726 & 4.5 & 0.9 & $(2.8-6.2)$ \\
\hline Tulsa, Oklahoma & 1,197 & 5.1 & 0.7 & $(3.6-6.6)$ \\
\hline Tuscaloosa, Alabama & 430 & 8.5 & 1.4 & $(5.6-11.3$ \\
\hline Urban Honolulu, Hawaii & 2,503 & 4.8 & 0.6 & $(3.6-5.9$ \\
\hline Vineland-Bridgeton, New Jersey & 402 & N/A & $\mathrm{N} / \mathrm{A}$ & $(\mathrm{N} / \mathrm{A}-\mathrm{N} / \mathrm{A})$ \\
\hline $\begin{array}{l}\text { Virginia Beach-Norfolk-Newport News, } \\
\text { Virginia-North Carolina }\end{array}$ & 1,076 & 5.7 & 0.8 & $(4.0-7.3)$ \\
\hline $\begin{array}{l}\text { Warren-Troy-Farmington Hills, } \\
\text { Michigan* }\end{array}$ & 1,551 & 5.2 & 0.7 & $(3.8-6.7)$ \\
\hline $\begin{array}{l}\text { Washington-Arlington-Alexandria, } \\
\text { District of Columbia-Virginia- } \\
\text { Maryland-West Virginia** }\end{array}$ & 5,553 & 4.6 & 0.5 & $(3.7-5.5$ \\
\hline Wichita, Kansas & 1,669 & 5.3 & 0.7 & $(3.9-6.7)$ \\
\hline $\begin{array}{l}\text { Wilmington, Delaware-Maryland- } \\
\text { New Jersey* }\end{array}$ & 2,179 & 4.9 & 0.6 & $(3.8-6.1)$ \\
\hline Winston-Salem, North Carolina & 546 & 5.6 & 1.2 & $(3.3-7.8$ \\
\hline Worcester, Massachusetts-Connecticut & 1,983 & 4.2 & 0.7 & $(2.8-5.6)$ \\
\hline Yakima, Washington & 364 & N/A & $\mathrm{N} / \mathrm{A}$ & $(\mathrm{N} / \mathrm{A}-\mathrm{N} / \mathrm{A}$ \\
\hline $\begin{array}{l}\text { Youngstown-Warren-Boardman, } \\
\text { Ohio-Pennsylvania }\end{array}$ & 740 & 3.2 & 0.9 & $(1.4-5.0$ \\
\hline Median & & 4.4 & & \\
\hline Range & & $2.1-9.3$ & & \\
\hline
\end{tabular}

Abbreviations: $\mathrm{Cl}=$ confidence interval; $\mathrm{MMSA}=$ metropolitan and micropolitan statistical area; $\mathrm{SE}=$ standard error.

* Metropolitan division.

${ }^{\dagger}$ Estimate not available (N/A) if the unweighted sample size for the denominator was $<50$ or if the relative standard error is $>0.3$. 
TABLE 69. Estimated prevalence of adults aged $\geq 45$ years who have ever been told by a health care professional that they had a stroke, by county - Behavioral Risk Factor Surveillance System, United States, 2012

\begin{tabular}{|c|c|c|c|c|}
\hline County & $\begin{array}{l}\text { Sample } \\
\text { size }\end{array}$ & $\%$ & SE & $95 \% \mathrm{Cl}$ \\
\hline Jefferson County, Alabama & 646 & 5.2 & 1.0 & $(3.2-7.2)$ \\
\hline Madison County, Alabama & 355 & $N / A^{*}$ & $\mathrm{~N} / \mathrm{A}$ & $(\mathrm{N} / \mathrm{A}-\mathrm{N} / \mathrm{A})$ \\
\hline Mobile County, Alabama & 641 & 9.3 & 1.7 & $(6.0-12.6)$ \\
\hline Anchorage Municipality, Alaska & 483 & 3.7 & 0.9 & $(1.9-5.5)$ \\
\hline Fairbanks North Star Borough, Alaska & 328 & N/A & N/A & $(\mathrm{N} / \mathrm{A}-\mathrm{N} / \mathrm{A})$ \\
\hline Matanuska-Susitna Borough, Alaska & 378 & 4.3 & 1.1 & $(2.2-6.3)$ \\
\hline Maricopa County, Arizona & 1,383 & 4.4 & 0.7 & $(3.1-5.7)$ \\
\hline Pima County, Arizona & 726 & 4.5 & 0.9 & $(2.8-6.2)$ \\
\hline Pulaski County, Arkansas & 561 & 4.7 & 1.1 & $(2.6-6.7)$ \\
\hline Alameda County, California & 408 & N/A & N/A & $(\mathrm{N} / \mathrm{A}-\mathrm{N} / \mathrm{A})$ \\
\hline Los Angeles County, California & 2,125 & 3.4 & 0.5 & $(2.4-4.4)$ \\
\hline Orange County, California & 677 & N/A & N/A & $(\mathrm{N} / \mathrm{A}-\mathrm{N} / \mathrm{A})$ \\
\hline Riverside County, California & 561 & 3.7 & 1.0 & $(1.8-5.6)$ \\
\hline Sacramento County, California & 425 & 5.5 & 1.6 & $(2.4-8.6)$ \\
\hline San Bernardino County, California & 438 & 3.3 & 1.0 & $(1.4-5.2)$ \\
\hline San Diego County, California & 728 & 3.2 & 0.8 & $(1.6-4.8)$ \\
\hline Santa Clara County, California & 405 & N/A & $\mathrm{N} / \mathrm{A}$ & $(\mathrm{N} / \mathrm{A}-\mathrm{N} / \mathrm{A})$ \\
\hline Adams County, Colorado & 504 & 4.8 & 1.1 & $(2.6-7.0)$ \\
\hline Arapahoe County, Colorado & 557 & 4.3 & 1.0 & $(2.3-6.2)$ \\
\hline Boulder County, Colorado & 354 & N/A & N/A & $(\mathrm{N} / \mathrm{A}-\mathrm{N} / \mathrm{A})$ \\
\hline Denver County, Colorado & 574 & 2.5 & 0.7 & $(1.1-3.8)$ \\
\hline Douglas County, Colorado & 335 & $\mathrm{~N} / \mathrm{A}$ & $\mathrm{N} / \mathrm{A}$ & $(\mathrm{N} / \mathrm{A}-\mathrm{N} / \mathrm{A})$ \\
\hline El Paso County, Colorado & 638 & 3.0 & 0.7 & $(1.7-4.4)$ \\
\hline Jefferson County, Colorado & 793 & 2.6 & 0.5 & $(1.6-3.6)$ \\
\hline Larimer County, Colorado & 412 & 4.4 & 1.1 & $(2.2-6.5)$ \\
\hline Weld County, Colorado & 337 & 4.0 & 1.2 & $(1.7-6.3)$ \\
\hline Fairfield County, Connecticut & 1,446 & 2.8 & 0.5 & $(1.8-3.9)$ \\
\hline Hartford County, Connecticut & 1,390 & 3.4 & 0.5 & $(2.4-4.4)$ \\
\hline Litchfield County, Connecticut & 506 & 4.1 & 1.0 & $(2.1-6.0)$ \\
\hline New Haven County, Connecticut & 1,435 & 3.7 & 0.6 & $(2.6-4.9)$ \\
\hline New London County, Connecticut & 757 & 3.3 & 0.8 & $(1.8-4.8)$ \\
\hline Kent County, Delaware & 1,030 & 6.9 & 1.2 & $(4.5-9.3)$ \\
\hline New Castle County, Delaware & 1,490 & 4.7 & 0.7 & $(3.4-6.0)$ \\
\hline Sussex County, Delaware & 1,114 & 5.8 & 0.8 & $(4.3-7.4)$ \\
\hline $\begin{array}{l}\text { District of Columbia, } \\
\text { District of Columbia }\end{array}$ & 2,662 & 7.0 & 0.9 & $(5.2-8.8)$ \\
\hline Broward County, Florida & 382 & N/A & $\mathrm{N} / \mathrm{A}$ & $(\mathrm{N} / \mathrm{A}-\mathrm{N} / \mathrm{A})$ \\
\hline Miami-Dade County, Florida & 552 & N/A & $\mathrm{N} / \mathrm{A}$ & $(\mathrm{N} / \mathrm{A}-\mathrm{N} / \mathrm{A})$ \\
\hline Hawaii County, Hawaii & 939 & 5.2 & 1.2 & $(2.9-7.4)$ \\
\hline Honolulu County, Hawaii & 2,503 & 4.8 & 0.6 & $(3.6-5.9)$ \\
\hline Kauai County, Hawaii & 532 & 2.8 & 0.8 & $(1.2-4.4)$ \\
\hline Maui County, Hawaii & 853 & 3.4 & 0.7 & $(2.0-4.8)$ \\
\hline Ada County, Idaho & 578 & 4.5 & 1.2 & $(2.2-6.9)$ \\
\hline Canyon County, Idaho & 367 & 6.4 & 1.9 & $(2.6-10.1)$ \\
\hline Cook County, Illinois & 984 & 3.7 & 0.7 & $(2.4-5.1)$ \\
\hline Lake County, Indiana & 665 & 5.6 & 1.3 & $(3.1-8.1)$ \\
\hline Marion County, Indiana & 856 & 5.4 & 0.9 & $(3.6-7.2)$ \\
\hline Polk County, lowa & 542 & 4.7 & 1.0 & $(2.8-6.6)$ \\
\hline Johnson County, Kansas & 1,582 & 3.4 & 0.5 & $(2.4-4.3)$ \\
\hline Sedgwick County, Kansas & 1,230 & 5.4 & 0.9 & $(3.7-7.1)$ \\
\hline Shawnee County, Kansas & 539 & 3.7 & 0.9 & $(2.0-5.5)$ \\
\hline Wyandotte County, Kansas & 671 & 6.8 & 1.5 & $(3.9-9.7)$ \\
\hline Jefferson County, Kentucky & 1,238 & 6.4 & 1.2 & $(4.1-8.7)$ \\
\hline East Baton Rouge Parish, Louisiana & 398 & 4.9 & 1.3 & $(2.4-7.4)$ \\
\hline Androscoggin County, Maine & 466 & 8.0 & 1.6 & $(4.9-11.1)$ \\
\hline Aroostook County, Maine & 409 & 6.5 & 1.5 & $(3.6-9.4)$ \\
\hline Cumberland County, Maine & 1,253 & 3.7 & 0.8 & $(2.2-5.2)$ \\
\hline Kennebec County, Maine & 578 & 3.4 & 0.9 & $(1.8-5.1)$ \\
\hline Penobscot County, Maine & 658 & 4.4 & 1.0 & $(2.5-6.4)$ \\
\hline York County, Maine & 865 & 3.7 & 0.7 & $(2.3-5.1)$ \\
\hline
\end{tabular}

See table footnotes on page 129.
TABLE 69. (Continued) Estimated prevalence of adults aged $\geq 45$ years who have ever been told by a health care professional that they had a stroke, by county - Behavioral Risk Factor Surveillance System, United States, 2012

\begin{tabular}{|c|c|c|c|c|}
\hline County & $\begin{array}{c}\text { Sample } \\
\text { size }\end{array}$ & $\%$ & SE & $95 \% \mathrm{Cl}$ \\
\hline Anne Arundel County, Maryland & 640 & 4.7 & 1.0 & $(2.8-6.5)$ \\
\hline Baltimore County, Maryland & 1,095 & 4.5 & 0.7 & $(3.1-5.8)$ \\
\hline Charles County, Maryland & 357 & 4.9 & 1.3 & $(2.3-7.5)$ \\
\hline Frederick County, Maryland & 576 & 4.4 & 0.9 & $(2.6-6.1)$ \\
\hline Montgomery County, Maryland & 1,123 & 1.5 & 0.4 & $(0.8-2.2)$ \\
\hline Prince George's County, Maryland & 803 & 5.0 & 1.3 & $(2.5-7.5)$ \\
\hline Washington County, Maryland & 419 & 3.5 & 0.9 & $(1.6-5.4)$ \\
\hline Baltimore city, Maryland & 534 & 6.7 & 1.6 & $(3.6-9.8)$ \\
\hline Barnstable County, Massachusetts & 466 & 3.8 & 1.0 & $(1.8-5.9)$ \\
\hline Bristol County, Massachusetts & 1,937 & 3.8 & 0.6 & $(2.5-5.1)$ \\
\hline Essex County, Massachusetts & 1,887 & 3.1 & 0.5 & $(2.1-4.2)$ \\
\hline Hampden County, Massachusetts & 1,399 & 4.5 & 0.8 & $(3.0-6.0)$ \\
\hline Middlesex County, Massachusetts & 2,844 & 2.9 & 0.4 & $(2.1-3.6)$ \\
\hline Norfolk County, Massachusetts & 1,253 & 1.7 & 0.5 & $(0.8-2.6)$ \\
\hline Plymouth County, Massachusetts & 1,320 & 3.8 & 1.0 & $(1.9-5.7)$ \\
\hline Suffolk County, Massachusetts & 1,426 & 2.9 & 0.5 & $(1.8-3.9)$ \\
\hline Worcester County, Massachusetts & 1,819 & 4.0 & 0.8 & $(2.5-5.5)$ \\
\hline Kent County, Michigan & 389 & N/A & $\mathrm{N} / \mathrm{A}$ & $(\mathrm{N} / \mathrm{A}-\mathrm{N} / \mathrm{A})$ \\
\hline Macomb County, Michigan & 418 & 7.2 & 1.5 & $(4.2-10.2)$ \\
\hline Oakland County, Michigan & 852 & 4.9 & 1.1 & $(2.7-7.1)$ \\
\hline Wayne County, Michigan & 1,599 & 5.8 & 0.8 & $(4.3-7.4)$ \\
\hline Anoka County, Minnesota & 361 & N/A & $\mathrm{N} / \mathrm{A}$ & $(\mathrm{N} / \mathrm{A}-\mathrm{N} / \mathrm{A})$ \\
\hline Dakota County, Minnesota & 422 & N/A & $\mathrm{N} / \mathrm{A}$ & $(\mathrm{N} / \mathrm{A}-\mathrm{N} / \mathrm{A})$ \\
\hline Hennepin County, Minnesota & 2,189 & 4.6 & 1.0 & $(2.7-6.6)$ \\
\hline Ramsey County, Minnesota & 1,443 & 4.9 & 1.2 & $(2.6-7.2)$ \\
\hline Jackson County, Missouri & 668 & 5.5 & 1.3 & $(2.9-8.1)$ \\
\hline St. Louis County, Missouri & 668 & 4.8 & 1.3 & $(2.3-7.3)$ \\
\hline Cascade County, Montana & 539 & 6.6 & 1.2 & $(4.2-9.1)$ \\
\hline Flathead County, Montana & 398 & 4.2 & 1.1 & $(2.0-6.5)$ \\
\hline Hill County, Montana & 432 & 2.8 & 0.7 & $(1.4-4.2)$ \\
\hline Lake County, Montana & 701 & 5.3 & 1.0 & $(3.4-7.2)$ \\
\hline Missoula County, Montana & 539 & 5.1 & 1.1 & $(3.0-7.3)$ \\
\hline Yellowstone County, Montana & 474 & 4.3 & 0.9 & $(2.5-6.1)$ \\
\hline Dakota County, Nebraska & 573 & 7.0 & 2.0 & $(3.1-10.9)$ \\
\hline Douglas County, Nebraska & 2,273 & 4.3 & 0.5 & $(3.3-5.2)$ \\
\hline Hall County, Nebraska & 379 & 3.7 & 0.9 & $(1.9-5.5)$ \\
\hline Lancaster County, Nebraska & 778 & 3.4 & 0.7 & $(2.0-4.8)$ \\
\hline Lincoln County, Nebraska & 473 & 4.6 & 1.1 & $(2.5-6.7)$ \\
\hline Sarpy County, Nebraska & 726 & 3.4 & 0.8 & $(1.8-5.0)$ \\
\hline Scotts Bluff County, Nebraska & 475 & 3.1 & 0.8 & $(1.5-4.7)$ \\
\hline Clark County, Nevada & 1,206 & 5.9 & 1.0 & $(4.0-7.8)$ \\
\hline Washoe County, Nevada & 1,080 & 2.2 & 0.4 & $(1.4-3.1)$ \\
\hline Belknap County, New Hampshire & 469 & 4.4 & 1.3 & $(1.9-6.9)$ \\
\hline Carroll County, New Hampshire & 458 & 2.9 & 0.8 & $(1.4-4.4)$ \\
\hline Cheshire County, New Hampshire & 439 & N/A & $\mathrm{N} / \mathrm{A}$ & $(\mathrm{N} / \mathrm{A}-\mathrm{N} / \mathrm{A})$ \\
\hline Coos County, New Hampshire & 433 & 4.4 & 1.1 & $(2.1-6.6)$ \\
\hline Grafton County, New Hampshire & 440 & 3.3 & 0.8 & $(1.8-4.8)$ \\
\hline Hillsborough County, New Hampshire & 1,366 & 3.5 & 0.6 & $(2.3-4.7)$ \\
\hline Merrimack County, New Hampshire & 522 & 3.9 & 1.1 & $(1.7-6.1)$ \\
\hline Rockingham County, New Hampshire & 785 & 2.5 & 0.6 & $(1.4-3.6)$ \\
\hline Strafford County, New Hampshire & 477 & 4.9 & 1.2 & $(2.6-7.3)$ \\
\hline Atlantic County, New Jersey & 786 & 4.8 & 1.0 & $(2.8-6.7)$ \\
\hline Bergen County, New Jersey & 606 & 2.5 & 0.7 & $(1.1-3.9)$ \\
\hline Burlington County, New Jersey & 486 & 4.7 & 1.4 & $(2.0-7.4)$ \\
\hline Camden County, New Jersey & 516 & N/A & $\mathrm{N} / \mathrm{A}$ & $(\mathrm{N} / \mathrm{A}-\mathrm{N} / \mathrm{A})$ \\
\hline Cape May County, New Jersey & 454 & 6.0 & 1.1 & $(3.8-8.2)$ \\
\hline Cumberland County, New Jersey & 402 & N/A & $\mathrm{N} / \mathrm{A}$ & $(\mathrm{N} / \mathrm{A}-\mathrm{N} / \mathrm{A})$ \\
\hline Essex County, New Jersey & 792 & 5.2 & 1.0 & $(3.2-7.2)$ \\
\hline Gloucester County, New Jersey & 401 & 4.1 & 1.1 & $(2.0-6.3)$ \\
\hline Hudson County, New Jersey & 741 & 4.2 & 0.9 & $(2.4-6.0)$ \\
\hline
\end{tabular}

See table footnotes on page 129. 
TABLE 69. (Continued) Estimated prevalence of adults aged $\geq 45$ years who have ever been told by a health care professional that they had a stroke, by county - Behavioral Risk Factor Surveillance System, United States, 2012

\begin{tabular}{|c|c|c|c|c|}
\hline County & $\begin{array}{l}\text { Sample } \\
\text { size }\end{array}$ & $\%$ & SE & $95 \% \mathrm{Cl}$ \\
\hline Hunterdon County, New Jersey & 437 & N/A & N/A & $(\mathrm{N} / \mathrm{A}-\mathrm{N} / \mathrm{A})$ \\
\hline Mercer County, New Jersey & 407 & $\mathrm{~N} / \mathrm{A}$ & N/A & $(\mathrm{N} / \mathrm{A}-\mathrm{N} / \mathrm{A})$ \\
\hline Middlesex County, New Jersey & 518 & 3.3 & 0.9 & $(1.6-5.0)$ \\
\hline Monmouth County, New Jersey & 507 & $\mathrm{~N} / \mathrm{A}$ & N/A & $(\mathrm{N} / \mathrm{A}-\mathrm{N} / \mathrm{A})$ \\
\hline Morris County, New Jersey & 610 & 3.0 & 0.9 & $(1.3-4.7)$ \\
\hline Ocean County, New Jersey & 477 & 5.4 & 1.3 & $(2.8-8.0)$ \\
\hline Passaic County, New Jersey & 407 & N/A & $\mathrm{N} / \mathrm{A}$ & $(\mathrm{N} / \mathrm{A}-\mathrm{N} / \mathrm{A})$ \\
\hline Salem County, New Jersey & 445 & 7.4 & 1.6 & $(4.2-10.6)$ \\
\hline Somerset County, New Jersey & 453 & N/A & N/A & $(\mathrm{N} / \mathrm{A}-\mathrm{N} / \mathrm{A})$ \\
\hline Sussex County, New Jersey & 423 & 4.1 & 1.0 & $(2.0-6.1)$ \\
\hline Union County, New Jersey & 440 & 3.3 & 0.9 & $(1.4-5.1)$ \\
\hline Warren County, New Jersey & 419 & N/A & N/A & $(\mathrm{N} / \mathrm{A}-\mathrm{N} / \mathrm{A})$ \\
\hline Bernalillo County, New Mexico & 1,285 & 4.8 & 0.7 & $(3.4-6.1)$ \\
\hline Dona Ana County, New Mexico & 474 & 4.7 & 1.1 & $(2.5-6.9)$ \\
\hline Sandoval County, New Mexico & 482 & $\mathrm{~N} / \mathrm{A}$ & N/A & $(\mathrm{N} / \mathrm{A}-\mathrm{N} / \mathrm{A})$ \\
\hline San Juan County, New Mexico & 424 & 4.6 & 1.3 & 7.1) \\
\hline Santa Fe County, New Mexico & 505 & N/A & $\mathrm{N} / \mathrm{A}$ & $(\mathrm{N} / \mathrm{A}$ \\
\hline Kings County, New York & 208 & $\mathrm{~N} / \mathrm{A}$ & $\mathrm{N} / \mathrm{A}$ & $(\mathrm{N} / \mathrm{A}-\mathrm{N} / \mathrm{A})$ \\
\hline Guilford County, North Carolina & 331 & N/A & N/A & $(\mathrm{N} / \mathrm{A}-\mathrm{N} / \mathrm{A})$ \\
\hline Mecklenburg County, North Carolina & 360 & N/A & $\mathrm{N} / \mathrm{A}$ & $(\mathrm{N} / \mathrm{A}-\mathrm{N} / \mathrm{A})$ \\
\hline Robeson County, North Carolina & 402 & $\mathrm{~N} / \mathrm{A}$ & $\mathrm{N} / \mathrm{A}$ & $(\mathrm{N} / \mathrm{A}-\mathrm{N} / \mathrm{A})$ \\
\hline Wake County, North Carolina & 358 & N/A & N/A & $(\mathrm{N} / \mathrm{A}-\mathrm{N} / \mathrm{A})$ \\
\hline Burleigh County, North Dakota & 398 & 8.2 & 1.9 & $(4.5-12.0)$ \\
\hline Cass County, North Dakota & 578 & N/A & $\mathrm{N} / \mathrm{A}$ & $(\mathrm{N} / \mathrm{A}-\mathrm{N} / \mathrm{A})$ \\
\hline Cuyahoga County, Ohio & 586 & 4.5 & 1.0 & $(2.7-6.4)$ \\
\hline Franklin County, Ohio & 478 & 4.6 & 1.1 & $(2.5-6.7)$ \\
\hline Hamilton County, Ohio & 509 & 6.0 & 1.1 & $(3.9-8.0)$ \\
\hline Lorain County, Ohio & 495 & 6.2 & 1.4 & $(3.6-8.9)$ \\
\hline Lucas County, Ohio & 465 & 5.4 & 1.2 & $(2.9-7.8)$ \\
\hline Mahoning County, Ohio & 488 & N/A & N/A & $(\mathrm{N} / \mathrm{A}-\mathrm{N} / \mathrm{A})$ \\
\hline Montgomery County, Ohio & 491 & 4.7 & 1.2 & $(2.4-7.1)$ \\
\hline Stark County, Ohio & 482 & 5.9 & 1.3 & $(3.4-8.4)$ \\
\hline Summit County, Ohio & 479 & 5.9 & 1.4 & $(3.2-8.7)$ \\
\hline Oklahoma County, Oklahoma & 727 & 6.0 & 1.0 & $(4.1-7.8)$ \\
\hline Tulsa County, Oklahoma & 803 & 3.9 & 0.7 & $(2.5-5.3)$ \\
\hline Clackamas County, Oregon & 386 & N/A & N/A & $(\mathrm{N} / \mathrm{A}-\mathrm{N} / \mathrm{A})$ \\
\hline Lane County, Oregon & 391 & 4.3 & 1.1 & $(2.1-6.5)$ \\
\hline Multnomah County, Oregon & 600 & 5.4 & 1.1 & $(3.3-7.6)$ \\
\hline Washington County, Oregon & 414 & 4.3 & 1.2 & $(2.1-6.6)$ \\
\hline Allegheny County, Pennsylvania & 1,372 & 5.4 & 0.7 & $(3.9-6.8)$ \\
\hline Bradford County, Pennsylvania & 1,457 & 4.2 & 0.6 & $(3.0-5.4)$ \\
\hline Montgomery County, Pennsylvania & 347 & N/A & $\mathrm{N} / \mathrm{A}$ & $(\mathrm{N} / \mathrm{A}-\mathrm{N} / \mathrm{A})$ \\
\hline Philadelphia County, Pennsylvania & 1,373 & 6.5 & 0.9 & $(4.8-8.2)$ \\
\hline Pike County, Pennsylvania & 1,536 & 3.7 & 0.5 & $(2.7-4.7)$ \\
\hline Kent County, Rhode Island & 584 & 2.9 & 0.8 & $(1.4-4.4)$ \\
\hline Providence County, Rhode Island & 2,294 & 4.3 & 0.6 & $(3.2-5.4)$ \\
\hline Washington County, Rhode Island & 517 & 5.0 & 1.1 & $(2.8-7.2)$ \\
\hline Aiken County, South Carolina & 426 & 7.2 & 1.7 & $(4.0-10.5)$ \\
\hline Beaufort County, South Carolina & 688 & 2.1 & 0.5 & $(1.1-3.1)$ \\
\hline
\end{tabular}

TABLE 69. (Continued) Estimated prevalence of adults aged $\geq 45$ years who have ever been told by a health care professional that they had a stroke, by county - Behavioral Risk Factor Surveillance System, United States, 2012

\begin{tabular}{|c|c|c|c|c|}
\hline County & $\begin{array}{c}\text { Sample } \\
\text { size }\end{array}$ & $\%$ & SE & $95 \% \mathrm{Cl}$ \\
\hline Charleston County, South Carolina & 736 & 6.5 & 1.3 & $(4.0-9.0)$ \\
\hline Greenville County, South Carolina & 572 & 6.4 & 1.3 & $(3.9-9.0)$ \\
\hline Horry County, South Carolina & 577 & 5.7 & 1.1 & $(3.6-7.8)$ \\
\hline Richland County, South Carolina & 657 & 4.0 & 0.9 & $(2.2-5.8)$ \\
\hline Spartanburg County, South Carolina & 467 & 9.0 & 1.9 & $(5.2-12.7)$ \\
\hline Lincoln County, South Dakota & 312 & $\mathrm{~N} / \mathrm{A}$ & $\mathrm{N} / \mathrm{A}$ & $(\mathrm{N} / \mathrm{A}-\mathrm{N} / \mathrm{A})$ \\
\hline Minnehaha County, South Dakota & 468 & 3.8 & 1.0 & $(1.9-5.7)$ \\
\hline Pennington County, South Dakota & 357 & 5.3 & 1.2 & $(2.9-7.6)$ \\
\hline Davidson County, Tennessee & 364 & 6.2 & 1.5 & $(3.2-9.2)$ \\
\hline Shelby County, Tennessee & 329 & 8.3 & 1.8 & $(4.7-11.9)$ \\
\hline Bexar County, Texas & 374 & 3.0 & 0.8 & $(1.5-4.5)$ \\
\hline Dallas County, Texas & 288 & $\mathrm{~N} / \mathrm{A}$ & $\mathrm{N} / \mathrm{A}$ & $(\mathrm{N} / \mathrm{A}-\mathrm{N} / \mathrm{A})$ \\
\hline El Paso County, Texas & 437 & $\mathrm{~N} / \mathrm{A}$ & $\mathrm{N} / \mathrm{A}$ & $(\mathrm{N} / \mathrm{A}-\mathrm{N} / \mathrm{A})$ \\
\hline Harris County, Texas & 387 & 4.4 & 1.0 & $(2.4-6.3)$ \\
\hline Hidalgo County, Texas & 384 & $\mathrm{~N} / \mathrm{A}$ & $\mathrm{N} / \mathrm{A}$ & $(\mathrm{N} / \mathrm{A}-\mathrm{N} / \mathrm{A})$ \\
\hline Tarrant County, Texas & 384 & $\mathrm{~N} / \mathrm{A}$ & $\mathrm{N} / \mathrm{A}$ & $(\mathrm{N} / \mathrm{A}-\mathrm{N} / \mathrm{A})$ \\
\hline Travis County, Texas & 738 & 3.8 & 0.9 & $(2.1-5.5)$ \\
\hline Davis County, Utah & 656 & 4.3 & 0.9 & $(2.6-6.1)$ \\
\hline Salt Lake County, Utah & 2,540 & 3.9 & 0.4 & $(3.0-4.7)$ \\
\hline Tooele County, Utah & 359 & N/A & $\mathrm{N} / \mathrm{A}$ & $(\mathrm{N} / \mathrm{A}-\mathrm{N} / \mathrm{A}$ \\
\hline Utah County, Utah & 848 & 4.1 & 0.7 & $(2.8-5.5)$ \\
\hline Wasatch County, Utah & 356 & $\mathrm{~N} / \mathrm{A}$ & N/A & $(\mathrm{N} / \mathrm{A}-\mathrm{N} / \mathrm{A})$ \\
\hline Weber County, Utah & 679 & 3.0 & 0.8 & $(1.6-4.5)$ \\
\hline Chittenden County, Vermont & 589 & 3.6 & 0.8 & $(2.1-5.2)$ \\
\hline Rutland County, Vermont & 470 & 3.8 & 1.0 & $(1.9-5.7)$ \\
\hline Washington County, Vermont & 386 & $\mathrm{~N} / \mathrm{A}$ & $\mathrm{N} / \mathrm{A}$ & $(\mathrm{N} / \mathrm{A}-\mathrm{N} / \mathrm{A})$ \\
\hline Windsor County, Vermont & 422 & 2.2 & 0.6 & $(1.0-3.4)$ \\
\hline Fairfax County, Virginia & 459 & $\mathrm{~N} / \mathrm{A}$ & $\mathrm{N} / \mathrm{A}$ & $(\mathrm{N} / \mathrm{A}-\mathrm{N} / \mathrm{A})$ \\
\hline Clark County, Washington & 554 & 4.2 & 0.9 & $(2.4-6.0)$ \\
\hline King County, Washington & 2,644 & 3.6 & 0.5 & $(2.6-4.5)$ \\
\hline Kitsap County, Washington & 425 & 5.7 & 1.2 & $(3.5-8.0)$ \\
\hline Pierce County, Washington & 809 & 4.4 & 0.8 & $(2.8-6.0)$ \\
\hline Snohomish County, Washington & 801 & 3.5 & 0.9 & $(1.8-5.2)$ \\
\hline Spokane County, Washington & 714 & 4.6 & 0.8 & $(3.1-6.2)$ \\
\hline Thurston County, Washington & 382 & 4.1 & 1.1 & $(1.9-6.3)$ \\
\hline Whatcom County, Washington & 612 & 3.4 & 0.7 & $(2.0-4.8)$ \\
\hline Yakima County, Washington & 364 & $\mathrm{~N} / \mathrm{A}$ & $\mathrm{N} / \mathrm{A}$ & $(\mathrm{N} / \mathrm{A}-\mathrm{N} / \mathrm{A})$ \\
\hline Kanawha County, West Virginia & 440 & 5.4 & 1.1 & $(3.2-7.6)$ \\
\hline Milwaukee County, Wisconsin & 656 & 5.0 & 1.2 & $(2.7-7.3)$ \\
\hline Laramie County, Wyoming & 748 & 3.5 & 0.7 & $(2.1-4.9)$ \\
\hline Natrona County, Wyoming & 646 & 4.8 & 1.1 & $(2.5-7.0)$ \\
\hline San Juan Municipio, Puerto Rico & 423 & $\mathrm{~N} / \mathrm{A}$ & $\mathrm{N} / \mathrm{A}$ & $(\mathrm{N} / \mathrm{A}-\mathrm{N} / \mathrm{A})$ \\
\hline Median & & 4.4 & & \\
\hline Range & & $1.5-9.3$ & & \\
\hline
\end{tabular}

Abbreviations: $\mathrm{Cl}=$ confidence interval; $\mathrm{SE}=$ standard error.

* Estimate not available (N/A) if the unweighted sample size for the denominator was $<50$ or if the relative standard error is $>0.3$. 
TABLE 70. Estimated prevalence of adults aged $\geq 18$ years who reported limited activities because of physical, mental, or emotional problems, by state/territory - Behavioral Risk Factor Surveillance System, United States, 2012

\begin{tabular}{|c|c|c|c|c|}
\hline State/Territory & $\begin{array}{l}\text { Sample } \\
\text { size }\end{array}$ & $\%$ & SE & $95 \% \mathrm{Cl}$ \\
\hline Alabama & 8,935 & 28.0 & 0.7 & $(26.7-29.3)$ \\
\hline Alaska & 4,288 & 20.5 & 0.8 & $(18.9-22.2)$ \\
\hline Arizona & 7,177 & 21.1 & 0.8 & $(19.6-22.6)$ \\
\hline Arkansas & 5,106 & 25.0 & 0.8 & $(23.5-26.5)$ \\
\hline California & 13,467 & 19.5 & 0.5 & $(18.6-20.4)$ \\
\hline Colorado & 11,721 & 19.6 & 0.5 & $(18.6-20.5)$ \\
\hline Connecticut & 8,645 & 18.6 & 0.6 & $(17.5-19.8)$ \\
\hline Delaware & 5,163 & 17.5 & 0.7 & $(16.2-18.9)$ \\
\hline District of Columbia & 3,756 & 18.1 & 1.0 & $(16.0-20.1)$ \\
\hline Florida & 7,485 & 22.4 & 0.7 & $(21.0-23.8)$ \\
\hline Georgia & 5,910 & 20.1 & 0.7 & $(18.7-21.4)$ \\
\hline Hawaii & 7,524 & 16.3 & 0.6 & $(15.1-17.6)$ \\
\hline Idaho & 5,828 & 24.0 & 0.9 & $(22.2-25.9)$ \\
\hline Illinois & 5,539 & 19.0 & 0.8 & $(17.5-20.5)$ \\
\hline Indiana & 8,513 & 21.3 & 0.6 & $(20.2-22.4)$ \\
\hline lowa & 7,022 & 18.6 & 0.5 & $(17.5-19.6)$ \\
\hline Kansas & 11,724 & 20.3 & 0.5 & $(19.4-21.3)$ \\
\hline Kentucky & 10,912 & 26.2 & 0.6 & $(24.9-27.4)$ \\
\hline Louisiana & 9,004 & 23.1 & 0.7 & $(21.7-24.4)$ \\
\hline Maine & 9,822 & 23.0 & 0.5 & $(22.0-24.1)$ \\
\hline Maryland & 12,516 & 16.7 & 0.5 & $(15.7-17.8)$ \\
\hline Massachusetts & 20,836 & 18.1 & 0.4 & $(17.3-18.9)$ \\
\hline Michigan & 10,390 & 22.3 & 0.5 & $(21.3-23.4)$ \\
\hline Minnesota & 11,942 & 16.2 & 0.4 & $(15.3-17.0)$ \\
\hline Mississippi & 7,716 & 24.6 & 0.6 & $(23.4-25.9)$ \\
\hline Missouri & 6,679 & 23.7 & 0.7 & $(22.3-25.1)$ \\
\hline Montana & 8,585 & 23.2 & 0.6 & $(22.1-24.4)$ \\
\hline Nebraska & 19,018 & 18.4 & 0.4 & $(17.7-19.2)$ \\
\hline Nevada & 4,782 & 19.2 & 0.8 & $(17.7-20.8)$ \\
\hline New Hampshire & 7,437 & 21.3 & 0.6 & $(20.0-22.5)$ \\
\hline New Jersey & 15,115 & 15.4 & 0.4 & $(14.6-16.2)$ \\
\hline New Mexico & 8,660 & 22.6 & 0.6 & $(21.5-23.7)$ \\
\hline New York & 5,950 & 19.2 & 0.7 & $(17.8-20.7)$ \\
\hline North Carolina & 11,782 & 19.7 & 0.4 & (18.9-20.6) \\
\hline North Dakota & 4,829 & 16.2 & 0.7 & $(14.9-17.6)$ \\
\hline Ohio & 12,677 & 20.3 & 0.5 & $(19.3-21.2)$ \\
\hline Oklahoma & 7,957 & 25.7 & 0.6 & $(24.5-26.9)$ \\
\hline Oregon & 5,166 & 26.4 & 0.8 & (24.9-28.0) \\
\hline Pennsylvania & 19,657 & 20.1 & 0.5 & $(19.2-21.0)$ \\
\hline Rhode Island & 5,393 & 20.0 & 0.7 & (18.6-21.5) \\
\hline South Carolina & 12,584 & 23.0 & 0.5 & $(22.0-24.0)$ \\
\hline South Dakota & 7,820 & 20.0 & 0.7 & $(18.6-21.4)$ \\
\hline Tennessee & 6,827 & 23.1 & 0.7 & $(21.8-24.4)$ \\
\hline Texas & 8,996 & 17.9 & 0.5 & $(16.8-18.9)$ \\
\hline Utah & 12,199 & 19.1 & 0.4 & $(18.2-19.9)$ \\
\hline Vermont & 5,972 & 19.7 & 0.6 & $(18.5-21.0)$ \\
\hline Virginia & 7,176 & 19.0 & 0.6 & $(17.8-20.1)$ \\
\hline Washington & 15,142 & 23.7 & 0.5 & $(22.8-24.6)$ \\
\hline West Virginia & 5,394 & 28.6 & 0.7 & $(27.2-30.1)$ \\
\hline Wisconsin & 5,142 & 19.2 & 0.8 & $(17.6-20.8)$ \\
\hline Wyoming & 6,186 & 19.1 & 0.7 & $(17.6-20.5)$ \\
\hline Guam & 2,003 & 15.0 & 1.0 & (12.9-17.0) \\
\hline Puerto Rico & 6,286 & 19.0 & 0.6 & $(17.8-20.1)$ \\
\hline Median & & 20.0 & & \\
\hline Range & & $15.0-28.6$ & & \\
\hline
\end{tabular}

Abbreviations: $\mathrm{Cl}=$ confidence interval; $\mathrm{SE}=$ standard error.
TABLE 71. Estimated prevalence of adults aged $\geq 18$ years who reported limited activities because of physical, mental, or emotional problems, by metropolitan and micropolitan statistical area Behavioral Risk Factor Surveillance System, United States, 2012

\begin{tabular}{|c|c|c|c|c|}
\hline MMSA(s) & $\begin{array}{l}\text { Sample } \\
\text { size }\end{array}$ & $\%$ & SE & $95 \% \mathrm{Cl}$ \\
\hline Aguadilla-Isabela, Puerto Rico & 549 & 20.5 & 2.0 & $(16.6-24.4)$ \\
\hline Akron, Ohio & 724 & 20.8 & 2.0 & $(16.9-24.7)$ \\
\hline Albuquerque, New Mexico & 3,224 & 21.7 & 0.9 & $(20.0-23.4)$ \\
\hline $\begin{array}{l}\text { Allentown-Bethlehem-Easton, } \\
\text { Pennsylvania-New Jersey }\end{array}$ & 1,319 & 19.3 & 1.5 & $(16.3-22.3)$ \\
\hline Anaheim-Santa Ana-Irvine, California* & 965 & 15.0 & 1.6 & $(11.9-18.2)$ \\
\hline Anchorage, Alaska & 1,489 & 19.8 & 1.3 & $(17.4-22.3)$ \\
\hline Asheville, North Carolina & 591 & 24.9 & 2.2 & $(20.7-29.2)$ \\
\hline $\begin{array}{l}\text { Atlanta-Sandy Springs-Roswell, } \\
\text { Georgia }\end{array}$ & 2,460 & 17.8 & 1.0 & $(15.7-19.8)$ \\
\hline Atlantic City-Hammonton, New Jersey & 988 & 21.7 & 1.8 & $(18.1-25.2)$ \\
\hline $\begin{array}{l}\text { Augusta-Richmond County, Georgia- } \\
\text { South Carolina }\end{array}$ & 1,008 & 24.0 & 2.6 & $(19.0-29.0)$ \\
\hline Augusta-Waterville, Maine & 826 & 24.3 & 1.8 & $(20.9-27.8)$ \\
\hline Austin-Round Rock, Texas & 1,372 & 17.5 & 1.5 & $(14.6-20.4)$ \\
\hline $\begin{array}{l}\text { Baltimore-Columbia-Towson, } \\
\text { Maryland }\end{array}$ & 4,626 & 18.0 & 0.8 & $(16.4-19.6)$ \\
\hline Bangor, Maine & 925 & 24.3 & 1.7 & $(21.0-27.5)$ \\
\hline Barnstable Town, Massachusetts & 541 & 19.7 & 2.1 & $(15.6-23.9)$ \\
\hline Barre, Vermont & 508 & 17.7 & 2.0 & $(13.8-21.6)$ \\
\hline Baton Rouge, Louisiana & 1,382 & 20.6 & 1.5 & $(17.6-23.5)$ \\
\hline Bellingham, Washington & 841 & 23.9 & 2.1 & $(19.7-28.1)$ \\
\hline Berlin, New Hampshire-Vermont & 699 & 28.1 & 2.7 & $(22.9-33.3)$ \\
\hline Billings, Montana & 843 & 22.5 & 1.6 & $(19.3-25.7)$ \\
\hline Birmingham-Hoover, Alabama & 1,800 & 25.4 & 1.4 & $(22.7-28.1)$ \\
\hline Bismarck, North Dakota & 816 & 17.3 & 1.7 & $(14.0-20.6)$ \\
\hline Boise City, Idaho & 1,470 & 23.6 & 1.6 & $(20.4-26.8)$ \\
\hline Boston, Massachusetts* & 5,677 & 16.2 & 0.7 & $(14.8-17.7)$ \\
\hline Boulder, Colorado & 494 & 20.0 & 2.1 & $(15.8-24.2)$ \\
\hline Bremerton-Silverdale, Washington & 561 & 27.0 & 2.3 & $(22.4-31.5)$ \\
\hline $\begin{array}{l}\text { Bridgeport-Stamford-Norwalk, } \\
\text { Connecticut }\end{array}$ & 2,145 & 16.8 & 1.2 & $(14.5-19.1)$ \\
\hline Burlington-South Burlington, Vermont & 1,502 & 17.3 & 1.1 & $(15.1-19.6)$ \\
\hline $\begin{array}{l}\text { Cambridge-Newton-Framingham, } \\
\text { Massachusetts* }\end{array}$ & 6,619 & 16.4 & 0.6 & $(15.1-17.6)$ \\
\hline Camden, New Jersey* & 1,930 & 18.8 & 1.2 & $(16.4-21.2)$ \\
\hline Canton-Massillon, Ohio & 666 & 20.0 & 2.0 & $(16.0-23.9)$ \\
\hline Casper, Wyoming & 819 & 23.0 & 2.1 & $(18.8-27.2)$ \\
\hline Cedar Rapids, lowa & 573 & 17.0 & 1.7 & $(13.7-20.4)$ \\
\hline Charleston, West Virginia & 767 & 27.8 & 1.8 & $(24.2-31.4)$ \\
\hline $\begin{array}{l}\text { Charleston-North Charleston, } \\
\text { South Carolina }\end{array}$ & 1,680 & 21.1 & 1.4 & $(18.4-23.8)$ \\
\hline $\begin{array}{l}\text { Charlotte-Concord-Gastonia, } \\
\text { North Carolina-South Carolina }\end{array}$ & 2,499 & 18.4 & 1.0 & $(16.4-20.4)$ \\
\hline Chattanooga, Tennessee-Georgia & 611 & 21.4 & 2.2 & $(17.2-25.7)$ \\
\hline Cheyenne, Wyoming & 942 & 20.5 & 1.9 & $(16.7-24.2)$ \\
\hline $\begin{array}{l}\text { Chicago-Naperville-Elgin, } \\
\text { Illinois-Indiana-Wisconsin }\end{array}$ & 3,696 & 16.7 & 0.9 & $(15.0-18.4)$ \\
\hline Cincinnati, Ohio-Kentucky-Indiana & 2,317 & 20.2 & 1.1 & $(18.1-22.3)$ \\
\hline $\begin{array}{l}\text { Claremont-Lebanon, New } \\
\text { Hampshire-Vermont }\end{array}$ & 1,931 & 20.6 & 1.2 & $(18.2-23.0)$ \\
\hline Cleveland-Elyria, Ohio & 1,699 & 19.8 & 1.3 & $(17.2-22.4)$ \\
\hline Colorado Springs, Colorado & 1,118 & 20.7 & 1.5 & $(17.8-23.6)$ \\
\hline Columbia, South Carolina & 1,782 & 20.4 & 1.4 & $(17.7-23.1)$ \\
\hline Columbus, Ohio & 1,564 & 19.3 & 1.3 & $(16.8-21.8)$ \\
\hline Concord, New Hampshire & 694 & 24.1 & 2.2 & $(19.7-28.5)$ \\
\hline Dallas-Plano-Irving, Texas* & 892 & 15.4 & 1.4 & $(12.7-18.2)$ \\
\hline Dayton, Ohio & 836 & 19.6 & 1.7 & $(16.2-22.9)$ \\
\hline Denver-Aurora-Lakewood, Colorado & 4,650 & 18.5 & 0.7 & $(17.2-19.9)$ \\
\hline Des Moines-West Des Moines, lowa & 1,141 & 19.1 & 1.4 & $(16.4-21.8)$ \\
\hline
\end{tabular}

See table footnotes on page 132. 
TABLE 71. (Continued) Estimated prevalence of adults aged $\geq 18$ years who reported limited activities because of physical, mental, or emotional problems, by metropolitan and micropolitan statistical area — Behavioral Risk Factor Surveillance System, United States, 2012

\begin{tabular}{|c|c|c|c|c|}
\hline MMSA(s) & $\begin{array}{l}\text { Sample } \\
\text { size }\end{array}$ & $\%$ & SE & $95 \% \mathrm{Cl}$ \\
\hline Detroit-Dearborn-Livonia, Michigan* & 2,190 & 24.3 & 1.4 & $(21.5-27.1)$ \\
\hline Dover, Delaware & 1,438 & 18.1 & 1.4 & $(15.5-20.8)$ \\
\hline Duluth, Minnesota-Wisconsin & 510 & 18.5 & 2.1 & $(14.4-22.6)$ \\
\hline Durham-Chapel Hill, North Carolina & 789 & 16.4 & 1.4 & $(13.6-19.2)$ \\
\hline El Paso, Texas & 619 & 16.1 & 2.0 & $(12.2-20.0)$ \\
\hline Eugene, Oregon & 515 & 31.7 & 2.6 & $(26.6-36.9)$ \\
\hline Fairbanks, Alaska & 595 & 24.7 & 2.4 & $(19.9-29.5)$ \\
\hline Fargo, North Dakota-Minnesota & 930 & 16.1 & 1.6 & $(12.9-19.3)$ \\
\hline Farmington, New Mexico & 636 & 22.0 & 2.0 & $(18.1-25.9)$ \\
\hline Fayetteville, North Carolina & 502 & 20.3 & 2.1 & $(16.1-24.4)$ \\
\hline $\begin{array}{l}\text { Fayetteville-Springdale-Rogers, } \\
\text { Arkansas-Missouri }\end{array}$ & 801 & 22.9 & 2.1 & $(18.7-27.1)$ \\
\hline Fort Collins, Colorado & 576 & 18.0 & 1.8 & $(14.5-21.6)$ \\
\hline Fort Wayne, Indiana & 527 & 20.4 & 2.3 & $(16.0-24.9)$ \\
\hline Fort Worth-Arlington, Texas* & 717 & 18.7 & 1.8 & $(15.3-22.2)$ \\
\hline Grand Island, Nebraska & 851 & 16.5 & 1.4 & $(13.7-19.3)$ \\
\hline Grand Rapids-Wyoming, Michigan & 887 & 18.8 & 1.7 & $(15.5-22.1)$ \\
\hline Great Falls, Montana & 703 & 26.2 & 2.1 & $(22.1-30.3)$ \\
\hline Greeley, Colorado & 514 & 17.2 & 1.9 & $(13.5-20.9)$ \\
\hline $\begin{array}{l}\text { Greensboro-High Point, } \\
\text { North Carolina }\end{array}$ & 804 & 20.1 & 1.7 & $(16.9-23.4)$ \\
\hline $\begin{array}{l}\text { Greenville-Anderson-Mauldin, } \\
\text { South Carolina }\end{array}$ & 1,668 & 24.6 & 1.4 & $(21.9-27.2)$ \\
\hline Gulfport-Biloxi-Pascagoula, Mississippi & 740 & 29.9 & 2.1 & $(25.8-34.0)$ \\
\hline $\begin{array}{l}\text { Hagerstown-Martinsburg, Maryland- } \\
\text { West Virginia }\end{array}$ & 752 & 21.1 & 2.2 & $(16.7-25.4)$ \\
\hline Harrisburg-Carlisle, Pennsylvania & 665 & 22.2 & 1.9 & $(18.6-25.9)$ \\
\hline $\begin{array}{l}\text { Hartford-West Hartford-East Hartford, } \\
\text { Connecticut }\end{array}$ & 2,636 & 18.3 & 0.9 & $(16.5-20.2)$ \\
\hline Heber, Utah & 503 & 12.0 & 1.8 & $(8.5-15.5)$ \\
\hline Hilo, Hawaii & 1,342 & 20.1 & 1.6 & $(17.0-23.1)$ \\
\hline $\begin{array}{l}\text { Hilton Head Island-Bluffton-Beaufort, } \\
\text { South Carolina }\end{array}$ & 925 & 17.1 & 1.8 & $(13.5-20.7)$ \\
\hline $\begin{array}{l}\text { Houston-The Woodlands-Sugar Land, } \\
\text { Texas }\end{array}$ & 1,123 & 16.1 & 1.3 & $(13.6-18.6)$ \\
\hline $\begin{array}{l}\text { Huntington-Ashland, West } \\
\text { Virginia-Kentucky-Ohio }\end{array}$ & 1,102 & 28.4 & 1.7 & $(25.2-31.7)$ \\
\hline Huntsville, Alabama & 611 & 23.1 & 2.2 & $(18.8-27.4)$ \\
\hline Idaho Falls, Idaho & 531 & 23.7 & 3.0 & $(17.8-29.6)$ \\
\hline $\begin{array}{l}\text { Indianapolis-Carmel-Anderson, } \\
\text { Indiana }\end{array}$ & 2,161 & 19.9 & 1.1 & $(17.8-22.0)$ \\
\hline Jackson, Mississippi & 912 & 20.8 & 1.6 & $(17.7-24.0)$ \\
\hline Jacksonville, Florida & 512 & 21.3 & 2.5 & $(16.4-26.2)$ \\
\hline Kahului-Wailuku-Lahaina, Hawaii & 1,210 & 18.4 & 1.5 & $(15.4-21.3)$ \\
\hline Kalispell, Montana & 551 & 24.2 & 2.1 & $(20.0-28.4)$ \\
\hline Kansas City, Missouri-Kansas & 4,697 & 21.1 & 1.0 & $(19.1-23.1)$ \\
\hline Kapaa, Hawaii & 662 & 17.6 & 2.2 & $(13.3-21.9)$ \\
\hline Keene, New Hampshire & 540 & 16.8 & 2.0 & $(12.8-20.8)$ \\
\hline Kennewick-Richland, Washington & 527 & 19.8 & 2.1 & $(15.7-23.9)$ \\
\hline $\begin{array}{l}\text { Kingsport-Bristol-Bristol, } \\
\text { Tennessee-Virginia }\end{array}$ & 550 & 28.6 & 2.7 & $(23.4-33.9)$ \\
\hline Knoxville, Tennessee & 814 & 25.3 & 1.9 & $(21.5-29.1)$ \\
\hline Laconia, New Hampshire & 556 & 22.4 & 2.5 & $(17.6-27.3)$ \\
\hline Lafayette, Louisiana & 556 & 18.0 & 2.1 & $(14.0-22.1)$ \\
\hline Las Cruces, New Mexico & 702 & 22.3 & 2.0 & $(18.3-26.3)$ \\
\hline $\begin{array}{l}\text { Las Vegas-Henderson-Paradise, } \\
\text { Nevada }\end{array}$ & 1,990 & 18.1 & 1.0 & $(16.2-20.1)$ \\
\hline Lewiston-Auburn, Maine & 694 & 23.8 & 1.9 & $(20.1-27.5)$ \\
\hline Lexington-Fayette, Kentucky & 521 & 21.1 & 2.2 & $(16.8-25.3)$ \\
\hline Lincoln, Nebraska & 1,662 & 15.8 & 1.0 & $(13.8-17.8)$ \\
\hline
\end{tabular}

See table footnotes on page 132.
TABLE 71. (Continued) Estimated prevalence of adults aged $\geq 18$ years who reported limited activities because of physical, mental, or emotional problems, by metropolitan and micropolitan statistical area — Behavioral Risk Factor Surveillance System, United States, 2012

\begin{tabular}{|c|c|c|c|c|}
\hline MMSA(s) & $\begin{array}{l}\text { Sample } \\
\text { size }\end{array}$ & $\%$ & SE & $95 \% \mathrm{Cl}$ \\
\hline $\begin{array}{l}\text { Little Rock-North Little Rock-Conway, } \\
\text { Arkansas }\end{array}$ & 1,153 & 22.6 & 1.5 & $(19.6-25.6)$ \\
\hline Logan, Utah-Idaho & 502 & 14.8 & 2.1 & $(10.7-18.8)$ \\
\hline $\begin{array}{l}\text { Los Angeles-Long Beach-Glendale, } \\
\text { California* }\end{array}$ & 3,165 & 17.7 & 0.9 & $(15.8-19.5)$ \\
\hline $\begin{array}{l}\text { Louisville/Jefferson County, } \\
\text { Kentucky-Indiana }\end{array}$ & 2,109 & 23.4 & 1.4 & $(20.7-26.1)$ \\
\hline Lumberton, North Carolina & 538 & 29.0 & 3.7 & $(21.8-36.3)$ \\
\hline Manchester-Nashua, New Hampshire & 1,890 & 20.9 & 1.1 & $(18.6-23.1)$ \\
\hline McAllen-Edinburg-Mission, Texas & 615 & 19.1 & 2.4 & $(14.5-23.8)$ \\
\hline $\begin{array}{l}\text { Memphis, } \\
\text { Tennessee-Mississippi-Arkansas }\end{array}$ & 1,275 & 18.1 & 1.4 & $(15.4-20.9)$ \\
\hline $\begin{array}{l}\text { Miami-Fort Lauderdale-West Palm } \\
\text { Beach, Florida }\end{array}$ & 1,627 & 18.4 & 1.4 & $(15.7-21.1)$ \\
\hline $\begin{array}{l}\text { Milwaukee-Waukesha-West Allis, } \\
\text { Wisconsin }\end{array}$ & 1,206 & 18.4 & 1.7 & $(15.0-21.8)$ \\
\hline $\begin{array}{l}\text { Minneapolis-St. Paul-Bloomington, } \\
\text { Minnesota-Wisconsin }\end{array}$ & 7,680 & 15.8 & 0.6 & $(14.7-17.0)$ \\
\hline Missoula, Montana & 778 & 24.7 & 1.9 & $(21.0-28.4)$ \\
\hline Mobile, Alabama & 810 & 28.4 & 2.3 & $(23.8-33.0)$ \\
\hline Montgomery, Alabama & 531 & 29.7 & 2.7 & $(24.3-35.0)$ \\
\hline $\begin{array}{l}\text { Montgomery County-Bucks County- } \\
\text { Chester County, Pennsylvania* }\end{array}$ & 1,299 & 17.7 & 1.4 & $(14.9-20.5)$ \\
\hline $\begin{array}{l}\text { Myrtle Beach-Conway-North Myrtle } \\
\text { Beach, South Carolina-North Carolina }\end{array}$ & 929 & 22.8 & 1.7 & $(19.5-26.1)$ \\
\hline $\begin{array}{l}\text { Nashville-Davidson-Murfreesboro- } \\
\text { Franklin, Tennessee }\end{array}$ & 1,304 & 18.4 & 1.3 & $(15.9-20.8)$ \\
\hline $\begin{array}{l}\text { Nassau County-Suffolk County, } \\
\text { New York* }\end{array}$ & 885 & 15.3 & 1.5 & $(12.5-18.2)$ \\
\hline Newark, New Jersey-Pennsylvania* & 6,367 & 14.2 & 0.6 & $(13.0-15.5)$ \\
\hline New Haven-Milford, Connecticut & 1,986 & 20.8 & 1.3 & $(18.3-23.3)$ \\
\hline New Orleans-Metairie, Louisiana & 1,264 & 21.1 & 1.5 & $(18.1-24.0)$ \\
\hline $\begin{array}{l}\text { New York-Jersey City-White Plains, } \\
\text { New York-New Jersey* }\end{array}$ & 7,106 & 17.1 & 0.8 & $(15.4-18.7)$ \\
\hline Norfolk, Nebraska & 567 & 16.8 & 1.8 & $(13.2-20.4)$ \\
\hline North Platte, Nebraska & 608 & 21.1 & 2.4 & $(16.5-25.8)$ \\
\hline Norwich-New London, Connecticut & 981 & 18.3 & 1.9 & $(14.6-21.9)$ \\
\hline $\begin{array}{l}\text { Oakland-Hayward-Berkeley, } \\
\text { California* }\end{array}$ & 913 & 17.9 & 1.9 & $(14.2-21.7)$ \\
\hline Ocean City, New Jersey & 533 & 25.1 & 3.1 & $(19.1-31.2)$ \\
\hline Ogden-Clearfield, Utah & 2,392 & 20.3 & 1.0 & $(18.4-22.3)$ \\
\hline Oklahoma City, Oklahoma & 2,422 & 24.2 & 1.1 & $(22.1-26.3)$ \\
\hline Olympia-Tumwater, Washington & 509 & 26.8 & 2.6 & $(21.6-31.9)$ \\
\hline Omaha-Council Bluffs, Nebraska-lowa & 5,524 & 18.4 & 0.7 & $(17.1-19.7)$ \\
\hline Orlando-Kissimmee-Sanford, Florida & 556 & 24.5 & 2.9 & $(18.8-30.1)$ \\
\hline Philadelphia, Pennsylvania* & 2,408 & 20.2 & 1.1 & $(18.1-22.3)$ \\
\hline Phoenix-Mesa-Scottsdale, Arizona & 2,555 & 20.1 & 1.0 & $(18.0-22.1)$ \\
\hline Pittsburgh, Pennsylvania & 3,319 & 20.8 & 0.9 & $(19.1-22.5)$ \\
\hline Ponce, Puerto Rico & 547 & 19.6 & 2.0 & $(15.7-23.5)$ \\
\hline Portland-South Portland, Maine & 3,299 & 20.7 & 0.8 & $(19.1-22.4)$ \\
\hline $\begin{array}{l}\text { Portland-Vancouver-Hillsboro, } \\
\text { Oregon-Washington }\end{array}$ & 3,051 & 22.8 & 1.0 & $(20.9-24.7)$ \\
\hline $\begin{array}{l}\text { Providence-Warwick, Rhode } \\
\text { Island-Massachusetts }\end{array}$ & 7,944 & 20.1 & 0.7 & $(18.8-21.4)$ \\
\hline Provo-Orem, Utah & 1,721 & 18.3 & 1.1 & $(16.1-20.5)$ \\
\hline Raleigh, North Carolina & 939 & 13.3 & 1.2 & $(10.9-15.7)$ \\
\hline Rapid City, South Dakota & 1,045 & 25.4 & 1.8 & $(21.9-28.8)$ \\
\hline Reno, Nevada & 1,488 & 22.2 & 1.6 & $(19.1-25.2)$ \\
\hline Richmond, Virginia & 982 & 15.9 & 1.4 & $(13.1-18.7)$ \\
\hline
\end{tabular}

See table footnotes on page 132. 
TABLE 71. (Continued) Estimated prevalence of adults aged $\geq 18$ years who reported limited activities because of physical, mental, or emotional problems, by metropolitan and micropolitan statistical area — Behavioral Risk Factor Surveillance System, United States, 2012

\begin{tabular}{|c|c|c|c|c|}
\hline MMSA(s) & $\begin{array}{l}\text { Sample } \\
\text { size }\end{array}$ & $\%$ & SE & $95 \% \mathrm{Cl}$ \\
\hline $\begin{array}{l}\text { Riverside-San Bernardino-Ontario, } \\
\text { California }\end{array}$ & 1,409 & 21.1 & 1.5 & $(18.2-24.0)$ \\
\hline $\begin{array}{l}\text { Rockingham County-Strafford County, } \\
\text { New Hampshire* }\end{array}$ & 1,652 & 20.4 & 1.3 & $(17.9-22.9)$ \\
\hline Rutland, Vermont & 587 & 21.3 & 2.1 & $(17.2-25.5)$ \\
\hline $\begin{array}{l}\text { Sacramento-Roseville-Arden-Arcade, } \\
\text { California }\end{array}$ & 935 & 20.2 & 1.7 & $(17.0-23.5)$ \\
\hline St. Louis, Missouri-Illinois & 2,046 & 21.4 & 1.4 & $(18.8-24.1)$ \\
\hline Salisbury, Maryland-Delaware & 2,098 & 20.6 & 1.5 & $(17.6-23.6)$ \\
\hline Salt Lake City, Utah & 4,519 & 19.2 & 0.7 & $(17.8-20.6)$ \\
\hline San Antonio-New Braunfels, Texas & 769 & 17.5 & 1.6 & $(14.3-20.7)$ \\
\hline San Diego-Carlsbad, California & 1,056 & 18.6 & 1.6 & $(15.5-21.7)$ \\
\hline $\begin{array}{l}\text { San Francisco-Redwood City-South } \\
\text { San Francisco, California* }\end{array}$ & 543 & 18.0 & 2.2 & $(13.6-22.4)$ \\
\hline $\begin{array}{l}\text { San Jose-Sunnyvale-Santa Clara, } \\
\text { California }\end{array}$ & 641 & 15.6 & 1.9 & $(11.9-19.2)$ \\
\hline San Juan-Carolina-Caguas, Puerto Rico & 3,940 & 17.7 & 0.7 & $(16.3-19.0)$ \\
\hline Santa Fe, New Mexico & 686 & 20.1 & 1.8 & $(16.5-23.6)$ \\
\hline Sayre, Pennsylvania & 1,835 & 18.8 & 1.4 & $(16.1-21.5)$ \\
\hline Scottsbluff, Nebraska & 630 & 22.1 & 2.4 & $(17.3-26.9)$ \\
\hline $\begin{array}{l}\text { Scranton-Wilkes-Barre-Hazleton, } \\
\text { Pennsylvania }\end{array}$ & 734 & 23.4 & 2.0 & $(19.4-27.4)$ \\
\hline Seattle-Bellevue-Everett, Washington* & 5,045 & 20.7 & 0.7 & $(19.3-22.1)$ \\
\hline Shreveport-Bossier City, Louisiana & 574 & 22.1 & 2.1 & $(18.0-26.3)$ \\
\hline $\begin{array}{l}\text { Silver Spring-Frederick-Rockville, } \\
\text { Maryland* }\end{array}$ & 2,235 & 15.7 & 1.1 & $(13.6-17.8)$ \\
\hline $\begin{array}{l}\text { Sioux City, lowa-Nebraska- } \\
\text { South Dakota }\end{array}$ & 1,191 & 21.0 & 2.5 & $(16.1-25.9)$ \\
\hline Sioux Falls, South Dakota & 1,461 & 16.4 & 1.3 & $(13.9-18.9)$ \\
\hline Spartanburg, South Carolina & 701 & 26.1 & 2.4 & $(21.4-30.8)$ \\
\hline Spokane-Spokane Valley, Washington & 1,087 & 27.4 & 1.7 & $(24.1-30.8)$ \\
\hline Springfield, Massachusetts & 2,276 & 22.9 & 1.3 & $(20.4-25.5)$ \\
\hline
\end{tabular}

TABLE 71. (Continued) Estimated prevalence of adults aged $\geq 18$ years who reported limited activities because of physical, mental, or emotional problems, by metropolitan and micropolitan statistical area — Behavioral Risk Factor Surveillance System, United States, 2012

\begin{tabular}{|c|c|c|c|c|}
\hline MMSA(s) & $\begin{array}{l}\text { Sample } \\
\text { size }\end{array}$ & $\%$ & SE & $95 \% \mathrm{Cl}$ \\
\hline Tacoma-Lakewood, Washington* & 1,170 & 27.2 & 1.7 & $(23.9-30.4)$ \\
\hline $\begin{array}{l}\text { Tampa-St. Petersburg-Clearwater, } \\
\text { Florida }\end{array}$ & 817 & 21.7 & 1.8 & $(18.1-25.3)$ \\
\hline Toledo, Ohio & 948 & 18.2 & 1.6 & $(15.0-21.4)$ \\
\hline Topeka, Kansas & 1,087 & 25.2 & 1.7 & $(21.9-28.5)$ \\
\hline Torrington, Connecticut & 655 & 18.3 & 2.0 & $(14.3-22.3)$ \\
\hline Trenton, New Jersey & 560 & 14.1 & 1.6 & $(10.9-17.2)$ \\
\hline Tucson, Arizona & 983 & 24.7 & 1.9 & $(21.1-28.4)$ \\
\hline Tulsa, Oklahoma & 1,733 & 23.8 & 1.2 & $(21.5-26.2)$ \\
\hline Tuscaloosa, Alabama & 574 & 28.2 & 2.9 & $(22.4-33.9)$ \\
\hline Urban Honolulu, Hawaii & 4,310 & 15.2 & 0.8 & $(13.6-16.8)$ \\
\hline Vineland-Bridgeton, New Jersey & 540 & 18.7 & 2.2 & $(14.3-23.1)$ \\
\hline $\begin{array}{l}\text { Virginia Beach-Norfolk-Newport News, } \\
\text { Virginia-North Carolina }\end{array}$ & 1,472 & 22.0 & 1.5 & $(19.0-25.0)$ \\
\hline $\begin{array}{l}\text { Warren-Troy-Farmington Hills, } \\
\text { Michigan* }\end{array}$ & 2,160 & 20.4 & 1.1 & $(18.3-22.5)$ \\
\hline $\begin{array}{l}\text { Washington-Arlington-Alexandria, } \\
\text { District of Columbia-Virginia- } \\
\text { Maryland-West Virginia* }\end{array}$ & 8,025 & 14.7 & 0.6 & $(13.5-16.0)$ \\
\hline Wichita, Kansas & 2,339 & 19.5 & 1.0 & $(17.5-21.4)$ \\
\hline $\begin{array}{l}\text { Wilmington, Delaware-Maryland- } \\
\text { New Jersey* }\end{array}$ & 3,209 & 16.7 & 0.8 & $(15.1-18.4)$ \\
\hline Winston-Salem, North Carolina & 790 & 22.0 & 1.8 & $(18.5-25.4)$ \\
\hline Worcester, Massachusetts-Connecticut & 2,745 & 19.2 & 1.0 & $(17.2-21.3)$ \\
\hline Yakima, Washington & 528 & 22.3 & 2.4 & $(17.5-27.1)$ \\
\hline $\begin{array}{l}\text { Youngstown-Warren-Boardman, } \\
\text { Ohio-Pennsylvania }\end{array}$ & 905 & 22.7 & 2.2 & $(18.4-26.9)$ \\
\hline Median & & 20.3 & & \\
\hline Range & & $12.0-31.7$ & & \\
\hline
\end{tabular}

Abbreviations: $\mathrm{Cl}=$ confidence interval; $\mathrm{MMSA}=$ metropolitan and micropolitan statistical area; $\mathrm{SE}=$ standard error.

* Metropolitan division. 
TABLE 72. Estimated prevalence of adults aged $\geq 18$ years who reported limited activities because of physical, mental, or emotional problems, by county - Behavioral Risk Factor Surveillance System, United States, 2012

\begin{tabular}{|c|c|c|c|c|}
\hline County & $\begin{array}{l}\text { Sample } \\
\text { size }\end{array}$ & $\%$ & SE & $95 \% \mathrm{Cl}$ \\
\hline Jefferson County, Alabama & 945 & 22.4 & 1.6 & $(19.3-25.6)$ \\
\hline Madison County, Alabama & 510 & 22.0 & 2.3 & $(17.5-26.5)$ \\
\hline Mobile County, Alabama & 810 & 28.4 & 2.3 & $(23.8-33.0)$ \\
\hline Anchorage Municipality, Alaska & 894 & 18.5 & 1.5 & $(15.6-21.4)$ \\
\hline Fairbanks North Star Borough, Alaska & 595 & 24.7 & 2.4 & $(19.9-29.5)$ \\
\hline Matanuska-Susitna Borough, Alaska & 595 & 23.6 & 2.1 & $(19.6-27.6)$ \\
\hline Maricopa County, Arizona & 2,080 & 20.3 & 1.1 & $(18.2-22.5)$ \\
\hline Pima County, Arizona & 983 & 24.7 & 1.9 & $(21.1-28.4)$ \\
\hline Pulaski County, Arkansas & 738 & 22.1 & 2.0 & $(18.2-26.0)$ \\
\hline Alameda County, California & 583 & 17.5 & 2.3 & $(12.9-22.0)$ \\
\hline Los Angeles County, California & 3,165 & 17.7 & 0.9 & $(15.9-19.5)$ \\
\hline Orange County, California & 965 & 15.1 & 1.6 & $(11.9-18.2)$ \\
\hline Riverside County, California & 753 & 20.2 & 1.9 & $(16.5-23.9)$ \\
\hline Sacramento County, California & 565 & 21.4 & 2.2 & $(17.0-25.7)$ \\
\hline San Bernardino County, California & 656 & 21.2 & 2.1 & $(17.0-25.3)$ \\
\hline San Diego County, California & 1,056 & 18.6 & 1.6 & $(15.5-21.7)$ \\
\hline Santa Clara County, California & 623 & 15.5 & 1.9 & $(11.9-19.2)$ \\
\hline Adams County, Colorado & 778 & 20.6 & 1.7 & $(17.2-24.1)$ \\
\hline Arapahoe County, Colorado & 827 & 18.4 & 1.6 & $(15.2-21.6)$ \\
\hline Boulder County, Colorado & 494 & 20.0 & 2.1 & $(15.8-24.2)$ \\
\hline Denver County, Colorado & 930 & 18.5 & 1.4 & $(15.7-21.3)$ \\
\hline Douglas County, Colorado & 525 & 13.2 & 1.7 & $(9.9-16.5)$ \\
\hline El Paso County, Colorado & 984 & 20.5 & 1.5 & $(17.5-23.5)$ \\
\hline Jefferson County, Colorado & 1,084 & 19.1 & 1.4 & $(16.3-21.9)$ \\
\hline Larimer County, Colorado & 576 & 18.0 & 1.8 & $(14.5-21.6)$ \\
\hline Weld County, Colorado & 514 & 17.2 & 1.9 & $(13.5-21.0)$ \\
\hline Fairfield County, Connecticut & 2,145 & 16.8 & 1.2 & $(14.5-19.1)$ \\
\hline Hartford County, Connecticut & 1,957 & 18.9 & 1.1 & $(16.7-21.1)$ \\
\hline Litchfield County, Connecticut & 655 & 18.3 & .0 & $(14.3-22.3)$ \\
\hline New Haven County, Connecticut & 1,986 & 20.8 & 1.3 & $(18.3-23.3)$ \\
\hline New London County, Connecticut & 981 & 18.3 & 1.9 & $(14.6-21.9)$ \\
\hline Kent County, Delaware & 1,438 & 18.1 & 1.4 & $(15.5-20.8)$ \\
\hline New Castle County, Delaware & 2,328 & 16.2 & 0.9 & $(14.4-18.1)$ \\
\hline Sussex County, Delaware & 1,397 & 20.2 & 1.4 & $(17.5-22.9)$ \\
\hline $\begin{array}{l}\text { District of Columbia, } \\
\text { District of Columbia }\end{array}$ & 3,756 & 18.0 & 1.1 & $(15.9-20.1)$ \\
\hline Broward County, Florida & 519 & 18.2 & 2.5 & $(13.3-23.1)$ \\
\hline Miami-Dade County, Florida & 789 & 17.1 & 2.0 & $(13.2-21.0)$ \\
\hline Hawaii County, Hawaii & 1,342 & 20.1 & 1.6 & $(17.0-23.1)$ \\
\hline Honolulu County, Hawaii & 4,310 & 15.2 & 0.8 & $(13.6-16.8)$ \\
\hline Kauai County, Hawaii & 662 & 17.6 & 2.2 & $(13.3-21.9)$ \\
\hline Maui County, Hawaii & 1,210 & 18.4 & 1.5 & $(15.4-21.3)$ \\
\hline Ada County, Idaho & 803 & 21.7 & 2.0 & $(17.7-25.7)$ \\
\hline Canyon County, Idaho & 498 & 26.5 & 3.2 & $(20.3-32.7)$ \\
\hline Cook County, Illinois & 1,494 & 17.4 & 1.3 & $(14.9-19.9)$ \\
\hline Lake County, Indiana & 874 & 23.7 & 2.6 & $(18.6-28.7)$ \\
\hline Marion County, Indiana & 1,258 & 17.9 & 1.3 & $(15.4-20.5)$ \\
\hline Polk County, lowa & 794 & 19.8 & 1.6 & $(16.5-23.0)$ \\
\hline Johnson County, Kansas & 2,160 & 15.9 & 1.0 & $(13.9-17.8)$ \\
\hline Sedgwick County, Kansas & 1,783 & 19.0 & 1.1 & $(16.9-21.1)$ \\
\hline Shawnee County, Kansas & 771 & 24.6 & 1.9 & $(20.9-28.3)$ \\
\hline Wyandotte County, Kansas & 869 & 27.5 & 2.6 & $(22.3-32.7)$ \\
\hline Jefferson County, Kentucky & 1,632 & 24.3 & 1.8 & $(20.8-27.7)$ \\
\hline East Baton Rouge Parish, Louisiana & 568 & 17.9 & 2.0 & $(14.0-21.9)$ \\
\hline Androscoggin County, Maine & 694 & 23.8 & 1.9 & $(20.1-27.5)$ \\
\hline Aroostook County, Maine & 533 & 28.3 & 2.4 & $(23.7-32.9)$ \\
\hline Cumberland County, Maine & 1,738 & 20.2 & 1.1 & $(17.9-22.4)$ \\
\hline Kennebec County, Maine & 826 & 24.3 & 1.8 & $(20.9-27.8)$ \\
\hline Penobscot County, Maine & 925 & 24.3 & 1.7 & $(21.0-27.5)$ \\
\hline York County, Maine & 1,180 & 21.7 & 1.4 & $(19.0-24.4)$ \\
\hline
\end{tabular}

See table footnotes on page 134 .
TABLE 72. (Continued) Estimated prevalence of adults aged $\geq 18$ years who reported limited activities because of physical, mental, or emotional problems, by county - Behavioral Risk Factor Surveillance System, United States, 2012

\begin{tabular}{|c|c|c|c|c|}
\hline County & $\begin{array}{c}\text { Sample } \\
\text { size }\end{array}$ & $\%$ & SE & $95 \% \mathrm{Cl}$ \\
\hline Anne Arundel County, Maryland & 883 & 18.5 & 1.8 & $(14.9-22.1)$ \\
\hline Baltimore County, Maryland & 1,493 & 17.9 & 1.4 & $(15.3-20.6)$ \\
\hline Charles County, Maryland & 499 & 14.6 & 1.9 & $(10.8-18.3)$ \\
\hline Frederick County, Maryland & 746 & 18.4 & 2.4 & $(13.6-23.1)$ \\
\hline Montgomery County, Maryland & 1,489 & 15.1 & 1.2 & $(12.8-17.5)$ \\
\hline Prince George's County, Maryland & 1,119 & 11.7 & 1.5 & $(8.9-14.6)$ \\
\hline Washington County, Maryland & 530 & 20.9 & 3.0 & $(14.9-26.8)$ \\
\hline Baltimore city, Maryland & 723 & 21.4 & 2.2 & $(17.1-25.7)$ \\
\hline Barnstable County, Massachusetts & 541 & 19.7 & 2.1 & $(15.6-23.9)$ \\
\hline Bristol County, Massachusetts & 2,551 & 21.3 & 1.4 & $(18.5-24.0)$ \\
\hline Essex County, Massachusetts & 2,480 & 18.9 & 1.3 & $(16.4-21.5)$ \\
\hline Hampden County, Massachusetts & 1,899 & 25.5 & 1.6 & $(22.4-28.6)$ \\
\hline Middlesex County, Massachusetts & 4,139 & 15.3 & 0.8 & $(13.8-16.8)$ \\
\hline Norfolk County, Massachusetts & 1,694 & 14.3 & 1.2 & $(12.0-16.7)$ \\
\hline Plymouth County, Massachusetts & 1,757 & 20.1 & 1.6 & $(16.9-23.3)$ \\
\hline Suffolk County, Massachusetts & 2,226 & 15.9 & 1.1 & $(13.8-18.0)$ \\
\hline Worcester County, Massachusetts & 2,503 & 18.9 & 1.1 & $(16.7-21.0)$ \\
\hline Kent County, Michigan & 522 & 16.8 & 1.8 & $(13.2-20.4)$ \\
\hline Macomb County, Michigan & 608 & 20.7 & 2.0 & $(16.9-24.6)$ \\
\hline Oakland County, Michigan & 1,153 & 18.6 & 1.4 & $(15.9-21.3)$ \\
\hline Wayne County, Michigan & 2,190 & 24.3 & 1.4 & $(21.5-27.1)$ \\
\hline Anoka County, Minnesota & 531 & 16.7 & 2.0 & $(12.8-20.7)$ \\
\hline Dakota County, Minnesota & 659 & 14.4 & 1.6 & $(11.4-17.5)$ \\
\hline Hennepin County, Minnesota & 3,189 & 14.8 & 0.9 & $(13.1-16.5)$ \\
\hline Ramsey County, Minnesota & 1,902 & 19.1 & 1.8 & $(15.6-22.6)$ \\
\hline Jackson County, Missouri & 865 & 22.0 & 2.1 & $(17.9-26.1)$ \\
\hline St. Louis County, Missouri & 946 & 20.5 & 1.7 & $(17.1-23.8)$ \\
\hline Cascade County, Montana & 703 & 26.2 & 2.1 & $(22.1-30.3)$ \\
\hline Flathead County, Montana & 551 & 24.2 & 2.1 & $(20.0-28.4)$ \\
\hline Hill County, Montana & 576 & 22.2 & 2.4 & $(17.5-27.0)$ \\
\hline Lake County, Montana & 888 & 28.5 & 2.4 & $(23.8-33.1)$ \\
\hline Missoula County, Montana & 778 & 24.7 & 1.9 & $(21.0-28.4)$ \\
\hline Yellowstone County, Montana & 747 & 21.9 & 1.7 & $(18.6-25.2)$ \\
\hline Dakota County, Nebraska & 728 & 18.4 & 2.7 & $(13.1-23.8)$ \\
\hline Douglas County, Nebraska & 3,531 & 20.1 & 0.9 & $(18.4-21.7)$ \\
\hline Hall County, Nebraska & 534 & 17.0 & 1.8 & $(13.4-20.5)$ \\
\hline Lancaster County, Nebraska & 1,440 & 15.8 & 1.1 & $(13.7-17.9)$ \\
\hline Lincoln County, Nebraska & 584 & 21.4 & 2.4 & $(16.6-26.1)$ \\
\hline Sarpy County, Nebraska & 1,165 & 16.2 & 1.4 & $(13.5-18.9)$ \\
\hline Scotts Bluff County, Nebraska & 578 & 23.1 & 2.5 & $(18.1-28.0)$ \\
\hline Clark County, Nevada & 1,990 & 18.1 & 1.0 & $(16.2-20.1)$ \\
\hline Washoe County, Nevada & 1,469 & 22.2 & 1.6 & $(19.1-25.2)$ \\
\hline Belknap County, New Hampshire & 556 & 22.4 & 2.5 & $(17.6-27.3)$ \\
\hline Carroll County, New Hampshire & 525 & 22.0 & 2.4 & $(17.2-26.7)$ \\
\hline Cheshire County, New Hampshire & 540 & 16.8 & 2.0 & $(12.8-20.8)$ \\
\hline Coos County, New Hampshire & 530 & 28.0 & 3.1 & $(21.9-34.2)$ \\
\hline Grafton County, New Hampshire & 562 & 22.8 & 2.4 & $(18.1-27.4)$ \\
\hline Hillsborough County, New Hampshire & 1,890 & 20.9 & 1.1 & $(18.6-23.1)$ \\
\hline Merrimack County, New Hampshire & 694 & 24.1 & 2.2 & $(19.7-28.5)$ \\
\hline Rockingham County, New Hampshire & 1,038 & 18.8 & 1.5 & $(15.8-21.8)$ \\
\hline Strafford County, New Hampshire & 614 & 24.2 & 2.3 & $(19.7-28.8)$ \\
\hline Atlantic County, New Jersey & 988 & 21.7 & 1.8 & $(18.1-25.2)$ \\
\hline Bergen County, New Jersey & 901 & 14.0 & 1.5 & $(11.0-16.9)$ \\
\hline Burlington County, New Jersey & 668 & 16.8 & 2.1 & $(12.7-20.9)$ \\
\hline Camden County, New Jersey & 722 & 20.5 & 2.1 & $(16.4-24.6)$ \\
\hline Cape May County, New Jersey & 533 & 25.1 & 3.1 & $(19.1-31.2)$ \\
\hline Cumberland County, New Jersey & 540 & 18.7 & 2.3 & $(14.3-23.1)$ \\
\hline Essex County, New Jersey & 1,299 & 15.6 & 1.2 & $(13.2-17.9)$ \\
\hline Gloucester County, New Jersey & 540 & 20.2 & 2.3 & $(15.8-24.6)$ \\
\hline Hudson County, New Jersey & 1,242 & 14.2 & 1.3 & $(11.7-16.7)$ \\
\hline
\end{tabular}

See table footnotes on page 134 . 
TABLE 72. (Continued) Estimated prevalence of adults aged $\geq 18$ years who reported limited activities because of physical, mental, or emotional problems, by county - Behavioral Risk Factor Surveillance System, United States, 2012

\begin{tabular}{|c|c|c|c|c|}
\hline County & $\begin{array}{l}\text { Sample } \\
\text { size }\end{array}$ & $\%$ & SE & $95 \% \mathrm{Cl}$ \\
\hline Hunterdon County, New Jersey & 548 & 13.0 & 1.8 & $(9.6-16.5)$ \\
\hline Mercer County, New Jersey & 560 & 14.1 & 1.6 & $(10.9-17.2)$ \\
\hline Middlesex County, New Jersey & 862 & 12.2 & 1.4 & $(9.5-15.0)$ \\
\hline Monmouth County, New Jersey & 688 & 15.3 & 1.7 & $(11.9-18.7)$ \\
\hline Morris County, New Jersey & 822 & 11.8 & 1.3 & $(9.3-14.4)$ \\
\hline Ocean County, New Jersey & 633 & 16.2 & 1.7 & $(12.9-19.6)$ \\
\hline Passaic County, New Jersey & 656 & 14.2 & 1.8 & $(10.8-17.7)$ \\
\hline Salem County, New Jersey & 571 & 23.8 & 2.4 & $(19.0-28.5)$ \\
\hline Somerset County, New Jersey & 612 & 12.8 & 1.8 & $(9.3-16.2)$ \\
\hline Sussex County, New Jersey & 532 & 19.6 & 2.3 & $(15.0-24.1)$ \\
\hline Union County, New Jersey & 681 & 13.3 & 1.5 & $(10.3-16.3)$ \\
\hline Warren County, New Jersey & 517 & 18.8 & 2.4 & $(14.1-23.5)$ \\
\hline Bernalillo County, New Mexico & 2,037 & 21.8 & 1.1 & $(19.7-23.9)$ \\
\hline Dona Ana County, New Mexico & 702 & 22.3 & 2.0 & $(18.3-26.3)$ \\
\hline Sandoval County, New Mexico & 644 & 19.0 & 1.9 & $(15.3-22.7)$ \\
\hline San Juan County, New Mexico & 636 & 22.0 & 2.0 & $(18.1-25.9)$ \\
\hline Santa Fe County, New Mexico & 686 & 20.1 & 1.8 & $(16.6-23.6)$ \\
\hline Kings County, New York & 482 & 17.3 & 2.4 & $(12.7-22.0)$ \\
\hline Guilford County, North Carolina & 510 & 17.1 & 2.0 & $(13.2-21.0)$ \\
\hline Mecklenburg County, North Carolina & 721 & 16.4 & 1.9 & $(12.8-20.1)$ \\
\hline Robeson County, North Carolina & 538 & 29.1 & 3.7 & $(21.8-36.3)$ \\
\hline Wake County, North Carolina & 696 & 11.3 & 1.3 & $(8.7-14.0)$ \\
\hline Burleigh County, North Dakota & 565 & 18.5 & 2.2 & $(14.2-22.8)$ \\
\hline Cass County, North Dakota & 834 & 15.5 & 1.6 & $(12.3-18.8)$ \\
\hline Cuyahoga County, Ohio & 809 & 22.6 & 1.9 & $(19.0-26.3)$ \\
\hline Franklin County, Ohio & 814 & 19.6 & 1.8 & $(16.1-23.2)$ \\
\hline Hamilton County, Ohio & 751 & 18.0 & 1.7 & $(14.7-21.3)$ \\
\hline Lorain County, Ohio & 597 & 18.0 & 2.4 & $(13.2-22.8)$ \\
\hline Lucas County, Ohio & 609 & 19.0 & 2.1 & $(15.0-23.1)$ \\
\hline Mahoning County, Ohio & 573 & 24.9 & 3.1 & $(18.9-30.9)$ \\
\hline Montgomery County, Ohio & 649 & 21.1 & 2.0 & $(17.2-24.9)$ \\
\hline Stark County, Ohio & 609 & 19.5 & 2.1 & $(15.3-23.6)$ \\
\hline Summit County, Ohio & 612 & 21.8 & 2.3 & $(17.4-26.3)$ \\
\hline Oklahoma County, Oklahoma & 1,181 & 23.2 & 1.4 & $(20.3-26.0)$ \\
\hline Tulsa County, Oklahoma & 1,198 & 21.1 & 1.4 & $(18.4-23.8)$ \\
\hline Clackamas County, Oregon & 497 & 24.0 & 2.7 & $(18.8-29.2)$ \\
\hline Lane County, Oregon & 515 & 31.7 & 2.6 & $(26.6-36.9)$ \\
\hline Multnomah County, Oregon & 872 & 24.3 & 1.9 & $(20.6-28.0)$ \\
\hline Washington County, Oregon & 605 & 20.1 & 2.0 & $(16.2-23.9)$ \\
\hline Allegheny County, Pennsylvania & 1,859 & 19.5 & 1.1 & $(17.2-21.7)$ \\
\hline Bradford County, Pennsylvania & 1,835 & 18.8 & 1.4 & $(16.1-21.5)$ \\
\hline Montgomery County, Pennsylvania & 542 & 16.1 & 1.9 & $(12.4-19.9)$ \\
\hline Philadelphia County, Pennsylvania & 2,017 & 21.4 & 1.2 & $(19.0-23.7)$ \\
\hline Pike County, Pennsylvania & 1,873 & 20.3 & 1.5 & $(17.3-23.2)$ \\
\hline Kent County, Rhode Island & 805 & 22.9 & 2.0 & $(18.9-26.8)$ \\
\hline Providence County, Rhode Island & 3,289 & 20.3 & 0.9 & $(18.4-22.1)$ \\
\hline Washington County, Rhode Island & 646 & 15.5 & 2.0 & $(11.6-19.4)$ \\
\hline
\end{tabular}

TABLE 72. (Continued) Estimated prevalence of adults aged $\geq 18$ years who reported limited activities because of physical, mental, or emotional problems, by county - Behavioral Risk Factor Surveillance System, United States, 2012

\begin{tabular}{|c|c|c|c|c|}
\hline County & $\begin{array}{l}\text { Sample } \\
\text { size }\end{array}$ & $\%$ & SE & $95 \% \mathrm{Cl}$ \\
\hline Aiken County, South Carolina & 545 & 25.0 & 2.5 & $(20.1-29.8)$ \\
\hline Beaufort County, South Carolina & 796 & 16.7 & 1.9 & $(12.9-20.5)$ \\
\hline Charleston County, South Carolina & 1,000 & 20.9 & 1.8 & $(17.3-24.4)$ \\
\hline Greenville County, South Carolina & 891 & 23.1 & 1.8 & $(19.5-26.6)$ \\
\hline Horry County, South Carolina & 761 & 22.0 & 1.8 & $(18.6-25.5)$ \\
\hline Richland County, South Carolina & 955 & 17.0 & 1.6 & $(13.8-20.2)$ \\
\hline Spartanburg County, South Carolina & 647 & 25.9 & 2.5 & $(21.0-30.8)$ \\
\hline Lincoln County, South Dakota & 498 & 13.7 & 2.1 & $(9.5-18.0)$ \\
\hline Minnehaha County, South Dakota & 875 & 17.5 & 1.6 & $(14.4-20.6)$ \\
\hline Pennington County, South Dakota & 603 & 25.5 & 2.1 & $(21.5-29.5)$ \\
\hline Davidson County, Tennessee & 540 & 17.1 & 1.9 & $(13.4-20.9)$ \\
\hline Shelby County, Tennessee & 515 & 15.5 & 1.8 & $(12.0-19.1)$ \\
\hline Bexar County, Texas & 578 & 17.1 & 1.9 & $(13.4-20.8)$ \\
\hline Dallas County, Texas & 503 & 17.0 & 2.0 & $(13.1-20.9)$ \\
\hline El Paso County, Texas & 618 & 16.1 & 2.0 & $(12.2-20.0)$ \\
\hline Harris County, Texas & 757 & 16.8 & 1.6 & $(13.7-19.9)$ \\
\hline Hidalgo County, Texas & 615 & 19.1 & 2.4 & $(14.5-23.8)$ \\
\hline Tarrant County, Texas & 563 & 18.9 & 2.0 & $(15.0-22.8)$ \\
\hline Travis County, Texas & 1,035 & 15.8 & 1.7 & $(12.6-19.1)$ \\
\hline Davis County, Utah & 1,126 & 18.7 & 1.4 & $(16.1-21.4)$ \\
\hline Salt Lake County, Utah & 3,971 & 19.2 & 0.7 & $(17.7-20.6)$ \\
\hline Tooele County, Utah & 548 & 18.9 & 2.8 & $(13.4-24.4)$ \\
\hline Utah County, Utah & 1,654 & 18.4 & 1.1 & $(16.1-20.6)$ \\
\hline Wasatch County, Utah & 503 & 12.0 & 1.8 & $(8.5-15.5)$ \\
\hline Weber County, Utah & 1,032 & 22.2 & 1.6 & $(19.1-25.3)$ \\
\hline Chittenden County, Vermont & 905 & 16.6 & 1.4 & $(13.9-19.3)$ \\
\hline Rutland County, Vermont & 587 & 21.3 & 2.1 & $(17.2-25.5)$ \\
\hline Washington County, Vermont & 508 & 17.7 & 2.0 & $(13.8-21.6)$ \\
\hline Windsor County, Vermont & 535 & 19.7 & 2.1 & $(15.5-23.8)$ \\
\hline Fairfax County, Virginia & 708 & 14.1 & 1.6 & $(10.9-17.2)$ \\
\hline Clark County, Washington & 777 & 23.0 & 1.8 & $(19.6-26.5)$ \\
\hline King County, Washington & 3,882 & 20.0 & 0.8 & $(18.4-21.6)$ \\
\hline Kitsap County, Washington & 561 & 27.0 & 2.3 & $(22.4-31.5)$ \\
\hline Pierce County, Washington & 1,170 & 27.2 & 1.7 & $(23.9-30.4)$ \\
\hline Snohomish County, Washington & 1,163 & 22.6 & 1.5 & $(19.6-25.6)$ \\
\hline Spokane County, Washington & 941 & 27.1 & 1.9 & $(23.5-30.7)$ \\
\hline Thurston County, Washington & 509 & 26.8 & 2.6 & $(21.6-31.9)$ \\
\hline Whatcom County, Washington & 841 & 23.9 & 2.1 & $(19.7-28.1)$ \\
\hline Yakima County, Washington & 528 & 22.3 & 2.4 & $(17.5-27.1)$ \\
\hline Kanawha County, West Virginia & 639 & 27.7 & 2.0 & $(23.7-31.7)$ \\
\hline Milwaukee County, Wisconsin & 916 & 21.0 & 2.1 & $(16.8-25.2)$ \\
\hline Laramie County, Wyoming & 942 & 20.5 & 1.9 & $(16.7-24.2)$ \\
\hline Natrona County, Wyoming & 819 & 23.0 & 2.2 & $(18.8-27.2)$ \\
\hline San Juan Municipio, Puerto Rico & 664 & 15.2 & 1.6 & $(12.0-18.5)$ \\
\hline Median & & 19.5 & & \\
\hline Range & & $11.3-31.7$ & & \\
\hline
\end{tabular}

Abbreviations: $\mathrm{Cl}=$ confidence interval; $\mathrm{SE}=$ standard error. 
TABLE 73. Estimated prevalence of adults aged $\geq 18$ years who required use of special equipment* because of any health problem, by state/territory - Behavioral Risk Factor Surveillance System, United States, 2012

\begin{tabular}{|c|c|c|c|c|}
\hline State/Territory & $\begin{array}{c}\text { Sample } \\
\text { size }\end{array}$ & $\%$ & SE & $95 \% \mathrm{Cl}$ \\
\hline Alabama & 8,966 & 11.1 & 0.5 & $(10.2-12.0)$ \\
\hline Alaska & 4,309 & 7.2 & 0.5 & $(6.2-8.2)$ \\
\hline Arizona & 7,189 & 8.1 & 0.4 & $(7.2-8.9)$ \\
\hline Arkansas & 5,113 & 10.2 & 0.5 & $(9.2-11.2)$ \\
\hline California & 13,474 & 6.9 & 0.3 & $(6.4-7.5)$ \\
\hline Colorado & 11,755 & 6.4 & 0.3 & $(5.8-6.9)$ \\
\hline Connecticut & 8,662 & 8.5 & 0.4 & $(7.8-9.2)$ \\
\hline Delaware & 5,161 & 8.4 & 0.5 & $(7.4-9.4)$ \\
\hline District of Columbia & 3,766 & 9.2 & 0.6 & $(8.0-10.4)$ \\
\hline Florida & 7,512 & 8.9 & 0.4 & $(8.0-9.7)$ \\
\hline Georgia & 5,921 & 7.9 & 0.4 & $(7.1-8.7)$ \\
\hline Hawaii & 7,531 & 6.8 & 0.4 & $(5.9-7.6)$ \\
\hline Idaho & 5,840 & 7.4 & 0.5 & $(6.4-8.4)$ \\
\hline Illinois & 5,537 & 7.9 & 0.5 & $(6.9-8.9)$ \\
\hline Indiana & 8,548 & 8.5 & 0.4 & $(7.8-9.2)$ \\
\hline lowa & 7,042 & 7.2 & 0.3 & $(6.6-7.8)$ \\
\hline Kansas & 11,753 & 7.9 & 0.3 & $(7.3-8.4)$ \\
\hline Kentucky & 10,933 & 11.6 & 0.5 & $(10.7-12.5)$ \\
\hline Louisiana & 9,028 & 9.2 & 0.4 & $(8.4-10.1)$ \\
\hline Maine & 9,867 & 8.4 & 0.3 & $(7.7-9.0)$ \\
\hline Maryland & 12,530 & 7.0 & 0.3 & $(6.4-7.6)$ \\
\hline Massachusetts & 20,904 & 6.9 & 0.3 & $(6.4-7.4)$ \\
\hline Michigan & 10,395 & 8.7 & 0.3 & $(8.1-9.4)$ \\
\hline Minnesota & 11,964 & 5.8 & 0.3 & $(5.3-6.3)$ \\
\hline Mississippi & 7,736 & 10.2 & 0.4 & $(9.4-11.0)$ \\
\hline Missouri & 6,693 & 10.4 & 0.5 & $(9.5-11.4)$ \\
\hline Montana & 8,605 & 7.9 & 0.4 & $(7.2-8.6)$ \\
\hline Nebraska & 19,053 & 6.8 & 0.2 & $(6.4-7.3)$ \\
\hline Nevada & 4,794 & 7.7 & 0.5 & $(6.6-8.7)$ \\
\hline New Hampshire & 7,459 & 7.1 & 0.4 & $(6.4-7.8)$ \\
\hline New Jersey & 15,142 & 6.4 & 0.3 & $(5.9-6.9)$ \\
\hline New Mexico & 8,670 & 9.6 & 0.4 & $(8.9-10.3)$ \\
\hline New York & 5,958 & 8.3 & 0.5 & $(7.3-9.3)$ \\
\hline North Carolina & 11,815 & 8.0 & 0.3 & $(7.5-8.6)$ \\
\hline North Dakota & 4,842 & 5.8 & 0.4 & $(5.1-6.6)$ \\
\hline Ohio & 12,704 & 8.0 & 0.3 & $(7.5-8.6)$ \\
\hline Oklahoma & 7,960 & 9.2 & 0.4 & $(8.5-9.9)$ \\
\hline Oregon & 5,204 & 9.1 & 0.5 & $(8.1-10.0)$ \\
\hline Pennsylvania & 19,707 & 8.6 & 0.3 & $(8.0-9.2)$ \\
\hline Rhode Island & 5,414 & 8.7 & 0.5 & $(7.8-9.7)$ \\
\hline South Carolina & 12,644 & 9.6 & 0.4 & $(8.9-10.3)$ \\
\hline South Dakota & 7,824 & 7.2 & 0.4 & $(6.4-8.0)$ \\
\hline Tennessee & 6,835 & 9.3 & 0.4 & $(8.4-10.1)$ \\
\hline Texas & 9,021 & 7.3 & 0.3 & $(6.6-7.9)$ \\
\hline Utah & 12,238 & 5.8 & 0.2 & $(5.4-6.3)$ \\
\hline Vermont & 6,001 & 6.6 & 0.3 & $(5.9-7.2)$ \\
\hline Virginia & 7,184 & 7.9 & 0.4 & $(7.2-8.6)$ \\
\hline Washington & 15,231 & 8.1 & 0.3 & $(7.6-8.6)$ \\
\hline West Virginia & 5,402 & 11.2 & 0.4 & $(10.3-12.0)$ \\
\hline Wisconsin & 5,148 & 7.3 & 0.5 & $(6.4-8.3)$ \\
\hline Wyoming & 6,190 & 7.2 & 0.4 & $(6.3-8.0)$ \\
\hline Guam & 2,002 & 4.8 & 0.7 & $(3.5-6.1)$ \\
\hline Puerto Rico & 6,298 & 9.7 & 0.4 & $(8.9-10.6)$ \\
\hline Median & & 8.0 & & \\
\hline Range & & $4.8-11.6$ & & \\
\hline
\end{tabular}

Abbreviations: $\mathrm{Cl}=$ confidence interval; $\mathrm{SE}=$ standard error.

*Including use of a cane, wheelchair, special bed, or special telephone, occasionally or in certain circumstances.
TABLE 74. Estimated prevalence of adults aged $\geq 18$ years who required use of special equipment* because of any health problem, by metropolitan and micropolitan statistical area - Behavioral Risk Factor Surveillance System, United States, 2012

\begin{tabular}{|c|c|c|c|c|}
\hline MMSA(s) & $\begin{array}{l}\text { Sample } \\
\text { size }\end{array}$ & $\%$ & SE & $95 \% \mathrm{Cl}$ \\
\hline Aguadilla-Isabela, Puerto Rico & 550 & 9.3 & 1.3 & $(6.7-11.8)$ \\
\hline Akron, Ohio & 725 & 9.1 & 1.1 & $(6.9-11.3)$ \\
\hline Albuquerque, New Mexico & 3,220 & 8.8 & 0.6 & (7.7-9.9) \\
\hline $\begin{array}{l}\text { Allentown-Bethlehem-Easton, } \\
\text { Pennsylvania-New Jersey }\end{array}$ & 1,322 & 8.6 & 1.0 & $(6.6-10.6)$ \\
\hline Anaheim-Santa Ana-Irvine, California ${ }^{\dagger}$ & 968 & 4.8 & 0.8 & $(3.1-6.4)$ \\
\hline Anchorage, Alaska & 1,490 & 7.1 & 0.7 & $(5.7-8.6)$ \\
\hline Asheville, North Carolina & 593 & 9.5 & 1.4 & $(6.8-12.2)$ \\
\hline $\begin{array}{l}\text { Atlanta-Sandy Springs-Roswell, } \\
\text { Georgia }\end{array}$ & 2,467 & 6.4 & 0.6 & $(5.3-7.6)$ \\
\hline Atlantic City-Hammonton, New Jersey & 991 & 7.1 & 0.9 & $(5.3-8.8)$ \\
\hline $\begin{array}{l}\text { Augusta-Richmond County, Georgia- } \\
\text { South Carolina }\end{array}$ & 1,013 & 11.8 & 1.8 & $(8.3-15.2)$ \\
\hline Augusta-Waterville, Maine & 828 & 10.2 & 1.2 & $(7.9-12.5)$ \\
\hline Austin-Round Rock, Texas & 1,379 & 4.8 & 0.6 & $(3.7-5.9)$ \\
\hline $\begin{array}{l}\text { Baltimore-Columbia-Towson, } \\
\text { Maryland }\end{array}$ & 4,634 & 7.6 & 0.5 & $(6.6-8.5)$ \\
\hline Bangor, Maine & 927 & 8.3 & 1.0 & $(6.3-10.3)$ \\
\hline Barnstable Town, Massachusetts & 540 & 5.6 & 1.0 & $(3.7-7.5)$ \\
\hline Barre, Vermont & 514 & 5.4 & 1.0 & $(3.5-7.3)$ \\
\hline Baton Rouge, Louisiana & 1,382 & 9.5 & 1.0 & $(7.6-11.5)$ \\
\hline Bellingham, Washington & 845 & 7.6 & 1.0 & $(5.6-9.7)$ \\
\hline Berlin, New Hampshire-Vermont & 706 & 11.9 & 2.1 & $(7.8-16.1)$ \\
\hline Billings, Montana & 844 & 8.8 & 1.1 & $(6.6-11.0)$ \\
\hline Birmingham-Hoover, Alabama & 1,807 & 10.7 & 1.0 & $(8.7-12.6)$ \\
\hline Bismarck, North Dakota & 819 & 6.8 & 1.1 & $(4.7-8.9)$ \\
\hline Boise City, Idaho & 1,465 & 6.2 & 0.8 & $(4.7-7.7)$ \\
\hline Boston, Massachusetts $^{\dagger}$ & 5,686 & 6.5 & 0.4 & $(5.7-7.4)$ \\
\hline Boulder, Colorado & 498 & 4.0 & 0.9 & $(2.3-5.7)$ \\
\hline Bremerton-Silverdale, Washington & 562 & 9.9 & 1.4 & $(7.2-12.6)$ \\
\hline $\begin{array}{l}\text { Bridgeport-Stamford-Norwalk, } \\
\text { Connecticut }\end{array}$ & 2,145 & 7.7 & 0.8 & $(6.2-9.3)$ \\
\hline Burlington-South Burlington, Vermont & 1,506 & 5.3 & 0.6 & $(4.1-6.5)$ \\
\hline $\begin{array}{l}\text { Cambridge-Newton-Framingham, } \\
\text { Massachusetts }{ }^{\dagger}\end{array}$ & 6,647 & 6.6 & 0.4 & $(5.7-7.4)$ \\
\hline Camden, New Jersey ${ }^{\dagger}$ & 1,930 & 7.3 & 0.6 & $(6.1-8.6)$ \\
\hline Canton-Massillon, Ohio & 665 & 7.1 & 1.1 & $(4.9-9.2)$ \\
\hline Casper, Wyoming & 818 & 7.8 & 1.0 & $(5.8-9.7)$ \\
\hline Cedar Rapids, lowa & 577 & 6.4 & 0.9 & $(4.5-8.2)$ \\
\hline Charleston, West Virginia & 771 & 12.4 & 1.3 & $(9.9-15.0)$ \\
\hline $\begin{array}{l}\text { Charleston-North Charleston, } \\
\text { South Carolina }\end{array}$ & 1,695 & 9.9 & 1.0 & $(8.0-11.8)$ \\
\hline $\begin{array}{l}\text { Charlotte-Concord-Gastonia, } \\
\text { North Carolina-South Carolina }\end{array}$ & 2,510 & 6.9 & 0.6 & $(5.8-8.1)$ \\
\hline Chattanooga, Tennessee-Georgia & 611 & 8.5 & 1.2 & $(6.1-10.8)$ \\
\hline Cheyenne, Wyoming & 943 & 8.4 & 1.2 & $(6.0-10.8)$ \\
\hline $\begin{array}{l}\text { Chicago-Naperville-Elgin, } \\
\text { Illinois-Indiana-Wisconsin }\end{array}$ & 3,696 & 7.5 & 0.6 & $(6.3-8.7)$ \\
\hline Cincinnati, Ohio-Kentucky-Indiana & 2,323 & 8.3 & 0.7 & $(6.9-9.7)$ \\
\hline $\begin{array}{l}\text { Claremont-Lebanon, New } \\
\text { Hampshire-Vermont }\end{array}$ & 1,936 & 8.0 & 0.7 & $(6.6-9.4)$ \\
\hline Cleveland-Elyria, Ohio & 1,703 & 8.7 & 0.9 & $(7.0-10.5)$ \\
\hline Colorado Springs, Colorado & 1,119 & 7.5 & 0.8 & $(5.9-9.1)$ \\
\hline Columbia, South Carolina & 1,782 & 9.3 & 0.9 & $(7.4-11.1)$ \\
\hline Columbus, Ohio & 1,568 & 7.2 & 0.8 & $(5.7-8.7)$ \\
\hline Concord, New Hampshire & 701 & 7.4 & 1.2 & $(5.1-9.7)$ \\
\hline Dallas-Plano-Irving, Texas ${ }^{\dagger}$ & 896 & 6.4 & 0.8 & $(4.8-8.0)$ \\
\hline Dayton, Ohio & 839 & 6.8 & 0.9 & $(5.0-8.5)$ \\
\hline Denver-Aurora-Lakewood, Colorado & 4,660 & 6.3 & 0.4 & $(5.5-7.1)$ \\
\hline Des Moines-West Des Moines, lowa & 1,145 & 8.1 & 0.9 & $(6.3-9.8)$ \\
\hline
\end{tabular}

See table footnotes on page 137. 
TABLE 74. (Continued) Estimated prevalence of adults aged $\geq 18$ years who required use of special equipment* because of any health problem, by metropolitan and micropolitan statistical area Behavioral Risk Factor Surveillance System, United States, 2012

\begin{tabular}{|c|c|c|c|c|}
\hline MMSA(s) & $\begin{array}{l}\text { Sample } \\
\text { size }\end{array}$ & $\%$ & SE & $95 \% \mathrm{Cl}$ \\
\hline Detroit-Dearborn-Livonia, Michigan $^{\dagger}$ & 2,188 & 11.4 & 0.9 & $(9.6-13.2)$ \\
\hline Dover, Delaware & 1,440 & 9.7 & 1.1 & $(7.5-11.9)$ \\
\hline Duluth, Minnesota-Wisconsin & 509 & 7.6 & 1.4 & $(4.9-10.3)$ \\
\hline Durham-Chapel Hill, North Carolina & 790 & 5.2 & 0.8 & $(3.7-6.7)$ \\
\hline El Paso, Texas & 619 & 7.7 & 1.3 & $(5.1-10.3)$ \\
\hline Eugene, Oregon & 516 & 9.7 & 1.4 & $(6.9-12.5)$ \\
\hline Fairbanks, Alaska & 596 & 8.3 & 1.6 & $(5.2-11.5)$ \\
\hline Fargo, North Dakota-Minnesota & 932 & 5.5 & 0.9 & $(3.8-7.2)$ \\
\hline Farmington, New Mexico & 640 & 8.9 & 1.3 & $(6.4-11.5)$ \\
\hline Fayetteville, North Carolina & 502 & 10.2 & 1.6 & $(7.2-13.3)$ \\
\hline $\begin{array}{l}\text { Fayetteville-Springdale-Rogers, } \\
\text { Arkansas-Missouri }\end{array}$ & 798 & 8.1 & 1.1 & $(5.8-10.3)$ \\
\hline Fort Collins, Colorado & 576 & 5.7 & 0.9 & $(3.9-7.4)$ \\
\hline Fort Wayne, Indiana & 529 & 9.6 & 1.8 & $(6.1-13.2)$ \\
\hline Fort Worth-Arlington, Texas ${ }^{\dagger}$ & 719 & 7.4 & 1.1 & $(5.3-9.6)$ \\
\hline Grand Island, Nebraska & 853 & 6.8 & 0.9 & $(5.0-8.5)$ \\
\hline Grand Rapids-Wyoming, Michigan & 888 & 6.9 & 1.1 & $(4.6-9.1)$ \\
\hline Great Falls, Montana & 707 & 8.5 & 1.1 & $(6.4-10.6)$ \\
\hline Greeley, Colorado & 514 & 4.9 & 0.9 & $(3.2-6.6)$ \\
\hline $\begin{array}{l}\text { Greensboro-High Point, } \\
\text { North Carolina }\end{array}$ & 804 & 7.6 & 0.9 & $(5.7-9.4)$ \\
\hline $\begin{array}{l}\text { Greenville-Anderson-Mauldin, } \\
\text { South Carolina }\end{array}$ & 1,676 & 9.2 & 0.8 & $(7.5-10.8)$ \\
\hline Gulfport-Biloxi-Pascagoula, Mississippi & 740 & 12.3 & 1.3 & $(9.7-14.9)$ \\
\hline $\begin{array}{l}\text { Hagerstown-Martinsburg, Maryland- } \\
\text { West Virginia }\end{array}$ & 752 & 10.0 & 1.7 & $(6.7-13.3)$ \\
\hline Harrisburg-Carlisle, Pennsylvania & 664 & 8.3 & 1.4 & $(5.6-10.9)$ \\
\hline $\begin{array}{l}\text { Hartford-West Hartford-East Hartford, } \\
\text { Connecticut }\end{array}$ & 2,646 & 8.0 & 0.6 & $(6.7-9.2)$ \\
\hline Heber, Utah & 504 & 4.2 & 1.1 & $(2.1-6.4)$ \\
\hline Hilo, Hawaii & 1,346 & 6.4 & 0.8 & $(4.9-8.0)$ \\
\hline $\begin{array}{l}\text { Hilton Head Island-Bluffton-Beaufort, } \\
\text { South Carolina }\end{array}$ & 925 & 6.8 & 1.1 & $(4.7-8.9)$ \\
\hline $\begin{array}{l}\text { Houston-The Woodlands-Sugar Land, } \\
\text { Texas }\end{array}$ & 1,124 & 7.2 & 0.9 & $(5.4-9.0)$ \\
\hline $\begin{array}{l}\text { Huntington-Ashland, West } \\
\text { Virginia-Kentucky-Ohio }\end{array}$ & 1,103 & 12.1 & 1.1 & $(9.9-14.4)$ \\
\hline Huntsville, Alabama & 611 & 9.1 & 1.4 & $(6.3-11.8)$ \\
\hline Idaho Falls, Idaho & 535 & 7.7 & 1.8 & $(4.2-11.3)$ \\
\hline $\begin{array}{l}\text { Indianapolis-Carmel-Anderson, } \\
\text { Indiana }\end{array}$ & 2,168 & 8.1 & 0.7 & $(6.8-9.4)$ \\
\hline Jackson, Mississippi & 911 & 9.9 & 1.1 & $(7.7-12.1)$ \\
\hline Jacksonville, Florida & 513 & 8.3 & 1.3 & $(5.9-10.8)$ \\
\hline Kahului-Wailuku-Lahaina, Hawaii & 1,212 & 6.2 & 1.0 & $(4.3-8.2)$ \\
\hline Kalispell, Montana & 555 & 8.7 & 1.4 & $(5.9-11.5)$ \\
\hline Kansas City, Missouri-Kansas & 4,705 & 9.0 & 0.7 & $(7.6-10.5)$ \\
\hline Kapaa, Hawaii & 663 & 5.6 & 1.1 & $(3.5-7.7)$ \\
\hline Keene, New Hampshire & 543 & 7.0 & 1.6 & $(3.9-10.2)$ \\
\hline Kennewick-Richland, Washington & 536 & 8.2 & 1.4 & $(5.5-10.9)$ \\
\hline $\begin{array}{l}\text { Kingsport-Bristol-Bristol, } \\
\text { Tennessee-Virginia }\end{array}$ & 549 & 14.7 & 2.6 & $(9.6-19.8)$ \\
\hline Knoxville, Tennessee & 816 & 8.1 & 1.0 & $(6.2-10.1)$ \\
\hline Laconia, New Hampshire & 559 & 6.4 & 1.1 & $(4.2-8.6)$ \\
\hline Lafayette, Louisiana & 556 & 6.8 & 1.4 & $(4.1-9.5)$ \\
\hline Las Cruces, New Mexico & 701 & 10.7 & 1.4 & $(7.9-13.4)$ \\
\hline $\begin{array}{l}\text { Las Vegas-Henderson-Paradise, } \\
\text { Nevada }\end{array}$ & 993 & 8.3 & 0.7 & $(6.9-9.6)$ \\
\hline Lewiston-Auburn, Maine & 699 & 9.8 & 1.3 & $(7.3-12.3)$ \\
\hline Lexington-Fayette, Kentucky & 519 & 11.1 & 1.8 & $(7.5-14.6)$ \\
\hline Lincoln, Nebraska & 1,661 & 6.0 & 0.7 & $(4.7-7.3)$ \\
\hline
\end{tabular}

See table footnotes on page 137.
TABLE 74. (Continued) Estimated prevalence of adults aged $\geq 18$ years who required use of special equipment* because of any health problem, by metropolitan and micropolitan statistical area Behavioral Risk Factor Surveillance System, United States, 2012

\begin{tabular}{|c|c|c|c|c|}
\hline MMSA(s) & $\begin{array}{l}\text { Sample } \\
\text { size }\end{array}$ & $\%$ & SE & $95 \% \mathrm{Cl}$ \\
\hline $\begin{array}{l}\text { Little Rock-North Little Rock-Conway, } \\
\text { Arkansas }\end{array}$ & 1,155 & 9.0 & 0.9 & $(7.2-10.8)$ \\
\hline Logan, Utah-Idaho & 504 & 5.1 & 1.3 & $(2.5-7.7)$ \\
\hline $\begin{array}{l}\text { Los Angeles-Long Beach-Glendale, } \\
\text { California }^{\dagger}\end{array}$ & 3,170 & 6.7 & 0.6 & $(5.5-7.8)$ \\
\hline $\begin{array}{l}\text { Louisville/Jefferson County, } \\
\text { Kentucky-Indiana }\end{array}$ & 2,107 & 11.0 & 1.0 & $(9.0-13.0)$ \\
\hline Lumberton, North Carolina & 539 & 13.3 & 2.7 & $(8.0-18.7)$ \\
\hline Manchester-Nashua, New Hampshire & 1,890 & 6.9 & 0.6 & $(5.7-8.1)$ \\
\hline McAllen-Edinburg-Mission, Texas & 617 & 9.5 & 1.7 & $(6.2-12.7)$ \\
\hline $\begin{array}{l}\text { Memphis, } \\
\text { Tennessee-Mississippi-Arkansas }\end{array}$ & 1,285 & 10.7 & 1.2 & $(8.4-13.0)$ \\
\hline $\begin{array}{l}\text { Miami-Fort Lauderdale-West Palm } \\
\text { Beach, Florida }\end{array}$ & 1,637 & 6.7 & 0.8 & $(5.1-8.2)$ \\
\hline $\begin{array}{l}\text { Milwaukee-Waukesha-West Allis, } \\
\text { Wisconsin }\end{array}$ & 1,206 & 7.8 & 1.0 & $(5.9-9.7)$ \\
\hline $\begin{array}{l}\text { Minneapolis-St. Paul-Bloomington, } \\
\text { Minnesota-Wisconsin }\end{array}$ & 7,690 & 5.4 & 0.3 & $(4.8-6.0)$ \\
\hline Missoula, Montana & 778 & 6.9 & 1.1 & $(4.8-9.0)$ \\
\hline Mobile, Alabama & 814 & 12.1 & 1.6 & $(9.0-15.1)$ \\
\hline Montgomery, Alabama & 532 & 12.8 & 1.8 & $(9.3-16.3)$ \\
\hline $\begin{array}{l}\text { Montgomery County-Bucks County- } \\
\text { Chester County, Pennsylvania }{ }^{\dagger}\end{array}$ & 1,300 & 7.1 & 1.0 & $(5.2-9.0)$ \\
\hline $\begin{array}{l}\text { Myrtle Beach-Conway-North Myrtle } \\
\text { Beach, South Carolina-North Carolina }\end{array}$ & 941 & 7.2 & 0.9 & $(5.5-8.9)$ \\
\hline $\begin{array}{l}\text { Nashville-Davidson-Murfreesboro- } \\
\text { Franklin, Tennessee }\end{array}$ & 1,302 & 6.5 & 0.7 & $(5.0-7.9)$ \\
\hline $\begin{array}{l}\text { Nassau County-Suffolk County, } \\
\text { New York }\end{array}$ & 891 & 5.8 & 0.9 & $(4.1-7.5)$ \\
\hline Newark, New Jersey-Pennsylvania ${ }^{\dagger}$ & 6,384 & 6.2 & 0.5 & $(5.3-7.1)$ \\
\hline New Haven-Milford, Connecticut & 1,984 & 9.7 & 0.8 & $(8.1-11.3)$ \\
\hline New Orleans-Metairie, Louisiana & 1,266 & 7.7 & 0.8 & $(6.0-9.3)$ \\
\hline $\begin{array}{l}\text { New York-Jersey City-White Plains, } \\
\text { New York-New Jersey }{ }^{\dagger}\end{array}$ & 7,109 & 8.2 & 0.6 & $(7.0-9.4)$ \\
\hline Norfolk, Nebraska & 568 & 7.7 & 1.1 & $(5.5-10.0)$ \\
\hline North Platte, Nebraska & 611 & 6.8 & 1.2 & $(4.4-9.2)$ \\
\hline Norwich-New London, Connecticut & 989 & 9.4 & 1.6 & $(6.4-12.5)$ \\
\hline $\begin{array}{l}\text { Oakland-Hayward-Berkeley, } \\
\text { California }^{\dagger}\end{array}$ & 914 & 7.0 & 1.2 & $(4.6-9.3)$ \\
\hline Ocean City, New Jersey & 534 & 9.4 & 1.6 & $(6.2-12.6)$ \\
\hline Ogden-Clearfield, Utah & 2,396 & 6.2 & 0.5 & $(5.2-7.3)$ \\
\hline Oklahoma City, Oklahoma & 2,425 & 9.2 & 0.7 & $(7.8-10.6)$ \\
\hline Olympia-Tumwater, Washington & 515 & 13.6 & 2.0 & $(9.6-17.5)$ \\
\hline Omaha-Council Bluffs, Nebraska-lowa & 5,534 & 6.8 & 0.4 & $(6.0-7.6)$ \\
\hline Orlando-Kissimmee-Sanford, Florida & 555 & 8.1 & 1.4 & $(5.4-10.8)$ \\
\hline Philadelphia, Pennsylvania ${ }^{\dagger}$ & 2,415 & 9.0 & 0.7 & $(7.6-10.3)$ \\
\hline Phoenix-Mesa-Scottsdale, Arizona & 2,555 & 7.3 & 0.6 & $(6.1-8.5)$ \\
\hline Pittsburgh, Pennsylvania & 3,321 & 9.4 & 0.6 & $(8.3-10.6)$ \\
\hline Ponce, Puerto Rico & 548 & 11.4 & 1.7 & $(8.1-14.6)$ \\
\hline Portland-South Portland, Maine & 3,312 & 7.2 & 0.5 & $(6.3-8.2)$ \\
\hline $\begin{array}{l}\text { Portland-Vancouver-Hillsboro, } \\
\text { Oregon-Washington }\end{array}$ & 3,084 & 7.2 & 0.6 & $(6.1-8.3)$ \\
\hline $\begin{array}{l}\text { Providence-Warwick, Rhode } \\
\text { Island-Massachusetts }\end{array}$ & 7,980 & 8.4 & 0.4 & $(7.6-9.2)$ \\
\hline Provo-Orem, Utah & 1,721 & 5.2 & 0.6 & $(4.0-6.4)$ \\
\hline Raleigh, North Carolina & 939 & 6.2 & 0.8 & $(4.6-7.8)$ \\
\hline Rapid City, South Dakota & 1,049 & 10.0 & 1.1 & $(7.7-12.2)$ \\
\hline Reno, Nevada & 1,494 & 5.9 & 0.8 & $(4.3-7.4)$ \\
\hline Richmond, Virginia & 987 & 5.9 & 0.8 & $(4.3-7.4)$ \\
\hline
\end{tabular}

See table footnotes on page 137. 
TABLE 74. (Continued) Estimated prevalence of adults aged $\geq 18$ years who required use of special equipment* because of any health problem, by metropolitan and micropolitan statistical area Behavioral Risk Factor Surveillance System, United States, 2012

\begin{tabular}{|c|c|c|c|c|}
\hline MMSA(s) & $\begin{array}{l}\text { Sample } \\
\text { size }\end{array}$ & $\%$ & SE & $95 \% \mathrm{Cl}$ \\
\hline $\begin{array}{l}\text { Riverside-San Bernardino-Ontario, } \\
\text { California }\end{array}$ & 1,409 & 7.2 & 0.8 & $(5.7-8.8)$ \\
\hline $\begin{array}{l}\text { Rockingham County-Strafford County, } \\
\text { New Hampshire }\end{array}$ & 1,653 & 6.4 & 0.6 & $(5.2-7.6)$ \\
\hline Rutland, Vermont & 590 & 8.6 & 1.3 & $(6.1-11.2)$ \\
\hline $\begin{array}{l}\text { Sacramento-Roseville-Arden-Arcade, } \\
\text { California }\end{array}$ & 934 & 8.1 & 1.1 & $(6.0-10.2)$ \\
\hline St. Louis, Missouri-Illinois & 2,046 & 8.5 & 0.7 & $(7.0-9.9)$ \\
\hline Salisbury, Maryland-Delaware & 2,100 & 9.6 & 0.8 & $(8.1-11.2)$ \\
\hline Salt Lake City, Utah & 4,546 & 5.8 & 0.4 & $(5.0-6.5)$ \\
\hline San Antonio-New Braunfels, Texas & 774 & 7.8 & 1.0 & $(5.8-9.9)$ \\
\hline San Diego-Carlsbad, California & 1,056 & 6.2 & 0.8 & $(4.6-7.8)$ \\
\hline $\begin{array}{l}\text { San Francisco-Redwood City-South } \\
\text { San Francisco, California }{ }^{\dagger}\end{array}$ & 543 & 7.7 & 1.5 & $(4.7-10.6)$ \\
\hline $\begin{array}{l}\text { San Jose-Sunnyvale-Santa Clara, } \\
\text { California }\end{array}$ & 639 & 5.1 & 0.9 & $(3.4-6.8)$ \\
\hline San Juan-Carolina-Caguas, Puerto Rico & 3,946 & 9.2 & 0.5 & $(8.2-10.3)$ \\
\hline Santa Fe, New Mexico & 690 & 8.5 & 1.2 & $(6.1-10.8)$ \\
\hline Sayre, Pennsylvania & 1,845 & 7.9 & 0.7 & $(6.4-9.3)$ \\
\hline Scottsbluff, Nebraska & 630 & 7.8 & 1.3 & $(5.4-10.3)$ \\
\hline $\begin{array}{l}\text { Scranton-Wilkes-Barre-Hazleton, } \\
\text { Pennsylvania }\end{array}$ & 733 & 12.7 & 2.2 & $(8.3-17.1)$ \\
\hline Seattle-Bellevue-Everett, Washington ${ }^{\dagger}$ & 5,071 & 6.8 & 0.4 & $(5.9-7.6)$ \\
\hline Shreveport-Bossier City, Louisiana & 577 & 8.8 & 1.3 & $(6.2-11.4)$ \\
\hline $\begin{array}{l}\text { Silver Spring-Frederick-Rockville, } \\
\text { Maryland }{ }^{\dagger}\end{array}$ & 2,240 & 5.5 & 0.6 & $(4.4-6.6)$ \\
\hline $\begin{array}{l}\text { Sioux City, lowa-Nebraska- } \\
\text { South Dakota }\end{array}$ & 1,193 & 7.4 & 1.3 & $(4.8-9.9)$ \\
\hline Sioux Falls, South Dakota & 1,456 & 5.9 & 0.8 & $(4.3-7.5)$ \\
\hline Spartanburg, South Carolina & 702 & 10.5 & 1.8 & $(7.0-13.9)$ \\
\hline Spokane-Spokane Valley, Washington & 1,090 & 7.4 & 0.8 & $(5.8-9.0)$ \\
\hline Springfield, Massachusetts & 2,288 & 8.4 & 0.9 & $(6.7-10.1)$ \\
\hline Tacoma-Lakewood, Washington ${ }^{\dagger}$ & 1,174 & 8.4 & 0.9 & $(6.6-10.1)$ \\
\hline
\end{tabular}

TABLE 74. (Continued) Estimated prevalence of adults aged $\geq 18$ years who required use of special equipment* because of any health problem, by metropolitan and micropolitan statistical area Behavioral Risk Factor Surveillance System, United States, 2012

\begin{tabular}{|c|c|c|c|c|}
\hline MMSA(s) & $\begin{array}{l}\text { Sample } \\
\text { size }\end{array}$ & $\%$ & SE & $95 \% \mathrm{Cl}$ \\
\hline $\begin{array}{l}\text { Tampa-St. Petersburg-Clearwater, } \\
\text { Florida }\end{array}$ & 818 & 9.1 & 1.3 & $(6.6-11.6)$ \\
\hline Toledo, Ohio & 948 & 8.5 & 1.0 & $(6.4-10.5)$ \\
\hline Topeka, Kansas & 1,088 & 8.3 & 0.9 & $(6.5-10.0)$ \\
\hline Torrington, Connecticut & 655 & 7.9 & 1.2 & $(5.6-10.2)$ \\
\hline Trenton, New Jersey & 564 & 5.5 & 1.0 & $(3.5-7.6)$ \\
\hline Tucson, Arizona & 981 & 8.8 & 1.0 & $(6.8-10.8)$ \\
\hline Tulsa, Oklahoma & 1,729 & 7.3 & 0.6 & $(6.1-8.5)$ \\
\hline Tuscaloosa, Alabama & 579 & 9.6 & 1.7 & $(6.2-12.9)$ \\
\hline Urban Honolulu, Hawaii & 4,310 & 6.9 & 0.6 & $(5.8-8.1)$ \\
\hline Vineland-Bridgeton, New Jersey & 543 & 6.6 & 1.0 & $(4.6-8.7)$ \\
\hline $\begin{array}{l}\text { Virginia Beach-Norfolk-Newport News, } \\
\text { Virginia-North Carolina }\end{array}$ & 1,472 & 9.1 & 1.0 & $(7.1-11.0)$ \\
\hline $\begin{array}{l}\text { Warren-Troy-Farmington Hills, } \\
\text { Michigan }{ }^{\dagger}\end{array}$ & 2,165 & 8.0 & 0.7 & $(6.6-9.3)$ \\
\hline $\begin{array}{l}\text { Washington-Arlington-Alexandria, } \\
\text { District of Columbia-Virginia- } \\
\text { Maryland-West Virginia }{ }^{\dagger}\end{array}$ & 8,036 & 6.4 & 0.4 & $(5.7-7.1)$ \\
\hline Wichita, Kansas & 2,344 & 8.0 & 0.6 & $(6.7-9.2)$ \\
\hline $\begin{array}{l}\text { Wilmington, Delaware-Maryland- } \\
\text { New Jersey }{ }^{\dagger}\end{array}$ & 3,206 & 7.2 & 0.6 & $(6.1-8.3)$ \\
\hline Winston-Salem, North Carolina & 794 & 8.9 & 1.1 & $(6.8-11.0)$ \\
\hline Worcester, Massachusetts-Connecticut & 2,746 & 7.2 & 0.6 & $(6.0-8.4)$ \\
\hline Yakima, Washington & 531 & 8.8 & 1.5 & $(5.8-11.8)$ \\
\hline $\begin{array}{l}\text { Youngstown-Warren-Boardman, } \\
\text { Ohio-Pennsylvania }\end{array}$ & 908 & 10.0 & 1.6 & $(6.9-13.2)$ \\
\hline Median & & 7.8 & & \\
\hline Range & & $4.0-14.7$ & & \\
\hline
\end{tabular}

Abbreviations: $\mathrm{Cl}=$ confidence interval; $\mathrm{MMSA}=$ metropolitan and micropolitan statistical area; $\mathrm{SE}=$ standard error.

* Including use of a cane, wheelchair, special bed, or special telephone, occasionally or in certain circumstances.

${ }^{\dagger}$ Metropolitan division. 
TABLE 75. Estimated prevalence of adults aged $\geq 18$ years who required use of special equipment* because of any health problem, by county - Behavioral Risk Factor Surveillance System, United States, 2012

\begin{tabular}{|c|c|c|c|c|}
\hline County & $\begin{array}{l}\text { Sample } \\
\text { size }\end{array}$ & $\%$ & SE & 95\% Cl \\
\hline Jefferson County, Alabama & 951 & 10.0 & 1.1 & $(7.9-12.0)$ \\
\hline Madison County, Alabama & 508 & 8.1 & 1.5 & $(5.1-11.1)$ \\
\hline Mobile County, Alabama & 814 & 12.1 & 1.6 & $(9.0-15.1)$ \\
\hline Anchorage Municipality, Alaska & 893 & 5.9 & 0.8 & $(4.3-7.4)$ \\
\hline Fairbanks North Star Borough, Alaska & 596 & 8.3 & 1.6 & $(5.2-11.5)$ \\
\hline Matanuska-Susitna Borough, Alaska & 597 & 11.1 & 1.6 & $(8.0-14.1)$ \\
\hline Maricopa County, Arizona & 2,077 & 7.4 & 0.7 & $(6.1-8.7)$ \\
\hline Pima County, Arizona & 981 & 8.8 & 1.0 & $(6.8-10.9)$ \\
\hline Pulaski County, Arkansas & 743 & 10.5 & 1.4 & $(7.8-13.2)$ \\
\hline Alameda County, California & 583 & 7.7 & 1.6 & $(4.4-10.9)$ \\
\hline Los Angeles County, California & 3,170 & 6.7 & 0.6 & $(5.6-7.8)$ \\
\hline Orange County, California & 968 & 4.8 & 0.8 & $(3.2-6.5)$ \\
\hline Riverside County, California & 753 & 7.5 & 1.1 & $(5.4-9.7)$ \\
\hline Sacramento County, California & 564 & 9.1 & 1.5 & $(6.2-12.0)$ \\
\hline San Bernardino County, California & 656 & 6.9 & 1.2 & $(4.6-9.3)$ \\
\hline San Diego County, California & 1,056 & 6.2 & 0.8 & $(4.6-7.8)$ \\
\hline Santa Clara County, California & 621 & 5.0 & 0.9 & $(3.3-6.7)$ \\
\hline Adams County, Colorado & 780 & 6.7 & 0.9 & $(4.9-8.5)$ \\
\hline Arapahoe County, Colorado & 830 & 6.3 & 1.0 & $(4.4-8.2)$ \\
\hline Boulder County, Colorado & 498 & 4.0 & 0.9 & $(2.3-5.7)$ \\
\hline Denver County, Colorado & 932 & 7.6 & 0.9 & $(5.9-9.3)$ \\
\hline Douglas County, Colorado & 526 & 2.8 & 0.7 & $(1.4-4.2)$ \\
\hline El Paso County, Colorado & 984 & 7.6 & 0.9 & $(5.9-9.3)$ \\
\hline Jefferson County, Colorado & 1,085 & 6.1 & 0.8 & $(4.4-7.7)$ \\
\hline Larimer County, Colorado & 576 & 5.7 & 0.9 & $(3.9-7.4)$ \\
\hline Weld County, Colorado & 514 & 4.9 & 0.9 & $(3.2-6.6)$ \\
\hline Fairfield County, Connecticut & 2,145 & 7.7 & 0.8 & $(6.2-9.3)$ \\
\hline Hartford County, Connecticut & 1,969 & 7.7 & 0.7 & $(6.4-9.0)$ \\
\hline Litchfield County, Connecticut & 655 & 7.9 & 1.2 & $(5.6-10.2)$ \\
\hline New Haven County, Connecticut & 1,984 & 9.7 & 0.8 & $(8.1-11.4)$ \\
\hline New London County, Connecticut & 989 & 9.5 & 1.6 & $(6.4-12.5)$ \\
\hline Kent County, Delaware & 1,440 & 9.7 & 1.1 & $(7.5-11.9)$ \\
\hline New Castle County, Delaware & 2,325 & 7.1 & 0.6 & $(5.9-8.3)$ \\
\hline Sussex County, Delaware & 1,396 & 10.6 & 1.0 & $(8.7-12.6)$ \\
\hline $\begin{array}{l}\text { District of Columbia, } \\
\text { District of Columbia }\end{array}$ & 3,766 & 9.2 & 0.6 & $(8.0-10.4)$ \\
\hline Broward County, Florida & 519 & 6.9 & 1.4 & $(4.1-9.7)$ \\
\hline Miami-Dade County, Florida & 797 & 5.8 & 1.0 & $(3.9-7.7)$ \\
\hline Hawaii County, Hawaii & 1,346 & 6.4 & 0.8 & $(4.9-8.0)$ \\
\hline Honolulu County, Hawaii & 4,310 & 6.9 & 0.6 & $(5.8-8.1)$ \\
\hline Kauai County, Hawaii & 663 & 5.6 & 1.1 & $(3.5-7.7)$ \\
\hline Maui County, Hawaii & 1,212 & 6.2 & 1.0 & $(4.3-8.2)$ \\
\hline Ada County, Idaho & 800 & 6.1 & 1.1 & $(4.0-8.1)$ \\
\hline Canyon County, Idaho & 497 & 6.7 & 1.3 & $(4.1-9.3)$ \\
\hline Cook County, Illinois & 1,492 & 8.3 & 0.9 & $(6.6-10.0)$ \\
\hline Lake County, Indiana & 877 & 10.0 & 1.3 & $(7.5-12.5)$ \\
\hline Marion County, Indiana & 1,263 & 8.8 & 0.9 & $(7.0-10.6)$ \\
\hline Polk County, lowa & 798 & 8.3 & 1.1 & $(6.2-10.4)$ \\
\hline Johnson County, Kansas & 2,165 & 5.5 & 0.6 & $(4.4-6.5)$ \\
\hline Sedgwick County, Kansas & 1,786 & 8.0 & 0.7 & $(6.5-9.4)$ \\
\hline Shawnee County, Kansas & 771 & 8.8 & 1.1 & $(6.5-11.0)$ \\
\hline Wyandotte County, Kansas & 871 & 11.7 & 1.5 & $(8.7-14.6)$ \\
\hline Jefferson County, Kentucky & 1,629 & 12.0 & 1.3 & $(9.4-14.6)$ \\
\hline East Baton Rouge Parish, Louisiana & 569 & 9.2 & 1.3 & $(6.8-11.7)$ \\
\hline Androscoggin County, Maine & 699 & 9.8 & 1.3 & $(7.3-12.3)$ \\
\hline Aroostook County, Maine & 534 & 9.9 & 1.5 & $(7.0-12.9)$ \\
\hline Cumberland County, Maine & 1,747 & 6.8 & 0.7 & $(5.5-8.2)$ \\
\hline Kennebec County, Maine & 828 & 10.2 & 1.2 & $(7.9-12.5)$ \\
\hline Penobscot County, Maine & 927 & 8.3 & 1.0 & $(6.3-10.3)$ \\
\hline
\end{tabular}

See table footnotes on page 139.
TABLE 75. (Continued) Estimated prevalence of adults aged $\geq 18$ years who required use of special equipment* because of any health problem, by county - Behavioral Risk Factor Surveillance System, United States, 2012

\begin{tabular}{|c|c|c|c|c|}
\hline County & $\begin{array}{l}\text { Sample } \\
\text { size }\end{array}$ & $\%$ & SE & $95 \% \mathrm{Cl}$ \\
\hline York County, Maine & 1,184 & 8.0 & 0.9 & $(6.3-9.7)$ \\
\hline Anne Arundel County, Maryland & 883 & 7.2 & 1.1 & $(5.0-9.3)$ \\
\hline Baltimore County, Maryland & 1,496 & 8.3 & 0.9 & $(6.6-10.0)$ \\
\hline Charles County, Maryland & 502 & $\mathrm{~N} / \mathrm{A}^{\dagger}$ & $\mathrm{N} / \mathrm{A}$ & $(\mathrm{N} / \mathrm{A}-\mathrm{N} / \mathrm{A})$ \\
\hline Frederick County, Maryland & 746 & 5.8 & 0.9 & $(4.0-7.6)$ \\
\hline Montgomery County, Maryland & 1,494 & 5.3 & 0.7 & $(4.0-6.6)$ \\
\hline Prince George's County, Maryland & 1,118 & 5.9 & 0.8 & $(4.4-7.4)$ \\
\hline Washington County, Maryland & 530 & 10.2 & 2.4 & $(5.6-14.8)$ \\
\hline Baltimore city, Maryland & 725 & 10.5 & 1.4 & $(7.7-13.2)$ \\
\hline Barnstable County, Massachusetts & 540 & 5.6 & 1.0 & $(3.7-7.5)$ \\
\hline Bristol County, Massachusetts & 2,566 & 8.6 & 0.8 & $(6.9-10.2)$ \\
\hline Essex County, Massachusetts & 2,492 & 7.6 & 0.8 & $(5.9-9.2)$ \\
\hline Hampden County, Massachusetts & 1,910 & 9.5 & 0.9 & $(7.7-11.3)$ \\
\hline Middlesex County, Massachusetts & 4,155 & 6.1 & 0.5 & $(5.2-7.0)$ \\
\hline Norfolk County, Massachusetts & 1,695 & 6.3 & 0.7 & $(4.8-7.7)$ \\
\hline Plymouth County, Massachusetts & 1,762 & 6.2 & 0.9 & $(4.5-8.0)$ \\
\hline Suffolk County, Massachusetts & 2,229 & 7.2 & 0.7 & $(5.8-8.6)$ \\
\hline Worcester County, Massachusetts & 2,503 & 7.0 & 0.6 & $(5.7-8.2)$ \\
\hline Kent County, Michigan & 522 & 7.1 & 1.8 & $(3.7-10.5)$ \\
\hline Macomb County, Michigan & 609 & 9.2 & 1.3 & $(6.6-11.8)$ \\
\hline Oakland County, Michigan & 1,156 & 7.0 & 0.8 & $(5.4-8.7)$ \\
\hline Wayne County, Michigan & 2,188 & 11.4 & 0.9 & $(9.6-13.2)$ \\
\hline Anoka County, Minnesota & 534 & 4.1 & 0.9 & $(2.3-5.9)$ \\
\hline Dakota County, Minnesota & 662 & 4.7 & 0.9 & $(2.9-6.5)$ \\
\hline Hennepin County, Minnesota & 3,188 & 5.1 & 0.4 & $(4.2-5.9)$ \\
\hline Ramsey County, Minnesota & 1,905 & 8.6 & 1.5 & $(5.6-11.5)$ \\
\hline Jackson County, Missouri & 867 & 9.5 & 1.4 & $(6.8-12.2)$ \\
\hline St. Louis County, Missouri & 945 & 10.8 & 1.3 & $(8.2-13.3)$ \\
\hline Cascade County, Montana & 707 & 8.5 & 1.1 & $(6.4-10.6)$ \\
\hline Flathead County, Montana & 555 & 8.7 & 1.4 & $(5.9-11.5)$ \\
\hline Hill County, Montana & 577 & 7.1 & 1.3 & $(4.6-9.6)$ \\
\hline Lake County, Montana & 891 & 7.5 & 0.9 & $(5.8-9.2)$ \\
\hline Missoula County, Montana & 778 & 6.9 & 1.1 & $(4.8-9.0)$ \\
\hline Yellowstone County, Montana & 748 & 8.7 & 1.1 & $(6.4-10.9)$ \\
\hline Dakota County, Nebraska & 730 & 6.3 & 1.2 & $(3.8-8.7)$ \\
\hline Douglas County, Nebraska & 3,537 & 7.0 & 0.5 & $(6.1-8.0)$ \\
\hline Hall County, Nebraska & 533 & 6.9 & 1.1 & $(4.7-9.1)$ \\
\hline Lancaster County, Nebraska & 1,439 & 6.0 & 0.7 & $(4.6-7.3)$ \\
\hline Lincoln County, Nebraska & 587 & 6.7 & 1.2 & $(4.3-9.1)$ \\
\hline Sarpy County, Nebraska & 1,169 & 6.4 & 0.9 & $(4.7-8.1)$ \\
\hline Scotts Bluff County, Nebraska & 577 & 8.0 & 1.4 & $(5.3-10.7)$ \\
\hline Clark County, Nevada & 1,993 & 8.3 & 0.7 & $(6.9-9.6)$ \\
\hline Washoe County, Nevada & 1,475 & 5.9 & 0.8 & $(4.3-7.4)$ \\
\hline Belknap County, New Hampshire & 559 & 6.4 & 1.1 & $(4.2-8.7)$ \\
\hline Carroll County, New Hampshire & 527 & 7.3 & 1.4 & $(4.7-10.0)$ \\
\hline Cheshire County, New Hampshire & 543 & 7.0 & 1.6 & $(3.9-10.2)$ \\
\hline Coos County, New Hampshire & 536 & 12.4 & 2.6 & $(7.3-17.5)$ \\
\hline Grafton County, New Hampshire & 561 & 8.5 & 1.3 & $(5.9-11.0)$ \\
\hline Hillsborough County, New Hampshire & 1,890 & 6.9 & 0.6 & $(5.7-8.1)$ \\
\hline Merrimack County, New Hampshire & 701 & 7.4 & 1.2 & $(5.1-9.7)$ \\
\hline Rockingham County, New Hampshire & 1,037 & 5.6 & 0.7 & $(4.2-7.0)$ \\
\hline Strafford County, New Hampshire & 616 & 8.1 & 1.1 & $(5.9-10.4)$ \\
\hline Atlantic County, New Jersey & 991 & 7.1 & 0.9 & $(5.3-8.8)$ \\
\hline Bergen County, New Jersey & 905 & 4.7 & 0.8 & $(3.2-6.2)$ \\
\hline Burlington County, New Jersey & 666 & 6.6 & 0.9 & $(4.7-8.4)$ \\
\hline Camden County, New Jersey & 722 & 7.6 & 0.9 & $(5.8-9.5)$ \\
\hline Cape May County, New Jersey & 534 & 9.4 & 1.6 & $(6.2-12.6)$ \\
\hline Cumberland County, New Jersey & 543 & 6.6 & 1.0 & $(4.6-8.7)$ \\
\hline Essex County, New Jersey & 1,307 & 6.6 & 0.7 & $(5.2-8.1)$ \\
\hline
\end{tabular}

See table footnotes on page 139. 
TABLE 75. (Continued) Estimated prevalence of adults aged $\geq 18$ years who required use of special equipment* because of any health problem, by county - Behavioral Risk Factor Surveillance System, United States, 2012

\begin{tabular}{|c|c|c|c|c|}
\hline County & $\begin{array}{l}\text { Sample } \\
\text { size }\end{array}$ & $\%$ & SE & $95 \% \mathrm{Cl}$ \\
\hline Gloucester County, New Jersey & 542 & 7.9 & 1.5 & $(5.0-10.8)$ \\
\hline Hudson County, New Jersey & 1,239 & 7.1 & 0.9 & $(5.4-8.9)$ \\
\hline Hunterdon County, New Jersey & 550 & 3.4 & 0.7 & $(2.0-4.7)$ \\
\hline Mercer County, New Jersey & 564 & 5.6 & 1.1 & $(3.5-7.6)$ \\
\hline Middlesex County, New Jersey & 860 & 5.8 & 1.0 & $(3.8-7.9)$ \\
\hline Monmouth County, New Jersey & 688 & 5.1 & 1.0 & $(3.2-7.0)$ \\
\hline Morris County, New Jersey & 824 & 5.6 & 0.9 & $(3.7-7.5)$ \\
\hline Ocean County, New Jersey & 638 & 9.3 & 1.3 & $(6.7-11.8)$ \\
\hline Passaic County, New Jersey & 652 & 5.1 & 0.9 & $(3.2-6.9)$ \\
\hline Salem County, New Jersey & 572 & 10.4 & 1.5 & $(7.5-13.2)$ \\
\hline Somerset County, New Jersey & 612 & 5.3 & 1.1 & $(3.1-7.5)$ \\
\hline Sussex County, New Jersey & 532 & 8.3 & 1.8 & $(4.8-11.8)$ \\
\hline Union County, New Jersey & 683 & 6.4 & 1.3 & $(3.8-9.0)$ \\
\hline Warren County, New Jersey & 518 & 6.9 & 1.2 & $(4.6-9.3)$ \\
\hline Bernalillo County, New Mexico & 2,036 & 9.1 & 0.7 & $(7.8-10.5)$ \\
\hline Dona Ana County, New Mexico & 701 & 10.7 & 1.4 & $(7.9-13.4)$ \\
\hline Sandoval County, New Mexico & 640 & 6.0 & 1.0 & $(4.0-8.0)$ \\
\hline San Juan County, New Mexico & 640 & 8.9 & 1.3 & $(6.4-11.5)$ \\
\hline Santa Fe County, New Mexico & 690 & 8.5 & 1.2 & $(6.1-10.8)$ \\
\hline Kings County, New York & 487 & 9.3 & 2.0 & $(5.4-13.2)$ \\
\hline Guilford County, North Carolina & 509 & 5.8 & 0.9 & $(4.0-7.7)$ \\
\hline Mecklenburg County, North Carolina & 724 & 5.0 & 0.9 & $(3.3-6.8)$ \\
\hline Robeson County, North Carolina & 539 & 13.3 & 2.7 & $(8.0-18.7)$ \\
\hline Wake County, North Carolina & 695 & 5.6 & 0.9 & $(3.8-7.4)$ \\
\hline Burleigh County, North Dakota & 567 & 7.2 & 1.3 & $(4.6-9.9)$ \\
\hline Cass County, North Dakota & 836 & 5.2 & 0.9 & $(3.4-7.0)$ \\
\hline Cuyahoga County, Ohio & 810 & 11.0 & 1.4 & $(8.2-13.7)$ \\
\hline Franklin County, Ohio & 818 & 7.8 & 1.1 & $(5.7-10.0)$ \\
\hline Hamilton County, Ohio & 754 & 9.2 & 1.3 & $(6.7-11.7)$ \\
\hline Lorain County, Ohio & 600 & 7.5 & 1.3 & $(4.9-10.0)$ \\
\hline Lucas County, Ohio & 607 & 8.7 & 1.3 & $(6.2-11.2)$ \\
\hline Mahoning County, Ohio & 576 & 7.8 & 1.3 & $(5.2-10.3)$ \\
\hline Montgomery County, Ohio & 649 & 7.4 & 1.0 & $(5.3-9.4)$ \\
\hline Stark County, Ohio & 608 & 6.5 & 1.1 & $(4.4-8.6)$ \\
\hline Summit County, Ohio & 613 & 9.9 & 1.3 & $(7.3-12.5)$ \\
\hline Oklahoma County, Oklahoma & 1,185 & 9.3 & 0.9 & $(7.5-11.1)$ \\
\hline Tulsa County, Oklahoma & 1,193 & 6.6 & 0.7 & $(5.2-7.9)$ \\
\hline Clackamas County, Oregon & 503 & 7.1 & 1.8 & $(3.6-10.6)$ \\
\hline Lane County, Oregon & 516 & 9.7 & 1.4 & $(6.9-12.5)$ \\
\hline Multnomah County, Oregon & 880 & 6.5 & 0.9 & $(4.7-8.3)$ \\
\hline Washington County, Oregon & 609 & 7.2 & 1.3 & $(4.8-9.7)$ \\
\hline Allegheny County, Pennsylvania & 1,862 & 9.2 & 0.8 & $(7.7-10.8)$ \\
\hline Bradford County, Pennsylvania & 1,845 & 7.9 & 0.7 & $(6.4-9.3)$ \\
\hline Montgomery County, Pennsylvania & 540 & 5.9 & 1.1 & $(3.8-8.0)$ \\
\hline Philadelphia County, Pennsylvania & 2,024 & 9.6 & 0.8 & $(8.1-11.1)$ \\
\hline Pike County, Pennsylvania & 1,876 & 8.0 & 0.7 & $(6.6-9.4)$ \\
\hline Kent County, Rhode Island & 807 & 8.8 & 1.1 & $(6.7-11.0)$ \\
\hline Providence County, Rhode Islan & 3,299 & 8.9 & 0.6 & $(7.7-10.2)$ \\
\hline Washington County, Rhode Island & 651 & 5.8 & 1.0 & $(3.9-7.7)$ \\
\hline Aiken County, South Carolina & 549 & 11.4 & 1.7 & $(8.1-14.8)$ \\
\hline
\end{tabular}

TABLE 75. (Continued) Estimated prevalence of adults aged $\geq 18$ years who required use of special equipment* because of any health problem, by county - Behavioral Risk Factor Surveillance System, United States, 2012

\begin{tabular}{|c|c|c|c|c|}
\hline County & $\begin{array}{l}\text { Sample } \\
\text { size }\end{array}$ & $\%$ & SE & $95 \% \mathrm{Cl}$ \\
\hline Beaufort County, South Carolina & 794 & 7.0 & 1.2 & $(4.6-9.4)$ \\
\hline Charleston County, South Carolina & 1,005 & 8.5 & 1.2 & $(6.3-10.8)$ \\
\hline Greenville County, South Carolina & 896 & 8.3 & 1.1 & $(6.1-10.4)$ \\
\hline Horry County, South Carolina & 771 & 7.3 & 0.9 & $(5.5-9.2)$ \\
\hline Richland County, South Carolina & 956 & 8.0 & 1.0 & $(5.9-10.0)$ \\
\hline Spartanburg County, South Carolina & 649 & 11.0 & 1.9 & $(7.2-14.8)$ \\
\hline Lincoln County, South Dakota & 494 & $\mathrm{~N} / \mathrm{A}$ & $\mathrm{N} / \mathrm{A}$ & $(\mathrm{N} / \mathrm{A}-\mathrm{N} / \mathrm{A})$ \\
\hline Minnehaha County, South Dakota & 874 & 6.3 & 0.9 & $(4.5-8.1)$ \\
\hline Pennington County, South Dakota & 605 & 9.5 & 1.3 & $(7.0-11.9)$ \\
\hline Davidson County, Tennessee & 539 & 6.7 & 1.2 & $(4.4-9.0)$ \\
\hline Shelby County, Tennessee & 520 & 11.4 & 1.7 & $(8.1-14.7)$ \\
\hline Bexar County, Texas & 582 & 8.0 & 1.2 & $(5.6-10.4)$ \\
\hline Dallas County, Texas & 504 & 6.7 & 1.1 & $(4.6-8.9)$ \\
\hline El Paso County, Texas & 618 & 7.7 & 1.3 & $(5.1-10.3)$ \\
\hline Harris County, Texas & 756 & 7.5 & 1.1 & $(5.3-9.7)$ \\
\hline Hidalgo County, Texas & 617 & 9.5 & 1.7 & $(6.2-12.7)$ \\
\hline Tarrant County, Texas & 565 & 7.6 & 1.2 & $(5.2-10.0)$ \\
\hline Travis County, Texas & 1,043 & 4.5 & 0.6 & $(3.4-5.6)$ \\
\hline Davis County, Utah & 1,128 & 5.8 & 0.7 & $(4.4-7.2)$ \\
\hline Salt Lake County, Utah & 3,991 & 5.8 & 0.4 & $(5.0-6.5)$ \\
\hline Tooele County, Utah & 555 & 5.8 & 1.2 & $(3.5-8.2)$ \\
\hline Utah County, Utah & 1,654 & 5.2 & 0.6 & $(4.0-6.4)$ \\
\hline Wasatch County, Utah & 504 & 4.2 & 1.1 & $(2.1-6.4)$ \\
\hline Weber County, Utah & 1,034 & 6.2 & 0.8 & $(4.5-7.8)$ \\
\hline Chittenden County, Vermont & 906 & 4.7 & 0.7 & $(3.3-6.1)$ \\
\hline Rutland County, Vermont & 590 & 8.7 & 1.3 & $(6.1-11.2)$ \\
\hline Washington County, Vermont & 514 & 5.4 & 1.0 & $(3.5-7.3)$ \\
\hline Windsor County, Vermont & 537 & 6.3 & 1.0 & $(4.3-8.3)$ \\
\hline Fairfax County, Virginia & 706 & 5.1 & 0.9 & $(3.3-6.9)$ \\
\hline Clark County, Washington & 786 & 8.5 & 1.1 & $(6.3-10.7)$ \\
\hline King County, Washington & 3,907 & 6.6 & 0.5 & $(5.7-7.5)$ \\
\hline Kitsap County, Washington & 562 & 9.9 & 1.4 & $(7.2-12.6)$ \\
\hline Pierce County, Washington & 1,174 & 8.4 & 0.9 & $(6.6-10.2)$ \\
\hline Snohomish County, Washington & 1,164 & 7.3 & 0.9 & $(5.5-9.2)$ \\
\hline Spokane County, Washington & 943 & 7.0 & 0.9 & $(5.3-8.7)$ \\
\hline Thurston County, Washington & 515 & 13.6 & 2.0 & $(9.6-17.5)$ \\
\hline Whatcom County, Washington & 845 & 7.6 & 1.1 & $(5.6-9.7)$ \\
\hline Yakima County, Washington & 531 & 8.8 & 1.5 & $(5.8-11.8)$ \\
\hline Kanawha County, West Virginia & 641 & 12.9 & 1.5 & $(9.9-15.8)$ \\
\hline Milwaukee County, Wisconsin & 917 & 10.2 & 1.3 & $(7.6-12.8)$ \\
\hline Laramie County, Wyoming & 943 & 8.4 & 1.2 & $(6.1-10.8)$ \\
\hline Natrona County, Wyoming & 818 & 7.8 & 1.0 & $(5.8-9.7)$ \\
\hline San Juan Municipio, Puerto Rico & 665 & 8.6 & 1.3 & $(6.1-11.2)$ \\
\hline Median & & 7.5 & & \\
\hline Range & & $2.8-13$ & & \\
\hline
\end{tabular}

Abbreviations: $\mathrm{Cl}=$ confidence interval; $\mathrm{SE}=$ standard error.

* Including use of a cane, wheelchair, special bed, or special telephone, occasionally or in certain circumstances.

† Estimate not available (N/A) if the unweighted sample size for the denominator was $<50$ or if the relative standard error is $>0.3$. 


The Morbidity and Mortality Weekly Report (MMWR) Series is prepared by the Centers for Disease Control and Prevention (CDC) and is available free of charge in electronic format. To receive an electronic copy each week, visit MMWR's free subscription page at http://www.cdc.gov/mmwr/mmwrsubscribe.html. Paper copy subscriptions are available through the Superintendent of Documents, U.S. Government Printing Office, Washington, DC 20402; telephone 202-512-1800.

Readers who have difficulty accessing this PDF file may access the HTML file at http://www.cdc.gov/mmwr/volumes/65/ss/ss6504a1.htm?s_cid=ss6504a1_w. Address all inquiries about the $M M W R$ Series, including material to be considered for publication, to Executive Editor, MMWR Series, Mailstop E-90, CDC, 1600 Clifton Rd., N.E., Atlanta, GA 30329-4027 or to mmwrq@cdc.gov.

All material in the MMWR Series is in the public domain and may be used and reprinted without permission; citation as to source, however, is appreciated. Use of trade names and commercial sources is for identification only and does not imply endorsement by the U.S. Department of Health and Human Services.

References to non-CDC sites on the Internet are provided as a service to $M M W R$ readers and do not constitute or imply endorsement of these organizations or their programs by CDC or the U.S. Department of Health and Human Services. CDC is not responsible for the content of these sites. URL addresses listed in $M M W R$ were current as of the date of publication.

ISSN: 1546-0738 (Print) 